


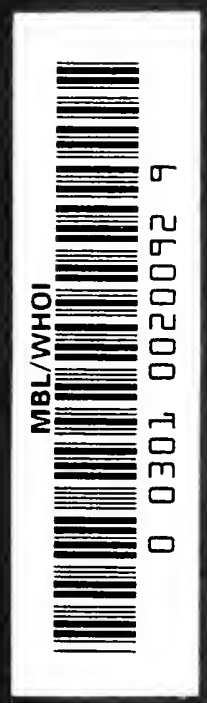





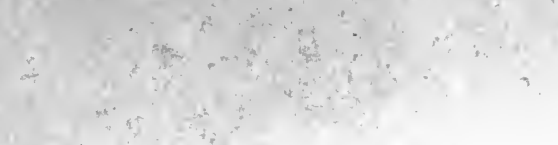

$3 \quad 1$. 



\title{
ELEMENTS
}

OF

\section{Physiological Psychology}

\author{
A TREATISE OF THE ACTIVITIES AND NATURE \\ OF THE MIND
}

FROM THE PHYSICAL AND EXPERIMIENTAL POINT OF VIEW

BY

GEORGE T. LADD

PROFESSOR OF PHILOSOPHY IN YALE UNIVERSITY

NEW YORK

CHARLES SCRIBNER'S SONS

1887 
Coprright, 1887, BY

CHARLES SCRIBNER'S SONS

Thowr

PRINTING AND BOOKBINOINO COMPANY, NEW YORK. 


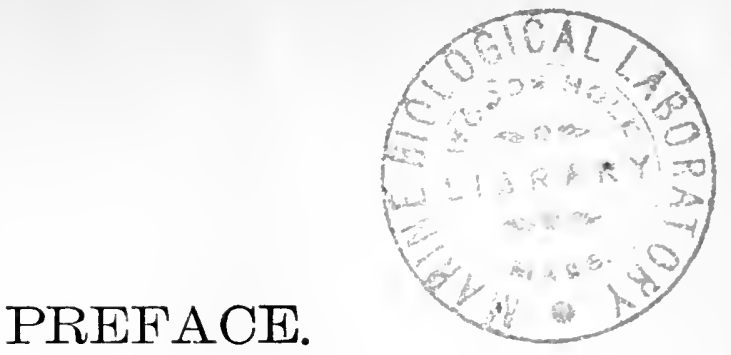

There can be no doubt that an important movement in psychology has arisen in recent times through the effort to approach the phenomena of mind from the experimental and physiological point of view. Different students of psychological science will estimate differently both the net result already reached by this effort and the promise of further additions to the sum of our knowledge from continued investigation of the same kind. Some writers have certainly indulged in extravagant claims as to the past triumphs of socalled Physiological Psychology, and in equally extraragant expectations as to its future discoveries. On the other hand, a larger number, perhaps, have been inclined either to fear or to depreciate every attempt to mingle the methods, laws, and speculations of the physical sciences with the study of the human soul. These latter apparently anticipate that some discovery in the localization of cerebral function, or in psychometry, may jeopard the birthright of man as a spiritual and rational being. Or possibly they wish to regard the soul as separated, by nature and with respect to its modes of action, from the material body in such a way as to render it impossible to understand more of the one by learning more about the other.

As a result of some years of study of the general subject, I express with considerable confidence the opinion that there is no ground for extravagant claims or expectations, and still less ground for any fear of consequences. In all cases of new and somewhat rankly growing scientific enterprises, it is much the better way to waive the discussion of actual or possible achievements, as well as of welcomed or dreaded revelations of new truth, and proceed at once to the business on hand. It is proposed in this book to follow this better way. It will be the task of the book itself to set forth the assured or alleged results of Physiological Psychology; and this will be done at 
every step with such degree of assurance as belongs to the evidence hitherto attainable upon the particular subject discussed. With declamation, either in attack or defence of the "old psychology," of the "introspective method," etc., one may dispense without serious loss.

The study of the phenomena of consciousness by the method here proposed necessarily requires some acquaintance with a considerable circuit of sciences which are not usually all alike elosely allied. The number of scholars who can form opinions with equal freedom and confidence in all of these sciences is very small. Moreover, since all psycho-physical laws are supposed-as the very term indicates-to govern the correlations of phenomena of consciousness with phenomena of the nerrous system, a peculiar mystery belongs to much of the domain within which psycho-physical science is compelled to more. These facts may fitly, on the one hand, excite caution in the writer; and, on the other hand, excuse him for many inevitable failures to set forth with perfect definiteness and confidence the conclusions he has to propose. Much will be said that must be accepted as provisional, as only probably true. Much room must also be made for conjecture and speculation. What is most important, however, is that conjecture should not be put forth as ascertained fact, or speculation as unquestioned law.

It would have been a great assistance to me if I had had more predecessors in the path which I am to take. But with the exception of Wundt's masterly work (Grundzüge der physiologischen Psychologie, second edition in 1880), no one book has attempted to cover, even in a summary way, the entire ground. The number of monographs, however, which have dealt with individual questions subordinate to, or part of, the main inquiry is very great. These two facts also render the attempt at a general survey of Physiological Psychology for readers of English both peculiarly attractive and peculiarly difficult. I can only indulge the hope that $I$ have done something toward breaking this path and rendering it easier and more secure, both for myself and for others, in the future.

The investigators and authors to whom I am under obligations for material upon the rarious questions discussed, or statements made, in this book are by no means all mentioned by name. Of course, much of what is said on the structure of the nerrous system, and on the phenomena of sensation and perception, has already become part of that general fund of facts and laws which belongs alike to all students of the subject. But by quoting certain author- 
ities in the text, and by a few (in comparison with the number which might have been cited) references in foot-notes, I have connected some of the discoveries and views of modern psycho-physical science with their authors. These may serve somewhat as guide to those persons who wish to pursue such studies still further.

I am under particular obligations to Dr. James K. Thacher, Professor of Physiology in the Yale Medical School, for valuable assistance in that description of the Nervous Mechanism, its structure and functions, which the First Part of the book contains. If I lave escaped the mistake of assuming to teach more than is really known upon this subject, it has been in large measure due to his friendly and skilful guidance. Valuable assistance has also been received from Russell H. Chittenden, Professor of Physiological Chemistry, and Charles S. Hastings, Professor of Physics-both of the Sheffield Scientific School.

The method and arrangement of the book have been chosen so as to fit it for use, both as a text-book by special students of the subjects of which it treats, and also by the general reader who is interested in knowing what results have been reached by the more modern-and even the latest-psycho-physical researches.

\section{George T. Ladd.}

Yale University, New Havex, February, 1887.

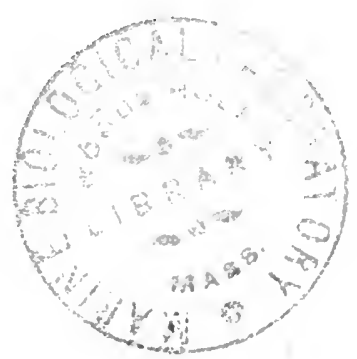





\section{TABLE OF CONTENTS.}

INTRODUCTION PAGE

PART FIRST.

THE NERVOUS MECHANISM.

\section{CHAPTER I.}

The Elements of the Nervous System.............. 17-55

$\S \S 1-4$, General Function of the Nervons System.- $\$ \S 5-16$, Chemieal Constitution of the Nervous Elements. - $\$ 17-30$, Structural Form of the Nervous Elements.- $\$ \$ 31-36$, Common Properties of the Nervous Elements.

\section{CHAPTER II.}

Combination of the Nervous Elements into a SysteM..... 56-101

$\S \S 1-3$, Threefold Plan of the Nervous System. $-\$ 4$, The Sympathetic and Cerebro-spinal Systems. $-\$ 5$, Membranes of Brain and Spinal Cord.- $\$ \$ 6-12$, Structure of the Spinal Cord.- $\$$ 13-14, General Arrangement of the Encephalon.- $\$$ 15, Structure of the Medulla Oblongata. $-\$ 16$, Structure of the Cerebellum.- $\$ 17$, Structure of the Pons Varolii.- $\$ 18-24$, Structure of the Cerebrum.$\$ \S 25-27$, Cortex of the Cerebral Hemispheres. - $\$ \$ 28-29$, Arrangement of the Nerve-Tracts. $-\$ 30$, The Cranial and Spinal Nerves.

\section{CHAPTER III}

The Nerves as Conductors..................... 102-129

$\S \S 1-3$, General Office of the Nerves. $-\$ 4$, The Nerve-Muscle Machine.-\$§ 5-8, The Conditions of Neural Action.-_\$ 9-19, Phenomena induced in the Nerves by different Stimuli.-_\$20-23. Electrical and other Processes in the excited Nerve-Stretch.-\$\$24-26, Laws

\section{1}


of the Nerve-Commotion. $-\$ 27$, Speed of the Nerve-Commotion.$\S 28$, Effect of Section. $-\S 29$, Nervous Conduction in the Central Organs. - $50-32$, Paths of Conduction in the Spinal Cord.- $53-35$, Paths of Conduction in the Brain.

\section{CHAPTER IV.}

Automatic axd Reflex Funcions of the Central Organs.. 130-162

$\$ S$ 1-2, Nature and Kinds of Reflex Action.- $\$ S$ 3-5, The Spinal Cord as a Central Organ. - $5-9$, Laws of Spinal Reflexes. $-\$ 10$, Irregular Automatism of the Cord. $-\$ 11$, Centres of the Cord.-\$12, Excitability of the Cord.— $\$ 13$, Inbibition of the Cord. -14 . The Brain as a Central Organ._- 15-16, Functions of the Medulla Oblongata - $\$ \$ 17-19$, Centres of the Medulla Oblongata. $-\$ 20$, Influence of the Cerebral Lobes. $-\$ 21$, Functions of the Cerebellum.$\$ \$ 22-2 \pi$, Functions of the Basal Ganglia_- $\$ 28$, Gray Matter of the Third Ventricle.

\section{CHAPTER V.}

End-Organs of the Nervous System................. 163-197

§§ 1-2, Characteristics of the End.Organs. - $\$ 3$, The Kinds of EndOrgans. - $\$ 4-5$, The End-Organs of Smell.- $\$ \S 6-7$, The End-Organs of Taste. $-\$ 8-10$, The End-Organs of Touch. $-\$ 11$, The EndOrgan of Sight.- $5-12$, Tunics, Media, and Appendages of the Eye.- $₫ 17$, The Mechanism of Accommodation._-S 18-21, Structure and Functions of the Retina.- $\$ 22$, Photo-Chemistry of Vision.$\$ \S 23-26$, External and Middle Ear. - $\$ 27$, Structure of the Labyrinth. $-\$ 28$, End-Apparatus of the Vestibule. - $\$ 29$, The Organ of Corti.$\$ \S 30-31$, Problem solved by the Labyrinth. - $\$ 32$, End-Organs of Motion.

\section{CHAPTER VI.}

The Development of the Nervous Mechanism........... 198-213

$\$ 1$, Nature of Embryonic Life. $\$ S$ 2-5, Earliest Development of the Ovum. - $\leqslant$ 6-8, Blastodermic Layers and their Differentia. $-\leqslant \leqslant 9-$ 11, Head-Fold and Brain-Vesicles. $-\$ 12$, Development of Cranial and Spinal Nerres.- $\$ S$ 13-15, Subsequent Development of the Brain. - 5 16-17, Development of Eye and Ear.- 18 , Histogenetic Changes in the Embryo. $-\$ 19$, Conclusions.

\section{CHAPTER VII.}

Mechanical Theory of the Nervous Srstem........... 214-236

$\S 1$, Machine-like Nature of the Body.- $\$ \$ 2-6$, The Nerrous System as a Mechanism. $-\$ 7$, Summation and Interference in Nerves.$\$ 8$. Evidence from the Electrical Phenomena. $\$ 9$, Theory of du Bois-Reymond.- $\$$ 10-11, Theory of Hermann.—S 12-13, Theory of Wundt. - $\$$ 14-15, General Conclusions as to a Mechanical Theory. 


\section{PART SECOND.}

\section{CORRELATIONS OF THE NERVOUS MECH- ANISM AND THE MIND.}

\section{CHAPTER I.}

PAGE

Time Localization of Cerebral Function

239-262

\$S 1-3, Proofs of the Brain's Special Significance.-S 4-7, The Brain as a Measure of Intelligence.-- $\$$, Special Significance of the Cerebral Hemispheres.-S 9-10, The Question of Localization.$\$ \$ 11-13$, The History of Discovery. - $\$ 14-16$, The Evidence from Experiment. $-\$ 17$, The Evidence from Pathology. $-\$ 18$, The Evidence from Anatomy. $-\$ 19$, True Method of Investigation.

\section{CHAPTER II.}

The Localization of Cerebral Fuxctiox [Continued]....... 263-302

$\S \S 1-4$, Difficulties from Negative Cases. - $\$ 5-6$, Experiments in Stimulation. - $\$ 7$, Experiments in Extirpation.- $\leq \leqslant 8$ 8-9, Nature of so-called Motor Centres. - $\$$ 10-15, Method and Results of Exner.$\S 16$, Confirmatory Conclusions from other Sources. $-\$ 17$, The Evidence of Histology. - 18 , Relation of Motion and Sensibility. - $\$ \$ 19-$ 21, Visual and Auditory Centres of Ferrier and Munk. - 22 , Exner's Cerebral Field of Vision. - $\$ 23$, Relations between the Retinas and the Cerebrum. $-\$ 24$, Localization of Smell and Taste.- $\$ \$ 25-$ 27, The Phenomena of Aphasia. - $\$ 28$, Cerebral Lesions in Aphasia. $-\$ 29$, Conjectures as to the Frontal Lobes. - $\$ 30$, Negative Conclusions of Goltz. $-\$ 31$, Conclusion as to three leading Principles.

\section{CHAPTER III.}

Tile Qdality of Sensations.

$\S \$ 1-5$, Sensations and Things. $-\$ 6$, The Subjects investigated.$\$ 7$, Specific Energy of the Nerves. - $\$ 8-11$, Sensations of Smell. - $\$ \$ 12-15$, Sensations of Taste. - $\$ \$ 16-17$, The Varieties of Sound. - $\$ 18-20$, The Pitch of Tones._ $\$ 21-22$, The Composition of Clangs. $-\S 23$, Analysis of Sounds by the Ear.

\section{CHAPTER IV.}

The Quality of Sensations [Continned]............... 325-355

$\S 1$, Analysis of Sensations of Sight. $-\$ \$ 2-3$, The Stimulus of Sight. $-\$ 4$, Relation of Quality and Quantity. $-\$ \S 5-8$, The Different Color- 
Tones. $-\$ 9$, The Complementary Colors.-\$§ 10-13, Conditions of Changes in Color. - $\$ 14$, Phenomena of Contrast. - $\$ \$ 15-17$, Theories of Visual Sensations. $-\S 18$, Symbolism of Visual Sensations. $-\$ 19$, Sensations of the Skin.- $\$ 20$, The Muscular Sensations.- $\$ 21$, Sensations of Pressure. -5 22-24, Sensations of Temperature. $-\$ 25$, Specific Energy of the Nerves.

\section{CHAPTER V.}

The Qdantity of Sensations...................... 356-381

\$ 1-3, Distinction of Variations in Quantity. - $\$ \$ 4$ 4-5, The Measurement of Sensations. - $\$ 6$, Nature of the Least Observable Difference. $-\S 7$, The Determining of the Limits. $-\$ 8$, Methods of Experiment. $-\S 9$, Statement of Weber's Lav. $-\S 10$, Measurement of Sensations of Pressure. -511 , Measurement of Sensations of Temperature.- $\$ \$ 12-15$, The Intensity of Sounds.- $\$$ 16-18, The Intensity of Visua! Sensations. $-\$ 19-21$, Measurement of Taste and Smell._- 22-23, Value of Weber's Law.

\section{CHAPTER VI.}

The Presentations of Sense....................... 382-419

$\S \S 1-2$, Sensations and Things. $-\$ 3$, General Nature of the Presentations of Seuse. - $\$ \$ 4-5$, Laws of the Synthesis of Sensations.\$ 6-7, Nativistic and Empiristic Theories. $-\$ 8-11$, Nature of the Spatial Series. - $\$$ 12-15, The Theory of Local Signs. $-\$ 16$, The Stages of Perception. - $\$ 17$, Perceptions of Smell. $-\$ 18$, Perceptions of Taste.- $\$ \S 19-20$, Perceptions of Hearing. $-\$ \S 21-22$, Sense of Locality by the Skin.-_§ 23-25, Weber's Sensation-Circles._\$ 26, The Discernment of Motion.-- $\$ 27$, Localizing of Temperature-Sensations. $-\$ 28$, Localizing of Muscular Sensations.- $\$ \$ 29-30$, Construction of the Field of Touch. $-\$ 31$, Feelings of Double Contact.

\section{CHAPTER VII.}

The Presentations of Sense [Continued] .............. 420-467

$\S 1$, General Principles applied to the Eye. - $\$ 2$, Data or Motifs of Vision.- $\$$ 3-6, Nature of the Primary Retinal Field. $\$ \$ 7$, Value of the Retinal Elements. - $\$ s-9$, Motions of the Eye.- $\$$ 10, The Law of Listing. - $\$ 11$, Meridians of the Field of Vision. - $\$ 12$, Effect of Accommodation.- $\$ \$ 13-16$, Single and Double Images.- $\$ 17$, The Fixation of Attention.- $\$ \$ 18-20$, Stereoscopic Vision and Vision of Perspective.-SS 21-23, The Use of Secondary Helps. - $\$ \$$ 24-25, General Office of Experience.- $\$ \$ 26-28$, Judgment of Spatial Extension and Relations. $\longrightarrow$ 29, Visual Perception of Motion. $-\$ \$ 30-34$, Errors of Sense. $-\S 35$, Development of Visual Perception. 


\section{CHAPTER VIII.}

$\S \S 1-2$, Time-Form as belonging to Mental Phenomena.- $\$$ 3-4, Elements of Physiological and Psycho-physical Time._\$ 5-7, Effect of the Inertia of the Nervous System. - 8-12, Nature and Length of Simple Reaction-Time.- $\$$ 13-15, Methods for discovering Apperception-Time. - $\$ \S 16-18$, Iength of Apperception-Time.- $\$ \$ 10$ 20, Nature of Will-Time.- $\$ \$ 21-23$, Subjective Estimate of Time.$\$ \$ 24-25$, Reaction-Time of complex Mental Processes. - $\$ 26$, The Circuit of Consciousness. - $\$ 27$, Various Influences upon Psycho-physical Time. $-\$ 28$, Conclusions.

\section{CHAPTER IX.}

Feelings and Motions.

$\$ 1$, Nature of the Inquiry. - $\$ \S 2-4$, Physiological Theory of the Nature of Feeling. - $\$ \$ 5-7$, Psychological Theory of the Nature of Feeling. - $\$ \$ 8-9$, Classification of the Feelings.- $\$ \$ 10-11$, Characteristics of all Feeling. - $\$ 12$, Physical A pparatus of Feeling. - $\$ 13-$ 14, Nature of Common Feeling. - $\$ \$ 15-18$, Feelings of Sensation.$\$ \$ 19-21$, The Emotions. - $\$ 22$, Mental Moods._- $\$ 23-24$, The Æsthetic Feelings. - $\$ 25$, The Intellectual Feelings._\$ 26, The Feeling of Effort.- $\$ \$ 27-31$, Voluntary and Involuntary Movements.

\section{CHAPTER $\mathrm{X}$.}

Physical Basis of the Higher factlties. . . . . . . . . . . 532-559

$\S \S 1-3$, The Method of Investigation. - $\$ \S 4-6$, Physiological Basis of Acts of Will. - $\$ \$ 7-9$, The Will in Attention.- $\$ \$ 10-11$, Cerebral Processes in Attention. $-\$ 12$, Freedom of Will. $-\$ 13$, Physical Basis of Consciousness. - $\$ 13-14$, Physiological Basis of Memory. - $\$ 15-$ 17, Memory as Retentive.- $\$ 3$ 18-19, Memory as Reproductive. $\S \S 20-21$, Organic Memory.-. $\$$ 22, Memory as a Psychical Process. $-\$ 23$, Physical Basis of Conception.

\section{CHAPTER XI.}

Certatn Statical Relations of the Body axd Mental Phe-

NOMENA............................... 560-582

$\$ \S 1-3$, Popular Estimate of the Relations of Body and Mind.$\S \S 3-9$, Relations Dependent on Age and Development.- $\$$ 10-12, Relations Dependent on Sex. - $\$ 13$, Characteristics of different Races. $-\$ 14$, The Theory of Temperament. $-\$ \$ 15-17$, Kinds of Temperament. $-\S 18$, Physical Basis of Temperament.- $\$ 19$, General Corre. lations of Body and Miud. 


\section{PART THIRD. \\ THE NATURE OF THE MIND.}

\section{CHAPTER I.}

The Faculties of tire Mind, and its Unity

$\S \S 1-4$, The Method of Investigation.- $\$ \S 5-6$, Mental Phenomena and Cerebral Changes. - 7-9, Physical Theory of the Cause of Psychical States. $-\$ 10$, The Subject of Psychical States._- $\$ 11-13$, Variety of the Phenomena of Consciousness. - $\$ \$ 14-16$, Classification of Psychical States. - $\$ 17$, Nature of the Mental Faculties. - $\$ \$ 18-22$, The Unity of Consciousness.

\section{CHAPTER II.}

The Development of the Mind.................. 614-632

$\S \S 1-2$, Genetic Study of the Mind.- $\$ \S 3-5$, Reality of Mental Development.- $\$$ 6-8, Stages of Mental Development.- $\$ \$$ 9-10, Dependence of Mental on Physical Growth._\$11, Psychical Factors in Development.- $\$ \S 12-16$, The Theory of Mental Evolution.

\section{CHAPTER III.}

Real Connection of Bratn and Mind ................6633-667

$\S 1$, General Question of a Connection of Brain and Mind.- $\S \S 2-6$, The Brain as the "Seat" of the Mind. - $\$$ 7-9, The Brain as the "Organ" of the Mind.- $\$ 10$, Special "Bond" between Brain and Mind.- $\$ 11$, Figurative Connection of Brain with Mind. $-\$ 12$. Causal Relation of Brain and Mind.- $\$ \$ 13-14$, Occasionalism and Pre.established Harmony. - $\$ 15$, Positivism and Monism.- $\$ 16$, The Position of Dualism. - $\$ 17$, Conservation and Correlation of Energy.- $\$ 18-$ 24, The Causal Nexus declared Valid.

CE.AP'TER IV.

The Mind as Real Being .......................... 668-688

$\S 1$, The Metaphysical Treatment of Mind.- $\$ \S 2-8$, The Mind as a Real Being.- $\$ \S 9-10$, The Spirituality of Mind.- $\$ \S 11-15$, The Unity of Mind. $-\$ 16$, First and Last Things of the Mind. 


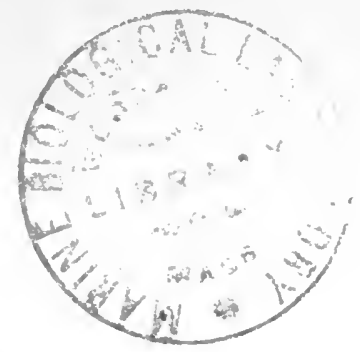

\section{PHYSIOLOGICAL PSYCHOLOGY.}

\section{INTRODUCTION.}

§1. A clear conception of Physiological Psychology requires some special knowledge of the nature and methods of those two sciences, the results of whose investigation it endeavors to combine. These sciences are, of course, Psychology and Physiology-the latter being understood in a broad way as including also various applications of the general theory of physics to the functions of the animal organism. But as the form taken by this compound term would itself seem to indicate, the two do not stand upon precisely the same level in effecting this combination, whether we consider the end that the one science into which both enter desires to reach, or the means that it employs to reach the end. For the noun ("psychology") in the compound term may be said more particularly to define the end desired; the adjective ("physiological") the character of the means which it is proposed especially to employ. Hence "Physiological Psychology" can scarcely claim to be an independent science, or even a definite branch of the science of psychology in general. It is rather to be regarded simply as psychology approached and studied from a certain-the so-called "physiological "-side or point of view. It is necessary, then, in the first place, to define what we understand by the science of psychology, and how it is proposed to treat this science as subject to the plyysiological method, and as approached by means of physiological experimentation and researches.

\$2. Perhaps the most common definition of psychology, up to the present time, has regarded it as "the science of the human soul." If this definition had always been given, on beginning the pursuit of the science, only in a provisional way, and with the implied or open confession that it is the business of psychology itself to demonstrate the existence of a particular entity called "the soul," and 
to show how this entity is needed to explain the phenomena of con. sciousness, then little valid objection could have been made to it. But such has by no neans been the case. For example, one writer on the subject (Drbal), at the very commencement of his treatise, asserts that "psychology is the science of the lhuman soul as the real foundation of the spiritual life ;" and another (Erdmann) cleclares that " the subject-matter of psychology is the subjective spirit," meaning by this term the liuman soul. Objections have, therefore, been more or less fitly and forcefully urged against this definition as ordinarily employed. It has been said that clearly we have no right to assume any such entity as the soul ; and even that a careful study of all the phenomena-especially by the experimental and physiological method-does not compel or induce us to conclude that sucl entity exists. It has been claimed, especially of late, that there may be a "psychology without a soul," and, indeed, that this kind of psychology is alone worthy of being considered truly scientific. Further objestion to the same definition has been made in other quarters, because it seems to regard the question as settled, whether man lias not more than one subject (or "ground") of the manifold phenomena called psychical ; whether, in fact, he may not be the fortumate possessor of both an "animal" and a "rational" soul, etc. It would be aside from the course of our inquiries to consider these objections in detail at this time; or to state at any length how far we are inclined to agree with them and how far to express dissent. They may all be, for the present, set aside by stating the course of procedure which the study of psychology from the plyysiological point of view seems to us plainly to recommend.

The satisfactory definition of any science is often one of the latest and most difficult achievements of that science. When such definition is placed at the beginning of an investigation, it must often really inclucle results reached only by going carefully and repeatedly over the entire ground of the scicnce. In all such cases the learner of the science is quite unable fully to comprehend the definition, or to understand the positions upon various disputed questions which it may really involve. In general, then, it is better that the earliest so-called definition should be simply a description of that class of phenomena which it is proposed, as far as possible, to isolate for purposes of inquiry. This remark applies with peculiar force to psychology, both on account of such objections as those mentioned above, and also on account of certain difficulties inherent in the subject itself. Accordingly, it will serve our purpose best to "define" this science simply by ascribing to it a certain more or less 
definite sphere of phenomena. Thus we shall consider psycliology as that science which has for its primary subject of investigation all the phenomena of human consciousness, or of the sentient life of man. If the term "sentience" be employed as preferable to consciousness, it must be understood as equivalent to consciousness in the broader sense of the latter word. This definition, or rather description, plainly implies an acquaintance experimentally with certain phenomena that cannot, strictly speaking, be defined. These are the phenomena of consciousness ; and one result of all our subsequent iuvestigations will be to show us that consciousness and its primary phenomena can nerer be defined. The definition of psychology need not, however, be understood to imply the real existence of any one entity such as a soul:

Nerertheless it would be very inconvenient, not to say impossible, to begin and continue the investigation of psychical phenomena, using only roundabout phrases through fear of implying the real existence of some spiritual entity called the Soul or the Mind. In some sort there cannot be any description, much less any scientific study, of the phenomena of consciousness without implying somewhat which requires us to use a word like these. In all languages, and in the constant everyday use of them all, men in stating and describing the phenomena of their own sentient life employ such terms as "I" and "me," and place in a kind of contrast with them such other terms as "thou" and "he" or "it." Inasmuch as recollection, and the assumption of some lind of continuous personal identity, enter into all their experience, and underlie all their relations with each other and with the physical world which surrounds them, they are compelled to use language implying a permanent subject of the phenomena of consciousness. No one doubts as to his right to ascribe to himseif the phenomena of his oun consciousness; and as well to ascribe certain other phenomena, which are not attributed to himself as their subject, to other subjects (so-called "persons"), which he supposes to have, each one, a consciousness of his own. No one doubts that this subject is in every case somehow the same with itself from hour to hour and day to day, and even from year to year. In all the earlier part of this treatise the word "mind" will be employed simply as the equivalent of the subject (which all language as expressive of universal experience necessarily recognizes) of the phenomena of consciousness. In other words, whatever all men inevitably mean by the word "I" (the empirical ego of philosophy), whenever they say $I$ think, or feel, or intend this or that; and whatever they understand others to mean by using similar language-thus much, and 
no more, we propose at first to include under the term "mind." This term is preferred to the word "soul," in part out of concession to the prejudices to which allusion has already been made, and in part because it seems to admit of the handling which it is proposed to give to it subsequently, with more freedom from entangling alliances with ethical, social, and religious ideas. In other words, we wish to begin and continue as far as possible upon purely scientific grounds. And when, subsequently, these grounds are in part abandoned for certain fields of rational speculation, we wish to have the connection between the two kept open and unimpeded.

§3. In accordance with what has already been said concerning the nature of psychology, we may define Physiological Psychology as the science which investigates the phenomena of human consciousness from the "physiological" point of view or method of approach. Remembering the cautions which have already been expressed, we may also say that it is the science of the human mind as investigated by means of its relations to the human physical organism. A more accurate definition, however, requires that something further should be sail concerning the nature and method of that science which furnishes the aljective to our compound term. Human Physiology is the science of the functions (or modes of the behavior in its correlated action) of the human physical organism. As studied at present it implies an acquaintance with the fields of gross and special microscopic anatomy (histology), of embryology and the general doctrine of development, of biology,-including the allied phenomena of plant life,- - of molecular physics and chemistry as related to the structure and action of the bodily tissues, and of other forms of kindred knowledge. It is only a relatively small part of this vast domain, howerer, with which Physiological Psychology has directly to deal ; for it is only a part of the human organism which has any direct relation to the phenomena of consciousness. As will appear subsequently, it is with the nervous system alone that our science has its chief immediate concern. Indeed it might be described - though in a still somewhat indefinite, but more full and complete, way-as the science which investigates the correlations that exist between the structure and functions of the human nervous mechanism and the phenomena of consciousness, and which derives therefrom conclusions as to the laws and nature of the mind.

$\S 4$. Plyysiology is compelled, from its very mature as a physical science, to regard the nervous system as a mechanism. Physiological Psychology, inasmuch as it relies so largely upon physiology for its data and method and points of view, is also required to consider 
this system in the same way. Those unique relations in which the structure and functions of the nervous substance of the body stand to the phenomena of the mind cannot deter the investigator from assuming toward it the so-called mechanical point of view. Physiology presents psychology with a description of this nervous substance as a vast and complex system of material molecules, which are acted upon by different forms of the energy of nature outside (external stimuli), and by intimate changes in the contiguous molecules of the other substances of the body (internal stimuli); and which behave as they do on account of the influences thus received, as well as on account of their own molecular constitution and arrangement. But all this is the description of a material mechanism. The word "mechanisn" is preferable to the word " machine" for describing such a system of interacting molecules as constitute the living nervous substance, because we attach to the latter word the mental picture of something which has a certain magnitude and rigidity of parts that act and react upon each other in a palpable way under the ordinary laws of mechanics. A steam-engine is a machine whose parts may be seen to push and pull and turn each other after the ordinary fashion of all levers and wheels. But the molecules of the steam, from the activity of which all the motion of the rigid and ponderous parts of this machine is derived, are no less material and governed by physical law in their changing relations to each other than are the masses of the machine itself. The interaction of the minute particles of the steam falls more fitly, however, under the conception of mechanism. Indeed, it is only as falling under this general conception that these molecules admit of any scientific treatment at all. Now it is not our purpose to begin the consideration of the human nervous system by debating the question, how completely it falls under the conception of mechanism, and whether some other conception be not needed to supplement this when the unique relations of this system to the phenomena of the mind are taken into account. Whatever is to be said upon such a question must appear in its proper place in the order adopted for the discussion of the general subject. Physiological Psyclology, however, can scarcely establish itself at all unless it be willing to receive from the proper one of the two sciences which enter into it that conception of the nervous system at which this science has arrived as the result of the most successful modern researches. As far as the nervous system admits of being subjected at all to scientific treatment, for the purpose of attaining a more complete knowledge of the nature of its functions, it is necessarily considered as a complex molecular mechanism. We shall, then, receive, in a grateful 
and docile manner, all that the noble science of human physiology has to teach us, under the guidance of the conception of a mechanism, both directly concerning the manner in which the nervous matter of the human body performs its wonderful functions, and more indireetly concerning the relations in which these functions stand to the phenomena of conseiousness.

§5. Physiological Psychology-it is by this time apparent-partakes of the nature and methods of two seiences that differ widely from each other. One is a scienee which involves introspection; for it is only by the method of introspection that the real and present facts of human consciousness can be reached. The other is a physical science, and involves external observation to detcrmine the external faets of the structure, development, and functions of a physical mechanism. Two sets of phenomena must then be examined in their relations to each other, and, so far as possible, the laws (or permanent modes) of these relations pointed out. It is due to this fact, in part, that both the peculiar difficulties and the peculiar interest and value of psycho-physical researches are so great.

In every science a beginning is first made by ascertaining and comparing together all the important phenomena; the laws, or regular modes of the occurrence of the phenomena in relation to each other, are then investigated ; and, finally, certain conclusions are drawn concerning the nature and significance of those real beings which reason compels us to assume as permanent subjects of the different classes of phenomena. In its effort to establish itself upon a scientific basis, Physiological Psychology has no ehoice but to follow essentially the same method of procedure. In its case, however, as has already been remarked, the phenomena which are to be ascertained and compared belong to two orders that obviously differ greatly from each other; and the laws which it is sought to discorer are laws which maintain themselves between these two orders of phenomena. The phenomena of the nerrous system, like all physical phenomena, consist in changes in the constitution and mutual relation of material masses and molecules. They are, then, of a kind to be related to each other, under the conception of mechanism, inside of the nervous system and of the entire human body; and also, outside of the body, to the various forms of physical energy in nature which act upon these masses and molecules. But the psychical phenomena are states of consciousness, constantly shifting modes of the behavior of that subject which we have agreed-as much as possible without involving any premature assumptions-to call the Mind. Still the above-mentioned two orders of phenomena 
are obviously to a large extent related to each other ; they mar, in fact, be said to be correlated in a unique manner. The constant forms of this correlation constitute the laws for the discovery of which Physiological Psychology undertakes its special researches. It endeavors to bring the two orders of plienomena face to face, to look at them as they stand thus related to each other, and, as far as possible, to unite them in terms of a uniform character, under law.

It might seem that simply to attempt the accomplishment of the task just described should satisfy all legitimate demands. And, indeed, no little protest has of late been made against any attempt on the part of scientific psychology (and how much more when studied from the physiological and experimental point of riew) to proceed further than this. All inquirers have been warned, not only against introducing metaphysical assumptions into the beginnings of psychology, but also against allowing any admixture of the two during the investigations pursued by the latter. We have, indeed, just agreed that metaphysical assumptions as to the nature of mind should prejudice as little as possible our statement of psychological facts and laws. But if the warning against so-called "metaphysics" be understood to mean that inquiry must be stopped when the phenomena and their uniform modes of relation have been enumerated, and that no venture must be made upon any discussions or conclusions regarding the real nature of the subject of them all (the mind), such warning may very well be quietly disregarded. What we are chiefly interested in, on undertaking all psychological investigation, is the real nature-the permanent characteristics, the claims to be a substantial existence, a spiritual unity-and the origin and destiny of the mind. To assume as little as possible concerning all this, at the first, is simply a matter of wise reserre and self-control in the interests of scientific investigation. We feel no hesitancy, howerer, in announcing our intention, ultimately, to draw whatever conclusions seem to us legitimate and desirable concerning many of these so-called "metaphysical" inquiries. Psychology-no less truly when studied from the phrsiological and experimental point of view-has the undoubted right, and is under the obligation, to contribute as much as possible toward the solution of these inquiries. Nor do observation and wide reading show that the advocates of "psychology without a soul," and freed from all metaphysics, are at all certain to aroid drawing conclusions, not to say introducing illegitimate assumptions, upon these very same inquiries. In brief, Physiological Psychology has the right, which belongs to it as a science, to introduce whatever conclusions as to the nature of mind follow legitimately from its 
discussions of phenomena and laws. It has eren a right to indulge in well-founded and reasonable speculation. Such things are not necessarily objectionable when indulged in by any of the more purely physical sciences. Indeed, there is not one of these sciences which would not look comparatively bare and unattractive if wholly stripped of its more or less questionable inferences, its metaphysical assumptions, its guessings, and speculations.

\&6. The remarks immediately foregoing serve to indicate what are the principal Divisions of this work. The First Part will consist of a description of the structure and functions of the Nerrous System. This system will there be considered under the conception of a mechanism, and as far as possible without any direct or indirect reference to the phenomena of consciousness as determined by introspection. The Second Part will describe the various classes of correlations which exist between the phenomena of the nerrous mechanism and mental phenomena. It will also attempt to state what is known of the laws which maintain themselres over these various classes. No attempt will be made, however, to describe and discuss any of the phenomena which may be classed as abnormal, or as consisting (so far as they are psychical) in so-called " disturbances of consciousness," except when reference to such abnormal phenomena is necessary in order to explain those which are called ordinary or normal. The phenomena of insanity, delirium, hypnotism, somnambulism, ecstasy, mind-reading, spiritualism, and even of sleep and dreaming, will therefore be definitely excluded. The chief reason for such exclusion is to be found in a lack of space, it being difficult even to bring within the limits of a single volume a sufficiently thorough discussion of the more ordinary plenomena with which Physiological Psychology is called upon to deal.

The various correlations of the mind and the nervous mechanism (of which the Second Part treats) may be conveniently considered under several principal groups or classes. The first of these includes more particularly such relations as can be established between the condition and activity of the supreme nervous centres and the phenomena of conscious sensation and volition. Most of what can be said at present upon this point may be summed up in the discussion of the localization of cerebral function, as taken in connection with the description of the automatic and reflex action of these centres considered as parts of the nervous mechanism. The second class of these correlations covers all the phenomena with which psycho-physics (in the more precise use of the term) attempts to deal. It discusses the relations which exist between the quality, 
quantity, combination, and order of succession in time, of the various stimuli which act upon the nerrous system, and the kind, magnitude, composite result, and time-relations of the mental phenomena. Hence the significance of the term psycho-physics. As Physiological Psychology is ordinarily and legitimately treated, it includes these more specially psycho-physical researches. Another class of these correlations covers certain related phenomena of mind and body as dependent upon age, sex, race, etc.

Besides the foregoing groups, or classes, certain observations which have more or less of scientific confirmation and value, may be made regarding the physical basis of the feelings and volitions controlling the bodily members, and of the higher faculties of memory, association of ileas, etc. The Third Part will fitly introduce, at the close of the psycho-physical researches, the presentation of such conclusions as may be legitimately gathered, or more speculatively inferred, concerning the nature (considered as a real being) of the human mind. The justification of the order and extent of the entire discussion, and especially of the Third Part as a whole, has already been given to some extent; the rest must be left to the progress and result of the discussion itself.

$\S 7$. It has already been said that the peculiarity of Physiological Psychology, considered as a branch of the general science of mind, consists largely in the method of its approach to its subject. Attention must now be more specifically called to this method as necessarily partaking of the methods of the two sciences whose researches it undertakes to combine. The method of physiology, which is in general that of external observation as employed in all the physical sciences, should be applied only when supplemented by the many delicate and accurate instruments of observation now at command, and guarded and checked by that accumulation of experience concerning the best ways of studying nature and concerning her ways of working which the whole body of such sciences has made. On the other hand, the method of psychology has ordinarily been defined as solely the method of introspection or selfconsciousness. These two methods are obviously very different. It would not be strange, then, if the science which finds it necessary to combine the two should experience some special difficulty. This difficulty has, however, more often been exaggerated than explained and (what is quite possible) for the most part removed.

Our present purpose does not require that we should examine at length the question whether the introspective method is the only one possible in psychology. Scarcely more is necessary than the statement of the bearing of this question upon the inquiries it 
is proposed to make. There should in general be no mystery or arrogant assumption about the use of such words as "science" and "scientific method." Science is nothing but knowledge-real, verifiable, and systematic. Scientific method is nothing but the way of arriving at such knowledge. Now, although Physiological Psychology brings the investigator face to face with some of the most interesting and distinctive mysteries, it is not, as a science, to be regarded as especially mysterious. Inasmuch as its specific business is to ascertain and combine, under definite laws, two widely differing classes of facts (facts of the human nervous mechanism and facts of human consciousness) it is, of course, compelled, first of all, to ascertain both kinds of facts. The phenomena of consciousness, as primary facts, can be ascertained in no other way than in and by consciousness itself. Whatever fault may be found with the socalled introspective method in psychology, on account of its alleged inaccuracy, lack of scientific and progressive quality, etc., from the very nature of the case no other way of ascertaining what the phenomena of consciousness in themselves are can ever take the place of the direct examination of consciousness. And there is no way of directly examining consciousness but the way of being conscious one's self. On the other hand, it is perfectly obvious to students of psychology and of its history (on grounds which need not be stated here) that the scientific treatment of the facts of consciousness can never be, to any satisfactory extent, accomplished by introspection alone. For psychology, in order to make valid its claim to be a science, must not merely display the alleged facts of individual mental experience; it must treat these facts analytically, must resolve them into their ultimate factors, and trace the stages of their development from what is simpler to what is more complex; it must also show on all sides their connections and causes, thus placing the phenomena of the mind as much as possible in interaction with the rest of the world. It is because human physiology can contribute largely to such scientific treatment (as distinguished from the mere observation, grouping, and cataloguing) of the phenomena of the mind that it is entitled to be consiciered as furnishing one distinctive and fruitful branch of psychological researches.

$\S 8$. The following statements will, accordingly, be found to bold good concerning the method of Physiological Psychology. It must employ faithfully the methods distinctive of both the two sciences which it eudeavors to combine. Facts as to the structure and functions of the nervous mechanism, and as to the effect upon it of various kinds of physical energy acting as stimuli, must be ascertained by external observation. In general they must be accepted 
by us as contributed from the modern science of human physiology. The primary facts of consciousness must be ascertained from consciousness itself; or, since they have already been for a long time subjected to this form of observation, and tabulated, compared, and classified, they may be accepted from the science of introspective psychology. Care must be taken, however, to make sure that all alleged psychical facts are really facts; but upon this point, again, there is no other way of making sure than in and through consciousness. The principal laws and inferences also of introspective psychology may be accepted (at least in a provisional way) on beginning the study of Physiological Psychology. The final result of such study will doubtless be, not only to supplement and explain, but also to modify and correct, the statement of these laws and inferences. But here, as in other scientific research, we are obliged to work our way through many mistakes, obscurities, and other obstacles, progressively nearer the complete and verifiable knowledge of the truth.

Furthermore, from the nature of the case, Physiological Psychology takes its point of starting from the facts and laws of physiology as reached by the method of external observation. This follows necessarily from the relation in which the two sciences of physiology and psychology stand as entering into the proposed combination. The enlargement of our knowledge of the latter is the end to be reached; but the former is to give us the way by which, and the guidance under which, the approach to this end must be made.

It will also become evident, in the course of the following investigation, that we are seldom or never able to proceed directly with the work of comparing the immediate physical antecedents or consequents of the mental phenomena with these phenomena themselves, and so of drawing conclusions at once as to the laws by which the two classes of facts are connected. Such immediate antecedents and consequents are hid in the inexplorable recesses of the living and molecularly active brain. It is seldom, indeed, that our direct observation can approach within the tenth, or it may be within the hundredth, remore of what goes on in these recesses. We are obliged to examine the physical phenomena from a greater distance and in a more indirect way. For example, physics can inform us what combinations of what wave-lengths of the vibration of ether fall on the eye when a certain form of conscious sensation, which we call "yellow" or "red" or "blue" arises; physiology can locate the nervous elements of the retina upon which the waves fall, can conjecture something as to the chemical changes there produced, 
and trace doubtfully the paths along which the resulting nerrous impulses rise to the brain and diffuse themselves orer certain of its areas; psycho-physics can tell approximately the relations in which the varying quantities of the stimulus stand to the resulting degrees of the sensatiors. But in all this we are still at a great distance from the enjornent of those opportunities which would seem necessary to make the science of Physiological Psychology as comprehensive and exact as could readily be wished. As a rule, certain kinds and amounts of physical energy, more or less definitely measurable, are known to be acting on the peripheral parts of the body, and the next series of observed facts is the emergence in consciousness of a psychical experience quite unlike all kinds of physical energy. To be sure, Fechner's ' couception of psycho-physics is that it treats those "physical activities which are the bearers (Träger) or conditions of the psychical, and accordingly stand in direct functional relation with them;" or again, "psycho-physies is an exact doctrine of the relations of function or dependence between body and soul-of the universals that lie between the bodily and spiritual, the physical and psychical world." But it will be seen that of such physical activities we have little or no assured knowledge; although we hare the best of grounds for believing that such activities exist, and that they stand in important relations under law with the facts of the conscious psychical life.

It follows, then, that Physiological Psychology is, pre-eminently, first experimental and then speculative; it can never become strictly demonstrative, or eren deductive to any considerable extent. That a strictly demonstrative science of the relations between the structure and functions of the nervous mechanism and the phenomena of consciousness is impossible, we might argue from the most ordinary experience. To infer from certain movements of material molecules that certain facts of consciousness must take place, under the most universal laws of all Being, involves a lind and amount of knowledge of which we cannot even clearly conceive.

In brief, our proper course will be, first, to explain, as completely as possible, the structure and functions of the nerrous mechanism; and then to set forth, as fully as the present means at disposal will permit, the various relations in which its action under stimuli stand to the phenomena of the mind. In attempting the latter problem we shall come upon a few, but only a few, general statements of fact which deserve to be spoken of as laws in any strict meaning of the word.

${ }^{1}$ Elemente d. Psychophysik, pp. 8 and 10. Leipzig, 1860. 
§9. If the eorrectness of the remarks last made be admitted, the inquiry may be raised: What justification has this so-called science of Physiological Psychology for the large claims which it has made of late; and, indeed, what right has it to exist as a special discipline at all? The full answer to the call for self-justification must be rnade by the actual achievements of the science itself. It will be better, then, to leave it to the convictions of the reader when the presentation of these achievements shall have been made. But even at this point an appeal may be taken to certain facts. We have already repeatedly conceded the fact that we are to investigate the phenomena of consciousness (that is, study psychology) by a special method rather than try to establish an independent science or even separate branch of the general science of mind. The demand for a justification is then reduced to this-Is there valid reason for studying psychology in this particular way ; for approaching its domain through the researches and conclusions of plyysiology? To such a question there can be but one intelligent answer. There is an abundance of valid reason.

The history of modern investigation, and the conclusions of the modern science of man, both plysical and psychological, empliasize the necessity of studying his nature and development as that of a living unity. Such science shows man to be at the head of a series of physical and psychical existences; he cannot be understood as he is, in his whole nature and in his place within nature at large, without taking both sides of this living unity into account. For man is known to himself as body and mind-and not as bodiless spirit or a mindless congeries of moving molecules. That the structure and functions of the body, especially of the nerrous mechanism, and the activities of the mind, are extensively and intimately correlated, is a fact beyond all doubt. It is the particular task of Physiological Psychology to show in what manner, and to what extent, such correlation exists. Moreover, there are few questions more interesting, from a philosophical and an ethical point of view, than such as the following: What is the nature of mind, considered in the light of its correlations with the body? and, Do the so-called physiological and the so-called psychical phenomena belong to one subject, or to more than one? But these and similar questions can be scientifically answered only by giving a speculative treatment to the conclusions of psycho-physical investigation.

In brief, it may be said that introspective psychology, important as its results have been, and indispensable as its method is, has shown its incompetency to deal with many of the most interesting inquiries which it has itself raised. On the other hand, psychology 
as pursued by the experimental and physiological method has already thrown a flood of fresh light upon many of these inquiries. We may affirm with Wundt,' without fear of successful contradiction: "Psychology is compelled to make use of objective changes in order, by means of the influences which they exert on our consciousness, to establish the subjective properties and laws of that consciousuess." On this fact and on the real achievements of the method we confidently rest its claims to serious and permanent consideration.

${ }^{1}$ Art. "Ueber psychophysiken Methoden," Philosoptische Studien, 1881, heft 1, p. 4. 
PART FIRST.

THE NERVOUS MECHANISM. 



\section{CHAPTER I.}

\section{THE ELENENTS OF THE NERVOUS SYSTEM.}

\&1. Iv all forms of animal life, except the rers lowest, the presence and activity of a mervous system constitutes the chief characteristic of their difference from all the more nearly corresponding forms of plant life. Both animals and plants are organisms, and their structure-regarded as a whole composed of an indefinite number of material masses or particles, which move with reference to each other for the accomplishment of a common piece of work -may be considered as a "natural mechanism." Both hare material parts of superior firmness, adapted to divide off and to support their softer parts. Plants, as well as animals, are possessed of liring, and, more especially, of contractile tissue ; they are therefore capable of the functions of nutrition, of propagation, and of that so-called automatic motion which is thought to be a fundamental property of protoplasm. As is well known, science is not yet able always to distinguish between the lowest forms of animal and the lowest forms of plant life. But nervous tissue and its functions do not belong to the vegetable kingdom ; on the contrary, the possession and use of at least a rudimentary mechanism of nerve-fibres and nerve-cells are found in most members of the animal kingdom.

It is true that, even in the case of animals which do possess a nervous system, the structure and functions of the nervous tissue are very closely related to those of the merely contractile tissue. Thus the muscular tissue of the animal might seem to be a connecting-link between its own nerrous tissue and the contractile tissue of the plant. For the motor nerves, at least, are anatomically connected by means of their end-plates with the contractile substance of the muscular fibre, and the result of modern experimentation, with both muscles and nerres, has been to make clear many features of resemblance between them. On the other hand, even the isolated nervous elements, when subjected to the same experimental tests as those which are used to determine the fundamental properties of contractile tissue, exhibit certain marked differences of behavior; while the functions of such elements, 
when combined into a very simple nervous system, are altogether unique. Moreover, as the nerrous system of the animal becomes more elaborate and complex, and especially as its central organs-spinal cord and brain-are relatively developed, other new and wonderful functions are seen to be connected with it. In the case of the supcrior vertebrate animals, and especially of man, the significance of this particular form of a physical mechanism becomes, therefore, vastly increased. Thus the minute structure of the nerrous mechanism invites the student of chemistry, molecular: physics, and listology, to investigations of the greatest interest and yet of extreme difficulty; while the functions of this mechanism are so curiously and intimately connected with the phenomena, not merely of all higher animal life, but also of human consciousness, that inquiry into them is, among all physical inquiries, the one of unparalleled intellectual interest and importance.

$\S 2$. It will be the work of this entire treatise to set forth in some detail the unique functions of the human nerrous mechanism, to which allusion has just been made. For the present a very general and somewhat indefinite statement of these functions must suffice. In general, and somewhat indefinitely, it may be said, then, that the one great fuuction of the nervous system is to concatenate (or link together into a whole) the manifold elements, both pliysical and psycho-physical, which enter into the twofold life of man. Different and distant parts of the body, whether they belong to the same or to different so-called systems (as, for example, the circulatory, the secretory, the digestive, the muscular), are bound together, and made to exercise their functions in reciprocal dependence and for common ends, by the nervous mechanism. The whole body is also linked to the external world, and kept in either unconscious or conscious adjustment to the changeful play of its forces, by the same mechanism. And further, the development of the mental life, at least in all its more primitive and fundamental factors, is mediated by the nerrous system. For it is certainly in connection with the exercise of nerrous functions that sensation takes place; and, by development and combination of the sensations, all our perceptions of the so-called "Things" of the external world. It is the nervous mechanism which unites the entire body with the physical stimuli of the external world, on the one hand, and, on the other, with the primitive activities of mind. What relation the nerrous functions have, and whether they have any direct relation at all, to memory, judgment, and the higher activities of mind in general, we do not now even inquire.

The siguificance of the above-mentioned function of "concatena- 
tion," so far as it concerns the different and distant parts of the body, might be illustrated in many ways. Inasmuch as the plant is an organism, there is a reciprocal dependence of the structure and action of all its parts. But each part of the plant acts directly and slowly on only contiguous parts in effecting the distribution of the fluids, upon the spread of which the life and growth of the plant depend. In the case of the animal, howerer, an effect produced in one part of the body may quickly spread to other distant parts by the mediation of the nervous system. The circulation of the blood is made to affect, and to be affected by, the state of the skin and the muscles, the state of the respiratory organs, or the state of the mind's feeling as determined by the ideas before the mind. A draught of cold air, for example, strikes some peripheral portion of the body; the heart and lungs modify their activities, the muscles contract, and a shudder runs through the physical framework; the secretions are disturbed, and the mind is, perhaps, seized with a vague feeling of fear. Such a complex effect of the stimulus of cold on some region of the skin has been brought about by the action of the nervous system, with its peripheral end-organs, conducting nerre-fibres, and nervous centres. Or, again, the seeing of some sight or the hearing of some sound is followed by ideas and emotions of shame, or of fear, or of joy. A complex co-ordination of the muscles then takes place, so as to move the limbs in running, to give or ward off a blow, to extend the hand in greeting, to lift up or bow down the head. In this case, also, the action of heart and lungs and secretory organs is greatly modified; the capillary circulation is altered, and the cheeks are blanched or reddened; the pupils and lachrymal ducts of the eyes are moved; the very hair of the head seems to sympathize with the state of the mind. Thus, changes which involve the functions of almost all the tissues and organs of the body are accomplished by the mediation of the nerrous mechanism. Unlike the modifications in expression of function which take place in the plant, they are accomplished with what seems a practical instantaneousness. The complexity of the reciprocal changes which characterize the life of the ligher animals is due to the general functions of the nerrous system; the speed with which the changes are accomplished is dependent upon the laws of the propagation of nervous impulses within that system.

Further illustration of this general office of the mechanism of nerve-fibres and nerve-cells in "concatenating" the manifold elements of physical and psycho-physical life may well be left to the progress of our examination.

8. The application of the term " mechanism" to the nervous sys- 
tem of man has already (see p. $4 \mathrm{ff}$.) been partially explained and justified. We now describe the elementary parts of such a system as considered from the same general point of view which induces us to apply this term to the structure and functions of the entire system. In order to do this, it is necessary to speak, first, of the structure, and, second, of the function of these parts, regarded as the fundamental and distinguishing factors of a complex mechanism. That is to say, two inquiries must be made: What is the composition and form of those ultimate structures called nervous elements, into which microscopic anatomy analyzes the nerrous system? and, What can such structures $d o$ which fits them to act as parts of a "mechanism" like that of the nerrous system? It is obvious that the answers to these inquiries lie at the very entrance upon the way toward a complete science of the nervous mechanism. But even if the fullest imaginable answers were already attained, much would remain to be done in order to perfect the science. Histology would still have to inform us precisely how the elements are combined into the manifold organs of a system. Physiology would have to discover the laws according to which the functions of the elements are modified, when they act as thus combined. - Of course, to know completely the nature, number, and properties of all the individual factors of a mechanical system, and to know also precisely how those factors are combined into the system, as well as how their modes of behavior are affected by such a combination, would be to have a complete science of such system.

A strictly deductive science of the molecular motion, and consequent function of the elements of the nerrous mechanism, is, indeed, a conceivable attainment. But it need scarcely be said that we are indefinitely far from, not only the attainment, but even the reasonable prospect of such a complete physical science of the nervous system. None of the questions raised respecting the structure and functions of its elements, whether considered apart or in combination, can be answered with complete satisfaction. Moreover, the scientific study and description of the nervous mechanism is compelled from the first to pursue a somewhat different path from that open to many forms of physical science. The direct path to the complete science of the subject is impassable; it is rendered impassable by the most fundamental and universal of our experiences respecting the nature of the phenomena of the nervous system. The immediate effects of the molecular changes which take place in the nervous elements, even when isolated as much as possible, can only with difficulty be made the subject of direct observation. Histology has enormous difficulties to overcome in its effort 
to describe how these elements are combined in the living human body, and physiology has like difficulties in the way of its effort to determine the functions of those organs which are constructed by means of such combination. Only the beginning of a theory which shall correlate that mode of molecular motion which is peculiar to nervous matter with other modes of the motion of matter has yet been made.

In spite of the foregoing concessions, a careful study of the elements of the nervous system is the indispensable mode of approach to the subject of physiological psychology. It is these elements which, when variously combined, constitute all the organs of the system ; it is they which, when acting in combination, do all the work of the system.

$\S 4$. The Elements of the Nervous System of Man, as elements, do not differ in any essential known respect from those of other vertebrate animals. Upon this subject, then, histology with its microscope, and physiology with its experimentation, can describe the nerve-fibres and nerve-cells of other animals, and then safely draw certain inferences from them which will apply to the case of man. It is, however, the development of enlarged or of new organs by the combination of these elements, and the development and elaboration of function as dependent upon such organs, which constitute the difference between the nerrous system of man and that of the lower animals. It is here that histology meets with its supreme difficulties and its most interesting problems; it is here that physiology is most insecure when trying to carry over to the structure and functions of the human nervous mechanism the conclusions which have been reached by experiments upon the lower animals. On the contrary, the nerve-fibres and nerve-cells of these animals are, in most respects, perfectly competent to tell us all we need to know regarding the nerve-fibres and nerve-cells of man. In describing the constitution, structure, and function of the nerrous elements, therefore, it will not generally be necessary to pay attention to the specific animal form from which the description is taken. In other words, the discussion of the nervous elements belongs to the most general histology and physiology of the nervous system.

\&5. The elements of the nervous mechanism require to be considered in three ways: (1) as respects their chemical constitution; (2) as respects their formal structure ; (3) as respects their general physiological function.

$\S 6$. The Chemistry of the Nervous System is of necessity in an exceedingly unsatisfactory condition. The facts concerning which perfect certainty is attainable are very few in number; the bearing 
of those facts on our theory of nerre-function is both slight and disputable. Physiological chemistry is in general encompassed with many difficulties. These difficulties are not due simply to the complex constitution of most of the substances with which it has to deal. They are also very largely due to the fact that these substances are products of life; and living tissue cannot be at the same time kept in normal condition and subjected to the handling necessary for chemical analysis. As soon as it is no longer alive, or at any rate long before any chemical analysis can be completed, the constitution of such tissue is changed. However carefully the chemical elements, the constituents, which enter into the nervous substance may be preserved, their constitution, their chemical arrangement and behavior, cannot be preserved. It is impossible - for example-for the chemist even to determine the specific gravity of uncoagulated blood, "except by operating with extreme expedition and at temperatures below $0^{\circ} \mathrm{C}$."

Moreover, the difficulties which belong to the chemistry of all living tissue are especially great in the case of the nerrous tissues. In their natural state the proximate principles which compose these tissues are very complex and unstable compounds. To obtain specific portions or kinds of nervous substance free from foreign ingredients - as, for example, the axis-cylinder of the nerves, or the rods and cones of the retina-is by no means always easy. The analysis of such substance, when once the substance is obtained, is often extremely tedious in respect to process, and doubtful in respect to result. Nevertheless, the principal conclusions, which may be accepted with considerable confidence in their correctness, are as follows :

§7. Nerrous Matter is of two kinds, called white or fibrous, and gray or vesicular, which differ not only in color and microscopic structure, but also in specific gravity and chemical constitution. The specific gravity of the white nervous matter is greater than that of the gray. Danilewski ${ }^{1}$ found the specific gravity of the gray matter in man to vary from 1.02927 to 1.03854 ; that of the white matter from 1.03902 to 1.04334. Others (as Bastian, W. Krause, and L. Fischer) calculate the mean specific gravity of the gray matter at about 1.031, of the white at 1.036-1.040. This difference in the weight of the two is chiefly due to the difference in the relative amount of water and of solids which they contain. Of 100 parts of each, from the brain of the ox, the gray matter was found to be

${ }^{1}$ See Med. Centralbl., xviii., p. 241, as cited by Drechsel, with apparent confidence, in Hermann's Handbuch der Physiologie, V., i., p. 577. Leipzig, 1883. 
composed of 81.60 parts of water and 18.40 of solids; the white, of 68.35 of water and 31.65 of solids. The amount of water is also larger in the brain of the young animal than in that of the adult. The brain of the foetus was found by Weisbach to consist of from 87.9 to 92.6 parts of water. The amount of water entering into the composition of the different parts of the central nervous system is unequal. The following is a tabulated statement ${ }^{1}$ of the facts to which allusion has just been made :

Proportion of Water in One Hundred Parts.

\begin{tabular}{|c|c|c|c|}
\hline & Age, 20 to 30. & Age, 30 to 50. & Age, 70 to 94. \\
\hline White substance of the brain & 69.56 & 68.31 & 72.61 \\
\hline Gray substance of the brain... & 83.36 & 83.60 & 84.78 \\
\hline Cerebellum & 78.83 & 77.87 & 80.34 \\
\hline Pons Varolii . & 73.46 & 72.55 & 72.74 \\
\hline Medulla oblongata...... . & 74.43 & 73.25 & 73.62 \\
\hline
\end{tabular}

The amount of water varies in the different regions of the spinal cord. Bernhardt found a smaller proportion of water in the cervical (73.05 per cent.) than in the lumbar (76.04) region of the cord. The gray matter also contains more of albumen, lecithin, and lactic acid than the white, and less of cholesterin, fat, and protagon.

$\$ 8$. Of the solids contained in the matter of the nerve-centres, more than one-half in the gray, and about one-quarter in the white, consist of certain proteid or albuminous substances. Bodies of this general class are the only ones never absent from the active living cells ; ther therefore exist in the primordial structures of all vegetable and animal organisms, and occupy a peculiar place among organic proximate principles. Of these proteid substances found in the nerve-centres very little is as yet known. Gamgee ${ }^{2}$ mentions three such substances-one soluble in water and probably derived from the gray matter, another a globulin-like body which is dissolved by a teu per cent. solution of common salt, still another a myosin-like body which remains in solution when a ten per cent. salt solution of brain is boiled.

§9. Three other non-phosphorized bodies of different classes from that above mentioned are found in nervous tissues: these are Cholesterin, Neurokeratin, and, more doubtfully, Cerebrin. Cho-

' Derived from Weisbach's observations, and found in Gamgee, Physiological Chemistry of the Animal Body, i., p. 445. London, 1880.

${ }^{2}$ Physiological Chemistry, i., p. 423 ; see, also, the article of D. Petrowsky, "Zusammensetzung der grauen und der weissen Substanz des Gehirns," Pflüger's Archiv, vii., p. 367 . 
lesterin is among the most abundant of the constituents of the nervous tissues-especially of the white matter of the cerebro-spinal axis and of the nerves. It is a "monad alcohol," the only alcohol which occurs in the human body in a free state. On account of its solubility in ether, cold or hot, and in warm alcohol, cholesterin finds its way into both ethereal and alcoholic extracts of the nervous tissues. It is a non-nitrogenous body, crystallizing in beautiful white crystals, which, when separated pure from its solutions in ether or chloroform, takes the shape of fine needles, and when separated from alcohol takes the shape of rhombic tables. It is supposed to exist preformed in the brain. Its formula is $\mathrm{C}_{26} \mathrm{H}_{44} \mathrm{O}+\mathrm{H}_{2} \mathrm{O}$.

Neurokeratin is most easily derived by treating the medullated nerve-fibres with boiling alcohol and ether, so as to extract the fatty matters of the medullary sheath; in the place of this sheath there is left, as a kind of irregular framework, a highly refractile substance which resembles the horny matter of epidermis in its power of resistance to chemical agents. This substance is also found in the gray matter of the nerve-centres, and in the retinal epithelial cells and pigment cells of the choroid; but not in the non-medullated nerve-fibres. It contains nitrogen, 2.93 per cent. of sulphur, and leaves 1.6 per cent. of ash.

Cerebrin was announced by Müller, in 1858, as a non-phosphorized nitrogenous body, obtained from a precipitate from the brain when pounded up with baryta water to the consistence of thin milk and then boiled. He described it as a loose, white, very light powder, destitute of smell and taste, soluble in boiling alcohol and ether, but insoluble in water. $\mathrm{He}$ gare to it the formula $\mathrm{C}_{34} \mathrm{H}_{33} \mathrm{NO}_{6}$. Thudichum believes that brain matter contains a class of nitrogenous bodies free from phosphorus, to which he gives the name of "cerebrins." Gamgee, however, thinks it very unlikely that a body produced, like Muller's cerebrin, "by the prolonged action of a solution of boiling barium hydrate on so complex an organic mixture as brain should be a definite proximate principle of the unaltered organ;": but the same authority admits ${ }^{2}$ that the precipitate which separates itself from an alcoholic solution of brain contains, besides cholesterin, protagon, and the so-called lecithins, "a body for which we may retain the name of cerebrin."

Nuclein was discovered by Miescher in the nuclei of pus-corpuscles and in the yellow corpuscles of yolk of egg. Other observers subsequently obtained it from various other substances, especially from the nuclei of the red blood-corpuscles of birds and amphibia.

1 Physiological Chemistry, i., p. 439.
' Ibid., i., p. 433. 
Von Jaksch ${ }^{1}$ thinks he has discovered nuclein in the human brain. His claim seems to be credited by Drechsel. ${ }^{2}$ Its formula is given as $\mathrm{C}_{20} \mathrm{H}_{49} \mathrm{~N}_{3} \mathrm{P}_{3} \mathrm{O}_{22}$. But the rery existence of nuclein, as a definite body, has been denied by chemists like Worm-Müller and Gamgee; and the analyses of Von Jaksch do not agree with those obtained from other sources than the substance of the human brain. The whole question of nuclein must then be left in doubt.

$\S 10$. No other substances found in the nerrous system are, however, both so interesting and so difficult to consider, from the mixed chemical and psycho-physical point of riew, as certain complex phosphorized fats. The entire progress of our inquiry will make it obrious how inadequate and misleading is the use often made of such off-hand remarks as the celebrated dictum: "No thought without phosphorus." Yet it is doubtless true that the highly elaborate and unstable compounds containing phosphorus, which enter into the composition of nervous matter, have a significance for physiological and psychological researches such as belongs to no other material bodies. These complex bodies are especially characteristic of the centres of the nervous system. The strife of discorery and of confirmatory experiment has been chiefly carried on over the following three : Protagon, Lecithin, and Cerebrin. Of these three, however, probably only the two former are phosphorized bodies. The main question involved in controversy concerns the relation in which lecithin and cerebrin stand to protagon. Is protagon a definite proximate principle of the brain, and are lecithin and cerebrin bodies of ill-defined properties and doubtful claim to existence as proximate principles of the brain? or, are lecithin and cerebrin definite proximate principles, and is protagon a mechanical admixture of the two? The latter view of protagon has been held by Diaconow, Hoppe-Seyler, and Thudichum; on the contrary, its claims to the position of the "only well-characterized phosphorized proximate principle" of the brain as yet discorered have been defended (and, it may be said, apparently established) by the researches of Gamgee and others.

Protagon was discorered, as a new proximate principle that can be separated from the brain, in 1864, by Dr. Oscar Liebreich; his discovery was announced in a paper ${ }^{3}$ published in 1865 . This investigator gave to this substance the name which it still bears, as

\footnotetext{
I See article "Ueber das Vorkommen von Nuclein im Menschengehirn," Pflüger's Archiv, xiii., p. 469.

${ }^{2}$ In Hermann's Handb. d. Physiol., V., i., p. 578.

" "Ueber die chemische Beschaffenheit der Gehirnsubstanz," Annalen der Chemie und Pharmacie, cxxxiv., pp. 29-44.
} 
in his opinion the first to be definitely ascertained among the specific constituents of the brain ( $\pi \rho \omega \tau$ ayos, leading the van). He assigned to it the formula $\mathrm{C}_{116} \mathrm{H}_{241} \mathrm{~N}_{4} \mathrm{O}_{22} \mathrm{P}$. In spite of subsequent denials and disproofs of its existence, the extremely careful and often-repeated researches of Gamgee ${ }^{2}$ and Blankenhorn lave corroborated the discovery of Liebreich. The process by which protagon is obtained from the brain may be thus briefly described (the description will serve to illustrate in general the processes of physiological chemistry): Perfectly fresh ox's brains are freed from the blood and membranes, and are then digested for about a day in eighty-five per cent. alcohol ; from this fluid, when filtered, a quantity of white flocculent precipitate is obtained, and the cholesterin and other bodies soluble in ether are dissolved out; from the substance left undissolved, when dried and reduced to powder and digested for many hours with alcohol, and then filtered and cooled, microscopic crystals separate themselves, arranged for the most part in rosettes. The substance thus crystallized is protagon. It is considered by some chemists the easiest to obtain, and indeed the only rery well-established phosphorized proximate principle of the brain. Such a material substance is indeed a long way removed from the living nerrous mass, with its capacity for exercising such marvellous physiological and psycho-physical functions. But it is the best representative that chemistry can as yet present of a scientific result upon which to base any attempt to point out definite relations between psychical activities and the chemical constitution of those complex phosphorized fats which exist in the central nervous mechanism. The empirical formula of protagon, as given by Gamgee, is $\mathrm{C}_{160} \mathrm{H}_{308} \mathrm{~N}_{5} \mathrm{PO}_{35}$. It lias been made highly probable that protagon camnot be a compound or mixture of cerebrin and lecithin; it may, then, be announced as a proximate principle of the brain.

Lecithin is an organic phosphorized compound which exists in large quantities in ova, spermatozoa, etc., as well as in the nerrous tissues. It is described as a yellowish-white, waxy, very hygroscopic solid, which in thin layers shines with a silky lustre. It is soluble in ether and alcohol; on being stirred with water it forms a starch-like solution difficult to filter. Diaconow assigns to it the formula $\mathrm{C}_{44} \mathrm{H}_{00} \mathrm{NPO}_{3}+\mathrm{H}_{2} \mathrm{O}$. Gamgee supposes that the lecithin of Diaconow is only one of a group of similar bodies which possess a higher percentage of pliosphorus than protagon, and the "general smeary character's" of lecithin. We may, then, speak of "the lecithins" as highly phosphorized compounds existing in the matter of the brain.

${ }^{1}$ See his Pliysiological Chemistry, i., pp. 425-429 ; and article in the Journal of Plhysiology, ii., pp. 113-131. 
The various products of the decomposition of protagon and lecithin it is not necessary to describe. Neurin is the only one of these products which deserves for our purpose even to be named. It may be obtained from either protagon or lecithin. Dr. Thudichum's elaborate "Researches on the Chemical Constitution of the Brain" " conclude that at least three well-defined groups of phosphorized bodies may be separated from the brain; these are distinguished as (1) kephalins, (2) myelins, (3) lecithins. The existence of a group of bodies which may be termed "lecithins" has just above been declared probable. Thudichum thinks that all these bodies contain phosphoric acid combined proximately with glycerin, but " differ" in the manner in which they contain the nitrogen and the acid radicles which constitute the great bulk of their substance." The researches of Dr. Thudichum still await confirmation.

$\S 11$. In addition to the substances already mentioned, the brain contains certain extractive matters which are found also in other tissues, especially in muscle. Among these bodies are creatin, inosite, xanthin, and lactic acids.

\$12. The brain also contains an extremely small amount of inorganic matters-so small, indeed, that few facts can be relied on concerning it. The estimates of this amount vary from 0.1 to 1 per cent. of the fresh brain. Among such inorganic matters are alkaline phosphates and sulphates, chalk, magnesia, oxide of iron, etc. It is said that the ash of the gray matter has an alkaline reaction, that of the white matter an acid reaction, ${ }^{2}$ and that the reaction of the former during life is acid, while that of the latter is neutral or weak alkaline.

\&13. All quantitative analyses of the brain are exceedingly doubtful ; the older results are wholly worthless. The following table of Petrowsky, ${ }^{3}$ which gives the chief organic constituents of the brain of the ox, is an object of interest rather than of complete confidence :

\begin{tabular}{|c|c|c|}
\hline Substance. & Gray Matter. & White Matter. \\
\hline 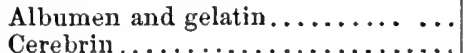 & $\begin{array}{l}55.37+\text { per cent. } \\
0.53+\text { “ }\end{array}$ & $\begin{array}{l}24.72+\text { per cent. } \\
9.55-\end{array}$ \\
\hline Lecithin & $17.24+\quad$ " & $9.90+$ \\
\hline Cholesteri & $18.68+$ & $51.91-$ \\
\hline solu & $6.71+$ & $3.34+$ \\
\hline Salts & $1.45+$ & $0.57+$ \\
\hline
\end{tabular}

1 Reports of Medical Officer of the Privy Council and Local Government Board, 1874, pp. $113 \mathrm{ff}$.

: See Gamgee, Physiological Chemistry, i., p. 445 ; Hermann, Handb. d. Physiol., V., i., p. 577.

3 "Zusammensetzung der grauen und der weissen Substanz des Gehirns," Pflüger's Archiv, vii., p. 367. 
$\S 14$. The specific chemistry of the elements of the nervous system, or of the various parts of such elements which histological science reveals, is yet more meagre and doubtful than its general chemistry. The micro-chemistry of the nerve-cells tells us simply that they are in the main protoplasmic, and therefore rich in proteid substances; and since an analysis of the two kinds of nervous matter shows that the gray is much the poorer in complex phosphorized constituents and in cholesterin, we conclude that the cells which enter so largely into the gray matter are also poor in the same substances. The different structural parts of the nerve-fibres have doubtless a different chemical constitution. This is proved by the difference in their appearance under the microscope, by the different action of reagents upon them, and, to some extent, by chemical analysis. The neurilemma, or membranous envelope of the nerve-fibres, like the sarcolemma, on prolonged boiling, yields gelatin. The axis-cylinder appears to be a mixture of proteid with complex fat-like bodies. The white substance of Schwann is rich in complex phosphorized fats, in cholesterin, and in the so-called cerebrins.

The researches of Kühne ${ }^{1}$ and others-for the most part his pupils-have developed certain interesting results with respect to the chemical constitution and chemicals change of the nervous tissue of the eye. Many of the various non-nervous parts of the ear and the eye have been carefully analyzed. The extremely small amount of such material which is obtainable for chemical analysis is one reason why so little can be known concerning the chemical constitution of the substance of the retina. It is said to have an acid reaction. It is a fair surmise, on general grounds, that it contains the same bodies as the ceutral nervous system. The two segments into which the rods and cones break up exhibit marked differences in their chemical as well as optical characters. The inner segments are composed of protoplasm of "a marvellous transparency." The outer limbs of the rods have an external envelope which agrees in its physical characters with neurokeratin. This envelope encloses a mixture of proteid bodies and of other substances similar to those found in the other nervous tissues.

$\S 15$. If knowledge of the chemical constitution of the nerrous system is so far behind what we could wish, knowledge of the chemical processes and chemical changes which are connected with the physiological functions of this system must be declared to be

${ }^{1}$ For a list of papers by Kühne and his pupils on this subject, see Gamgee, Physiological Chemistry, i., p. $462 \mathrm{f}$; and for an account by him of his researches and their results, see Hermanu, Handb. d. Physiol., I., i., pp. 235 ff. 
almost wholly wanting. Eren the beginnings of scientific general statements, or laws, respecting the relations between the chemical constitution of the nervous system and its various physiological activities have yet to be made. Different investigators will doubtless differ as to the prospect for such discovery in the future. When chemistry can deduce the molecular behavior of the most highly complex chemical compound from the nature and number of its component chemical elements, and physiology can definitely connect all the physiological functions of nervous matter with the molecular motions of its chemical constituents, we shall have the means for a strictly scientific solution of such problems.

$\S 16$. It need scarcely be said that we have little knowledge respecting the relation which exists between the chemical constitution and chemical processes of the nervous system, on the one hand, and, on the other, the phenomena of so-called mind.

Nevertheless, certain important general relations may be pointed out between the chemical nature of the nervous mechanism and its psycho-physical functions. The extremely high organization and chemically sensitive constitution of this mechanism are beyond doubt related to all its distinctive functions. Like every other natural material structure, the nerrous system is obriously adapted to its peculiar kind of work. Chemically considered, it appears as composed of a number of extremely complex and highly unstable compounds. It therefore holds in its chemical constitution a large amount of disposable energy ; this energy it yields readily when the equilibrium of its molecules is in any way disturbed. Within certain limits, it explodes with increasing surrender of its disposable energy as the number and intensity of the demands upon it are increased-very much as would a gun which should be arranged so as to go off with greater energy as the pressure of the finger on its trigger is repeated or increased.

It is probable that the substance of the nerves is the seat of a chemical synthesis, as the result of which still more complex bodies are constructed from the already complex alimentary material furnished by the blood; such bodies have a high value as combustibles, and thus, as has been said, possess a significant amount of disposable energy. The relation of a supply of oxygen to the nerre-centres is also important to notice. The nerve-fibres require comparatively a small amount of oxygen. It may be conjectured that in their case, as in the case of muscle-fibre, intra-molecular oxygen is of some use in preparing explosive materials. But at present we must be satisfied with conjecture on this point. On the contrary, the vascular nature of the central organs creates a pre- 
sumption that the chemical processes which have their seat in them require an abundance of oxygen. Experience confirms this presumption. The respiratory centre in the medulla oblongata is chiefly controlled in its action by the amount of oxygen which reaches it in the blood. The phenomena of conscionsness vanish when the supply of oxygenated blood is cut off from the brain.

Although we are still in the dark as to the precise significance of the visual purple, the phenomena which the study of it has brought to light are suggestive of unseen chemical processes that are set up in the retina, and so serve as stimulus for the fibrils of the optic nerve. In general we know that certain sensations are dependent upon the chemical constitution and activity of the various endorgans of sense.

Further researches will undoubtedly greatly enlarge our knowledge of those facts of relation which exist between the chemical constitution and changes of the nervous mechanism and the phenomena of psychical life. Perhaps such particular statements of fact may be gathered into such more general statements of fact, verifiable by experiment, as we consider sufficient to constitute scientifically established laws. But why certain chemical constitnents, when combined and changed in definite fashion, should be specifically connected witl certain conscious experiences will always remain an unanswerable inquiry.

$\S 17$. From the chemical constitution of the elements of the nervous system we now pass to their Structural Form. The science which must be our guide is no longer chemistry, but microscopic anatomy, or histology; this science furnishes us with a large amount of trustworthy information mingled with a still larger amount of conjecture and doubt. Even the number of those elements upon which histology is entitled to focus its microscope and declare that such, and no others, are capable of performing distinctively nervous functions can scarcely be said, as yet, to be placed beyond all doubt; neither can it be claimed that the microscope has yet demonstrated the ultimate structure of those two species of such elements the reality of whose nervous functions is beyond doubt.

It is customary to speak of nerre-fibres and ganglion-cells as the only structural elements of the nervous system. If, however, by the term "ganglion-cell" we intend only such bodies as histology usually describes under this type (for example, the so-called motor ganglion-cells of the spinal cord) the limitation is without sufficient warrant. For there are many cells, which almost certainly have some significance as parts of the nervous system, that differ in form very widely from the typical ganglion-cell. Moreover, by such 
an off-hand twofold division the important question is often silently passed by: What is the significance for the nerrous functions of that diffuse, finely granular substance, found in considerable quantity in the great nerve-centres, and called neuroglia, or nerve-cement (Nerven-kitt; Kittsubstanz)? This substance is most frequently classed with the connective tissue ; but, according to Henle, "it is at all events to be distinguished from counective tissue on account of its chemical properties." That certain microscopic forms of so-called "neuroglia" are largely unlike other forms recognized as being nerve-cells beyond doubt cannot be argued in proof of its inability to perform any of the strictly nerrous functions, except upon the basis of the assumption that we already know beyond reasonable question what are all the elementary structural forms of true nervous matter. But, as says Eckhard, " "if we start the inquiry, what formal elements of the brain and spinal cord take part in the activities of these (the nervous) organs, and in what way do they take part, we are able to give to it only a very unsatisfactory answer." We are not in a position, then, either to affirm or to deny absolutely the claim sometimes set up for the neuroglia, that it contains true nerrous elements.

It is best to recur to the facts which microscopic anatorny discloses as a basis for classifying the different structural elements of the nerrous system. These may be briefly described as follorrs: ${ }^{3}$ As to the true nerrous character of fibres of rarious kincls, not only as conducting bands between the nervous centres and the peripheral parts of the body, but also within the substance of these centres, there is no disputc. Nerve-fibres undoubtedly constitute one of the structural elements of the nerrous mechanism. Besides the nerve-fibres, histology distinguishes in the gray substance of the nervous centres-where all the structural elements of the nervous system are to be found in greatest abundance and variety-three other species of structural form. Such are $(a)$ certain cells, known more particularly as the "ganglionic nerve-cells." These cells (to be described more minutely hereafter) are irregular masses of finely granular protoplasm, possessed of a clear nucleus and one or more nucleoli, and sending off one or more processes.

(b) Corpuscles, consisting either of naked nuclei or of nuclei with only a small amount of surrounding protoplasm, and having various shapes sometimes difficult to nake out, are also found abundantly in the gray matter of certain nerrous centres. Such

'Anatomie des Menschen. Text, p. 306. Braunschweig, 1880.

2 Hermann, Handb. d. Physiol, II., ii., p. 15.

${ }^{3}$ Comp. Henle, Anatornie des Menschen. Text, p. 306. 
bodies are usually much smaller than the colls of undoubted nerrous character described above, many of them being scarcely more than $\frac{1}{230 \overline{0}}-\frac{1}{300 \overline{0}}$, or even $\frac{1}{50 \overline{0}}$, of an inch in diameter. Some of them, like the typical ganglionic cell, give off processes which are thought to be continuous with nerve-fibres. It is altogether probable that these cells of the second class differ only in their dimensions from the cells of the first class. In some places (for example, in the cortex of the cerebrum, or large brain) they appear to have the characteristics of transitional forms between the undeveloped granules of the same class and the more highly dereloped ganglioncells. In other places (as in the cerebellum) they form independent layers. They may be described as nuclei "invested by only a small quantity of cell-substance." ${ }^{1}$ Some are multipolar, some bipolar, some unipolar. Admitting, as we seem compelled to do on account of their resemblance to the typical form of the ganglionic nerve-cell, that some of these cells are true nerrous elements, it is impossible for listology to draw the line through the entire class, and so to affirm beyond doubt that any of them are without claim to be counted among such elements.

(c) The diffuse, finely granular substance, already referred to as so-called "neuroglia," which fills in the gaps between the nervefibres and the cells of the two preceding classes, constitutes the other form of matter observed in the nervous centres. It exists in quantity large enough to form an essential constituent of some localities of the brain and spinal cord. It is not always clear, howerer, to what this appearance of granular or molecular matter, in which the nerre-cells seem embedded, is due. According to some authorities, it may result from a confused interlacement of fine nervefibrils and fine ramifications of nerve-cells; or from the crushing of such nerrous matter in the process of examination." The neuroglia itself is described as a delicate reticulum, or network, in which certain small cells (neuroglia-cells) supposed to belong to the sustentacular tissue, and other more conspicuous cells, usually stellate in section (" cells of Deiters"), are found.

$\S 18$. Of the three foregoing linds of structural forms found in the gray nervous matter, it is perhaps safest to class the first two together under the term "nerve-cells." We should then have to remember how greatly these vary in size and formation-all the way from the naked, or almost naked, nucleus to the large ganglioncell with its many processes and complex microscopic structure.

${ }^{1}$ See Max Schultze in Stricker, Human and Comparative Histology, i., p. 183. London, $18 \% 0$.

'See Quain's Elements of Anatomy, ii., p. 149. London, 1882. 
The last of the three (neuroglia) may then be regarded as a sustentacular tissue; though with the confession that in the brain and spinal cord it is by no means always easy to distinguish sustentacular from true nervous tissue. ${ }^{1}$

Of the structures known as nerve-fibres and nerve-cells, histology enables us to give a further more minute, if not a complete, description ; it also excites our interest by making it possible to conjecture what is the regular anatomical relation between the two. When combined with physiological researches, histology also enables us to make considerable progress toward distinguishing the separate as well as the combined functions of these elements. We consider, then, with particular detail, the structure and functions of nerve-fibres and ganglionic nerve-cells.

\$19. What is ordinarily called a nerve appears to the naked eye, when dissected from an animal, as a cord of a whitish or grayish

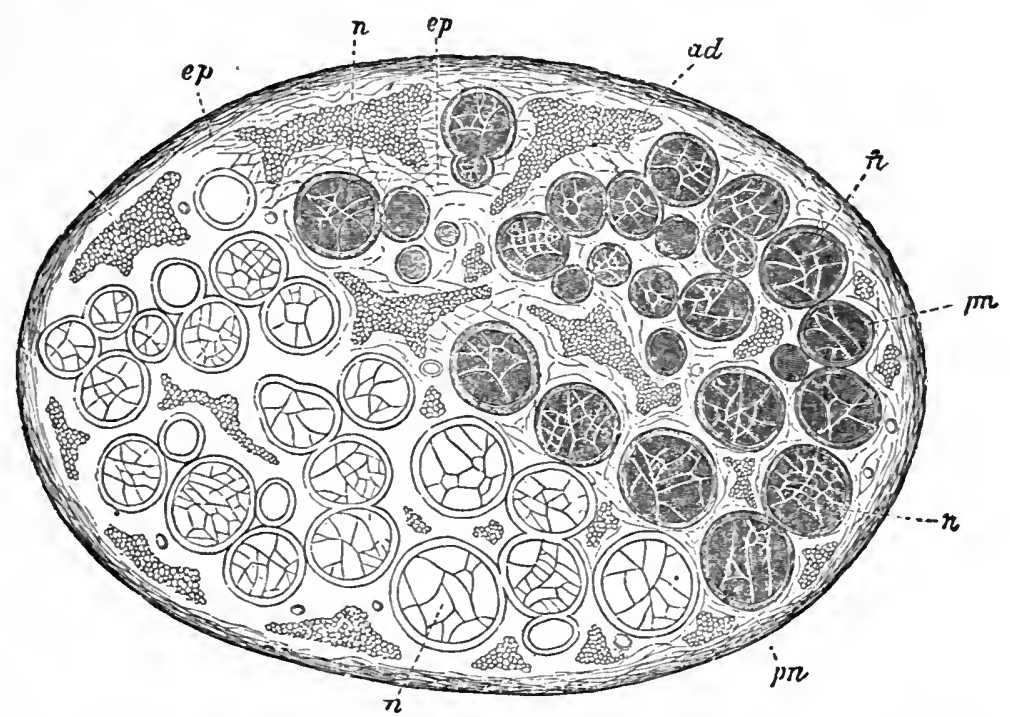

Frg. 1.-Cross-section of the Sciatic Nerve of Man. $8 /$. (After Key and Retzius.) The left lower half is schematic. $\boldsymbol{n}, \boldsymbol{n}$, Bundles of nerve-fibres, surrounded by $\boldsymbol{p}, \boldsymbol{p} n$, the perineurium: between them appears the connective tissue, epineurium $(e p, e p)$, and adipose substance (ad).

color, and of uniform structure. The nerve is really, however, one or more bundles, or fascicles, of a larger or smaller size, bound together by connective tissue. Accordingly, on following it toward its peripheral termination we find that it divides and subdivides until its subdivisions consist of a single nervous element called a

' Comp. Ranvier, Traité Technique d'Histologie, i., p. 717. Paris, 1875. 
Nerve-fibre. The bundles hare a special sheath formed of connective tissue (neurilemma, or perineurium), which in the finest branches becomes reduced to a single layer of cells cemented together edge to edge, and is called the "sheath of Henle." On following the fibres backward again toward the central organs, it is found that several of them are bound together to form a nervefascicle; a small amount of fibrillar connective tissue appears between the several fibres within the same sheath; the character of the sheath itself is changed, and it becomes attached to surrounding structures by a layer of connective tissue. It is the fibres into which the nerves break up on being followed toward their peripheral terminations, or by junction of which, successively, they are composed on being followed toward their central termination, that are to be considered as the true elements of the nerrous system.

$\$ 20$. Such nerve-fibres as compose the nerres which stretch from the central organs to the peripheral parts of vertebrate animals may readily be divided into two classes: one called medullated fibres or nerve-tubes, and the other non-medullated fibres or fibres of Remak. Nerves in which there is a large proportion of medullated fibres have a characteristic white or watery appearance; those in which only non-medullated fibres, or only a few medullated fibres, exist are grayish and slightly translucent. Vertebrates alone have the former. The medullated nerve-tubes belong particularly to the cerebro-spinal system, and are, therefore, of prime interest in psycho-physical researches; the fibres of Remak are very abundant in all the nerves belonging to the sympathetic system. This twofold division of nerve-fibres, while admitting of easy application to the constituent elements of the nerre-trunks, becomes more difficult when we attempt to earry it out within the complex nerrous matter of the central organs. Here Max Schultze ${ }^{3}$ points out several varieties of nerve-fibres. There are, first, those "very fine threads which lie on the extreme verge of microscopic mensuration," and which require an enlargement of from 500 to 800 diameters in order to be made visible. In such fibres no internal structure can be detected by the microscope. To these Schultze gives the name of "primitive nerve-fibrils." Second : certain very delicate transparent fibres of albuminous composition, and distinguished from the foregoing by their greater size and their manifest fibrillar structure, are found in the central organs. These are the so-called naked axis-cylinders. Both the foregoing, when invested with a medullary sheath, become converted into the third, or medullated, form of nerve-fibre. These fibres in the nerves, while running be-

' See Stricker's Iluman and Comparative Histology, i., pp., $147 \mathrm{ff}$. 
tween the central organs and the end-organs, become invested with a delicate membrane, and are thus converted into nerve-tubes of the well-known threefold structure. A fourth form of nerve-fibre occurs in the peripheral nerves, and is distinguished from the foregoing by the absence of the medullary sheath. This is the peripheral non-medullated fibre, or fibre of Remak, already alluded to. As they appear to the microscopist, then, on an examination of all the kinds of nerve-fibres which are found in all the different parts of the nervous system, the following table of varieties is proposed by Schultze :

I. Non-medullated
fibres. $\left\{\begin{array}{l}\text { 1. Primitive fibrils. } \\ \text { 2. Fasciculi of primitive fibrils. } \\ \text { 3. These last, with a sheath of Schwann. }\end{array}\right.$

II. Medullated fibres. $\left\{\begin{array}{l}\text { 1. Primitive fibrils with medullary sheath. } \\ 2 . \text { Fasciculi of primitive fibrils with such } \\ \text { sheath. } \\ \text { 3. These last, with a sheath of Schwann. }\end{array}\right.$

The exposition of Schultze, although of value in setting forth the variety of forms in which the nerve-fibre is actually found by the histologist, does not constitute an objection to the twofold division first proposed. On the contrary, it leads directly to such a division. For it will be noticed that both of the chief classes of fibres are regarded as composed of a number of primitive fibrils ; both are also regarded as becoming invested in their peripheral course with an outside membrane. The two classes, however, are really derived upon the basis of the fact that some of the primitive fibrils, whether they have already become invested with this membrane or not, possess a medullary sheath, and others do not. It is the presence or absence of this medullary sheath which constitutes the one important difference between the different classes of nervefibres.

\$21. Medullated nerve-fibres, or nerre-tubes, have a threefold structure. Such fibres, when separated by teasing with needles from the fascicle of nerve-fibres and examined under the microscope while still fresh, appear pellucid, with a central part and a border on each side, like a translucent liquid in a tube of translucent walls. The lines of this double contour are at first comparatively sharp and regular; lengthening the focus of the instrument obscures slightly the central part, and causes the parts on the border to appear brighter. Little by little the appearance of the fibres changes. The contours become irregular, and the substance (myelin) 
composing the borders becomes folded, striated, and granulated in appearance. The myelin wells over the sides of the ends of the fibres in irregular globular or contorted masses. Occasionally a pale filament may be seen projecting beyond the torn end of a fibre. Owing to the fact that various solutions have different effects

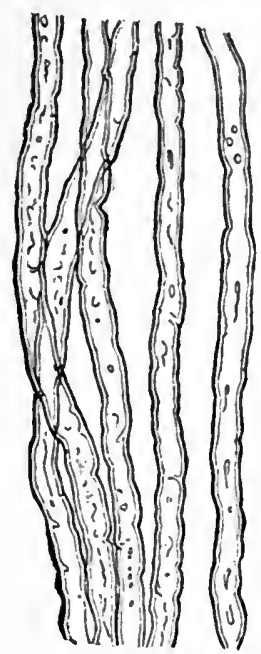

Fre. 2.-Medullated Nerve-fibres, with donble and irregular contour showing. (Schwalbe.) upon the different parts of the nerve-fibres, it is possible to prepare specimens which shall exhibit clearly their threefold structure. Thus, for example, a solution of picrocarminate of ammonia colors the central part of the fibre, or axis-cylinder, but not the myelin; whereas osmic acid stains the axis-cylinder slightly, the myelin thoroughly, but not the substance of the annular rings. By use, then, of various reagents, to color the nerve-fibres, and by numerous observations of them under various circumstances, their threefold nature in a living state is thought to be demonstrated. We distinguish, then, in the medullated fibres: (1) An outer membrane, extremely thin, pellucid, with nuclei in it, and called the primitive sheath or sheath of Schwann; (2) an interior layer of dimly granular, white, and highly refracting substance, semi liquid during life, and after death undergoing a process resembling coagulation-called the medullary sheath or white substance of Schwann; and (3) a cylindrical band of albuminous material, transparent, but with marks of fibrillation-called the axis-cylinder. Only the last is supposed to constitute the essential nerrous structure; for, as we have already seen, many nervefibres are simple naked axis-cylinders, and all fibres for a certain distance from the cells in which they originate are devoid of the medullary sheath. There is considerable evidence that the presence of this sheath depends upon the need of insulation only.

$\$ 22$. Besides the threefold longitudinal structure of the medullated nerve-fibre, we have to notice certain structural modifications that occur at intervals in its length. The peripheral nerve-tube does not run along as a regular cylinder, but places of constriction appear at certain points situated beneath the outer sheath; these constrictions are made at the expense of the medullary sheath or myelin. They are called annular constrictions or nodes of Ranvier; the portion of nerve-fibre included between two of these constrictions is called an interannular segment. At the constrictions the ends of the segments of the outer sheath are joined together by a 
$\mathbf{A}$

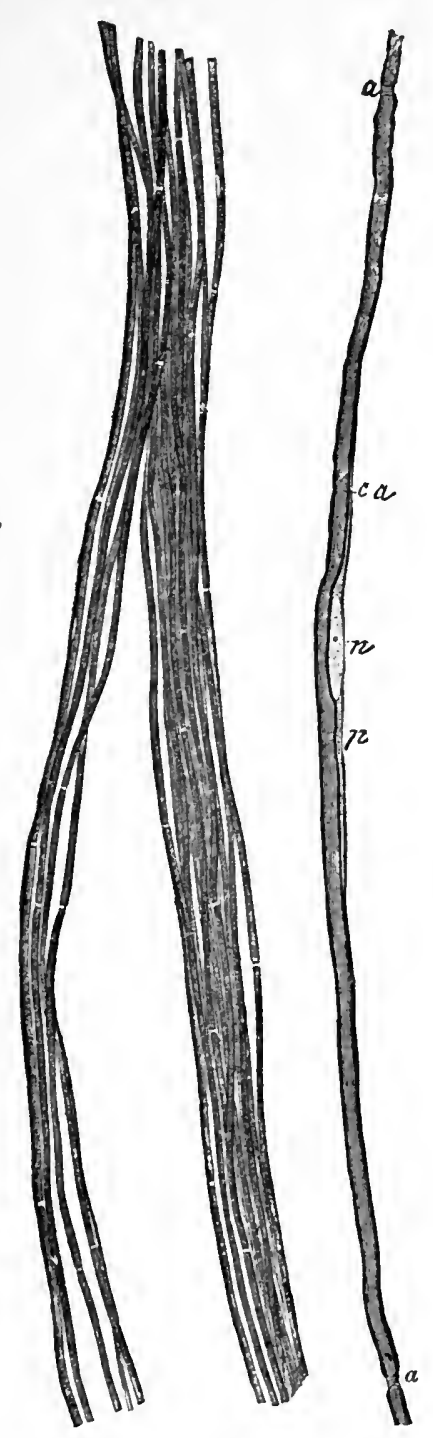

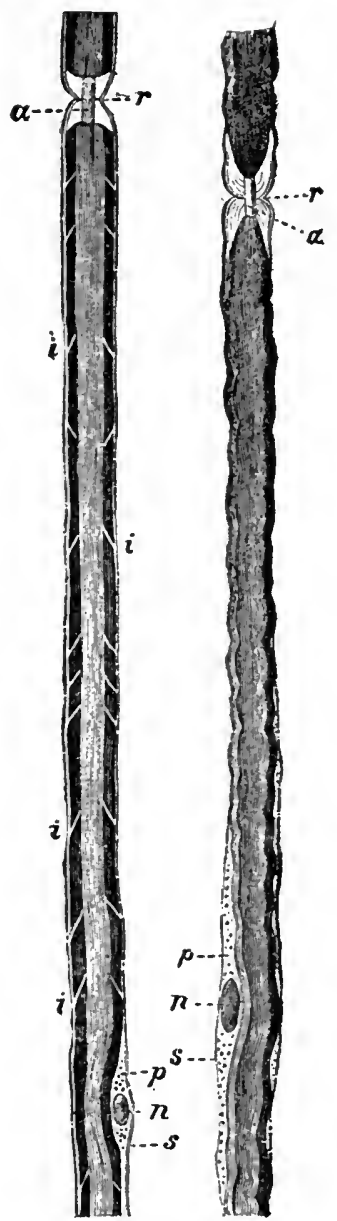

Fra. 3.-A, Medullated Nerve-fibres from the Sciatic of a Rabbit, stained with osmic acid, and dissociated in water. (Ranvier.)

B, Single Fibre Enlarged $400 \%, a, a$, Annular constrictions, or nodes of Ranvier, nearly midway between which is $n$, the nucleus, with protoplasm, $p$, surrounding it; $c a$, axis-cylinder.

Fia. 4.-Medullated Nerve-fibres. (Schwalbe.) $a$, Axis-cylinder; $\&$, sheath of Schwann: $n$ nucleus: $p, p$, grannlar substance at the polcs of the nuclens; $r, r$, Rauvier's nodes, where the medullary sheath is interrupted and. the axis-cylinder appears; $i, i$, incisures of Schmidt. 
thin layer of cementing substance which extends inward toward the axis-cylinder. These interannular segments of the nerve-fibre vary greatly in length. When several nerve-fibres lie parallel with each other, the segments of four or five of them often seem to have about the same length, and then the series appears interrupted by some segment considerably longer or shorter than the rest.

Each interannular segment of a nerve-fibre has a flattened elliptical nucleus, situated nearly equidistant between the two annular

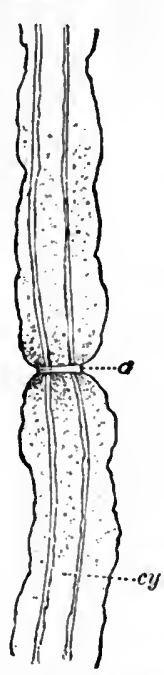

Fig. 5.-Medullated Nerve fibre from the Sciatic of an Adult Rabbit. $600 \%$. (Ranvier.) $a, \mathrm{An}$. nular constriction ; and cy. axis-cylinder with double contour showing. constrictions which limit the segment. This nucleus often has a nucleolus; between the nucleus and the myelin there exists a minute mass of protoplasm which is spread beneath the membrane of Schwann and fixed to it.

Scattered irregularly along each interannular segment are delicate lines or fissures which seem to run obliquely through the medullary sheath from the membrane on the surface of the nerve-fibre to the axis-cylinder. Their significance is not yet determined; they are called the "incisures of Schmidt." (See Fig. 4.)

$\S 23$. The complex microscopic structure of the medullated nerve-fibre, as described above-outer sheath, medullary sheath, axis-cylinder, interannular segments limited at each end by annular constrictions, nucleus and nucleolus, and incisures of Schmidt-cannot be consilered as "ultimate," even in the restricted sense in which we use the word as applied to what the eye can see by the aid of optical instruments. Other still more minute characteristics of its structure must be briefly mentioned, although with the understanding that their interpretation, and even their existence, is more doubtful than are the characteristics already described.

The fact that isolated axis-cylinders, when submitted to the action of picrocarminate of ammonia, are stained red along their median line, while an extremely thin homogeneous border is left comparatively uncolored, and the additional fact that minute flakes or scales sometimes seem to appear upon their surface, have led to the conjecture that the axis-cylinder has a double structure. The clear homogeneous border probably corresponds to the so-called "sheath of Mauthner." "

The "fibrillated" appearance of the axis-cylinder under the microscope has already been referred to as undoubted; but the exact

' See Ranvier, Traité Technique d'Histologie, i., pp. 738, 742. 
nature and the interpretation of this appearance are still matters of dispute. On account of the fact that the light must be passed through two or perhaps three cylinders in order to reach the interior structure of the nerve-fibre, its examination under the high powers of the microscope which are necessary to see this fibrillated structure is extremely difficult. In spite of this difficulty, however, Hans Schultze 'claims that the fibrils of the axis-cylinder can be
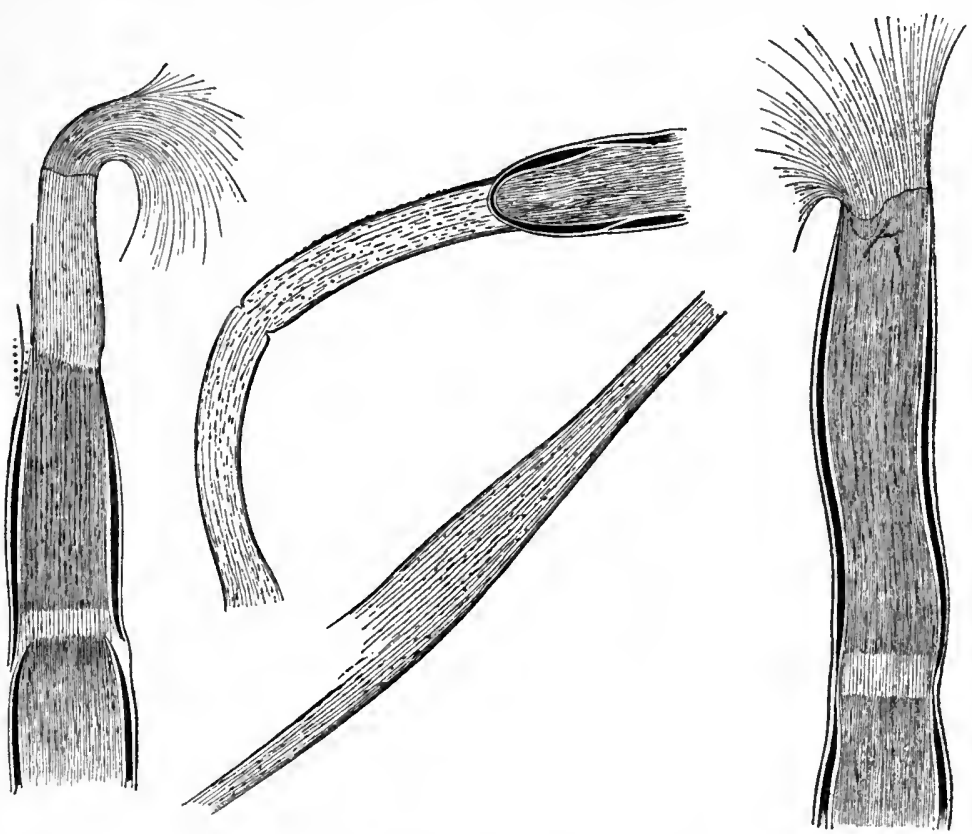

Frc. 6.-Fibrillated Appearance of the Axis-cylinders of Medullated Nerve-fibres. (Hans Schultze.)

distinctly traced in living fibres, when these are in process of forming and are still naked, or where they issue from the cells without a medullary sheath, or where they lose this sheath at the annular constrictions or in the peripheral end-plexuses. Various preparations of dead nerve-fibre, treated with different reagents, seem to demonstrate the same fibrillated structure. Moreover, from the fact that the nervous substance of the fibrils takes a carmine tinge, while the interfibrillary nucleated substance remains stained steelblue with the nitrate of silver, Schultze argues that the axis-cylinder consists of two chemical substances. The fibrillated appearance can, therefore, scarcely be considered as due to the arrangement of

${ }^{1}$ In the Archiv f. Anat. und Physiol., 1878, Anat. Abth., pp. 259-285. 
rows of granules in straight lines. ${ }^{1}$ According to T. W. Engelmann, ${ }^{2}$ in good preparations these fibrils appear distinct, and are never seen to anastomose or form a plexus of fibrils. By actual count the number of fibrils remains the same-at any rate, between any two annular constrictions; nor are they apparently interrupted in their course by these constrictions. The fibrils, as found in different nerve-fibres, seem not to differ in respect to size or closeness of
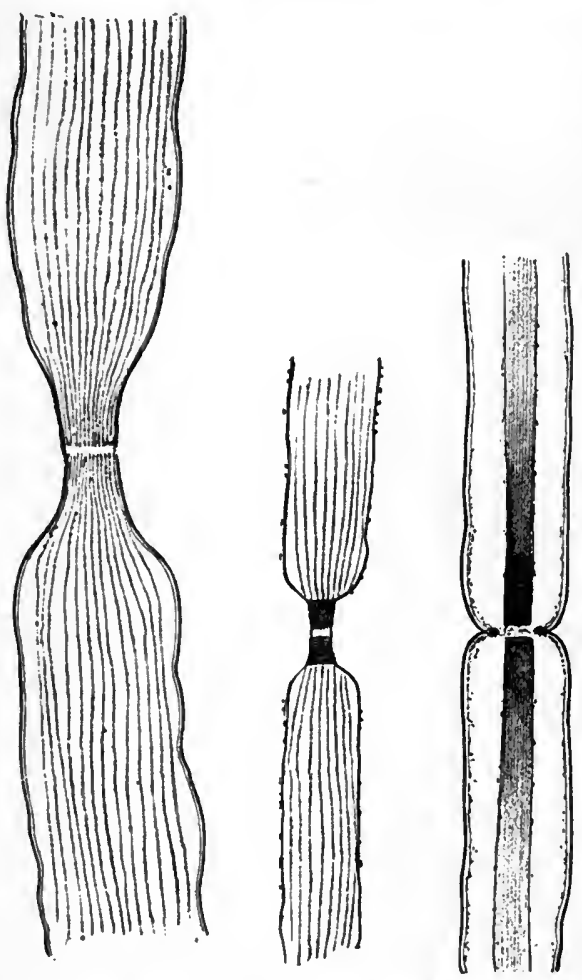

FIG. 7.-Fibrillated Axis-cylinders broken at the Nodes contact, but their number differs in nerve-fibres of different sizes. Engelmann counted about four hundred in the thickest fibres taken from the motor roots of the spinal cord of the frog. The closeness of their contact, and the smallness of their number, as compared with that of the fibrils into which the fibre breaks up at its peripheral terminations, make it difficult to see how these subdivisions of the axis-cylinder can have any separate function as the conductors of nervous impulses. Further information regarding them must be left to subsequent researches. (See Fig. 6.)

The strict continuity of the axis-cylinder through the annular constrictions may be called in question.

Engelmann found that, on being treated with nitrate of silver, the axis-cylinders, as a rule, were broken off at the annular constrictions or nodes. ${ }^{3}$ Out of a hundred cases of broken cylinders only four appeared where they had not parted in the middle of these constrictions. It is not to be inferred from this, however, that normal and living nerve-fibres are interrupted by a space of even mi-

'So article of H. D. Schmidt, in the Monthly Micr. Jour., 1874, vol. xii.

'Pflüger's Archiv, xxii., p. 26.

3 Pflüger's Archiv, xxii., pp. 1-24. 
croscopic proportions at these nodes; no such interruption appears. But it is by no means impossible that these fibres are to be regarded as composed of a number of annular segments cemented togethereach separate fibril placed exactly end to end with its fellow in the adjoining segments. Such an arrangement would accord with the theory which regards the segments as elongated and developed nerve-cells.

$\S 24$. Non-medullated nerve-fibres, or fibres of Remak, differ from those already described in that they do not possess a medullary sheath. They are grayish and translucent, longitudinally striated, with flattened elongated nuclei lying at frequent intervals upon their surface. When gathered together within a sheath of neurilemma, they are not placed side by side as are the medullated nervetubes; they are rather formed in the interior of the nerve, where they unite and divide and make an intricate plexus or network of fibres. They are grouped in larger bundles, sometimes alone, but more frequently in connection with medullated fibres. Their striated appearance is probably due to the fact that they, like the axis-cylinder of the medullated nerve-fibres, are composed of numerous fibrils. As has already been said, they belong to the sympathetic system.

$\S 25$. The size of different nerve-fibres in the human body varies greatly, according to their kind, position, and, perhaps, function. As a rule the non-medullated fibres are smaller than the medullated, the former being from $\frac{1}{6000}$ to $\frac{x}{8000}$ of an inch in diameter, and the latter (in the trunk and branches of the nerve) from $\frac{1}{1500}$ to $\frac{1}{300}$ of an inch. But this rule is not always followed. In the white matter of the cord the medullated

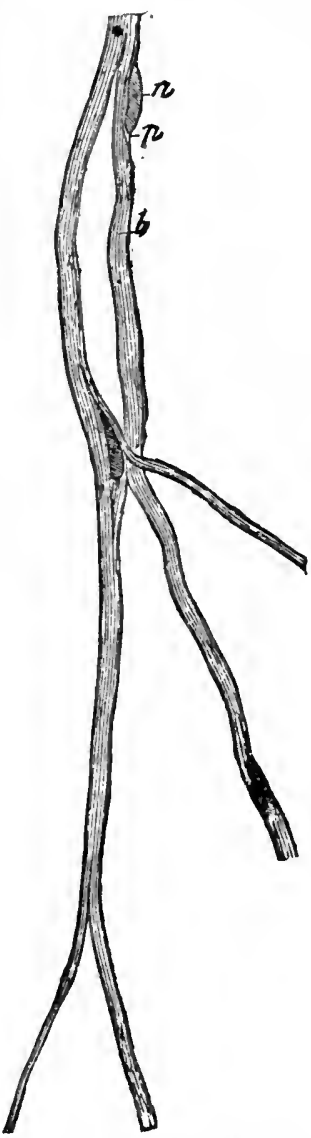

Fia. 8.-Fibres of Remak from the Pneumogastric of the Dog. $400 \%$. (Ranvier.) $n$, Nucleus with surrounding protoplasm, $p$; $b$, stria corresponding to fibrils.

fibres range in size from $\frac{1}{1200}$ to $\frac{1}{2000}$ of an inch, in parts of the anterior columns, and about $\frac{1}{7000}$ of an inch in those regions of the lateral and posterior columns which are nearest the gray matter of the cord. In the gray matter of the cord and brain the fibres are much finer-being from $\frac{1}{7000}$ to ${ }_{14} \frac{1}{1000}$ of an inch in diameter, or 
even of an almost immeasurable fineness ; they are finest of all in the superficial layers of the brain and in the nerves of special sense. In some instances the axis-cylinder may be not more than $\frac{10000}{1000}$ of an inch in diameter.

$\$ 26$. The number of fibres which enter into the composition of individual nerves also varies greatly. In the common motor nerve of the tongue it has been estimated at about five thousand, in that of the eyes at fifteen thousand, in the optic nerve at one hundred thousand at least.

\$27. So-called ganglion-cells, or nerve-cells, are the second of the two structural elements which can be more minutely described as undoubtedly belonging to the nervous system. These bodies vary greatly in size and shape, but they all show, when subjected to microscopic examination, certain well-recognized common characteristics. Nerve-cells are irregular masses of protoplasm, finely granular and delicately striated, with a large nucleus which is welldefined and resicular in appearance, and which usually contains a shining nucleolus ; they send off one or more processes. In the gray matter of the cord and brain they are embedded in the neuroglia or so-called "nerve-cement;" in the smaller nervous centres, such as the ganglia of the sympathetic and the ganglia on the posterior roots of the spiual cord, they are surrounded by a capsule of connective tissue.

\&28. Careful microscopic investigation of the nerve-cell with high magnifying powers of the instrument reveals the great complexity of its structure. In describing this complex structure the bipolar ganglion-cell of the fish may be considered as a common type. Such a cell is called by Max Schultze ${ }^{1} a$ " nucleated swelling of the axiscylinder." When found in the course of a nerve-tibre it appears at first sight as a complete interruption to the continuity of the fibre. Further examination is thought to show, however, that, when the fibre reaches the cell, the axis-cylinder loses its medullary sheath, and the fibrils which constitute the substance of the cylinder become dissociated, and continue their course over the surface of the "ganglionic globe" to its opposite pole; here they reunite and form a fibre identical with the one that approached the nearer pole of the cell. The "ganglionic globe" itself appears to be composed of granular substance. We may distinguish, then, in such a ganglion-cell these two parts: (1) a fibrillary covering, the fibrils of which are continuous with the fibrils of the axiscylinder on either side of the cell ; and (2) a granular globe containing near its surface a nucleus, within which one or more nucleoli

${ }^{1}$ In Stricker, Human and Comparative Histology, i., p. 174. 
appear. ${ }^{1}$ A repetition of these parts of the structure of the bipolar cell, it is claimed, may be expected and found in ganglionic nervecells in general.

A microscopic structure substantially like that of the bipolar ganglion-cell of the fish, as already described, is found to belong to the multipolar cells of the anterior horns of the spinal cord of man, and of the ox, or of other mammals. Among the many processes given out by such a cell, the researches of Deiters and of others have demonstrated that ordinarily only one becomes continuous with the axis-cylinder of the peripherally running nerve-fibre. This one, called the "prolongation (or process) of Deiters," has sometimes been distinctly seen to be fibrillated; and it is supposed that its fibrils are, as a rule, continuous with those of the axis-cylinder of the nerre-fibre. Hence it is called the "axis-cylinder process." The other processes from the cell also seem to be fibrillar; but the quantity of interfibrillar granular substance which they contain is greater than that in the axis-cylinder process. These fibrils ramify, anastomose with each other, and become lost in an intricate network of extremely minute nervous filaments. Orer the surface and within the interior of the "ganglionic globe" of the multipolar cell the fibrils of all these processes run in every direction with bewildering complexity. Their relation to one another, and to the rarious parts of the substance of the cell, cannot be said to be determined with any degree of certainty. Most of the fibrils appear only to traverse the ganglion-cells, but some of them, perhaps, originate within the cells. In the case

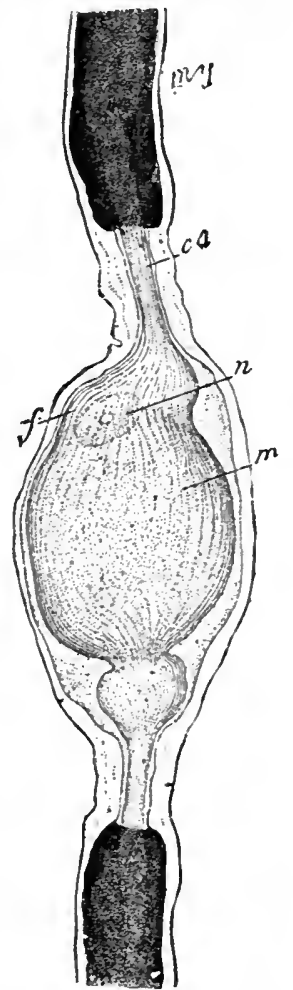

Fia. 9.-Nerve-cell from the Spinal Ganglion of the Ray. ${ }^{350} 1$. (Ranvier.) $m y$, Mednllary sheath of nerve-fibre, enclosing $c a$, the axis-cylinder, the fibrils of which $(f)$ separate and run over the ganglionic globe, $m ; n$, nucleus. of any thus originating, it is not as yet possible to say whether or not they arise out of the nuclei and nucleoli, and so, whether we may consider these parts of the cells as the special sources or centres of the nerve-fibrils, as Harless, Meynert, and others have done. ${ }^{3}$

${ }^{1}$ See Ranvier, Traité Technique d'Histologie, i., p. 712.

'See, on this whole subject, Max Schultze in Stricker, Human and Compara. tive Histology, i., pp. 172-187 ; Ranvier, Traité Technique d'Histologie, i., pp. $710 \mathrm{ff}$; and Hans Schultze, Archiv f. Anat. u. Physiol., 1878, pp. 259-285. 
§29. The variety of shapes taken by the nerve-cells has already been mentioned, as well as the fact that they may be classified as unipolar, bipolar, and multipolar. Some are nearly round ; others ovoidal, caudate, stellate, or shaped like a flask or the blade of a paddle. Still others appear somewhat like the foot of an animal with claws; while the branching processes of others give them the appearance of sprawling out irregularly in a half-score of different directions. To a certain extent the shape of the cells is characteristic of that region of the central nervous system where they are

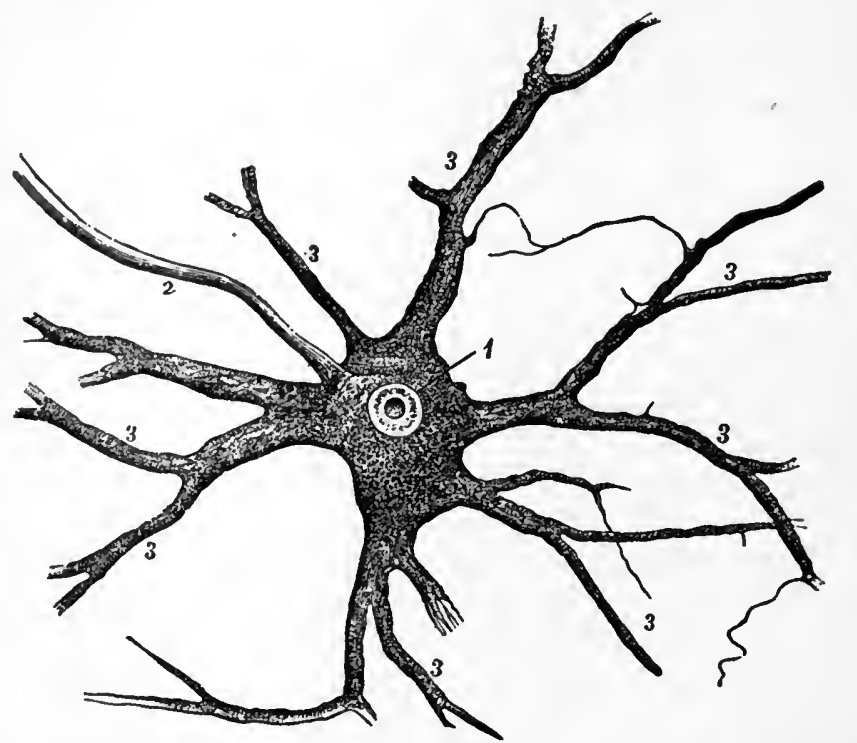

Fig. 10.-Mnltipolar Ganglion-cell from the Anterior Horn of the Gray Snbstance of the Spinal Cord of the Ox. (After Deiters.) 1, Nucleus; 2, axis-cylinder process; 3, 3, branching processes.

found, in most abundant numbers, embedded in the neuroglia. For example, large ganglion-cells of irregular shape, with branching processes, which have been called "motor," are found in the anterior horns of the gray matter of the spinal cord ; pyramidal cells of various sizes, with processes from both base and apex, are characteristic of the cortex of the cerebrum; and just at the inner edge of the gray cortical matter in the cerebellum appear irregular globular or ovoidal cells, which send off one or two branching processes toward the surface of the cerebrum. The ganglion-cells of the sympathetic also are usually globular or ovoidal, and have one or more processes which pierce their capsule and become non-medullated 
nerve-fibres. Unipolar cells are found in the spinal ganglia of the higher animals, bipolar in the spinal ganglia of fishes.

Nerve-cells vary in size as much as in sinape ; the limits may perhaps be given as from about $\frac{1}{250}$ to $\frac{1}{3500}$ of an inch. ${ }^{2}$ No special physiological significance can in any case be assigned to the shape of the nerve-cell; we are wholly ignorant of the meaning of such a variety of forms, and of the value of any particlar form in a given position. It is possible, however, that the large size of the so-called "motor-cells" of the anterior horns of the spinal cord is indicative of their special physiological function. We may also fairly incline to interpret the multiplication of ganglion-cells in the central parts of the nervous system as significant of the large amount and high quality of work which must be done by them within these centres. It is possible that the shape of the cells is largely due to the mechanical conditions which control their development within the embryo; but upon this subject we have scarcely any trustworthy information.

$\S 30$. The structure of the nerve-fibres and nerve-cells, and the nature of the histological relations which apparently exist between the two, have led to a captivating theory intended to reduce all the elements of the nervous mechanism to modifications of a single form. Extremely different in structure as the various parts of the nervous system obviously are, we are told that modern histological science refers them all, for their elements, to "one perfectly definite type ;" this type is the ganglionic nerve-cell. The important common characteristic, that they send out prolongations which become nerre-fibres, is assumed to belong to all such cells. The fibres are, accordingly, considered to be prolongations of the cells, and to be formed of substance like that of the source from which they appear to arise. Nerve-fibres may then be described as nervecells drawn out into an extremely long peduncle, which serves to connect them with other similar cells and fibres, or with certain muscular fibres which the nervous matter commands. This morphological theory of the nervous elements rests, however, upon a doubtful basis, and certain strong objections may be brought against it. We are probably warranted simply in asserting that both classes of these elements, like the other primary structural forms of the body, may be regarded as differentiations of one type (the cell) under conditions of which we are almost wholly ignorant.

There is accumulating evidence in favor of the view that nerve-

i See an article of $\mathbf{J}$. Hoffmann in the American Journal of Neurology and Psychiatry, August, 1883, pp. 432 ff.

${ }^{2}$ Ranvier, Traité Technique d'Histologie, i., p. 710. 
fibres are, in general, connected, both histologically and physiologically, with the nerve-cells. One of the processes of each cell may, therefore, as a rule, be regarded as continuous with the axis-cylinder of a nerve-fibre. It is true that this connection can by no means always be traced by the microscope. A score of years ago one investigator ' declared that, after having examined the gray matter of the spinal cord a great number of times, he had demonstrated this alleged connection only very rarely. Repeated observations since, of the improved modern kind, liave not done away with the comparative infrequency of the desired demonstration. But from the rery.nature of the case a great number of the nervefibres must have their connection with the cells broken off by the treatment they receive in preparation for examination. And the positive cases where such connection has been traced may fairly be said to have indicated the rule. Moreover, the facts of physiology (to which reference will be made subsequently) seem to favor such a view of the anatomical relation of these two elements of the nervous system.

Additional evidence upon this subject may perhaps be derived from the recent researches of $\mathrm{E}$. A. Birge. ${ }^{2}$ This investigator undertook the gigantic task of counting the nervous elements in the ganglia and roots of the spinal cord of a large number of frogs. $\mathrm{He}$ apparently discovered a general relation indicating some agreement in the number of the so-called motor-cells and the fibres alleged to originate from these cells. In one case (No.42) an actual count of ten motor-roots gave 5,734 fibres and 5,777 cells on the right, and 5,740 cells on the left side of the cord. Other results of counting, however, were by no means so favorable to the statement that the number of the fibres in the roots agrees exactly with the number of cells in the corresponding region of the cord. Nor could more complete results of this kind form any sufficient warrant for the conclusion that everywhere in the nervous system the number of fibres corresponds with the cells, or that the nerve-fibres all spring from the nerve-cells; much less, that they may be reduced to one form of such cells as to a perfectly definite type.

$\S 31$. The discussion of the chemical constitution and structural form of the elements of the nervous system introduces the question as to the Functions of these Elements. This question must be answered, if at all, by the science of physiology. And in view

1 Vulpian, see Leçons sur la Physiologie du Système Nerveux, p. 318, Lecture of July $9,1864$.

${ }^{2}$ Archiv f. Anat. u. Physiol., 1882, Physiolog. Abth., pp. 435-479, especially p. 471 . 
of our ignorance of the genuine nervous character of all other claimants to a place among the elements of the nervous system, our inquiry is narrowed to the following terms: What can nerve-fibres and ganglionic nerve-cells do? With the activities of these elements, as combined into the complex organs of the human nervous mechanism, the whole of our subsequent examination is designed to deal. We speak here very briefly of certain fundamental properties of the two nerve-elements already described-that is, of the nerve-fibres as gathered into bundles called nerves, and of the cells as collected into ganglia and connected with these nerves.

Nerves and nerve-cells have certain properties in common ; that is to say, within certain limits both can do the same things. Botll are capable of becoming the subjects of a specific kind of molecu. lar motion which we are entitled to consider as distinctively " neural," but about whose nature and mathematical or physical relations to other modes of the molecular motion of matter we are still almost totally ignorant. Both are also capable of propagating this distinctively " neural commotion" from one portion of their structure to another. In a word, both nerve-fibres and nerve-cells have the properties of Excitability and Conductivity; and the excitation and conduction of excitation which these nervous elements display are of a kind peculiar to themselves. It is the production, propagation, modification, and distribution of this distinctive nerve-commotion which constitutes the one constant function, or property, of the nervous elements, whether considered as isolated or as combined into organs. It is customary with some writers to speak of the production of psychical phenomena as the crowning function of the nerrous system. But whatever may be the view we shall find ourselves compelled to take of the relations between the location, quantity, quality, and combinations of this neural molecular motion and the phenomena of self-conscious life, from our present point of riew the utterances of such writers-if designed as anything other than figures of speech which need to be explained in detail to be even suggestive of those real facts and relations which they, in truth, only symbolize-are of little interest or value. We are speaking of a material structure, which, although alive and standing in altogether unique relations to psychical phenomena, is, nevertheless, in itself considered, nothing but a very complex collection of moving molecules. The peculiar form of molecular motion which characterizes this structure-namely, so-called "nerve-commotion"-is its unique function. Inasmuch as such nerve-commotion may be considered as originally set up in a single nervous element or group of elements, and then propagated from this initial point along cer- 
tain more or less definitely marked tracts to other elements or groups of elements, we may divide the one function into two-the function of excitation and the function of conduction.

$\S 32$. Nerve-commotion, or neural molecular action, is, of course, never an uncaused event. It begins at certain points in the nervous elements, where it is set agoing by the application of appropriate causes of excitation. The causes of the excitation of the nervous elements are called "stimuli." Stimuli are of two general kinds-external and internal. External stimuli comprise all such modes of the motion of matter as act upon the peripheral parts of the nervous system, and so produce within it a state of excitation or nerve-commotion; among these are light, heat, chemical changes, etc. Internal stimuli are such as act upon the nerve-cells of the central organs; they consist, in general, of changes in the blood produced by an increase or decrease of oxygen, the presence of drugs, etc. The susceptibility of a nerre to any form of external stimulus is called its "irritability;" and when a nerve will no longer respond to such stimulus by being thrown into a condition of excitation, it is said to have lost its irritability. As the word is generally used, then, the irritability of a nerve is its property of excitability under the action of some form of external stimulus. When excited by such stimulus it is said to be irritated. We shall use both sets of words, reserving the words "excitation" and "excitability "for the general state and function of all nerrous tissue considered as capable of a specific molecular commotion '-a nervecommotion.

$\S 33$. But although all the nervous elements may be said to have the properties of neural excitability and conductivity, important differences arise as to the conditions under which, and as to the modes in which, they exercise their functious when combined into a complex nerrous system. In the normal condition of such a system it is by no means all of its parts which are directly excitable, whether by external or by internal stimuli; nor is the effect of the excitation of both the elementary structural forms of such a system exactly the same. A single nerve may, indeed, be separated from the other parts of the nervous system, with a muscle attached, and may then be made to exercise its neural function in moving the muscle by being itself stimulated with different kinds of stimuli at different points along its course. But in their normal place and condition nerves are never excited by the direct application of

${ }^{1}$ It is a pity that we have in English no one word which can be used under all conditions, and compounded ad libntum, in order to designate a property, a process, a state, etc., as can the German word Erregen, Erregung, etc. 
stimuli ; they are always excited indirectly by the propagation to them of nerve-commotion which originates in the central organs or in the end-organs. The distinctive office of the nerres is, then, to act as conductors of molecular motion set up in themselves by the direct excitation of the nerrous elements in which they either centrally or peripherally terminate. Moreover, large portions of the central organs do not respond to the direct application of various kinds of stimuli to their surface. We are obliged, then, to suppose that many of the nerve-cells which compose these organs are excitable only by stimulation through the nerve-fibres that run into them. The case of the normal nervous system, with respect to its excitability, may, then, be briefly described in the following terms: The end-organs of sense are directly excitable by external stimuli, and each specific kind of end-organ which is characteristic of a particular sense is excitable only by the specific kind of stimuli appropriate to that sense. The afferent or centripetal nerves are excited only by the end-organs of sense ; their specific function is to conduct the nerve-commotion, started by the external stimuli in these end-organs, toward the central organs. The efferent or centrifugal nerves are not directly excited by either internal or external stimuli, but only by the central organs ; their specific function is to conduct the nerve-commotion started in them by the central organs to the muscles, glands, etc. - to the peripheral parts of the body which are to be mored through their excitation. The central nerve-cells themselves are excited either through the nerve-commotion brought to them by the afferent nerves or by internal stimuli. Nervecommotions are also said to arise in them automatically; but the facts covered by the term "automatic" require further distinctions to be made as to the functional activity of the different nerveelements.

$\S 34$. If the distinctive normal function of the nerres is the conducting of neural molecular motion between the central organs and the end-organs, the function of the ganglion-cells can by no means be pronounced so simple. These cells are, indeed, also conductors of nerve-commotion; within the central organs they form important parts of the tracts along which such commotion passes. They serve also as points for the division and redistribution of this commotion; they may be spoken of as switching-places in the system or network of tracts. In these "shunting-places" of the cell many lines of conduction meet; and the one of them taken by any impulse entering the cell may depend upon the relative amount of resistance offered by these lines. The work of the cell may then be considered as "re-directive." The office of the cell in distri- 
bution of the nerve-commotion may also be either to condense or to disperse it ; in the former case the distribution might be spoken spoken of as "associative," in the latter as "dissociative." " They may also intensify or diminish the nerve-commotion entering them. But the nerve-cells have also other functions, or forms of the one neural function, which have been classed as either (a) automatic, (b) reflex, or $(c)$ inhibitory.

(a.) Automatism, or the power of initiating the peculiar form of molecular motion known as "vital impulses," independently of the action of any discorerable stimulus from without, is one of the fundamental properties of protoplasm. An amœba, for example, is a minute mass of such protoplasm; it executes movements which cannot be wholly explained by reference to any changes in its environment. The difficulty of distinguishing automatic from reflex action in the spinal cord, and muscular from nervous automatism in the sporadic ganglia, need not concern us at present. According to Eckhard ${ }^{2}$ two kinds of this automatic function of the ganglion-cells may be distinguished-viz., the automatic-tonic and the automaticrhythmic. In the former case the control of the cells over the muscular structures by means of the efferent nerves is irregular; in the latter this control results in the nearly simultaneous contraction of the same set of such structures, repeated at regular intervals; as is the case in the movements of the heart and lungs. In neither case, however, can we form any clear conception of the origin within the cells of this neural commotion, of the nature of the forces at work to produce it, or of the changes in material that are involved in it. We can only say that as yet we know no reasons lying outside of the structure and activities of the living nerve-cells themselves which will account for the starting of the excitation. In this sense, at least, such neural action is "automatic."

(b.) The reflex function of the ganglion-cells admits of a somewhat more detailed and satisfactory statement; but the phenomena and laws of reflex nervous action are properly discussed as belonging to the central organs of the nervous system. It is enough, at present, to note that the great changes which take place in the character of nervous impulses, when, after entering the central organs by the afferent tracts, they are, as it is said, "reflected" from those organs along the efferent tracts, are indubitable evidence of the specific molecular activity of the ganglion-cells. For the afferent impulses are, in fact, not simply reflected in these cells;

${ }^{1}$ See A. Hill, The Plan of the Central Nervous System, p. 2. Cambridge, 1885.

2 Hermann's Handb. d. Physiol., II., ii., p. $19 \mathrm{f}$. 
they are greatly modified as to their number, intensity, character, and distribution. This modification is proof of profound molecular changes that are instituted in the substance of the cells themselves. It is one proof, among others, that a large expenditure of energy in the cells accompanies the transmutation of afferent into efferent impulses.

(c.) The function of inhibition, as ascribed to ganglion-cells, must be pronounced more doubtful in character than either of the two foregoing. It was found by Wundt ${ }^{1}$ that nerrous impulses are delayed on passing through the spinal ganglion. Such impulses seem also to consume an amount of time in travelling along or through the cord that cannot readily be accounted for as wholly due to the length of the nervous tracts which they thus traverse. But until our information is more precise as to the microscopic structure of the cord, and as to the tracts within it which the nervous impulses follow, we cannot say with confidence how much of this delay is due to molecular changes peculiar to the cells themselves. That the automatic and reflex functions of the medulla oblongata may be compounded, as it were, in such way as either to inhibit or to accelerate the action of the heart and lungs and muscular walls of the arteries, is a well-known fact. It has already been said that nerve-cells may diminish as well as intensify the nerve-commotion entering them. When afferent impulses reach the ganglion-cells of the centres, and find them already at work, such impulses result, according to circumstances, in either inhibiting or augmenting this activity. ${ }^{2}$ Moreover, the tone giren forth by a muscle, when tetanized by stimulating the nerve to which the muscle is attached with repeated induction-shocks, has the same number of vibrations per second as there are of such shocks; but the tone given forth by muscle tetanized through the spinal cord, or by action of the will, has a constant number of vibrations, namely, about nineteen per second. It would appear from this, also, that the central apparatus of nerve-cells controls the impulses which tetanize the muscle, according to the molecular structure and changes of those cells. In this sense, then, the cells may be said to exercise inhibitory functions under certain conditions.

$\S 35$. A consideration of the different effects produced by the conduction of nerrous impulses along the different nerves of the system would seem at first to justify the classification of the nerves according to the varieties of their functional activity. In this way

1 Untersuchungen zur Mechanik der Nerven, 18;6, Abth. ii., pp. $45 \mathrm{ff}$.

${ }^{2}$ Comp. Foster, A Text-book of Physiology, fourth edition, p. 134 . New York, 1880. 
we should distinguish the following classes: (a) nerves of motion controlling the muscular apparatus, whether of smooth or of striated muscular fibres; $(b)$ nerves of inhibition; $(c)$ nerves of secretion; (d) trophic nerves, or nerves which have a direct influence upon nutrition; $(e)$ centripetal nerves that have no sensory function; and, finally, $(f)$ sensory nerves, or those the excitation of which may result in conscious sensation. ${ }^{1}$

That the irritation of different nerves may have results so different as are indicated by the foregoing classes must indeed be admitted; but it is quite another question whether this difference is not wholly due to the sources of origin for the nerve-commotions sent along them, and to the structures in which it terminates, rather than to any difference in the essential physiological function of the nerves themselves. Just as the same electrical current may pass along the same kind of wire, and write a message, or ring a bell, or move the legs of a frog; just so the irritation of certain fibres of the pneumogastric nerve results in controlling the motion of the heart; the irritation of other nerves seems to bave an immediate metabolic effect in directing the secretory processes; that of still others profoundly modifies the nutrition of the portions of the body to which they are distributed. All these effects are in appearance greatly unlike the movement of a muscle under stimulation from a nerve. With regard to the influence of the nerves on nutrition (their trophic function) it is not necessary, in order to account for it, that some specific action of a particular kind of nerves should be assumed. We should suppose, of course, that the chemical processes in which nutrition consists would be changed in character by the molecular changes in the tissue which irritating any of the nervefibres running into it would inevitably bring about.

Further consideration of the six possible classes of nerves given above reveals the fact that they may all be reduced to two, according to the direction in which their function of conducting nervecommotion is exercised. The first four conduct it outward from the nervous centres, and are therefore called "efferent;" the last two conduct it inward toward the nervous centres, and are therefore called " afferent." Into these two kinds all nerves are customarily divided, so far as their physiological function is concerned.

$\S 36$. The further question now arises, Whether the general physiological function of these two principal classes of nerves differs in kind as well as in direction; or are afferent and efferent nerves to be identified so far as their specific neural function is concerned? Inasmuch as every nerve-fibre, in the normal condition of the ner-

${ }^{1}$ Comp. Sigmund Mayer, in Hermann, Handb. d. Physiol., II., i., pp. $200 \mathrm{ff}$. 
vous system, is a stretch of nervous matter between two terminations-a point of origin and a point of issue for the state of excitation-it might, at first, seem simpler to consider it as intrinsically capable of propagating nerve-commotion in one direction only. It would be concluded, then, that the behavior of afferent and efferent nerres, when stimulated, is essentially different with respect to their molecular processes. Certain phenomena are sometimes urged in favor of such a conclusion.

The application of heat to an efferent (or motor) nerre causes no contraction in the muscle which the nerve supplies; heat does not appear to be a stimulus of such nerres. On the contrary, Grützner ${ }^{2}$ concluded that heating the different kinds of afferent nerves to from about $115^{\circ}$ to $125^{\circ} \mathrm{Fahr}$. does excite them. The passage of a constant current along an efferent nerve, so long as this current does not suddenly change in strength, does not stimulate this nerve so that the muscle contracts; but such a current does excite nervous impulses in a sensory nerre. Moreover, certain chemical substances are said to act as stimuli on efferent nerves which hare no such effect upon sensory nerves.

On the other hand, the rate of conduction in both afferent and efferent fibres, under similar conditions, is about the same. The laws which evince the behavior of nerves under stimulation by electricity, and which are most relied upon as a basis for a mechanical theory of the nervous system, are largely the same for both kinds of fibres. There is a large amount of scientific information, called "general physiology of the nerves," which looks in the direction of identifying the molecular processes in the two classes of nerrefibres. This is true in particular of the remarkable phenomenon known as the "negative variation" of the nerve-current. Moreover, the marked difference (referred to abore) in the results obtained by stimulating motor nerves on the one hand, and sensory nerves on the other; is plainly, to a great extent, due to the difference in the sources of the stimulation; the former are excited by the central organs, the latter by the end-organs of sense. The molecular structure of these two sets of organs, and their consequent molecular motion when acted upon by the appropriate stimuli, differ widely; we do not, then, need to assume a specific difference in the function of the connecting nerre-strands in order to account for a marked difference in the results. Thus it may be assumed that molecular disturbances, which would be quite powerless to stir the sluggish muscle-fibres when transmitted to them by a motor nerve, would occasion profound changes in the more sensitive

'Pflüger's Archiv, xvii., p. 215. 
structure of the ganglion-cells when transmitted to the latter by a sensory nerre.

Various attempts have been made to demonstrate, experimentally, that motor and sensory nerves can perform each other's functions. Such experiments have not yet been altogether successful. They consist, in general, of attempts to unite by healing the central part of a divided sensory nerve and the peripheral part of a divided motor nerre, and then to show that the nerve thus united discharges certain sensory or motor functions, as the case may be. Philipeaux and Vulpian,' after various rather unsuccessful attempts of Flourens, Bidder, Schiff, and others, succeeded in uniting the central portion of the lingual (or sensory gustatory) nerve of young dogs with the peripheral end of the hypoglossal (motor nerve of the tongue) on the same side. Stimulation of the lingual nerve above the point of union then produced contractions in the hypoglossal of the same side, and that even when the lingual was divided high up so as to preclude any reflex action. But the action obtained was found to be apparently due to the chorda (motor) fibres present in the lingual. In 1863 Bert succeeded in reversing the course of the nerve-fibres in the tail of a rat, by bending this appendage over and implanting its end in the animal's back. After healing had taken place, the transplanted tail was cut off near its origin, and found to be sensitive- of course, in the reverse direction of the nerve-fibres from the natural one. This experiment would seem, then, to show that sensory nerve-fibres, when reversed, can transmit sensory impulses in the direction which was formerly centrifugal. The experiments of Kühne ${ }^{2}$ and others upon the intramuscular ramifications of the nerve-fibres in the sartorius muscle of the frog point in the same direction. If the broad end of this muscle be divided by a longitudinal slit into a forked shape, then stimulation of one of the two tines of the fork beyond their division will stimulate the fibrils of the other tine; that is, the minute twigs of the motor nerve in the tine which is directly stimulated have acted centripetally, and the excitation has then descended the twigs of the other tine.

For all the foregoing, and for other reasons, we seem warrnnted in assuming that there is no such specific difference in the function of the two kinds of nerves as is dependent upon the peculiar structure or molecular processes of each kind. Both afferent and

${ }^{1}$ See Vulpian, Leçons sur la Physiologie du Système Nerveux, etc., pp. $274 \mathrm{ff}$. ; and comp. the remarks of Hermann, Handb. d. Physiol., II., i., pp. $10 \mathrm{ff}$, and of Foster, Text-book of Physiology, pp. 503-508.

${ }^{2}$ Archiv f. Anat., Physiol., etc., 1859, pp. 595 ff. 
efferent nerves are probably capable of the same kind of molecular commotion called nerrous excitation, and of conducting this commotion in either direction. The marked difference in the results of the exercise of this function in the two cases is probably due chiefly to the difference in the organs from which the excitation of the nerve starts, and into which it is discharged. With respect to neural molecular disturbances, all nerves are excitable, conductors of excitation, and exciters of nerve-cells and muscle-fibres. And if to this description we add the statement that nerve-cells can, acting automatically, originate this nerve-commotion, can modify its character profoundly as it passes through them, and distribute it in various directions, we state, in the most general form, what is at present known as to the functions of the nervous elements. 


\section{CHAPTER II.}

\section{COMBINATION OF THE NERVOUS ELEMENTS INTO A SYSTEM.}

\&1. In the last chapter the nervous elements were considered, as far as possible, without reference to their combination for the accomplishment of a common work. Regarded as isolated, and as possessed only of those properties which belong to all living matter of the peculiar chemical constitution and structural form which are described by the word "nervous," these elements are of great interest to physiological and psycho-physical researches. But in their normal position and activity the nerve-fibres and nerve-cells are always combined into certain organs, which are then arranged in a symmetrical whole. Thus combined they are dependent upon each other for the parts which they play in the entire system. The condition and function of each element are thus determined by the condition and function of the rest. One part of this system excites another, or modifies the excitation received from another. We are unable to isolate perfectly any one of these elements, and so study its normal functions apart. It is, indeed, possible to dissect out a nerve with a muscle attached, to keep it alive for a time, and thus to inquire what an isolated nerve will do. In this way many of the most important discoveries in the general physiology of the nerves have been made. But every nerve is itself a compound of nervous elements which have been placed for purposes of experiment under abnormal conditions. The action of the nerve-cells, even when gathered into small masses called ganglia, is not open to direct inspection. Moreover, when different tracts of nerves, or different regions in the central organs where ganglion-cells abound, are partially isolated by being laid bare for the direct application of stimulus, just so far as they are separated from the system they are in abnormal condition and show abnormal results; and just so far as they are normal in condition and function they are still connected with the system. It is the mutual condition and reciprocal action of the elements, when combined into this totality, which constitute 
the nervous mechanism. A brief description of the manner of this combination is, then, indispensable at this point.

$\S 2$. It will be of great service toward understanding such a description if it is begun under the guidance of some appropriate idea. Nerve-fibres and nerve-cells exist in enormous numbers within the human nerrous system, and are combined in different proportions to make the different organs of this system. The significance of the combination appears only in the light of reflection upon the amount and kind of work which is to be done. The office of the nerrous mechanism has been said (p. $18 \mathrm{f}$.) to be that of " concatenating" all the functions of the living body in accordance with the complex internal and external conditions to which it is subject. But in the case of any of the higher auimals, and especially in the case of man, this one office requires the doing of a quantity and variety of work that are proportionate to the complexity of these conditions. How shall such a quantity and variety of work be done? To answer this question may be said-speaking figuratively-to be the problem before the nervous system. The actual arrangement of the elements of this system, in the exercise of their reciprocally conditioned activities, is the solution of the problem. As in all very complex questions of this sort, so this particular problem is solved by a wise division of labor.

The manner in which the human nerrous mechanism is developed as a response to the before-mentioned problem is made clear by considering, in the first place, a much simpler form of the same problem. The simple protoplasmic speck called an amœba may be considered as a living molecular mechanism. It appears, even under the higher powers of the microscope, as almost wholly, if not quite, composed of undifferentiated protoplasm, in the midst of which, as a rule, lies a single nucleus. If differentiated at all, it may be observed to have a somewhat solid external layer, called an ectosarc, and a more fluid granular interior, called endosarc. But minute and almost structureless as it appears, the amœba is really composed of a great number of molecules that are undergoing constant change; and it is capable of exercising several wonderful functions that do not belong to any non-living collection of molecules. Its substance is metabolic, respiratory, reproductive. The protoplasm of the amœba is the subject of constant chemical alterations, by which the old protoplasm is broken up and its products cast off, while new protoplasm is formed. Oxygen is assumed by this substance and carbonic acid excreted. The unit which is constituted by the amœba may, by fission (or by other means), divide into two parts, each of which becomes a fresh unit. But more important for our 
purpose is the fact that the amœba is irritable and automatic. It is almost unceasingly in motion. It is living matter; and when acted on by stimuli, it suffers an explosion of energy which generally results in a change of place and form. Inasmuch as these peculiar "amœboid" movements seem substantially identical with those which occur in a muscle and result in its contraction, the animalcule may be said to be contractile. But inasmuch as some of these movements caunot be ascribed to irritation of the external molecules of the amœba by the surrounding medium, but seem rather to be due to energy set free in consequence of unknown internal changes, we call it automatic. We say, "it has a will of its own." Thus does the molecular mechanism of this small bit of protoplasm, under the stimulus of changes in the pressure and temperature of its medium, and in accordance with the unknown laws of its internal self-originating changes, solve the problem pre. sented to it.

Let it be supposed that the problem becomes more complicated, and the animal structure which is to solve it correspondingly complex. The metabolic function of the animal may then be assigned to a separate system of structures; and the closely related secretory and excretory functions as well. The reproductive function may then also acquire its own peculiar organs. The muscles perform morements in masses because they retain in an eminent degree the " amœboid" contractility. But the property of being irritable and automatic becomes the special endowment of the nervous system. All these different systems, in order that they may be moved in united masses, are then adjusted to a mechanical framework (of indifferent value so far as really vital changes are concerned) of cartilitge, bone, etc.

But the eminently irritable and automatic system of molecules called nervous must undergo a further differentiation of function. In the structureless protoplasm of the amœba, the external molecules are, of course, the ones primarily to be affected by the external stimuli. It is with the internal molecules, on the other hand, that the changes called "automatic" begin. But the continual flux of its protoplasmic substance indicates that, in its simplest form, any of the molecules of the animalcule may in turn act either as irritable or as automatic. The primary differentiation of this substance into ectosarc and endosarc points, however, to a division of labor.

By this primary differentiation of the substance of the animal, one cell, or group of cells, becomes more eminently irritable, another automatic. The former has thus been fitted for the spe- 
cial work of responding to external stimuli by vital impulses; the latter for that of initiating so-called automatic impulses. The position of the former in the animal mechanism will then naturally be at the surface, where it can be acted upou by the appropriate external stimuli; the position of the latter will naturally be withdrawn from the surface, where it can be protected from such stimuli and left undisturbed for action that is either automatic or excited by only internal stimuli. But if the two linds of substance are to perform one work, although by division of labor, they must be connected; that is, the eminently irritable protoplasm of the surface must be joined by irritable protoplasmic material with the eminently automatic protoplasm of the interior. Three sets of organs are then called for in this rudimentary differentiation of the nervous substance: (1) superficial cells susceptible to external stimuli ; (2) central and eminently automatic cells, also susceptible to internal stimuli ; (3) a strand of irritable protoplasm connecting the two.

Yet one more step in the distribution of functions between the irritable and the automatic protoplasm of the complex animal organism must be taken, in order to reach the fundamental triple arrangement of a nervous system. The system of eminently contractile tissue called muscular must be brought into connection with the parts already described. In order that the more lighly organized animal may, like the amœba, both have and exercise "a will of its own," certain of its muscle-fibres must be placed under the control of the central and automatic cells. In order, also, that the entire muscular system may feel the reflex influence of external stimuli, and so, by co-ordinated contractions adapt the organs of the body to the changes of its environment, the muscle-fibres must be indirectly connected, through the automatic cells, with such superficial cells as are sensitive to these stimuli. The nervous system, therefore, in its most fundamental form consists of these three sets of contrivances with their respective functions: $(A)$ sensitive cells upon the surface of the body ; $(B)$ central cells that are both automatic and modifiers and distributers of sensory impulses; $(C)$ connecting cords, or strands, that can convey the nervous impulses either centripetally from $A$ to $B$, or centrifugally from $B$ to the contractile muscular tissues of the body.

Higher developments of this triple-formed fundamental type of a nervous system are reached by further differentiations of $A, B$, and $C$. If various kinds of stimuli are to act upon this system, then the sensitive cells upon the surface $(A)$ must be modified into various external organs of sense; and with these organs the ter- 
minations of the centripetal or sensory nervous strands must be variously connected. The terminations of the centrifugal or motor nervous strands may also be variously modified so as to connect with and control the contractile tissue of many sets of muscles. The central cells may be variously grouped and arranged, with functions more or less localized, so as to receive, modify, and distribute, in manifold ways, the different sensory impulses; and so as to co-ordinate these impulses for definite results in the peripheral parts of the body. Other such central cells may become more particularly related to the phenomena of conscious sensation and volition. Such a highly developed nervous system will then consist of the following parts : (A) End-organs of Sense, like the skin, the eye, and the ear ; $\left(A^{\prime}\right)$ End-organs of Motion, like the so-called motor end-plates and terminal nerve-bulbs; $(B)$ Central Organs, like the various peripheral and sporadic ganglia, the spinal cord, and brain, in which may come to exist $(b)$ certain portions more distinctively automatic, $\left(b^{\prime}\right)$ certain others more concerned in receiving and distributing reflexly the sensory impulses, and $\left(b^{\prime \prime}\right)$ still others more particularly connected with the phenomena of consciousness; and $(C)$ Conducting Nerves, which will be either $(c)$ centripetal, afferent, and sensory, or $\left(c^{\prime}\right)$ centrifugal, efferent, and motor, designed to connect the central organs and the end-organs. We are now to consider the details with which such a highly developed nervous system is actually constructed in the case of man. Our guides will, of course, be anatomy and histology.

§ 3. In the manner already described (Chapter I., § 19) the individual nerve-fibres are collected and bound together in fascicles or groups of fascicles, called nerves, and in larger bundles or nervetrunks. The nerve-cells are grouped into minute masses of nervous matter, such as the sporadic ganglia found in the sinus, auricular walls, and auriculo-ventricular groove of the heart; or they are gathered into larger bodies, intersected with most intricate ramifications of the nerves and interspersed with the finely granular substance called neuroglia, such as constitute the various parts of the brain and spinal cord.

$\S 4$. The nerves and ganglionic masses of nervous matter in the human body are arranged in two great systems, the Sympathetic and the Cerebro-spinal. The Sympathetic Nervous System consists of a pair of nerrous cords, situated one on each side of the spinal column; of three main plexuses, situated in the cavities of the thorax and abdomen ; of a great number of smaller ganglia, lying in relation with the viscera of the same cavities, and widely distributed over the body, especially in connection with the vascular sys- 
tem; and of a great multitude of fine distributory nerves. Each of the two cords consists of a number of ganglia united by intermediate nerves. In the other regions of the spinal column the number of these ganglia equals that of the vertebræ (sacral 5 , lumbar 5 , thoracic or dorsal 12), but in the neck (cervical) there are only 3. From this gangliated cord a communicating and a distributory series of nerve-branches are derived. By the communicating branches-each of which contains not only non-medullated nervefibres from the sympathetic system to the cerebro-spinal nerves, but also medullated fibres from the cerebro-spinal to the sympathetic-the two systems are brought into close anatomical and physiological relation, and a kind of double interchange takes place between them. The distributory branches of nerves in the sympathetic system bring the gangliated cord into connection with the blood-ressels and viscera of the body. The involuntary muscles in the coats of these vessels and in the walls of the viscera are thus bound together, and through the sympathetic fibres brought under the control of the cerebro-spinal axis. The three main plexuses referred to are collections of nerve-cells and a dense plexiform arrangement of nerve-fibres. One of them is situated at the base of the heart, to which it gives off branches that wind around that organ and penetrate its muscular substance; another is placed at the upper part of the abdominal cavity, and gives origin to numerous plexiform branches that supply the viscera of the abdomen; the third is in front of the last lumbar vertebra, and supplies the vaso-motor nerves and nerves of the muscular coats and mucous membranes of the various organs in that region of the body. Further details in the anatomy of the sympathetic nervous system are of little interest to psycho-physical studies. To such studies it is of great interest, however, to know that this system forms a bond between the sensations, emotions, and ideas which have their physical basis in the molecular condition of the cerebro-spinal centres, and those various organs in the thoracic and abdominal regions whose condition is so closely related to such psychical states. The effect of certain emotions, for example, upon the condition of the circulation, digestion, etc., is too well known to require a lengthy statement.

$\S 5$. The Brain and Spinal Cord are the great centres of the cerebro-spinal system. These bodies are situated in the bony cavity of the skull and spinal column. They have three Coverings or Membranes, the innermost one of which is directly united with the surface of the nervous substance, and sends numerous processes into its interior. (1) The Dura Mater, which is the membrane lying 


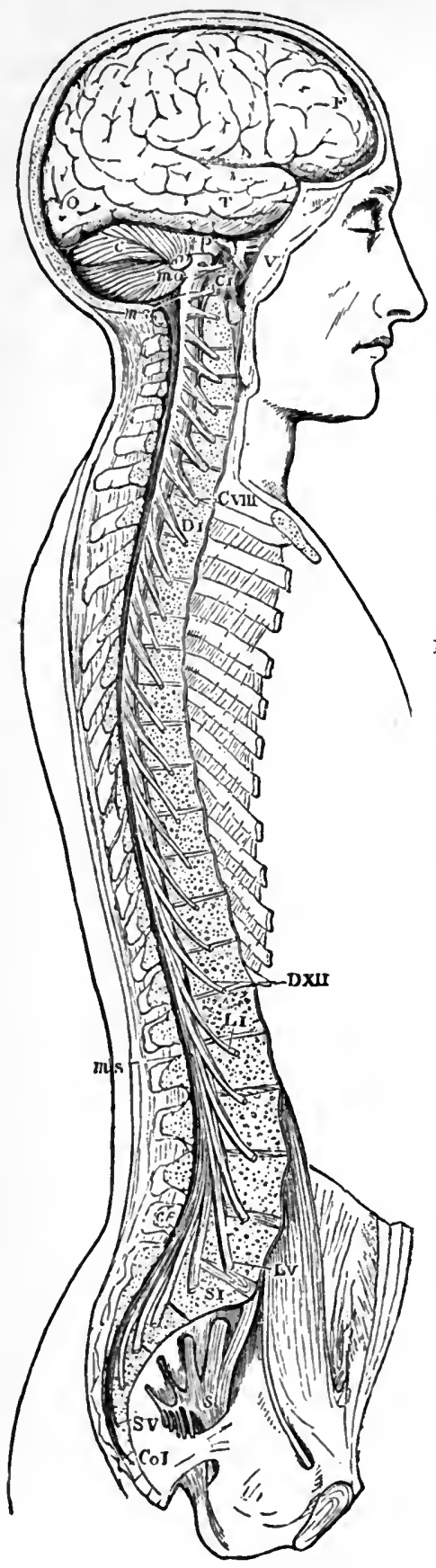

FIG. 11.-View of the Cerebro-spinal Axis. (After Bonrgery.) 1/s. The right half of the cranium and trunk has been removed, and the roots of the spinal nerves dissected out and laid on their several vertebre. F, T, O, cerebrum ; C, cerebellum : $P$, pons Varolii ; $m o$, medulla oblongats; $m s, m s$, upper and lower extremities of the spinal marrow. Cl. to CVIII, are cervieal nerves; DI. to DXII., dorsal ; LI. to LV., lumbar : SI. to SV., sacral ; CoI., coccygeal. 
next to the wall of the bony cavity, is tough, white, fibrous, and of structure somewhat different in the cranial from the spinal cavity. In the former position it is identical with the inner periosteum of the bones of the skull ; on passing into the spinal column, however, the periosteum divides into two or more lamellæ, the innermost of which is prolonged into the cylindrical tube that includes the spinal cord. Three processes of the dura mater divide-only incompletely - the cavity of the skull into two symmetrical halves and into an upper and lower space: (a) the falx cerebri, a sickleshaped process between the two hemispheres of the large brain;

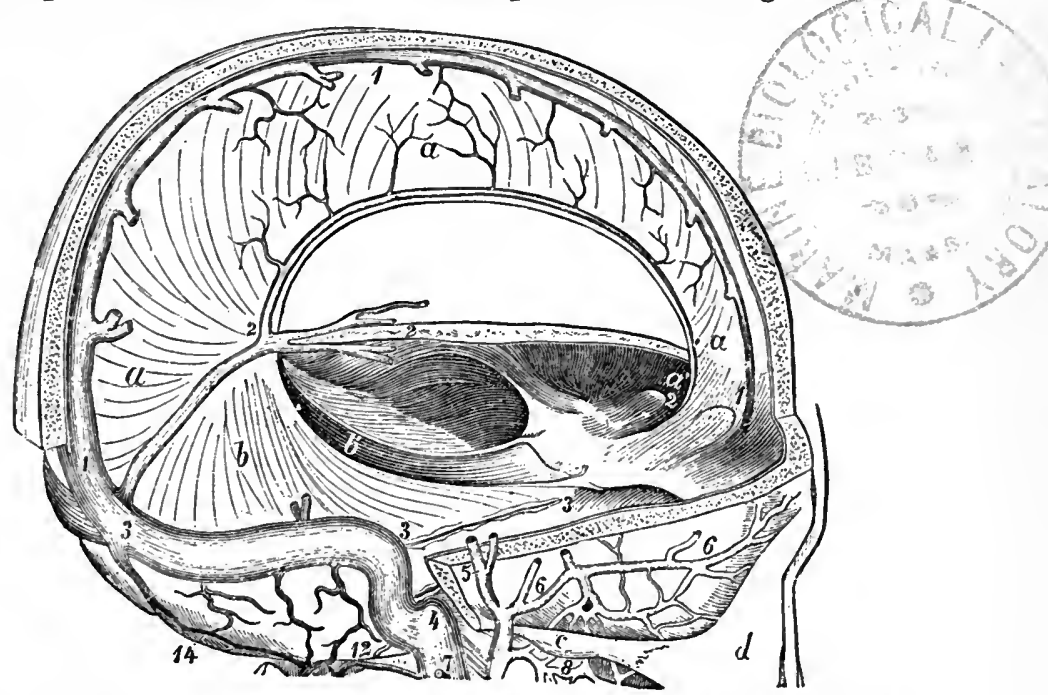

Fig. 12.-The Cranium opened to show the Falx Cerebri and Tentorium Cerebelli, and the Places of Exit for the Cranial Blood-vessels. $X_{2}$. (Schwalbe.) $a, a$, Fulx ; $b, b$, the tentorium ; 3, 3, Sinus transversus, and 2 to 3 , Sinus rectns, receiving from in front the Vena magna Galena. 4 , internal jugular vein; 5 , superficial temporal vein; and 6 , middle temporal vein.

(b) the falx cerebelli, a similar process between the two lateral lobes of the cerebellum, or small brain; and $(c)$ the tentorium cerebelli, an arched process over the cerebellum separating it from the back portions of the large brain. The fluid necessary to fill up the gaps and smooth over the surfaces of the closed area made by the dura mater is contained in the intercommunicating spaces of the membrane lying next inward and called (2) Arachnoid; this membrane is transparent and of delicate connective tissue. Toward the dura mater it presents a smooth, firm surface, like that of a serous membrane, and is covered by a layer of scaly endothelium; this layer is reflected on to the roots of the spinal and cranial nerves, and becomes continuous with the lining of the dura mater when the 
nerves pierce the latter membrane. The space below this surface is called subarachnoid ; the subarachnoid or cerebro-spinal fluid (already referred to as filling the intercommunicating compartments into which this space is dirided by bundles of delicate areolar tissue) is alkaline and poor in albumen. (3) The Pia Mater is a vascular membrane, a minute network of fine branches of arteries and veins held together by delicate connective tissue. These ramifications of the blood-vessels in the pia mater are on their way to or from the nervous substance of the spinal cord and brain. The membrane, therefore, closely invests this substance, being, however, more intimately attached to the cord than to the brain. Unlike the arachnoid membrane, the pia mater dips into the fissures between the convolutions of the cerebrum. It also sends its prolongations, not only into the fissures of the cord, but also, as slender bands (trabeculae) from its inner surface, into the columns of the cord. These trabeculæ branch and anastomose within the white substance of the cord like the midrib of a leaf. The pia mater is well supplied with nerves.

$\mathrm{By}$ these three membranes the nervous masses of the cerebrospinal system are protected, held together and in place with a soft and yielding but sufficiently firm pressure, and nourished by the blood. This great nervous system, as a whole, consists of the central organs-spinal cord and brain-and of various roots, divisions, and branches of spinal and cranial, or encephalic nerves.

86. The Spinal Cord, or Medulla Spinalis, extends in the spinal canal from the aperture in the cranial cavity (foramen magnum), above which it is continuous with the medulla oblongata, downward to opposite the body of the first lumbar vertebra, where, after tapering off, it is spun out into a slender thread of gray nervous substance (filum terminale) that lies in the axis of the sacral canal. Its length is from fifteen to eighteen inches; its weight, when divested of membranes and nerves, about an ounce and a half, or not far from one thirty-third of that of the brain. It is nearly cylindrical in shape, its front and back surfaces being somewhat flattened; it has two considerable enlargements of its girth-an upper (cervical), from which arise the nerves that supply the upper limbs; and a lower (lumbar), which supplies the lower limbs with nerves.

$\S 7$. The external structure of the spinal cord requires us to notice (1) the Fissures which almost completely divide it for its whole length into right and left (lateral) halves, and are, therefore, fitly called "median;" of these fissures $(a)$ the one in front (anterior median) is somewhat broader than $(b)$ the one behind (posterior 


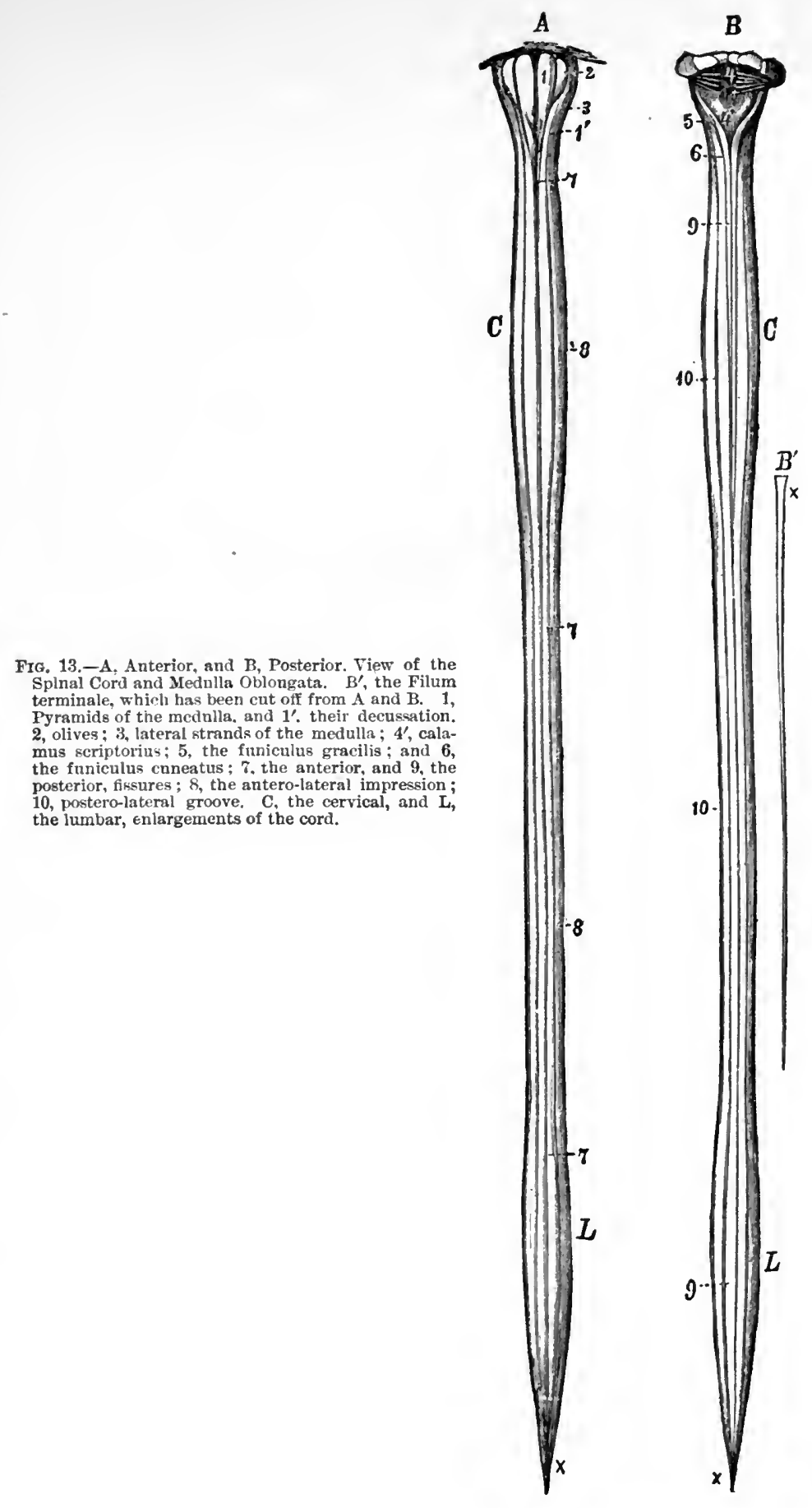


median). Both are filled to their bottom with processes of the pia mater; and the sides of the posterior fissure are bound closely together by the same membrane.

Each of these symmetrical and nearly half-cylindrical halves of the cord is subdivided by the lines of the entrance of the posterior and anterior nerve-roots into (2) three Columns: (a) the anterior, which lies betwcen the anterior median fissure and the anterior roots; $(b)$ the posterior, which lies between the posterior median fissure and the posterior roots; and $(c)$ the lateral column, which lies at the side of the cord between the other two columns.

(3) The Commissures of the spinal cord are two bands of ner-

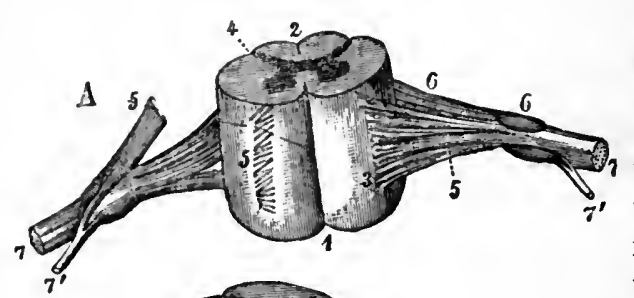
vous matter which unite its halves, thus preventing it from being com. pletely separated into two portions by the fissures. The one in front, at the bottom of the anterior median fissure, is composed of transverse nerve-fibres and is called $(a)$ the anterior white commissure; the one behind, at the bottom of the posterior fissure, is (b) the posterior gray commissure. The gray commissure is nearly twice as large as the

Fic. 14.-A, Anterior, and B, Lateral, View of a Portion of the Cord from the Cervical Region. $\%$. (Schwalbe.) 1, Anterior median, and 2, posterior median. fissures. At 3 is the $n$ tero-lateral impression, over which spread the anterior roots (5). The posterior roots $(6)$, with thejr ganglion $\left(6^{\prime}\right)$, arise from the postero-lateral groove, and uniting with the anterior roots form the compound nerve $(\boldsymbol{\gamma})$. white, except at the cervical and lumbar enlargements of the cord, where the white is

larger. ${ }^{1}$ Along its whole length the gray commissure incloses a circular or elliptical eanal (central canal), whose diameter is about one-twenty-fifth of an inch and which is lined by ciliated cells. Near the central canal lies a thin layer of gelatinous substance. The rest of the gray commissure consists for the most part of extremely fine nerve-fibres devoid of medullary sheath; while the white commissure is composed of medullated fibres. The thickness of the 
commissures is, as a rule, proportional to the size of the corresponding nerve-roots; their form, as they pass into the lateral parts of the cord, varies in different sections of its length.

§8. Transverse sections of the spinal cord show us that, as its external appearance would indicate, the substance of which it is composed is arranged in two symmetrical halves, almost, but not quite separated by the median fissures. This substance, like that of all the nervous centres, consists of both white and gray nervous matter. The former is external and composes the columns of the cord; while the latter is internal and is surrounded by the white. The relative amount of the two kinds of nervous matter varies in the different parts of the cord. At its beginning from the filum terminale scarcely any white matter appears ; the amount of such matter, however, increases from below upward, and is largest in the cervical part of the cord. The amount of gray matter is greatest in the upper and lower enlargements of the cord.

The gray columns on either side of the cord, together with the commissures which unite them, form a figure somewhat like a large Roman $x$, with diverging sides; but the lateral masses of these crescent-shaped bodies are narrower in the thoracic (or dorsal) region, and broader in the cervical and lumbar enlargements. Sometimes the figure is rather like that of a large $\chi$, or a pair of butterflies' wings. The two limbs of each side of the figure into which the gray colunins are thus formed are called (4) Horns; (a) the anterior horn is rounded, $(b)$ the posterior long and narrow. The division into anterior, posterior, and lateral columns, which is well marked

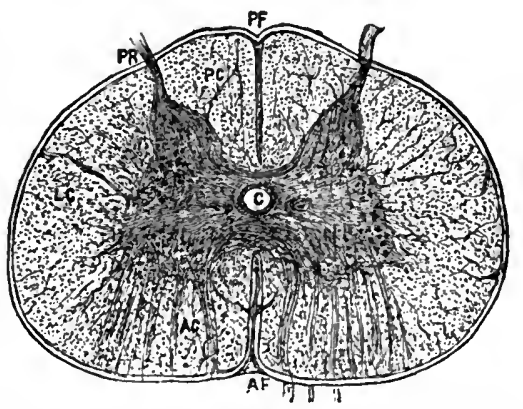

FIf. 15.-Transverse Section through the Spinal Cord. AF, antero-median, and PF, postero-median fissures; $1^{3} \mathrm{C}$, posterior, LC, lateral, and $\mathrm{AC}$, anterior columns; $A R$, anterior, and $P R$, posterior nerveroots: C, central canal of cord, with its columnar endothelial lining. 'T"he pia mater is shown investing the cord, sendirg processes into the anterior and posterior fissures, as well as delieate prolongations into the columns. The crescentic arrangement of the gray matter is shown by the darker shaded portion.

on the external surface of the spinal cord, is gradually lost as we pass inward toward the central gray substance. Of the two horns of each side, the anterior has the appearance of "spongy substance," the posterior of a kernel of such substance surrounded by gelatinous substance.

\$9. Careful study of the spinal cord with the higher powers of the microscope has enabled histologists to describe with further 
details the manner in which the nervous elements, both fibrillar and granular, are arranged within the connective substance.

The White Substance of the spinal cord, besides connective tis-

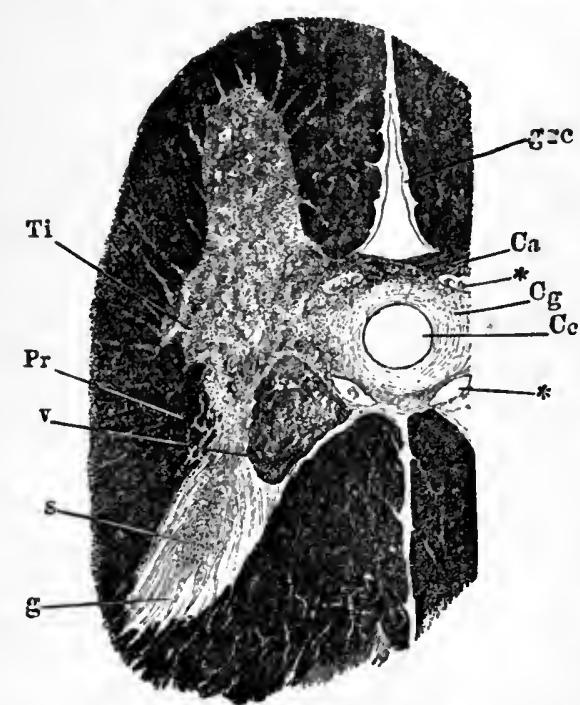

FIo. 16.-Section of Dorsal Part of the Spinal Cord showing the Gray Matter of the Horns, 20\%. (Ilenle.) $\mathrm{Ca}$, anterior white, and $\mathrm{Cg}$. gray conmissnre; Cc, central canal; v, vesicular column; s, spongy substance of the posterior horn, surrounded by g. gelatinous substance; $12 r$, reticular process; $\mathrm{Ti}$, intermedian lateral tract.

ones lying inward near the gray matter. sue and lymph- and bloodvessels, is composed of nerve-fibres of comparatively large or of medium size. The essential constit. uent of these fibres is the axis-cylinder, the diameter of which is generally onethird or one-fourth of their breadth. When fully developed, they are rarely or never without a medullary sheath, but probably hare no neurilemma. Their diameter is not constant; the thickest fibres $\left(\frac{1}{2^{\frac{1}{00}}}\right.$ to $\frac{1}{2000}$ of an inch) are found in the outer portions of the anterior columns, where their size is tolerably uniform. In the lateral columns the nerve-fibres vary greatly in size, the finer they increase in thickness as they approach the posterior gray commissure. In the upper thoracic, and through the whole of the cervical, region, there is found a wedge-shaped bundle of fine fibres that is separated off from the posterior columns toward the middle line of the cord by a strong septum ; this is called fasciculus gracilis, or "column of Goll."

The direction of some of the nerve-fibres in the white substance of the cord is vertical, of others, horizontal, of still others, oblique. The vertical fibres are most abundant, are united with a parallel arrangement into fascicles of various sizes, and ascend toward the brain. Horizontal fibres in the white substance of the spinal cord are of two kinds-commissural fibres and fibres of the roots. The fibres of the white commissure run horizontally along the median border of the gray matter of the horns, and become interwoven with the vertical bundles of the anterior columns. Most of them pass from the substance of the anterior horn of one side across to the 
anterior column of the other side. The fibres of the posterior spinal roots run in a nearly horizontal direction inward; they divide into anastomosing bundles so minute and so intricately interworen with the vertical fibres of the posterior column that their course is difficult to trace. Part of them (the lateral ones) run directly into the substantia gelatinosa of the posterior horns, and are, perhaps, continuous with the axis-cylinder processes of the nerve-cells 'of its spongy kernel ; part of them appear to enter the gray substance of these horns only after curving and running a variable dis-

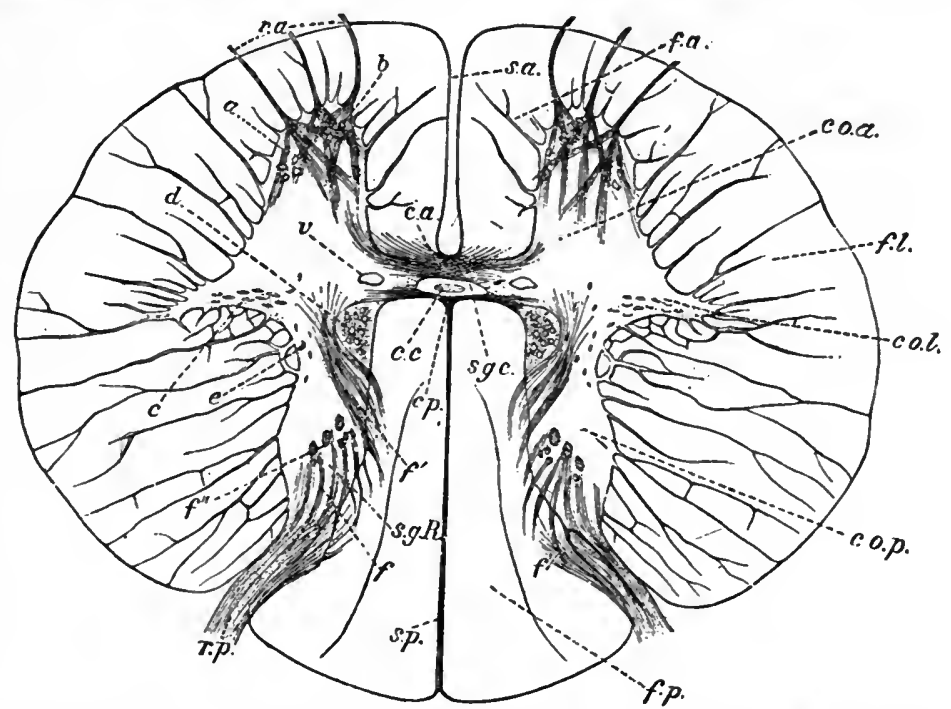

Fig. 17.-Section of the Spinal Cord at the Level of the Eighth Pair of Dorsal Nerves. 8/. (Sehematic, from Schwalbe.) 8.a., anterior fissure; s.p., posterior septum (or fissure); $c_{.} a_{\text {., }}$, anterior, and $\epsilon_{.}$., posterior, commísgures : c.c., central canal ; co.a, anterior horn ; co.l., lateral horn; co.p., posterior horu; $a$, anterior lateral, and $b$, anterior median cells; $c$, cells of the lateral horn; $d$, coltumns of Clarke; $e$, solitary cells of the posterior horn ; r.a., the anterior, and $r . p$., the posterior, roots; $f$, bundle of fibres of the posterior horn; and $f^{\prime}$, bundle of the posterior column ; $f^{\prime}$, longitudinal fibres of the posterior horn; $8 . g . R$., gelatiuous substance of Rolando; f.a., anterior, f.l., lateral, and f.p., posterior, columns.

tance upward, or perhaps downward, in the posterior columns. The fibres of the anterior roots of the spinal cord traverse its white substance obliquely; some of them enter the gray matter of the anterior horns on the same side, where they probably become continuous with the axis-cylinder processes of its large ganglion-cells; others of thein pass through the anterior commissure to the other side of the cord; still others pass into the lateral columns and the posterior horns.

The Gray Substance of the spinal cord, in addition to the same constituents as those of the white substance, has numerous nerve- 
cells. Its nerve-fibres, which form the chief part of its mass, and are generally non-medullated, differ from those of the white substance in that they frequently subdivide and thus become attenuated into extremely minute plexuses. The ganglion-cells of the spinal cord are multipolar, and give off two kinds of processes; one an unbranched axis-cylinder process and the others branching processes, both being of a fibrillated character (comp. Chap. I., \$\$ 28 and 29). The unbranclied processes of the ganglion-cells of the anterior horns are probably continuous with the axis-cylinders of the nerve-fibres of the anterior spinal roots. Of most of the similar processes from cells in the posterior horns we cannot yet make the same affirmation. The branching processes of the nerve-cells were traced by Gerlach ' until he thought himself able to affirm that their finest ramifications participate in those plexuses of nervefibres which he regards as an essential constituent of the gray substance of the cord. Henle ${ }^{2}$ and others consider the fate of these processes to be still unknown.

Characteristic groups of ganglion-cells occur at various places in the sections of the gray matter of the spinal cord. In the anterior horns of the cervical and lumbar regions are three groups of large cells; one of these is on the side of the horn (lateral), one farther to the front, one on its median border. They all coalesce in the anterior horns of the thoracic region. In the anterior horns also occur isolated nerve-cells of different sizes. The middle part of the gray lateral halves of the spinal cord contains, in parts of the cervical and thoracic regions, isolated groups of cells; one important group is situated at the inner angle of the base of the posterior horn, and is called the "columns of Clarke." The other nerve-cells of the posterior horns are small, and are not collected into groups, but are distributed through that part of the substance of the horns which is also traversed by the above-mentioned fine plexuses of nerve-fibres (see Fig. 17).

$\S 10$. By careful counting, E. A. Birge ${ }^{3}$ ascertained the number of the elements in the spinal cords of several frogs. From his conclusions something may perhaps be gained toward forming a better conception of this organ. In seven cases Birge found that the number of fibres in the anterior roots varied from 5,984 in the smallest animal to 11,468 in the largest ; the number increasing at the rate of about one thousand four hundred and fifty motor fibres to each added ounce of weight ( 51.5 to the gram). The diameter of the fibres was

${ }^{1}$ See in Stricker's Human and Comparative Histology, ii., pp. $352 \mathrm{ff}$.

'Anat. des Menschen. Text, pp. $310 \mathrm{ff}$.

8 Archir f. Anat. u. Physiol, 1882, Physiolog. Abth., pp. 435-479. 
also found to be much enlarged, according to the size and weight of the animal; and the average diameter widely different in the different nerve-roots. For example, it varied from 3,550 tibres, in the serentl pair of nerves, to 14,133 in the tenth pair, for a cross-section one twenty-fifth of an inch square. So, too, were the socalled motor-cells of the anterior gray columns found to vary from 4,871 to 11,517 , according to the weight of the animal. It was found that the large masses of cells lie in two principal groups, corresponding to the cervical and lumbar enlargements of the cord.

$\$ 11$. It would be of great interest to our inquiries if it were possible to give a complete description of the tracts of the nervefibres in their passage along the spinal cord; but it is impossible for the microscope to unravel them, and the evidence of physiology is (as we shall see subsequently), somewhat doubtful and even conflicting. Of late, however, certain of these paths have been traced with considerable certainty by combining the methods of embryological and pathological observation. In the development of the spinal cord, the medullary substance of the nerve-fibres along certain tracts of the white columns is formed later, so as to render them distinguishable in cross-sections. Moreover, when the nervefibres are separated from their place of origin, degeneration of their elements takes place. The place of the degenerated nervous substance is taken by connective tissue, which behaves differently under the influence of staining fluids. By following the course of this degeneration toward their periphery, the paths of conduction in the nerves may be traced. Some time ago, Tïrck ${ }^{1}$ attempted to mark out certain motor tracts in the brain by using this process of degeneration as his guide. Our great authority at present on the paths of the nerve-fibres in the spinal cord and brain, as ascertained chiefly by the former of these methods, is the work of Flechsig. ${ }^{2}$

Two tracts in the antero-lateral columns, which extend along the greater part of the spinal cord and into certain parts of the brain, are thus quite certainly made out. From their upper connections they have been named the pyramidal tract (or tracts) and the direct lateral cerebellar tract. The former is directly traceable down from the anterior pyramid of the medulla oblongata. Most of the fibres of this tract cross over in the extreme upper part of the cord, and pass down it in the back part of the lateral column as a compact

${ }^{1}$ Sitzgsb. d. Kaiserl. Acad., vi., pp. 303 ff.

${ }^{2}$ Die Leitungsbahnen im Gehirn u. Rückenmark d. Menschen. Leipzig, 1876. 
bundle. This crossed (or lateral) part of the pyramidal tract can be traced as far as the third or fourth pair of the sacral nerves. But some of the fibres from the pyramids of the medulla do not

I.
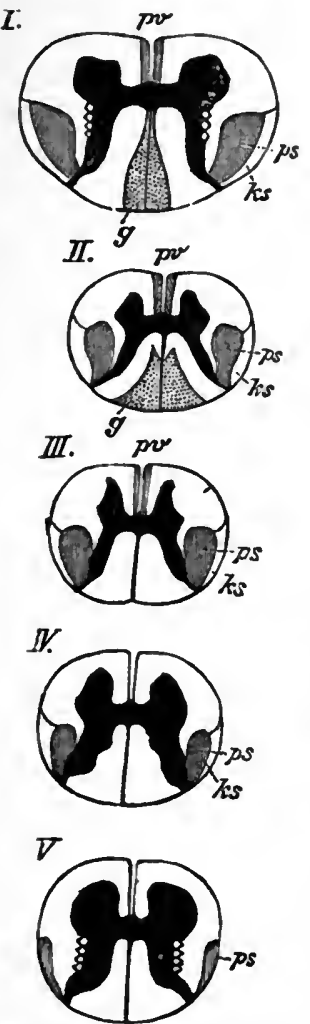

FIa. 18.- Sections through the Spinal Cord at different elevations, to show the tracts of White Substance. I., elevation of the sixth cervical nerves. $I I$, of the third: $I I I .$. of the sixth ; and $I V$. of the twelfth, dorsal nerves; and $V$. of the fourth lnmbar nerves; $p v$, uncrossed (or anterior) pyramidal tract ps, crossed (or lateral) pyramidal tract; $k s$, direct late ral cerebellar tract; $g$, tract of Goll.

cross in the upper part of the cord. These form the uncrossed (or anterior) part of the pyramidal tract; this part gradually diminishes as it passes downward, and ceases in the dorsal region of the cord. The direct lateral cerebellar tract lies between the lateral pyramidal tract and the outer surface of the cord. It disappears in the lumbar region. It is thought that the rest of the anterior column of the cord, besides the anterior pyramidal tract, may be, for the most part, commissural in nature-that is, it serves to bind together the two halves of the cord on the same level, or somewhat obliquely those lying slightly below or slightly above.

In the posterior white column a tract can be traced as far downward as the middle of the dorsal region of the cord ; this is the one already referred to as the "tract (or column) of Goll."

$\S 12$. The spinal cord is, therefore, shown to be a mechanism composed by combining the nervous elements so as to serve the great purpose of conducting nerve-commotion and acting as a series of reflex and automatic centres. In it we find tracts of connected nervous elements for the movement of ascending and descending nervous impulses. It is also a column or pile of nerrous centres, each one of which may have a particular value for particular functions; but which are also all bound together, up and down, right and left, and obliquely, so as to act unitedly under a certain control from each other and from the central organs lying above. It is especially strong in nervecells, just where it needs to be so-namely, at the enlargements, where it sends off nerves to the upper and lower limbs. Its paths for the passage and diffusion of molecular disturbance are indefinitely numerous, and their intricacy extremely great. It has groups 
of nerve-elements, such as belong to the central organs generally, of ganglion-cells embedded in neuroglia; it has special local mechanisms within, and yet connected with its general mechanism. It is adapted to do a large amount and variety of work through its pairs of nerves, without calling upon the higher nervous centres; it is constructed so as to act like a system of relays, not only transmitting, but also modifying, inhibiting, enhancing, and distributing the impulses which it receives, both from the more central and from the peripheral portions of the cerebro-spinal system.

$\S 13$. The same elements of nerve-fibres and nerve-cells, in conjunction with connective tissue and neuroglia, and enveloped in the three inclosing membranes (dura mater, arachnoid, and pia mater) already described, are combined with an increased variety and complexity of arrangement to form those intercranial central organs with which the upper end of the spinal cord is continuous. Here, too, these elements are gathered into fascicles of nerve-fibres which converge, or diverge, and run their courses in various directions, and into ganglionic masses, in which, besides the nerve-fibres, nerve-cells and diffused finely granular substance of a doubtful physiological character are found. Uniformity of elementary parts, together with the greatest intricacy of arrangement, prevails, abore all other regions of the body, in the structure of the brain. The significance of the elements and elementary parts can, therefore, only be understood when they are considered in the localities and relations to other parts which are assigned them by this so intricate arrangement.

§ 14. The Encephalon, or Brain, in the most extended sense of the word, includes all that portion of the central nerrous axis which is contained within the cavity of the skull. This grand mass of nervous matter may be divided into several parts, somewhat differently marked off according to the point of view from which the division proceeds. The division proposed by Meynert ${ }^{1}$ - to which reference will be made later-is based upon the supposed physiological significance of the different parts, and upon their arrangement so as to discharge the functions of conduction and "susceptibility to impressions." For, as this authority rightly claims, "a purely histological description" is of comparatively little service in comprehending the meaning of the architecture of the brain. We shall, first of all, however, describe briefly the contents of the cranial cavity, as it appears both to the unaided eye and under the microscope, without reference to theory.

${ }^{1}$ In Stricker, Human and Comparative Histology, ii., pp. 367 ff. 
On remoring the entire brain from the skull, the following four divisions of its mass engage the attention of even the inexperienced observer. Inmediately above the section by which it has been separated from the spinal cord, and appearing as an enlarged prolongation of the cord, is ( $I_{\text {. }}$ ) the Medulla Oblongata. Covering the upper back part of this organ, and extending beyond it on both sides, with its surface divided into small lobes by furrows, is (II.) the Cerebellum, or little or hinder brain. Swelling out in front of and above the medulla is (III.) the Pons Varolii, or so-called "bridge" of the brain. While in two hemispheres separated by a deep fissure, above both pons and cerebellum, and filling the larger part of the cranial cavity, is seen (IV.) the Cerebrum, or large brain, or

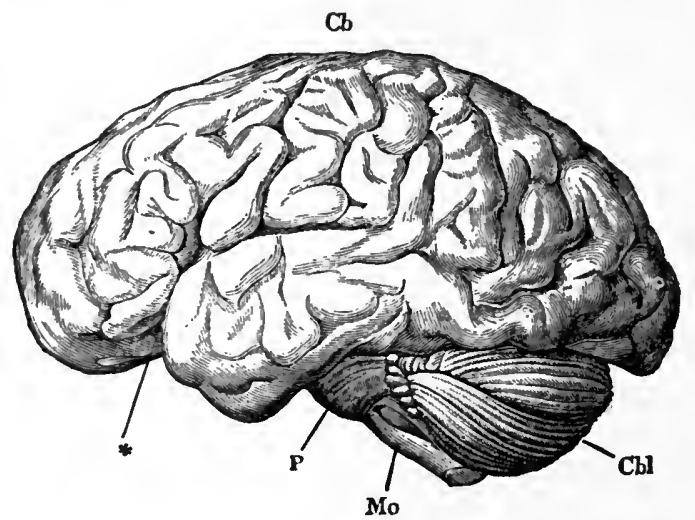

FIG. 19.-View of the Brain in Profilc. 1/3. (Henle.) Cb, cerebrum; Cot, cerebellnm: $M o$, medulla oblongata; $P$, pons Varoli,

brain proper. These divisions are all readily distinguishable on the external surfaces of the Encephalon.

On laying the encephalic mass open, however, certain bodies of nerrous matter are disclosed that have been concealed beneath the cerebellum and the cerebrum, and that-although ordinarily regarded as parts of the latter-are scarcely to be included in any one of the four main divisions of the brain. We shall describe in order the organs just named.

$\S 15$. I. The Medulla Oblongata is somewhat pyramidal in form, about one and one-fourth inch in length, from three-fourths to one inch broad in its widest part, and one-half inch thick; it extends from the spinal aperture of the cranial cavity (foramen magnum) to the lower border of the pons Varolii. It is continuous with the spinal cord, and somewhat resembles it in the divisions of its external surface. Its anterior pyramids appear superficially continu- 
ous with the anterior columns of the cord ; its lateral area shows upon its upper end an oval-shaped elevation called the "olivary body ;" its posterior tracts also appear continuous with the posterior columns of the cord. Just outside the upper portion of each posterior tract, and behind the olive, ascends to the cerebellum a strong tract named the "restiform body." That portion of the posterior column of the upper cord (alrendy referred to, $\mathrm{p}$. 68) which is marked off from the rest by a septum of pia mater, is continued up into the medulla oblongata, and becomes more strongly marked. It is known as the $f u$ niculus gracilis; and when traced still farther upward is seen to broaden out into an expansion called the clava. A prolongation of the posterior lateral column also gradually

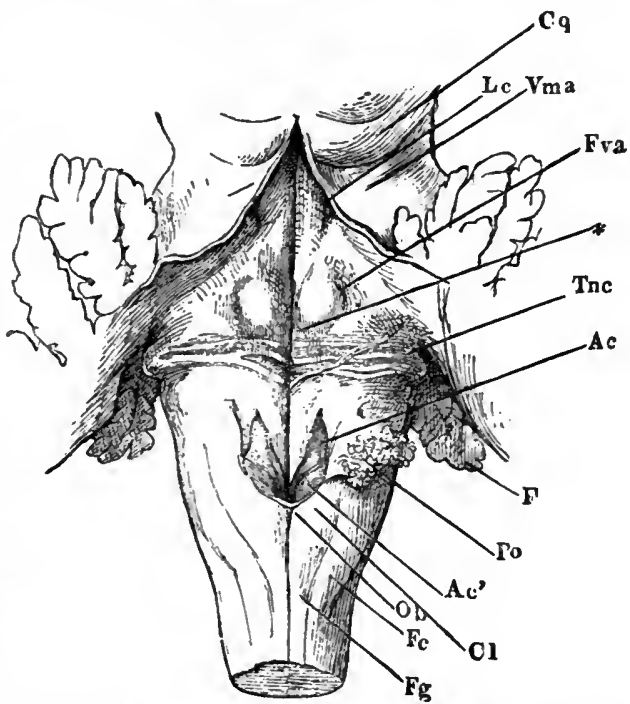

FIG. 20.-Back View of the Medulla Oblongata, the Cerebellum being removed. (Henle.) Cq. corpus quadrigeminum; Lc, locus coeruleus; F, flocculus of the cerebellum; Ac, ala cinerea ; and $\mathrm{Ac}$ 'Stilling's nucleus accessorius; $\mathrm{Cl}$, clava ; $\mathrm{Fc}$, funiculus cuneatus; $\mathrm{Fg}$, funiculus gracilis.

expands as it ascends, so that it accuires a "wedge-shape" form, and is accordingly known as the cuneate funiculus.

The medulla oblongata, like the spinal cord, is composed of white and gray nervous matter; it differs from the cord, however, in having its gray matter not confined to the central part, but gathered more into special masses or nuclei. A redistribution of the nerve-elements takes place in the medulla, and their arrangement becomes more complex. An important part of this redistribution is accomplished by the divergence of the posterior tracts and restiform bodies, which opens up the central gray mass, and lets it come to the surface between the sides of the surrounding white matter. Looking at this redistribution as it appears from below, the elements of the cord may be said to be spread out and increased by the addition of new elements; looking at it as it appears from above, the two great nerve-tracts of the cerebrum (tegmentum and crusta of the crus cerebri), and the tract of the cerebellum, may be 
said to be gathered up in the medulla, and compressed so as to form in the cord a continuous and symmetrical medullary investment for its central gray matter.

The intimate structure of this organ is exceedingly complicated; much of it is doubtful, and as yet impossible to make out satisfactorily. The two important considerations are (1) to trace the nerre-fibres as they ascend through the medulla from the various columns of the cord, and (2) to locate the particular collections of gray matter, whether as continuous with those of the cord or as consisting of independent masses.

The rarious tracts of White Matter in the medulla oblongata, although they superficially appear to be prolongations of the col-

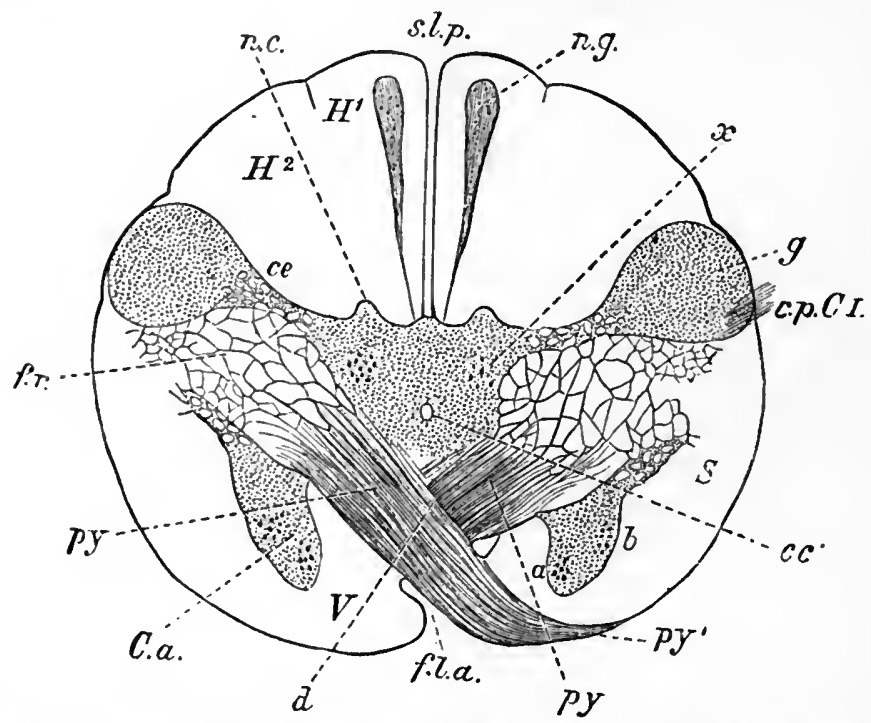

FIG. 21.-Section showing the Decussation of the Pyramids at the point where the Spinal Cord passes into the Medulla Oblongata. $\%$. (Schwalbe.) f.l.a., Iongitudinal anterior fissure, through which the bundles of pyramidal fibres $\left(p y, p y^{\prime}\right)$, are crossing over at $d$ : $V$, anterior, ard $S$. lateral pyramids: $C . a_{\text {.n }}$ anterior horn with groups of ganglion-cells, $a$ and $b ; c c$. central canal; $f . r$., formutio reticnlaris; $c e$, the ncck, and $g$, the head, of the posterior horn; n.c., nucleus of the funiculus cuneatus; and $n . g$. of the funiculus gracilis; $H^{3}$, funiculus gracilis; $I^{2}$, funiculus cuneatus; $x$, group of ganglion cells.

umns of the spinal cord, are really so to a small extent only. This fact is most clearly made obvious by a comparison of successive transverse sections. A large bundle of fibres, which in the cord lies in the posterior part of the lateral column (see p. $71 \mathrm{f}$.), pushes its way obliquely through the gray matter of the anterior horn, and passes in front of the central canal to the pyranid of the opposite side. The crossing of this bundle, as seen in the anterior median 
fissure at the lower part of the medulla, is called the "decussation of the pyramids." The abrupt passage of so many fibres through it breaks up the anterior horn, separates part of it from the rest, and pushes this separated part over to one side, so that it comes to lie close to a part of the posterior horn. The latter also becomes gradually shifted sidewise by an increase in the size of the posterior. tracts, so that it comes to lie almost at right angles to the posterior median fissure; its head enlarges and approaches close to the surface, where it forms a projection (funiculus of Rolando), and, higher up, a distinct swelling (tuberele of Rolando). Tracing the principal bundles of fibres on their course from the columns of the spinal cord upward through the medulla oblongata, we find (in accordance with what has already been said) that the posterior column forms the substance of the three posterior funiculi of the medulla-namely, gracilis, cuneatus, and funiculus of Rolando: a considerable part of the lateral column (the lateral pyramidal tract, see p. 72) passes into the opposite pyramid of the medulla, and ascends in it toward the cerebrum in company with a small part of the anterior column of the same side; while another part of the lateral column (the direct lateral cerebellar tract) passes at about the middle of the medulla obliquely backward to the restiform body, and the rest of it dips under the olives, and is continued toward the corpora quadrigeminum and optic thalamus. Most of the anterior column dips under the pyramid, and passes upward toward the cerebrum, but part is continued into the pyramid of the same side.

Curved fibres may also be seen running their course in the plane of the different transverse sections-some superficial, some deep (arciform or arcuate fibres).

As the medulla is a bilateral organ, its halves are bound together by commissural fibres, which run obliquely and decussate in the mesial plane, forming a well-marked band called raphé. In addition to the fibres of the medulla oblongata which are continuous with those of the spinal cord, others originate within the organ itself. It is a centre of origin for several pairs of encephalic nerves.

The Gray Matter of the medulla oblongata is, in part, continuous with that of the cord, and in part consists of independent masses. The former part is, as we have seen, broken up and rearranged by the decussation of the pyramids. The fate of the posterior horns and of the central gray substance has already been described. The substance of the anterior horns becomes divided into many little masses by the nerve-fibres that traverse it, so as to form a coarse network of nervous matter (formatio reticularis) containing nerve- 
cells, and intersected by bundles of fibres. In the upper part of the organ its interior gray matter appears upon the floor of the fourth ventricle, into which the central canal dilates. Four special

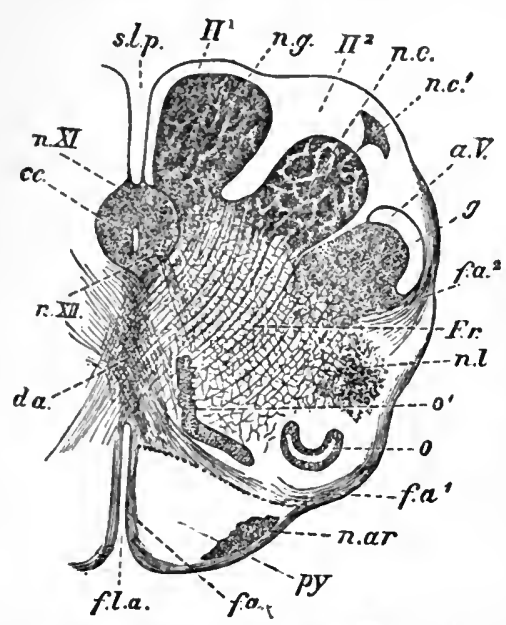

FIa. 22.-Section showing Gray Matter of the Medulla Oblongata, in the region of the upper f.l.a., anterior, and s.l.p., posterior, fissures; $n, X I$. and $n . X I I$. nuclei of the vagus accessorius and hypogloseal nerves; $d . a$., so-called upper crossing of the pyramids; $p y$. anterior pyramid in which is n.ar, the nucleus arcifornis; $o$, beginning of the olivary nucleus: $o^{1}$, accessory olivary nuclens; $\boldsymbol{F} . \boldsymbol{r}$., formatio reticularis; $g$, substantia gelatinosa; $f . a ., f \cdot a .1, f . a .{ }^{2}$, arciform fibres. crossing of the Pyramids, 4/1. (Schwalbe.)

kernels or nuclei, of gelatinous appearance and containing few multipolar nerre-cells, are to be noted in each half of the medulla. These are (1) the nucle. us arciformis, which is situated just beneath the pia mater, at the front of the anterior pyramid ; (2) the nucleus olivaris, or dentate body (corpus dentatum), which is within the inferior olive, a mass of gray matter folded in a zigzag or denticulated manner, forming a sort of capsule through the openings of which closely packed masses of fibres run into the surrounding space; (3) the nucleus olivaris accessorius, a smaller gray mass lying on the outside of the dentate body; and (4) the nucleus pyramidalis (sometimes also called " inner accessory nucleus" of the olive), lying on the inside of the same body. Another kind of collections of gray matter in the medulla consists of those groups of multipolar cells to which the nerves that have here their so-called roots of origin can be traced. These cells resemble those of the gray columns of the cord-the larger ones apparently being connected with the roots of the motor nerves, the smaller with those of the sensory. It may be assumed that some of their processes are continuous with the axis-cylinders of the fibres of the nerve-roots, and that others serve to place the medulla in direct connection with the cerebrum; positive demonstration of these as. sumptions, however, requires further histological researches. The nerre-nuclei in the medulla receive their name from the nerves whose fibres originate in them.

\$16. II. In the Cerebellum, or Little Brain, the general arrangement of the two kinds of nervous matter is the reverse of that of the spinal cord and the medulla oblongata: the gray matter is external, the white internal. More precisely, the cerebellum is 
a white or medullary mass rising out of three large bundles or stalks of nerve-fibres on each side, and enveloped with a covering of gray nervous matter. Like the other organs of the cerebrospinal system, it is a bilateral structure. These stalks of nerves connect the cerebellum with three other organs, with parts of which they are continuous. Considered as connections, they are called the "peduncles" or crura of the cerebellum. Of the three peduncles, (1) one (inferior peduncle) on each half of the organ is identical with the restiform fascicle which ascends from the medulla to the cerebellum; (2) another (superior peduncle), similar to the first in size, passes forward over the anterior end of the fourth ventricle, and connects the cerebellum with the tegmentum of the crus ; (3) a third (niddle peduncle) passes down on each side into the pons. This middle peduncle forms the larger portion of the white core of the organ. In addition to the fibres from these three sets of peduncles, this core is in part constituted by others which arise in the cerebellum itself; some of the latter connect together the different regions of the organ lying above or below each other, some unite the opposite and symmetrical regions of its hemispheres.

The interior relations of the fibres from the three peduncles are, on account of the extreme intricacy of their course, not yet fully made out. United in the white core of the cerebellum, they form a rather uniform mass, which is interrupted, however, by certain nuclei of a gelatinous appearance. Within either hemisphere, and to be disclosed by cutting through it a little to the outer side of the median lobe, is a mass of nervous matter arranged like the dentate body of the medulla oblongata; it is the corpus dentatum of the cerebellum. Other smaller, round, or oblate masses of gray matter are found toward the middle of the core from the dentate body.

The arrangement of the gray matter which forms the rind or cortex of the cerebellum is somewhat peculiar; its characteristics are best seen by examining a cross-section. It is thus found that this cortical gray substance is arranged in thin plates, or lamellæ, which are penetrated by prolongations of the white matter of the core ; these prolongations branch off into the interior of the lamellæ, and give to the cortex the arborescent appearance known by the name of "arbor vitce." The primary branches of this tree-like prolongation of the white matter of the core within the gray mattex of the cortex stand either perpendicular or a little inclined to the surface of the core. The smaller branches run from one side to another transversely or forward in concave curves. 
The external surface of the cerebellum presents two hemispheres, or lateral lobes, united by a central lobe called the vermiform process. This central lobe on its upper (or tentorial) surface is a mere elevation, but the "vermiform" character of its lower (or occipital) surface is well defined. The process here lies at the bottom of a deep fossa (vallecula). From the middle peduncle of each hemisphere a large horizontal fissure extends backward along its onter border, and divides the hemisphere into its tentorial and occipital

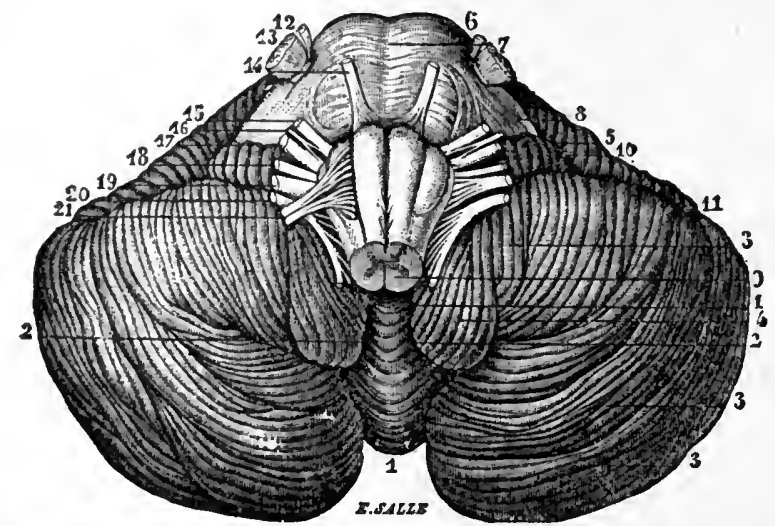

Fic. 23.-Lower Surface of Cerebelium. \%3. (After Sappey.) 1, inferior vermiform process; 2 , 2, vallecula: 5 , flocculus; 6 , pons Varolii ; 8 , middle peduncle of the Cerebellum: 9 , medulla oblongata. Varions pairs of nerves are seen thus: 12 and 13, roots of fifth pair; 14, sixth pair; 15, facial nerve; 17 , auditory; 18, glosso-pharyngeal; 19 , pneumo-gastric; 20, spinal nccessory ; 21 , hypoglossal.

surfaces. Each of these surfaces is divided by fissures into smaller lobes or lobules.

In the gray matter of the cortex of the cerebellum three distinct layers of nervous substance may be distinguished. Of these the pure gray layer is the most external; it is sometimes called the "molecular layer." It consists of an extremely delicate framework of connective tissue, in which, together with nuclei of the connective tissue, a few roundish cells and minute fibres of nervous structure appear. The middle layer is cellular and composed of a single irregular row of large ganglion-cells, called "cells or corpuscles of Purkinje." Comparatively large processes from these cells branch into and ramify within the outer layer. According to most observers (Kölliker, Deiters, and others) each of the cells sends a single medullated and unbranched process inward, which becomes continuous with the axis-cylinder of a fibre of the medullary portion of this organ; but according to Stilling there are several branches from each, which divide to form a network in the internal layer. This 
layer is rust-colored and merges gradually into the white substance of the core; it appears to contain multitudes of granules, with a well-defined nucleus surrounded by branching protoplasm. The nature of the granules is not known; they have been considered by some as elements of sustentacular tissue, by others as lymphcorpuscles, by others as multipolar nerve-corpuscles.

The cerebellum is thus constituted by a complex arrangement of the nerrous elements as a kind of side mechanism of the nervous systern, lying out of the course of its direct tracts and yet bound by nervous cords (the peduncles) in all directions to the other organs of the brain.

\$17. III. The Pons Varolii, or Bridge of the Brain, has its principal office in the mechanism of the central organs of the cranial cavity as a meeting-and switching-place of nerve-tracts between other organs; but it is also itself a central organ, as well as a centre of origin for certain nerve-fibres. The pons is really a thicken-

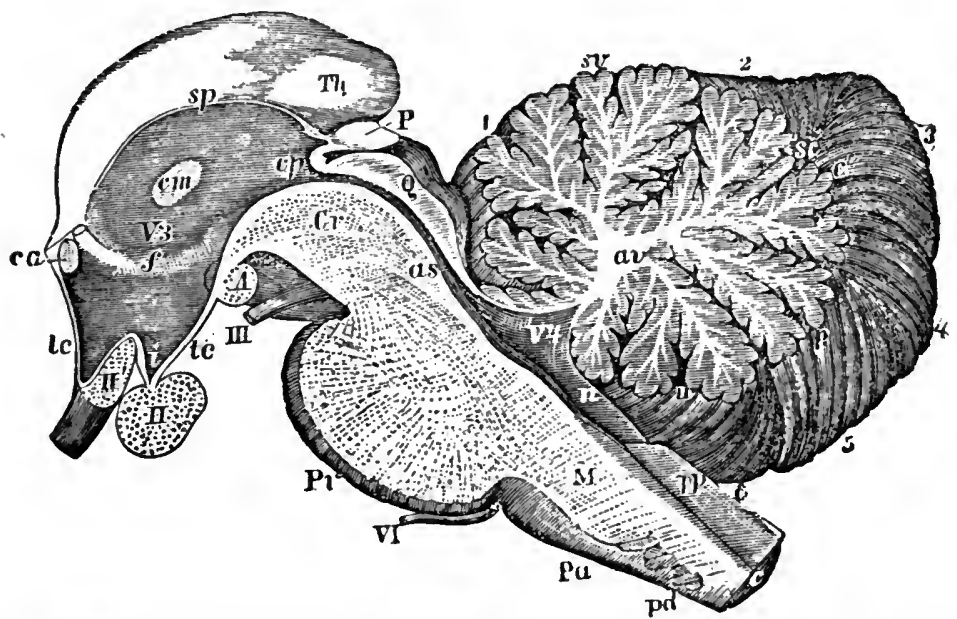

F1G. 24. - Median Section through the Stem of the Brain. (After Reichert.) $\boldsymbol{M}$, medulla oblongata ; of which $P a$ are the pyramids, decussating at $p d ; c$, central canal ; $p p$, restiform body : $P v$, pons Varolii : V4, fourth ventricie. $a v$, arbor vita of the cerebellum; $p$, pyramid; $u$, uvula : $n$, nodule; $a s$, aqueduct of Sylvius; $C r$, crus cerebri : $Q$, corpora quadrigemina; $P$, pineal gland : Th, optic thalamus, Commissures; $c a$, the anterior; $\mathrm{cm}$, the mollis; and $c p$, the posterior. $V 3$, the third ventricle; $A$, corpus albicans; $t c$, tuber cinereum; $i$, infundibulum.

ing of the ventral wall of the fourth ventricle, composed of the middle peduncles of the cerebellum encircling and partly blending with the continuation upward of the medulla oblongata. Its superficial fibres on the ventral surface are transverse in their general direction; but the middle fibres pass directly across, the lower ascend slightly, and the superior are more curved, and descend obliquely 
to reach the crus cerebelli. On removing these superficial fibres the prolonged fibres of the anterior pyramids are exposed to view. These, as they ascend through the pons, are intersected by the transverse fibres. At the lower part of the organ, behind the fibres from the anterior pyramids, a special set of transverse fibres (trapezium) begins at a collection of gray matter (superior olivary nucleus) on one side, and crosses the middle line to ascend to the cerebellum on the other side.

Nuclei of gray matter with small multipolar nerve-cells are found everywhere between the fibres of the ventral part of the pons. Many of its transverse fibres are probably connected with these cells. The posterior portion of this organ is chiefly constituted by a continuation upward of the formatio reticularis, and of the gray matter of the medulla oblongata. In the reticular formation two or three important collections of nerve-cells lie embedded. One of these is the "superior olivary nucleus," which lies behind the outer part of the trapezium and gives origin to some of its nervefibres. Of the other nuclei in this region, one gives origin to the seventh or facial nerve, and others to portions of the fifth nerve.

$\S 18$. IV. The Cerebrum, or Large Brain, much exceeds in size all the other contents of the cranial cavity; but it surpasses them more especially in the variety and complexity of the arrangement here given to the nervous elements; while its significance for the inquiries of Physiological Psychology is altogether unique.

As ordinarily described, this nervous mass includes a considerable number of organs, which vary in structure, relations, and physiological functions. Besides the hemispheres of the cerebrum, and the great ganglia (corpora striata and optic thalami) which lie at their base, custom includes in this term certain bodies that appear connected with the lower surface of the mass, viz., the corpora quadrigemina, pineal gland, crura cerebri, etc.

\$19. The Cerebrum is of ovoid shape and is divided-above, in front, and behind-into two hemispheres by a deep median longitudinal fissure. If these hemispheres are drawn asunder by opening this fissure, they are seen to be connected at its bottom by a broad white band of nerrous matter, the corpus callosum. The outer surface of each hemisphere is convex and fitted to the concave inner side of the bones of the skull; the inner surface along the median fissure is flat, and separated from the corresponding surface of the other hemisphere by a process of the dura mater (falx cerebri) ; its under surface is separated from the cerebellum and the pons by another process of the same membrane (tentorium). From the front of the pons the large white nervous cords, called 
cerebral peduncles, or crura cerebri, pass upward and forward to connect the cerebrum with the organs lying below it. Around each crus winds a flat band, the optic tract; these tracts come together in front to form the optic commissure from which the two optic nerres; arise. The lozenge-shaped space enclosed by the crura cerebri, the optic tracts, and optic commissure, contains a

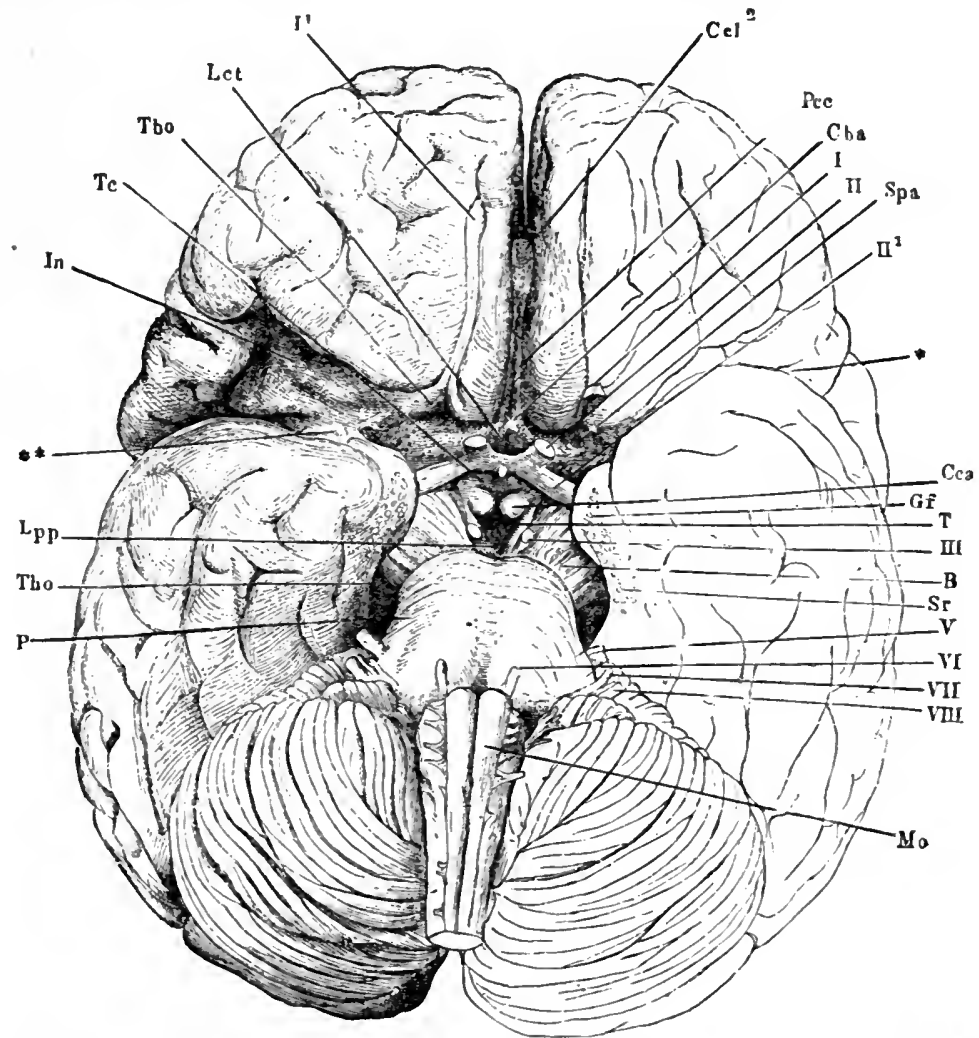

Fig. 25.- Under Aspect of the Brain. (Henle.) B, basis of the crura cerebri ; Cca, corpora albicantia: $I^{1}$, olfactory bulb; $1 I^{3}$, optic tract; Tc, tnber cinereum; Lpp, posterior perforated space: Ccl, corpus callosum : Lct, lamina cinerea terminalis: Spa, anterior perforated space ; T, tegmentum ; Tho, thalamus opticus; $\mathrm{P}$, pons; Mo, medulla oblongata ; I. to Vill., first to eighth pair of cranial nerves.

gray layer (posterior perforated space), two small white bodies (corpora albicantia), and a gray nodule (tuber cinereum) which is joined to a small reddish-gray oval mass (pituitary body) by a conical process of gray matter (infundibulum). In front of the optic commissure is a thin layer of gray substance (lamina cinerea); and on each side of the deep longitudinal fissure stretches the olfactory tract, 
with its bulb. The intercranial part of this "nerve" is now known really to be a projecting portion of the brain. All these structures, together with the cut ends of the several pairs of cranial nerves, may be seen upon the under surface of the cerebrum.

$\S 20$. The upper surface of the cerebral hemispheres presents the appearance of gray nervous matter arranged in folds which are called "convolutions" or" gyri. These convolutions are separated by " fissures" or sulci of varying depth, some of which are so constant and strongly marked that their presence is employed to divide the surface of the lemispheres into lobes, while others, less strongly marked, separate from each other the convolutions of the same lobe. It is the arrangement of the convolutions, with their separating fissures, which gives the hemispheres of the brain their: characteristic appearance, and which fits them for their unique functions in the economy of the nerrous mechanism.

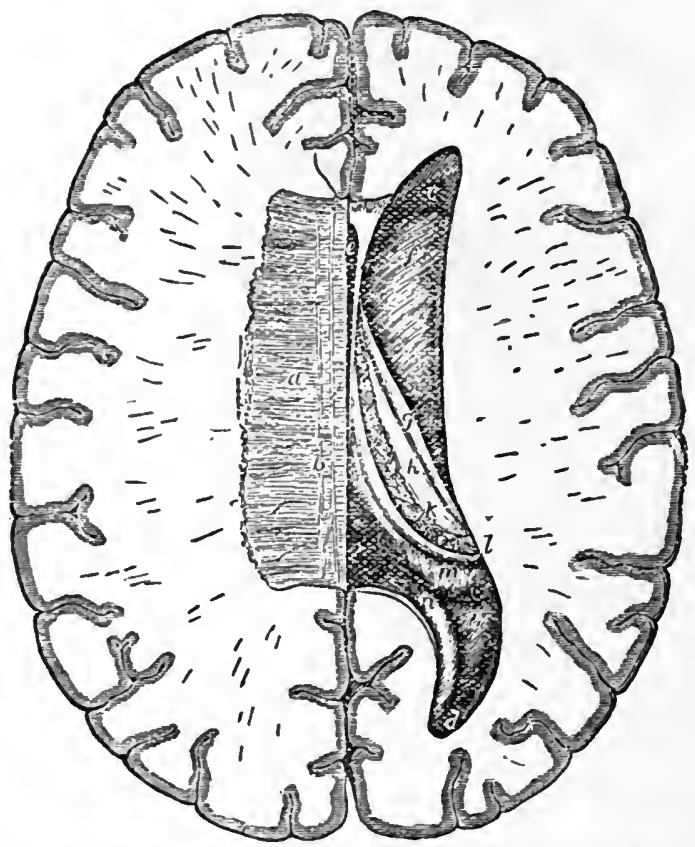

FIG. 26. - To show the Right Ventricle and the I-fL Jalf of the Corpus Callosum. $a$, transverse fibres, and $b$, longitudinal fibres of corpus callosum ; $c$, anterior, and $d$, posterior cornua of lateral ventricle: $e$, septum lucidum: $f$, corpus striatum; $g$, ten:a setnicircularis; $h$, optic thulamus: $k$, choroil plexus; l, tenia hippocampi; $m$, hippocampus major; $n$, hippocampus minor; o, eminentia collateralis.

$\$ 21$. By cutting off successive slices from the upper part of the hemispheres their general internal structure may be seen. It re- 
sembles the plan upon which the cerebellum is constructed. A core of white nervous matter is surrounded by a shell or cortex of gray; the two lateral halves of the core are bound together by a strong band of fibres, usually described as commissural (corpus callosum), which is itself overlapped by one of the most marked conrolutions of the brain (gyrus fornicatus). By cutting still deeper it is found that the corpus callosum forms the roof of a space in the interior of each hemispherc (the lateral ventricles). These two cavities or ventricles are moistened by a serous fluid and separated by a thin transparent wall (septum lucidum). The roof of another cavity, the third ventricle, is formed by an expanded fold of the pia mater (velum interpositum), the margins of which are fringed by the socalled "choroid plexuses;" the latter contain the minute arteries which sup-

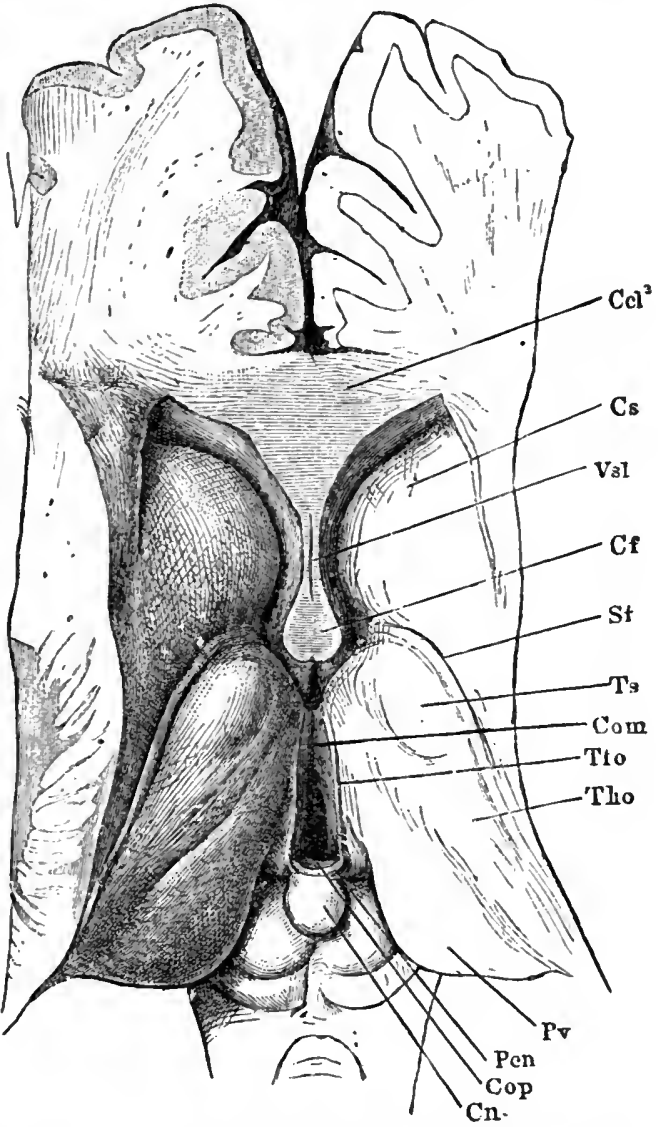

Fig. 27,-Basal Ganglia of the Cerebrum seen from above. (Henle.) $\mathrm{Ccl}$, genu of the corpus callosum: Cs, corpus striatum; Vsl, ventricle of the septum lucidum : Cf, column of the fornix ; St, stria terminalis: Tho, optic thalamus: and Ts, its anterior tubercle : Com, middle commissure between the thalami and over the third ventricle; $\mathrm{Pv}$, pulvinar; $\mathrm{Cn}$, conarium or pincal gland; Cop, corpus quadrigeminum.

ply the nerrous structures of this region. Each lateral ventricle is divided into a central space and three curved prolongations or cornua; of the cornua, one (the anterior) extends forward and outward toward the front part of the cerebrum; one (the posterior) curves backward, outward, and then inward; and the third (the descending) curves backward, outward, downward, and then forward and inward. 
On the floor of each lateral ventricle the exposed portions of the great basal ganglia of the cerebrum are visible. A large pear-shaped body of gray color is here seen with its broad extremity direeted forward into the anterior cornu of the ventricle and its narrow end outward and backward.' This body, on account of the striped

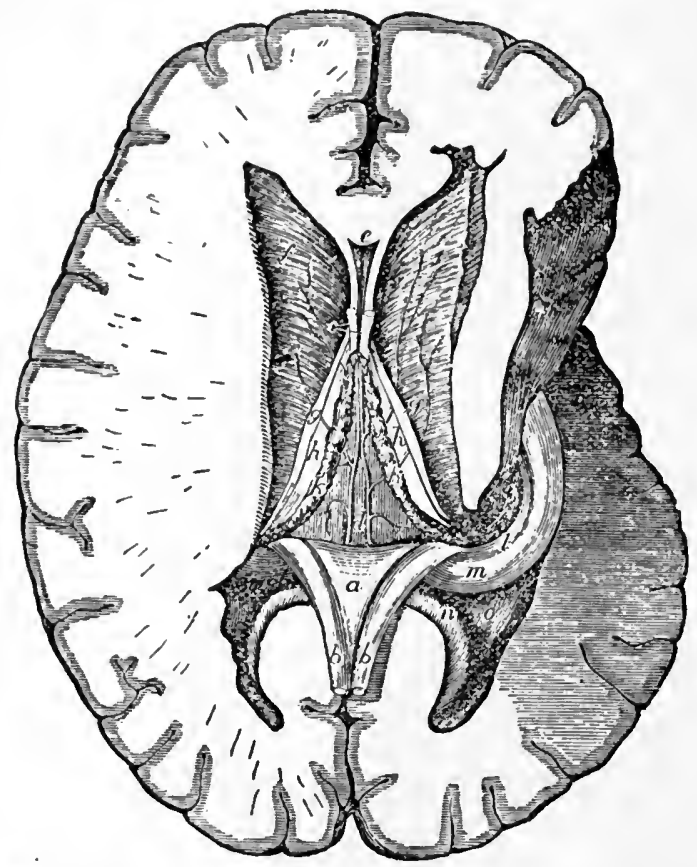

FIG. 28.--A Deeper Dissection of the Lateral Ventricle, and of the Velum Interpositum. $a$, nn . der surface of corpus callosum, turned back; $b, b$, posterior pillars of the fornix, turned back ; c. $c$. anterior pillars of the fornix; $d$, velum interpositum and veins of Galen : $\boldsymbol{e}$, fifth ventricle : $f . f$. corpus striatum ; $g, g$, trenia semicircularis; $h, h$, optic thalamus; $k$, choroid plexus ; $l$, tania hippocampi; $m$, hippocampus major in descending corru; $\boldsymbol{n}$, hippocampus minor; $o$, eminentia collateralis.

appearance which it presents when eut open, is called a "striate body" (corpus striatum). It consists of two masses, the upper one of which (nucleus caudatus) projects into the lateral ventricle; the lower one is embedded in the white substanee of the hemisphere and forms the principal part of the body (nucleus lenticularis). The two are separated by a layer of white matter ealled the "interual eapsule." Between the diverging portions of the striate bodies are the oblong

1 Dalton (Topographical Anatomy of the Brain, Philadelphia, 1885, ii., p. 76) and others speak as though the caudate nucleus alone were to be called corpus striatum, the nucleus lenticularis by this name; and the two corsidered as separate bodies. 
or somewhat ovoid masses of the "optic thalami." Each thalamus rests upon and partially embraces one of the crura cerebri ; its median surface forms the side wall of the third ventricle, and upon its outer and back part are two small elevations, one on each side of the optic tract (corpora geniculata, internum and externum). In the depression between each striate body and the optic thalamus is a narrow, whitish, semitransparent band of medullary substance (tania semicircularis). Along the entire length of the floor of the descending cornu of the ventricle is a white eminence (hippocampus major or cornu Ammonis) which is the inner surface of the gyrus fornicatus doubled upon itself like a horn. An arch-shaped band of nerve-fibres, consisting of two lateral halves, which, in front, form two pillars that descend to the base of the cerebrum and become the corpora albicantia, and which diverge behind into two pillars that descend with the descending cornu of the ventricle and connect with a convolution of the brain (gyrus hippocampi), is situated beneath the corpus callosum; it is called the fornix. Behind and between the optic thalami, and resting on the back surface of the crura cerebri, are four rounded eminences in two pairs, called corpora quadrigemina; the front pair are the nates, the back pair, testes.

$\S 22$. Without mentioning other more minute subdivisions, superficial or internal, in the structure of the cerebrum as seen by the unaided eye, we now consider the arrangement of the nerve-fibres and nerve-cells in the more important organs already named.

Of the fascicles of nerve-fibres belonging to the cerebrum, some connect it with the lower organs of the encephalon; some connect together its hemispheres; some join different structures in the same hemisphere; some are roots of origin for certain nerves.

The fibres of the crura cerebri-those strong peduncles of the brain that ascend

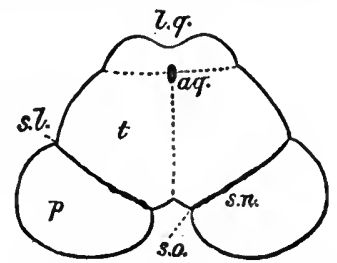

FIG. 29.-Section through the Mid-brain. (Schwalbe.) aq., aqueduct of Sylvius;, s.n., substantia nigra ; $p$, crusta of the crus cerebri ; $t$, tegmentum of the crus cerebri. from the pons to the optic thalami and the striate bodies-are arranged in two groups (crusta and tegmentum) separated by the gray matter of the substantia nigra. An important part of the fibres of the crusta, or front part (pes) of the crus, is continuous with the longitudinal fibres of the pons which come from the pyramids of the medulla ; it receives some fibres from the gray matter of the substantia nigra. Many of the fibres of the crusta terminate in the nuclei of the striate bodies; but some radiate upward through the internal capsule directly to the gray cortex of the 
cerebrum. (Comp. Fig. 24.) Some of the more diffused fibres of the tegmentum, or back and deeper part of the crus, are probably continued from the anterior column of the cord, and may be traced above to the optic thalami. Others of its fibres are collected into more well-defined tracts; one of the most important of which comes from the superior peduncle of the cerebellum, and has already been traced as it passes forward over the anterior end of the fourth ventricle (see p. 79). The formatio reticularis is continued into the tegmentum; the latter, therefore, has a considerable amount of gray matter containing nerve-cells. Some of its fibres arise in these cells. The superior peduncle of the cerebellum as. scends, crosses over to the other side beneath the Sylvian aqueduct, and terminates in a collection of large pigmented cells (the nucleus of the tegmentum or red nucleus).

The intimate structure of the striate bodies is not as yet entirely made out. On its deeper side, which is turned toward the internal capsule, the nucleus caudatus receives from the capsule several bundles of fibres. According to Meynert, some of these bundles serve to connect this nucleus downward with the peduncle of the cerebrum, some upward with its cortex; but, according to Wernicke, it is doubtful if any of them pass to the white matter of the hemispheres, or come directly (that is, without traversing the lenticular nucleus) from the crusta. All parts of the nucleus lenticularis are pervaded with white fibres. Some pass into its inner zone from the adjacent part of the internal capsule; some connect it with the caudate nucleus; some pass from it into the corona radiata, and then to the cerebral cortex. These nuclei appear to have a special connection with the frontal and parietal lobes, but also with some convolutions of the temporal lobe and the island of Reil. The gray matter of this organ is composed of delicate connective tissue, with "free nuclei sparingly distributed through it." The nervecells of the nucleus caudatus are multipolar and of two sizes; some are about $\frac{1}{800}$ inch in diameter with many processes, but most are much smaller ( $\frac{x}{1600}$ inch). Between the fibres of the gray matter of the nucleus lenticularis are many cells with yellow pigment in them.

The three collections of gray matter-the locus niger, and the caudate and lenticular nuclei of the striate body-with the nerve-fibres which originate in them and bind them together, have been held to constitute a connected chain of nervous organs, to which the name "ganglia of the crusta" has been given by Meynert. Recently, however, this relation of the corpora striata as "basal ganglia," or "middle-men " between the spinal cord and the cerebral cortex, has 
been called in question by Wernicke and A. Hill. The latter argues, ${ }^{2}$ chiefly on morphological grounds, that the nucleus caudatus should be separated from the optic thalamus, and connected immediately with the cortex. This connection, he thinks, is favored by the nature of its development, by its minute structure, which differs from that of the thalamus, and by its resemblance to another nucleus (the amygdaloid) which has an undoubted origin from the cortex.

$\S 23$. Another chain of nerrous organs, leading between the pons

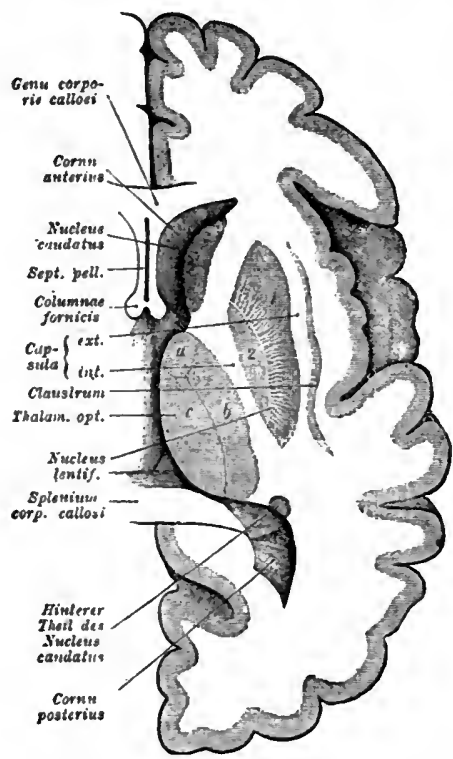

FIG. 30.

Varolii and the hemispheres of the brain, consists of the tegmentum of the crus and its ganglia-the red nucleus (already described), corpus subthalamicon, the corpora geniculata, and the optic thalami.

The arrangement of the nerrous elements in the external corpus

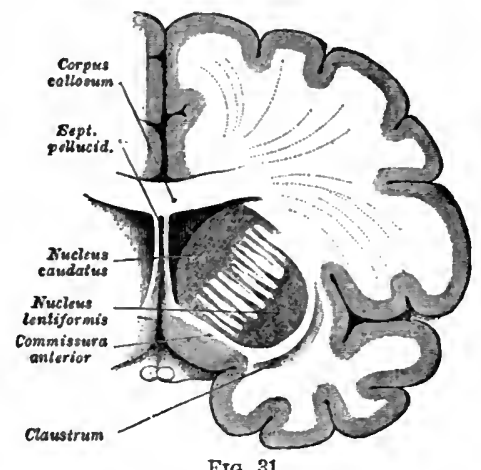

FIc. 31.

These and the following two Figures show the arrangement of the whlte and gray substance in the interior of the cerebrum. (All four are from Gegenbaur.)

Fig. 30.-Horizontal Section through the Right Hemisphere.

Fig. 31.-Frontal Section through the Cerebrum in front of the Fornix. Posterior snrface of the section displayed.

geniculatum is peculiar: it consists of alternate layers of white and gray matter, as though occasioned by laying a lamina of the gray between two medullary laminæ, and then folding them in a zigzag manner. The nerve-cells of this organ are from $\frac{1}{300}$ to $\frac{1}{800}$ of an inch in length, and $\frac{1}{1600}$ of an inch in breadth; they are coarsely granular and pigmented.

The optic thalamus is a mass of gray matter, with multipolar and fusiform cells, traversed by nerve-fibres. This gray matter is partially subdivided into two parts, an inner and an outer nucleus.

1 The Plan of the Central Nervous System, pp. 25 ff. 
Its free surface (inner and upper) is covered by a layer of white fibres. On its outer surface is the white matter of the internal capsule, formed by fibres diverging from the crusta into the hemispheres. All along this surface fibres radiate from the interior of this organ, and mingle with those of the internal capsule on their way to the cerebral hemispheres. Those in front pass to the frontal lobe; those in the middle pass to the back part of the same lobe and to the parietal lobe; those behind to the temporo-sphenoidal and occipital lobes.

"The external and under surfaces of the thalamus are not free, but are united with the other parts of the brain. The under surface is united with the tegmental part of the crus cerebri, while the external surface is covered by white substance, that is formed

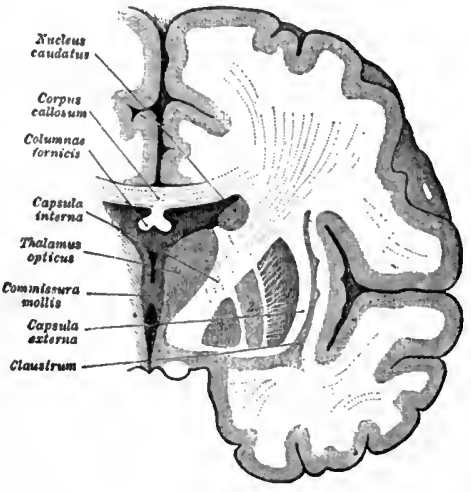

FIG, 32.

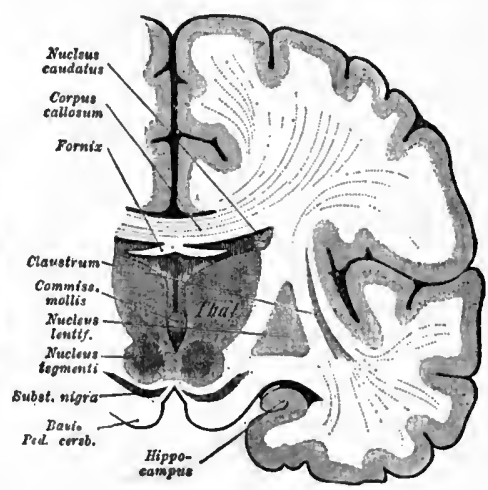

FIG. 33.

FIG. 32.-Frontai Section through the Right Hemisphere of the Cerebrum in front of the Com-

Missura Mollis. Posterior surface of section displayed.
Fio. 33. - Frontal Section through the Cerebrum back of the Commissura Mollis. Front surface of section displayed.

of fibres of the crusta, which here diverge into the substance of the hemisphere and pass between the thalamus and the lenticular nucleus, forming the so-called 'internal capsule" "' (comp. p. $87 \mathrm{f}$.). The cells of its substance average about $\frac{1}{80}$ inch in length and 25 in breadth; their long axis is parallel to the course of the nerve-fascicles. According to a recent authority, ${ }^{2}$ the thalamus is the primary centre of the optic nerve, and is also connected with the olfactory nerve-originally by way of the fornix.

The nervous substance of the corpora quadrigemina consists mainly of gray matter covered externally with a thin layer of nerve-fibres. In the interior of the upper or front pair the most

1 Quain's Anatomy, ninth edition, ii., p. 324.

A. Hill, The Plan of the Central Nervous System, pp. $20 \mathrm{ff}$. 
characteristic portion of this organ is found; it is a layer of fine nerve-fibres running longitudinally, between which are small, scattered nerve-cells. In the external strata of these bodies multipolar cells are abundant; in their interior, at the sides of the Sylvian aqueduct, is a collection of gray matter which forms a continuation of the lining of the third ventricle. The nerve-cells in the corpora quadrigemina vary greatly in size. Most of those in the superficial strata are small; but in the deeper strata some of them reach the maximum of nearly $\frac{1}{250}$ of an inch. The centres of origin for the third and fourth nerves are in that nervous structure of fine fibrils and fusiform cells which lies along the Sylvian aqueduct.

$\S 24$. The arrangement of the nervous elements in all the basal ganglia, as connected with the cerebral peduncles, indicates the nature of the mechanism of this region of the brain. It is constructed so as to co-ordinate all the nerve-tracts of motion with those of sense, and thus give to these ganglia important reflex and automatic powers over the sensory-motor apparatus, while subordinating them to the control of the nervous centres of the cerebral cortex that lie farther above.

To the highest and dominating nerrous centres in the cerebral hemispheres the paths of nervous impulse are laid from the basal ganglia by that blossoming out, as it were, of the nerve-fibres on their way to the white core of the cerebrum, which is called the "corona radiata." The corona is formed by the fibres that radiate from the striate bodies, from the optic thalami, and the internal capsule, into the convolutions of the lobes of the hemispheres.

$\$ 25$. The combination of the nervous elements into the preeminently complex mechanism of the convolutions of the human cerebrum may be described from two points of view; the first is that from which their various external surfaces may be regarded by the unaided eye, the second that which histology assumes when it examines under the microscope various sections made from layers of their substance.

The details of the external aspect of the convolutions vary so much in each individual, and even in the two hemispheres of the same brain, that the only chance of bringing order out of this apparent confusion is to discover what is general, and for the most part constant, in the midst of what is particular and subject to change. In making such discovery the study of embryology is especially important. Certain sulci and their corresponding gyri appear with a marked regularity in the earlier and more fundamental stages of the development of the foetal brain. So, too, does the examination of the surfaces of the hemispheres of the adult brain show certain degrees of 
strength with which the sulci and gyri are distinguishable, and thus enable the investigator to divide them into so-called primary, secondary, and even tertiary classes. Bischoff, Ecker, and others have aptly compared the primary gyri to the large mountain ranges whose sinuosities give to an entire region its characteristic features; the secondary gyri are like those subordinate ranges which are brought into existence through the formation of longitudinal valleys (secondary sulci) in the main ranges; while the tertiary convolutions may be compared to the small spurs which run out into the valleys between the principal ranges and from their sides. Only the primary gyri are, as a rule, pretty regularly disposed.

It is by means of the primary sulci that the surfaces of the hemispheres of the brain have been divided by modern anatomy into five territories or Lobes. ${ }^{1}$ The frontier lines of these lobes, however, are clearly laid down only on some of the surfaces, while on other surfaces the lobes encroach on each other without distinct boundaries. The five lobes are called Frontal, Parietal, Temporosphenoidal (also Temporal or Sphenoidal), Occipital, and Central, or Insula, or Island of Reil ; the latter does not stand in immediate relation with the walls of the skull. The Frontal Lobe is divided from the parietal on its upper and lateral surface by the Fissure of Rolando (sulcus centralis); and on its lower surface from the temporal lobe by the horizontal branch of the Fissure of Sylvius. The Parietal Lobe is divided from the temporal for the greater part by the Fissure of Sylvius, and from the occipital-on its median surface completely, but on its upper surface only very incompletely -by the parieto-occipital fissure. The Temporo-sphenoidal lobe is distinctly marked off from the frontal and parietal, as already described; while the boundary line between it and the occipital lobe is ill defined. The Island of Reil lies concealed between the frontal, parietal, and temporo-sphenoidal lobes; its surface, when exposed by drawing aside the margin of the Sylvian Fissure, shows a few short convolutions which radiate forward, upward, and backward from a central spot on the lower surface. The occipital, tem. poro-sphenoidal, and frontal lobes, all have three principal convolutions arranged in parallel tiers (superior, middle, and inferior); in the frontal lobes these three spring from the anterior part of the ascending convolution just in front of the Fissure of Rolando (that is, from the gyrus centralis anterior) and run forward to the front end of the cerebrum.

1 The convolutions are here described in dependence upon the work of Ecker, The Convolutions of the Human Brain. London, 1873. 
\$26. A few of the most important of the sulci and gyri need separate mention; the accompanying diagrams will make clear

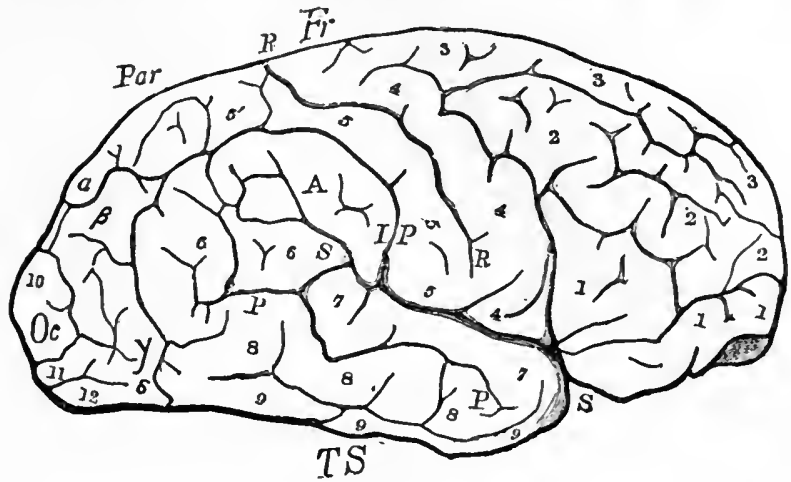

FIG. 34.

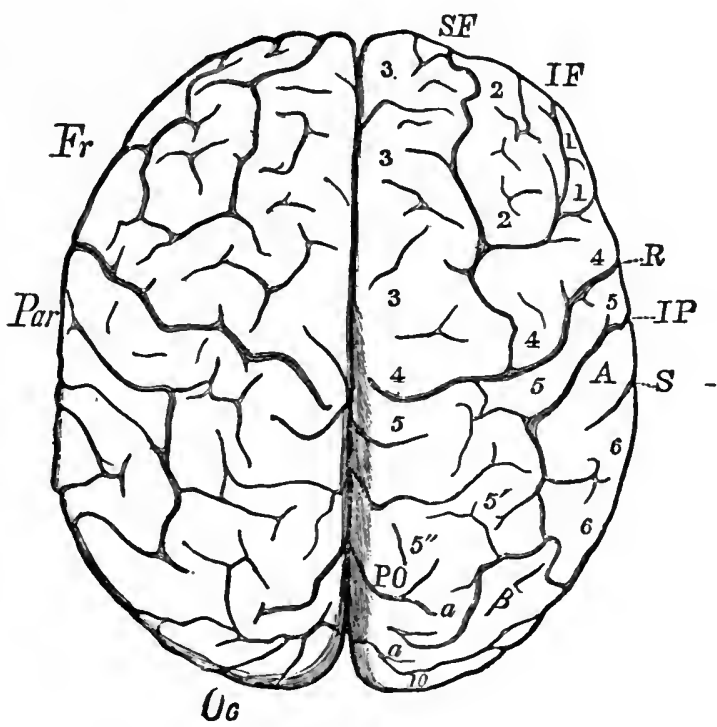

Fig. 35.

Figs. 34 and 35.-Profle and Vertex Views of Cerebrum. Fr, the frontal lobe; Par, parietal; $O c$, occipital: $T s$, temporo-sphenoidal lobe; SS, Sylvian fissnre: RR, fissure of Rolando ; PO, parieto-occipital fiasnre; IP, intra-parietal fissure; $\mathrm{PP}$. Parallel flssure: SF and IF, superoand infero-frontal fissures; $1,1,1$, inferior, $2,2,2$. middle, and $3,3,3$, mperior frontal convolutions: 4, 4, ascending frontal convolution: 5, 5, 5, ascending parietal, 5', posterc-parietal, and 6,6 , angular convolutions: $A$, supra-marginal, or convolution of the parietal eminence ; 7 , 7. superior, \&, 8, 8. middle, and $9,9,9$, inferior temporo-sphenoidal convolutions; 10 , superior, 11 , middle, and 12 , inferior occipital convolutions; $\alpha, \beta, \gamma, \delta$, four annectent convolutions.

further details (see Figs. 34, 35, and 36). Among the sulci which bound the main territories of the cerebral hemispheres the Fissure 
of Sylvius is much the most important. "It can," says Ecker, " in nowise be considered in the same category as the rest of the sulci on the surface of the brain." The other sulci may be regarded as mere folds of the cerebral cortex; the Fissure of Sylvius, on the contrary, is made by folding the entire hemisphere into an arch, with its concave surface downward, about the point of entrance of the crus cerebri. This fissure exists in the foetal brain at the third month. "It arises," say Foster and Balfour," " at the time when the hemispleres, owing to their growth in front of and behind the corpora striata, have assumed somewhat the form of a bean." The Fissure of Rolando is also always present in the human brain. It makes its appearance in the foetus as early as the end of the fifth month. It is rarely, if ever, bridged over by a secondary gyrus; it, therefore, forms a point of departure in the examination of all the convolutions. It is bounded for its entire length by two

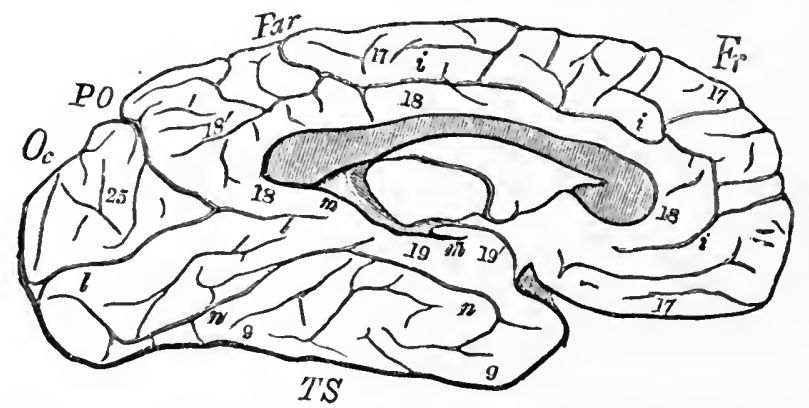

FIG. 36.-Convolntlons of the Inner and Tentorial Surfaces of the Left Hemisphere. $i$, $i, i$, calloso-marginal fissure, $l, l$, calcarine fissure; $m, m$, hippocampal flssure; $n, n$, colluteral fissure: PO, parieto-occipital fissure; 17,17 , marginal convolution ; 18, 18, gyrus fornicatus; 18 , qnaililateral lobule; 19 , hippocampal gyrus; 19 , its recurved end ; 25 , occipital lobule; 9,9 , inferior temporo-sphenoidal convolution.

important convolutions (the anterior and posterior central or ascending frontal and ascending parietal), which at both of its ends connect together in the form of an arch. The fissure which separates the parietal from the occipital lobe (parieto-occipital) and the one which runs from before backward through the parietal lobe (intra-parietal) are also to be mentioned among the more important. The intraparietal fissure, on the convexity of the parietal lobe, includes, between it and the median line, the upper parietal convolution, and embraces in its downward and outward bend the angular convolution. The latter convolution, and the marginal convolution form the inferior parietal lobule.

1 Elements of Embryology, p. 384. London, 1883. 
Besides the superior, middle, and inferior convolutions of the frontal, temporo-sphenoidal, and occipital lobes, and the two central convolutions on each side of the Fissure of Rolando, the following, which belong to the median aspect of the hemispheres, are to be noted in particular. The convolution which arches around the corpus callosum, and is separated from the median aspect of the first frontal convolution by a deep and constant fissure (the sulcus calloso-marginalis) is called from its shape, gyrus fornicatus. The back end of this convolution curves downward and then forward, under the name of gyrus hippocampi, to the inner tip of the temporal lobe. The passage of the former convolution, without break, into the latter, Ecker considers one of the most important differences between the hemispheres of the brain of man and those of the ape.

$\S 27$. Although the general arrangement of gray nervous matter upon the surface, and of white matter within, is adhered to in all parts of the cerebral cortex, the form and disposition of the cells in the gray matter differ in different regions, and also in different layers of the same regions. But its most common form, which is that seen in the convolutions of the parietal lobe, corresponds to what Meynert" has called "the general or five-laminated type of the cortex of the cerebrum." There are, as a rule, that is to say, five layers or laminx to be discovered in the gray matter of the cerebral cortex. The thickness of the entire cortex, thus composed, is, in the adult, from $\frac{1}{1^{2}}$ to $\frac{1}{8}$ of an inch. The first of the layers consists of a matrix, in which delicate nerve-fibrils run parallel to the surface and interlace with a few small globular or elongated branching nerve-cells scattered here and there. The nature of this matrix has been the subject of dispute; by some it is looked upon as connective tissue (Kölliker), by others as neuroglia (Virchow). The second and third layers contain a large number of pyramidal, or spindle-shaped cells ; of these layers the third is the broadest, and contains the largest (but fewest) cells. The cells of the second larer are about $.2 \frac{1}{500}$ of an inch in diameter, and are closely pressed together to form its substance ; but in the third layer they augment gradually in size until they reach a diameter of $\frac{1}{1000}$, to perhaps $\frac{1}{60}$ of an inch, with their long axes perpendicular to the cortical surface. The fourth layer contains large numbers of small, globular, and irregularly-shaped and branching cells ; the fifth, spindle-shaped bodies with long tapering processes, and also a certain number of smaller irregular cells. This innermost layer consists chiefly of a compact accumulation of cells which give

' In Stricker's Human and Comparative Anatomy, ii., p. 381. 
off lateral processes. Gerlach discovers here, as in the spinal cord (see $\S 9$ ), a very ininute network with which these processes are apparently contiuuous. It is also an assumption, verified by direct observation of some cases, and by the general analogy of the nerrous system, that many of the extremely attenuated nerve-fibrils which

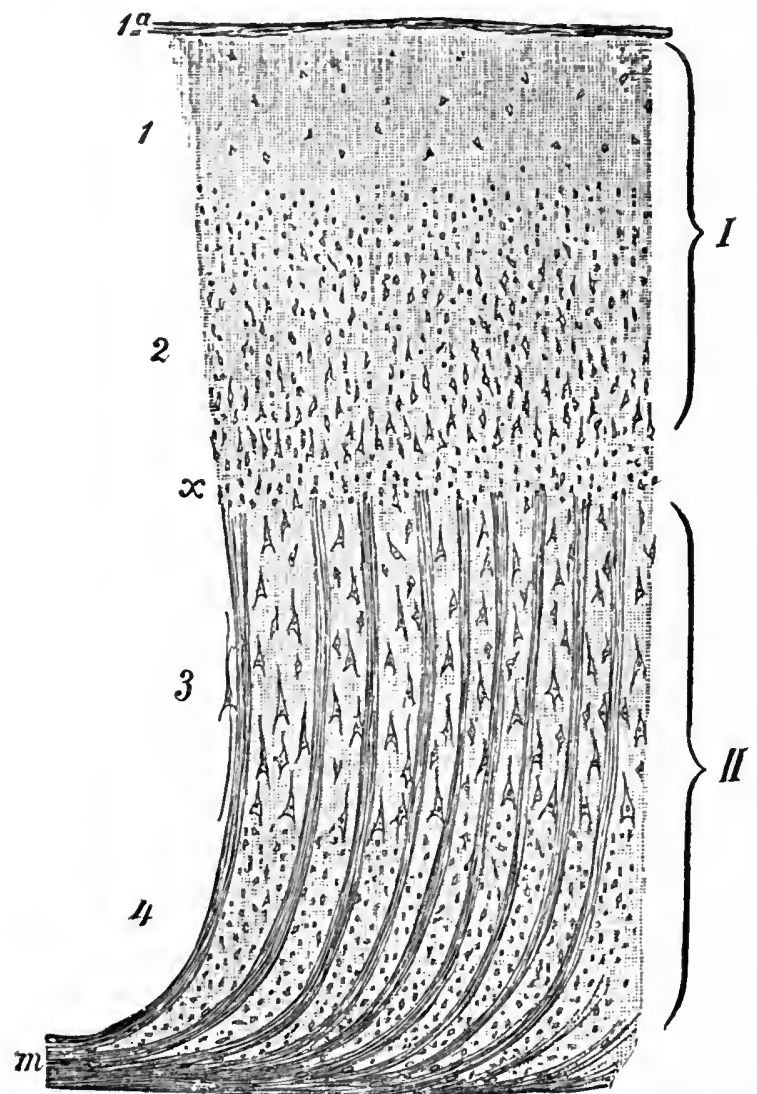

Frg. 37.-Section through the Cerebral Cortex of Man, prepared with Osmic Acid 45/1. (Schwalbe) $I$, principal external, and $I I$, internal, layer; $x$, layer lying as a limit between the two : $m$, medullary substance sending out bundles of nerve-fibres into $I I ; 1$, layer poor in cella, but with an extcrnal plexns of nerve-fibres $\left(1 a_{1} ; 2\right.$, layer of small, and 3 , of large, pyramidal cells ; 4, inner layer of small nerve-cells.

radiate from the white core of the convolutions are continuous with the basal axis-cylinder processes of the cells in the layers of gray substance. Small rounded corpuscles and small stellate cells, so pellucid that they seem to be only free nuclei, are contained in the neuroglia of the gray substance of the cerebral cortex. It is doubt- 
ful whether these are true nerrous elements or not. The number of nerve-cells in the cortical substance is very great. In a portion of this substance, only one millimeter square and $\frac{1}{10}$ millimeter thick, 100 to 120 have, on an average, been counted. ${ }^{2}$

Modifications of the arrangement which prevails in most of the gray substance of the hemispheres of the brain are found in certain regions. In the cortex of the occipital lobe the number of layers is increased by the intercalation of additional granule layers to seven or eight. In the cortex of the Island of Reil, and of the convolutions bounding the Fissure of Sylvius, a large proportion of fusiform cells is found. In the fourth layer of the cerebral cortex of the $\mathrm{dog}$, in the region which Hitzig considered to be motor, Betz discovered certain cells lying in scattered groups, with two large and several small processes; these cells, on acccount of their great size, he called "giant-cells." Similar" cells, have been found by him in certain regions of the human cerebral cortex-namely, in the entire anterior central, and the upper end of the posterior central convolutions, and along the lobe which is prolonged backward from the two.

$\S 28$. The white substance of the hemispheres of the brain may all be considered as originating in its cortical gray substance; but the nerve-fibres of which it is composed constitute three classes, according to the destination of the fascicles into which the fibres are gathered. These three are the down-going or peduncular, the commissural, and the arcuate (or fibre proprice). It is the business of the peduncular system to connect the cerebrum with the lower parts of the encephalon. This system, called the corona radiata, is narrowed into the internal capsule and continued downward to the crura cerebri ; its diminished size shows that a considerable portion of its fibres have entered into the optic thalami and striate bodies. But it is also probable that many fibres of the crusta pass directly into the brain's medullary centre, and through this to its gray cortex, without entering these ganglia. Of such tracts the best known is the pyramidal (probably motor). According to Flechsig and others, this is traceable through the internal capsule and corona radiata to certain frontal and parietal convolutions. Another tract, traceable directly to the convolutions of the cortex, passes from the external part of the crusta into the white matter of the occipital lobe (so-called direct sensory tract). The fibres which come from the tegmentum, and are lost, for the most part, in the thalamus and the subthalamic region, stream outward from the other side of this organ, join the general system of the corona radi-

'See Luys, The Brain and its Functions, p. 17. New York, 1882. 
ata, and diverge to nearly every part of the hemispheres ; but especially to the temporo-sphenoidal and occipital lobes (probably sensory).

The commissural system of fibres has hitherto universally been supposed to connect the two hemispheres of the brain ; but Professor Hamilton, of Aberdeen, and others, have recently called this statement in question. The principal tract of such fibres is in the corpus callosum. Since this commissure lies in a plane above that of the corona radiata, the two systems of fibres intersect each other on their way to the convolutions of the cerebral hemispheres. A smaller commissure (the anterior) passes below the lenticular nuclei of the striate bodies and connects the convolutions around the Sylvian fissure-binding together the right and left temporo-sphenoidal lobes; it also furnishes a root of origin for the olfactory nerve.

The arcuate fibres extend over more or less territory on the same side, and connect the gray matter of adjacent, or more or less distant, convolutions in the same hemisphere-" a garland-like interwearing" of two convolutions around the sulcus between them. In certain localities, where the fascicles into which these fibres are gathered are strongly marked, they have received special names; such are the fasciculus uncinatus which crosses the bottom of the Sylvian fissure and connects the convolutions of the frontal with those of the temporo-sphenoidal lobe; the fillet of the gyrus fornicatus, extending longitudinally in that conrolution; the longitudinal inferior fasciculus, connecting the convolutions of the occipital and temporo-sphenoidal lobes. Such fibres are sometimes called longitudiral or collateral fibres. It is by the commissural and arcuate fibres that the inuumerable ganglion-cells and nerve-granules of the cortex are bound into a unity of form and of function. The processes of the cells anastomose, and are thus united with immediately adjoining cells by means of a gray fibre-plexus. The axiscylinder processes become continuous with the medullated fibres, which, gathered into bundles (the fasciculi of the arcuate fibres), line as a continuous layer the inner surface of the cortex. In this manner the nervous elements of that crowning mechanism, which is known as the chief glory of man's nervous system, are made to exhibit a manifolduess, and at the same time a unity of structure, suggestive of a common service joined with diversity of mode in which the service is rendered.

$\$ 29$. The view of Meynert-to which reference has already been made (p. 73)-regards the gray masses and converging and diverging tracts of the cerebro-spinal nerrous mechanism as a "Projec- 
tion System" (or rather as a series of "projection systems"), which is capped and dominated by the hemispheres of the cerebrum. The sensory nerves may thus be figuratively described as the "feelers," and the motor nerves as the "arms" of its cortical gray matter. This matter is both a "sensory shell," upon which the centripetal nerve-commotions gather and dispose themselves; and is also the "motor shell" in which certain centrifugal motions originate. It is, therefore, an internal "Projection Field" for the muscular" sys-

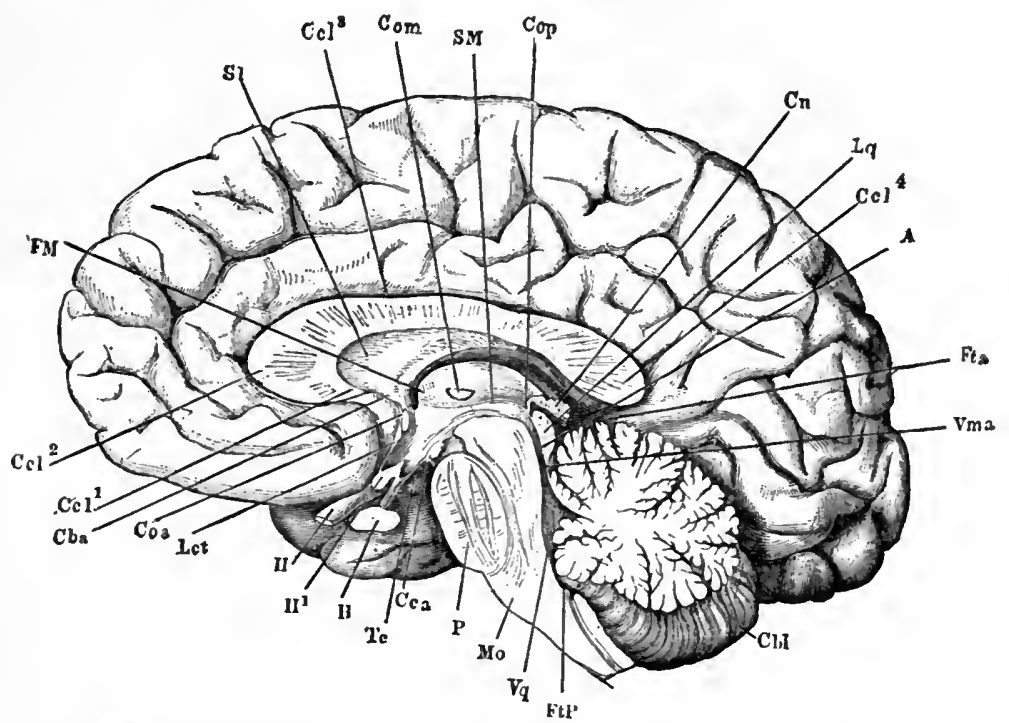

FIG. 38.-Jedinn Section of the Brain. A, aqueduct of Sylvius; Cba, white commissure; Chl, cerebellum : Cca, corpus albicans; Ccl, corpus callosum, of which the different parts are Ccl'. rostrum, $\mathrm{Ccl}^{2}$, the genu, $\mathrm{Ccl}^{3}$, the body, and $\mathrm{Ccl}^{4}$, splenium; $\mathrm{Cn}$, conarium, or pineal gland ; Coa, anterior, Com, middle, and Cop, posterior, commissures; FM, foramell of Monro; Fta, the antcrior, and $\mathrm{Ftn}$, the posterior, transverse fissures; Lct, lamina cinerea terminalis; and Lq, lamina of the corpora quadrigemina; Mo, medulla oblongata ; P, pons Varolii : SI, septum lucidum; SM, sulcus of Monro; Tc, tuber cinereum; Vma, anterior velum mednllare; $\nabla$, fourth ventricle; $1 \mathrm{I}$, optic nerve; II $^{1}$, chiasm of the optic nerve.

tem. The gray masses of the brain below its hemispheres (with the exception of the internal tubular mass) may-according to Meynert-be described as either $(a)$ "Interruption Masses" of the projection system, or as belonging to (b) the "Region of Reduction" of the mass of this system. It is in these lower gray masses that the great bull of the nerve-tracts (the corona radiata) coming from the cortex of the cerebrum are not only broken and interrupted in their course, but are also greatly reduced in size. ${ }^{1}$ The

'For a clear and concise summary of Meynert's entire view, see Quain's Anatomy, ninth edition, ii., pp. $3 \% 0 \mathrm{ff}$. 
functional significance of this relation in which the cerebral cortex stands to all the rest of the nervous mechanism will appear more clearly further on in the discussion.

$\S 30$. The cerebro-spinal axis, or central nerrous mechanism of the cavity of the spinal column and skull, is connected with the end-organs of motion and of sense by thirty-one pairs of Spinal, and twelve pairs of Cranial or Encephalic Nerves.

The thirty-one pairs of Spinal Nerves originate in the spinal cord and pass out of the spinal canal through the openings called

$\Lambda$

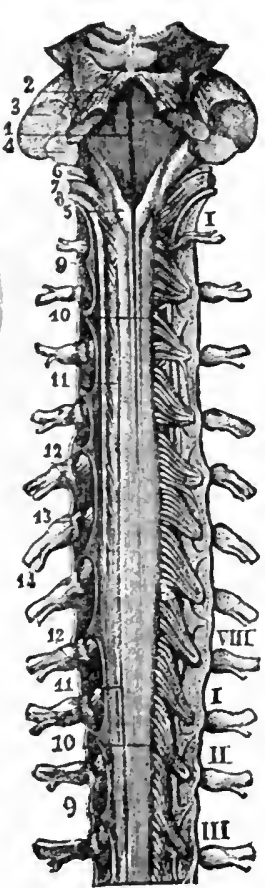

B

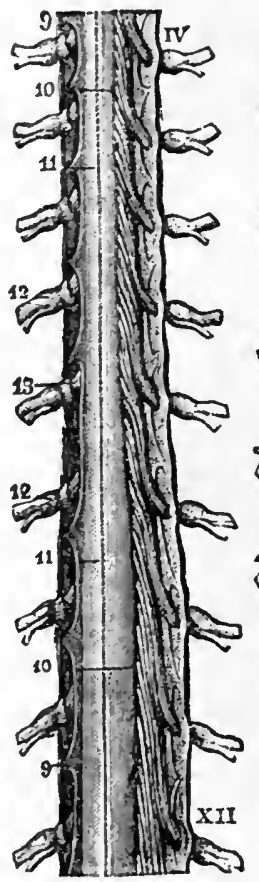

C

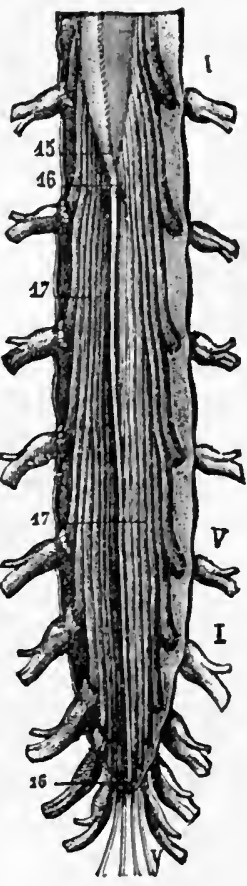

FIg. 39.-Posterior View of the Spinal Cord with its Nerves, 1/. (After Sappey.) I-VIII in A are cervical: I, II, and III in A, and IV-XII in 1 , dorsal; the last in B, und clown to V in C, lumbar: I-V in C, sacral : 10, 11, origin of the posterior roots; 11,11 , posterior median fitsure ; 12, 12, spinal ganglia ; 13,13, united nerve ; 15, tapering of the lower end, becoming 16 , 16 , the filum terminale; 17 , cauda equina.

"intervertebral foramina." Of the entire number-enumerating from above-eight pairs are cervical, twelve thoracic or dorsal, five lumbar, five sacral, one coccygeal. Each nerve arises from the side of the cord by two roots, an anterior and a posterior. The posterior root has a swelling or ganglion upon it, the anterior has none; the former is composed of sensory nerve-fibres, the latter, 
of motor nerve-fibres. The ganglion of the posterior roots contains unipolar nerve-cells. The roots themselves vary, in the different regions of the cord, both as respects direction and length. Immediately outside the ganglion the anterior root joins the posterior, and the united nerve-containing a mixture of motor and sensory fibres-soon after separates into two divisions, that are formed of elements from each root and that are distributed, one upon the back and the other upon the front and sides, to all parts of the trunk and limbs.

Of the twelve pairs (adopting the Continental instead of the English division) of Cranial Nerves, which arise from the base of the encephalon and pass through the openings (foramina) in the floor of the cranial cavity, three groups may be distinguished: $(a)$ the sensory nerves, or nerves of special sense; $(b)$ the motor nerres; (c) the mixed nerves, which contain both sensory and motor fibres. To the first group belong the olfactory nerve (first pair), the optic (second pair), and the auditory (eighth pair); to the second group belong the nerves that supply the principal muscles of the eyeball (oculo-motor, third pair), the superior oblique (trochlear, fourth pair), and external rectus (abducent, sixth pair), muscles of the eye, the muscles of facial expression (seventh pair), the muscles of the tongue (hypoglossal, twelfth pair), and the spinal accessory nerre (eleventh pair) ; to the third group belong the three nerves which are so widely distributed over the mucous membranes and muscles of the face, tongue, pharynx, and internal organs-namely, the trigeminus (fifth pair), the glossopharyngeal (ninth pair), and the pneumogastric or vagus (tenth päir).

$\$ 31$. It is, then, by a process of differentiation of a few comparatively simple elements, and of infinitely varied arrangement and combination of the elements thus differentiated, that the elaborate mechanism of the human nerrous system is constructed, and made fit for the great variety of interconnected functions which it is called upon to perform. Material atoms are chemically united into the complex and unstable molecules of which nervous matter is composed. These molecules are arranged into the structural forms of nerve-fibres and nerve-cells; and the latter, at least, are modified in form according to their locatiou, and perhaps also function. The elements are combined into conducting cords, end-organs, and central organs, according to the threefold plan of a nerrous system ; and the organs are arranged, in the case of man, with an intricacy of relations which can be only very inadequately described.

The description of the mechanism being finished, we consider more in detail what it can do. 


\section{CHAPTER III.}

\section{THE NERVES AS CONDUCTORS.}

\$1. Iv that threefold economy of organs which characterizes the developed nervous mechanism, the office of propagating the neural process between the central organs and the end-organs has been assigned to the nerves. The power to originate this process under the action of external stimuli, although experiment shows that it belongs to the nerves, is not exercised by them while in their normal place within the mechanism. It is the office of the end-organs to transmute the physical molecular processes, which are their stimuli, into the physiological and neural process, and hand it over, as it were, to these conducting cords. But the office of the nerves as conductors is, of course, not like that of a tube which conducts along its channel some lind of fluid, nor is it like that of the wire or bellmetal which is thrown into vibration throughout. It is a molecular commotion which, when started at any point in the nerves, moves in both directions from point to point along its course. The intimate connection between the two functions of excitation and conduction becomes, then, at once apparent. Indeed, excitation may be considered as the setting up of the process of conduction; conduction as the uninterrupted continuance, or propagation from point to point, successively, of the process of excitation. Each minute subdivision of the nerve, then, must be regarded as constituting, in some sort, a source or centre of stimulation with respect to its neighboring subdivisions. If the nerve-commotion is to move along the nerve $N$, between two distant portions of its structure, $a$ and $z$, then $a$ must act upon its neighbor $b$ as a stimulus, $b$ upon $c$, and so on successively until $y$ is found stimulating $z$, and the process of progressive excitation or conduction is complete.

$\S 2$. It follows from what has just been said that, in considering the nerves as conductors, the conditions and laws of the origination of that process of excitation which they conduct must be taken into account. It is neither necessary nor convenient, however, to carry throughout a distinction between the two functions-the excitability and the conductivity-of the nerves; it is better to regard them 
as one process from somewhat different points of view. The arising and progressive movement of a unique molecular commotion constitutes the distinctively neural action or function of the nerves. And since this so-called nerve-commotion has eluded all the attempts hitherto made to discover its more intimate nature, and to bring it under a strict theory, we must be content with describing the following three classes of facts: (1) The Conditions of the process ; (2) the Phenomena evoked with it, or as part of it, by different kinds of stimuli ; (3) the Laws of its propagation.

What is called "the general physiology of nerves" attempts to consider their action while excluding the influence upon it from the central organs and the end-organs. That is, the function of the nerves, as we now consider it, is exercised under abnormal conditions. It has been objected to the view which regards each element of the nerve as the stimulus of neighboring elements, that the effects of direct artificial stimulation must differ in important respects from those obtained by stimulation in the normal way. For example, Ziemssen and others have shown that the crushed nerve of an animal, or the paralyzed nerve of a man, may be made to set up a nerve-process by reflex stimulation when it will no longer respond to stimulation applied directly to its trunk. And Grünhagen affirms that after a stretch of nerre has been reduced by the effects of carbonic acid to a lower degree of excitability under direct stimulation, it will still propagate through itself the excitation set up elsewhere with undiminished force. Such facts, however, only prove that the application of stimuli to the nerve for purposes of experiment is a very rough and ineffective way compared with nature's method of preparing the stimuli by the modifying influence of the nervous tissues themselves. They do not prove that the neural process is not fundamentally the same in whatever way it is brought about. On the contrary, there is abundant evidence to show that this abnormal activity, when carefully studied, will give us the key to the normal function of the nerves. The advantages of simplifying the problem by experiments upon isolated nerves are too great, and the fund of valid information thus obtained is too important for us to neglect the method proposed. The science called "general physiology of the nerves" is, indeed, very largely built upon experiments with the motor nerves of frogs; and, of course, it may be said that frogs, with respect to their nervous systerns as otherwise, are very unlike men. But with respect to the character of that specific molecular process which is set up in the nerve when excited, frogs appear to be essentially the same as men. At any rate, we have no physical means adequate for detecting any 
essential differences. In other words, nerves are nerves the world over ; and what they do as nerves simply, is essentially one thing in all cases. What they do in their vastly different arrangements and connections with central organs and end-organs differs vastly in different cases.

\&3. The view that each element of every nerve, irrespective of its kind or specific place in the animal mechanism, can only stimulate its neighbor and be stimulated by its neighbor, suggests another interesting inquiry. Is this stimulus of the nerve-elements, this effect in exciting contiguous elements, anislogous to any of the so-called external stimuli? Or, in other words, the inquiry may be raised: Is the process of nerre-commotion in the nerves similar to, or identical with, any of those molecular processes which act as indirect stimuli upon the nerves through the end-organs? In answering this question it has long been customary to ally nerve-commotion with electricity. In a posthumous work by the mathematician Hausen, in 1743, it was first proposed to consider the efficient principle of nervous action as identical with that of the electrical machine. ' Exactly a century later (1843) du Bois-Reymond announced the discovery of an electrical current in unexcited nerves (the so-called "current of rest"). Since then the phenomena of this current, of the negative variation of the nerve, and of electrotonus -all discovered almost simultaneously by the same investigatorhave been the subject of much painstaking research. This research has resulted in showing that important differences exist between the neural process and that of the electrical current, and in making more and more clear the impossibility of forming a purely electrical theory of the nervous functions. On the other hand, it has also revealed many important similarities between the two. It is by experiment with the effect of electrical currents, of different kinds and directions, and under varying conditions, that the science of general physiology of the nerves has been built up.

$\$ 4$. In order to understand the general results of experiment upon the nerves, the nature and use of the so-called Nerve-muscle Machine must be understood.

A "nerve-muscle preparation" consists of muscle freshly taken from the living animal with its attached nerve dissected out; for example, the gastrocnemius muscle of the frog with the attached sciatic nerve. Such a preparation may be kept alive for some time in a moist chamber. By tha simple contrivance of connecting the

${ }^{1}$ See in du Bois-Reymond's great work. the history of opinions on this point : Untersuchungen über thierische Electricität, II., i., pp. 209 ff. Berlin, 1849. 
end of the muscle with a lever, arming the lever with some means of making a mark-either pen, or bristle, or needle-and bringing its point thus armed to bear on a rapidly travelling surface (plain paper, or smoked paper, or glass), the time and amount of the contractions of the muscle may be recorded. The most refined means for noting the exact instant when the stimulus is applied, and also the state of the effects produced at every succeeding instant of their duration, are of first importance. The nerve may be stimulated with different kinds, degrees, and directions of the electrical current (or with other forms of stimuli) at any points preferred in its stretch, and under a great variety of conditions with respect to temperature, moisture, mechanical pressure or stricture, integrity and vitality of its structure, etc.; and the effects of such stimulations upon the contractions of the muscle may be noted and compared as they have been recorded. Means for testing the most delicate and rapid changes in the electrical or thermometric conditions of the nerve may be applied to it at any point of its stretch. Variations and refinements of experiments essentially the same may be almost indefinitely multiplied; the experiments may be repeated, and verified or corrected, by the same observer or by others. Inasmuch as the preparation is both muscle and nerve, an acquaintance with the behavior of the muscle, and with the laws of its contraction, is necessary in order that it may be known how much of the complex phenomena is to be ascribed to the functional activity of muscle, how much to that of nerve. But into a statement of the general laws of contractile tissues, and of the nature and explanation of the behavior of muscle when irritated, we cannot enter. ${ }^{1}$

Certain terms in constant use to describe the methods and results of experiments with the nerve-muscle machine also require a brief explanation. The line traced by the armed end of the lever, as it rises and falls with the contractions of the muscle, is known as the "muscle-curve." In so far as it shows changes that are due to the condition of the attached nerve, or to the quality, intensity, and order of the stimulations applied to that nerve, this curve is a measure of the process of neural excitation and conduction. If the electrical current flows with the course of the motor nervestretch-that is, from the central toward the neripheral parts-it is called "descending," or direct; if in the opposite direction "ascending," or inverse. The current to be detected in an unex-

${ }^{1}$ For a description of the method and results of experimenting with the nerve-muscle preparation, more accessible to the general reader than the books to which reference will chietly be made, see Foster's Text-book of Physiology, pp. 43 ff. 
cited nerve (a nerve, that is, whose functional activity is not at the time in exercise on account of the application of any kind of stimlus) is called a " natural current," or a " current of rest." The current produced by stimulating the nerve, and so calling into exercise its plyysiological function, is a "current of action." When a single induction-shock, or a number of such shocks repeated at sufficient intervals, is sent through a nerve-stretch, the contractile spasm of the muscle in response to each shock shows that a single "nervous impulse" is passing along the nerve. When the single stimulations are repeated with sufficient rapidity, the single spasms fuse into one apparently continuous effort, known as "tetanus," or "tetanic contraction." The term "tetauus" applies primarily to the muscle only; but the application of rapidly repeated shocks to the nerve, such as would produce "tetanic contraction " of the muscle, may be called the "tetanization of a nerve." The contraction which follows the closing of the current is called the "making contraction," or "closing contraction;" that which follows its opening, the "breaking" or "opening" contraction.

$\$ 5$. Of the conditions under which alone the nerve is capable of exercising its function of neurility the most important are these three : Vitality, Oxygen, and Recovery from previous exhaustion.

A nerve cannot act as a conductor of the neural process unless it is vital ; but the death of the nerve is not necessarily simultaneous with that of the body from which it is taken, or of the muscle to which it is attached. On the contrary, by careful treatment witl respect to moisture and temperature, and by guarding it from mechanical or chemical injury, it may be preserved alive for some time after excision. The indirect irritability of the muscle through the excised nerve attached to it frequently continues in warmblooded animals and in high temperature not longer than about an hour ; in the frog and in a low temperature it may last for several days. The nerves of the summer frog are much more perishable than those of the same animal in winter. A nerve is, of course, alive as long as it will excite the muscle to contract. But the nerve is not necessarily dead when the attached muscle no longer responds to its excitation; the failure may be due to the death of the very sensitive and perishable end-organs which connect the two. Hermann ' considered that the existence of electrical phenomena in the nerres of rabbits showed the nerves to be alive for several hours after they would no longer stimulate the muscle, and also after the muscle itself could not be irritated directly. Nerves may even be alive after they cease to exhibit electrical phenomena that can be

${ }^{1}$ Handb. d. Physiol., II., i., p. 120. 
detected by the most delicate tests available. It is possible that the capacity for excitation may linger after the capacity for conducting the excitation is lost. Since the nerve, unlike the muscle, has no death-rigor, we cannot say just when it is wholly dead.

During the stages of dying, nerves exhibit two interesting changes of excitability. Immediately after it is severed from the body the irritability of the nerve increases temporarily, and afterward diminishes by successive degrees until it is wholly lost. The course of these changes in its irritability is found to be different for different parts of the same nerve-stretch. It was discorered by Valli and Ritter ${ }^{2}$ that a nerve which has once ceased to stimulate its attached muscle to contract will again excite muscular contraction if the electrodes be applied farther down its stretch; therefore the lower portion of the nerve-stretch seems to preserve a given degree of vitality for the longest time. From this fact "Valli's principle" has been derived: Nerves die from the centre to the periphery. The temporary increase of the irritability of the excised nerve belongs indeed to its entire stretch ; but it appears first in the upper part. This fact is connected with the important influence which the cross-section of a nerve has upon its electrical and neural condition. As to the reason for this increase of nervous excitability which accompanies the first stage in the dying of the nerve, we are quite in the dark.

§6. Closely allied to the foregoing changes are those which take place in the structure and functional activity of a nerve that remains in the living animal organism after having been separated from the central organs. Such a nerve, after a time, completely loses its irritability. Two investigators, Günther and Schön, ${ }^{2}$ found this time to be, in the case of rabbits or dogs, about three or four days; in a cold-blooded animal like the frog, the time may be prolonged to a week, or even more. The law of increased irritability, produced in the entire nerve-stretch, but first manifested in the portion nearest the cross-section, immediately after separation from the central organ, holds good for most observations on nerves cut in situ; its application is obvious, however, only to the case of the motor nerves. In 1850 Waller announced ${ }^{3}$ the discovery that the anatomical changes (a fatty or granular degeneration) which take place in the nerve-fibres after being severed from the central organs proceed from the place of section to the extreme peripheral portion

${ }^{1}$ See in du Bois-Reymond's Untersuchungen, etc., i., pp. $321 \mathrm{ff}$.

${ }^{2}$ See the Archiv f. Anat. Physiol., etc., 1840, p. 270.

'In Philosophical Transactions, 1850, ii., p. 423; and see, also, Archiv f. Anat. u. Physiol., 1852, p. 392. 
of the fibre; and that the sensory nerves do not degenerate in their peripheral, but in their central portion, when the posterior roots are cut above the ganglion. The central portion of the nerve, when cut at a point lying toward the periphery from the ganglion, may be shown (in the case of the sensory nerves, which alone admit of being experimented upon for this purpose) to retain its irritability for a long time, although it finally loses it through lack of exercise. A cut nerve remaining in situ may be regenerated, and so regain its functional powers. Regeneration takes place by the axis-cylinders growing out from the central portion and running into and between the sheaths of Schwann of the peripheral portion; it is accomplished, then, by the influence of the central organs. The irritability of the nerve returns as its structure is regenerated. According to some investigators its conductivity is regained earlier than its power of local irritability. Duchenne 'and others claim that the influence of the will is the first form of stimulus to regain control of regenerated motor fibres.

§. Oxygen, as furnished by the circulation of the arterial blood, is the second condition for the performance by the nerves of their distinctive functions. But nerves, as compared with the central organs or end-organs of the nerrous system, or eveu with the muscles, are relatively independent of the presence of oxygen. Indeed, since the muscle is so much more sensitive to changes in the quality of the blood, and is supplied by the same arteries that supply the attached nerres; and since the irritability of the nerve is tested by the vital contraction of the muscle-it is difficult to determine by experiment the exact effect upon the nerves of withdrawing from them the oxygen of the blood. The irritability of the nerves continues about as long in a moist racuum, or in indifferent gases, as in the air. What little is known of the chemical processes which take place in the nerves confirms the view that they are relatively independent of the presence of oxygen; and the experi. ments of Severini, who thinks that he has discovered a restorative effect of ozone (if not of ordinary oxygen) upon these organs when dying, are not yet fully confirmed. It may be argued, however, from the marked dependence of the other forms of nervous tissue upon a supply of arterial blood, as well as from the general theory of the nerrous system, that the presence of some oxygen is a necessary condition of the functional activity of the nerves.

§. Exhaustion is a condition of the nerves recovery from which is necessary in order that they may exercise their normal functions ; but exhaustion of the nerves is difficult to distinguish experimen-

${ }^{1}$ Traité de l'électrisation localisée, second edition. Paris, 1861. 
tally from exhaustion of the central organs or of the end-organs. The experiments of du Bois-Reymond upon the negative variation of the nerve-current under repeated irritation give us the first item of the desired proof. The variation under these circumstances becomes constantly weaker. By ingeniously separating the proofs of exhaustion in the muscle from those of exhaustion in the nerve, Bernstein ${ }^{1}$ has shown that the latter comes on much more slowly than the former; and that by far the greater amount of the effects attributed to exhaustion in the nerve-muscle mac'sine belong to the muscle-element of this machine. When tired, however, the nerve recovers more slowly than the muscle. Nervecells-and therefore the central organs and end-organs of the nervous mechanism-tire much more easily and quickly than nerrefibres. Indeed, according to Hermann, ${ }^{2}$ it is conceivable that all the phenomena of exhaustion which take place in the normal experience of the nervous system belong really to the organs connected with the nerves rather than to the nerves themselres. When we are tired nervously, it is not ordinarily the nerres that are tired. And yet the law of the exhaustion and recorery of functional activity doubtless belongs to normal, as it does to excised, nerve-fibres.

$\$ 9$. The various classes of phenomena which are eroked in connection with the starting and propagation of nerre-commotion along a nerve-stretch will be consiclered from two points of riew : First, as regards their dependence upon the character, amount, and method of the application of the stimuli which are used; and, second, as indicative of certain processes-chemical, thermic, electrical, etc. - set up in the nerves themselves. We shall thus, as far as possible, avoid repetition.

$\S 10$. The mechanical properties of the nerves are of little interest to psycho-physical researches; and comparatively little concerning their physiological functions has been learned by the application to them of mechanical stimuli. The elasticity of nerves in the dead body was found by Wertheim to follow the same laws as that of the muscle-their absolute ductility is less than that of muscle; their cohesion greater. All kinds of mechanical attacks on the nerves excite them, and are followed by pain in the case of sensory nerves, contraction of the muscles in the case of motor nerres. By rapid shocks of this kind-for example, with a toothed wheel or a hammer-tetanus may be produced. A certain suddenness of influence is, in general, necessary to the effect. Yet Fontana succeeded in cutting nerves very quickly with a sharp linife without producing any muscular contraction. Pressure of a nerve

'In Pflüger's Archiv, xv., p. 289 f. $\quad$ 'Handb. d. Physiol., II., i., p. 135. 
may be increased very gradually to a high degree without exciting it; but its power of conductivity is thus temporarily suspended. Very moderate pressure or slight traction of the nerve lias been found by several investigators to increase, at least for a moment, the irritability of the nerve ; and perhaps, also, the speed of conduction in it. All neural function is, of course, destroyed by any considerable mechanical injury of the nerve, such as often happens by stricture or pressure from a swelling.

$\$ 11$. Thermic influences upon the phenomena of the neural process are very marked and important. On the other hand, almost nothing is known as to the specific heat of nerres or as to their power to conduct heat. Hermann thinks it probable that the latter is different in the two main directions of the fibres. The results of experiment differ as to the degree of heat which is necessary to act upon the nerres as a stimulus. Valentin, the first observer in this line, found that dipping the motor nerves of frogs in water heated to about $100^{\circ}$ Fahr. $\left(38^{\circ} \mathrm{C}\right.$. $)$ caused contractions; but Eckhard obtained such results only from temperatures above $150.8^{\circ}$ to $154.4^{\circ}$, or below $25^{\circ}$ to $22^{\circ}$-that is, temperatures that are either deadly or permanently injurious to the nerve. Nor, according to the latter, is the nerve excited by changes in temperature as it is by changes in the electrical current. Slighter changes near the dead-line may have an effect to excite the nerve; but considerable changes in the medium temperatures, as a rule, have no such effect. It is the opinion of some, however, that such thermic changes, when marked and sudden, may act as a stimulus to motor nerves. It was shown by $\mathrm{E}$. $\mathrm{H}$. Weber ${ }^{1}$ that heat and cold have no effect in producing sensations when applied directly to the sensory nerre-trunks of man.

While there is little evidence, then, to show the direct excitatory effect of heat upon the nerves, there is no doubt whatever as to the importance of thermic influences upon their excitability and conductivity. High degrees of temperature may destroy the power of the nerve to perform its functions, but without killing it. Warmth increases the immediate expenditure of energy in an excised nerve, and so hastens its death; cold delays this expenditure, and so conserves the nerve. The limit of this increased irritability of the nerve under the influence of heat is reached at about $122^{\circ}$ Fahr. ; as the degree of heat applied rises from this point toward $150^{\circ}$, its effect is rapidly felt in causing the death of the nerve. Sudden cooling from about $68^{\circ}$ down to $50^{\circ}$ may produce a tem-

'In Wagner's Handwörterb. d. Physiol., III., ii., pp. 496, 578; and Archiv f. Anat., Plysiol., etc., 1847 , p. 342,1849, p. 273. 
porary rise of irritability; but, in general, cooling below $59^{\circ}$ diminishes the irritability of nerres. The effect of temperature upon the speed of conduction will be referred to elsewhere.

\&12. Chemical influences have, for the most part, surprisingly little effect upon the irritability and conductivity of the nerves, especially in view of their great sensitiveness to other external influences. Such indifference is probably due to the protection of the nerve by its membranes. The effect of most chemical agents, when long continued, is to destroy the nerve without irritating it; but some agents in a concentrated form act upon it as stimuli. The researches of Eckhard, Kölliker, and Kühne have given us most of the information we have upon this matter. Only two points need mention here. First: Changes of the amount of water in the substance of the nerve affect its functional activity. Drying the nerve produces contractions ending in tetanus; although, according to some authorities, these effects do not follow if the drying be very sudden. A slight amount of drying raises temporarily the irritability of the nerve. The amount of the decrease of water necessary to produce contractions in the attached muscle is given by Birkner at four to eight per cent. of the weight of the nerve; irritability ceases, although the dried nerre is not dead, with a loss of forty per cent. Others, however, give the latter figure as between eight per cent. and nineteen per cent. Swelling the nerve in water or other indifferent fluids decreases its irritability slowly to the point of entire cessation.

Second: The effect of certain acid and alkaline solutions upon the nerve is much like that of drying it. Various neutral salt solutions, and free alkalies in solution, produce strong muscular contractions, ending in tetanus and death. Certain organic substances in concentrated solutions-for example, urea, sugar, and glycerine-irritate the nerve ; so, according to most observers, does alcohol of from ninety per cent. to eighty per cent. The law seems to be, that all chemical stimulation of the nerres is closely connected with the destruction of the nerrous tissue.

\$13. The phenomena eroked by applying the stimulus of electricity to the nerve-muscle machine are very numerous and difficult of disentanglement, since they depend upon such a variety of changing conditions. Following is a very brief statement of some of the more important of such phenomena, in as far as they relate to the direct excitatory effect of this stimulus, and also to its effect in modifying the excitability of the nerve. ${ }^{1}$

\footnotetext{
${ }^{1}$ Here, as throughout the subject of the general physiology of the nerves, the chief reliance has been placed upon Hermann, Handb. d. Physiol., II., i.
} 
The resistance which living nerves offer to the electrical current does not differ much from that of living muscle; it is given by most authorities as somewhat greater. According to Weber's investigation its resistance is about 50,000,000 times as great as that of copper wire. According to Harless, the conductivity of the nerve is on the averige about 14.86 times that of distilled water. Hermann found the conductivity to be much greater in the longitudinal than in the transverse direction of the nerve.

As to the direct excitatory effect upon the nerve of constant currents and of their variations, the main principle is that formulated by du Bois-Reymond in 1845.' This principle may be stated as follows: The excitatory effect of the constant current, as judged by the contraction-curve of the muscle, does not correspond to the absolute value of the intensity of the current at each moment, but to the change in this value from one moment to another; and the effect is greater the less the time in which changes of the same magnitude in the current occur, or the greater their magnitude in the same length of time. The essential fact is that constant currents, while they remain constant, do not irritate the nerve; variations in such currents do irritate it. The variation may be either from zero or to zero (the making or the breaking of the current), but it must have a certain degree of suddenness to be of any effect. Hence induction-shocks are, relatively to their actual strength, much more effective than the constant current in exciting the nerve. Great difficulties, however, stand in the way of stating definitely the relations that exist between variations in the strength of the constant current and changes in the excitation of the nerve produced by these variations; Hermann, indeed, pronounces the difficulties "insuperable."

It is not absolutely certain that the constant current itself, apart from variations in its strength, has any excitatory effect upon the sensory nerves. The sensory effects produced by such a current,for example, pain in the skin, roaring in the ears, sensations of light and color, electrical taste, giddiness (as when the current is passed transversely through the head at the mastoid processes), etc. - are due to the end-organs and the central organs. It is perhaps probable that such a current itself may produce tetanus in certain nerres; but the effect is very small compared with that produced by variations of this current. Pflüger found tetanus pro-

'In a paper communicated to the Physiological Society in Berlin, August 8th, of that year; see, also, his Untersuchungen über thierische Electricität, I., p. 258 . 
duced by weak currents of about the order of the so-called musclecurrent; but not by strong ones.

$\S 14$. The excitatory effect of the constant current is dependent upon its direction. If three grades of strength are assigned to all such currents-namely, weak, medium, and strong-the results of all the experimenters will be found to agree as to the dependence of the effect of medium and strong currents upon their direction; as to the case of weak currents, authorities differ. The following table, given by Pfluger, ${ }^{1}$ states the conclusion agreed to by the larger number of observers:

\begin{tabular}{|c|c|c|c|c|}
\hline \multirow{2}{*}{ Strength of Current. } & \multicolumn{2}{|c|}{ Ascending Current. } & \multicolumn{2}{|c|}{ Descending Current. } \\
\hline & Making. & Breaking. & Making. & Breaking. \\
\hline 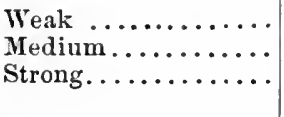 & $\begin{array}{l}\text { Contraction. } \\
\text { Contraction. } \\
\text { Rest. }\end{array}$ & $\begin{array}{l}\text { Rest. } \\
\text { Contraction. } \\
\text { Contraction. }\end{array}$ & $\begin{array}{l}\text { Contraction. } \\
\text { Contraction. } \\
\text { Contraction. }\end{array}$ & $\begin{array}{l}\text { Rest. } \\
\text { Contraction. } \\
\text { Rest or weak } \\
\text { contraction. }\end{array}$ \\
\hline
\end{tabular}

The results here tabulated are obtained by experimenting with the excised motor nerves of frogs. In experiments with the sensory nerves, or with any of the nerves while remaining in the living animal, the conditions become so complicated that satisfactory results in confirmation of Pfluger's conclusions have not yet been reached.

§ 15. The excitatory effect of the constant current is also dependent upon its absolute strength. Du Bois-Reymond, after discovering his law, proceeded to raise the inquiry, whether the height of the current upon which the variation is piled up, as it were, has any influence upon its effect. Various attempts to answer the inquiry have been made ; but the discovery of Pflüger's law of electrotonus has, according to Hermann, ${ }^{2}$ changed the form of the question to the following: What influence upon the excitatory effect of increasing catalectrotonus and diminishing anelectrotonus does the absolute amount of existing electrotonus have? In this form it will be referred to again.

$\S 16$. The excitatory effect of the electrical current is influenced by the length of the nerve-stretch through which it flows. From the beginning of electro-physiology the opinion has prevailed that the excitatory effect is increased by the length of the nerve-stretch. This view accords theoretically with deductions from Pfliger's law of electrotonus. The experimental proof, however, is somewhat vacillating; in part, doubtless, on account of the admixture of 'See Untersuchungen über die Physiologie d. Electrotonus, p. 453. Ber-
'in, 1859. 
different local conditions where different considerable lengths of a nerve are passed through. Different investigators have found the increase of irritability in the nerve, as dependent upon its length, confined within different limits; one has fixed the limit at from $\frac{1}{12}$ to $\frac{1}{6}$ inch, another at from $\frac{1}{4}$ to $\frac{2}{5}$ inch. Willy found the rule, in general, to hold good only for descending currents.

$\S 17$. The excitatory effect of a constant current is influenced by the angle between the axis of the nerve and the direction of the current. After considerable experimentation, with varying results, the more modern researches have, according to careful experiments made by Albrecht and A. Meyer, in the laboratory of Hermann, ${ }^{2}$ confirmed the opinion of Galvani: The electrical current does not excite the nerve when it flows precisely at right angles to the nerve's axis.

$\S 18$. The duration of the current also influences its effect as a stimulus.

Attention has already been called to the exhausting effects of long-continued stimulation of the nerve, whether by electricity or otherwise. But can a shock be so brief as not to stimulate the nerve at all? The reason why very brief currents, on breaking the circuit, are not followed by a contraction of the muscle is obviously to be found in the fact that the condition of anelectrotonus, on which the breaking contraction depends, has not had time to develop itself. But J. König, working under Helmholtz's direction, found that currents which would produce the making but not the breaking contraction, provided they had sufficient duration, produced no contractions at all if they lasted only 0.001 of a second. On increasing the duration of the current the strength of the contractions increased also, until at $0.017-0.018$ of a second they reached the same height as that of the contraction produced by the corresponding constant current. It may be said, then, that the electrical current must act upon a nerve for at least about $0.001 \frac{1}{2}$ of a second in order to excite it. The nerve on being cooled becomes more sluggish in its response to the stimulus; at the freezing-point it requires a duration of nearly 0.02 of a second for the stimulus to start it into action.

$\S 19$. Besides the direct excitatory effect upon the nerve of electrical currents, we have to consider their effect in modifying the action of the nerve under stimuli, whether electrical or of some other kind. If a nerve-stretch is under the influence of a constant current which is being passed through it, the effect of stimuli, when applied to any part of the nerve and judged by sensation or

${ }^{1}$ See his Handb. d. Physiol., II., i., p. 81 f. 
muscular contraction, is increased. This changed condition of the nerve with respect to its excitability, which the electrical current produces, is called "Electrotonus." The term was introduced into plyysiology by du Bois-Reymond, who was preceded in his investigations by Ritter, Nobili, and Matteucci, and followed by Valentin, Eckhard, and others. It is Pflüger, however, who is entitled to have his name permanently attached to the law of electrotonus; for it is he who most thoroughly analyzed the facts, separated the variables from the constants, and gave scientific form to the result. It is found that the modified excitability of the electrotonized nerve (that is, of the nerve which has been thrown by the passage of the electrical current into this modified condition of excitability) is not uniform through its entire stretch, but is greatest in the immediate region where the electrodes are applied. Noreover, it differs at the two electrodes-the condition at the anode (or positive pole) from that at the cathode (or negative pole). It differs, also, for that part of the stretch which lies between the electrodes as compared with that which is outside of the electrodes. Pflüger's law states the whole case as follows: The excitability of a nerve under the action of the constant current is increased in the catelectrotonized region (that is, on both sides of the negative electrode), and diminished in the anelectrotonized region (that is, on both sides of the positive electrode). This law is declared by Hermann ' to hold good of all kinds of stimulus, and in all eases-with the only apparent exception of the suprapolar region of an ascending current.

This electrotonic effect of the constant current, like its direct excitatory effect, is influenced by the strength of the current, by its making and breaking, and by the length of the stretch through which it flows. The change in the excitability of the electrotonized nerve increases with the strength of the current, from the lowest observable point until it soon reaches a maximum; after this maximum is reached, further increase of electrotonus is to be recognized only by the expanding of this condition over the extrapolar parts of the nerve-stretch. Electrotonus increases also with the length of the nerve-stretch affected; but this relation also finally reaches a maximum. Electrotonic changes in the catelectrotonic region occur immediately upon making the current; they then speedily but slightly increase, and more slowly diminish again. The anelectrotonic condition develops and extends itself comparatively slowly, reaches a maximum, and then gradually falls off again. The immediate consequence of breaking the current is to increase the electrotonic condition of the nerve in the anelectrotonic

${ }^{1}$ Handb. d. Physiol., II., i., p. 43. 
region, and very briefly to decrease it in the catelectrotonic region; the former increase gradually vanishes; the latter decrease is followed, after a few seconds, by an increase which lasts from onehalf a minute to fifteen minutes.

The so-called "laws of electrotonus" are almost wholly based upon experiments with the motor nerves of frogs. Great and even insuperable difficulties stand in the way of proving experimentally its application to sensory nerves, or to the nerves of living and selfconscious man. The conditions of influence-from the central organs and end-organs, from sensation and will-upon the nerves in such cases are so complicated as to baffle all attempts to analyze them by means of direct experimentation.

Further consideration of electrotonus, and of its bearing upon a mechanical theory of the nerves, must be for the present postponed.

$\$ 20$. The phenomena evoked in connection with the starting and propagating of nerve-commotion along a nerve-stretch may be presented-in the second place (see $\S 9$ )-as indicative of certain Processes set up within the nerves themselves. That the effect of a constant current is not exhausted in direct excitation of the nerve is proved by the changed condition of excitability which it also produces.

No mechanical process that can be made directly appreciable by the senses or accurately measured by mechanical means, like that which takes place in the contracting muscle, occurs in the nerve when excited to its physiological activity by means of appropriate stimuli. Whatever changes then take place in it are invisible and impalpable.

$\S 21$. Nor are we much better able, on the ground of experimental tests, to affirm the existence of any thermic process in connection with the excitation of the nerves. If any rise of temperature in the nerve is caused by the application of stimulus, it is exceedingly small. Helmholz, ${ }^{1}$ in connection with his investigations into the heating of the muscle when in a state of tetanus, could detect no development of heat in the nerve, although his means would have revealed a change of only a few thousandths of a degree. On the other hand, Schiff and Heidenhain both detected a rise of temperature in the brain due to nervous excitation. But it is still a question how far this fact indicates anything more than change in the distribution of the arterial blood. Moreover, the former of the two observers failed to obtain any evidence of heating in the cerebellum by sensory excitation. The ease of the ${ }^{1}$ Archiv f. Anat., Physiol., etc., 1848, p. 158. 
conducting nerve-cords and that of the cellular tissue of the central organs may very likely be different in this regard.

$\$ 22$. Nor have any chemical processes been indubitably proved to occur in the nerves as an accompaniment or result of the exercise of their physiological function. The only experimental evidence of such a process is the change of reaction which some observers have found. Funke and others have asserted that, not only a certain time after death, but also after exertion as caused by cramping produced with strychnine-poisoning, the nerves show an acid reaction. But Heidenhain and other observers contest this alleged fact. Other assertions of chemical changes set up in the nerves by exciting them are even more uncertain. Ranke's theory of a "respiration of the nerves" is quite without any sufficient experimental proof; and so is his claim that an absorption of the water of the nervous tissue results from tetanus. If any chemical changes are produced in the nerve by exciting it, they are like the thermic-exceedingly small. This fact is proved by the almost complete independence of the nerve with respect to the oxygen of the arterial blood, and by the absence of any observable changes in its temperature when functionally active. But here again we must distinguish between the case of the nerves as conductors and that of the nervous tissue of the central organs.

$\S 23$. Evidence of the electrical process in nerves functionally active is not wanting. It was not, however, until the discovery of du Bois-Reymond, announced in 1843, that any experimental evidence had been obtained to show the existence of electrical currents in the nerves, although it had previously been conjectured that the distinctively neural process is a phase of electricity. This experimenter found that in the case of the nerve, as in that of the muscle, the cross-section artificially made is negative toward the longitudinal surface of the nerve-stretch. Weak longitudinal currents also show themselves between the two cross-sections of a nerre-stretch thus prepared. The current outside the nerve-stretch may be considered as completed by a current in the nerve-stretch from its cut end to the equator. This current (called "natural nerve-current," or "current of rest") is the same in the sensory, the motor, and the mixed nerves of the same animal ; but its electro-motive force is greater the larger and thicker the nerve. Its absolute strength in the sciatic nerve of the frog is given by du Bois-Reymond as 0.022 of a Daniell's cell, but by Engelmann as 0.046. It gradually becomes extinct in the nerves of the dead body, but it continues for some time after their irritability is lost.

The same discoverer, du Bois-Reymond, found that the current 
of rest is diminished in energy by tetanizing the nerve-stretch with an electrical current. That is, if when one of the electrodes is placed at the equator, and the other at the cut end of a nerve-stretch, the needle of the galvanometer inclicates the passage of a so-called current of rest, and then the muscle to which the nerve is attached be tetanized by passing an interrupted current through the nerve, the needle will swing back toward zero. This variation is called the "negative variation" of the nerve-current. It may be produced by chemical and mechanical, as well as by electrical, stimulation; and when the nerre is no longer irritable the negative variation sinks to zero. It shows, therefore, that the electro-motive force of the nerve is diminished by the nerve being excited; and the degree of the negative variation is a measure of this diminution, although it does not wholly nullify the so-called current of rest. The negative variation of the electrical current in the nerve is closely connected with the nerve-commotion which is started and conducted in the nerre. Since the excitation of the nerve is known to be progressive, or of a wave-like character, the nature of this connection, according to Hermann, may be more definitely stated as follows: The electrical condition of each excited place in a nervestretch is negative toward all the places of the same nerve-fibre that are unexcited. Hence, between any two points in a nervefibre, while the nerve-commotion is passing over the distance, two phases of the current of action occur; the first phase is in the same direction as the course of the wave of excitation, the second is in the opposite direction.

$\S 24$. The Laws which are known to govern the starting and propagation of nerve-commotion along the nerves as conductors are few in number; they deal chiefly with relations between the magnitude of the stimulus and the amount of the resulting impulses, and with the conditions for, and speed of, the unbroken propagation of these impulses.

The relations which exist between the magnitude of the stimulus applied to the nerves in their normal condition and the amount of resulting nerrous impulse cannot be given with accuracy. For, in the first place, there is no absolute measure for either of the two values which it is desired to compare. Of the various stimuli which act upon the nerve-fibres, electricity is the only one that admits of even a fairly approximate measurement as an excitant of these fibres; and the excitatory effect of electricity does not vary in direct proportion to the strength of the current, but in proportion to the changes in its strength. With reference to attaining a direct measurement of the amount of the process set up in the 
nerve by the stimulus, we seem to be in a yet more helpless condition. The effect of this process is almost wholly manifested in the organs with which the nerve is connected rather than in the nerve itself. There is evidence in the case of the nerres, however, as in that of the muscles, that their excitation consists in the setting free, by the stimulus, of potential energy due to the molecular constitution of the nerve itself. But the exact nature of this energy, and of its mathematical relations, both to the stimulus and to the resulting energy called forth in the organs connected with the nerve, we shall probably never discover. Still further; as Grützner ${ }^{2}$ and others have shown, the same kind and clegree of stimulus produces different effects when applied to different nerves.

$\S 25$. Allowing for the uncertain factors, however, some approximate statement may be ventured as to the relation between the magnitude of the stimulus and that of the resulting nerre-commotion. Measuring the amount of the process in the nerve by the resulting contraction in the muscle, Hermann ${ }^{2}$ found that this amount increases, at first rapidly and then more slowly, with the increase of the stimulus. According to Fick, ${ }^{3}$ the height to which the lever is raised by the contractions of the muscle raries, within certain limits, in direct proportion to the amount of the stimulus. The last-mentioned observer also noted two remarkable phenomena: (1) On increasing the amount of the stimulus beyond the point necessary to produce the first maximum contraction, another stage is reached in which the effect further increases, in proportion to the stimulus, until a second maximum is gained. (2) In some circumstances, after reaching the first maximum, the effect diminishes with the increase of the stimulus, then rises on further increase, until it attains a second maximum.

The effect of several excitations may be supposed to pass along the nerre as separate waves of nerve-commotion; but in order to keep the waves separate the interval between them must be more than about $\frac{1}{10 \overline{0}}$ of a second (the fraction differing for different nerres, different animals, etc.), otherwise they fuse in the muscle and tetanus results. The combined effects of stimulations having the requisite interval may be piled up, or summed up, in the nerve, and be seen in superimposed contractions of the muscle. Two simultaneous excitations of the same place of the nerve-stretch are thus "summed up" as long as the maximum of excitation is not reached; the two are, in fact, one. If the cathodes of the two

\footnotetext{
' Pflüger's Archiv, xvii., pp. 215 ff.; and xxv., pp. $255 \mathrm{ff}$.

${ }^{2}$ See Archiv f. Anat. u. Physiol., 1861, p. 392.

${ }^{3}$ Untersuchungen über electrische Nervenreizung. Braunschweig, 1864.
} 
exciting currents unite, the same effect takes place. A similar result may be gained by combining the effects of two different kinds of stimuli-as, for example, electricity and the drying off of the nerve.

$\S 26$. In a rough way the specific excitability also of different nerves, or of different localities in the same nerve, may be discovered. Harless found that the excitability of the nerves of the frog is twenty-two times as great in winter as in summer. In the cut nerve it is greater near the artificial cross-section. Many observers have contended that the excitability of the normal nerve diminishes toward its peripheral portion. Matteucci investigated the subject of local differences of excitability in the sensory nerves; more recently Rutherford ${ }^{1}$ discovered that the reflex effects of stimulating a sensory nerve are greater the nearer the central - organ the stimulus is applied. Finally, Helmholtz ${ }^{2}$ and Hermann ${ }^{3}$ - observed that the lower part of the nerve-stretch is more excitable under the action of an ascending, the upper under that of a descending induction-current.

$\S 27$. The Speed with which the process of conduction takes place in the nerves has been determined with considerable accuracy, under a variety of circumstances; this, notwithstanding the fact that the physiologist Joh. Müller ${ }^{4}$ declared it to be forever impossible no longer ago than 1844 . In only 1850, however, Helmholtz ${ }^{5}$ announced that he had succeeded in measuring the speed of nervous impulses in the motor nerves of the frog. The rate he found to be 26.4 meters, or about 86.6 feet, per second. Another series of investigations, in which the pendulum-myograph was used, gave a result about 3 feet larger $(27.25 \mathrm{~m}$.). Subsequent investigators have substantially confirmed the figures of Helmholtz. Bernstein, by a still different method of measurement, found that the speed of conduction in the nerves varies between 25 and 33 meters. In the motor nerves of man the number was still later fixed by Helmholtz and Baxt at 33.9 meters, or about 111 feet, per second. Von Wittich found it to be about 98.5 feet per second. The complexity of the elements which enter into the measurement of the speed of nervous impulses in the sensory nerves makes it nearly impossible to obtain satisfactory results by experiment. And so far as the calculations take into account changes produced in the

${ }^{1}$ See Journal of Anat. and Physiol., 1871, v., pp. $329 \mathrm{ff}$.

${ }^{2}$ Archiv f. Anat. u. Physiol., 1850, p. 337.

${ }^{3}$ Pflüger's Archiv, viii., p. 261; and xvi., p. 262.

'See his Handbuch der Physiologie, i., pp. 581 ff. Coblenz, 1844.

See Archiv f. Anat. u. Physiol., 1850, pp. 276-364. 
nervous centres with accompanying phenomena of sensation and attention, their discussion belongs elsewhere. Of the four factors that enter into the entire time ("reaction-time") which elapses between the application of stimulus to a sensory nerve and the resulting contraction of the muscle-namely, (1) time of conduction in the sensory nerve ; (2) processes in the central organs ; (3) time of conduction in the motor nerve; and (4) latent period of the muscle -it is difficult to disentangle the factor (No. (1)) required by the attempt at analysis. Hirsch, by experimenting with stimuli applied to the skin at different distances from the brain, found the speed of conduction in the sensory nerves of man to be about 111.5 feet per second-a result in exceedingly close agreement with the figure obtained by Helmholtz for the motor nerves. Schelske used another method of measurement; by applying the stimulus to the groin and the foot, and recording the difference of time in the two classes of cases, he obtained results varying between 25.294 and 32.608 meters per second. Others have given figures differing more or less widely from those just stated. The general conclusions, however, favor numbers lying between 98 and 131 feet per second as giving the speed of conduction in the sensory nerves of man.

The speed of conduction in all nerves depends upon several varying conditions, such as their temperature, the strength of the stimulus, length of the nerve-stretch, and its electrical condition. Experiments in winter give different results from those in summer. In the motor nerves of man the rate can be made, by changes of temperature, to vary from about 98 feet to 295 feet per second. It has been disputed by different observers whether the speed of conduction is dependent in any degree upon the strength of the stimulus; and even Hermann considers the question undecided. But Vintschgau ${ }^{1}$ has recently shown, as the result of a large number of carefully conducted experiments, that as soon as the stimulus rises above a certain limit of intensity, the speed of the nervous impulses increases with the increase of the intensity of the stimulus. This limit depends, however, upon the direction of the current, upon whether it is a making or a breaking current, upon the animal chosen for experiment, etc. Whether the speed of the nervous im. pulses is directly dependent upon the length of the nerve-stretch is scarcely decided beyond doubt. The effort of the science, general "nerve-physiology," is directed toward showing how these variations in speed, as experimentally determined, may be explained from the laws of Electrotonus.

${ }^{2}$ In Pflüger's Archiv, 1883, xxx., pp. 17 ff. 
$\S 28$. Finally, it should be remembered that the fact of any propagation of nervous impulses whatever presupposes the continuity, integrity, and isolation of the nerve tract along which the impulses move. The slightest separation of the substance of the nerre by cross-section, even when the cut ends are left in the closest mechanical contact, destroys the unity of the nerve's physiological function. The ancients knew that tying the nerve prevented its action; they explained the fact by saying that the flow of nervous fluid was thus hindered. So also does the fineness of the localization which belongs to the organs of motion, but especially to those of sense, as well as the fact that partial section of a nerve only lames part of the field cared for by that nerre, indicate the physiological isolation of the nerve-fibre during its course between end-organs and central organs. Since the result of stimulating a given nerve is in quality invariably the same, it would seem that the law of the "specific energy" of each nervous element (to which we shall refer elsewhere) is connected with the assumptions necessary to explain the phenomena attendant upon the starting and propagating of nervous impulses in the conducting nerves.

$\S 29$. Inasmuch as the central organs are to a large extent composed of nerves, a complete account of the nerves as conductors should include a description of the nature of that nerve-commotion which is propagated from point to point along the nervous elements within these organs, and of the paths or tracts along which it passes. But unfortunately our knowledge upon these matters is exceedingly scanty and uncertain. This is in part due to the fact that the influence of the ganglion-cells, with which the nerve-fibres are mixed to form the central organs, profoundly modifies the neural processes of excitation and conduction. The subject belongs, then, to a consideration of the functions of the central organs rather than of the nerves alone. Certain statements, however, may most fitly be given in this connection.

When speaking of conduction in the spinal cord or brain we are not to think of a nerve-commotion as always moving along one fixed path, after the analogy of the far simpler case of the nerve in the nerve-muscle machine. It is true that the nerve-fibre in its normal place in the body runs insulated, as it were, between the spinal cord and the end-organ at the periphery. But the spinal cord itself does not act as a perfectly isomorphic medium. The very complex structure of this organ, in which nerve-fibres and nerre-cells are intricately interwoven, has already shown us that it is not adapted to act as such a medium. The case of the brain is 
even clearer. It accords, therefore, with the structure of all central organs, that we should find the speed of conduction slower in them than in the peripheral nerves. Exner ${ }^{1}$ calculated, from the delay which sensory impulses experience in the cord of man, that their speed there is not more than about $26 \frac{1}{4}$ feet ( 8 meters) per second. The speed of the motor impulses in the cord he gives doubtfully as varying between 36 feet and 49 feet (11 to 12 and 14 to 15 meters). These numbers are substantially confirmed by the conclusions of Burckhardt ( 8 to 14 meters). The latter also maintains that the speed of the impulses which occasion sensations of touch is greater than that of those which occasion pain (as 27 to 50 meters compared with 8 to 14). It has also been observed that, in some cases of persons with disease of the posterior strands of the spinal cord, sensations of pain arise in consciousness notably later than those of touch. But the interpretation of all these phenomena is complicated with questions of the cerebral functions; for sensations of pain are pre-eminently of cerebral origin. Moreover, we can have but meagre confidence in our ability to tell with any precision the length of the paths by which nervous impulses travel in the spinal cord of man. The fact observed by du Bois-Reymond, that the ribrations of the muscle tetanized through the cord are less than would be expected from the number of shocks giren by the stimulus, and the fact discovered by Helmholtz, that muscle when tetanized by an act of will has a uniform tone indicating nineteen vibrations to the second (the rate of vibration into which the muscle is thrown by direct stimulation of the motor nerve, on the contrary, corresponding to the number of shocks), show the profound effect of the central organs over the nerrous impulses. Although, then, the experimental evidence is not perfectly conclusive, it, on the whole, confirms what we should expect from the anatomical structure of the spinal cord, as to the complexity and relative slowness of conduction in this organ.

$\S 30$. Various attempts have been made by experimental physiology to demonstrate the paths of conduction in the spinal cord. The evidence from histology on this difficult subject has already been given (p. $71 \mathrm{f}$.). It is not always easy to make the two lines of evidence coincide. As to one point of experimental physiology, however, no doubt has existed since the "epoch-making discovery" of Sir Charles Bell and Magendie. The sensory fibres enter the spinal cord by the posterior root, the motor fibres by the anterior. The demonstration of this fact is performed by dividing these roots, respectively, and observing the results. When a pos-

${ }^{1}$ Pflüger's Archiv, vii., pp. $632 \mathrm{ff}$; ; and compare Ibid., viii., pp. $532 \mathrm{ff}$. 
terior root is divided all the structures supplied by the same nerve lose their sensibility ; while the muscles supplied by its corresponding anterior root continue to be thrown into action by the will and by reflex stimulation. Moreover, stimulation of the central end of the posterior root thus divided produces sensory effects, but stimulation of its peripheral end produces no motion. When an anterior root is divided, on the contrary, the muscles supplied by its nerves cannot be made to act either by volition or by reflex stimulation; but no sensory paralysis is produced. Moreover, stimulation of the peripheral end of the nerve will now throw the muscles into contraction, but stimulation of the central end will produce no effects. An exception to the exclusively motor effects of the peripheral end occurs in certain cases of so-called "recurrent sensibility;" the sensibility shown in these cases is probably due to the fact that a few sensory fibres from the posterior root, after running a short distance in the mixed nerve, turn back and run upward in the anterior root. The proof is then complete, so far as the direct motor paths to the striated muscles, and the specifically sensory paths which conduct impulses to the cerebral hemispheres, are concerned. According to Sigmund Mayer' it does not necessarily follow, however, that only centripetal impulses are conducted by the posterior, and only centrifugal by the anterior roots.

§31. The general arrangement of the motor paths in that part of the spinal cord, on the same side, where they enter by the anterior roots of the nerves, and of the sensory paths in the posterior part of the cord, is maintained throughout. In man, that is to say, the impulses pass up or down the cord in that region of it at which they leave, or by which they enter with, the anterior or the posterior roots. But histology shows that the two halves of the cord are anatomically connected by the commissures, and that every part of each half is bound with other parts of the same half, both up and down and to and fro. Physiology, too, indicates that the paths of sensory impulse undergo a partial crossing from right to left, and from left to right. For, after complete section of one lateral half of the cord, complete loss of sensibility of either side in that part of the body which is supplied by those nerves of the same side that enter the cord below the place of section does not result. The effects that do result depend upon the animal chosen for experiment, and upon the height at which the section is made. Experiments upon the lower animals seem also to show that in their case a partial crossing of the motor paths takes place in the

'Hermann's Handb. d. Physiol., II., i., p. 217. 
spinal cord ; the evidence from pathology makes it doubtful whether in man any crossing from side to side occurs in the voluntary motor paths, at least below a point very high up in the neck. All the evidence shows that in the lateral columns both sensory and motor paths are to be found.

$\S 32$. In addition to the general statement just made, experimental physiology has little to say confirming or correcting the conclusions of histology (see p. $71 \mathrm{f}$.) as to the paths of neural impulses in the spinal cord of man.' Experiments which attempt to make a section, either of all the fibres in the anterior columns, leaving all the other fibres intact, or of all the other columns, leaving the fibres in the anterior columns intact, can never, indeed, be quite sure of their success. But, on the whole, their results are confirmatory of the statements made in the last article. Some investigators have endeavored to solve the same problem by directly stimulating the fibres of the different columns in such manner as to confine, as far as possible, the influence of the excitatory current (or other stimulus) to certain definitely selected fibres, and so to exclude all reflex action. It is found that no reaction, indicative of any sensory impulses whatever, follows the stimulation of the central ends of the anterior white columns of the spinal cord; but stimulation of the peripheral ends of these same columns may be followed by muscular contraction, sometimes (so Longet and Kürschner found) when mechanical stimuli are used, but oftener with weak electrical currents. Careful cutting of these columns is followed by no signs of pain.

On the other hand, stimulation of the central cut ends of the posterior columns produces signs of pain, and other sensory effects; for this purpose Longet has used electrical, and Eigenbrodt and Schiff mechanical stimulation. According to Schiff and others the entire cord can be cut through from before back to the posterior columns, and if these are left the animal will retain the sense of feeling. As to a further differentiation of the sensory function of these columns, different experimenters do not agree. Some would confine their function to impulses that give rise to sensations of touch, on the ground that animals, the substance of whose cord has been entirely cut through with the exception of the posterior columns, retain their sensations of touch, but loose their susceptibility to pain from impressions made on the surfaces whose nerves enter the cord below the place of section. Impulses which give

${ }^{1}$ Comp. the generalizations of Eckhard in the chapter on "Verlauf d. motorischen u. sensiblen Innervationswege im Rückenmarke," Hermann, Handb. d. Physiol., II., ii., pp. 148 ff. 
rise to sensations of pain must therefore pass elsewhere than by the posterior strands; that is, chiefly by the gray matter of the cord. According to others, however, these strands conduct sensory processes only in so far as they serve for the passage through them of the nerves from the sensory roots ; it is, then, the gray substance of the cord which conducts these processes along upward. In addition to the more marked sensory effects of stimulating the posterior columns, some experimenters get effects which they interpret as showing the presence of motor, and even of voluntary motor, paths in these columns. Stilling, for example, found that voluntary motions occurred after one entire anterior half of the cord had been cut through. But in the absence of proof that no motor paths in the lateral columns were left intact by his experiments, and in view of the fact that a crossing of such paths may take place in some of the animals, the evidence is not conclusive. Moreover, Türck and others have found that the posterior white columns may be entirely cut through without causing motor disturbances.

In the lateral columns of the cord, paths of both motor and sensory impulses are probably to be found. As to the case of motor paths there is, indeed, no reasonable doubt-at least there is no dispute. Ludwig and Woroschiloff found that, in the case of the rabbit, voluntary movements of the hinder extremities took place even after section of the anterior and posterior strands, and of the gray matter of the cord in the cervical region. As to the proofs of sensory paths in the lateral columns, the evidence is somewhat conflicting. Longet and Stilling discovered no proof of their existence ; Schiff pronounces the matter doubtful ; Türck found that unmistakable signs of pain followed the cutting of these portions of the cord. Experiments upon animals and pathological observation, however, on the whole, confirm the view that the sensory are mixed with the motor paths in the lateral columns. As Wundt ${ }^{2}$ expresses the apparent truth-in the side strands of the cord a part of the system of motor fibres is shoved off toward the limits of the posterior columns and surrounded on all sides by branches of the sensory tract.

It must be borne in mind that the function of neither the motor nor the sensory tracts is such that a nerve-commotion, when started in one of the columns, must necessarily run its course by the shortest path in that one column, or else not be propagated at all to its destination. Both histology and physiological experiment indicate that the interlacing of the nerve-fibres, and the interruption of their course with nerre-cells, provide various secondary paths in ${ }^{1}$ Grundzuige d. physiolog. Psychologie, i., p. 101. Leipzig, 1880. 
addition to that which may be called the primary or chief. MIoreover, the gray substance of the cord not only distributes, but also carries forward the nervous impulses. After entire half-section of the cord the sensory tracts of the other half still seem able, in a partially substitutionary way, to accomplish the work normal to both sides. And even in the case of the voluntary motor tracts in man's spinal cord, though such a work of substitution does not take place, we cannot affirm that the paths of voluntary innervation for a definite set of muscles are invariably the same through their entire length. A certain latitude of movement from the straightforward course of the impulse undoubtedly exists even in such a case.

\&33. Difficult as it is for experimental physiology to deal with the tracing of those paths along which the sensory and motor impulses flow in the spinal cord, it is much more so within the nervous mass which fills the cranial cavity. Both the structure and the functions of the cerebrum, as a group of chief central organs, make it nearly impossible experimentally to distinguish between paths of voluntary and paths of merely reflex motion; or even to conjecture where, within its substance, impulses that have been moring along some more clearly defined tract may not divide and, subdivide indefinitely, or-conversely-impulses that enter along: several converging paths be concentrated, as it were, into one or two that are more definitely fixed.

The evidence by which histology has succeeded in tracing certain tracts through the brain, from the medulla oblongata to the convolutions of the cerebral cortex, has been presented at sufficient length in the last chapter (see pp. 76 f., 87 f., and 97 f.). The fuller discussion of the evidence from experimental physiology concerning the same subject will more properly appear in subsequent chapters upon the automatic and reflex functions of the central organs and upon the localization of cerebral function. Certain tracts which pass directly from the crusta through the internal capsule, without entering the basal ganglia, into the frontal and parietal convolutions have already been referred to as probably motor. Others which come from the tegmentum, enter the thalamus and subthalamic region, and emerge after being redistributed to find their way especially to the tempero-sphenoidal and occipital lobes, have been declared, in all probability, to be sensory. With this statement, so far as the motor tracts are concerned, we shall see that the conclusions of experimental physiology accord very well.

$\$ 34$. But our assured kwowledge from experiment concerning the paths by which sensory impulses travel in the brain is exceedingly 
meagre. These paths are probably much more numerous and intricate than those along which the motor impulses are propagated. Moreover, we can seldom draw conclusions with safety concerning the sensations of the lower animals; we therefore largely lose our help from experiment upon them to determine these sensory paths. The phenomena connected with all sensory disturbances are exceedingly complicated, and the conclusions they seem to warrant are often conflicting. For example, the effect of destroying a sensory nerve-tract in the head does not consist simply in the destruction or laming of some one definite function. On the contrary, if a sensory cranial nerve is severed, the various different functions of feeling pain, of pressure, and temperature, and the power of localizing, in the region supplied by the nerve are all lost. But disease of the cerebro-spinal axis may impair one or more of these functions, and leave the others intact, in a given region of the periphery. Anæsthetics also may obliterate the sense of pain while leaving that of contact relatively unimpaired.

Still more difficult of comprehension from the point of view furnished by the general physiology of the nerves are the degrees of tenacity with which different sensory functions, even when administered by the same sensory nerve, are combined. Loss of the sense of temperature and of the muscular sense rarely or never occur separately; but muscular sense not infrequently disappears and the sensitiveness of the skin to pressure is retained. Upon such phenomena we have little clear light to throw. It can simply be said that the distribution of the sensory nerves within the central organs must be enormously complicated, and that we have absolutely no knowledge as to any differences in the kinds, or velocity, or paths, of the nerre-commotions there, that will help us to account for the facts. Yet such differences in the sensations doubtless rest upon differences in the nerve-commotion that causes them, within that inner projection-system of sensory impressions which is furnished by the cortex of the cerebrum.

It has already been seen that the paths of sensory impulses cross orer more or less completely within the spinal cord. They also, like the paths of motor impulses, cross in the region where the nerve-fibres in general decussate-namely, in the pons varolii and medulla oblongata. Experiment and pathology both show that the principal paths of sensory impulses from all the peripheral parts of the trunk of the body and from its mucous membrane lie close to those of the motor impulses in the white nervous substance surrounding the basal ganglia. Effusions of blood in this region not only cause hemiplegia, but also produce more or less impair- 
ment of the different modifications of touch, both in the skin and in the mucous membrane. According to some authorities, lesions in the same region often so impair the muscular sense that the contraction of the muscles which is produced by electrical stimulation is no longer felt. Veyssière and other's suppose that injuries to the white fibrous matter of the crura cerebri, the internal capsule, and the foot of the corona radiata, invariably produce a loss of sensibility on one side of the body; while those which are more definitely confined to the striate body have this effect only imperfectly and for a time-the amount of the effect depending upon the amount of the adjoining white substance which is involved in the injury. This view, like many others on the general subject, is doubtful.

§35. Attempts have been made to localize the paths of sensory impulses in the optic thalami and those of motor impulses in the striate bodies; and in counection with this view it has been held that the former are chiefly concerned in the elaboration of sensory impulses (as sensory ganglionic centres), and the latter in the elaboration of motor impulses (as motor centres). This theory has been wrought out (with much rhetoric and conjecture) by J. Luys. ${ }^{1}$ Luys finds in the optic thalami four centres which-lying in order, one behind the other in an antero-posterior line-conduct and "condense" respectively the olfactory, the visual, the tactual, and the auditory impressions; the corpora striata perform a similar office for the motor impulses. The sensory impressions which come from the periphery, therefore, all run through the optic thalami, according to this theory, in order that they may be "intellectualized" (whaterer that may mean); the motor through the striate bodies, in order that they may be "materialized." It is enough in this connection to say that no such complete distinction of function in the basal ganglia, whether as conductors or as central organs, has yet been made out. It is true, however, that the paths in the crusta and in and surrounding the striate bodies are probably mainly motor, while those in the tegmentum and in and around the optic thalami are mainly sensory. The tendency of the most recent investigation is toward placing more emphasis upon the fibrous nerve-matter surrounding these organs as furnishing paths for the conduction of both kinds of impulses.

\footnotetext{
${ }^{1}$ Recherches anatomiques, physiologiques, et pathologiques sur les Centres Nerveux, 1865; and The Brain and its Functions, New York, 1882. 


\section{CHAPTER IV.}

\section{AUTOMATIC AND REFLEX FUNCTIONS OF THE CENTRAL ORGANS.}

\$1. When a physiological function is occasioned in a peripheral nerve, independently of a so-called act of will, by the stimulation of some other peripheral nerve, this function is said to be "reflex." Such a reflex function of the nerve is regularly brought about, however, by the mediation of a collection of ganglion-cells and interlacing nerve-fibres, known as a central organ. In other words, the secondary stimulation of one peripheral nerve, through a central organ, as a result of a primary stimulation of some other peripheral nerve, is a reflex action of the nervous elements. The entire cerebro-spinal axis is a pile of nervous centres, increasing, on the whole, in complexity of structure and of function from below upward, which, with the nerve-tracts running into and out of it, constitutes a complicated mechanism capable of an indefinite variety of such reflex functions. But the spinal cord and the medulla oblongata are the special seat of many such functions. On the other hand, all excitations of the nervous system which originate in the nervous centres themselves-that is as distinguished from being called out there by the nerve-commotion brought to them through the afferent nerves-are called "automatic." The word automatic must doubtless often be used to conceal our ignorance of the real origin of a neural process. And doubtless, also, many processes which, on first inspection, appear to be automatic, may be discovered, or suspected, to be in reality reflex. But, as far as our information goes at present, not only morements of the muscles through the stimulating of the efferent nerves connected with them, but also the inhibiting of such movements, and the rise of sensations, must be ascribed to the automatic action of the central organs. Changes in the vital conditions to which these organs are subjected by their immediate surroundings, and especially changes in the condition of the blood with which they are supplied, ordinarily constitute the internals timuli to which they respond by exercising their peculiar functions. Automatic activities belong distinctively to the central ganglia of the brain; it is more difficult to 
vindicate their existence in the spinal cord. In general, it is by no means easy confidently to distinguish between the purely reflex and the purely automatic action of particular central organs. The two forms of action are doubtless uniformly blended; so that what is accomplished by any central organ depends both upon its own internal condition and molecular activity at the moment when the sensory impulse reaches it, and also upon the character of that impulse. Inasmuch as it is a vital molecular mechanism connected by an indefinite number of ties with other similar mechanisms, the central organ constantly acts both reflexly and automatically.

§ 2. It follows, therefore, that several kinds of reflex action are theoretically supposable in the nerrous system. When motor nerves are stimulated in a secondary way through a central organ, by applying stimulus to the sensory nerve-endings, the effect may be called reflex-motor. If an excitation of a motor nerve were transferred, without action of the will, to one or more senscry paths, such a conversion of nervous action might be called reflex-sensory. In this way the attempt has been made to explain the feeling of weariness in the muscles when they have been orerexerted, or the feeling which we describe as that of a limb being "asleep." It has also been proposed to speak of "co-motor reflexes," in cases where two motor nerves are assumed to be reciprocally combined in their influence, through a central organ; or of "co-sensory," in cases where the same relation is sustained by two sensory nerves. As an example of the latter, attention has been called to the sensation which is felt in the nose when trying to look at the sun. Examples of the three last classes of alleged reflex functions of the nerrous system are, however, for the most part very doubtful; or they admit of explanation by recognized causes in another way. ${ }^{\prime}$ It is only conceruing the laws of the first class of reflex actions-the reflex-motor or sensory-motor-that we have assured scientific evidence. The reflex function of a central organ may be defined, then, as being (at least in its simplest form) the "conversion" or "reflexion" of a sensory impulse into a motor excitation. We must guard ourselves carefully, however, against the misconception that lurks in these words: the effect of the central organ is never that of merely converting or reflecting a nerve-commotion from one perfectly definite sensory path to an equally definite motor path. No such simple figure of speech will serve to describe its function.

§3. The spinal cord-complex as its structure and functions are-is much the simplest and most accessible for experimental purposes of any of the organs of the cerebro-spinal system; it is

${ }^{1}$ Comp. Eckhard in Hermann's Handb. d. Physiol., II., ii., pp. $23 \mathrm{ff}$. 
pre-eminently the seat of unconscious reflex-motor functions. It is a column or pile of centres, bound together for the reception of sensory impulses by its posterior roots and for redistributing them, as modified by its own molecular structure and condition, through the efferent fibres of the anterior roots. Such is its office as an organ of reflex action in distinction from its office as an organ for conducting neural impulses. We consider, then, in the first place, the Spinal Cord as a Central Organ.

$\S 4$. As the "nerve-muscle machine" is a preparation for testing experimentally the laws of the action of the nerves as conductors, so preparations may be made for testing the laws of the reflex and automatic functions of the spinal cord, by separating that organ from the brain by section below the medulla oblongata. For the purpose of experiment, the "brainless frog" is the most convenient of such preparations and the most fruitful of results.' If the flank of such a frog be lightly touched, the resulting reflex motion will be limited to a slight twitching of the muscles that lie immediately beneath the spot on the skin thus stimulated. If its legs be stretched out and one of them pinched, all the segments of the limb thus irritated will be rapidly flexed in the definite purposeful way necessary to withdraw it from the irritation. If the skin of the region near the anal orifice be piuched, a new combination of muscular contractions will take place and a different form of defensive movements will result: the feet will be drawn up toward the spot irritated and the legs brusquely extended, as though to push away the irritating agent. If the stimulus applied to the skin of one hind leg be increased by forcibly pinching it, the resulting reflex motions may involve the fore leg of the same side, then the hind leg and fore leg of the opposide side, and finally almost all the muscles of the body. Moreover, changes in the character of these reflex motor activities take place which are plainly adapted to provide for changes in the animal's circumstances. For if the right flank of a brainless frog be irritated with a drop of acid, and at the same time the right leg be held (the member which, if unhindered, would be, almost without exception, used in the attempt to remove the irritation), or the right foot cut off, the left' foot may be used for the same purpose of defence.

Phenomena, similar to those obtained in the case of the frog, are obtained from other brainless animals. Thus the decapitated salamander, when the skin of one of its sides is pinched, will bend this side into concave shape in order to withdraw it. Not succeed-

\footnotetext{
'For detailed information see Vulpian, Leçons sur la Physiologie du Système Nerveux, pp. 311-465.
} 
ing in this way, it will make a morement with its foot as though to push away the cause of the irritation. In the case of the higher animals the reflexes of the spinal cord appear, on first inspection, to be comparatively feeble and lacking in purposeful charncter. The mammal, for a relatively long time after the division of the cord from the brain, exhibits only very imperfect reactions in parts of the body supplied by nerves which spring from the cord below the point of its section. But if the animal be kept alive for some time, and even without any physiological union of the severed parts, more strong, varied, and complex movements will follow upon the stimulation of the sensory nerves of those parts. Immediately after the spinal cord of a dog is divided low down in the dorsal region, the hind limbs hang limp and motionless; irritating the skin calls forth only feeble and irregular morements, or none at all. But after some weeks or months have elapsed, reactions resembling those already described in the case of the frog (taking into account, of course, the difference in the structure and normal functions of the two animals) begin to appear. The lind limbs, instead of remaining motionless, will, when the animal is held so that they are pendent, be drawn up and let down again with a kind of regular rhythm, as a result of the constant stimulation of their motor nerves by the sensory nerres, through the spinal cord. Moreover, it is found that the breed, age, sex, and training of the animal determine the character of these brainless reflex movements. That is to say, the spinal cord, as a nervous mechanism, embodies in its very structure and functions all the peculiarities due to these causes. And when its activities are elicited through the stimulus which, arising in many various regions, flows in upon it along the sensory nerve-tracts, or through some stronger but limited impulse occasioned by the application of stimulus to a particular spot on the skin with a definite degree of energy, these activities bear the character both of the stimulation and of the mediating central organ.

\$5. Little need be added to what has already been said (Chap. II., $\S 9$ ), in description of that mechanism of the cord to which the foregoing remarkable functions are referred. Earlier investigators 'assumed the existence of a special system of sensory and motor nerve-fibres, with connecting nerve-cells, designed and appropriated solely for executing these reflex-motor activities. That the motor tracts for reflex movements are to a certain extent distinct in the spinal cord from those devoted to specifically voluntary activities, there seems to be good reason for affirming; but the older

'For example, Marshall Hall, in his New Memoir on the Nerrous System. London, 1843. 
supposition, that there are double tracts,-one connected with conscious and voluntary reaction upon sensation, and one connected with unconscious and involuntary, or merely reflex-motor, reaction,-between the spinal cord and the end-organs of sensation and motion, is almost certainly incorrect. It seems antecedently very improbable that every spot of the skin should be equipped with such a twofold outfit of both kinds of nerve-fibres. No particular nerres which serve merely for reflex-motor functions, and which have no connection either with conscious sensation or with voluntary motion, can be pointed out.

What happens with respect to conscious sensation-the rise of it or its failure to rise-depends rather upon the effect of the stimulus on the end-organ, and upon the condition in which that stimulus finds the central organ on its arrival there. In considering that mechanism of the spinal cord which comes into use when it acts as a central organ in all the reflex-motor activities belonging to it, the office of the ganglion-cells is usually made prominent. And it can be definitely proved that these cells are an important part of the reflex mechanism of the cord. But the extremely delicate network of interlacing nerve-fibres in which the processes of these cells lose themselves also bears an important part in the same functions. Precisely what elements of the central substance alone act, and precisely how the elements act that do act, it is impossible to say.

$\S 6$. The following laws embody the most important general results of experiment upon the reflex-motor functions of the spinal cord, as applied to a variety of animals under a great number of changing conditions and circumstances.

The primary stimulation of the sensory nerves must have a certain degree of strength and suddenness in order to produce a secondary excitation of the motor nerves through the centres of the spinal cord. This is true of all the different kinds of stimuli by application of which spinal reflexes can be obtained. Continuous irritation of the skin, if very slowly increased, may be carried to the extent necessary to destroy its sensitive surface, without giving rise to any reflex movements; but a less degree of stimulus, if suddenly applied, will call forth such movements. Different chemical substances, when used as irritants, produce effects dependent upon the strength of the solution. Thus a weak solution $\left(\frac{1}{5}-\frac{4}{5} \%\right)$ of sulphuric acid is recommended by some experimenters; and it is asserted that in this way exactly the same reflex movements, as respects hind and degree, can be repeatedly got from the same nerve-preparation, with a machine-like regularity. Each chem- 
ical stimulus has its lower limit of concentration which will produce any reflex movement, and also its latent period. The time of the latent period for weak solutions of sulphuric acid is said by Baxt to increase nearly in geometrical ratio, while the concentration of the acid diminishes in arithmetical ratio. The chemical stimulus, like the mechanical, can be so slowly increased in strength as to produce no effect. The same thing is also true of thermic stimulus. A decapitated frog may be placed in water, and the water gradually heated to the point at which heat-rigidity sets in, without showing any reflex activity. This fact, however, may be in part ascribed to the direct effect of the heat, diffused from the skin upon the central organ. The same law which renders stimulus inoperative, when very gradually increasing in strength, applies to the use of the electrical current. Repetition of the shocks is much more effective than a slow increase in the strength of the current. Single induction-currents are relatively powerless, and produce no effect unless they have a high degree of strength. Frequent interruptions greatly increase the efficiency of the constant current in producing reflex movements. It would seem, then, that a kind of summation of afferent impulses may take place in the spinal cord; that is to say, the repeated excitation of the nerrous centre starts a nerve-commotion in its substance, which gathers intensity until it breaks over, as it were, into the adjoining motor tracts. We can scarcely affirm, however, that such summation of many impulses is necessary to start off the nerrous centre, as it were, since the single making of the constant current, or a single strong inductionshock, may be followed by a number of reflex movements.

\& 7. The speed of reflex processes is apparently increased by increasing the strength of the stimulus. We have already spoken (p. 123) of the delay which the process of conduction suffers in the spinal cord when passing longitudinally. The time of cross-conduction also in the cord seems to be a function of the strength of the stimulus. Exner ${ }^{2}$ calculated by an experiment, which consisted in causing one eyelid to move by stimulating the other, that the time consumed in the specifically central operations of the reflex act can be made to vary between 0.055 and 0.047 of a second by increasing the strength of the stimulus. Rosenthal ${ }^{3}$ and others have found that the time for any reflex act diminishes considerably with the increase of the strength of the stimulus ; is greater in transrerse than in longitudinal conduction; and is much increased by ex-

${ }^{1}$ Quoted in Hermann, Handb. d. Physiol., II., ii., p. 29.

${ }^{2}$ See Pflüger's Archiv, viii., p. 530 ff.

${ }^{3}$ Monatsbericht d. Berlin. Acad., 1873, p. 104. 
haustion of the cord. With very strong stimuli it becomes almost too brief for observation. W Wundt, ${ }^{1}$ however, denies that the time of the reflex act is dependent upon the strength of the stimulus; on the contrary, he affirms that the time is either very little or none at all affected by changes in strength of the stimulus, or else is even changed in the contrary direction to that required by the alleged law of Exner and Rosenthal.

$\S 8$. The condition of the spinal cord, at the time when it receives the impulses of the sensory nerves, undoubtedly cletermines to a large extent the character of the resulting reflex motions. Lesion increases the excitability of the part below the lesion, and this-for example, in the case of reflex movements of the posterior limbs-according to the amount of the cord removed from the portion of it lying anterior to its nerrous connections with these limbs. ${ }^{2}$ Marked effects are also produced by certain drugs, as strychnine, chloroform, aconite, quinine, etc. Of these drugs, some heighten and some depress its excitability. In an animal slightly poisoned with strychnine, the excitability of the cord is more or less heightened; and in cases of strong poisoning with the same drug, the least stimulation may call forth a condition of tetanus or convulsive cramping extending to the whole body. Two ways of explaining this effect upon the mechanism of the central organ are possible : one, that the excitability of those portions of this organ which mediate between the sensory and motor impulses is so much increased by the poison that, on being stimulated, they explode their molecular energy, as it were, and cause it to be diffused with great strength into unaccustomed paths; the other, that the effect of the poison is to diminish the resistance along all the network of paths, both habitual and unaccustomed, in the spinal cord. Between these two explanations Eckhard ${ }^{3}$ will not decide; Rosenthal seems to prefer the former, Foster ${ }^{4}$ and others the latter. Chloroform and various other anæsthetics diminish the reflex action of the cord. As to the effect of changes in temperature, and in electrical condition, upon the spinal reflexes, the conclusions of different experimenters are somewhat divergent. This power of the nervous mechanism is, as we have already seen, retained longer in low than in high temperatures. According to Cayrade, when the temperature of the whole cord is raised, the reflex movements, however produced, become more energetic and the single con-

${ }^{1}$ Mechanik d. Nerven, abth. ii., pp. 14 ff. Stuttgart, 1876.

${ }^{2}$ Vulpian, Leçons, etc., p. 438.

${ }^{3}$ In Hermann, Handb. d. Physiol., II., ii., p. 42.

4 Text-book of Physiology, p. 602. 
tractions last longer. Another observer found a temporary rise of excitability, followed by a depression, on heating sections of the cord between $75^{\circ}$ and $158^{\circ} \mathrm{Fahr}$. On the other hand, some observers are of the opinion that cold increases the excitability of the cord. In experimenting with the electrical current it is very difficult to distinguish between its effect upon the central organ as the mediating mechanism and the effect of the same stimulus upon the nerve-roots and nerve-paths between which the mediation occurs.

\$9. The locality to which the stimulus is applied has a marked influence in determining the extent and character of the resulting reflex movements. The most important difference of all is that found by stimulating some spot of the skin, and then comparing the resulting reflex action with what follows upon the application of the same stimulus to the trunk of the nerve which is distributed to that region of the skin. The simple nervous impulses, which result from stimulating the afferent nerre-fibres directly, call forth irregular spasms in a few muscles only; the complicated nervous impulses, which result from applying the same stimulus to the skin, are followed by extended movement of many muscles directed toward definite ends. Moreover, it is much more easy to produce reflex action by a slight pressure on the skin than by even strong induction-shocks when applied to the nerve-trunk. By separating a small bit of skin from that surrounding it on the back of a brainless frog, while taking care not to injure the nerves that attach it to the body, the foregoing difference may be made strikingly clear in an experimental way. ${ }^{1}$ What particular reflex actions will be eroked by the stimulus is, in each case, dependent upon the particular locality of the skin to which the stimulus is applied. Such facts suggest the truth that the entire mechanism of the cord is broken up into centres of activity, which, however, are in close molecular relation with each other, and which are of a somewhat expansive nature.

In view of the foregoing truths Pfluger ${ }^{2}$ has formulated the following laws of relation between the stimulation and the resulting reflex action: (a) In the case of a spinal cord from which the medulla oblongata is wholly severed, all reflex motion confined to one side of the body is due to stimulation of that side. (b) Reflex movements of both sides never occur in a diagonal direction; that is to say, stimulating one hind limb can never evoke reflex move-

'See the article of Fick and Erlenmeyer in Pfliiger's Archiv, iii., p. 326.

"In his work, Ueber d. seusorischen Functionen d. Ruickenmarks. Berlin, 1853. 
ment of that limb and of the fore limb of the opposite side. ${ }^{1}(c)$ If reflex action is called out in the limbs of both sides, and such action is stronger on one side than on the other, then it is stronger on the side stimulated. (d) If the motor effects of the stimulation show that the excitation has been "irradiated," as it were, from one centre to another, then such morement of irradiation is always downward toward the medulla oblongata in the brain, and upward in the cord toward the same organ. It is by no means certain, however, that these formulas (especially the second-No. $b$ ) admit of no exceptions which are involved in the peculiar structure and functions of the cords of certain animals. But the general rule appears to be, that the excitation of a sensory nerve with a slight degree of stimulus gives rise to reflex movements which originate in the cord on the same side, at about the same altitude as that at which the sensory impulses enter the cord; with an increased amount of stimulus, it gives rise to those also that arise in the other half of the cord at the same altitude; with a still greater amount, to those which arise above and below on both sides of the cord, with the preference given to the same side. That is, the molecular disturbance, as it is dispersed or radiated, passes from the cells and network of fibres situated near together on the same side of the cord, first to those on the other side of the cord at the same altitude, and then diffuses itself on both sides up and down the cord. ${ }^{2}$ Accordingly, it is only after allowing for a difference in the obstacles to be overeome along the different paths anatomically open to any nervecommotion in the spinal cord, that we can adopt the declaration of Luchsinger: ${ }^{3}$ When an excitation is started anywhere in the spinal cord, it radiates from this point in all directions, but with diminishing intensity. Hence the title which Flourens and Vulpian, ${ }^{4}$ following him, have giren to the spinal cord- "the organ for the dispersion of irritations."

$\S 10$. Besides such undoubted reflex action as the foregoing, other cases where the spinal cord controls the muscles of the body are less certainly of a purely reflex character. Indeed, for some such cases the title of "automatic" has been employed. The cord is not capalle of "irregular automatism"-that is, of spontaneous excitation like that which takes place in the higher nerrous centres

${ }^{1}$ See the observations of Luchsinger, which seem to show that in some animals-as, e.g., the salamander, turtle, and even dogs, when under the influence of ether-cross reflexes in violation of Pflüger's law do sometimes occur Pflüger's Archiv, xxii., pp. $179 \mathrm{ff}$.

¿ Compare Wundt, Grundzüge d. plıysiol. Psychologie, i., pp. 103 and 109.

${ }^{3}$ Pflüger's Archiv, xxii., p. 178.

${ }^{4}$ Leçons sur la Physiologie, etc., p. 404. 
on volition. If a brainless frog, for example, be kept in a condition of perfect equilibrium with respect to stimulus, it will remain wholly motionless. But the cord of such an animal will continue to influence certain muscles of the body through the motor nerves, even in cases where sensory impulses are difficult or impossible to trace. What is called the "tonic action" of the cord upon the skeletal and sphincter muscles, or the smooth muscles of the arteries, is a chief illustration of this influence. The fact that such tonic action does not contract all the muscles connected with the cord at the same time, or any one set of them with the same energy as any other, throws some suspicion on its alleged automatic character. A careful sifting of the evidence rather induces us to ascribe this influence to the constant reflex action of stimulus from subtle changes in the external circumstances in which the animal is placed. Moreover, the sensory nerves in the muscles and tendons, as well as in the skin and organs of special sense, may occasion the rise and continuance of such reflex action. Different investigators, almost without exception, have failed to notice any lengthening of a muscle (or loss of its tone) when the nerve going to it is severed from the cord. That this so-called "tonic" influence is largely reflex-motor is also shown by the fact that the tone of the muscles is lost when the skin corering them is removed, or when the posterior root which furnishes sensory impulses for the motor nerves connected with them is cut. Brondgeest has shown that, when a decapitated frog is hung up after having the sciatic plexus cut on one side, the leg is more flexed (that is, the muscles have more of tone) on the other side. But the same flaccid condition of the muscles can be produced by cutting only the posterior (or sensory) roots of this plexus. This observer is satisfied that the contraction of the muscles in the uninjured limb is due to stimulation from the nerves of the skin; the tonic action of the cord on the skeletal muscles is, therefore, reflex. The only objection to considering the tone of the sphincter muscles reflex lies in the fact that this tone continues to exist after all other reflex-motor action has been suppressed by narcotics; but our knowledge of the nervous mechanism which controls these muscles is not sufficiently complete to make it certain that we have excluded all possible forms of reflex influence.

Of the marked influence of the nervous system upon the calibre of the arteries, and through this upon the character of the circulation of the blood, there is abundant evidence. Besides the main vaso-motor centres in the medulla oblongata, certain parts of the spinal cord are capable of acting as such centres. Circulation may continue with regularity in a beheaded frog; but the remoral 
also of any considerable part of the cord affects the circulation through the loss of tone in the blood-vessels which it occasions. The mechanisms for expanding and contracting the arteries are apparently interlaced with those for contracting the skeletal muscles, in all portions of the cord. But their chief work undoubtedly consists in transforming afferent impulses into efferent vasomotor impulses directed toward the dilatation or constriction of the arteries. Whether they are capable of automatic action-in the sense in which the medulla oblongata seems to be thus capableis a question we need not discuss in detail here.

$\S 11$. The facts already alluded to, and others similar, form the basis for the assumption of "Centres" in the spinal cord. In general, the application of a given amount of stimulus to a definite group of sensory nerves calls forth reflex-motor activities in definite groups of muscles by means of a certain region of the cord. What groups of muscles are thus moved depends upon the amount of the stimulus and the locality of its application. This fact is due to dispersion of that nerve-commotion which is set up at different points in the course of the cord by the excitation of those points through the sensory nerves. That is to say, the mechanism of this central organ is so constructed as to connect the sensory with the motor tracts, more favorably in some regions than in others. Such regions are the so-called reflex centres of the spinal cord. If, however, a more or less constant flow of motor impulses takes place from any region, and this flow is due to molecular activity not occasioned by the sensory nerve-fibres of the region, then such region may also be called an automatic centre. Nothing would seem to prevent the same region from acting as both a reflex and an automatic centre. The general principle may then be formulated as follows: "The spinal cord is the proximate centre, the proximate physiological hearth of excitation, for all the nerves that originate from it." This principle has been defended and illustrated with many researches by Legallois, Volkmann, Pflüger, Goltz, Luchsinger, and others. In accordance with it, and especially since the "epochmaking" experiments of Goltz upon the spinal cord of dogs, many functions which were formerly ascribed to the brain have. been shown to have their proximate centre in the spinal-cord. In ac. cordance with the same principle, it is discovered that different animals have different spinal centres varying in relation to their peripheral structure and their habits. ${ }^{1}$

${ }^{1}$ Compare the results of the researches of Iangendorff in the Archiv $f$. Anat. u. Physiol., Physiolog. Abth., 1880, pp. 518 ff., and 1881, pp. 519 ff.; and of Luchsinger in Pflüger's Archiv, xxii., pp. $158 \mathrm{ff}$., and xxiii., pp. $308 \mathrm{ff}$. 
In illustration of the last point the following facts may be mentioned: By the sufficiently long-continued and strong stimulation of any portion of the skin of a decapitated frog, reflex morements may be induced in all of its muscles. With rabbits, however, a reflex action of one hind leg can be caused by stimulating the sensory nerves of a fore leg, only in case a portion of the medulla oblongata (at least about one-third) be left attached to the cord. With the cord alone, the stimulation of one hind leg fails to excite action in either of the fore limbs. By using great care and artificial respiration, Luchsinger ${ }^{1}$ succeeded in obtaining what he calls a "trotting reflex" from the spinal cord-after being completely severed from the medulla oblongata_-of several young animals with which that form of movement is natural. Thus the diagonal opposite extremities of goats and cats were moved in response to even such weak stimulation as passive motion of the fore leg, gentle pressure, and weak electrical currents. In general, then, it would seem that the spinal cord of every animal is a series of connected mechanisms, which are arranged so as to move the muscles of the body, either under the control of the higher nervous centres or in response to stimulation entering it at any point through the sensory peripheral nerves, in accordance with the specific structure and habits of the animal.

Many of the chief special centres connected with the organic and vital functions are located in the medulla oblongata; those connected with the co-ordination of impressions of the special senses and muscular action belong to the still superior portions of the cerebro-spinal system. But the spinal cord also contains mechanisms which serve as centres of both these kinds. ${ }^{2}$ Their location, howerer, is so much a matter of the special physiology of particular species of animals, and is so indirectly connected with the inquiries of physiological psychology, that it is unnecessary to add anything further upon the subject.

\$12. The question whether the spinal cord is excitable as a whole, and in its several parts, by artificial stimulation, has been much debated. Its direct excitability as a whole is denied by

\footnotetext{
${ }^{1}$ See Pflüger's Archiv, xxviii., pp. 65 ff.

${ }^{2}$ Besides the vaso-motor centres already referred to, those for micturition, defecation, erection, parturition, etc., may also be mentioned. Goltz, in his celebrated researches in 1874 (see Pfluger's Archiv, viii., pp. $474 \mathrm{ff}$.), showed that normal micturition may take place in a dog in which the lumbar region has been completely severed from the dorsal region. The influence of the cerebral centres seems, however, to be necessary to cause a steady increase or decrease of the action of the sphincter ani. The cilio-spinal centre, located by Budge at the seventh and eighth cervical roots, is more doubtful.
} 
Schiff, ${ }^{1}$ who declares that the motions obtained by stimulating any part of the cord with electricity comprise only those muscles which are physiologically related, to the exclusion of those which are anatomically contiguous through the stimulated part of the cord. A strong local stimulus, he affirms, produces just the same reflex motions as those which are accustomed to arise on occasion of an extended irritation of the skin at the places to which the nerves issuing from this locality of the cord are distributed. It is inferred, then, that the resulting motions are obtained only reflexly, by involving the sensory nerve-roots. But that certain longitudinal parts of the cord can be directly stimulated seems capable of demonstration. For Fick and Engelken ${ }^{2}$ found that movements of the muscles were obtained when the anterior columns were isolated from the rest of the cord for a considerable distance and then stimulated. Luchsinger's ${ }^{3}$ experiments, moreover, contradict the conclusions of Schiff; and Mendelssohn ${ }^{4}$ found that the reaction-time of the anterior half, and especially of the anterior columns of the cord, was uniformly less than the reaction-time of its posterior columns. The latter also found that weaker stimuli would suffice to excite motion when applied to the anterior columns. But, according to Schiff ${ }^{5}$ again, the cord contains no motor elements that are directly excitable except the central paths of the nerve-roots. He also agrees with van Deen in denying that the gray matter of the cord can be made, by direct stimulation, to originate either motor or sensory impulses. It affords paths, however, for the transmission of both these kinds of impulse when once started by the other nervous elements. Schiff accordingly speaks of the posterior gray columns, and of those parts of the posterior white columns which are not direct prolongations of the nerve-roots, as "cesthesodic." The corresponding parts of the anterior cord he calls "kinesodic." The sensitiveness of the posterior columns which others discover on experiment he regards as only indirect. Vulpian, ${ }^{6}$ on the contrary, agrees with Bell, Magendie, Flourens, and Longet, in holding that, while the gray matter is absolutely inexcitable and the posterior columns very excitable, the anterior columns possess only a moderate degree of excitability.

${ }^{1}$ See, especially, articles in Pfliger's Archiv, xxviii., pp. 537-555, and xxix., pp. $537-555$.

2 Du Bois-Reymond's Archiv, 1867, p. 198; and Pflüger's Archiv, ii., p. 414.

${ }^{3}$ Pfluger's Archiv, xxii., pp. 169-176.

${ }^{4}$ Archiv f. Anat. u. Physiol., 1883, Physiolog. Abth., pp. 282 ff.

- Pflüger's Archiv, xxix., p. 598.

${ }^{6}$ Leçons sur la Physiologie du Système nerveux, p. 362. 
By an ingenious arrangement for applying the mechanical stimulus of pricks from an extremely fine needle-point to definitely circumscribed spots in the spinal cord of the frog, E. A. Birge ${ }^{2}$ seems to have demonstrated the susceptibility of the ganglion-cells to direct stimulus. Pricking these cells produces movements in definitely located groups of muscles; and the te tanusis invariably confined to the muscles of the same side as that of the cells stimulated, unless (as inicroseopic examination shows) the effect of the needle has reached certain cells on the other side. Birge also found that different regions of a single cross-section of the cord are excitable in different degrees; the region from the posterior fissure to the median line of the gray matter being most inactive, and that of the large ganglion-cells in the anterior horn sinvariably being able to produce tetanus.

In view of such conflict of testimony it can only be said that certain longitudinal parts of the spinal cord are plainly susceptible to direct stimulation, but at present it is difficult to decide which parts, exclusively, are sensitive.

$\S 13$. Thus far the spinal cord has been considered as a series of related centres, that act automatically or reflexly when separated from the brain. But in its normal condition the cord always acts, of course, under the influence of the brain. The brain thus exercises a profound modifying influence over the automatic and reflex activities of the inferior organ. The cord alone can be depended upon, as it were, to respond with great regularity, in the form of definite reflex movements, to a given amount of stimulus, when applied at a given locality. But the action of the brain, when attached to the cord, interferes with this regularity, so that the expected muscular movements may not result when the stimulus is applied. They are then said to be inhibited by the action of the brain. The phenomena of "inhibition," when connected with volition, are familiar enough ; for example, one may voluntarily restrain those movements of one's legs which the cord, if left to itself, would produce as the result of tickling the soles of the feet.

But the brain without conscious volition exercises the same inhibitory action over the spinal cord. If a frog is suspended by the head, and its legs allowed to dip into a vessel of dilute acid, the interval between the contact of the acid and the withdrawal of the legs is considerably lengthened when the spinal cord remains undivided below the medulla oblongata; that is to say, the cord alone withdraws the legs quicker than the cord when influenced, or inhibited, by the brain. The interval between the application of the

${ }^{1}$ Archiv f. Anat. u. Physiol., 1882, Physiolog. Abth., pp. 481-489. 
acid and the contraction of the muscles can also be prolonged, when the brain is still connected with the cord, by applying chemical irritation at the same time to the optic lobes; that is to say, the cord is hindered from performing its reflex-motor function by the stimulation, and consequent influence upon itself, of the ligher nervous centre. Moreover, if at the time that one leg of a brainless frog is dipped into the acid, the sciatic nerve of the other is strongly stimulated with an interrupted current, the same prolongation of the period of incubation will be observed ; in some cases, indeed, the reflex act will not take place at all. In discussing the reciprocal relations of the higher centres of the brain, we shall discover many phenomena similar to the foregoing. All these centres may exercise this so-called " inhibitory" action upon other centres, according to their several physiological connections. The phenomena of inlibition are not, therefore, confined to the influence of the brain on the spinal cord.

Elaborate attempts have been made to point out a special mechanism of inlibition. Thus Setschenow ${ }^{1}$ has adrocated the view that localized inhibitory centres exist in the brain, and that the depressing effect travels by certain definite tracts in the spinal cord. But on this subject our doubts are entitled to go even beyond the remark of Ferrier: " "The nature of the inhibitory mechanism is exceedingly obscure." We cannot be said to have sufficient grounds for assuming the existence of any such specific mechanism. In general, nerve-commotions modify each other within the central organs; they either facilitate and increase, or inhibit and diminish, each other's effect, according to the structure and functions of the organs, the amount and kind of stimulus thrown in upon them from without, and the exact condition in which this stimulus finds them. The inlibition of the cord by the brain is, theu, only a special case under the general molecular theory of the nervous mechanism. The factors entering into every such case- will very likely always prove too varied and complex to be analyzed with complete success.

$\$ 14$. On passing from the spinal cord into the brain, the difficulty of defining the specific functions-whether automatic or reflex-of the different central organs becomes greatly increased. The phenomena are vastly more complicated, and the methods of analyzing them experimentally much less readily applied. The

${ }^{1}$ Ueber d. Hemmungsmechanismen f. d. Reflexthätigkeit im Gehirn d. Frosches, Berlin, 1863 ; and other papers.

${ }^{2}$ Functions of the Brain, London, 1876, p. 18, where he refers to the elaborate paper on Inhibition in the West Riding Reports, vol. iv., by Dr. L. Brunton. 
most complex portions of the nervous substance, in respect both to structure and to function, are most completely withdrawn from the use of strictly scientific methods of research. What is known, however, of the anatomical structure and connections of the different organs of the brain, and of the paths along which the nervous impulses are propagated between them, prepares the way for the more specific physiology of each organ. The methods of such physiological research are in general these two: Observation of the results which follow the application of stimulus to each of the encephalic organs, or to any definite locality in each; and observation of the results which follow the total extirpation or lesion of these organs, or of any portion of each. Of course, both of these methods are almost wholly applicable only to the lower animals. In using the method of stimulation, the stimulus cannot be applied to the nervous substance of the brain without a certain amount of injury to that substance. To stimulate any of the cranial organs with precision they must be exposed; those that lie deepest cannot be exposed without injury to other organs and the death of the animal. Moreover, it is difficult precisely to circumscribe the application of the stimulus. Just that form of stimulus which is most convenient, effective, and fruitful in results-namely, the electrical current-is liable to diffuse its direct effects beyond the region which it is desired to circumscribe. When no result follows the application of the current to a definite locality of the nervous substance, the failure may be due to the weakness of the stimulus, or to the fact that this particular centre is at the moment inliibited by its condition or by the activity of some connected centre. When a result does follow, it may be that this particular result is due to the direct or indirect stimulation of some other so-called centre, or to the stimulus hitting, by diffusion or otherwise, some of the contiguous sensory or motor nerve-tracts.

Objection may also be raised against the nature of the argument by which an inference is drawn from the facts gained by the second of the above-mentioned methods. Such argument not only assumes that the activities which remain, when some of the organs of the brain are partially or wholly destroyed, belong to those organs that remain, but also that those activities which have disappeared belong to the organs that have disappeared. Both of these assumptions are, however, doubtful, when we come to apply them to the organs in their normal condition and connections under the action of natural stimuli; the latter of the two is particularly doubtful. In a word, the different mechanisms of the human brain, in their normal condition and relations, constitute an in- 
ter-dependent and intimately related system; what each so-called organ or centre does, or can do, depends not only upon its own structure and condition at the time, but also upon the condition and behavior of the other organs and centres at the same time. Such interdependence extends not only to those divisions which gross anatomy can mark off and consider under the name "the organs of the brain," nor simply to those minuter subdivisions which histology can distinguish by aid of the microscope ; it doubtless also extends to the last details of that molecular mechanism which the brain-substance is. These details are different for every individual animal, and for every individual case. Specific differences belonging to the different species of animal life, as well as those idiosyncrasies with which pathology is familiar, must alike be recognized. It is by no means strange, then, that the physiology of the brain is able only very slowly and imperfectly to win from nature the truth, and to remove the reproach of apparently conflicting facts.

In spite of the above-mentioned difficulties certain results may be claimed as resting upon more or less of clear evidence regarding the specific automatic and reflex-motor functions of those intercranial organs that lie inferior to the cerebral hemispheres. The case of these hemispheres themselves will be subsequently considered in detail. For they are those portions of the nervous mechanism about the immediate correlation of which with the phenomena of consciousness there can be no doubt. Since we are now considering the nerrous system and its central organs merely as a physical mechanism, we definitely rule out, as far as possible, all allusion to any special relation between it and the phenomena of self-conscious mind.

$\S 15$. Besides the spinal cord, the Medulla Oblongata is the central organ concerning whose automatic and reflex-motor functions the largest amount of precise information exists. The reflex-motor functions of this organ are more intricate and of a ligher order than those belonging primarily to the cord. They are especially such as stand related to the vital functions of the lieart and bloodvessels; to respiration and its allied movements of the organs in coughing and sneezing, etc.; to the movements of the muscles in swallowing and vomiting; to the mimetic movements of laughing, weeping, etc. Among the different movements in the execution of which the medulla oblongata is concerned, some are more purely reflex and some less so. Thus one cannot swallow if the sensory tracts from the throat to this central organ are broken; but the movements of the heart and lungs continue after the reflex- 
motor paths to them are destroyed. Sensory stimulations of the medulla oblongata, as a rule, occasion reflex movements by secondary stimulation of a number of motor tracts. Swallowing, sneezing, coughing, shedding of tears, changes in respiration and in the movements of the heart, contortions of the countenance, may all be occasioned, through the mediation of this organ, by one and the same sensory impulse. There is also a marked difference in the extent of the domain over which the motor results of stimulating the different sensory paths connected with the medulla spread themselves. Stimulation of the optic nerve occasions only very limited reflex movements, such as the winking of the eyes, the secretion of a few tears, and a slight tendency to sneeze. Stimulation of the nerves of taste extends over a wider area of motor tracts; that of the palate and larynx still wider.

$\S 16$. The most important reflex centres of the medulla oblongata are also automatic; of such centres he chief are those connected with breatling, the movements of the heart, and the innervation of the blood-vessels. The excitation in these cases must be considered as a neural process arising within the central organ itself. The cause of its origin is doubtless to be found in the changes that occur in the supply and character of the blood. Not only all abnormal conditions of respiration, like dyspnœa and apnoa, but also the rhythm of normal respiration, are dependent upon the changing condition of the blood with respect to its more or less perfect oxidation. The stimulus to action of the respiratory centre in the medulla, from the condition of the blood, may be in part reflexly applied through the peripheral ends of the afferent nerves in various parts of the body; but the main effect is doubtless produced by the direct action of the blood on this centre. Its rhythmic nervous action may then very well be dependent upon the rhythmic action of the lungs, and upon the resulting periodic reoxidation of the blood. For the nervous substance of the medulla oblongata seems to be peculiarly susceptible to the condition of the blood.

\$17. This small central organ into which the spinal cord expands on entering the skull may then be said to be thickly crowded with reflex and automatic centres. To speak of the more important will best serve to exhibit what is known of its mechanism.

The respiratory centre was first located by Flourens in that part of the medulla oblongata which serves as the place of origin for the vagus nerve, and then more definitely in the $\mathbf{V}$-shaped apex of the fourth ventricle, or beak of the calamus scriptorius. Since 
extirpation or injury of this small portion of the nervous substance, when all other parts of the body are left intact, causes immediate and final cessation of respiration, Flourens called it the "vital knot" (nœeud vital). Foster" locates this centre below the vaso-motor centre, and between it and the calamus scriptorius. Schiff concludes that it is double, and lies on either side in the region of the anterior part of the ala cinerea; the function of each side, he thinks, is separate. In case of need it may be shifted slightly backward toward the spinal cord. The efforts of Gierke ${ }^{2}$ to fix it in a definite group of ganglion-cells were not successful. With this same centre all the modifications of respiration in sighing, sobbing, yawning, crying, laughing, coughing, sneezing, and hiccoughing are connected.

A nervous centre intimately connected with the vaso-motor system of the different parts of the body exists in the middle part of ${ }^{\circ}$ the medulla oblongata. Since we cannot examine experimentally the effect upon the action of this centre which would be produced by severing all the afferent nerves that lead into it, we cannot demonstrate directly how much of its action is automatic, how much reflex. It is probably both automatic and reflex. But the removal of the parts in front of the medulla, inclusive of the corpora quadrigemina, exercises no perceptible influence on the bloodpressure. The principal vaso-motor centres in the brain are then found in this portion of the medulla oblongata. Through it reflex motions are called forth of the most different kinds, and involving muscles widely separated from each other and from the region of the skin where the stimulus is applied. Witness the effect of a draught of air upon the circulation of the blood. The arteries of a rabbit's ear can be made to contract by stimulating any one of more than a half-dozen different sensory nerves, including the sciatic plexus. In this same central organ must be located the so-called cardio-inhibitory centre. In cases where the heart is stopped by sudden and great emotion, or by severe pain, the stimulus probably reaches the medulla from the hemispheres of the brain.

The centre of deglutition lies in the medulla higher up than that of respiration. If this part of the organ be destroyed, swallowing is impossible. This centre has been located in the floor of the fourth ventricle. In the floor of the same ventricle, and in the adjoining region, are probably located centres for different secretions-as, for example, of spittle, or sweat, of tears, and possibly of the pancreatic and other digestive juices. The connection of

1 Text-Book of Physiology, p. 370.

${ }^{2}$ See Pfliuger's Archiv, vii., pp. $583 \mathrm{ff}$. 
various sensations and emotions with these secretions is too familiar to need description. A central mechanism for winking the eyes Exner would place near the beak of the calamus scriptorius. The central mechanism for the reflex movement of the muscles of the csophagus and stomach also lies in the medulla oblongata. Of the centre for the production of artificial diabetes, and of other more conjectural centres which are packed within this small bit of nerrous matter, scarcely more than an inch in length, we do not need to speak.

$\S 18$. The alleged functions of the medulla oblongata in the coordination of the movements of the skeletal muscles ally this organ more closely with certain other inferior parts of the brain. The preparation of a frog which has retained this organ, in addition to the spinal cord, although without any of the rest of the brain, will execute movements of the muscles that are not possible for the cord alone. It will not, indeed, move spontaneously; it still requires external stimulation to start the mechanism of such a preparation. Under such stimulation, however, it will assume a position natural to it in an uninjured state. When laid on its back it will make efforts-generally unsuccessful-to turn over. The movements of the limbs with which it responds to various sensory impulses are more complicated than those executed by the spinal cord alone; they even resemble crawling motions or short leaps. Placed in the water, what is left of the animal will swim; and if its motions are less perfect than those of the perfect frog, they are much more so than those of the cord alone. It is doubtful whether, when placed beneath the water, it will ascend to the surface to breathe, or make efforts to escape from water gradually heated to about $104^{\circ}$ Fahr.,- - as will the animal that retains its cerebellum and optic lobes.

Reflex movements of considerable complexity can also be executed by mammals that have been deprived of all the encephalic centres above the medulla. Vulpian claims that a young rat in this condition will emit a cry, as of pain, when its toes are pinched. Such a mechanism will swallow and execute certain co-ordinated morements of the limbs. Infants whose nerrous centres above the medulla are undereloped will perform the associated movements of sucking when put to the breast. Moreover, the effects of lesion of the centres of the medulla are very marked in respect to the co-ordination of motion. Rolando observed that convulsive morements followed extensive injury of this central organ. More recent researches seem to show that the seat of these epileptiform movements is at the place of union between the medulla and the pons; 
it can, therefore, scarcely be located in either alone." One-sided lesions are followed by certain so-called " forced" and rotary movements of the head, and eyes, and trunk. Such effects are most likely to be produced when the injury affects the region of the tuberculum acusticum. In the opinion of Bechterew ${ }^{2}$ the olivary bodies are in relation with the gray matter of the third ventricle, and with the semicircular canals, as central organs for the co-ordination of the muscles used in balancing according to impressions of touch. It would then be one chief function of the medulla to secure equipoise through these sensory impressions. On the whole, it appenrs certain that considerable work in co-ordinating the muscular movements falls upon its mechanisms. Of such work it is probable that the movements concerned in articulate speech are a part. Any indirect relation which it may have to the production of those sensations and images which are woven into our dreams does not belong in this connection.

§19. The associations among the different centres of the medulla oblongata are curious enough; they involve an extremely intricate physiological apparatus. Some of these centres are indirectly connected with psychical activities. They are not all alike excitable; they are not all voluntarily so. Thus we can voluntarily control, within certain limits, the movements of the lungs, but not those of the heart and blood-vessels; we can cough, but cannot sneeze, at will. Some of their functions are associated together regularly; some of them seldom; some never. Swallowing is not necessarily connected with the activity of the other centres, unless it be with that for the secretion of saliva; it takes place, however, during arrest of respiration. The excitation of no other centre necessarily affects this centre. The secretion of saliva is constantly connected with a change in the circulation through the submaxillary glands.

$\S 20$. An animal which possesses all, or a considerable part of the other nervous mechanisms of the brain that lie below the cerebral hemispheres is capable of executing movements which differ greatly from those already described as belonging to the spinal cord and medulla oblongata. Very few of the movements of such a preparation are, indeed, even apparently spontaneous; for almost all of them a definite form and degree of stimulus acting on the sensory surfaces can be assigned. We are inclined, then, to suspect that those movements which are apparently spontaneous are really due to some stimulation from without the central organs

'See Eckhard, in Hermann, Handb. d. Physiol., II., ii., p. 98.

${ }^{2}$ Pflüger's Archiv, xxxi., pp. $479 \mathrm{ff}$., and xxix., p. $258 \mathrm{f}$. 
which has escaped our observation. But the range of reflex-motor activities which an animal deprived simply of its cerebral hemispheres will execute, in response to appropriate stimuli, is very great; it may be said to include every form of movement possible for the uniujured animal. The statement is, therefore, warranted by all our knowledge of the facts, that the medulla, pons, crura cerebri, cerebellum, corpora quadrigemina (or optic lobes), and basal ganglia generally are the special mechanism for co-ordinating the movements of the muscles with the various impulses of sense.

A frog from which the cerebral lobes have been removed will respond to appropriate stimuli with all the movements of which a perfect frog is capable. It will swim, leap, and crawl. When placed on its back, it will easily and at once regain its natural position. When placed on a tilting board, it will constantly adjust the position of its body so as to maintain an equilibrium. It will croak with the regularity of a music-box when its flanks are gently stroked. Thrown into the water it will swim with great regularity of motion until it is exhausted or finds something-as a small piece of wood placed in contact with it-upon which it can crawl. When submerged in the water, it will rise to the surface for air; it will not, like a mere spinal cord, remain quietly in water the temperature of which is gradually raised, but will make violent efforts to escape. It is guided by the light, for it avoids objects that cast a strong shadow. On the other hand, it appears stupid; it pars no attention to the flies that are placed near it; by careful exclusion of all stimuli it may be kept motionless for hours. We cannot argue from this, however, that it is without sensations, for it may not be hungry; and Heubel ${ }^{1}$ asserts that a sound frog may, with careful manipulation, be made to lie still upon its back for a long time.

Similar phenomena occur in the case of the mammal whose cerebral hemispheres have been removed. The rabbit or rat thus operated upon will stand and run and leap. Placed on its back, it will regain its feet. It will follow with its head a bright light held in front of it; it will start and tremble, or run, at a shrill or loud noise. It will utter a prolonged cry when pinched. Its muscular motions are obviously co-ordinated in response to sensory impulses from the organs of touch, hearing, and sight. The bird thus operated upon will easily regain its feet when laid upon its side or back, and will stand in a natural and easy posture. It will tuck its head under its wings, clean its feathers, and pick up corn 'Pflüger's Archiv, xiv., pp. 162 ff. 
or drink water presented to its beak. Thrown into the air, it will fly with considerable precision for some distance, and in its tlight will guide itself, though imperfectly, so as to avoid obstacles in its way. It will start at sharp sounds or flashes of light. Such animals have on the whole the appearance of being sleepy and stupid rather than of being deprived of any of their powers for co-ordinating sensation and motion. We conclude, then, that the organs which such animals possess are functionally capable of exercising all these powers of co-ordination; we do not at present raise the question whether this implies the existence of psychical phenomena or not. The phenomena which follow the partial loss of the cerebral hemispheres in the higher mammals confirm the same conclusion.

It is much more difficult, however, to assign the special place which belongs to each of the organs that lie between the medulla oblongata and the cerebral hemispheres, under their general function as already stated. They are all very intimately related; act to a large extent dependently ; can, within certain limits, assume each other's functions; and have largely the same connections with the peripheral organs of sense and of motion, and the same work to do as mediating between the two.

$\S 21$. It is impossible to determine the special functions of the Cerebellum, so conflicting is the testimony of different experimenters. A high degree of probability, however, attaches itself to the statement that this organ is largely concerned in the co-ordination of motion; although such statement cannot be held to exhaust its functions. The more specific theory of Wundt ${ }^{1}$ - "It is the central organ that brings such movements of the animal's body as are excited by impulses from the cerebrum, into accord with its situation as a whole in space"-is more doubtful, precisely because it is more specific. Comparative anatomy seems to show that the office of the cerebellum in some animals differs from its office in man; reasoning from the former to the latter is, therefore, especially precarious. Moreover, its functions are so closely connected anatomically with those of the pons, the crura cerebri, and the medulla, that it is difficult precisely to separate its work from that done by these organs.

Testimony as to the result of the extirpation or lesion of the cerebellum is very conflicting. Apparently almost the entire length and breadth of its surface (in the direction of the posterior bones of the skull), and not only the gray matter, but also the white, as far as near the bifurcation of its strands, may be removed without ' Grundzüge d. physiologische Psychologie, i., p. 201. 
any observable result. ${ }^{1}$ On approaching the middle of its thickness and removing the strands connected with the middle peduncles, disturbances of motion begin and increase rapidly in proportion to the amount of substance removed. Most of these disturbances, if the animal recovers well, prove to be only temporary; they are, therefore, probably due largely to traumatic excitation. Permanent disturbances, however, occur when the injuries reach the lower third of the organ, or when they are confined to this third. Vulpian accordingly concludes that the disturbance of gait which results from injury of the cerebellum, is due to the irritation of its more profound white parts or of the adjoining cerebral isthmus. But Schiff believes that the mass of the organ, apart from locality, has a definite influence upon the co-ordination of the bodily movements ; though what that influence is cannot yet be clearly defined. The influence of locality seems to be considerable upon the effect which results from lesions in a given amount of the cerebellar substance; but since this influence is much more marked near the connections of the cerebellum with other contiguous organs, some observers attribute it largely or wholly to the injury-by extension of the lesion, by pressure, or by inflammation-of these organs. Thus the place of its union with the medulla oblongata and the regions near the crura cerebri are especially important. But Schiff found, in experimenting upon mammals, that complete vertical section of the cerebellum, in the exact median line of the vermiform process, and removal with the knife or pincers of the entire substance, with the exception of the flocculi and the parts external to the peduncles; produced no appreciable loss of the power of co-ordination.

The effect of one-sided lesions of the cerebellum in the disturbance of motion seems to be, as a rule, much more certain and marked than that of symmetrical lesions of both sides. Schiff, indeed, asserts that when a bilateral lesion is perfectly symmetrical it produces no impairment whatever of the functions of motion. But the entire evidence from experiment shows that sudden lesion of one hemisphere of this organ is almost uniformly followed by at least temporary impairment of the motor functions. Section of the middle peduncle of the cerebellum of a bird or mammal almost always occasions so-called "forced" movements; the animal rolls around its own longitudinal axis, generally, though not invariably, toward the injured side. Nystagmus, or the peculiar rolling movement of the eyes suggestive of vertigo, and strabismus, take place

${ }^{1}$ Compare Vulpian, Leçons sur la Physiologie, etc., pp. $603 \mathrm{ff} . ;$ Eckhard, in Hermann, Handb. d. Physiol. II., ii., pp. 102 ff.; Schiff, in Pflüger's Archiv., xxxii., pp. $427 \mathrm{ff}$; and Ferrier, Functions of the Brain, pp. 85-123. 
in such cases. One eye may be moved inward and downward, the other outward and upward. Hitzig ${ }^{2}$ and Ferrier ${ }^{2}$ found the same results to follow injury of the lateral lobe. The latter observed that strong stinulation of the cerebellar surface with the interrupted current causes associated movements of the eyes and head and limbs, in eats and dogs and monkeys. But these effects may be largely due to the connection of the cerebellum with the medulla oblongata.

The evidence from pathological cases in man conflicts, to a considerable extent, with the conclusions which we might hasten to derive from experiment upon the animals. According to Vulpian ${ }^{3}$ it is by no means rare to have unilateral lesions of the cerebellum followed by no paralysis of either side. In a great number of such cases no genuine hemiplegia results ; the resulting enfeeblement of motion, moreorer, is as often on the same as on the opposite side. M. Andral is said to have made a collection of ninety-three cases of diseases of the cerebellum, in only one of which ataxy was observed in any marked way. In most cases where crossed hemiplegia does result, Vulpian thinks it due to the destruction or compression of the adjacent parts, especially the roots of the cerebellar peduncles. The same authority denies that the superficial parts of this organ are excitable, or that lesion of them is followed by pain or by convulsions of the body, face, or eyes. Such results do, however, follow excitation and lesion of its deeper parts, in proportion to the degree of approach to the peduncles. The discrepancy between experiment and pathology may perhaps be removed, at least in part, by remembering that the injury is sudden in the one case and not in the other. Moreover, few of the pathological cases are clearly enough defined to serve as a sure basis for conclusions. Some of them, howerer, would seem to warrant certain inferences. More than fifty years since, the well-known case of the girl Alexandrine Labrosse was reported by Combette, ${ }^{4}$ and afterward made known to students of physiology generally by Longet. This girl was found, on post mortem, to have no cerebellum; in its stead was a gelatinous membrane attached to the medulla by two peduncles of like construction. A true pons was also wanting, but no loss of substance seemed to have taken place here. Yet she could co-ordinate all the limbs voluntarily, and had the full use of

${ }^{1}$ Untersuchungen über d. Gehirn, pp. $198 \mathrm{ff}$.

${ }^{2}$ Functions of the Brain, p. $106 \mathrm{f}$.

${ }^{3}$ Leçons sur la Physiologie, etc., p. $607 \mathrm{f}$.

' Revue médicale, II., p. 57 (1831).

${ }^{5}$ Anatomie et Physiologie du Système nerveux, I., p. 764 (1842). 
all the senses. She was, however, subject to falling (se laissait tomber souvent) and spoke imperfectly. Bouillaud has reported another case of an adult whose entire cerebellum was changed into a brown purulent mass; this patient could walk, though in a tottering and insecure way. Vulpian ' also describes a case which came under his own observation. A woman, dying at the age of sixty-nine, after twenty years in the hospital of La Salpêtrière, was found to have suffered an entire atroplyy of all the cortical gray substance of the cerebellum. This patient preserved great muscular vigor, and could co-ordinate all the muscles; but her "locomotion" was disordered and difficult.

On the whole, then, it must be admitted that the evidence concerning the specific functions of the cerebellum of mammals, and especially of man, is not such as to warrant us in making definite affirmations. Scarcely a single case can be adduced in which it is not possible to maintain that the motor disturbances which followed lesion or excitation of this organ should be ascribed to an indirect effect upon contiguous organs. Yet the coincidence of evidence from several different lines gives sufficient support to the view that the functions of the cerebellum are in some way connected with the balancing, and therefore with the precise and secure locomotion of the body in space. More definitely, with reference to the nature of this connection, it is not possible to speak confidently. No disturbance of the senses of hearing, of sight, or of muscular feeling, can be shown to follow injuries of this organ where other parts of the brain are not involved; on the contrary, all these senses appear to have been perfect in certain cases of the complete absence of this organ. The only disturbance of sensibility which frequently follows affections of the cerebellum is vertigo; the same symptom can be produced by passing a current of electricity throngh the back part of the head, or by the effusions of blood in this region which are sometimes occasioned by alcohol. Vulpian and others are, howerer, probably right in holding that the result is only indirectly to be ascribed to this organ. Indeed the view of Schiff has much in its favor: this riew maintains that the aberration of motion due to lesion of the cerebellum should not be called a loss of co-ordination at all, since all the limbs may be moved in exactly the right relations necessary to carry the body forward or to maintain its equipoise; but the precision of the motion is impaired, because the nervous impulses from this organ that innervate the neighboring groups of muscles are not rightly adjusted to each other in amount along the different tracts. The

${ }^{1}$ Leçons sur la Physiologie, etc., p. 629. 
balance of the innervating cells is destroyed; and the result is a loss of nice adjustment of the amount of innervation sent to the particular muscles employed in equipoise and locomotion.

It scarcely need be added that modern physiology distinctly disproves the hypothesis of Gall, who connected the sexual instinct with the cerebellum. There is no good evidence that the hinder brain directly participates in any way in those activities of the nervous system which are immediately correlated with psychical phenomena, whether of emotion, instinct, or intelligence.

$\S 22$. The functions of only three other parts of the encephalon require consideration in this connection; these are the corpora quadrigemina, the optic thalami, and the corpora striata. The crura cerebri and the pons Varolii are, as we have already seen, significant chiefly as organs of conduction. So far as they have also the intermediating functions of central organs, it is not possible to treat of them otherwise than as concerned in that general reflex-motor mechanism which occupies all this region of the brain.

\& 23. Experiments upon the Corpora Quadrigemina are rendered especially difficult by the small size and deep situation of these organs; they cannot easily be exposed for stimulation without great effusion of blood, or subjected to lesion without extending the injury to contiguous parts. These difficulties render conclusions from the effect of stimulating or extirpating the corresponding organs (optic lobes) of the frog more than usually precarious. There is no doubt, however, as to some special connection between the corpora quadrigemina and sensory impulses of sight ; such connection is, then, of course, to be extended to those motor activities that are dependent upon the sensory impulses of sight. Flourens and many subsequent observers have found that one-sided extirpation of the optic lobes of birds, or of the corpora quadrigemina of mammals, with the cerebral hemispheres intact, produces blindness in the opposite eye. The amount of this blindness is different in different animals, as the decussation of the fibres in the optic chiasm is more or less complete in different animals. In the rabbit such decussation appears to be complete; in the cat and dog incomplete. The fact that hemianopsia in both eyes is connected with disease of one side of the brain is an evidence that it is incomplete in man also. Moreover, when the brain is removed in front of the corpora quadrigemina, and these organs left intact, the animal can still guide and co-ordinate its motions in response to visual impulses. (We do not in this place consider whether we are warranted in calling these impulses "sensations"-not to say "perceptions"-of sight.) These organs are, then, in some sort, central 
organs of sight. Since they are connected by nerve-tracts with the cortex of the cerebrum, motor innervation in response to stimulus from the optic nerve may arise either immediately in the corpora quadrigemina themselves or in the gray matter of the cortex. We may therefore suppose, with Wundt,' that destruction of the cerebral substance abolishes only those movements of the muscles, in response to the stimulus of light, which involve complicated co-ordinations with other excitations of sense, or with earlier established experience. It is scarcely allowable, however, to locate this special relation to visual impulses definitely in the substance of the corpora quadrigemina considered as isolated from the optic thalami, the optic tracts, and the gray matter at the floor of the third ventricle. There is sound sense in Eckhard's ${ }^{2}$ remark that the functions commonly attributed to these bodies should rather be ascribed to the region in which they lie. The nates (or anterior pair) seem to be more especially connected with the sensory, and the testes (or posterior pair) with the motor activities of sight.

Abnormal movements of a "forced" nature, and impairment of the power of co-ordination, follow the injury or extirpation of the corpora quadrigemina. These phenomena may be due in part to the loss of guidance by visual impressions; but they are probably due chiefly to the extension of the effects of the injury to the crura cerebri and other surrounding parts. The optic lobes, according to Goltz, are the principal central mechanism for the croaking of the frog deprived of its hemispheres. Vulpian ${ }^{3}$ makes a distinction between a merely reflex-motor cry and the plaintive utterance of an animal (e.g., the rabbit) which retains these organs and the pons Varolii. Ferrier, ${ }^{4}$ however, was unable to make the distinction so clearly. The latter observer found that very marked plenomenasuch as dilating the pupils, clenching the jaws, retraction of the ears and angles of the mouth, extending the legs, etc.-followed the stimulation of these organs with an electrical current, in the case of eats and dogs. But his experiments do not enable us to say how much of all this belongs to the specific function of the corpora quadrigemina as central organs, and how much to the irritation of the nerve-tracts in all the surrounding region. While we seem warranted in connecting these organs with the cerebellum, medulla, and pons, as concerned in the co-ordination of motions necessary for equipoise and locomotion, it is not safe at present to attempt a more precise localization of function.

${ }^{1}$ Physiologische Psychologie, i., p. 184.

'In Hermann's Handb. d. Physiol., II., ii., p. 131.

${ }^{3}$ Leçons sur la Physiologie, etc., p. 541 f.

Functions of the Brain, p. 76. 
§24. The office of the so-called basal ganglia-Optic Thalami and Corpora Striata-in that "projection-system" which connects the cerebral hemispheres with the periphery of the body, has already been spoken of; one chief function of these ganglia has usually been held to be that of acting the part "of middlemen between the cerebral convolutions and the rest of the brain." 1 But they both have further functions as specifically eentral organs in coordinating the movements of the body according to impressions of sense. It is difficult, if not impossible, however, to define precisely what these functions are. Some special relation of the optic thalami to impressions of sight must be admitted. The fact that animals deprived of the cerebral hemispheres are capable of complex co-ordination of their muscles as reflex effects of visual impressions, seems to indicate that the mechanism of the optic thalami is associated with that of the corpora quadrigemina in performing this function. In mammals complete extirpation of the posterior portion of one thalamus results in permanent expansion of the pupil of the opposite eye ; and Renzi was confident that injury of the upper surface of the anterior portion occasioned blindness. Lussana and Lemoigne found blindness in the opposite eye to be the invariable result of lesion of one thalamus. Cases of the disturbance of vision, or even of complete blindness, have been observed in human patients as the apparent result of ciisorganization of this organ. It must be admitted, however, that the significance of the optic thalami for vision may be due simply to the fact that certain fibres of the optic nerve have their origin in it, and are rendered inoperative by injuring it. Experiments and pathological cases connecting the optic thalami with the sensations of smell and taste are more doubtful and eonflicting. Ferrier ${ }^{2}$ concludes that lesions in and around this organ destroy the cutaneous sensation of the opposite side of the body in the monkey; Veyssière found the same thing true in dogs. But Nothnagel found that no effect upon sensation followed the destruction of these organs in the rabbit. Not a few cases of disease of the optic thalami in man seem to point to some connection with tactile impressions; other cases, however, are decidedly unfavorable to this view. On the basis of this rather meagre evidence Wundt ${ }^{3}$ is willing to rest the theory that the optic thalami are special eentres for the reflexmotions of touch; by the same theory he also accounts for the disturbances of motion which follow injury to these organs. $\mathrm{He}$

${ }^{1}$ See Foster, Text-Book of Physiology, p. 653.

${ }^{2}$ Functions of the Brain, pp. $238 \mathrm{ff}$.

${ }^{3}$ Physiologische Psychologie, i., p. 188. 
thinks it probable, nevertheless, that their function is not exhausted by this description. "Forced" positions and movements, and various other marks of impaired motor activities, follow the experimental lesion of these organs. But such disturbances largely or wholly vanish after a brief time, although they can be again called out by stimulation. They occur, as a rule, only when the lesion affects the posterior part of the thalamus, or the edges of the opening leading from above into the third ventricle. Most of the phenomena may be explained as clue to the working of a mechanism that has been stimulated to abnormal activity by the mechanical irritation due to the extirpation. ${ }^{1}$ We can scarcely, then, be any more explicit than to quote the remark of Vulpian, made some years since ${ }^{2}$ "We know nothing of the special functions of the optic thalami."

\&25. The special motor significance of the Corpora Striata is undoubted; although we cannot go to the length of holding that these bodies are concerned only in the elaboration and downward transmission of efferent impulses. Ferrier ${ }^{3}$ and others have observed that stimulating these bodies with an interrupted current produces strong convulsive movements of the opposite side of the body; with a very powerful stimulus the whole side is drawn into an arch. No such effect could be produced by stimulating the optic thalami. Ferrier holds" that "in man and the monkey there is little, if any, difference perceptible between the complete destruction of the cortical motor-centres and destruction of the corpus striatum." Vivisection of this organ is sometimes followed by hasty forward running motions. Lesions of the striate bodies, in the case of the animals, are usually followed by laming of the limbs of the opposite side; sometimes, however, no pathological symptoms result. As a rule, in the case of man, paralysis of the arms and legs of the opposite side follows disease of these organs. Here, as elsewhere in this region of the nervous system, a certain suddenness of the disturbance appears necessary to secure any marked result. Some experiments seem to point to a difference in the effects of injury to the two main nuclei of the corpora striata. Nothnagel asserted that all mechanical injury to the mucleus lenticularis of one side results in laming of the opposite side: destruction of this nucleus on both sides brings the animal into nearly the same condition as the removal of the cerebral hemispheres. But voluntary movements persisted after complete destruction of both the nuclei caudati of the rabbit.

\footnotetext{
${ }^{1}$ Comp. Eckhard in Hermann, Handb. d. Physiol., II., ii., p. 125 f.

${ }^{2}$ Leçons sur la Physiologie, etc., p. 659.

${ }^{3}$ Functions of the Brain, p. 161.

${ }^{4}$ Ibid., p. 249.
} 
There is much evidence, then, to show that the corpora striata are, as compared with the optic thalami, more especially connected with motor activities. Wundt ${ }^{1}$ considers them to be pre-eminently significant as ganglia for the co-ordination of those motor impulses which are derived from the cerebellum and the cerebrum. The relative importance which they seem to have in the higher, as compared with the lower, animals (the monkey and man as compared with the rabbit, etc.) he thinks is like that of all the anterior portions of the brain; such portions are in general, more significant in man than in the other animals. Wundt's view has considerable in its support-among other things, the fact that, in case of lesions of the striate bodies, voluntary motions, or those motions whose motor innervation originates above these organs, seem to suffer most. But we positively must not adopt without qualification the statement $^{2}$ that the corpora striata are exclusively motor, and the optic thalami exclusively sensory. In addition to what has already been said (p. 129) to eaution one against this view, it may now be added that numerous cases are recorded where injury, apparently confined to one corpus striatum, has resulted in loss of feeling on the opposite side; and other cases where disease, apparently confined to one optic thalamus, has caused loss of motion as well as of sensation. Moreover, the chief motor effects of injury to the striate bodies (if not all of them) may be due to the fact that the descending motor tracts are necessarily involved in the injury, rather than to any special motor function belonging to these bodies as a central organ. Another theory of the office of the striate bodies rejects entirely the view which regards them as in any true sense basal ganglia, with either specially motor or specially sensory functions; and regards them as belonging to the cerebral hemispheres, rather than subordinate to the hemisplieres in function. ${ }^{3}$ But inasmuch as this theory has its principal support, of a physiological kind, from a single case of an idiot's brain, in which these bodies were of nearly normal size, while the cortex was deficient in the motor regions and the base of the brain in general small, it can scarcely be regarded as sufficiently confirmed.

\&26. The researches of the last few years have tended to show that some special relation exists between the nervous substance of the organs lying at the base of the cerebrum, and the temperature

${ }^{1}$ Physiologische Psychologie, i., p. 193 f.

${ }^{2}$ As propounded by Carpenter and Todd, and apparently adopted by Ferrier, Functions of the Brain, $252 \mathrm{f}$.

${ }^{3}$ See A. Hill, The Plan of the Central Nerrous System, p. 276; and Journal of Anat. and Pliysiol., July, 1885. 
of the body. The earlier observations ${ }^{1}$ pointed out the limits betreen the medulla oblongata and the pons as a region, lesion of which was followed by a sudden and large rise of temperature. Still later, other observers ascribed raso-motor functions to the optic thalami, ${ }^{2}$ or asserted the existence of raso-motor fibres in the crura cerebri (so Budge). In $1884, \mathrm{~J}$. Ott pointed out that cutting the corpora striata is speedily followed by a marked rise of temperature. Yet more recently two experimenters, ${ }^{3}$ working together, have arrived at certain conclusions based upon a large number of experiments, chiefly on rabbits, but also on guinea-pigs and dogs. They discover that, while the cortical substance can be subjected to the most severe and extended lesions without producing a feverish rise of temperature, puncturing the brain at the juncture of the sagittal and coronal sutures, down to the level of the striate bodies or deeper, invariably produces a marked rise of temperature. If the lesion only hits the striate bodies (especially the medial side, near Nothnagel's nodus cursorius) the coming-on of the fever is slow and gradual; but if the needle is carried further toward the base of the brain, the ferer springs up at once and reaches a maximum in two to four hours. In what way these organs act as "ferer-centres," or precisely what nervous elements are chiefly involved in the action, has not yet been made clear.

$\S 27$. Eckhard ${ }^{4}$ is inclined to lay down the law that in all vertebrates the mechanisms for a change of place lie rather in the anterior part of this general region-corpora quadrigemina, etc.; while those for maintaining the upright posture and the equipoise of the - body are localized in the region of the pons, cerebellum, and medulla oblongata.

\$28. It should be added that almost all observers have hitherto failed to attach sufficient importance to the central functions of the gray matter which lines the floor and walls of the third ventricle. Bechterew ${ }^{5}$ has recently contributed the results of rery important experiments to determine the specific function of this central nervous substance. He finds that frogs retain the function of balancing even when the optic lobes are crushed, if no injury is done to the gray substance of the third ventricle or to the crura

${ }^{1}$ By Tschetschichin, in Archiv. f. Anat. u. Physiol., 1866, pp. 151 ff.; and Schreiber, Pflüger's Archiv., viii., pp. $576 \mathrm{ff}$.

${ }^{2}$ Lussana and Christiani (No. 16 of the Verhandlungen d. physiolog. Gesellschaft zu Berlin, 1883-84).

${ }^{3}$ Ed. Aronsohn and J. Sachs: See Pflüger's Archiv., xxxvii. (1885), pp. $232 \mathrm{ff}$.

${ }^{4}$ In Hermann's Handb. d. Physiol., II., ii., p. 138.

${ }^{5}$ Pflüger's Archiv, xxxi. (1883), pp. $479 \mathrm{ff}$. 
cerebri ; they lose this function, however, when a section is made into the third ventricle. Birds (hens and pigeons), also, show the same loss of function when a lesion is produced by running a very fine needle into the carity of this ventricle. In the case of dogs, Bechterew considers himself able to localize the function of equipoise precisely, and to point out the special effect of injury done to different definitely fixed localities. For example, bilateral lesion of the lateral or postero-lateral parts of the wall of the third ventricle results in the impairment or loss of equipoise and co-ordinated motion on both sides of the body: the lost function is regained only after a long time, and then but partially. In none of these cases were any of the phenomena of motor laming of the extremities apparent, or any very marked disturbance of sensation. This gray matter of the third ventricle operates, Bechterew thinks, in connection with the olivary bodies for the co-ordination of motor impulses in response to sensations of touch, and with the semicircular canals in response to sensations of sound. It is especially important also in equipoise through visual impulse connected with the changes in the axial direction of the eyes. Thus all the abovementioned organs operate with the cerebellum as complex and correlated mechanisms for keeping the body balanced in response to changing sensory impulses.

We stop at this point in our ascending review of the automatic and reflex-motor functions of the central mechanisms. For distinctly psycho-physical and psychological questions the most important of the activities of the nerrous mechanism still await our examination; these are the activities of the cerebral hemispheres. But nothing is known as to the molecular structure of these hemispheres, or as to their automatic and reflex-motor centres and activities, which adds anything of importance to the description of the nerrous system as a mechanism, or to the mechanical theory of its action. It is with such description and theory that we are now concerned. The correlations which exist between the structural condition, or physiological function of the nerrous system, and the phenomena of mind, are chiefly (if not wholly) capable of study as illustrated in the cerebral hemispheres. But the nature of the. nervous molecular machinery, and of its working as mere machinery, is understood, as far as our present information will permit, by an examination of the physiology of the spinal cord and of the intercranial ganglia lying below the hemispheres. As to the alleged psychical functions of these inferior organs we shall adduce further considerations when we come to consider such functions as belonging to the brain proper. 


\section{CHAPTER V.}

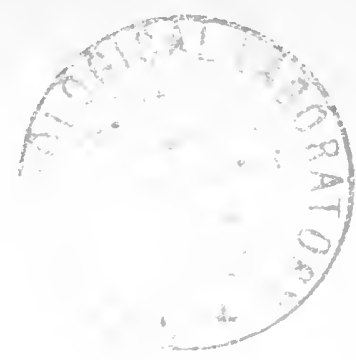

END-ORGANS OF THE NERVOUS SYSTEM.

\$1. Iv order to understand the end-organs it is necessary to refer again to the place which they hold in the threefold arrangement of the nervous mechanism (compare Chapter II., \& 2). In the general division of labor among its organs, certain cells situated at the surface of the body become especially sensitive to external stimuli. The special function of these cells accordingly becomes that of receiving the action of such stimuli, of modifying this action in accordance with their own peculiar structure, and thus of setting up in the conducting nerves the neural process which is propagated to the ceutral organs. It is obvious, then, that the structure and grouping of the superficial cells must bear some definite relation both to the external stimulus and also to the nerve-fibres which convey inward the nervous impulse occasioned by it. The end-organs of sense may then all be described as special adaptations of the superficial cells to the different kinds of stimuli. With such special adaptations the peripheral terminations of the nerrefibres must be connected. For the end-organs, as it were, look both outward and inward. They act as mediators between those different modes of external molecular motion which can occasion sensations in us, and the nerves which convey the results of this motion, when it has been changed into a nerve-commotion, onward to the central organs.

\&2. In the end-organs of the special senses the fibrils of the sensory nerves, as a rule, terminate in cellular structures which have the morphological significance of metamorphosed epithelial cells. The end-organs of smell and taste show this characteristic development most clearly. These end-organs are, in general, made up of cells which, posteriorly, pass into nerve-threads that are gathered to. gether into the sensory nerve of the special sense; and which, anteriorly, pass into conical or fusiform processes. The simplest type of an end-organ may then be described as follows: A hair-like process extending outward, and connected by a sensitive cell with a nervous filament extending inward. Such processes are probably 
extremely sensitive to external stimuli; and perhaps peculiarly so to the chemical changes which, at least in the case of three of the special senses (smell, taste, and sight), are their immediate excitants.

All the end-organs of sense may be regarded as modifications of the type described above. Only a small part, however, of what are ordinarily called "the organs of the special senses" (e.g., the nose, the mouth, the ear, the eye, the skin) belongs, strictly speaking, to the nerrous system. By far the greater part consists of mechanical contrivances, designed to prepare the external stimuli and conduct to the true nervous apparatus the impulses they occasion. These non-nervous mechanical contrivances, however, modify the nature of the stimulus in so important a manner as to merit some brief description in our consideration of the nervous mechanism.

§ 3. Besides the end-organs of sense, histology points out another kind of terminal apparatus. The efferent nerves, in order that they may stimulate the muscles, must have some special form of attachment to them. Special contrivances for connecting the motor nerves and the muscles are actually discoverable. We distinguish, then, two classes of end-organs : first, End-organs of Sense, and, second, End-organs of Motion.

§ 4. Among the end-organs of sense, those of Smell have been least successfully investigated. That portion of the mucous membrane of the nose which clothes the upper region of the nasal cavity and is marked by a brown-yellow color-the region of the expansion of the olfactory nerve-is called "regio olfactoria;" it contains the end-organs of smell. Here Ecker and Eckhardt (in 1855) discovered two different kinds of cells ; but we are indebted to Max Schultze for the first detailed description of them. The epithelial portion of the olfactory organ is supposed to be constructed upon the same type in all the vertebrate animals. Of the two kinds of cells which the lastmentioned investigator described, one is called "epithelial," the other "olfactory." The epithelial cells are the larger, have an oval nucleus of considerable size, and extend through the whole epithelial layer. Their external half appears more or less cylindrical or columnar (at least in the Triton and Proteus), and is described by some observers ' as striated longitudinally. The form of the inner half of these cells is varied. The olfactory cells are spindle-shaped, with a large, round nucleus, and very long, fine processes. The external process is elongated into a stiff hair, at least in many cases, although Schultze considers that in man the olfactory cells have no cilia.

' See Professor Babuchin in Stricker's Human and Comparative Histology, iii., p. $207 \mathrm{f}$. 
These cells are surrounded by the epithelial cells. Most physiologists follow Schultze in holding that the two kinds of cells are distinct both in form and in function, and that only the "olfactory" cells are connected with the end-fibrils of the nerve of smell; Exner ${ }^{1}$ and others, however, believe that the distinction is not a fixed one. In his opinion the structure of one is merged into that of the other, and both are connected, though in a different manner, with the subepithelial net-work in which the fibres of the olfactory nerve are lost. The exact histological relation of the fibrils of the olfactory nerve to the epithelium of the regio olfactoria is not yet made out. It is probable, lowever, that the finest of these fibrils, after penetrating the epithelial layer, closely embrace the large epithelial cells and enter into connection with the inner extremities of the olfactory cells. According to Exner, the fibres of the nerve do not pass orer directly into the processes of the end-organ cells, but are lost in a net-work whose interstices are filled up with granules of nervous matter. The first pair of cranial nerves, the ol. factorius, which, as ive have already seen on p. 84, is really a lobe of the brain itself, is the specific nerve of smell.

\&5. The contrivance for applying the stimulus to the end-organs of smell is very simple; in general it is only necessary that a current of air, in which the stimulating particles float, shall be drawn through the nasal passages over the mucous membrane of the regio olfactoria. Even ammomia and camphor, when placed under the nos-

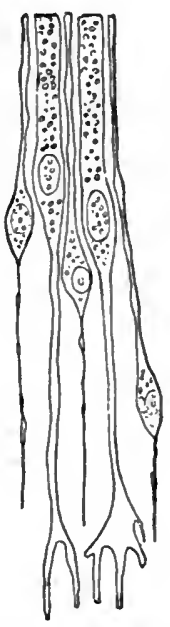
trils, have no smell so long as the breath is held or drawn through the mouth. In quiet inspiration much the greater part of the current of air is conducted to the pharynx directly, and comparatively little reaches the ridge situated above the nasal dam at the back of the nose, where the end-organs of smell are placed. In full inspiration, and still more when short and deep draughts are drawn through the nasal passages, a considerable amount of the air is forced over the sensory parts. By snuffing we increase the amount of air drawn into the region by first creating a partial vacuum in its cavity. In expiration the breathing passage is so located as to carry nearly all the air past the sensory parts without striking them. For this reason smelling is almost exclusively confined to ${ }^{1}$ Sitzgsber. d. Wiener. Acad., lxiii., p. 44 f. and lxv., p 7 f. 
inspiration ; it has been disputed whether the current of expiration can be smelled at all. But Debrou showed that the odor of orange blossoms, when water tinctured with them has been drunk, can be detected in the expired air. The current wlich passes through the anterior part of the nasal passages seems to be the more important. This is probably the reason why the loss of the nose is so frequently attended with loss of the sense of smell.

$\S 6$. The end-organs of Taste are situated in certain papillae, found on the upper surface of the root of the tongue, on the borders and apex of the tongue, and in some cases on the anterior portion of the soft palate. These papillae of the tongue, are the papillo circumvallate and the papillee fungiformes. The lateral portions of the former are pre-eminently the regions of the mucous menbrane of the tongue where the end-organs of taste are found. The same organs are also found more sparsely distributed in the fungiform papilla. The circumvallate papillæ are composed of connective tissue, which is invested by a pavement epithelium arranged in laminæe. The epithelial layer is thinner than elsewhere at the sides of the papilla, in which the end-organs of taste (gustatory flasks or bulbs) form a zone that extends upward to about the level at which the papilla are no longer protected by their lateral wall. In the fungiform papilla the end-organs appear in the epithelium which covers their upper surface, and in the side surfaces. A. Hoffmann also found them in the papillæ of the region of the soft palate. It is more doubtful whether they exist, as has been alleged, on the epiglottis. The papilloe filiformes, which are sometimes classed with the two others, probably have nothing to do with sensations of taste. ${ }^{\prime}$

Methods of experimenting to discover what surfaces are sensitive to taste arc not easily made exact, because the stimulus must be in solution to excite the end-organs, and because the nature of the excitatory changes is chemical. There is scarcely a spot from the lips to the stomach which some physiologist has not described as belonging to the organ of taste. But the regions where the abovedescribed papillæ, with their gustatory flasks, are found, are doubtless the principal-and probably they are the only-sensitive surfaces. Considerable differences exist, however, among different species of animals, and even among different individual men-especially as to the sensitiveness of the tip and edges of the tongue, and of the anterior surface of the palate. All the evidence tends to show that the gustatory flasks are the sole end-organs of taste.

${ }^{1}$ Comp. Brücke, Vorlesungen über Physiologie, ii., p. 257; and von Vintschgau in Hermanu's Handb. d. Physiol., III., ii., p. 147. 
\& 7. The microscopic structure of the end-organs of taste is described in substantially the same way by all investigators, although these structures vary considerably, according to their position, and according to the different species of animals. In general they are like a glass knob with a short neck, and with its length somewhat greater than its greatest width. Hence they are called "gustatory knobs" or "bulbs" Fia. 41.-Gustatory Bulbs from the Lateral Gustatory (so Henle), or, better, “gus-

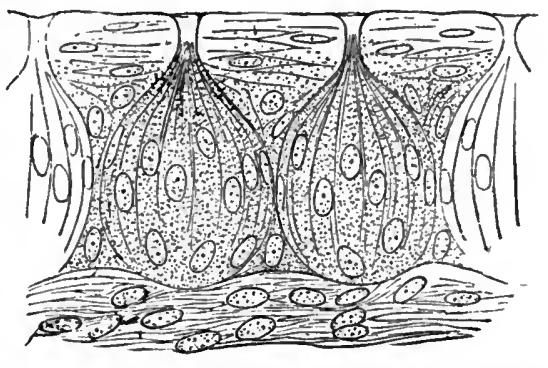
tatory flasks" (so M. Schultze). They occupy flask-shaped cavities of the epithelium, which they completely fill. Their lower or inner

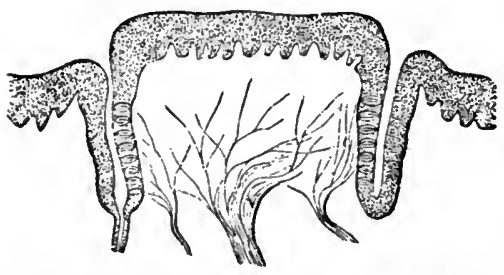

FIG. 42.-Transverse Section through a Papllla Cireumvallata of a Calf. Showing the arrangement and distribution of the gustatory buib. 26\%. (Engelmann.) part rests on the connective tissue of the mucous membrane; their upper and more slender part is surrounded by epithelial cells and has an opening, or pore, of from $\frac{x}{4000}$ to $\frac{1}{1250}$ of an incli in diameter, at the surface of the epithelium. The margin of this pore is usually formed by placing several cells together, but sometimes by a single cell which appears perforated with a round hole. Each of the gustatory flasks consists of from fifteen to thirty long, thin cells, arranged like the leaves of a bud in closely compressed rows around the axis.

All the gustatory flasks are composed of two kinds of cells: some are, essentially, epithelial cells, and have probably no direct connection with the nerves; the others are highly differentiated structures, are probably directly continuous with the nerve-fibrils and are thought to be true gustatory cells. The epithelial or investing-cells are long, narrow, spindle-shaped, bent, with a nucleus well marked ; the outward end is pointed, the central end branching. The gustatory cells are thin, long, and highly refractive of light, with nearly the whole body of the structure occupied by an elliptical nucleus. The body of the cell is elongated into two processes, of which the upper or peripheral is tolerably broad and bears a short and fine point like a hair or pencil-point. This point 
lies in a canal, in the epithelial layer, and rarely projects from the pore of the flask. The lower or central process of the cell is much attenuated, and usually divides into two branches. A direct con-

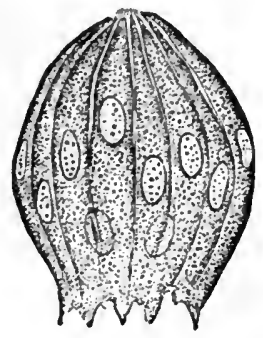

FIG, 43,-Isolated Gustatory Bulb. from the Lateral Gustatory Orinn of the Rabbit. ${ }^{600 \%}$. (Engelmanu.)
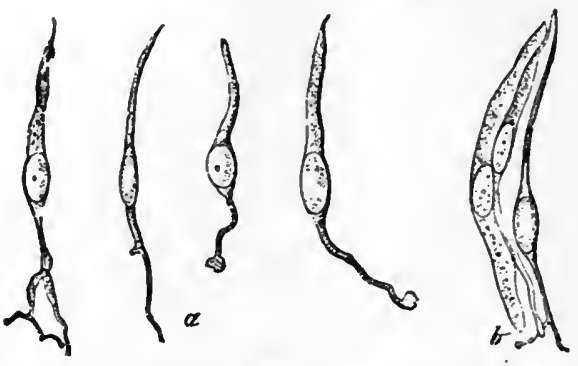

FIG. 44.- $a$. Isolated Gustatory Cells, from the Lateral Organ of the Rabbit; $b$, an Investiug and Two Gustatory Cells, isolated but still in connection. $60 \%$. (Engelmann.)

nection of these processes with the fibrils of the gustatory nerve is assumed by all investigators. The manner in which the nerve-fibres terminate within the papillæ is different in different animals.

The glosso-pharyngeal nerve is the principal nerve of taste. It is distributed to the back of the tongue, enters the circumvallate papillæ, where it forms a minute plexus, interspersed with nerve-cells, from which both medullated and non-medullated fibres pass to the base of the gustatory flasks. The lingual branch of the trigeminus has also some claims to be, in a minor degree, a nerve of taste. Schiff " considers it as designed for sour taste, with a slight sensitiveness to bitter also.

\& 8. In considering the end-organs of Touch, attention should be directed to the great variety of sensations which are grouped together under the word "touch," in the broadest meaning applicable to it. The question is thus raised whether any histological difference is to be detected in the nervous apparatus which may serve as a physical basis for the difference in the sensations. We may set aside for the present all consideration of the feelings of pain, of exertion and fatigue, and the so-called " common feeling" and "muscular sense." The question is thus reduced to this narrow form : Can histology point out two specifically distinct kinds of end-organs in the skin, one of which serves for sensations of temperature, and the other for sensations of pressure?

$\S 9$. Histological examination shows that the sensory nerves distributed to the skin-the general organ of touch-terminate in two

${ }^{1}$ Molesch. Unters., X., p. 406 f., as referred to by von Vintschgau in Hermann's Haußb. d. Physiol., III., ii., p. $171 \mathrm{f}$. 
ways, either in free end-fibrils or in special constructions called "tactile corpuscles" or "end-bulbs." The different varieties, all, however, essentially alike, of these special end-organs of touch have been named after as many different investigators. Their general office is that of modifying and multiplying the effect of the stimulus upon the nerve-fibres which terminate in them. The so-called "corpuscles of Pacini" were the first end-apparatus to be discovered in connection with the peripheral termination of the sensory nerves; they were seen more than one hundred and fifty years ago by Vater. In man they are constantly present in the subcutaneous connective tissue of the palms of the hand and of the soles of the feet; but are most numerous in the palmar surfaces of the fingers and toes, especially the third phalanges, although they occur in the neck, arms, etc. In some places they are visible to the naked eye as a minute grain of from $\frac{x}{20}$ to $\frac{1}{6}$ of an inch in diameter. They may be said to be nothing more than the ends of medullated nerve-fibres remarkably thickened. ${ }^{2}$ Each corpuscle consists of layers of counective tissue, arranged concentrically and more closely packed near the centre; these surround a cavity containing a soft nucleated material, into the interior of which the nerve penetrates. Here the nerrefibre, having become a naked axis-cylinder, appears to terminate in a little bulb. Examination with the highest

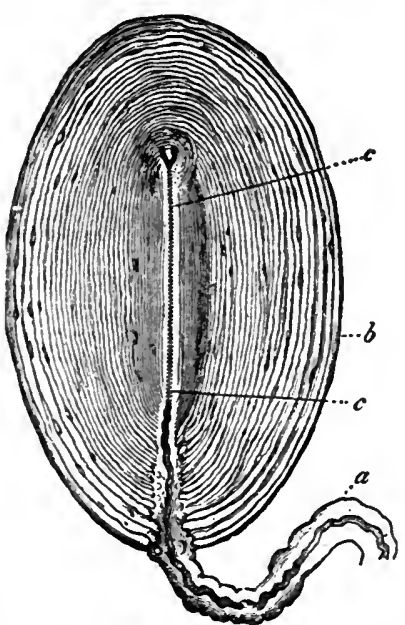

Fra. 45. - Corpuscle of Pacini (or Vater) from the Mesentery of the Cat. (After Frey.) $a$, nerve with its sheaths: $b$, system of tunics constituting the capsule of the corpuscle; $c$, axial canal, in which the nerve-fibre ends. powers of the microscope shows that the axis-cylinder of the fibre is fibrillated, and that the terminal bulb consists of finely granular substance.

Closely allied to the foregoing structures are the so-called "endbulbs of Krause." These are small capsules of connectire tissue in which nuclei can be detected. In them the nerve-fibrils of touch terminate either in a coiled mass or in a bulbous extremity. They are from $\frac{1}{250}$ to $\frac{1}{1000}$ of an inch in diameter, and exist in the con-

${ }^{1}$ On the different kinds of terminal corpuscles, a principal monograph is by Fr. Merkel, Ueber die Endigungen der sensiblen Nerven in der Haut der Wirbelthiere, Rostok, 1880.

"So Biesiadecki in Stricker's Human and Comparative Anatomy, ii., p. 232. 
junctiva of the eye, in the tongue, the lips, the floor of the buceal cavity, etc. The "corpuscles of Wagner" (or Meissner, who bas furnished most of the details) may be described as oval-shaped

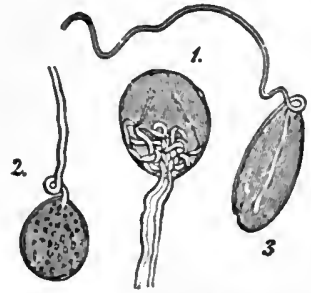

Fia. 46.-End-bulbs from the Conjunctiva of the Human Eye. (After Kölliker.) 1, has two nerve-fibres which form a coil within the end. bulb; 2, has a fatty core. The nerve-fibre of 3 ends within in the form of a knot. bodies, made up of superimposed laminæ and bearing some resemblance to a miniature fircone. The medullated nerve-fibres, like "creeping roots," wind beneath the cutaneous papillæ, and here and there penetrating them, terminate in the corpuscles. Within the corpuscles, according to Kölliker, the fibrils form two or three coils, and finally join together in loops. These tactile end-organs are most constant and numerous in the terminal phalanges of the fingers; they occur in smaller numbers on the palm and back of the hand, on the sole and back of the foot, and sometimes on the nipple, lips, etc. They are seated in the papillæ of the skin. Meissner counted four hundred papillæ in ${ }^{\frac{1}{5}} \overline{0}$ of an inch square on the third phalanx of the index-finger, and found

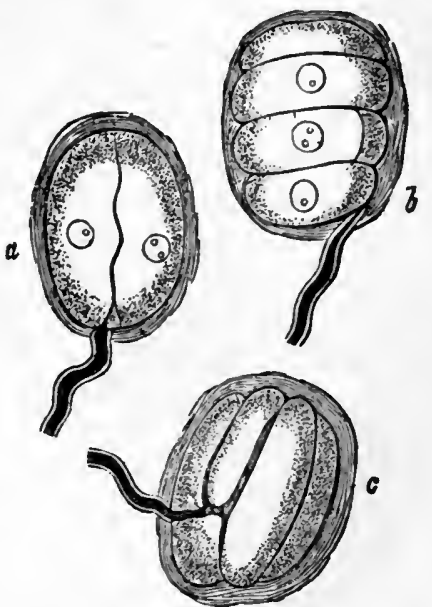

Fia. 47. -Corpuscles of Tonch. (After Frey.) $a$, from the soft skin of the duck's bill; $b$ and $c$, from the papillo of the tongue of the same animal.

these corpuscles in one hundred and eight of them. Their long diameter lies in the direction of the papillæ and extends from $\frac{1}{3} \frac{1}{80}$ to $\frac{1}{140}$ of an inch; they are about $\frac{1}{500}$ of an inch in thickness.

$\S 10$. Since the surface of the skin is in general sensitive to pressure and to temperature, it follows that the special structures described above as occurring in parts of this surface, cannot be the sole end-organs of touch. Modern histology has demonstrated the presence of an intricate plexus of non-medullated nerve-fibres which end in free extremities between the cells of the mucous layer. This terminal plexus of nerve-fibres is also the end-organ of so-called general sensibility and of touch.

None of the attempts litherto made to establish specific relations between the varieties in the structure of the tactile end-organs and the varieties of the sensations which they administer can be pronounced successful. Krause has tried to deduce from the construc- 
tion of the corpuscles of Pacini their fitness to act as the endorgans of pressure; but these corpuscles are wanting in many parts of the body that are sensitive to pressure. Wagner considered the corpuscles which bear his name to be special organs of touch. But it has been shown by Merkel that these corpuscles are nothing but aggregates of more elementary forms, the so-called " tactile cells." Some have argued that the end-bulbs of Krause and the corpuscles of Pacini are the organs of general feeling (sensus communis) ; but others, with more probability, assign this function to the free nerve-endings; while Merkel is of opinion that the latter are specifically concerned in sensations of temperature. Nothing is known on this point beyond the fact that the skin, within which the sensory nerve-fibres terminate, either in free ends or in special tactile corpuscles, is the organ for all the varieties of sensation brought under the most general meaning of the word "touch."

The more precise manner in which the terminal fibres of the nerves of touch stand related to the individual tactile cells is also still in doubt. Some investigators consider that the fibres enter into the very protoplasm of the cells (Merkel, Frey); others that they spread themselves on end-plates superimposed on the cells (Retzius, Ranvier).

\$11. With the exception perhaps of the ear, the Eye is by far the most elaborate and complicated of the end-organs of sense. This is true of those portions of it which are designed merely to bring the external stimulus to bear upon the nerrous structure, as well as of this structure itself. Considering it as a whole, we may say that the peripheral organ of sensatious of light and color is an optical instrument constructed on the plan of a water camera obscura, with a self-adjusting lens, and a concave, sensitive, nerrous membrane as a screen on which the image is formed.

$\S 12$. The eyeball consists of three coats or tunics inclosing three translucent refracting media. Since, however, the front part of the outer one of these coats is itself translucent and refracting, the number of refracting media in the eye is really four. (1) The first or external coat consists of two parts: $(a)$ the Sclerotic or posterior five-sixths part ("white of the eye"), which is a firm, fibrous membrane formed of connective tissue intermingled with elastic fibres ; and $(b)$ the Cornea, or translucent anterior one-sixth part, which is circular and convex in form, and covered with conjunctival epithelium. The comea rises and bulges in the middle like a watch-glass. (2) The second coat, or tunic of the eye, also consists of two parts: these are $(a)$ the Choroid coat, which comprises much its larger portion, is of a dark brown color, due to its 
pigment cells (except in the case of albinos), and is abundantly provided with nerves and blood-vessels ; and $(b)$ the Iris, a circular, flattened, disk-shaped diaphragm in front of the lens (the colored part of the visible eyeball), bathed with aqueous humor, and having in its centre a circular aperture called the "pupil" of the eye. The anterior border (corpus ciliare) around the iris consists of the

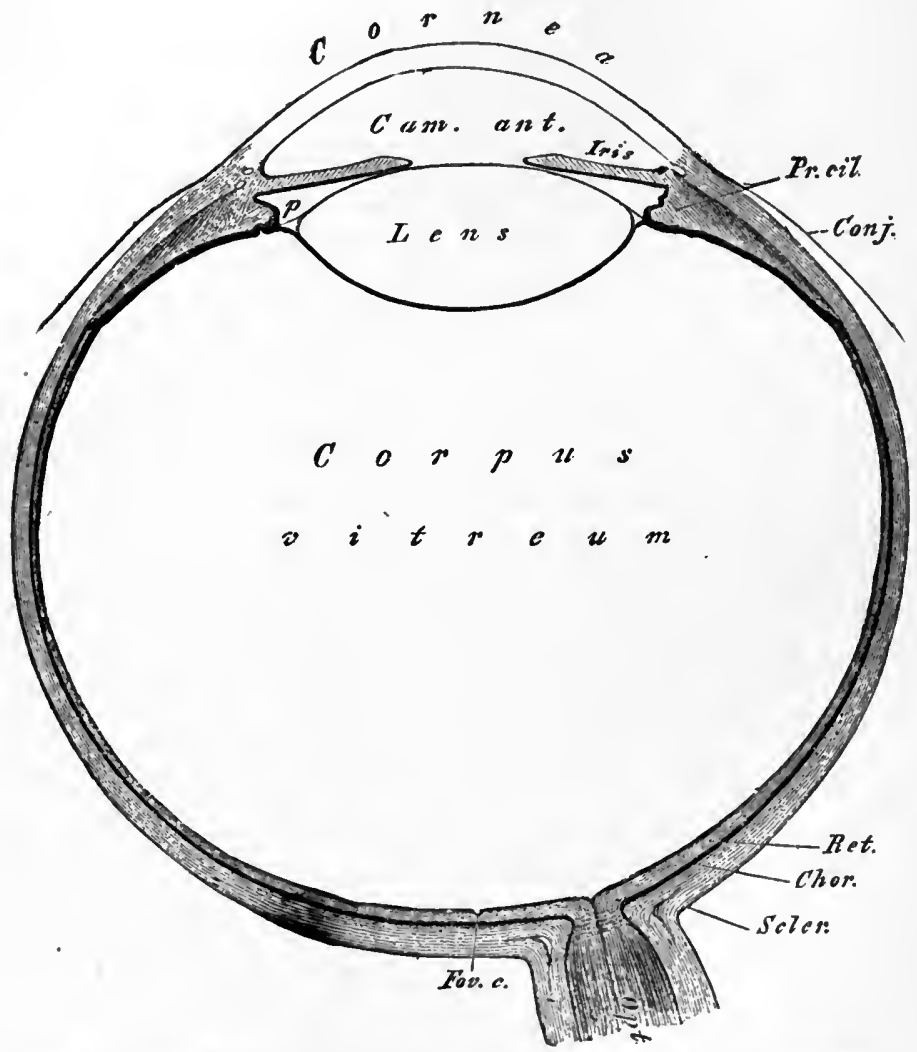

FIG. 48.-Horizontal Section through the Left Eje. 4/1. (Schematic, from Gegenbaur.)

ciliary muscle and the ciliary processes. (3) The Retina is the third or inner coat of the eye. It is a delicate membrane of exquisite transparency and almost perfect optical homogeneity; it has a highly complex structure, consisting of nine or ten layers, the truly nervous portions of which contain nerve-fibres, nerve-cells, and special end-organs, together with connective tissue and bloodvessels. The inner surface of the retina is moulded on the vitreous 
body, and it extends from the entrance of the optic nerve nearly as far forward as the ciliary processes.

$\S 13$. The eyeball has four translucent refracting media. The first of these-enumerating inward from the outside front-is (1) the Cornea, already spoken of as the anterior one-sixth of the outer coat of the eye. (2) The Aqueous Humor fills the space between the cornea and the lens, and is divided by the iris into two chambers, of which the front one is much the larger. It is limpid and watery; it holds in solution the salts of the bloodserum, with traces of organic substances. (3) The Crystalline Lens is situated between the iris and the vitreous body. It is a transparent biconvex lens, with its antero-posterior diameter about one-third less than the transverse diameter. It consists of a capsule and inclosed body. It is of "buttery consistency," composed, like an onion, of a number of easily separable layers. Each layer consists of fibres which, within the layer are, as a rule, radial. Between the entire ciliary part of the retina and the corresponding part of the vitreous humor is interposed a structureless membranous body, to which the edge of the lens is attached, and which radiates outward and maintains the lens in tension. It is called the suspensory ligament, (or Zonula of Zinn) and its office is very important in the accommodating of the eye to different distances. (4) The Vitreous Humor consists of a number of firm sheets or layers (lamellæ), between which fluid is contained, built into a body that is, optically considered, transparent and homogeneous. It occupies most of the space inclosed by the tunics of the eye. It is thought to be a gelatinous form of connective tissue, and is composed mostly of water with salts in solution, of proteids and mucin, fats and extractive matters-especially urea. Its peculiar structure is of little significance for the physiology of the eye.

$\S 14$. Of the appendages or accessory parts of the eye--such as the eyebrows, the eyelids, lachrymal apparatus, muscles of the eyeball-only the mechanism by which the eye is moved in its orbit has any special significance for physiological psychology. The building-up of a world of visible objects, and even the formation of a so-called "field of vision," is dependent upon the great mobility of the eye. The eyeball is moved in its bony socket, where it is embedded in a mass of fat as in a socket-joint, by six muscles, which are attached to it somewhat like the bridle to the horse's head. Four of these muscles spring from the bony wall near the point where the optic nerve enters, extend through the length of the socket and pass directly to the eyeball, where they are attached to it, one above, one below, one on the outer, and one 
on the inner side, (the recti; internus and externus, superior and inferior). In moving both eyes up or down, the same muscles in both contract simultaneously; in moving the eyes to the right, the outer

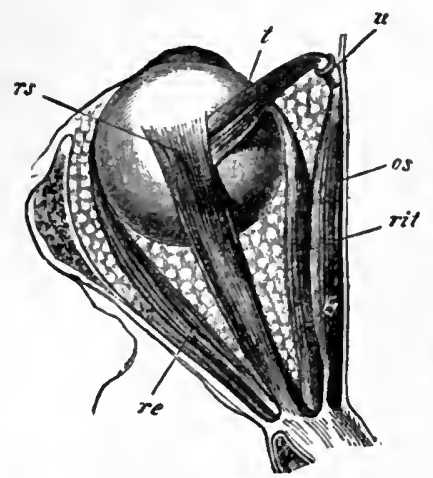

FIG. 49.-Muscles of the Left Human Eye, seen from above. $r s$, rectus superior ; re, rectus externus; and ril, rectus internus: 08 , superior oblique, with its tendon, $\ell$, whlch runs through the membranous pulley, $u$, at the inner wall of the cavity of the eyeball.

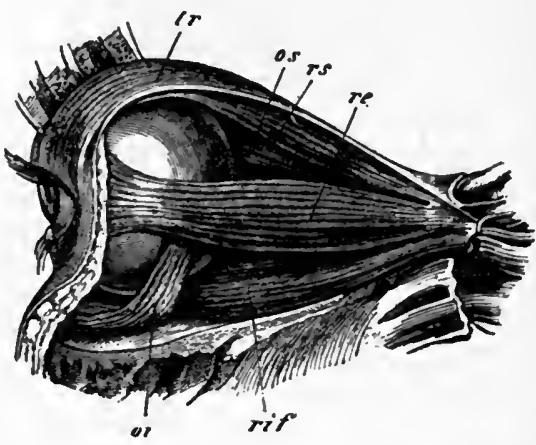

FIG. 50.-Muscles of the Left Hnman Eye, seen from the ontside. Ir, levator of the upper eyelid, which covers the rectus superior, $r s, r e, o s$, as in the preceding figure; rif, rectus inferior; $\omega t$, inferior oblique.

muscle of the right eye and the inner of the left, contract simultaneously (and vice versa); in turning both eyes inward to converge them upon a near object, the two inner muscles contract together. We cannot move the eyes so that the optical axes do not either meet or remain parallel ; we cannot look with one eye upward and the other downward, nor with one eve to the left and the other to the right; nor can we voluntarily turn the eyes farther apart than when their axes are parallel.

The other two of the six muscles of the eye are called oblique. Of these one is superior and internal ; it does not pass directly forward from its place of origin, at the posterior aperture through which the optic nerve enters to the eye, but first runs through a ring, then turns around, and is attached obliquely to the upper surface of the eyeball. The other oblique muscle begins at the inner wall in the socket, passes under the eye-ball, and is attached to it opposite to the superior oblique muscle. The two oblique muscles combine with the four recti to move the eyes in various directions which would be impossible for the latter alone.

$\S 15$. The problem which is to be solved by the end-organ of vision may be stated in a general form as follows: A mosaic of localized sensations must be so constructed that changes in the quantity, quality, local relation, and sequence of these sensations 
shall be quickly interpreted as indicative of the size, shape, locality, and motion of external visible objects. The most important part of the solution of this problem falls upon the nerrous structure of the retina. It is itself a mosaic of nerrous elements, the excitation of which may vary in quality, quantity, local coloring, and sequence of the different elements excited. But in order that the retina may exercise its function with the precision and delicacy of detail for which its structure fits it, the rays of light reflected from a single point of the surface of the visible object must excite a single one, or at most a small and definite group, of the retinal nervous elements. The sensations thus occasioned can then undergo a systematic arrangement by the mind. It is the work of the translucent refracting media of the eye to apply the stimulus to retinal elements exactly discriminated, and in an order corresponding to the object ; that is to say, the cornen, the humors of the eye, and the lens must form an image on the retina. To show the possibility of this by calculating how the general laws of optics apply to the special structure of the eye, as anatomy describes it, and to make the calculations accord approximately with the facts, has been the labor of a number of investigators, especially of Helmholtz and his pupils. To the results of this labor only a brief allusion must suffice.

$\S 16$. The four media of the eye constitute a system of refracting surfaces, each of which is separated from the one adjoining by a circular cut, as it were, in the whole refraction-substance. Especially is this true of the lens with its concentric layers. The "image" formed upon the first member of this system of surfaces, by its refraction of such bundles of rays, from the object, as all lie in a plane at right angles to the axis of the system, thus becomes an "object" for the second refracting surface of the system; and the image formed by the second an object for the third; and so on. The result of any number of such refractions will accordingly always be an image whose points lie in a plane at right angles to the axis of the system of refracting surfaces, and which, as a whole, is in true perspective to the original object. The last image and the object are geometrically similar.

In tracing the course of the rays of light through the refracting media of the eye, two things must be taken into the account: (1) the indices of refraction of these media, and (2) the geometrical form and position of all the limiting surfaces. (1) The means for attaining a knowledge of the former is by taking the average result of an examination of a number of eyes supposed to be normal. Fortunately for science, death has, for the first twenty-four hours, 
little or no effect in changing the indices of refraction of the eye. Krause ${ }^{1}$ found the mean index of refraction of the cornea to be $=$ 1.3507 , of the aqueous humor $=1.3420$, of the vitreous body $=$

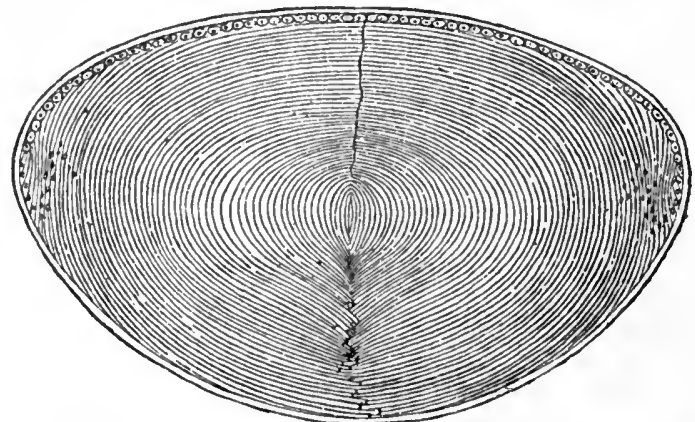

Fra. 51.-Median Section through the Axis of the Lens of the Eye. (Schematic, after Babuchin.)

1.3485. But Helmholtz (subsequent observers have agreed better with his result than with Krause's) found the two latter indices of

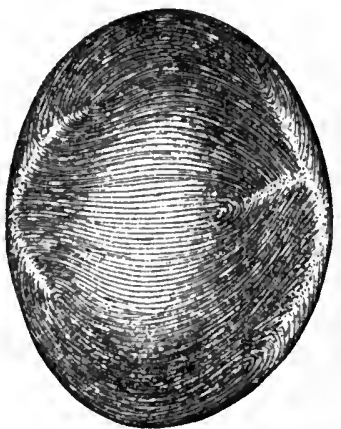

Fia. 52.-View of the Lens in Profile. $3 / \mathrm{s}$. (After Arnold.) refraction to be $=1.3365$ and $=1.3382$, respectively. The lens of the eye, especially, is not homogeneous throughout as to its index of refraction. Each layer has its own index, and the amount of the index of each layer increases regularly toward the kernel of the lens. The work of refraction done by the lens is, there. fore, greater even than that which could be done by a homogeneous lens with an index of refraction equal to that of the kernel, or' most highly refracting part of the lens.

(2) The position and form of the separating surfaces of the refracting media can be only approximately determined in the living eye. Three of these surfaces are of chief importance-the anterior surface of the cornea, and the anterior and posterior surfaces of the lens. The convexity of the first of these three is found to depart perceptibly from a sphere; it is greater toward its edge than at its vertex, where it resembles rather a section of an ellipsoid. The advantage of such a shape is seen in the fact that the images

'Krause's experiments refer to rays of the wave-length to which the brightest place in the solar spectrum corresponds; that is, to the place at the end of the first third, or quarter between $D$ and $E$. The refraction-index of water for these rays he assumed at $=1.33424$. 
formed when the pupil is expanded are thus made sharper than they could otherwise be. No observable refraction takes place on the posterior surface of the cornea, because the difference between the indices of refraction of the cornea and of the aqueous humor is so slight that the faint images from this surface vanish by proximity to the stronger ones refracted from the front part of the cornea.

$\S 17$. The power of altering the refracting conditions of the eye, so as to enable the media to form a single perfect image on the retina, for varying distances of the object, is called its porver of "accommodation" or adjustment. Plainly such adjustment of the eye caunot take place, like that of a camera obscura, by changing to any appreciable extent the distance of the lens from the screen on which the image is formed. It must therefore take place, either by increasing the indices of refraction of the media of the eye, or by increasing the curvature of one or more of the refracting surfaces. It is now known to be due to changes in the convexity of the lens, principally, if not wholly, of its anterior surface. The posterior apex of the lens remains unmoved. There are several methods of experiment which demonstrate that in accommodation for near distances the front of the lens becomes more strongly arched. When accommodation is taking place, the pupil may be seen not only to contract, but also to draw its edge forward. Helmholtz calculated the amount of this forward movement for two cases at about $\frac{1}{80}$ and $\frac{1}{59}$ of an inch, respectively. Moreover, by an ingenious contrivance the image reflected from the anterior surface of the lens may be watched as it becomes smaller and more distinct on adjustment for near distances, thus showing that the surface from which it is reflected has increased its curvature.

It is obvious that the mechanism for adjusting the eye must be under the brain's control, since adjustment is voluntary; and that it must consist of muscles which lie within the eyeball. The accepted hypothesis concerning the nature and action of this mechanism was first proposed by Helmholtz. This investigator assumes that the lens, when the eje is at rest, does not have the form which corresponds to a condition of equilibrium in its own elastic porwer. If it were not held in by its surroundings, it would be more arched than it is both before and behind. But it is kept flattened by the radial tension of the suspensory ligament; when this tension is withdrawn the lens becomes curved by the action of its own elasticity. The withdrarral of the tension is accomplished by the action of the ciliary muscle, the fibres of which have their point of fixation at the edge of the cornea, and run from here in the direction of a meridian toward the equator o fthe eye. When the ciliary muscle con- 
tracts, the free ends of its fibres are drawn toward its fixed ends on the edge of the cornea; the radial tension of the suspensory ligament is thus relaxed, and the lens is allowed to assume its natural form under the equipoise of its own elastic forces.

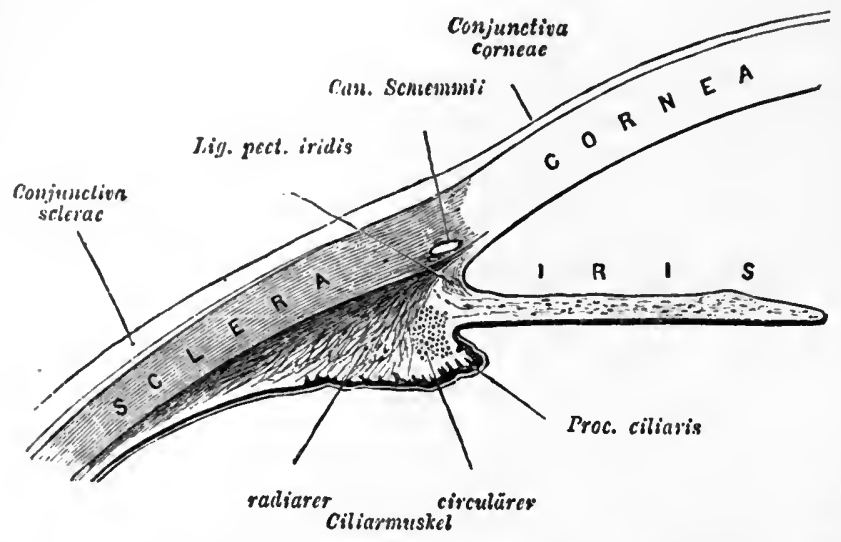

FIG. 53.-Sectional View of the Connections of the Cornea, Ciliary Mascle, Ciliary Processes, etc. $30 \%$. (Gegenbaur.)

The occulo-motor nerve furnishes the fibres that serve the ciliary muscle; these fibres run in the posterior strands of its roots. Their central place of origin is in the posterior part of the floor of the third ventricle ; stimulating the front division of this part produces accommodation of the lens; stimulating the back division of the same part produces contraction of the pupils. Stimulation still further back, where the third rentricle passes into the aqueduct of Sylvius, produces contraction of the internal rectus muscle of the eye ; and the innervation of this muscle is, of course, regularly connected with adjustment for near distances. Thus all the mechanism of accommodation, both that of the central organs and that of the end-organs, is made to work together for the production of an image upon the retina.

$\S 18$. Given the formation of the image upon the retina, it is further required in order to vision that this physical process should be changed into a physiological process. We now examine briefly the mechanism by which such a change is accomplished. [The reader is referred to the larger specific treatises for the detailed theory of the schematic, the emmetropic, the myopic, and the hypermetropic eye.] The retina, or inner tunic of the eye, contains the nerrous elements by whose action the system of refracted rays 
is changed into a mosaic of nerve-commotions. But light does not act as a stimulus to the nervous substance, either fibres or cells, unless it have an intensity which is nearly deadly to that substance. Since we are able to see the feeblest rays of the moon as reflected from white paper, the nervous excitation which is the condition of vision cannot be produced by the direct action of light on the nerve-fibres or nerve-cells of the eye. A photo-chemical substance and process, as well as a special end-apparatus, seems therefore to be necessarily involved in the problem which is given to the retina to solve.

$\S 19$. The nervous and other elements of the retina are arranged

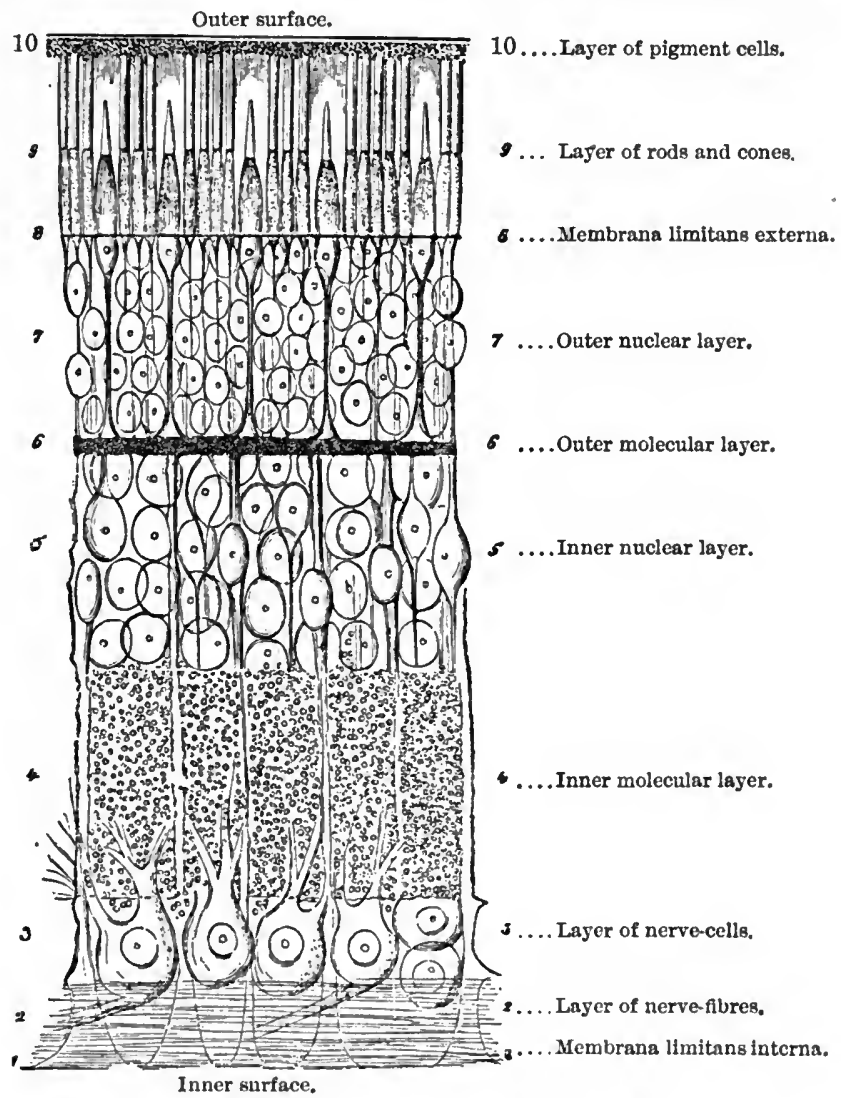

Fi6. 54.-Diagrammatic Section of the IIuman Retina. (Schultze.)

in the following ten layers, counting from within outward and backward: (1) the membrana limitans interna, which is the retinal 


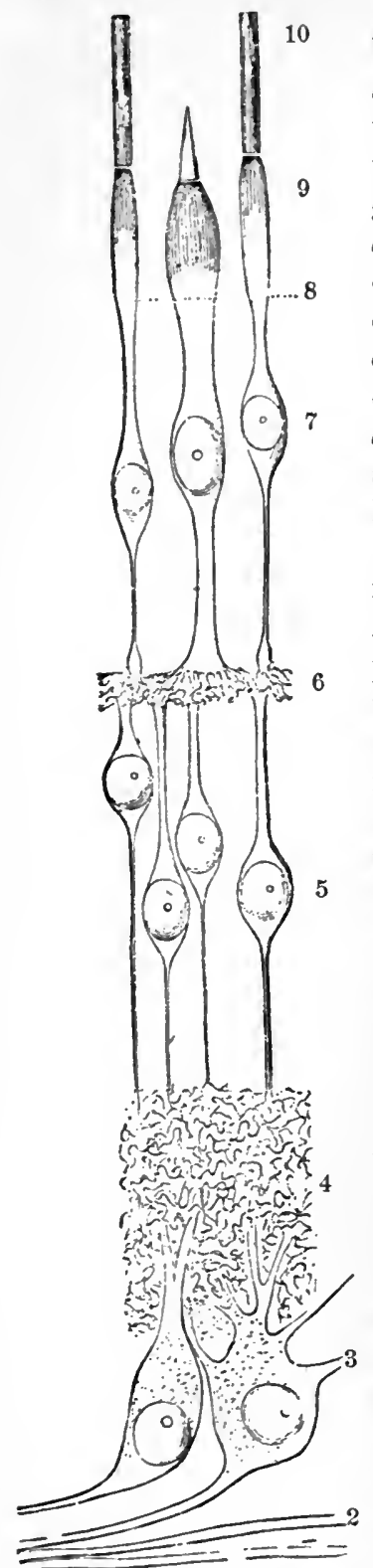

border toward the vitreous body; (2) the layer of optic nerve-fibres distributed from the papilla where this nerve breaks in through the tunics of the eye; (3) the ganglion-cell layer ; (4) the inner molecular layer; (5) the inner nuclear layer; (6) the outer molecular layer; (7) the outer nuclear layer; (8) the membrana limitans externa; (9) the bacillary layer, or layer of rods and cones; $(10)$ the pigment-epithelium layer. The membranes (Nos. (1) and (8)) are not really uninterrupted layers, but an extremely fine network.

By no means all the retirfal substance is nervous. Indeed, the numerous radial fibres (fibres of Müller) which seem to penetrate its entire thickness are now lield to be in great part elements of the supporting tissue; moreover, the whole connective substance is a kind of sponge-like tissue, in the gaps of which the true nerrous elements lie embedded. The gaps thus filled are especially large in the second, third, fifth, and seventh layers.

A description of the undoubtedly nervous elements of the retina includes the following particulars: (a) The retinal fibres of the optic nerve lie parallel to the surface, are non-medullated, and extremely fine ; in general, they are arranged in ray-like bundles, radiating on all sides from the place of the entrance of the nerve. The arrangement is special at the yellow spot, so as to surround, and not cover it. This nerve-fibre layer is thickest at the papilla of the retina, and diminishes continuously from this spot toward the ora serrata; at about one-third of the distance it becomes single. (b) The ganglion-cells, which form the principal part Frg. 55.-Diagrammatic represen- of layer No. 3, like the multipolar cells of tation of the Connections of the the rest of the cerebro-spinal system,
Nerve-flures in the Retina. (Schultze.) The numbers have have one large process of more trans-
the same reference as in Fig. 54 . 
lucent appearance. This process subdivides into fibrils of vanishing fineness, that enter and are lost in the next layer. At the yellow spot these cells are eight or ten deep; from this centre they diminish toward the ora serrata, where spaces are found between the cells. (c) The nerrous elements of the inner molecular layer (No. 4) are not clearly made out. They probably consist of extremely fine filaments, which are connected with the external processes of the ganglion-cells. (d) Most of the nucleus-like bodies of the inner nuclear layer (No. 5) are probably nervous. Each such body has two processes-one directed inward, the other outward. The former is thought to be connected with the filaments of the inner (No. 4), and the latter with those of the outer (No. 6) molecular layer. (e) In the outer molecular layer (No. 6) are nervous filaments, like those in No. 4, which are probably connected with the external processes of the inner nuclear layer. Here are also found numerous starshaped cells probably not nervous. $(f)$ In the outer nuclear layer (No. 7) the undoubtedly nervous elements preponderate. Each nucleus-like body in this layer is connected by a radial fibre with one of the nervous elements of the rod-and-cone layer (No. 9). These nuclear bodies are called rod-granules and cone-granules respectively, and are to be distin- Fra. 56.-Diagrammatic Section of the guished, not only by their connection with these elements, but also by their size and position; the latter are larger, and lie on the more external side of the layer. $(g)$ The layer of

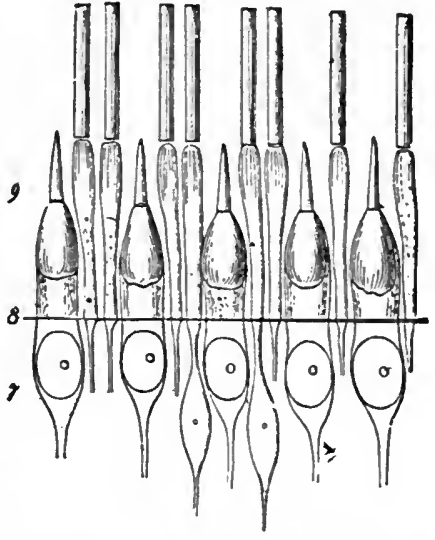
Posterior Part of the Retina of a Pig. $60 \%$. (Schnltze.) 7, part of outer ra. clear layer: 8 , membrana limitans externa; 9 , rods and cones. Each of the cones. which are in very close apposition, contains in its inner segment a highly refractile body, the function of which is nnknown. rods and cones (No. 9) consists of a multitude of elongated bodies arranged side by side, like rows of palisades, with their largest extension in the radial direction. These bodies are of two kindsone cylindrical, and called "rods of the retina," the other rather flask-shaped, and called "cones of the retina."

The rods extend the entire thickness of the layer, and are about $\frac{1}{350}$ inch in length, but the cones are shorter; the rods are about $\frac{1}{14000}$ inch in diameter, the smallest cones of the central depression $\frac{1}{10000}$ inch. The inner ends of both are continuous with the rod-fibres and cone-fibres of the outer nuclear layer. 
Each rod or cone is composed of an inner and outer segment or limb; the latter is highly refractile, the former only feebly so. The inner limbs appear under the microscope like a mass of protoplasm. The appearance of a most delicate longitudinal line in the inner and outer segments has led to the belief that a nervefibril is, as it were, drawn through their axis. The description

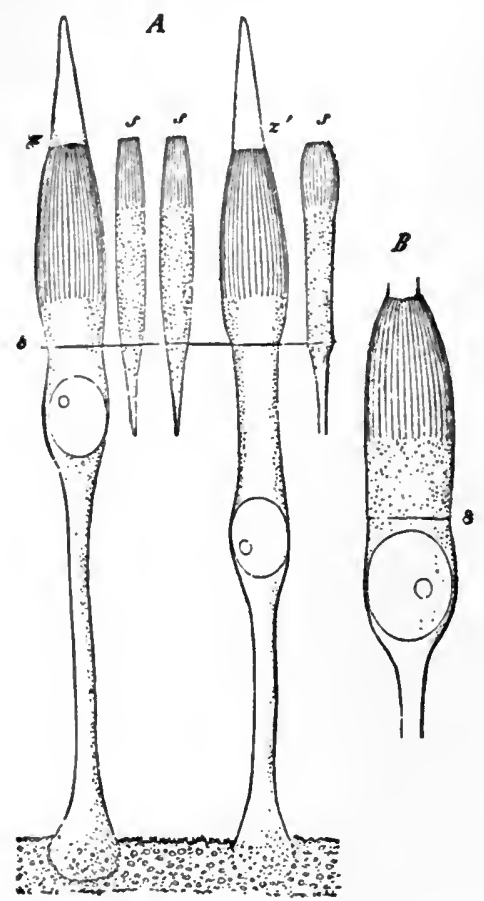

Fia. 57,-Rods and Cones of the Human Retina. (Schnltze.) $A$, showing inner segments of the rods, $s \& 8$, and of the cones, $z z^{\prime}:$ the latter in connection with the cone-nuclei and fibres as far as the onter molecular layer. $800 / . B$, inner segment of a cone with a cone-nucleus. $120 \%$. C, folated interior portion of a cone.

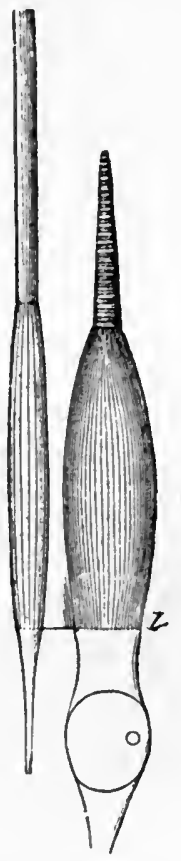

Fia. 58. - Rod and Cone from the Hinman Retina, preserved in perosmic acid, showing the fine fibres of the surface and the different lengths of the internal segment. $100 \%$. (Sehnltze.) The outer segment of the cone is broken into disks which are still adherent.

of the two shows that there is no essential anatomical difference between the rods and cones; nor are we able to distinguish any difference in their physiological significance. The distribution of the two elements is different for different parts of the retina. In the yellow spot only cones appear, but these are of more slender form, and of increased length, so that not less than one million are supposed to be set in a square $\frac{1}{10}$ inch $;{ }^{1}$ while not far from this

'See Le Conte, Sight, p. 58. New York, 1881. 
spot each cone is surrounded by a crown-shaped border of rods. Toward the ora serrata the cones become continually rarer. In close connection with the rods and cones stand the cells of the pigment-epithelium. These cells form a regular mosaic of flat, sixsided cells, which send out pigmented processes between the outer limbs of the rods and cones.

The fibres of the optic nerre are supposed to be
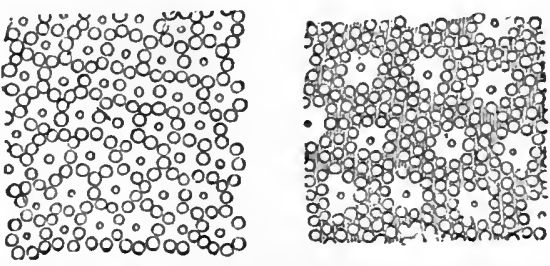

Figs. 59 and 60.-Superficial Aspect of the Arrangement of the Rods and Cones in the Retina. $80 \%$. (Schnltze.) The former is from the region of the macula lutea: the latter from the periphernl region. connected with the rods and cones by means of the ganglion.cells, and of the radial fibres in which the granules of the outer and inner nuclear layers are embedded.

$\$ 20$. Two minute portions of the inner surface of the retina require to be distinguished from the rest of its area ; the yellow spot (macula lutea) and the "blind spot" (papilla optica). The yellow spot

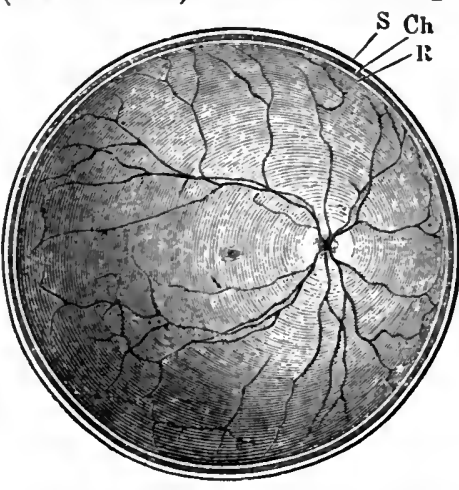

FIG. 61.-Equatorial Section of the Right Eye, showing the Papilla of the optic nerve, the Blood-vessels radiating from it, and the Macula lutea. $2 / 1$. (Henle.) S, sclerotic; $\mathrm{Ch}$, choroid; and $\mathrm{R}$, retina. is of oval shape, about $\frac{1}{16}$ of an inch in its long diameter, and has in the centre a depression called the fovea centralis. It is the place of clearest vision, and the physiological centre of the eye. About $\frac{1}{6}$ of an inch inside the eye from the middle of the yellow spot is the middle of the papilla, or place where the optic nerve breaks into the retina. The blind spot, or portion of the retina which can be experimentally shown to be inoperative in vision, has been proved by Helmboltz to correspond in both size and shape to that corered by this papilla. Its diameter is about $i_{12}^{1}$ or $\frac{1}{15}$ of an inch, varying considerably for different eyes. It is wanting in all the nervous elements.

\$ 21. In answer to the question, What elements of the retina are directly affected by the light? both anatomy and physiology refer to the layer of rods and cones. This layer alone possesses that mosaic nervous structure which appears to correspond to the demands made upon the end-apparatus of vision. It can be demon- 
strated that the waves of light pass through the structure of the retina, and that the nervous process must begin in the back part of this structure. Indeed, it is possible, by an experiment (devised by Purkinje), to perceive with one's own retina the aborescent figure formed by the shadow of the blood-ressels expanded upon its front part.

$\S 22$. We have already seen (Chapter I., $\S \S 14,15)$ that a chemical process may reasonably be conjectured to accompany the action of the nerves in general. Undoubtedly a photo-chemical process is concerned in vision. But after all the careful researches of many observers, especially of Kühne ${ }^{1}$ and his pupils-it is difficult to point to any results of chemical investigation which serve better to define the exact nature of the physiological action of the end-organs of the eye. The relation of the light to any chemical processes which may take place in the gray substance of the retina can be only indirect. The opto-chemical hypothesis must, therefore, regard the epithelial cells, with which the end-fibrils of the optic nerve are in physiological connection, as the bearers (Träger) of certain photo-chemically decomposable materials or visual substances (Sehstoffe) ; these substances, however, cannot excite chemically the irritable part of the visual cells-the protoplasm of the inner limbs of the rods and cones-without being themselves decomposed. Visual substance is necessarily some kind of matter easily decomposable by light, or chemically sensitive to light. The first process, then, in the excitation of the optic nerve, is the decomposition by the light of some substance found in certain epithelial elements of the retina. The second process is the action, as visual excitants (Sehreger), of the decomposition-products of the epithelial cells upon the protoplasm of the end-organs. But in order that such decomposition-products may act as excitants of the end-organs of vision, the visual substance must be rightly placed-that is, it must be in local connection with the protoplasm of the outer limbs of the rods and cones. The relation of the two last layers of the retina is such as to secure this necessary connection. We are as yet unable, however, to say what are the visual substances which the successful working of the opto-chemical hypothesis demands. The location of the pigmentum nigrum, and the changes produced in it by light, favor the conjecture that this substance is of the most fundamental and general importance for visual sensations. Visual purple may also be supposed to be a visual substance. The fact that light of different wave-lengths effects changes in this pig-

' The few statements here given are taken for the most part from the article of this investigator in Hermann's Handb. d. Physiol., III., i., pp. $235 \mathrm{ff}$. 
ment with different degrees of speed, suggests the view that it is related to the susceptibility of the eye for different colors. But since invertebrates do not have the visual purple; since the cones (a thing which no one doubts) see without this purple, and since the rods of some animals, such as hens and doves, and the rods of the ora serrata, perform their functions without it, this pigment can scarcely be said to be the only visual substance. The opto-chemical hypothesis, then, seems to require several colored visual substances. Moreover, since animals can see with bleached retinas, and albinos have the power of vision, we are compelled to assume also a colorless visual pigment. As to the nature of the chemical changes necessary to be produced in the protoplasm of the outer limbs of the rods and cones by the action of the decompositionproducts of the visual substances, we are quite ignorant.

$\S 23$. The end-organ of hearing is the Ear. But in this case, as in that of the eye, a very large part of the apparatus of sense is significant simply as a contrivance for applying the stimulus to the true end-organ, to the differentiations of epithelial cells and nervous cells connected with the terminal fibrils of the sensory nerve. The entire human ear consists of three parts, or ears ; namely, the external ear, the middle ear, or tympanum, and the inner ear, which is also called the "labyrinth," from its complex construction.

I. The External Ear-exclusive of the cartilaginous plate which is extended from the side of the head-consists of (a) the concha, a deep hollow, and (b) the external meatus, or passage leading from the bottom of this hollow to the drum of the ear. The concha is probably of little or no use in sharpening our perceptions of sound; for if a tube be inserted so as to secure a canal for the air to the drum of the ear, the entire concha may be filled with wax, and the result is to increase rather than diminish the sharpness of the sound. It is possible, however, that vibrations of more than one thousand in a second are concentrated by reflection ${ }^{1}$ from the concha. The external ear appears to be of some service in perceiving the direction of sound. Rinne's experiments seem to show that-as Harless ${ }^{2}$ thought--the cartilage of the ear can be thrown into sympathetic vibration with certain acoustic waves, and so reinforce the sound. At best, however such work done by the concha is small.

The most patent office of the external meatus is the protection of the ear-drum ; the passage is so curved that the drum cannot be

' See Hensen. Physiologie d. Gehörs, in Hermann's Handb. d. Physiol., III., ii., p. 23.

2Article Hören, in Wagner's Handwörterbuch d. Physiol., IV , 1853. 
reached from the outside in a straight line. Helmholtz called attention to the fact that certain tones of a high pitch resound strongly in the ear when the meatus is of normal length, but cease so to resound when its length is increased artificially. The meatus probably, therefore, modifies certain tones by its own resonant action-strengthening the high ones, and deadening the low, in some degree.

Various simple experiments-such as placing a resounding body in contact with the teeth-prove that the surrounding cranial bones conduct sound to the ear. It is probable, however, that the path of such conduction is not, for the most part, as was formerly supposed, directly to the inner ear by way of the cranial and petrous bones, but indirectly, through the ear-drum and bones of the middle ear to the fenestra ovalis. The amount of direct conduction possible, has not as yet been determined precisely.

$\S 24$. II. The Middle Ear, or Tympanum, is a chamber irregularly cuboidal in form, and situated in the temporal bone, between the bottom of the meatus and the inner ear. Its outer wall is (a)

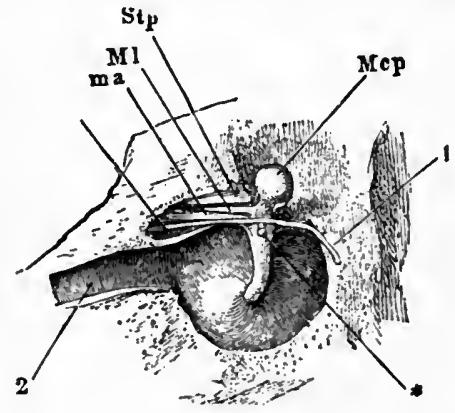

Fio. 62.-Drnm of the Right Ear with the Hammer, seen from the inside. $2 / 1$. (Henle.) 1, chorda tympani ; 2, Eustachian tube; *, tendon of the tensor tympani muscle cut off close to its insertion : $m a$, anterior ligsment of the malleus; if c p, its head; and II l, its long process. S t p, Spina tympanica posterior.

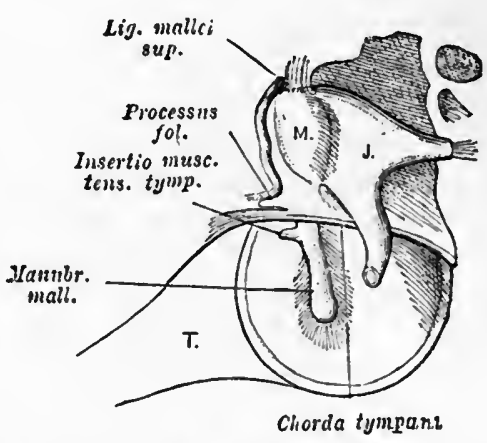

Fra. 63.-Side Wall of the Cavity of the Tym. panum, with the Hanmer (M) and the Anvil (J). The former shows the connection of its handle with the drum. T, Fustachian tube. $3 / 1$. (Gegenbaur.)

the membrana tympani, which consists of three layers-an external tegumentary, an internal mucous, and the intermediate membrana propria, composed of unyielding fibres arranged both radially and circularly. In the inner wall, which separates the tympanum from the labyrinth, are two openings or windows-the fenestra ovalis, which corresponds to the vestibule of the labyrinth, and the fenestra rotunda, which corresponds to the tympanic passage in the cochlea. Near its anterior part the tympanum opens into $(b)$ the Eustachian 
tube, a canal which communicates with the nasal compartment of the pharynx.

(c) The auditory bones are three in number, called Malleus, Incus, and Stapes, and arranged so as to form an irregular chain stretched across the cavity from the outer to the inner wall of the tympanum. The malleus has a head, separated by a constricted neck from an elongated handle; its handle is connected with the centre of the membrana tympani ; its head articulates with the incus. The incus has a body and two processes. On the front surface of the body is a saddle-shaped hollow, in which the head of the malleus fits; the short process is bound by a ligament to the posterior wall of the tympanum ; the long process ends in a rounded projection (os orbiculare) through which it articulates with the stapes. The stapes, or stirrup-shaped bone, has a head and neck, a base and two crura. The head articulates with the incus; from the constricted neck the two crura curve inward to the base, which is attached to the fenestra ovalis. These bones are moved on each other at their joints by $(d)$ two or three small muscles-the tensor tympani, the stapedius, and, more doubtfully, the laxator tympani. The first of these is inserted into the malleus, near the root, and serves to tighten the tympanic membrane by drawing the handle of the malleus

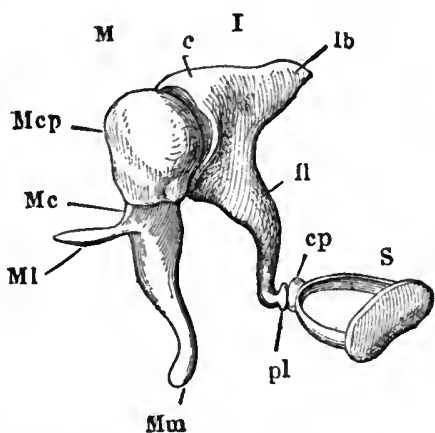

Fra. 64. - Bones of the Ear, as seen in their connection from in front. 4/1. (Henle.) I, Incus (anvil), of which Ib is the short, and II the long, process; $c$, its borly, anil pl, the process for articulation with the stapes (processus orbicularis.) M, Malleus (hammer), of which Mc is the neck, MIcp the head, II the long process, and IIm the mannbrinm; S, stapes (stirrup), with its capitulum, cp. inward; the stapedius is inserted into the neck of the stapes, but its function is doubtful-apparently it draws the stapes from the fenestra ovalis, and so diminishes the pressure of the chain of bones in that direction. The laxator tympani is inserted into the neck of the same bone, and its action has been supposed by some to be antagonistic to that of the tensor tympani; but its muscular character is now denied by most observers.

$\S 25$. The general office of the tympanum may be described as that of transmitting the acoustic waves to the inner ear, while at the same time modifying their character. Some modification is necessary in order that these waves may occasion such vibrations in the elements of the inner ear as shall be adapted for the excitation of its end-organs. The acoustic motion of the molecules of air, in the 
form in which it reaches the ear-drum, has a large amplitude, but a small degree of intensity. This motion must be changed into one of smaller amplitude and greater intensity ; and it must be transmitted, with as little loss as possible, to the fluids of the labyrinth. The transmitting vibrating media must also have the power of answering to the different tones of any pitch perceptible by the ear. The description of the manner in which this apparatus of membrane and bones solves so complicated a mechanical problem belongs to the physics of anatomy; it has been worked out with great detail by Helmholtz and others, although certain points still remain unsolved. We can here only indicate one or two particulars.

A flat membrane, evenly stretched, whose mass is small in proportion to the size of its superficies, is easily thrown into vibration by the impact of acoustic waves upon one of its sides. Such a membrane responds readily to tones which approach its own fundamental tone; but if divergent tones are sounded the membrane is unaffected. A motion which consists of a series of harmonious partial tones cannot then be repeated by such a membrane in the form in which the air brings it. If, then, the membrane of the tympanum were not so arranged and connected as to have no preponderating tone of its own, it could not be the medium of our hearing a great variety of tones. The property of taking up with the vibrations, as it were, of a large scale of tones is secured for the tympanum by its funnel-shaped form and by its being loaded. It is contracted inward into a depression of the right shape by means of the handle of the hammer; it is therefore unequally and only slightly stretched, and has no fundamental tone. It is also loaded with the auditory bones, which deprive it of every trace of such a tone and act as dampers to prevent long-continued ribrating. Moreover, since the apex of its funnel bulges inward, the force of the vibrations from all sides is concentrated in vibrations of greater intensity in the centre, where it is spent in setting the chain of earbones in motion.

The acoustic vibrations of the auditory bones, which are occasioned by the movements of the ear-drum, are not longitudinal, but transverse; they do not, however, resemble the vibrations of a stretched cord or a fixed pin. They do not vibrate by reason of their elasticity, but like very light small levers-vibrating as a system, with a simultaneous motion around a common axis. Direct observation of these bones in motion shows that their sympathetic vibrations vary greatly for tones of different pitch and sinilar intensity, from a scarcely observable motion to a surprisingly great elongation. 
The effect of the muscles of the tympanum upon the transmission of tones of different pitch is not as yet clearly demonstrated. In general, the stretching of the tensor muscle, within the limits which have thus far been investigated, seems to weaken the higher much less than the lower tones. But the tension of the drum under the influence of this muscle does not indicate the slightest change on passing from low to high tones. The stretching of the tendon of the stapedius muscle lias no observable influence on the acoustic vibrations of the tympanum.

$\S 26$. The Eustachian Tube, when in its normal position, is neither closely shut nor wide open. Its office is to effect a renewal of the air in the tympanum, to maintain the equilibrium of atmospheric pressure on both sides of the tympanic membrane, and to convey aray the fluids which collect in the tympanic cavity. If it remained open, so as to permit the acoustic waves of the air from the mouth to enter, our own voices would be heard as a roaring sound, and the passage of air inward and outward during respiration would affect the position and tension of the tympanic membrane. That it is opened, however, on swallowing, Valsalva proved two centuries ago. For if we keep the nose and mouth closed and then swallow, with the cheeks blown violently out, a feeling of pressure is felt in the ears and the hearing is weakened. These effects are due to the forcing of the air through the Eustachian tube into the tympanic cavity. The tube is thus of indirect service in respect to the physiological functions of the middle ear.

§ 27. III. The Internal Ear, or Labyrinth, is the complex organ in which the terminal fibrils of the auditory nerve are distributed and the end-organs of hearing situated. It lies in a series of cavities channelled out of the petrous bone. It consists of three parts -the Vestibule, the Semicircular Canals, and the Cochlea. In each osseous part a membranous part is suspended, corresponding to it in shape, but filling only a small portion of the bony cavity which contains it. It is in the labyrinth that the acoustic waves transmitted by the tympanum are analysed and clianged from a physical molecular process to a nerve-commotion, by the special endapparatus of hearing.

(A) The Vestibule is the central cavity of the internal ear ; it is the part of the labyrinth which appears first in animals and is most constant. The membranous vestibule is composed of two sac-like dilatations-the upper and larger of which is named utriculus, the lower sacculus. In its outer wall is the fenestra ovalis; its anterjor wall communicates with the scala vestibuli of the cochlea, and at its posterior wall the fine orifices of (B) the Semicircular Canals open 
into the utriculus. These canals are three in number, are bent so as to form nearly two-thirds of a circle, and are about an inch in length and ${ }_{2}^{\frac{x}{0}}$ of an inch in diameter. They are called the supe-

No. 1.

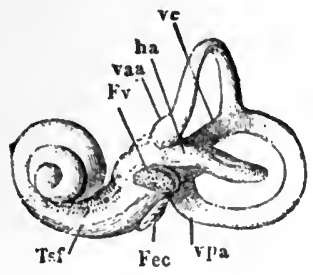

No. 2.

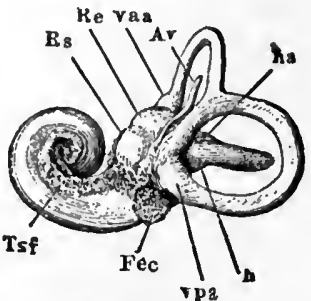

No. 3.

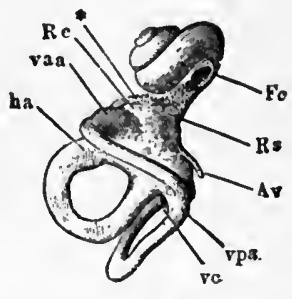

Fia. 65. No. 1. Osseons Labyrinth of the Left Ear, from below; No. 2, of the Right Ear, from the inside; No. 3, of the Left kar, from above. (Henle.) Av, aqueduet of vestibule; Fc. fossa of the cochlea; Fec, its fenestra (rotunda); Fv, fenestra of the vestibule (ovalts); ha, external ampulla; h, external semicircular canal; Tsf, tractus spiralis foraminosus; va, ampulla of the superior semicircular canal; vc, posterior semicircular canal ; and vpa, its ampulla.

rior, the posterior or vertical, and the external or horizontal canals. The coutiguous ends of the superior and posterior canals blend together and have a common orifice into the vestibule. They all

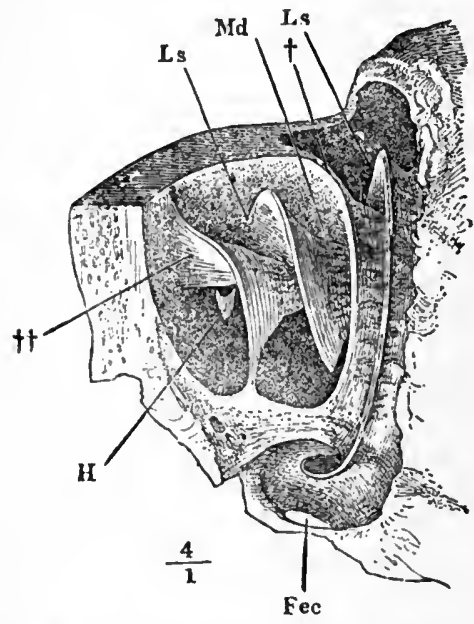

Fia. 66.-Osseons Cochlen of the Right Ear, exposed from in front. $4 / 1$. (Henle.) t, section of the division-wall of the eochlea; $t+$, npper end of the same. Fec, Fenestra; H, hamulus ; MId, modiolus; Ls, lamina spiralis.

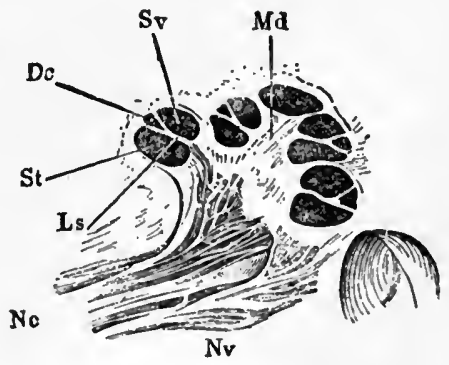

Fia. 67.-Cross-section throngh the Aconstic Nerve and the Cochlea. $\% / 1$. (Henle.) Nc, nerve of the cochlea; $N v$, jerve of the vestibule; $\mathrm{St}$, scala tympanl; Sv, scala vestibuli ; and between them the ductus cochlearis, Do, Ls and Md, as in preceding figure.

have a regular relative position, their planes being at right angles to each other. Near the vestibule they dilate to about twice their average dimmeter and form the so-called ampulloe. Both the osseous 
vestibule and the osseous canals contain a fluid (the perilymph), in which the membranous vestibule and canals are suspended; the membranous labyrinth is also distended with a similar fluid (the endolymph).

(C) The Cochlea is by far the most complex part of the labyrinth ; it is about $\frac{1}{4}$ of an inch long, and is shaped like the shell of a common snail. It, too, consists of a membranous sac embedded in the osseous cavity. The whole passage of the cochlea is imperfectly divided into two canals by a partition-wall of bone, which is wound $2 \frac{1}{2}$ times around an axis (the modiolus), from the base to the apex, somewhat like a spiral stair-case. It is called the osseous lamina spiralis. Of the two canals or passages thus formed, the one which faces the base of the cochlea is called the scala tympani; since it has its origin in the circular aperture (fenestra rotunda) which leads to the tympanic cavity. The other, which faces toward the apex, opens into the restibule, and is called the scala restibuli. At the apex of the cochlea these two scalix communicate with each other through a small hole (helicotrema). The division of the mem-

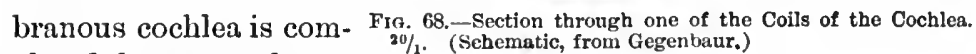
pleted by a membrane

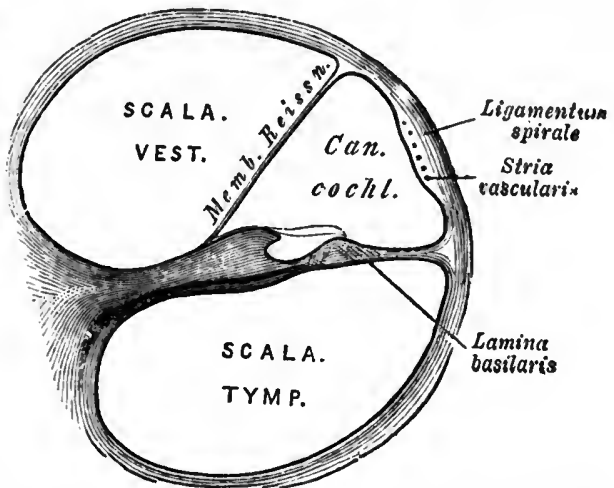
(the basilar membrane, or membranous spiral lamina), which bridges the interval between the free edge of the osseous spiral lamina and the outer wall of the passage ; it is attached to this wall by the spiral ligament. Another membrane (the membrane of Reissner) arises from a spiral crest (limbus, or crista spiralis) attached to the free edge of the osseous lamina, and extends to the spiral ligament, so as to form a small aqueduct between it and the basilar membrane (the scala intermedia, or ductus cochlearis, or canal of the cochlea). It is in the vestibule, in the ampulle of the canals, and in the scala intermedia that the nervous end-organs of hearing are to be found.

$\S 28$. The auditory nerve, on approaching the labyrinth, divides into a vestibular and a cochlear division. The former enters the restibule and subdivides into five branches-one for the utriculus, one for the sacculus, and one for each of the three ampulla. In 
each of these dilatations the membranous wall forms a projecting ridge, called the crista acoustica. The endothelial investment of the crista is elongated into columnar cells, intercalated between which are fusiform cells. Each of the latter, according to Max Schultze, and others, has the peripheral and the central process with which we are already familiar in the nerve-cells of other endorgans of sense. The peripheral process projects into the endo-

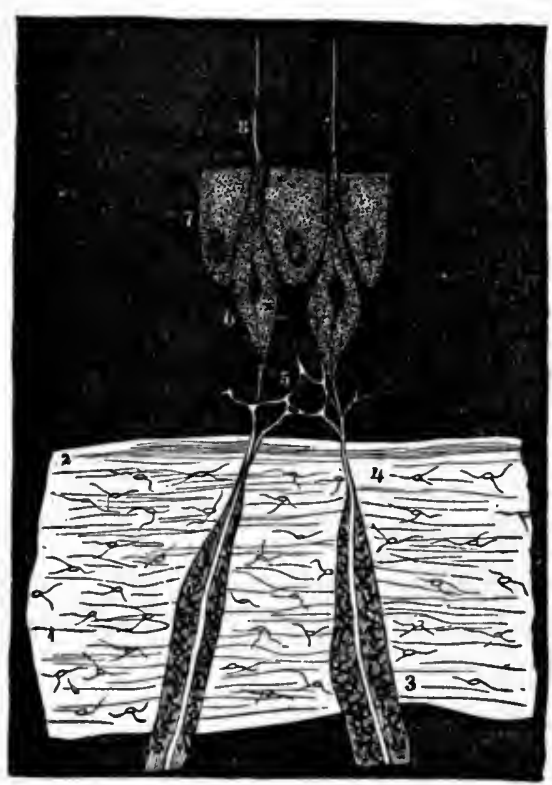

Fra. 69.- Scheme of the Nerve-endings in the Ampullæ. (After Rüdinger.) 1, membranous wail of the anupulle, with a structureless border. 2 ; through which the nervefibre, 3 , sends its axis-cylinder, $4 ; 5$, plexiform connection of the nerve-fibres; 6 , auditory cells; 7 , supporting cells ; 8 , auditory hairs.

\section{timate ends, however, be-} stones" (otoliths) appear in both saccule and utricle, embedded in a soft matrix and lying in contact with the nerve-epithelium. In the restibule the hair-like prolongations of the epithelial cells are more scanty than in the ampullæ.

$\S 29$. The terminal nerve-apparatus of the cochlea is even far more complicated and remarkable. The cochlear branch of the auditory nerve pierces the axis of the cochlea (modiolus) and gives off lateral branches which pass into the canals of the osseous spiral nembrane. Here they radiate to the membranous spiral lamina, and are connected with a ganglion of nerve-cells ; beyond the gan- 
glion they form a plexiform expansion, from which the delicate fibrils-losing their medullary sheath and becoming extremely fine axis-cylinders-pass through a gap in the edge of the lamina into the organ of Corti. The connection of their ultimate fibrils with the cone-cells of this organ may be assumed, but is difficult to demonstrate.

The organ of Corti is situated on that surface of the basilar membrane which is directed toward the ductus cochlearis. Its structure is a wonderful arrangement of cells. Some of these cells are curved, elongated, and placed in two groups-an inner and an outer. They are called the "rods," or "pillars," or "fibres of

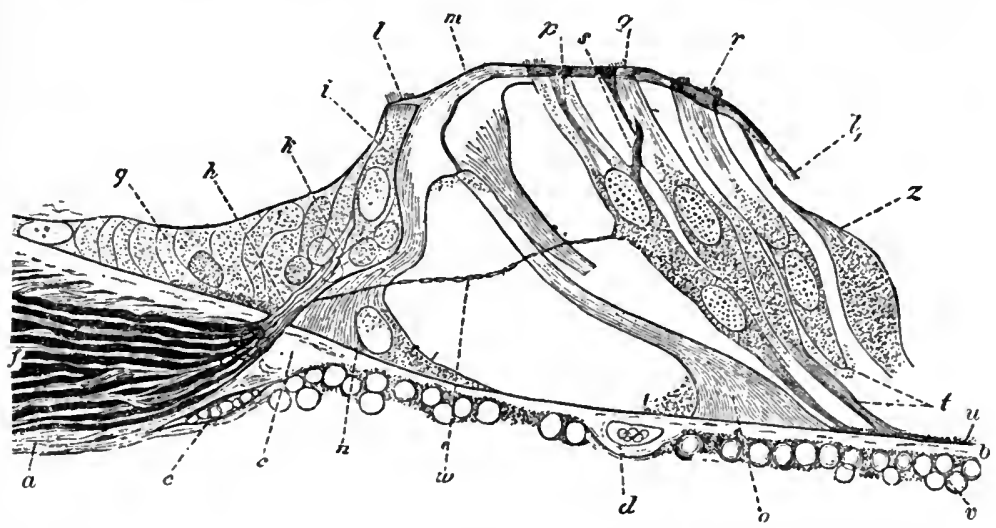

FIG. 70.-Organ of Corti in the Dog. $80 \%$. (Waldeyer.) b-c, homogeneous layer of the basilar membrane; $u$, its vestibular layer; $v$, its tympanal layer; $d$, blood-vessel; $f$, nerves in spiral lamina; $g$, epithelium of spiral groove: $h$, nerve-fibres passing toward inuer hair-cells, $i, \lambda ; l$, auditory haurlets on inner hair-cells; $i-d_{l}$, lamina reticularis; $m$, heads of the rods of Corti jointed together; the inner rod seen in its whole length; the outer one broken off; $n$, cell at base of inner rod; $p, q, r$, outer hair-cells; 8 , a cuticular process probably belonging to a cell of Deiters: $t$, lower ends of hair-cells, two being attached by cuticular processes to the basilar membrane; $v 0$, a nerve-fibril passing Into an outer hair-cell; $\varepsilon$, a sustentlcular cell of Deiters.

Corti." The cells of the inner group rest by a broad foot on the inner part of the basilar membrane, project obliquely forward and outward, and expand into a dilated head; the cells of the outer group rest in the same way, incline forward and inward, and fit into a depression in the head of the cells of the inner group. The two thus make a bow, which arches over an exceedingly minute canal (the canal of Corti) formed between them and the basilar membrane. These rods of Corti increase in length from the base to the apex of the cochlea. The basilar membrane is composed of fibres arranged in a transverse direction, so that each rod rests upon one, or upon a pair of these fibres. Internal and almost parallel to the inner group of rods is a row of compressed conical 
cells with short and stiff lair-like processes (inner hair-cells). External and almost parallel to the outer group are four or five rows of hair-cells (outer hair-cells) which are attached to the basilar membrane, while their other extremity projects as a brush of hairs through the reticular membrane (membrane of Kolliker). This latter membrane is a very delicate framework, perforated with holes, through which the hairs of the outer hair-cells project, and which extends from the inner rods to the external row of haircells. It acts as a support for the ends of these cells. The interval between the outer hair-cells and the spiral ligament is occupied by cells of a columnar form (the supporting cells of Hensen). The organ of Corti is covered over and separated from the endolymph of the ductus cochlearis by the so-called membrana tectoria.

$\S 30$. The problem before the labyrinth of the ear is in part the same as that solved by the tympanum, namely, the problem of conveying the acoustic waves to the true end-apparatus of hearing. The repeated shocks of the stirrup at the fenestra ovalis-and perhaps, in far less degree, the pulsations of air at the fenestra rotunda-produce waves in the fluid of the labyrinth. Any molecular oscillations of this fluid, thus occasioned, cannot, however, act directly as the appropriate stimulus of the sensations of sound. Since the dimensions of the whole mass thrown into vibration are so small in comparison with the length of the acoustic waves that the extension of the shock from the stirrup would be practically instantaneous tllroughout, and since the surrounding walls may be regarded as absolutely immovable by any such impact, the labyrinth-water would act as an incompressible fluid. It would, therefore, be unsuitable for the transmission of various kinds of acoustic wares. But different parts of the labyrinth are capable of yielding to the waves in the fluid caused by the repeated shocks of the stirrup. Four such places, into which, as they yield, the fluid of the labyrinth can retreat (as it were) are designated by Hensen ; ' these are the two openings of the aqueduct of the restibule, the mem. branes of the aqueduct of the cochlea, the pores of the blood-ressels in the bone, the membrane of the fenestra rotunda by bulging out into the tympanic cavity. Impulses started in the fluid of the labyrinth would thus result in its movement back and forth, so as to produce a friction of the encl-apparatus. This friction would be increased by the action of the otoliths, or minute calcareous particles, found in the fluid. Thus the waves started at the fenestra ovalis would be diffused over the vestibule and into the scala restibuli of the cochlea, where they would flow to its head, being

'In Hermann's Handb. d. Physiol., III., ii., p. 106. 
prevented by the separating membrane from entering the scala tympani. To what extent these waves flow through the helicotrema, or small hole at the apex of the cochlea, into the scala tympani, and what are the exact relations between the wares in this latter scala and those in the scala restibuli-cannot be stated confidently. Nor can the exact part of the basilar membrane at which the excitation of the end-organs by the oscillations of the structure begins, be indicated with certainty. This membrane is, however, undoubtedly thrown into vibration through the umequal pressure of the moving fluid; and by its vibration it excites the nerrous structures with which it is intimately connected.

§ 31. A still more difficult problem for the labyrinth to solve may be described in one word as a problem of "analysis." The inner ear is not, indeed, contrived so as to reproduce changes in the form of the acoustic oscillations, as such, after the manner in which these changes can be made apparent to the eye or to touch. But all our analogies for the analysis of composite tones-the " clangs" or musical notes of ordinary experience-are derived from the process of sympathetic vibrations. We are led, then, to inquire whether any part of the structure of the enr is capable of enough such sympathetic vibrations to account for the experience which we have in recognizing all the possible degrees of pitch in the scale of musical sounds. The structure must also be such as to receive the impressions produced by a number of simultaneous tones, composing a harmony. Moreover, it must be such as to represent tones that follow each other in rapid succession, as do the notes of a melody. The sympathetic vibratory apparatus of the labyrinth must therefore cease its vibrations immediately upon the cessation of the sounds in sympathy with which it vibrates. In other words, it must either have a damper, or be so constructed as to return at once to a state of rest without such a damper. It must be capable of being thus excited, and of returning to a state of rest, no fewer than five hundred times in a second, since the crackling of electric sparks, between which the interval is no more than .002 of a sccond, can be heard as distinct noises. Still further, the endapparatus of hearing must suffice for all kinds of noise, as distinguished from musical tones; and it is extremely difficult to see how the same apparatus which serves for the analysis of the clang can also suffice for all the various sensations of noise.

The manner is not known in which the auditory hairs and stones and cells of the vestibule and ampullæ, and the rods of Corti, the fibres of the basilar membrane, and the conical hair-cells of Deiters, in the cochlea, actually discharge the required functions. The 
structure of the end-apparatus in the restibule and semicircular canals is plainly not adapted to the analysis of musical tones. The otoliths found in the vestibule, and the hairs of the ampullæ, are not capable of regular sympathetic vibrations; moreover, they form no scale of structures corresponding to the scale of sensations of tone. This fact has led to the assumption that these organs are designed to act as the end-organs of noise instead of musical sound. The more complicated structures of the ductus cochlearis do seem, on the contrary, to be adapted for the required analytic functions. It was first argued by Helmholtz that the bows formed by the rods or fibres of Corti are enough in number to constitute such a scale of structures that this work of analysis can be assigned to them. Some three thousand of these fibres, arranged in rows upon the basilar membrane like the keys of a piano-forte, if distributed over seven octaves would give about thirty-three for a semitone. They might then be supposed to be elastic; and since they differ in size, to be tuned for particular sounds, so that the sympathetic vibration of each one of them corresponds to the sensation of a given tone. But the rods of Corti are stiff and not easily vibratory; and their office is probably simply to constitute a support for the hair-cells. Moreover, birds, which are undoubtedly capable of appreciating musical notes, have no rods of Corti.

Hensen has shown ${ }^{1}$ that the basilar membrane is itself in a good degree graded to pitch; its continuous structure and expansion in size from the beginning to the end of the ductus cochlearis encourage the assumption that its individual radii act like stretched strings to respond to the different tones, from the lowest to the highest. The calculations of Helmholtz have tended to confirm the view of Hensen. It is assumed, then, that the parts resting upon this membrane would be moved up and down, and that the excitation of the conical hair-cells-with which the terminal fibrils of the auditory nerve are supposed to be connected-is thus brought about. The number of the acoustic cells is claimed to be about great enough to correspond to the demands made upon the organ which shall be instrumental in the physical analysis required as a basis for the sensations of musical tones. The claim is at best doubtful. As Hensen himself remarks, ${ }^{2}$ the possibility is by no means excluded that the working of this complicated and delicate apparatus may be altogether different from that conjectured by all such theory. In other words, the physiology of the peripheral mechanism of hearing is as yet in a very incomplete and unsatisfactory state.

${ }^{1}$ Zeitschrift f. wiss. Zool., XIII., p. 481 f.

'In Hermann's Handb. d. Physiol., III., ii., p. $104 \mathrm{f}$. 
§ 32. A brief description of the End-Organs of Motion, or motor end-plates, will suffice for our purposes. In general, the terminations of the efferent nerves are connected either with electrical organs (as, for example, in the torpedo), or with secretory glands, or with the muscular fibre. We consider only the last of these three cases.

After an efferent nerve has entered the substance of the so-called voluntary or striated muscle, it subdivides among the individual muscular fibres, separating these fibres from each other. Such nerve-twigs usually lose their medullary sheath, and their axiscylinder splits up into fibrils, whose exact mode of termination has been much debated. It appears now to be demonstrated (by Kühne, Margo, Rouget, and others) that the axis-cylinder itself pierces the sarcolemma or sheath of the muscular fibre; that the neurilemma becomes continuous with the sarcolemma; ${ }^{1}$ and that the fibrils, into which the axis-cylinder divides, form a flat, branching mass within certain peculiar, disk-shaped bodies situated inside the sarcolemma, and called "motor end-plates." In the non-striated (or non-voluntary) muscles, the nerves divide and subdivide to form more and more minute plexuses of nerve-fibres, which are distributed in the connective tissue that separates the muscular fibres from each other. The exact relation between this extremely minute intramuscular network of fibrils and the nuclei of the cells of muscular "fibre" is not yet made out.

The shape and structure of the motor end-plates are different for different animals, and even for different muscles of the same animal. Indeed, the mode of the termination of the motor nerves in the muscle appears to be somewhat distinctive of the different parts of the muscular structure. Sometimes the axis-cylinders are somewhat enlarged, with strongly granular corpuscles attached or adjacent. Sometimes a granular mass with its nuclei forms a kind of base or floor for the terminal nerve-fibres ; and this eminence may be elongated, elliptical, or circular. But the character and variety of these forms are of no particular interest to psychology, even as approached from the physiological point of view.

1 'The question of histology is debated, whether the neurilemma actually becomes continuous with the sarcolemma. Strictly speaking, according to Kühne, it does not; but then, strictly speaking, it is not continuous with itself. It is, as we have seen ( $(9.36 \mathrm{f})$, divided by the annular constrictions into members which are separate structures. It is to be considered as fringed out on its edge and cemented to the sarcolemma. [See on this subject the monograph. Die Verbindung d. Nervenscheiden mit dem Sarkolemm, Separ ratabdruck aus der Zeitschrift für Biologie, by Kühne.] 


\section{CHAPTER VI.}

\section{THE DEVELOPMENT OF THE NERVOUS MECHANISM.}

§1. The life of the individual man, so far as it can be made an object of immediate observation and scientific description begins as an undifferentiated germ, without apparent distinction of bodily organs or of physical and psychical activities. This living germ undergoes a development. Before it can be subjected to ordinary inspection it has unfolded itself into an elaborate organism; and, in its normal relation to the other systems of this organism (muscular, respiratory, metabolic, reproductive, etc.), the nerrous system has acquired all its complex mechanism, consisting of an indefinite number of parts. What are the different stages of the development of this nerrous system, and what are the laws according to which its different factors and organs become differentiated, it belongs to the science of Embryology to describe. But it belongs to psychology to make such doubtful inferences as suggest themsel res concerning the psychical activities that are to be ascribed to the unfolding mind of the embryo. Psychology, indeed, attempts in such a case to form a picture of those earliest and most obscure mental states, the elements of which can no longer be reproduced or recombined in the developed consciousness of the adult. To this fact is due, in part, the doubt which clings to all such inferences. But this doubt is also due to the fact that embryology itself is so incomplete, even in respect to its possession of single facts, and yet more incomplete in respect to its power to set forth any system of general truths and laws.

Our knowledge of all the earlier states and changes of consciousness is wholly a matter of the interpretation of states and movements of the bodily organism, in terms of our own conscious mental experience. If, then, it were found that certain physical states and motions of the human embryo need for their interpretation the assumption of preceding or accompanying mental states, we should have the right to carry our psychological principles back to the life of this embryo-even back to its beginning in the undifferentiated germ from which the whole development proceeds. 
But as the case now stands, the proper physical science cannot claim to have furnished us with the requisite description of these antenatal-physical movements and states. Little use for the main purposes of Physiological Psychology, therefore, can be made of facts accessible as to the embryonic development of man. We might even seem warranted in passing by the whole subject with two or three general observations like the following: The two-fold life of man, both nervous mechanism and mind, begins in what is apparent only as a physical unity, in that system of moving molecules which constitutes the living germ. Out of this unity, and in indissoluble connection with it, the two-fold human life then progressively develops. The mechanism unfolds itself, increases the complexity of its molecular activities, runs its course of changes, and is broken up again into its material elements. The mind manifests itself in primitive activities, unfolds itself, increases the complexity of its psychical life, and then ceases to make itself known through the physical mechanism, when the mechanism itself is dissolved. And all the while the molecular mechanisin and the mind are most closely and mysteriously correlated in their development as a totality, and in their particular activities.

But in spite of the fact that embryology furnishes psychology with scanty material for any extended and trustworthy conclusions with regard to the earliest activities and development of the mind, at least a sketch of its principal outlines, so far as the nervous system is concerned, seems desirable. Of knowledge from direct observation concerning the early development of the human embryo there is exceedingly little: Yet the comparatively few facts which are indisputably known, throw considerable light upon the nature and functions of the human nervous mechanism. Moreover, in certain most important particulars there is good reason to believe that the earliest history of the development of the embryos of other animals is substantially like that of the human embryo. The very first things in the life of the chick-or better, one of the mammals - for example, may be described as probably holding good in all important respects for the life of man. And when those differences which are most strikingly human begin plainly to appear, they show what parts of the nervous system are most worthy of emphasis as distinctively connected with man's mental life. ${ }^{1}$

$\S 2$. The immature ovarian ovum of the common fowl-like that

${ }^{1}$ The following description is taken to a large extent, and in some places almost rerbatim, from Foster and Balfour's Elements of Embryology, London, 1883, and F. M. Balfour, Comparative Embryology, vol. ii., pp. 177 ff., London, 1881. 
of every other animal-presents the characters of a simple cell. It is seen to consist of a naked protoplasmic body which contains in its interior a nucleus (the germinal vesicle) and within this a nucleolus (the germinal spot). It is enclosed in a capsule of epithelium, called the "follicle," or "follicular membrane." As the ovum matures, the body of it grows in size and a number of granules make their appearance in the interior; while the outermost layer of the protoplasm remains free from them. But as the granules grow larger in the centre, other granules appear also in the periphery of the ovum. The germinal vesicle, during the growth of the ovum, travels toward the periphery where the protoplasm surrounding it remains comparatively free from granules. Accessory germinal spots make their appearance. The cells of the follicular membrane, which were at first arranged in a single row, now become two or more rows deep; and, whereas the immature orum is naked, its superficial layer is now converted into a radiately striated membrane. Still later, a second membrane appears between this striated membrane and the cells of the follicle; and the former disappearing as the ovum approaches maturity, the second membrane (called the "vitelline") remains alone. Other changes which take place after the ovum has ripened and has been discharged into the oviduct, it is not necessary to describe. They result in the formation of the accessory parts of the egg. The only essential constituent of the body of the ovum is an active living protoplasm.

§ 3. Impregnation takes place in the upper portion of the oviduct, and consists in the entrance of a single spermatozoon into the ovum, followed by the fusion of the two. The spermatozoon itself may be considered as a cell, the nucleus of which is its head. On entering the ovum, the substance of its tail becomes mingled with the protoplasm of the ovum; while the head enlarges, moves toward and fuses with a part of the substance of the ovum, thus constituting the nucleus of the impregnated egg. In this manner the physical and mental peculiarities of both parents are transmitted or carried over to the offspring by means of the actual fusion of substance derived from the bodies of both.

§4. A process known as segmentation or "yolk-cleavage" follows the fecundation of the ovum. This process consists in a successive division of the ovum into a number of cells, from which all the cells of the full-grown animal are, as it were, the lineal descendants. This process las many variations among the different animals. The chief peculiarity among the mammals is that the whole mass of the yolk is subject to this change.

By segmentation the germinal disk of the ovum is broken up 
into a large number of rounded segments of protoplasm, called the blastoderm. Of these segments those that lie uppermost are smaller than those beneath. The beginning of the two layers into which the blastoderm divides is thus made. The behavior of the nucleus formed by the union of substance from the male and the female, during the process of segmentation, has not been so satisfactorily traced ; it appears probable, however, that a process of division goes on in it also. Other nuclei, thought to be derived from the primitive nucleus, make their appearance immediately below the blastoderm. The distinction between the upper and lower layers of the blastoderm now becomes more obvious, for the segments of the former arrange themselves side by side, with their long axes vertical, as a membrane of columnar nucleated cells; while those of the latter continue granular and round, and form a close, irregular net-work of cells, whose nuclei are not easily seen.

$\S 5$. The principal difference between the ovum of a mammal and that of a bird depends upon the amount and distribution of the food-yolk. The ovum of the mammal is small-the human ovarian ovum being only from $\frac{1}{125}$ to $\frac{1}{50}$ of an inch in diameter-because it contains so little food-yolk; but this small supply is distributed un if ormly throughout. In consequence of the abovementioned difference, the ovum is able to Fros. 7 and $72 .-$ Fructified Human Egg of $12-13$ dayg, seen break up into seg-

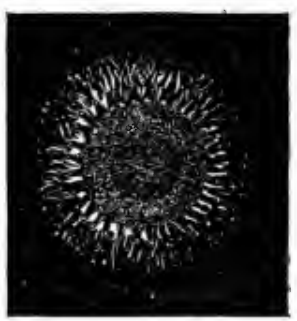

Fig. 71.

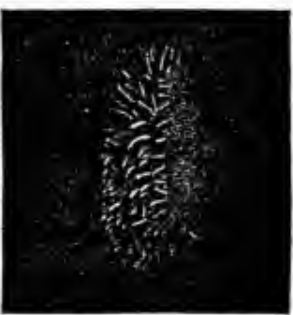

FIG. 72 what Reichert considers the embryonic area. ments through the whole of its protoplasmic mass. As the process of segmentation goes on, the differences among the ova of different species of animals become more clearly marked. For example, in the rabbit, although the details are differently described by different observers, at the close of the process of segmentation the ovum appears to be composed of "an outer layer of cubical hyaline cells, almost entirely surrounding an inner mass of highly granular, rounded, or polygonal cells." In a small circular area, however, the inner miss remains exposed. The outer cells soon close over the exposed spot (called by van Beneden, blastopore), and thus form a superficial layer. A narrow cavity then appears between the two layers, which extends so as to separate them completely, except in the 
region near to the spot originally exposed. The enlargement of the orum and of the cavity together, soon give the whole structure the appearance of a vesicle with a thin wall and a large central cavity. This vesicle is called the blastodermic vesicle. The greater part of its walls is composed of a single row of outer flattened cells; while an inner lens-shaped mass of cells appears attached to

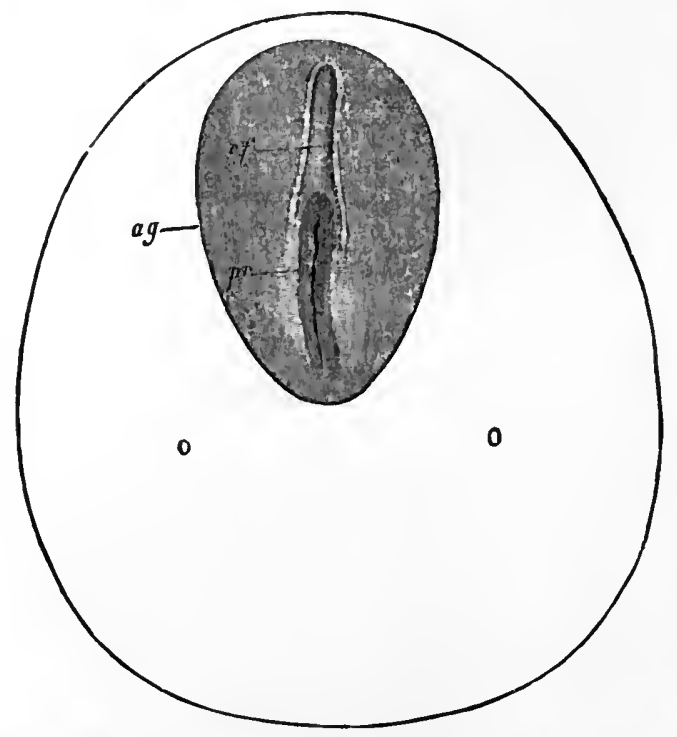

FIa. 73.-Vascular Area and Embryonic Area of the Embryo of a Rabbit, seven days old. 28/1, (Kölliker.) $o o$, the vascular or opaque area; $a g$, embryonic area; $p r$, primitive streak and groove; $r$, medullary groove.

a portion of the inner side of the outer layer. The "blastodermic vesicle" enlarges rapidly ; its inner mass of cells loses its lens-like shape, becomes flattened, and spreads out on the inner side of the outer layer. Its central part remains thicker and forms an opaque circular spot on the blastoderm, which is the beginning of the area where the embryo is to form (the embryonic area).

$\$ 6$. The immediately subsequent history of the development of the manmalian ovum, until the appearance of the so-called "primitive streak," is less perfectly understood: Foster and Balfour " ${ }^{1}$ speak of the following description as "tentative." In the embryonic area the cells of the inner mass become divided into two distinct strata, an upper one of rounded cells which lies close to the flattened outer layer, and a lower one of flattened cells (the "hypo-

\footnotetext{
${ }^{1}$ Elements of Embryology, p. $316 \mathrm{f}$.
} 
blast"). The former becomes fused with the outer layer, and thus gives rise to a layer of columnar cells (the "epiblast"). In this way the embryonic area consists of two layers of cells; the upper one of which is the epiblast, and the under one the hypoblast.

The blastoderm at first, then, consists of only two layers, which constitute a double-walled sac (the gastrula); but a third layer soon makes its appearance between the other two. These three layers-epiblast, mesoblast, and hypoblast-are called "germinal layers" and are found in the embryos of all forms of vertebrate, and most forms of invertebrate animals. The middle one, or mesoblast, arises from certain parts of the other two primitive layers, in a manner which need not be described. From these three germinal layers, all the different parts of the organism of the animal are developed. The history of the development of every animal in its earlier stages is, therefore, a narrative of the changes which take place in the three layers of the blastoderm. The hypoblast is the secretory layer; and from it almost all the epithelial lining of the alimentary tract and its glands is derived. The mesoblast is the source of the skeletal, muscular, and vascular systems, and of the connective tissue of all the parts of the body. But it is the epiblast which produces the central and peripheral nervous system, the epiciermis, and all the most important parts of the organs of sense. It is to the development of the epiblast exclusively, then, that we now direct our attention.

$\S 7$. The process of differentiating the layers of the embryo is intimately connected with another, which results in forming a

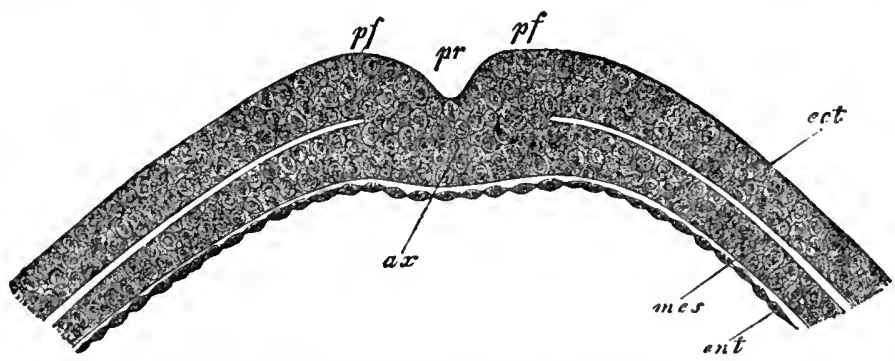

FIG. 74.-Primitive Streak of the Embryo of a Rabbit, eight dajs and nine hours old. $220 \%$. (Kölliker.) No medullary groove has yet been formed, ax, primitive streak: $m$, primitive groove : $p f$, primitlve fold; ect, ectoderm (or epiblast); mes, mesoderm (or mesoblast); ent, entoderm (lypoblist).

structure known as the primitive groove. This process is substantially alike in mammals and in birds. A short sickle-like thickening of the blastoderm, which afterward becomes a "narrow strap-like opacity"-due to a forward propagation (linear proliferation) of 
epiblast cells in a straight line-arises near the junction between the pellucid and the opaque areas of the blastoderm, and stretches inward upon the embryonic area; it is called the primitive streak. The median line of the primitive streak then shows a shallow furrow, running along its axis. This furrow is called the primitive groove. (Compare Fig. 73.)

\$. Now occurs the formation of the medullary groove. In that portion of the embryonic area which is in front of the primitive streak, the axial part of the epiblast thickens; two folds arise along the boundaries of a shallow median groove ; the folds meet in front, diverge behind, and then enclose between them the front part of the primitive streak. These are the medullary folds, and they constitute the first definite features of the embryo. The part bounded between these folds is called the "medullary plate;" its supreme importance in the embryo appears in the fact that it is the portion of the epiblast which gives rise to the central nervous system. At about the time of the development of the medullary groove (a little earlier) an important change is taking place in the constitution of the hypoblast in front of the primitive streak. An opaque line appears, as seen from the surface, and is continued forward from the front end of the streak, but stops short at a semicircular fold near the front part of the pellucid area. This fold is the future headfold of the embryo. The opaque line is due to a concentration of cells in the form of a cord; it is the beginning of what is known as the nolochord. It is to subsequent changes in connection with the notochord that we are to look for the development of the distinctively vertebral structure of the animal.

\$. From this point onward the shaping of recognizable parts of the embryo proceeds rapidly. The pellucid area, which was at first quite flat or slightly curved, has, in the process of its growth, suffered a "tucking in"-as it were-of a portion of the blastoderm, in the form of a crescent. It is this tuck which, when viewed from above, appears as a curved line marking the margin of the medullary groove. Thus the blastoderm is at this spot folded in the form of the reversed letter $\boldsymbol{Z}$; the fold is the one already referred to as the "head-fold." Of the two limbs of this $\mathbf{Z}$-fold, the upper is continually growing forward and the lower is continually growing backward. As the head-fold enlarges rapidly, the crescentic groove becomes deeper ; and at the same time, the overhanging margin of the groove rises up above the level of the blastoderm. The medullary folds meantime increase in beight and lean over from either side toward the middle line. They soon come in contact in the region which will afterward become the brain, and 
thus form a tubular canal (the medullary or neural canal), although they do not for some time coalesce. As the upper limb or head of the embryo becomes more prominent, the medullary folds close rapidly, and, in the region of the head quite coalesce. The open medullary groove is thus converted into a canal or tube, which is closed in front but remains open behind. The front end of this

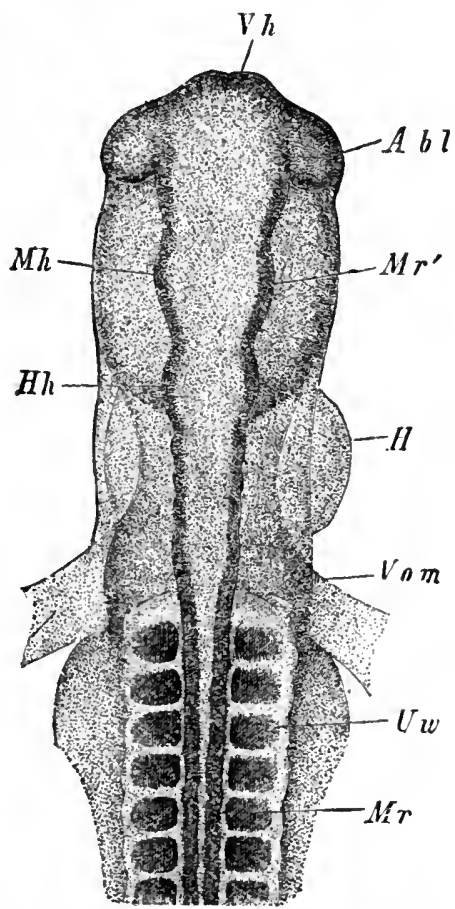

Fra. 75.-Fore-part of an Embryo-chick at the end of the seeond day, viewed from the Dorsal Side. $70 / 1$. (Kölliker.) $V h$, fore-brain; $A b l$, occular vesicles; $M h$, mid-brain; $H h$, hindbrain: $H$, part of the heart seen bulging to the right side; $V$ o m, vitelline veins; $M r r$ medullary canal, spinal part ; $\boldsymbol{M} \boldsymbol{r}^{\prime}$, mednllary wall of the mid-brain; $U w$, proto-vertebral somites.

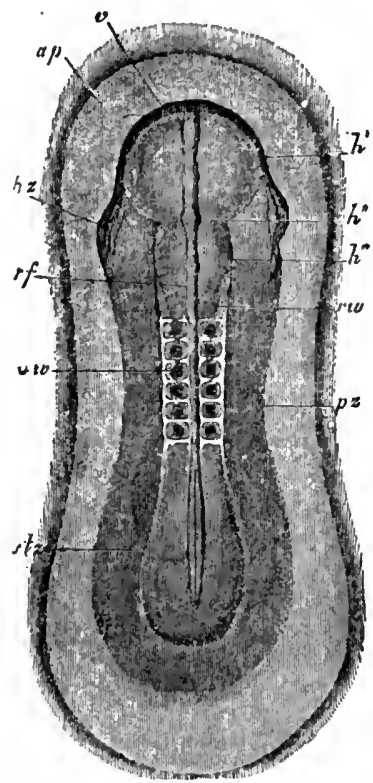

FIG. 76--Embryo of a Rabbit, eight days and fourteen hours old. $22.7 / 2$ (Kölliker.) $a p$. pellucid area; $v$. anterior edge of the circuit of the head; $h^{\prime}$, fore-brain; $h^{\prime \prime}$, region of later mid-brain: $h^{\prime \prime \prime}$, position of the hinder brain ; $h z$, position of the heart ; $r f$, mednllary groove: ro, medullary ridge: uw, mesoblastic somite; $p z$, lateral zone ; $s t z$, vertebral zone.

neural canal-having a more rapid growth than the rest-dilates into a small bulb or vesicle, the carity of which remains continuous with that of the rest of the canal, while its walls are similarly formed of epiblast. This bulb is the so-called first cerebral vesicle; and the lateral processes which soon grow out from its sides are called optic vesicles. Behind the first vesicle, a second, and afterward behind the second vesicle, a third is soon formed. Thus these three brain-buds, 
or germinal brains, are made. At the level of the hind end of the head, two shallow pits appear (the auditory pits) which are the rudiments of the organ of hearing. Thus the closing-up of the medullary canal has converted the original medullary groove into a neural tube; and three cerebral vesicles have been grown which are to develop into the fore-brain, the mid-brain, and the hindbrain.

\$ 10. The most important changes which now take place in the derelopment of the nerrous mechanism, are connected with the growth of the three cerebral vesicles and with the flexure of the medullary canal. The front portion of this canal-that is, the fore-brain with its resicles-in consequence of inequalities of
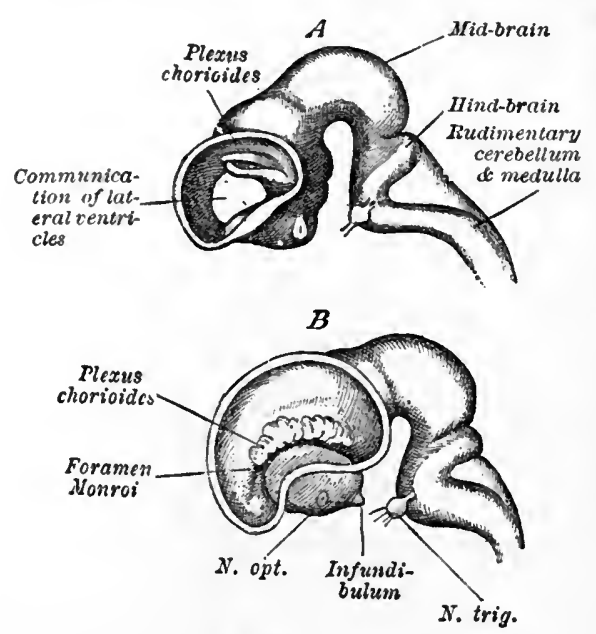

FIG. $7 \%$ - A, Brain of an Embryo of the Rabbit. $B$, Brain of an Embryo of the Ox. In both cases the side-wail of the left hemisphere is removed. (After Mihalkovics.) growth in the different parts of the brain, becomes bent downward; this is the commencement of the cranial flexure. As the flexure progresses, the front portion becomes more and more folded down, so that the second resicle, or midbrain, comes to project in front of it. From the front part of the forebrain the resicles of the cerebral hemispheres grow out and swell laterally, so as to make two buds corresponding to the two hemispheres of

the brain. Each of these side-buds has a cavity which is continuous belind with the cavity of the fore-brain ; each cavity becomes a lateral ventricle of the brain. The original vesicle of the fore-brain, having ceased to occupy its front position, is developed into the parts surrounding the third ventricle. In the hind-brain, or third cerebral resicle, the part nearest to the mid-brain becomes marked off by a constriction; the hind-brain is thus separated into two parts-the rudimentary cerebellum with the pons in front, the rudimentary medulla oblongata belind.

\$11. Various differentiations of the lining of the epiblast, which is inroluted along the cerebro-spinal cavity, take place. Through the length of the neural canal this lining is thickened at each 
side, so that the cavity is no longer circular, but resembles a narrow vertical slit. In the region of the cerebral hemispheres the sides and floor of the canal are much thickened, but in the region of the third and fourth ventricles, its roof becomes excessively thin, so as to form a membrane consisting of scarcely more than a single layer of cells.

\$12. Another important event, at about this stage in the development of the embryo, is the formation of the cranial and spinal nerves. The cranial nerres sprout out of a continuous band (the neural band), composed of two plates, which connects the dorsal edges of the neural canal with the external epiblast. This band separates from the epiblast and becomes a crest on the roof of the brain, with its two plates fused together. The crest extends forward as far as the roof of the mid-brain. As the roots of the cranial nerves grow centrifugally and become established, the crest connecting them is partially obliterated. The posterior roots of the spinal nerves are outgrowths of a series of median processes of cells that appear on the dorsal part of the cord. These outgrowths are symmetrically arranged, and attached to the walls of the cord ; but their original attachment is not permanent. Such rudimentary posterior spinal nerves divide subsequently into three portions $-a$ rounded portion nearest to the cord, an enlarged middle portion forming the rudiment of a ganglion, and a peripheral portion forming the commencement of the nerve. The origin of the anterior roots of the spinal nerves is less satisfactorily made out.

\$13. In the further development of the hind-brain the medulla oblongata undergoes changes of a somewhat complicated character. Its roof becomes extended and thinner; where the two lateral halves of the brain were at first united (at the raphe) a separation takes place, so that the sole union of the two sides is by a single row of cells. The thin roof of the fourth ventricle is thus formed. The floor of the whole hind-brain becomes thickened, and on its outer surface a layer of longitudinal non-medullated nerve-fibres appears. The roof of the anterior part of the hind-brain, which has become thickened instead of thinned out-thus forming the rudimentary cerebellum-is developed, first, by the formation of the median lobe (or vermiform process) and, afterward, by the swelling of its sides so as to constitute the cerebellar hemispheres.

\&14. The changes in the development of the mid-brain (or mesencephalon) are comparatively simple. When the cranial flexure has taken place, the mid-brain is left at the front end of the axis of the body, as a single vesicle with a vaulted roof and a curved 
floor, whose carity is known as the aqueduct of Sylvius. The corpora quadrigemina of the two sides are marked off from each other

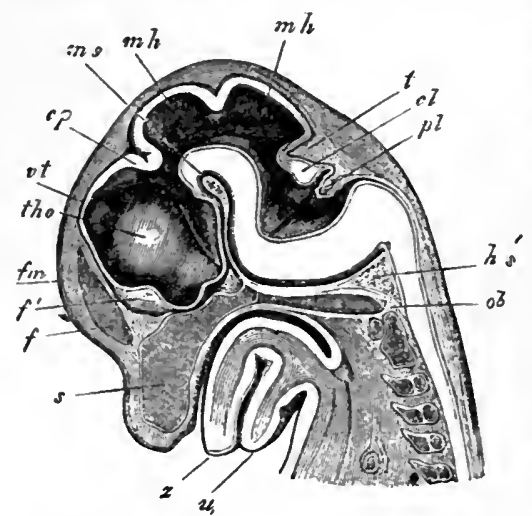

FIG. 78.-Head of the Embryo of a Sheep, cut through the middle. $3 / 1$. (Köliker.) $u$, under jaw; $z$, tongue ; 8 , septum narium; vccipitaie basilare; $t h$, thalamus opticus; $v t$, roof of the third ventricle: $c p$, posterior commissure; $m h$, mid-brain divided by a fold into two parts ; $f$, falx cerebri ; $f^{\prime}$, terminal plate of the fore-brain. At the prolongation of the line of $\mathrm{fm}$ is the foramen of Monro. $t$, tentorium cerebelli ; $c l$, cerebellum; $\boldsymbol{p} l$, plexus of the fourth ventricle.

floor gives rise to the optic chiasm and the origin of the optic nerves, and to the rudiment of the infundibulum; and its sides become thickened to form the optic thalami, while the interval between them enlarges toward the base and constitutes the cavity of the third ventricle. The more complicated changes which its roof undergoes give rise to the pineal gland and other small surrounding structures. It is the anterior and larger portion of the fore-brain which constitutes the rudiment of the cerebral hemispheres. In this cerebral rudiment, also, a floor and a roof may be distinguished. The former is developed into the principal basal ganglia, the striate bodies; the latter into the structures of the cerebral hemispheres proper. The formation of the striate bodies (corpora striata) is in fact due to thickenings of the walls of the floor of this furrow about the sixth month ; and about a month later a transverse depression separates the anterior (nates) and posterior (testes) pairs. The thickening of the floor of the mid-brain gives rise to the crura cerebri.

$\S 15$. Of the two divisions into which the fore-brain has already become divided, the posterior constitutes the socalled "thalamen-cephalon." This body is at first a simple vesicle, formed of spindleshaped cells, with walls of nearly uniform thickness. Its

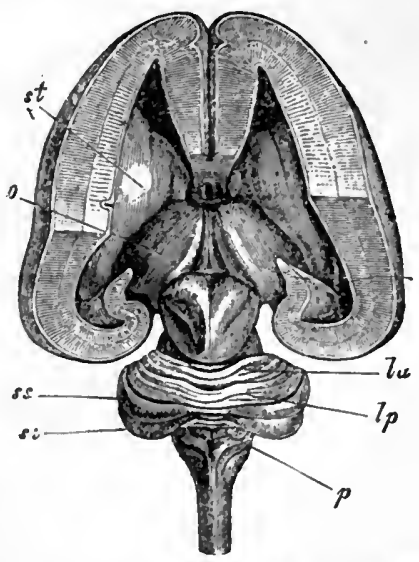

Fia. 79 -Brain of Human Embryo of five nionths, with Basal Ganglia laid bare. Natural size. (Kölliker.) $8 t$, corpus striatum; $o$, optic thalamns; $l a$, anterior lobe (lunatns) of the cerebellum, and $l p$, posterior lobe of the same ; 88 , semilunaris superior, and $s i$, inferior; $p$, pyramid.

by the appearance of a vertical 
rudiment. The laying of the commissures is the characteristic feature of the development of the mammalian hemispheres. These are the anterior commissure, the fornix, and the corpus callosum. But into the details of this process we do not need to enter. One characteristic of the embryonic development of mammals is the early enlargement of the cerebral hemispheres; in the humau embryo they are even by the tenth week much larger than all the other parts of the brain. At this time they are hollow bodies with comparatively thin upper walls, the lateral ventricles being dilated and communicating with each other through a wide opening, and with the third rentricle by the foramen of Monro. They grow from before backward, and thus cover up, one after the other, the optic thalami, corpora quadrigemina, and cerebellum. Their floor keeps on thickening, and thus the striate bodies become greatly enlarged, and project upward into the lateral ventricles, giving these cavities their arched form.

The following table, exhibits the rela-

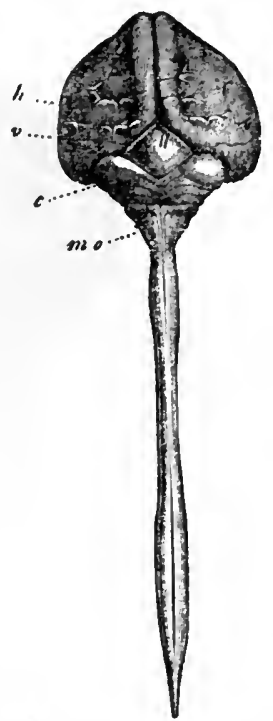

Frg. 80.-Brain and Spinal Cord of a Fœtns, four months old. (Kölliker.) $h$, hemispheres of the cerebrum; $m$, corpora quadrigemina (or mesencephalon); $c$, cerebellum; mo, medulla oblongata; s8, spinal cord with its brachial and crural enlargements. tions, with respect to their development, in which the different parts of the brain stand to its fundamental rudiments :

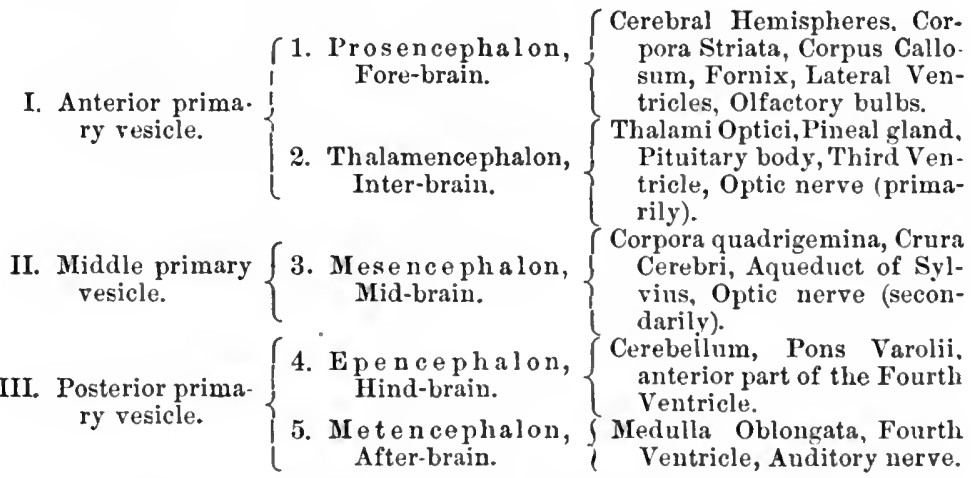

${ }^{1}$ Taken from Quain's Anatomy (Ninth Edition), II., p. 828. 
The more important convolutions and sulci of the cerebral hemispheres (those called "primitive") result from the folding of the

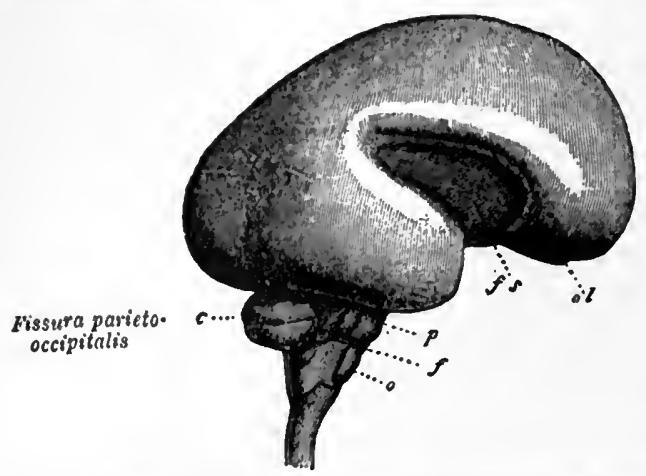

FIG. 81.-Brain of a Six-months Human Embryo. Natural size. (Kölliker.) ol, olfactory bnlb : $f 8$, fissure of Sylvius ; c, ccrebellum ; $p$, pons Varolif ; $f$, floceulns; 0 , olive. whole substance of the wall of the hemisphere; the less important (the so-called "secondary") consist merely of depressions and elevations of its more superficial portion. The former appear earlier-the first of the primitive sulci being the fissure of ${ }^{\circ}$ Sylvius, which is visi. ble before the end of the third month. By the end of the serenth month almost all the principal features of the cerebral hemispheres, both convolutions and sulci, are already fixed.

$\S 16$. The nervous parts of the eye are differentiations of certain lateral growths of the germinal brain-buds, called the "optic vesicles." The optic vesicles are outgrowths from the sides of the first cerebral vesicle, and are originally connected with it by short and wide stalks; at first they stand out at nearly right angles to the axis of the embryo. The stalks soon become narrower and thus form the rudinents of the optic nerves; ' at the same time the rudiments of the retina are formed from the vesicles themselves. The bulb of the optic vesicle is made into a cup with two walls by doubling it upon itself; thus a second optic vesicle or "optic cup" is produced, as distinguished from the original one. The lens of the eye is made by
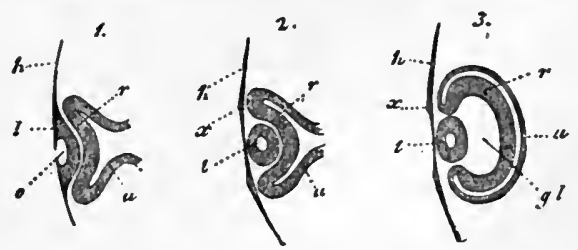

FIG. 82.--Longitndinal Sections of the Eye of an Embryo, in three stages. (From Remak.) 1, commencement of the formation of the lens, $l$, by depression of a part of $h$, the corneous layer: $u, r$, the primitive ocniar vesicle is donbled back on itself by the depression of the commencing lens. 2, the depression for the lens is now enclosed, with the lens beginning to be formed on the inner side: the optic vesicle is more folded baek. 3 , a third stage, in which the secondary optic vesicle, $g$ ?. begins to be formed.

thickening some of the superficial epiblast and involuting it in. ward over the front of the optic eup, or secondary optic vesicle.

${ }^{1}$ But His and Kölliker suppose these nerves to be formed by secondary emanation from the chiasm or nervous centre. 
This involution has at first the form of a pit, then of a closed sac with thick walls, then of a solid mass. The cavity between the two walls of the optic cup is closed up by bringing the walls into contact. The subsequent development of the different parts of the eye is conditioned upon the fact that the walls of the optic cup grow more rapidly than does the lens, and that their growth does not take place equally in all portions of the cup. It is by changes in the surrounding mesoblast, which takes on the character of an investment, that the outline of the eyebail is definitely formed (the choroid and sclerotic). The vitreous humor also is a mesoblastic product which is supposed to originate as a kind of transudation through the so-called choroid slit. Of the two walls, the inner or anterior is originally somewhat thicker; and since, in most parts of the cup it grows more rapidly, it constantly increases in relative thickness. But just in front of a line which afterward becomes the ora serrata, both layers soon cease to thicken and then completely coalesce; thus the hind portion or true retina becomes marked off from the ciliary ridges and the iris, while the wide opening of the optic cup is narrowed into a smaller orifice that constitutes the pupil. By differentiations of the inner or anterior wall of the hind portion of the optic cup-its cells multiplying rapidly and undergoing morphological changes while the wall is thickening-the different layers of the retina are formed. It is a significant fact that in its early stage this wall resembles the brain in its structure, and may be considered as a part of that organ. It is not necessary to enter into a more detailed description of the development of the different parts of the eye.

$\S 17$. The ear originally appears on either side of the hind-brain as an involution of the external epiblast, sunk in a mass of the mesoblast. It is then simply a shallow pit with a wide-open mouth. The mouth closes up and the pit then becomes a closed vesicle (the otic vesicle) which is lined with epiblast and surrounded by mesoblast. As the walls of this vesicle thicken, its cavity enlarges. The shape of the vesicle is at first nearly spherical, but it soon becomes triaugular, with the apex of the triangle directed inward and downward. It is by elongating this apex that the rudiment of the cochlear canal is formed. Part of the vesicle becomes stretched into a long, narrow, hollow process (the recessus vestibuli), and from the outer wall of the main body two protuberances grow, which are the rudiments of the vertical semicircular canals. These parts of the auditory labyrinth are soon more clearly defined. The cochlear canal is further elongated and curved; the recessus is also stretched out more; and from a new protuberance 
the horizontal canal is developed. Another protuberance, which becomes apparent at the inner commencement of the cochlear canal, is converted into the sacculus by being constricted on either side. The rest of the cavity, into which all the other parts open, may now be called the utriculus. Dilatations of the semicircular canals form the ampulla. When the cochlear canal has reached two and a half coils, the thickened epithelium of its lower surface forms a double ridge, from which the organ of Corti is developed. For the details of the structure of the labyrinth we refer to the previous description of this end-organ of sense.

$\S 18$. All the coarser differentiations of structure to which reference has thus far been made are only the expression-as it wereof certain histogenetic changes which have been secretly taking place. The laying down of delicate threads of nervous tissue, the proliferation of nerve-cells along definite lines of movement, have resulted in combining these elements by a living process into the organs of the nervous mechanism. The white matter of the cord is supposed to result from a differentiation of the outer parts of its superficial cells into longitudinal nerve-fibres; the latter remain, however, for a considerable time without their medullary sheath. The white matter first appears in four patches at the front and back of either side, in which the individual fibres seem like small dots. The gray matter of the cord is formed by a differentiation of the principal mass of the walls of the medullary canal. The outer cells first lose their epithelial-like arrangement, and then become converted into true nerve-cells, with prolongations that constitute nerve-fibres. The early listological character of the parts of the brain which lie back of the cerebral hemispheres is very similar to that of the spinal cord. In the floor of the hindbrain and mid-brain a superficial layer of delicate nerve-fibres is early formed. The cells internal to the nerve-fibres give rise to the epithelial layer which lines the cavities of the ventricles and to an outer layer of gray matter. In the fore-brain the walls of the hemispheres become divided into two layers, between which the fibres of the crura cerebri interpose themselves. The inner layer unites with these fibres to give rise to most of the white matter of the hemispheres; the outer layer of rounded cells becomes further differentiated into the outer part of the gray matter, which has comparatively few cells, and a deeper layer with numerous cells, the latter forming the principal mass of the gray matter of the cortex.

$\S 19$. The preceding description of the outlines of the development of the human nervous mechanism is derived, for the most 
part, from the study of other embryos than those of the human species. It is probably, however, substantially true for the latter also. It is valuable for the purposes of Physiological Psychology, chiefly as emphasizing what has already been said concerning the structure and functions of this mechanism in its developed form. The nature of the process by which the nervous system is developed, as well as the nature of the developed structure and its functions, as far as physical science can go at all, leads us in the direction of a mechanical theory. But in respect to both, such a theory is at present in an exceedingly fragmentary and uncertain condition. Further investigations may largely remove the present limitations. But the most complete theory possible can hardly be more than a statement of the order and extent of physical changes, the real causes and meaning of which it lies beyond the power of a mechanical theory to give.

The impreguated ovum does, indeed, become converted into the developed organism by an evolution that, at every step in its course, appears as an alteration in the arrangement of material molecules, under conditions derived from the original nature of the molecules themselves, from their necessary relations to each other, and from the action of their total environment. By division of that which was single into several parts, by bending of that which was straight, by stretcling in one direction and compressing elsewhere, by swelling and dilating in the various outlines under the influence of pressure, by folding and tucking in so as to close up an opening here and form another there, by laying down cells of the same kind in right lines or grouping them in masses, etc.-in brief, by motion of particles of matter in such way that the motion of each is conditioned upon that of the others, the nervous mechanism is built up. What it can accomplish in the way of further molecular motion, after it is thus built up, depends of course in large measure upon what it is made to be by the very process of building. How far it is possible even to propound a mechanical theory of the building process belongs to the speculations of embryologists to consider. It is our next problem to consider as a whole the fer data upon which it has been thought possible to base a mechanical theory of the behavior of the nervous system after it has once been constructed as a result of the embryonic process. 


\section{CHAPTER VII.}

\section{MECHANICAL THEORY OF THE NERVOUS SYSTEM.}

$\S 1$. The machine-like nature of much of the structure and movement of the human body does not escape the most ordinary observation. When this body, either as a whole or with respect to some of its parts, changes its position in space, its various masses support and act upon each other in essentially the same manner as the masses of matter which compose the parts of any machine constructed by human skill. Such movement is possible for it, because its framework of bones has a rigidity sufficient to sustain the other less rigid organs; and because the bones are so divided, and yet articulated, that they can assume different relations toward one another in accordance with the simplest principles of mechanics. The laws of the lever, of the pulley, the ball-and-socket joint, etc., need no modification when applied to this particular machine of the human body.

The action of certain other of its parts, which do not belong to the bony framework but which are of muscular or epithelial structure, is also plainly of the same machine-like character. The movement of the heart, for example, is in part to be explained as that of a pump with chambers and valves; and the flow of the blood through the arteries as that of a fluid pumped through conduits, of unlike and changeable sizes. So, too, the lungs may be, with considerable propriety, compared to bellows which alternately suck in and expel the surrounding atmosphere. The optics of the eye and the acoustics of the ear are special only so far as the structure of the organs makes necessary a special application of the general laws of those sciences. Moreover, the distribution of the fluids among the tissues of the body takes place under the laws which govern the distribution of fluids generally when separated by membranes which they can permeate. Nor is the chemistry of the same tissues and fluids by any means wholly unlike that with which the experiments of the laboratory make us familiar. When, however, we begin to speak of those changes of relative position which take place at extremely minute distances among the molecular ele- 
ments of which the larger masses of the body are composed, we seem compelled to drop the conception of a machine and to seek both another conception and another title.

The very attempt, then, to explain the motion of the more purely machine-like parts of the human body, leads us to consider certain activities of other parts for which the word "mechanism" is more appropriate. The movement of none of the more or less rigid organs of the body originates within these organs themselves. The changes of relative position in the parts, with which the ordinary laws of mechanics deal, imply antecedent molecular changes in other parts with which these laws cannot deal. The motion which finds its final expression in the swing of the arm, or of the leg, in the lifting of a weight, and even in the contraction of the heart, or in the rising and falling of the chest, does not begin in arm, or leg, or ribs, or diaphragm, or cardiac muscles. The change of position of so considerable masses of matter is but the summing-up of innumerable minute molecular changes which began within the body, but outside of the masses themselves. If, for example, we inquire as to what causes the bones to move-however strictly their motion, once begun, may follow the laws of mechanics-the answer is to be found in the pull of the tendons, or cord-like structures, which are attached to them. And if we then inquire, What causes the tendons to pull upon the bones by means of their attachment? the answer must be, That it is the contraction of the muscles which pulls upon the tendons.

The next step in following this chain of causes, however, introduces us to a different class of considerations from any of the foregoing. For we cannot say that the contraction of the muscles is caused by the pull of the nerves upon them. The morement of muscular fibre in contraction is an altogether different affair from the movement of the bones as they are pulled by the muscles; nor do the nerves act upon the muscles as the muscles act upon the tendons. The elasticity of the muscles is, indeed, a mechanical quality, like that of which we avail ourselves in the construction of machines. But the quality of elasticity does not fully explain the behavior of the so-called muscle-nerve machine when its muscular tissue is contracting or relaxing. Yet the living muscle, in itself considered, may certainly be looked upon as a molecular mechanism. It is a system of minute particles of matter which act upon each other at indefinitely small distances; and which, when any motion is set up at one part of it, propagates such motion according to laws that are given in the very constitution and arrangement of the particles themselves. This is precisely what we understand 
by a physical molecular mechanism. The office of the nerve with respect to the muscle is simply, as we know, to start that molecular activity which it is the function of the irritated muscle itself to exercise. The nerve, however, cannot perform its office of irritating the muscle without being in a state of molecular commotion called the "excitement" of the nerve. And, further, this excited condition of that part of the nerve which is in immediate contact with the muscle is itself a state of the nerre which has been propagated from a distant point of the nervous matter. All the machine-like movements of the masses of the body require us, therefore, to look for their origin in minute molecular changes that originate in its nervous elements. And for the further account of these neural molecular changes we are to look to a mechanical theory of the nervous system.

$\S 2$. The basis for a general view of the nervous system as a mechanism has been laid in all the preceding examination; and it cannot be denied that the results of this examination are such as to dispose us favorably toward the attempt to develop such a view into a complete mechanical theory. Physical science, as a matter of course, strives to establish such a theory. It knows no other way of considering any group of phenomena when brought before it for examination. To deny totally the application of the conception of a mechanism to the action of the nervous system would be to refuse to apply to its phenomena the same scientific treatment which we apply to all other physical phenomena. To limit, a priori, such application would be to restrict improperly, on merely theoretical grounds, the area of the phenomena with which such science is entitled to deal. The fact that molecular changes here are correlated with another class of phenomena which we call " mental," in no wise destroys the propriety of pushing our physical science of the nervous system to its furthest possible limits. The movements of all material bodies, whether in the elemental shape of the molecules, or in the shape of the same molecules when aggregated into masses, as well as the laws under which such bodies in morement act and react upon each other, constitute the legitimate sphere of physical science. But it is to a system of interacting molecules that the conception of mechanism especially applies. The aim of physical research with regard to any given system of this kind is, therefore, not accomplished until all the movements of its different parts are explained in the light of a consistent mechanical theory. This general principle of all physical science neither needs nor permits a special exception in the case of the human nerves, organs of sense, and brain. 
On the other hand, the very unsatisfactory condition of the data for a mechanical theory of the human nervous system has been implied in each of the preceding chapters. It will appear all the more plainly now as we present briefly a statement of two or three such theories in the form in which it has been found possible for different investigators to state and to defend them. Nor can we express much confidence that physics and physiology combined will ever be able to point to a complete theory of so intricate and delicate a mechanism as this nervous system. Moreover, we do not by any means affirm that a purely mechanical treatment, however complete, would of itself suffice to furnish a satisfactory understanding of all the phenomena ; or even that the phenomena in general could by any possibility be brought solely under the terms of such treatment. We only affirm the unrestricted right of physical science to attempt, in the light of the conception of mechanism, an explanation of the nervous system as well as of all other physical subjects; and also its right to its persistent faith that-So far as physical science can explain any such subject, all the special difficulties of the nervous system can be fitly considered only in this way.

§3. The chemical constitution and structural form of the elements of nervous matter require that the system which they compose should be regarded in the light of the conception of mechanism. It is true that physical science cannot give an accurate description of the chemical processes which take place in the formation of the nerve-fibres and nerve-cells, or during their functional activity; it cannot do so much as this for the living tissues generally. But it finds here the same chemical elements which exist elsewhere in nature, especially the four elements, oxygen, hydrogen, nitrogen, and carbon. It nowhere finds these elements behaving differently in the nervous system from the way in which it is their nature to behave elsewhere, under similar circumstances. And the fact that precisely similar circumstances do not occur to induce the same combination and interaction of these elements outside of the nerrous system, is traced back to its causes in a succession of occurrences that all have the character belonging to the chemistry of living tissues. We know of no sap which is suitable for forming organisms in general, but which is itself a perfectly homogeneous fluid. Nucleated granules in the very chemical constituents which give conditions to all the subsequent activity of the molecules, are revealed by microscopic examination of those cells from which the whole body springs. This fact, together with the character of the subsequent process, may lead some to insist that a certain special form of energy (called "vital force," or by some less obnoxious 
title), is marshalling the minute particles under its superior control. But such way of considering the phenomena-whether admissible or inadmissible - does not at all help us to dispense with the purely mechanical point of view. In the original living germ with which the organism began, and in all its subsequent development, every chemical change in nervous matter is nothing more than a movement of plyysical molecules, strictly under the conditions furnished by their constitution and previous arrangement.

The general significance of the chemical constitution of nervous matter, with reference to a mechanical theory of the nervous system, is by no means wholly obscure. It is obvious that all the energy expended in the movement of the body as a whole, or of its larger masses, originates in minute molecular changes. The latter changes have, of course, a direct relation to the chemical constitution of the tissues in which they occur. The muscular fibre can contract because its molecules admit of that rearrangement in which the contraction essentially consists ; for doing the amount of work implied in such rearrangement, this fibre is, of course, dependent upon its own chemical constitution. But the source of the excitation of the muscle is to be found in antecedent molecular changes within the nervous system; indeed, all the changes that are to be summed up in the work done by and within the rigid masses of the body have their origin here. It accords, then, with the mechanical conception of the nervous system that its chemistry should be just such as we have seen that it actually is. Nervous matter holds in store a large amount of energy that is easily disposable; of energy that will be yielded freely and rapidly if anything occurs to start the process within the system of molecules of which such matter is composed. For the molecules are of such kind as readily break up and recombine their elements in simpler forms ; in doing this they render kinetic a large amount of energy which they have previously held latent.

No mechanical theory of the nervous system can explain the meaning of all the various structural forms which the elements of this system assume. It cannot be told, for example, what peculiar place in the mechanism belongs to the different shapes of nervecells, bipolar, multipolar, stellate, etc. Nor can a complete picture be drawn of the differences in character which the nerve-commotion takes as it passes from the nerve-fibres to the nerve-cells, or from one nerve-cell to another. We can only insist upon the undoubted general fact that all these structural forms have whatever significance belongs to them, because they are themselves molecular structures, capable of undergoing, in relation to each other, those 
very changes in which the functional activity of the nervous system consists.

$\S 4$. There can be no doubt that the arrangement of the nervous elements into a system corresponds to the conception of mechanism. A certain work of "concatenating" the different physical systems of the body, and of adjusting its relations to the changes in its environment, requires to be accomplished. This problem demands a three-fold exercise of function; it is a problem in the construction of a mechanism. The nervous system actually is of threefold construction; its threefold construction is the answer which it practically makes to the above-mentioned problem. One part of the complex problem consists in the conversion of certain of those molecular motions which take place in nature outside of the living organism into molecular motion within the tissues of such organism. The solution of this part of the problem is furnished by the endorgans of the nervous system. The end-organs are those special mechanisms which are adapted to convert the molecular motions called stimuli into the molecular motions called neural excitation. That by far the larger portion of the eye and ear, for example, acts in a purely mechanical way, there is no doubt. It is the office of the great mass of the eye to transmit and refract the rays of light ; of the ear to transmit and condense the acoustic waves. But when the nervous elements of the retina and of the organ of Corti receive the physical processes transmitted to them, they transmute these physical processes into physiological neural processes; in doing this they act as special molecular mechanisms.

The second part of the complex problem before the nerrous system consists in the conduction in all necessary directions of these neural processes; only on this condition can distant parts of the nerrous system act, as it were, in view of each other, and thus the whole body be bound into a living unity under the influence of changes in its environment, and in the ideas and impulses of the mind. The nerre-fibres solve this part of the problem. This they do by acting as mechanisms, which have such a molecular constitution and function that a commotion, started at any point in the physical elements of the system, spreads from molecule to molecule, in accordance with the laws of the system.

The third part of the same complex problem requires for its solution structures and functions still more intricate and inexplicable. Incoming molecular disturbances must be modified and redistributed so as to give rise to outgoing molecular disturbances along definite tracts, in order that definite groups of muscles niay be made to contract. Only in this way can the whole physical organism, by a so- 
called reflex activity, adjust its condition, in view of the presence of given kinds and degrees of stimuli. Moreover, the vital functionsthe movements that control respiration, digestion, circulation of the blood and of other fluids, etc.-must be united so as to work to a common end, and with the modified forms and degrees of their respective energies, which the changing circumstances require. Still further, not only must the neural processes set up by the end-organs and conducted inward by the afferent nerves have a place of meeting in proximity with the centres of origin for the corresponding efferent impulses; but all the neural processes in this place of meeting must also be so modified and made mutually dependent that they can be correlated, under psycho-physical laws, with the processes of mind. It is the central organs which alone possess the molecular construction and functions necessary for such wonderful reflex and automatic activities. In their highest form-the hemispheres of the human brain-they solve the problem of providing a system of molecules, whose constitution and changes may be immediately related with the phenomena of mind. These central organs are extremely intricate physical structures. It cannot be pretended that even a beginning has been made toward a satisfactory theory of their functional activity considered as a special case in molecular physics. But this fact does not affect the confidence which is based upon what is known of physical structures in general, that in these organs, the changes which take place are essentially of the same order as are those with which the science of molecular physics has elsewhere to deal. They are modes of motion in which the behavior of each molecule, regarded as a constituent element of the system, is conditioned upon the constitution and behavior of the other members of the same system. That is to say, the central organs must be regarded in the light of the conception of mechanism.

$\S 5$. The general office of the nervous system may, then, be described in somewhat the following manner. The development of a rich and varied life, both animal and intellectual, requires a great store of sensations and of motions. The sensations are primarily designed to serve as signs of changes in the environment of the animal to which his condition must be adapted by movement of his bodily parts; but they are nlso to serve as a basis for intellectual attainment and development. The forces of external nature continually storm the peripheral parts of the animal's body. In order that any of these forces may act as the stimuli of sensations, they must be converted into molecular motions within the tissues of this body. In order, further, that the masses of the body may 
constantly be readjusted to the external changes of which the sensations are signs, the molecular motions must, in turn, be converted into movements of these masses. In other words, a process of constant interchange must take place between the animal organism and external nature.

Disturbances in one part of the body, by the play upon it of nature's energy, instead of becoming injurious or destructive, are thus made serviceable through inducing the needed disturbances of other parts of the same body. The equilibrium on which life depends is maintained. Moreover, the material necessary for selfconscious development, for a growing knowledge of the so-called outside world, is furnished through the conduction of these disturbances to their common meeting-places in the central organs.

To accomplish the general work of equilibrating the interaction of the different parts of the body, of readjusting its condition to the changing condition of its surroundings, some special construction and arrangement of material molecules is necessary. If the work is to be done in a highly elaborate way, a very intricate arrangement of an indefinitely great number of chemically complex molecules is necessary. Such an arrangement is the human nervous system. But just because its arrangement and function are of this kind, it is a "mechanism." As a highly complex molecular mechanism it utilizes the disturbances which arise from the environment. It binds together all the other systems of the body in living reciprocity of energies and functions. Its superficial parts are so constructed that they can be set in motion by various forms of physical energy - by light, heat, sound, chemical cinange, ete.; they are also adapted fitly to modify the impressions thus received. The molecules of its conducting nerves are so constituted and arranged that they can indicate the path along which the disturbance thus occasioned must pass; they can dictate the conditions and laws under which its course must be completed. The molecules of its central organs are capable of assuming inconceivably varied relations to each other, of thus transmuting and redistributing the nerve-commotions which reach them along the incoming tracts, and even (it would seem) of starting automatically outgoing disturbances in response to self-conscious sensations and ideas.

But all the foregoing offices of the nervous system are nothing but the movements of physical elements, in constant reciprocal dependence upon each other, though in response to excitations lying outside of the system itself. To move thus is the function of a molecular mechanism. So far as science can control the different parts of the nervous system for experimental purposes, it finds 
them behaving in such a manner as to make a plain demand for a physical and mechanical theory in explanation of their behavior.

$\S 6$. The foregoing description of the nervous system as a mechanism, like all similar descriptions, undoubtedly lacks scientific quality. It is neither exact nor in such form as to admit of experimental verification. It is largely based upon conjectures, full of gaps and assumptions; and were it pressed at every point for proof, it would be obliged to rely much upon general principles in mechanics (the special applications of which to the case in hand are by no means certain or obvious), and even to indulge in loopes and promises with reference to the future, rather than present demonstration. May we not know more precisely the nature of the molecular changes which constitute the functions of nerve-fibres and nerve-cells? Cannot physical science help us to complete these beginnings of a theory?

In answer to the question just raised we have already seen how little satisfaction is afforded on applying to the science of chemistry. On general principles of physical science there can be little doubt that the excitation and conduction of nerve-commotion is dependent upon a chemical change in the nervous tissue itself. Moreover, we know that the process of conduction in the nerve requires each of its molecules to act upon the neighboring elements as the condition of the process continuing. Nor can this process itself be a mere impartation of motion, from molecule to molecule ; on the contrary, the phenomena of electrotonus seem to show that it must also consist in the setting free of energy which exists latent within the molecules of the nerve-substance. These molecules contain, then, by virtue of their constitution, stored or potential energy which is converted into kinetic energy in the propagation of the process of excitation, and which is expended, in part, in either inhibiting or increasing the energy of that process. Such potential energy can scarcely be other than chemical.

Accordingly, we should be tempted to describe the process of progressive excitation of the nerve somewhat as follows: Every element of the nerve, by reason of its highly complex and unstable chemical constitution, contains a large store of energy ; the excitement of the nerve consists in the explosive decomposition successively of these elements of the nerve; and the result of the decomposition is the setting free of the stored energy to be expended in part in the excitation of the next adjoining elements. The process, then, is not altogether unlike the burning of a line of powder grains. Such an hypothesis, however, would at once have to answer several difficult questions. Why does not the whole of the explosive 
substance burn up, instead of only an amount of it approximately proportional to the strength of the stimulus which sets the process agoing? Analogies may indeed be found in the union of cllorine and hydrogen under the action of light. What checks the process in the nerve as a whole, and what limits it quantitatively in a different way at different points in its course, so as to give the phenomena of anelectrotonus and catelectrotonus ? (comp., Chap. III., $\$ 19$ f.). Moreover, direct observation has as yet discovered no indisputable evidence of functional chemical changes in the nerve-fibres. If such changes exist at all they are exceedingly small.

$\S 7$. Allusion has been made (p. 119 f.) to the fact that the effect of several excitations of a nerve-stretch is compounded, as it were, in the action of the attached muscle. That is to say, excitations which are simultaneous, or which follow each other with sufficient and not too great rapidity, are summed up in the nerve, like molecular waves of nerve-commotion piled upon each other. Besides such phenomena of "summation," there exist analagous phenomena of so-called "interference ;" and, further, of the facilitating effect which one excitation has upon others following it along the same paths of conduction, especially in the central organs. These and similar phenomena tempt us to consider the activity of the nerrous substance in terms of an exceedingly complex sum in the addition and subtraction of molecular disturbances of a wavelike character. Elaborate experiments have been made to determine the laws under which such summation or interference of electrical excitations takes place. Thus G. Valentin ${ }^{1}$ assumes that the case of the nerves comes under the general theory of molecular waves that may either be piled upon each other, or may interfere with each other. The interferences he calls "positive" when the currents are moving in the same direction, "negative" when they are moving in opposite directions; and such currents may, of course, be either ascending or descending. The character of the interferences is to be defined by the way in which the nerve-muscle machine responds to these four kinds of interference. The interference has a heightening effect (is erhöhende) when the result indicated by the behavior of the muscle is greater than the sum of two single effects from the partial excitations that are compounded; a depressing effect when the result is less than this sum. If the effect of the interference is such as to reduce the result to zero, it is called inhibitory. Valentin concludes that, in case of interferences of excitations from one and the same current (with respect

${ }^{1}$ In Pflüger's Archiv, vii. (1873), pp. 458-496, article on the Interferences of Electrical Excitations. 
to degree, direction, etc.), the character of the effects produced depends upon the original molecular constitution of the nerve. Just as its constitution is decisive with regard to the nature of the muscular contractions that follow a single excitation of the nerve, so is it also decisive with regard to the results of interference. These results, moreover, conform to the same laws after decapitation or poisoning as before. And further, the same rules govern in the case of interferences of two currents, if both the currents are of about the same degree of strength. Finally, according to Valentin, the same rules belong to the interferences that occur in cases of reflex action, or of the stimulation of motor nerves through the sensory, as those which apply to the direct stimulation of the motor nerves. It is apparent that the only net gain from the foregoing experiments consists in the information that the molecular constitution of the nerves themselves determines all the variable elements in the results of exciting them. But this would be an assumption fairly made by every attempt at a physical science of the nervous functions. And inasmuch as we can make no such verifiable statements concerning the nature of this molecular constitution as will serve the purposes of a precise mechanical theory, it is hard to see what advance has been gained toward the construction of such a theory.

The phenomena caused by the reciprocal action of different excitations within the central nervous system are, of course, much more complex and difficult to bring under a theory of molecular wave-like impulses, than are the phenomena of the comparatively simple nerve-muscle machine. A fortiori, molecular physics is unable to propose a satisfactory theory for the central organs. According to Exner, ${ }^{1}$ many of the phenomena are covered by the general principle that one excitation acts to facilitate or, as it were, smooth the path for others passing, after only a brief interval, along the same course. This principle he distinguishes from that of "summation," when applied to reflex action. The latter term Exner applies to the accumulation in the central organ of excitations which, taken singly, are too weak to produce any reflex motion, but which by their combined strength do produce such motion. The principle of "facilitation," however, refers to the condition of the central parts after the passage through them of a stimulus which has already called forth some reflex action. Exuer's experiments led him to conclude that the motor excitation of some one extremity from the brain (that is, by stimulating, in the brain, the so-called motor area of the extremity) facilitates the subsequent pas-

${ }^{1}$ Article in Pflüger's Archiv., xxviii., pp. $487 \mathrm{ff}$. 
sage of reflex stimulus affecting the same extremity; and, conversely, stimulating an extremity reflexly facilitates the passage of a subsequent motor excitation from the area of the brain to the same extremity. Thus, for example, the reflex motions of the foreleg of a rabbit, produced by stimulating the toes of that leg, were found to be increased in intensity if the so-called cerebral motor centre of the fore-leg was also stimulated. Different reflex excitations also may facilitate each other's effect in the same way. For example, the sensory stimulation of the left foot has the effect of facilitating the reflex act which, as it might appear, would relate only to the right foot and its motor area in the central organ; and such reflex action of the right foot facilitates the contraction set free in the same foot by stimulating the left-foot section of the spinal cord. Exner was unable, however, to obtain any inhibitory effect upon the motion of the extremities by stimulating various other places of the cortex of the cerebrum, or by stimulating the cerebellum. He also found that when one side of the cortex of the cerebrum is stimulated by electricity so as to produce a condition of tetanus in one extremity of the animal, the results of two excitations-one as a reflex from the foot and one directly from the same side of the brain-are compounded in a way which seems incompatible with any known form of the summation and interference of molecular wave-like disturbances.

Indeed (to return to the simpler case), Gritzner ${ }^{2}$ seems justified in saying that, strictly speaking, we cannot without qualification even represent what takes place when two currents of electricity act in combination upon a nerve, as though it were a matter of the addition or subtraction of their separate effects. For it is possible that an electrical current of an intensity equal to the amount of the natural nerve-current (current of rest $=a$ ) and the current used as stimulus (current of action $=b$ ), taken together $(a+b)$, will not excite a nerve that shows no current at all, although the latter $(b)$ alone will excite the nerve if just previously the former $(a)$ was present in the nerve. The currents already existing in the nerve, when the exciting current is applied, are, therefore, not simply added to or subtracted from the latter; but they produce molecular changes of an unknown kind which tend to induce the origination of so-called "cathodic" and "anodic" places in the nervethat is, places of exalted and places of depressed excitability. Thus a weaker current will excite the nerve when it is in a condition of exalted excitability ; a stronger current may fail to excite the nerve when in a condition of depressed excitability.

'See Pflüger's Archiv, xxviii., p. 144 f. 
How obscure and complicated are the molecular conditions connected with the excitation of the nerve is further shown by the effect of giving different treatments to the cross-section of the nerve. If the nerve is simply cut, its behavior under stimulation is different from that which occurs when it has been bound before the cross-section is made. Binding the nerve produces, for some minutes after cross-section, a large increase of its excitability in the immediate neighborhood of the injured place; this is true for all kinds of stimuli, including the electric current in both directions. From five to ten minutes subsequently, however, the making of the current in the opposite direction to the current induced by crosssection has frequently a diminished rather than an increased effect.

\& 8. On the whole, it would appear, then, that the ability to lay even a basis for a strictly scientific molecular theory of the nervous mechanism depends upon the ability satisfactorily to explain the electrical process in the nerres and their consequent behavior under electrical stimulation. It would by no means follow that a complete theory for the comparatively simple phenomena of the nervemuscle machine would furnish the sure clew, not to say the full explanation, of all the activities of the nervous system. On the contrary, the evidence is overwhelming that the working of the complete nervous mechanism involves other principles than those which may be deemed sufficient for the case of the single nerve and muscle when under electrical stimulation. But, plainly, the more complex case cannot be solved without first solving the far less complex one. And yet the simplest possible case of nervous molecular mechanism-the case that can be brought under the most favorable experimental conditions-has thus far proved to lie beyond our power to find a satisfactory scientific solution.

The two most important principles which must enter into any mechanical theory for explaining the behavior of nerves in relation to electricity are, according to Hermann: ' (1) the law of electrical excitation, and (2) the law of the so-called current of action. The phenomena upon which these laws are themselves based are chiefly the phenomena of electrotonus and the phenomena of negative variation.

It is a fact (see p. $114 \mathrm{f}$.) that the passage of the electrical current through a nerve-stretch produces in the nerve a changed condition of excitability called electrotonic. This condition is, however, different for different parts of the nerve-stretch. It is dependent upon the nearness of each part to the electrodes, it being greatest in their vicinity. It is dependent on the strength of the polarizing ${ }^{1}$ Handb. d. Physiol., II., i., p. 193. 
current and on the length of the stretch through which it flows. Its intensity is greater on the side of the anode than on the side of the cathode. The condition may be said to be one of increased excitability in the region of the cathode, of diminished excitability in the region of the anode. Helmboltz found that the time of the development of the electrotonic condition is not perceptibly later than that of the electrical current which excites it ; the condition originates at the moment of making, and ceases at the moment of breaking, the polarizing current. Du Bois-Reymond concludes, thereupon, that the electrotonic condition is spread over the nerve with a speed equal to that of the process of excitation.

It is also a fact (see p. $117 \mathrm{f}$.) that, in the case of the nerve-stretch as well as in that of the muscle, the galvanometer shows the passage of a current when one of the electrodes is placed at its cut end and the other at its equator. It is a fact that this so-called natural current, or current of rest, is diminished by the stimulation of the nerve with an interrupted current, or by other means of exciting it-the diminution being shown by the return of the needle of the galranometer toward the zero-point (the so-called "negative variation").

\$9. The two principal theories which have hitherto attempted to account for the above-mentioned facts are the theories of du Bois-Reymond and of Hermann. Du Bois-Reymond ${ }^{1}$ assumes that in the substance of the nerve there exists an arrangement of electro-motive molecules embedded in an imperfectly conducting medium. Each molecule is like a minute battery with positive and negative poles; and the molecules present their positive surfaces to the longitudinal surface of the nerve, their negative surfaces to the cut ends or transverse sections of the nerve. The presence of these molecules gives rise to currents in the medium which surrounds them. Owing to the imperfect conductivity of the medium, such currents flow in more or less concentric lines at some distance from each molecule. The current which exists in the nerres (exists, according to du Bois-Reymond, as natural to the nerve and previous to its injury by cross-section), and which is made obvious by the deflection of the needle of the attached galvanometer, may therefore be regarded as the resultant of the numerous unobservable currents belonging to the several molecules. In this way the so-called "current of rest" is to be explained. Du Bois-Reymond is forced to account for the fact that such natural currents are

\footnotetext{
1 The views of du Bois-Reymond are to be found in his Untersuchungen uber thierische Electricität, 1848-49, and Gesammelte Abhandlungen, etc., 1875-7\%.
} 
either exceedingly small or wholly wanting in an uninjured muscle by a very artificial lyppothesis as to a so-called parelectrononic region at the place where the ends of the muscle come into contact with the tendons. His theory of electrotonus and of the negative variation of the nerre-current is too complicated and doubtful to be even stated here; it is enough to say that his assumptions as to "peripolar" and "bipolar" molecules, and the effect of the electrical current in reversing the molecules, etc., have little to commend them to the practical workers in modern physics.

$\$ 10$. The theory which Hermann, ${ }^{2}$ and those who agree with him, would substitute for the theory of du Bois-Reymond takes its point of starting from a discovery made by Matteucci some years ago. In 1863 this truly great investigator noticed phenomena similar to those of the electrotonic condition of the nerves in over-spun wires moistened with a conducting fluid. If an electrical current is conducted to the moist covering of such a wire, the needle of the galvanometer shows along every part of the wire the presence of a current in the same direction with the primary current, but with the strength of the former diminishing as the distance increases from the points where the latter is applied to the wire. No such current arises, however, if the wire is made of amalgamated zine and its covering is moistened with a solution of sulphate of zinc. It appears, then, that the electrical condition of the wire, when a current is conducted to it, depends upon the limiting surfaces of its metal centre and of its moistened coveriug being polarizable. Very recently ${ }^{2}$ Hermann has, as he thinks, still further shown the possibility of explaining all the electrotonic properties of the nerves after the aualogy of Matteucci's discorery. A conductor consisting of a central and a covering substance, with polarizable limiting surfaces, as soon as a momentary electric current is conducted through any portion of it begins successively to exhibit a current of the same kind at every other place in it ; the more distant the place from the one to which the current is applied the later its appearance there, so that at the most distant places such current may begin after it has for some time ceased at the primary place. Now, in an analogous manner, every nerve-fibre may be assumed to consist of a centre and covering substance, with polarizable limiting surfaces. In the nerve-fibre the limiting surfaces needed for the theory are perhaps actually to be found between the axis-

${ }^{1}$ The views of Hermann may be found in his Untersuchungen zur Physiologie d. Muskeln u. Nerven, 1867-68, and in numerous later papers in Pflüger's Archiv.

${ }^{2}$ Pflüger's Archiv, 1885, xxxv., p. $23 \mathrm{f}$. 
cylinder and the medullary sheath. Grünhagen, ${ }^{1}$ however, affirms that the polarization of the limiting surfaces of the nerve-fibre is a consequence rather than a cause of the current in electrotonus. The first and fundamental cause of this current he considers to be the characteristic difference in the resistance, as conductors, of the kernel and the covering of the nerve-stretch.

The so-called " natural current," or " current of rest," Hermann does not consider it necessary to explain. What appears to be a natural current Hermann holds to be in all cases the result of injury. It is to be considered, then, as due to the peculiar molecular condition into which certain parts of a nerve-stretch are thrown by their mechanical or chemical destruction. In fact, whenever a nerve is cut across, or any of its fibres are injured, the molecules thus disturbed begin at once to die; they then become negative toward the other uninjured parts of the nerve. It is because of this change in the dying molecules that the electrical current is developed. But all the parts of a wholly untouched and unexcited nerve are, according to Hermann, "isoelectric." It is not necessary to give the experimental evidence by which this investigator strives to prove his opinion; it is enough to say that this evidence is strong, and nearly, if not quite, conclusive.

Accordingly, Hermann regards the negative variation as not due to the diminution of any current previously existing, but rather as a manifestation of the electro-motive forces which come into operation at the moment, and at the seat, of excitation. This current is, therefore, the only true "current of action." Its rise and flow are explained by the fact that every excited part of a nerve-stretch becomes negative toward all the other parts. As this wave passes along the nerve-fibre, each minute portion becomes first negative and then positive toward the adjoining minute portions; and hence the so-called "ad-terminal" and "ab-terminal currents" appear along the nerve-stretch as fast as successive parts of its substance reach their maximum of negativity. The excess of the ab-terminal over the ad-terminal current manifests itself as the so-called "negative variation."

The phenomena of electrotonus Hermann explains, as has already been said, upon the basis of Matteucci's experiments. An inner polarization, such as takes place between the wire and its moistened covering, takes place between the substance which constitutes the core of the nerve and one of its sheaths. The electrotonic current is, therefore, simply due to an escape of the polarizing current. It is wanting in the dead nerve, because the inner polariza- 
tion belongs only to the nerve in its living state ; it is stopped by ligature or by crushing, because the nervous substance is thus made into dead, indifferent substance, and the functional continuity of the nervous core is destroyed. His detailed explanation of "tetanic action-currents" and "phasic action-currents," and of the physiological phenomena of electrotonus and catelectrotonus, need not be repeated. The one principle to which Hermann would reduce all the electrical phenomena derived from the cut nervestretch is this: All excitable protoplasm, when dying or irritated, becomes negative toutard its own uninjured and unirritated parts. Such is the nature of its electro-motive reaction.

$\S 11$. Objections have been made to the theory of Hermann, but they can scarcely be said to be so formidable as those which he brings against the theory of du Bois-Reymond. The most forcible of them is, perhaps, the following: If the so-called currents of rest were due solely to the negativity of the dying portion of the substance, we should not expect that the current from the equator to the crosssection would be greater than the current from a point nearer the cross-section, seeing that the resistance is greater in the former case.

Hermann is himself ready to admit, ${ }^{1}$ however, that no simple scheme of polarization will fully satisfy the conditions of the problem offered by the behavior of the nerve-muscle machine under electrical stimulation. "The platinum wire, with its moist sheaths, is no model of the irritable nerve; it is only a model of its electrotonic properties." We must, therefore, after the discussion of all analogies resort again to the unknown molecular constitution and properties of the substance of the nerve, as being sui generis, for our explanation of its peculiar physiological properties. Its functions are a species of molecular change, connected, to be sure, both with chemical changes and with other mechanical changes of a wave-like character, and yet unlike them all ; and these molecular changes, when the nerve is excited, are propagated from point to point along its course with a speed and according to laws which have already been stated (see Chapter III.). But further than this we cannot as yet go with confidence in affirming a mechanical theory of even that simplest element of the nervous mechanism for experimental purposes-namely, the nerve attached to the muscle and constituting the nerve-muscle machine. ${ }^{2}$

\footnotetext{
'See Handb. d. Physiol., II., i., p. 195 f.

${ }^{2}$ Further information upon the two theories of Hermann and du Bois-Reymond may be found in Foster's Text-book of Physiology, pp. $101 \mathrm{ff}$. See, also, a brief statement of Hermann's theory in the Journal of Physiology, I., pp. 196-212.
} 
\$12. A confession of ignorance might fitly close the entire discussion as to the possibility at present of a precise mechanical theory of the nervous system. For on resuming the larger and more complicated inquiry, as to how the physiological functions of all the nervous organs in their mutual relations may be explained according to any known laws of molecular science, we are obliged to approach this inquiry with an acknowledged inability to deal satisfactorily even with the much simpler case of one of the elements of this system. The peculiar forms and laws of the molecular activity of the entire nervous mechanism certainly cannot be . understood until we are able to describe and explain the molecular activity of a single nerre-muscle machine. A statement of an elaborate theory, framed with a view to meet the whole case, by a distinguished authority, cannot fail, however, to possess a certain interest and value. Accordingly, we shall refer briefly to the theory of Wundt."

Wunclt begins his discussion of the mechanics (or molecular physies) of nervous substance by stating two possible ways of approaching the subject. It is conceivable that we might directly investigate the chemical and physical constitution of the nervous elements, and the changes they undergo in the exercise of their physiological functions, with a view to construct a theory of socalled nerve-force by induction from such investigation. But the preferable-because the much more promising-way of procedure is to assume that the processes which take place in the nervous system are modes of molecular motion connected with each other, and with the forces of external nature, under the general principles of molecular physics ; and then, arguing deductively, to make such a combination and application of these principles as will serve to meet all the demands of the case. It scarcely need be said that Wundt adopts the latter method.

Assuming, then, the general principles of molecular physics, and especially the law of the conservation of energy, it is possible to show how living beings may be brought under the control of these principles. Such beings, through the regularity with which the making and breaking of chemical combinations goes on within them, take a noteworthy part in the continuous process of interchanging potential and kinetic (inner and external) energy. It is the nervous system, in all the animals that have one, from which

1 To be found, in part, in his Untersuchungen zur Mechanik der Nerven, and in later and more complete form in the chapter (vi., Part 1.) "Physiologische Mechanik der Nervensubstanz," in his Grundzüge der physiologischen Psychologie. Leipzig, 1880. 
this process is controlled. The process itself is a species of combustion; the nervous system keeps going those functions which effect the process, regulates the setting free and distributing of the heat, and determines the muscles to movement. The source of the special activities of the nervous system itself lies in the nature of the chemical combinations which compose it.

The nervous system regarded as unaffected by stimuli-that is, as unexcited-may be theoretically compared to a fluid in a condition of equilibrium. But, in fact, the nervous system is never in a condition of perfect equilibrium. For, not only is there a ceaseless play of energy internal to this system, in which the atoms separate from the old combinations as nervous substance to form new combinations as the same substance; but a continuous process also goes on by which the molecules of the nervous substance are broken up to form less complex but more stable compounds. Moreover, the building of the nervous substance itself out of the nourishment brought to it is a process the reverse of that last mentioned; it is a process, that is to say, in which the more stable chemical compounds of other substance are broken up and their atoms used to form the more complex but more unstable molecules of the nervous substance. The process of change from the less stable to the more stable combinations represents the setting-free of stored or potential energy ; the reverse process represents the storing of energy and the vanishing of kinetic or actual energy. That energy which is made apparent by the former process Wundt calls "positive;" that which is stored up, when the more stable combination disappears, he calls "negative." Positive molecular energy of the nervous system is recognized as heat set free, as contraction of the muscles, etc. ; its negative molecular energy exists in the form of heat becoming latent, or of inhibitory action upon the course of excitation in the nerves, etc.

In accordance with the foregoing theory of positive and negative molecular energy, as due to the chemical activity of the nervous substance, Wundt would explain the process of excitation and conduction in the nerve-fibres. No simple conduction of motion, of course, takes place in the nerve ; but certain molecular processes, peculiar to the constitution of the nerve, are set up at one point by the stimulus, and are then conducted successively to other points along its stretch. In all cases when a nerve is irritated two classes of opposed effects are set up in its substance; the one is directed toward the production of external energy (secretion, stimulation of the ganglion-cells, movement of the muscles, etc.), the other toward the control of the energy thus set free. The former is positive, 
the latter negative or inhibitory. The general law for all excitation of the nerves is, that by the application of stimulus the positive as well as the negative molecular energy of the nervous substance is increased. Stimulating the nerve accelerates both the recombination of the atoms of its highly complex molecules in less complex but more stable forms, and also the escape of the atoms from these forms and their return to the more complex and less stable combinations. The renewal of the nerve depends upon the restitution of the more complex molecules; but the work which the nerve does external to itself depends upon that process of combustion in which the complex molecules break up and pass into more stable but less complex forms. The latter process involves, of course, the exhaustion of the nerve. External energy (work done outside of the nerve) can then only take place in case the positive molecular energy is more accelerated than the negative, by the application of the stimulus.

The entire sum of positive molecular energy which is set free when a nerve is irritated may be reckoned as distributed in three directions: a part is spent in the continuous excitation of the nerve ; another part becomes heat ; still another part is converted into negative molecular energy. In this way the peculiar molecular condition which the nerve-fibre leading from the peripheral region assumes, when it is irritated, is imparted to the central region of the nerve-cell.

$\S 13$. The application of the foregoing theory to the central organs of the nervous mechanism requires us to take account of the fact that a greater intensity of the stimulus is needed to move a muscle through a collection of ganglion-cells than directly by stimulating the nerve-fibre connected with the muscle. We are to conclude, then, that the nervous substance of the central parts offers a far greater resistance to the conduction of the process of excitation than is offered by the nerves themselves. On the other hand, the central organs are in a condition to develop within themselves a far greater amount of work; that is, to convert into kinetic form a vast sum of energy stored in their chemical constitution. The proofs which Wundt brings forward for this view are derived from the phenomena of summation of inhibition, and of so-called "reflex-poisons," etc. A detailed discussion of such phenomena leads to the conclusion that, when "summation" (compare pp. $223 \mathrm{ff}$.) takes place, the sereral excitations along the centripetal tracts have been conducted to different sensory central regions, and have then passed from them, as a result of their being simultaneously excited, over into the same motor elements of the 
central organ; but when "inhibition " takes place, such excitations have been conducted so as to come together and counteract each other in the same sensory central region. The external conditions of those relations which obtain among the different senses and sensations are to be found, partly in the constitution of the organs of sense, and partly in the nature of their respectire stimuli.

When speculating as to the molecular changes, with respect both to positive and also to negative energy, which take place in the central organs, our point of starting must be taken from a condition of equilibrium assumed to exist in their ganglion-cells. Excitation of the central organs, like irritation of the nerves, increases both kinds of nervous energy. But the positive molecular energy of the central organs is relatively little increased by a momentary excitation. The result of repeated excitation, however, is to make the positive conclition largely predominate in the whole central region. An excited ganglion-cell is in a condition analogous to that of the nerve-stretch at the anode when a constant current is passing through it. In the nerve, as a rule, the nervous substance is used up, and the process of storing energy goes on in only a very partial manner. In the cells the production of the complex molecules in which energy is stored predominates, as a rule.

The fundamental properties of nervous matter-mechanically considered-are (1) to receive external impressions in order by them to be determined in its own molecular condition; and (2) to transform potential energy into kinetic, partly under the immediate, and partly under the progressive, influence of these impressions.

Wundt also proposes an elaborate and highly speculative view of the molecular constitution and functions of the ganglion-cells. Every such cell possesses, he thinks, two regions (although the word "regions" is not to be interpreted locally). These regions are called "peripheral" and "central," because the former is assumed to stand in more intimate relations to the peripheral nervous substance, with respect to its own reactions under stimulation. Excitations which reach the central region of a ganglion-cell induce a propagation of the processes set up in this region to the other or peripheral region. In the same way do excitations which first touch the peripheral region necessitate the spreading of the form of molecular energy set free here over into the central region. When a process of excitation is frequently conducted in a definite direction through some ganglion-cell, such direction is favorably disposed toward the conduction of future excitations which may reach the same cell. Whether the excitation of any particular 
nerve-fibre connected with a ganglion-cell results in an excitatory or an inhibitory effect depends upon the nature of its connection with the cell.

But we refrain from further statement of $a$ theory so largely conjectural. Nothing remains but to repeat a confession of ignorance and of inability eren to suggest a satisfactory solution for so complex a problem in molecular physics as is offered by the human nervous system.

§14. A review of various molecular theories proposed to account for the nervous mechanism, either as a whole or in any of its parts, makes plain the important fact that such theories are all obliged to assume the origin and continuance of a peculiar molecular structure for this mechanism. In other words, no attempt to explain how the nervous system acts can avoid the conclusion that the determining factor in the explanation must be found in what the nervous system $i s$. The physiological functions of the nerre depart when the nerve dies. The nerre dies when it is severed from the ganglion-cell. Both cell and nerve must, therefore, constitute a living molecular unity, in order that their normal physiological functions may be performed. The explanation of these functions assumes the molecular constitution of the organs themselves. But how shall we explain, in accordance with the known laws of molecular physies, the origin and preservation of such a molecular constitution? It is the business of biology rather than of physiology to attempt an answer to this question. But the question itself asks from science the performance of a task no smaller than that of framing a mechanical theory of life. Biological science can, as yet, do little toward framing such a theory. Throughout our entire discussion of the nervous mechanism we have carefully avoided raising an inquiry as to the nature of life, as to the source and conditions of that very molecular constitution which determines the nature and working of this mechanism. We have simply assumed and argued that, taking the nervous system for what it really is and really does, its structure and functions admit of scientific explanation, so far as such explanation is possible at all, only when they are regarded as belonging to a molecular mechanism. The question of a mechanical theory for the origin and constitution of living organisms in general lies outside of the inquiries of Physiological Psychology.

$\$ 15$. One other important question has also thus far been avoided. What is the relation of the mind to the working of the nervous mechanism? Can the mind set this molecular mechanism at work, or can it in any way determine the character of its func- 
tions? As far as our consideration of the nervous system has gone hitherto, all might very well have been the same without the existence of a single act of conscious thought or feeling occurring in any relation whatever to this system. Given the molecular mechanism as it is constituted and conserved by the forces which control as long as life continues; and given the necessary impact of outside forces upon the end-organs, and the proper changes of blood within the central organs; and it has been assumed that this mechanism would exercise its functions in ways thus far described. But the consideration of another class of plenomena is now to be introduced; these are the phenomena of human consciousness, the phenomena of Mind. The question whether such phenomena can be true causes of any of the changes in the molecular mechanism is a part of the general question as to the correlations that exist between two classes of facts. The answer to such general question belongs to the following divisions of our work. 


\section{PART SECOND.}

\section{CORRELATIONS OF THE NERVOUS MECHANISM AND THE MIND.}





\section{CHAPTER I. \\ THE LOCALIZATION OF CEREBRAL FUNCTION.}

§1. Ordivary observation recognizes the fact that the phenomena of consciousness are more or less definitely correlated with the condition of the body. Certain alterations in our mental states, on account of the injury of any of its masses, as well as a constant dependence of those states upon the way some of the masses stand related to each other and to the outside world, impress the fact upon our daily experience. It is by no means so obvious that the nervous substance has any peculiar relation to the thoughts and feelings of the mind. For the functions of the nervous system are not exercised in giving information as to itself, its own condition and changes. By aid of these functions we have presented in con. sciousness a more or less clear picture of the condition and changes of the superficial parts of the body. In the same way a knowledge is gained of the successive states of tension belonging to the muscles in movement, and even-though rather obscurely-of the place and condition of the internal organs. But as long as they are healthy and excited with only a moderate intensity of their stimuli, the nerves do not even reveal their own existence; and when they are injured or unduly excited, the notice they furnish of the fact comes in the form of painful feeling which we have learned to localize, not in the nervous substance itself but in the adjacent parts of muscle and skin. Attention may be called, however, to the peripheral nerves by the accident or the dissectingknife which exposes them to sight. In the case of the central nervous organs, and especially in the case of the brain, there is little in ordinary experience which leads to a suspicion of their significance or even of their existence.

It is not very strange, then, that no general recognition of the supreme importance of the brain, in relation to the phenomena of consciousness, is to be found in early history. It is true that Plutarch $^{2}$ and Theophrastus ${ }^{2}$ inform us of the opinion of the

1 De Placitis Philosophorum, IV., 17, 1.

${ }^{2}$ De Sensu, $\$ 25 \mathrm{f}$. 
physician Alcmaeon, who is said to have been a younger contemporary of Pythagoras, and who regarded the brain as the common meeting-place of the senses. The same view is also ascribed to the celebrated Hippocrates. Later on Plato accepted it. But Aristotle, 'the greatest of all thinkers in antiquity, the son of a physician, especially educated in physical science, and well acquainted for the time in the dissection of animals, regarded the brain as a lump of cold substance, quite unfit to be the seat and organ of the sensus communis. This important office he ascribed rather to the heart. The brain he considered to be chiefly useful as the source of fluid for lubricating the eyes, etc.

§ 2. The opinion of Exner, ${ }^{2}$ however, who supposes that feeling in no way immediately informs us that we think with the head, still less with the brain or the cortex of the cerebrum, seems somewhat extreme. Concerning the contents of the cranial cavity, indeed, we get no direct information from the feelings connected with the exercise of its functions. But we certainly localize in the head certain phenomena of consciousness that are inextricably interwoven with the processes of thought. The act of attention results in feelings which indicate that the muscles of the eye are being innervated or in the more indefinite and diffused sense of strain produced by contracting the skin of the forehead and adjacent parts of the face. The special sensations of hearing, smelling, and tasting, which impress so strongly our conscious life, are frequently referred to the head. The same thing is true of many of the sensations of sight-particularly of such as appear when the eyes are closed, in the form of after-images, or spectra, or indefinite and changing color-spots, seated in the upper front part of the face. Moreover, that inchoate and sometimes half-articulated language, with which we support our trains of thought, even when we are not conscious of resorting to the expedient of "talking to ourselves," is felt to be going on within the head. When one has been engaged for some time in intense thought, or in enger and concentrated observation, one is suddenly made aware of more or less painful feelings which are somewhat indefinitely ascribed to the same cerebral region. Men commonly lean the lead upon the hand in supporting meditation; or rub it vigorously to awaken the powers of memory and reasoning; or stroke it to relieve the disagreeable sensations which follow severe mental excitement. Headache, of more or less intensity, thus becomes associated with

${ }^{1}$ See De Partibus Animalium, 652, b. 5; (II., 7); 656, b. 22 (II., 10); De Juvent., 467, b. 28 ; and De Anima. III., 1 and 2.

'See Hermann's Handb. d. Physiol., II., ii., p. 192. 
active exercise of the intellect. The head is wearied with thought ; and not only so, but also with intense physical exercise. The discomfort which bodily strain produces in the hinder regions of the head are an indication, although of only a very general kind, that processes have gone on in that locality which are of great importance to the succeeding states of consciousness. All this apparent testimony of immediate feeling is; doubtless, somewhat exaggerated in an age so distinctively "nervous" as our own ; and this fact may, perhaps, account in part for the inclination of the ancients to emphasize the more obvious connection of mental phenomena with the heart, and other lower visceral organs, to the neglect of all connection of these phenomena with the brain. But it cannot well be doubted that a certain amount of testimony from immediate feeling as to the important relation which exists between the state of mind and the contents of the cranial carity, belongs to all human experience.

Howerer uncertain the witness of immediate feeling upon the point in question may be, very little observation of others is needed to amplify and confirm its witness. We are not infrequently led to notice how quickly and profoundly the states of consciousness are changed by injuries to the brain. The effect of a blow upon the head in suspending consciousness is decisive of this question. The intimate local connection between the organs of sense and the brain leads naturally to the conclusion that the avenues of sensation and of perception have in the latter a kind of gathering-place, as it were. It is but a step from this conclusion to a recognition of the truth that the plyysiological significance of the contents of the cranial cavity consists in their affording a field upon which all the impressions of sense can meet together, and so furnish the basis and material of comparative thought. Indeed, it was this line of inquiry which probably led certain ancient anatomists, like Herophilus and Galen, to locate the soul, or psychical principle, in the brain.

§ 3. A great multitude of plyysical considerations, advanced by modern science, place beyond doubt the supreme importance of the brain in its influence upon the phenomena of consciousness. It has already been stated (Part I., Chapter III., § 7) that the free circulation of arterial blood, with its supply of oxygen, is a necessary condition for the fulfilment of the functions of all the central organs; this necessity is especially marked in the case of the brain. The stoppage of one of the great arteries leading to this organ, either by compression in the neck, or by embolism at some point along its course, at once produces profound disturbances and even complete cessation of consciousness. It has been calculated that, while the weight of the entire encephalon is 
only about one-forty-fifth of that of the body, the supply of blood used up there is not less than about one-eighth of the whole supply. This expenditure is indicative of the large amount of work done by the intercranial organs.

More delicate measurements seem to show that the temperature rises and falls in the whole cerebral area, or at particular circumscribed regions of the cortex, in close connection with the psychical activities. Thus Dr. Lombard found, by measurements with exact thermo-electric apparatus, that the temperature of the head during waking hours varies rapidly, though slightly (less than $\frac{1}{10 \pi}{ }^{\circ}$ C.) ; and that these variations "appear to be connected with different degrees of cerebral activity. . . . Every cause that attracts the attention-a noise, or the sight of some person or other object-produces elevation of temperature. An elevation of temperature also occurs under the influence of an emotion, or during an interesting reading aloud." Similar examiuations have been carried still further by Schiff, ${ }^{1}$ who has applied extremely delicate thermoscopic instruments directly to the cerebral substance of certain animals (comp. Part I., Chapter III., § 21). He finds that the arrival of sensorial impressions is followed by a rise of temperature, in certain special areas of the cortical substance, where - as he supposes-these impressions are diffused; he also concludes that any resulting psychical activity is itself connected with a still further rise of temperature than that which the sensorial impressions alone engender. Schiff's conclusions, therefore, point not only to the localization in the entire brain of functions connected with the phenomena of conscious psychical life, but also to some distribution of such functions among its various areas. In the same general direction are the conclusions of Byasson ${ }^{2}$ and others, as to an increase of waste in the tissues of this organ, which corresponds, to some extent, at least, with the amount of thought accomplished. This investigator found that the quantity of sulphates and phosphates excreted, in comparison with the quantity estimated as entering into his diet, was notably increased in proportion to the amount of his mental work. That is to say, in connection with an increase in the number and intensity of the psychical operations stands an increase in the functional activity of the cerebral cells, as shown by the expenditure of their phosphorized constituents. ${ }^{3}$

1 Archires de Physiologie, 1870, p. 451.

${ }^{2}$ In the Jour. d. Anat. de Robill, 1869, p. 557 f.

${ }^{3}$ See the chapter of Luys on the Physico-chemical Phenomena of Cerebral Activity; The Brain and its Functions. 
84. Comparative anatomy also indicates the importance of the relation between the size, structure, and functions of the intercranial nervous mass and the phenomena of mind. It shows, first of all, a general but indefinite correspondence between the size and weight of the brain of any species of animal, as compared with the weight of its entire mass, and the piace of the same species in the scale of intelligence. This fact is roughly exhibited by the following comparative table: ${ }^{1}$

RELATION OF THE WEIGHT OF THE BRAIN TO THE WEIGHT OF THE BODY.

\begin{tabular}{|c|c|c|}
\hline Tunny-fish & 37,440 & Finch. \\
\hline Land tortoise. & 2,240 & $1: 160$ \\
\hline Shad $\ldots \ldots \ldots \ldots \ldots 1$ & 1,837 & Pigeon.. \\
\hline Tadpole... & 720 & $1: 82$ \\
\hline Elephant.. & 500 & Gibbon \\
\hline Salamander. & 380 & Young cat \\
\hline Sheep............ & 351 & Sai-ape. \\
\hline
\end{tabular}

Doubtless other tables might be compiled which would lead to less satisfactory conclusions than the one given above. Even in this table we note that the elephant stands lower than the salamander or the sheep, both of which animals are, in fact, far inferior to the elephant in intelligence. Large allowance must also be made in certain cases for peculiarities of physical structure ; for exainple, the tortoise is rated lower than he would be were it not for his heary shell. The law itself is confessedly subject to remarkable and unexplained exceptions; at best it holds good only in a very general way. For example, the relative weight of the brain is not greatly different in the dolphin, in the baboon, and in man. It is much greater in the infancy and youth of the human species than in middle life or old age. In the male child at birth it is about as one to six or seren (according to Tiedemann, 1 to 5.85 in the male, and 1 to 6.5 in the female). The brain grows with great rapidity for the first few years-the increase during the first year being estimated at about one cubic centimeter daily. But the rest of the body increases so much more rapidly that by the end of the second year it is about 1:14; by the end of the third year, 1:18. It increases in absolute weight until well on into middle life, and then after middle life diminishes at about the average rate of one

${ }^{3}$ Taken from Hermann's Handb. d. Physiol., II., ii., p. 193, as compiled by Exner, on the basis of the works, in part of Carus, and in part of J. Miiller. The figures of comparative weight between the brain and the body are some. what differently given by other authorities. 
ounce in a decade. The average relative weight of the adult brain is one-fortieth or one-fiftieth. Tiedemann found that the relative weight of the brain is dependent upon the absolute weight of the body, and is relatively greatest with light persons. The human brain is, however, absolutely heavier than that of any of the animals except the elephant ( $8-10$ lbs.) and the whale (5-6 lbs.).

Much pains has been taken, by actually weighing different human brains, or by calculating their weight on the basis of careful cranial measurements, to establish a law connecting the amount of the intercranial nervous mass with the comparative intelligence of races and of individuals. ${ }^{2}$ The average weight of the brain of the adult European is, for the male, from 46 to 52 ounces; for the female, from 42 to 46 ounces. Boyd gives the average weight of the brain of the male, at the period of life when it is most developed (twenty-five to forty years of age), as 46.8 ounces (1,321 grams, 91 centigrams). This difference between the sexes is not wholly dependent on difference in bulk of body, but is an important sexual distinction. The brain of man is on the average ten per cent. above that of woman ; the difference in average stature is about eight per cent. Many human brains rise above the upper average ranges; others fall below the lower average ranges; and yet no marked peculiarities of mental development are necessarily connected with these variations. Considerable quantities of the substance of the brain may be lost (at any rate from some areas of the cortical surface) without perceptibly changing the mental life. In 278 cases of males, the maximum weight of brain was found to be 65 ounces, the minimum 34 ounces; in 191 cases of females, the maximum was 56 ounces, the minimum 31 ounces. $^{2}$ Numerous instances of large excess in the average weight of brain-mass by individuals eminent for intelligence are on record : for example, Byron scarcely under 79 ounces; Cromwell, only 77 grains less, or 78.8 ounces (although Vulpian thinks that the national spirit has exaggerated both these instances); Cuvier, 64.5 ounces; Abercrombie, 63 ounces; Spurzheim, 55 ounces ; Sir J. Y. Simpson, 54 ounces; Webster, 53.5 ounces; Agassiz, 53.4 ounces; Chalmers, 53 ounces. Other persong of eminence, however, have had brains of only average, or of under average weight; thus C. F. Hermann, 46.5 ounces, and J. F. L. Hausmann, 43.3 ounces. Moreover, brains of high weight not infrequently occur without evidence of unusual mental capacity, or even in the case of those mentally inferior. Record is

${ }^{1}$ On the relations of the Brain with respect to weight and mass, see Schwalbe, Lehrb. d. Neurologie, ii., pp. 589 ff. Erlangen, 1881.

' Results obtained by Sims, Clendinning, Tiedemaun, and J. Reid. 
made of four male brains, belonging to persons of no repute for intellectual ability, which ranged from 62.75 ounces to 61 ounces ; of another such, which weighed 60.75 ounces; of the brain of a boy of fourteen which weighed 60 ounces. In the West Riding Asylum ' for the Insane, out of 375 males examined, the weight of the brain in 30 cases was 55 ounces or upward; out of 300 females examined, in 26 cases it was 50 ounces or upward. Several persons afficted with dementia were found to have brains weighing more than 60 ounces. On the contrary, idiots, almost without exception, have brains far below the average in weight; as a rule, the brain of such an unfortunate does not weigh so much as 30 ounces. Cases of microcephalous idiots are on record whose brains weighed only 10.5 , or even 8.05 ounces. Here, again, howerer, singular exceptions must be admitted; for in a few cases the brains of idiots have reached the arerage weight, and have even, in rare cases, considerably surpassed it.

Although the data adduced to show that the arerage weiglit of brain in the more highly civilized races is greater than in the savage races, are by no means abundant or conclusive, yet they are sufficient to create a reasonably strong presumption in favor of this view. Calculating from the size of the cranial cavity, as ascertained by measurement of a large number of skulls, it is inferred that the arerare weight of brain in the African, Australian, and Oceanic races generally, falls from 1 to 4 ounces below that of the more highly civilized European. It is further noted that there is almost a complete absence of cases rising above the higher ranges-above 54 ounces, for example; and that there is not the same difference between the two sexes in the uncultivated as in the cultivated peoples. Davis calculated the average weight of brain among the Chinese to be about equal to that of the Caucasian race in Europe; among the Sandwich Islanders to be some thirty grams less. The surprisingly low weight of the brain of the Hindus is in part a function of their smaller weight and bulk of body. It may fairly be urged in objection, that by the method of measuring skulls taken somewhat at random we should be likely to find a noteworthy absence of such exceptional cases in certain quarters among the European races; and that the relative increase in size of the female brain among uneducated peoples is probably, in part at least, the result of the response of the nerrous system to the demand made upon it for the hard labor performed by the women among such peoples.

Any law which refers the intensity and range of the mental

${ }^{3}$ For these facts see the Encyclopædia Britannica, ninth ed., I., p. 879 f. 
activities directly to the size and weight of the nervous mass of the brain must, therefore, be held only very loosely. It is to be expected that many unexplained exceptions will meet us, whether we compare men with the other animals, or certain races of men with others, or individual men with one another. No intelligent physiologist would now think of making mere mass the test of mental capacity.

\$5. A more intimate relation of dependence exists between the amount of intelligence and the complex structure of the brain as arising to a large extent from the development of the cerebral hemispheres-that is, from their relative size and expanse, and from the number and depth of their convolutions. In other words, wealth of expanded and convoluted cerebral hemispheres is, in some general way, a measure of the richness and intensity of mental life. This conviction becomes stronger the more carefully the comparative anatomy of the cerebrum, and the development of the cerebral hemispheres in the human embryo, are examined. The forms of brain found permanently in fishes, amphibians, reptiles, birds, and the lower mammals, are extremely similiar to those shown in succession by the developing brain of the higher mammals, and especially of man. The most distinctive feature of man's superior brain is the marked development in the size, number, and depth of the convolutions of the hemisplieres. In fishes generally, both cerebrum and cerebellum are very small; but the ganglia connected with the organs of sense, especially of vision, are relatively large. In amphibia the cerebral hemispheres are relatively enlarged; are advanced backward still farther in reptiles; while in birds the vesicles of the mid-brain are partially hidden by the development of the hemispheres. In the lower mammals the enlargement of these same organs by growth backward continues, and their two parts become connected by a commissure; but they still remain comparatively meagre in size and simple in structure, without much distinction of lobes or division into convolutions. It is only in the most elaborately developed brains of the higher mammals that the occipital lobe enlarges backward so as to cover mid-brain, cerebellum, and medulla oblongata; and that the frontal lobe spreads forward over the nasal cavities so as to constitute a derelopment of forehead. Meantime the convolutions apparent on the cerebral surface increase in number and depth.

The theory suggested by comparative anatomy is confirmed by the probable view of Meynert, that the whole of this cortical region of the cerebrum is a great "projection-field" on which the sensory impulses are marshalled and systematically ordered (to serve, as it 
were, for the physical basis of mental phenomena), as they arrive from the peripheral regions and are distributed over the outgoing motor tracts. Certain striking exceptions to the principle of this theory must, however, be acknowledged. Within each great group of animals considerable variations occur in the degree of cerebral convolution, such that it cannot be said accurately to measure the degree of intelligence. For example, among mammals the insectivora have brains "poorest" in convolutions, the herbivora are "richest," and the carnivora stand between; the ruminants, although rather dull and incapable of being taught, have numerous and deep convolutions enough to rank them much higher than their real intelligence deserves. The marmoset, on the other hand, the relative weight of whose brain is as 1 to 18 , shows a comparatively smooth and non-convoluted surface, in striking contrast with that of other monkeys.

Trustworthy data are as yet wanting to place beyond doubt the probable opinion that the brains of less highly civilized races and less highly intellectual individuals are relatively poor in development of the cerebral hemispheres. The human embryo is, indeed, an illustration in miniature of the truth of this opinion; the older it becomes the more distinctly marked are the lobes of the cerebral hemispheres, and the more numerous and deep are their convolutions. The brains of idiots are said, as a rule, to be poor in convolutions; this fact is doubtless connected with the embryonic condition in which many of them have remained through arrested development. Hermann Wagner, ${ }^{1}$ on the basis of measurements made by his father, undertook to estimate the comparative total surface of the cerebral hemispheres of four brains, viz.: of two males of noteworthy intelligence (Gauss, the mathematician, and Fuchs, the physician), of a male laborer (Krebs), and of a female in middle life. By weighing carefully the amount of gold-foil laid on uniformly, which was required completely and closely to envelop all the convolutions of these brains, Wagner concluded that the area of concealed surface was, in each case, approximately equal to that exposed. The total surfaces of the four brains were thus found to measure-of Gauss, 2,196 square centimeters; of Fuchs, 2,210 ; of the woman, 2,041 ; of Krebs, 1,877. It is a tempting but rather insecure generalization which concludes from so few cases that the richness of the cerebral convolutions (the total surface, both that exposed and that concealed by the sulci), is a general direct measure of the intelligence.

\$6. Other interesting attempts have been made to measure the

${ }^{1}$ Maassbestimmungen d. Obertläche d. grossen Gehirns. Cassel, 1864. 
intelligence of the animal by the relative size and structure of the intercranial nervous mass, and so, definitely, to establish a direct relation between the two; we notice especially those of J. Müller, ${ }^{1}$ and of Meynert." The great physiologist, Muller, held that the position of an animal in the scale of intelligence may be estimated by comparing the hemispheres of his brain with the corpus quadrigeminum. According as the latter organ is relatively large, and lies behind the hemispheres, uncovered by them, the animal is low in the scale of intelligence; according as the hemispheres increase in size, and so envelop and bury beneath them the relatively small corpora quadrigemina, the animal stands high in that scale. This statement, however, scarcely covers anything more explicit than the general fact that relative increase of the cerebral hemispheres is indicative of progressive mental life. Meynert has pointed out other important relations between parts of the brain, by which he proposes to measure the intelligence. In the entire mass of the crura cerebri we may recognize two parts, an upper (tegmentum), which stands in direct connection with the optic thalami and the corpora quadrigemina, and a lower (crusta), which is connected through the lenticular nuclei of the striate bodies with the cerebrum. Now the greater the hemispheres are in comparison with the corpora quadrigemina, the greater must the mass of the crusta be in comparison with that of the tegmentum. The development of the pons Varolii is also essentially dependent on that of the crusta, for the fibres of the latter enter into the former; the arching of the pons is therefore connected with the development of the hemispheres. In general, then, the relative development of the entire tract represented by the crusta, or lower part of the crura cerebri, and the nucleus lenticularis, the fibres of which expand in the cerebrum, is--according to Meynert-a measure of an animal's intelligence. In man the mass of the crusta on the level of the corpora quadrigemina exceeds that of the tegmentum; in the other mammals the reverse is true. ${ }^{3}$

$\S 7$. The above-mentioned facts of comparative anatomy, with many others similar, show plainly the unique significance which the masses of the brain, and especially the cerebral hemispheres, have, as related to the phenomena of self-conscious mind. They may be supplemented and confirmed through other facts furnished by physiology, especially of the experimental kind. Upon this point,

'Handb. d. Physiol. d. Menschen, 1844, I., p. 702 f.

${ }^{2}$ Sitzgsber. d. Wiener Acad., LX., iii. (1869), pp. 447-462.

${ }^{2}$ For a brief but judicious discussion of this subject, see Brücke, Vorlesungen über Physiologie, 1884, II., pp. 52 ff. 
for the present, reference is simply made to the results of investigation as already set forth in Part I. (see especially Chapter IV.). Physiology demonstrates that the nerrous impulses, so far as they result in sensation, pass along centripetal tracts which converge from every portion of the periphery toward the brain; and that, so far as they result in motion following upon idea and volition, they pass along centrifugal tracts diverging from the same central masses. It thus confirms the same theory which studies of the anatomical structure of the nervous system suggest, namely, that in these masses, and especially upon the cortex of the cerebrum, is the common meeting-place of both kinds of impulses. The section or injury of any nerve-tract, even in the spinal cord, apart from indirect and secondary influences, does not affect the psychical functions. In such an event, the parts of the body lying peripherally from the point of interruption are simply withdrawn from all direct connection with sensations or volitions. Sensory impulses, then, no longer occasion sensations; ideas of motion and volitions to motion, of the parts thus disconnected, become of no effect in producing the customary result. It has also been made obvious that, in proportion as the masses of an animal's brain are remored or incapacitated from performing their functions, the evidences of a varied and complex mental experience are diminished. The simple spinal cord of a frog, acting as a nervous mechanism, will perform a few wonderful feats; joined with the medulla oblongata, optic lobes, and other lower parts of the brain, it will give largely increased signs of psychical phenomena; it would not be claimed, however, that the cerebral hemispheres of this animal-relatively insignificant as they are when compared with those of the higher animals - are of no special importance for its lighest psychical life. Essentially the same thing, though in more emphatic form, is true of all animals of a higher grade of intelligence.

$\S 8$. In the case of man, the cerebral hemispheres are, apparently, the only portions of the nervous system, between the size, condition, and molecular activity of which and the phenomena of consciousness there is a direct correlation. If, then, we are to speak of mental activities as "localized" at all, the locality must be in the cortex of the cerebrum. The position that, in the case of man, the spinal cord and all the intercranial organs below the cerebral hemispheres, are incapable of acting as the immediate physical basis of mental states, is confirmed even by those experiments upon other animals, which seem at first sight to discredit it. The hypothesis that consciousness has a seat in the spinal cord of the frog; that, in fact, we may properly speak of the decapitated animal as 
having a soul-has been urged by eminent physiologists (Pflüger, for example). That the cord alone is capable of various purposeful activities, such as serve, under certain circumstances, as signs of a psychical experience, may be demonstrated by experiment (comp. Part I., Chapter IV., $\$ \S 4 \mathrm{ff}$.). But unless one is prepared to maintain that all purposeful activity, as resulting from excited nervous substance, must be correlated with phenomena of conscious sensation and rolition, one can scarcely assume with confidence that such phenomena accompany the movements of the decapitated frog.

What the nervous mechanism will do, when set agoing by the appropriate stimuli, depends not only on its original structure, but also on its acquired habits of action. That this law holds good even for the mechanisin of the hemispheres of the brain is obvious from various facts. Stimulating those regions of the cerebral cortex which are connected with definite groups of muscles, in the case of the adult animal (for example, a dog), does not call out the same responses in the animal newly born (that is, under nine or ten days old). The case of the bird which has lost its cerebral hemispheres, and which executes motions by means of the lower basal ganglia, that seem to indicate a complex psychical life (comp. Part I., Chapter IV., $\$ 20$ ) is less easy of solution. Are we to consider such an animal still capable of "sensation," "perception," and "volition?" If this question means whether any phenomena continue to occur such as correspond to those conscious experiences of our own to which we apply the above-mentioned words, then we must confess our inability to answer it.

In general, we know extremely little of the conscious mental life of the lower animals. What we conjecture is wholly dependent on the interpretation, given in terms of our human consciousness, to motions of their bodies resembling those which express definite conscious states in ourselves. But a large part of our own bodily activity is ordinarily not definitely correlated with any conscious mental activity ; for example, breathing, winking, swallowing, changing the posture of the body in sleep and in states of profound meditation, and especially the very complex operations involved in walking, singing, playing on musical instruments, or handling a tool, etc. In all these and similar cases, we find that the intricate and purposeful play of the mechanism is by no means necessarily connected with a corresponding series of conscious sensations and volitions. But in proportion as the hemispheres of an animal's brain become relatively developed, not only their absolute but also their relative significance is increased. The influence 
of the brain proper upon the roluntary movements of an animal is greater, the higher the animal stands in the scale of cerebral development and of intelligence. A frog, or a fowl, deprived of its hemispheres, can do what is quite impossible for a dog or an ape in the same condition. If, then, man's nerrous mechanism, especially in case it has been trained to elaborate co-ordinated functions, can, without any corresponding accompaniment of mental phenomena, accomplish so much which appears significant of the most elaborate psychical activities; a fortiori, it is likely that we may make this mechanism, working without consciousness, account for most of what is done by the hen or pigeon without its cerebral hemispheres. Moreover, experimental physiology undoubtedly tends toward accounting more and more fully for the most complex bodily motions under the terms of physical mechanism.

The most marked result of an animal's loss of the cerebral hemispheres is the sudden and great, or total departure of its intelligence. This fact is, of course, confirmatory of the impression that the functions of these hemispheres, and of them alone, constitute the physical basis of its intelligence. We confess, however, our inability to affirm that the "psychical life" of every animal is inseparably bound to its continued possession of these organs. There may possibly be a varied psychical life of animals that have no brain. Yet in the case of the higher mammals, and especially in the case of man, we need not hesitate to affirm the probability of such an inseparable connection. The physical basis of the phenomena of human consciousness is pre-eminently, if not exclusively, the convoluted cortex of the cerebrum.

§. It is impossible, accordingly, to aroid raising the inquiry whether some more definite scheme of the localization of cerebral functions may not be discovered. The cerebral cortex is itself a very complex organ, or system of organs. Its different regions are marked by comparatively slight, and yet not insignificant, differences of structure; they stand in different local relations and nervous connections with one another and with the ganglia lying below. This outlying rind of gray nervous matter is, of course, not a homogeneous mass. It is made up of innumerable nervous elements combined in various ways and multiform connections. It may be regarded, then, as a complex of organs. The question therefore arises: Have the different members of this complex of organs different relations to definite motor activities in the peripheral regions, and to the various phenomena of conscious mental life? or, in other words: Have different parts of the cerebral hemispheres all the same office and value in relation to the life 
of sensation and voluntary motion? This is the question generally understood under the term-" the localization of cerebral function."

$\S 10$. Most of our definite knowledge concerning the functions of the other parts of the nerrous mechanism creates a presumption in favor of some localization of cerebral functions. All the different parts of this mechanism are, indeed, constructed by combining variously a few elements of essentially the same structure; all of them likewise are capable of exercising essentially the same neural functions. But each part of this mechanism has also its special functions. Thus we found that the different nerves become classified functionally; some are motor, voluntary or involuntary, some inbibitory, some secretory, some sensory, etc. Hints of a certain kind of classification may be discovered for the smaller ganglia or collections of nerve-cells. In making transverse sections of the cord, different regions with different functions appear. Considered longitudinally, the cord is capable of being more or less definitely divided into several so-called centres, with specifically different functions. Localized centres, where specific kinds of reflex-motor activity have their particular seats, are fairly crowded together in the medulla oblongata. All the lower parts of the encephalon appear subject, in a measure, to the principle of localization. Shall we, then, stop short in our attempts at differencing the functions of the locally separate parts of the nervous system just at the point where we reach the most complex and extended organ, or rather collection of organs, which this system contains?

\&11. Notwithstanding the strong presumption in favor of the localization of cerebral function, the beginnings of a successful attempt to establish this theory are only about fifteen years old. The doctrines of Gall, Spurzheim, and others in the older school of phrenologists, proved so inconclusive as to bring contempt upon subsequent attempts to divide the hemispheres of the brain into different functional areas. Moreover, certain indisputable facts seemed to render impossible the assured beginnings of a theory of cerebral localization. Considerable portions of the human brain, it was found, might be lost without destroying any one sensory or motor function. Moreover, the gray matter of the cerebral hemispheres, it was then thought, could not be directly excited by electricity or by other forms of stimuli. The greatest experimenters in physiology, such as Longet, Magendie, Flourens, Matteucci, van Deen, Budge, and Schiff, declared against the localizing of cerebral function. In 1842 Longet $^{1}$ affirmed that he had experimented upon

'Anatomie et physiologie du système nerveux, etc., Paris, 1842, i., p. $644 \mathrm{f}$. 
the cortical substance of dogs, rabbits, and kids, had irritated it me. chanically, cauterized it with potash, nitric acid, etc., and had passed galvanic currents through it in different directions, without obtaining any sign whatever of resulting muscular contraction. In the same year Flourens ${ }^{1}$ asserted, on the basis of numerous experiments in extirpation, that the lobes of the cerebrum perform their functions with their whole mass; that there is no special seat for any of the cerebral activities; and that even a small remnant of the hemispheres can serve all the uses of their collective functions.

So great was the authority of the distinguished names just mentioned, that their confident opinions gained general credence. The evidence brought forward by Broca and others seemed, however, to show some special connection between a single convolution of the frontal lobe and the complex activities of articulate speech; and the anatomist, Meynert, held the opinion that the structure and connections of the cerebrum show its anterior portion to be in general used for motor, its posterior for sensory functions. In 1867 Eckhard repeated the siynificant observation which had been made by Haller and Zinn more than a century before: namely, that, on removing parts of the cortical substance of an animal's brain, convulsive movements occur in its extremities.

$\S 12$. It was not until 1870 that the "epoch-making" experiments of Fritsch and Hitzig ${ }^{2}$ began the modern era of investigation into this subject. These observers announced the fact that the cerebral cortex of dogs is, at least in certain minute areas of it, excitable by electricity. They pointed out the further fact that, while some parts of the convexity of the cerebrum are capable of motor excitation and others not, the motor parts lie in general to the front, the non-motor to the rear of this convexity. By stimulating with an electrical current the so-called motor parts, co-ordinated contractions of the muscles in the opposite half of the body are obtained. Of such so-called "motor centres" they indicated, in their first announcement, the following five: One for the muscles of the neck, another for the extension and adduction of the forelimb, another for the bending and rotation of the same limb, another for the hind-limb, and lastly one for the face. From such facts they drew the conclusion that the principle announced by

1 Recherches expérimentales sur les propriétẻs et les fonctions du système nerveux, etc., p. $99 \mathrm{f}$.

${ }^{2}$ See the artiele by G. Fritsch and E. Hitzig in the Archiv f. Anat., Physiol., etc., 1870 , pp. $300-332$; and subsequent articles by Hitzig in the same Archiv, 1871, 1873,1874,1875, 1876 ; also his work, Untersuchungen über das Gehirn, Berlin, 1874. 
Flourens is demonstrably false. We must rather admit, say they, that " certainly several psychical functions, and probably all, are shown to have their point of entrance into matter or of origin from it at circumscribed centres of the cerebral cortex." 1 The same principle was subsequently defended at length by Hitzig, and the number of so-called cerebral centres increased. The most noteworthy facts which these experimenters first made clear and demonstrable have since been verified by many investigators. Many of these facts may, with care and skill, be verified by any observer. Dr. Ferrier in particular has used the method of Fritsch and Hitzig to map out the hemispheres of the brain of the monkey into no fewer than fifteen kinds of centres. The testimony of human pathology, and the evidence of comparative anatomy and of histology, have also been largely drawn upon either to confirm or to confute the conclusions originally based on experiments with animals. Before considering the conclusions themselves, it is necessary to understand the true nature and extent of the various kinds of evidence.

$\S 13$. Exner ${ }^{2}$ has well said that "a physiology of the cerebral cortex, in the sense in which there is a physiology of the muscle, etc., scarcely exists at the present time." The reasons for such a deficiency lie partly in the very nature of this organ and the place it holds within the animal economy ; as well as partly, perhaps, in certain prejudices which have hindered the physical theory of a material structure so intimately related to the action of the mind. The cerebral cortex of the animals is experimentally approached only by overcoming immense difficulties. Moreover, those physical and chemical processes of the cerebral substances, to which we must look for any strictly scientific understanding of its physiology, are placed almost utterly beyond reach of investigation. Reasoning must fill up with conjecture the great gaps that lie between a very complex series of physical occurrences, only a part of which are observable, on the one side, and on the other, an equally complex group of psychical occurrences. The latter belong to a different order of phenomena from the former; and, moreover, in the case of the lower animals-which must be selected almost exclusively for experiment-we know nothing of these psychical occurrences except through physical signs that are peculiarly liable to misinterpretation. The result is that our conclusions on the localization of cerebral function must be reached by considering a great multitude of complicated facts, many of which appear to take sides with contend-

${ }^{2}$ Archiv. f. Anat., Physiol., etc., 1870, p. 332.

'See Hermann's Handb. d. Physiol., II., ii., p. 189. 
ing champions of different theories who alike appeal to them. It is only by observing the directions in which the different lines of evidence seem to point in common, that we can reach even a probable opinion upon a few points.

$\S 14$. Three great lines of evidence, leading from three great groups of facts, must be considered. These are the evidence from experimentation, the evidence from pathology, and the evidence from histology and comparative anatomy. Each of the three has its peculiar advantages and value; each also its peculiar difficulties and dangers. It is only by regarding the combined testimony of the three that the highest probability at present possible can be attained.

Experimentation with a view to discover the localized functions of the cerebral cortex is of two kinds, stimulation and extirpation. Here, too, what has already been said (Part I., chap. IV., \$14) concerning the difficulties of the same mode of investigation in the sub-cerebral regions of the encephalon must be recalled and made more emphatic. All experiment by stimulation of certain areas of the hemispheres of the brain relies, of course, upon the argument that those areas whose stimulation is followed by the movement of definite groups of muscles are especially connected with such groups of muscles. The further assumption is likely to be made that these areas constitute the special organs which have control, as it were, of the same muscles. Since it seems to be a general principle that the sensory and motor nerve-tracts distributed to any region of the periphery come into tolerably close local relations to each other somewhere within the entire field of the cerebrum, it would seem to follow that some special connection exists between certain classes of sensations and volitions and the circumscribed areas of cortical substance pointed out by experiment. It should not be forgotten, however, that the excitation of any group of muscles, by applying stimulus to some area of the cerebral cortex, proves only that this area is somehow connected with such group of muscles. It still remains to be shown that sensory impulses, on arriving from such a peripheral portion of the body, serre as the physical basis for the psychical phenomena of sensation solely within this circumscribed central area ; or that conscious volitions, in order to be followed by motion in this peripheral portion, must give rise to the molecular commotion of the same area.

$\S 15$. By far the most efficient and manageable stimulus for experimenting upon the localization of cerebral function is the electrical current. Mechanical or chemical irritation may, however, be employed in certain cases. The use of the electrical current incurs, 
of course, the danger of its diffusion. Important objections, based upon this fact and upon other grounds connected with the use of electricity, have been raised to the conclusions of Hitzig.' To Hitzig's claim that the electrical currents which excite the so-called motor areas are "very weak," and therefore unable, at a very slight distance from the place of the application of the electrodes, to affect the nervous substance, Hermann replies that, on the contrary, considering the effect antecedently to be expected, these currents are "surprisingly strong," and that the brain, in diffusing the currents, must act like any other substance (e.g., a mass of copper) of similar form-that is to say, the distribution of such a current in the substance of the brain is a purely geometrical function of the form of this substance and of the position of the electrodes. Moreover, it is found that increasing the strength of the current applied to a so-called "motor area" invariably increases the size of the cortical region thrown into activity. That extra-polar conduction actually takes place in the substance of the brain has been shown by Dupuy, and by Carville and Duret; contraction of the muscle of the rheoscopic frog and deflection of the needle of the galvanometer, at remote distances from the electrodes, prove that the current passes along the whole extent of the cerebral hemisphere. The excitability of the cortical substance continues for hours after its exposure to the air, or after acids have completely destroyed its external third portion. If the cortical area be separated by a circular cut from all connection with the nervous substance below, it is still excitable with only a slight increase in the strength of the stimulus applied. Or if the gray substance of the surface be wholly removed, and the electrodes plunged in the blood of the cavity of one of these socalled motor areas, the customary results follow. Still further, the size of the circle within which the minimum amount of stimulus, when applied to certain gyri, will serve to excite the hind-limb of the animal, remains about the same whether the amount of cortical surface contained in the circle be largely increased by a sulcus crossing it, or not.

From facts like the foregoing it is argued that, while beyond question the application of a given amount of stimulus to certain gyri of the cortical surface will produce definite motor results, we caunot affirm those gyri to be the true cortical centres of such motion. Such gyri have accordingly been regarded by some as merely connected with the excitation of motion in a mechanical

${ }^{1}$ See especially the article of Hermann describing investigations under taken by him in company with von Borosnyai, Luchsinger, and others, Pflüger's Archiv (1875), x., pp. 77 ff. 
way, through their service in conducting the electrical stimulus to other regions of the brain, especially to the basal ganglia. The argument for the theory of localization would need to show, however, that the electrical current stimulates these areas immediately to the exercise of their central nervous functions, and does not simply pass through them to excite other nerrous matter lying beneath.

To the foregoing objections the adrocates of the theory of localization make the following among other replies: "The effect of irritation of the basal ganglia is capable of exact estimation ;" 1 and definite localized contraction of single groups of muscles, such as comes from stimulating certain areas of the cortical surface, does not follow from irritating the basal ganglia. Stimulation of other areas of the cortical surface which lie nearer to the basal gangliafor example, of the island of Reil, which immediately overlies the corpus striatum-causes no movements. On the contrary, it was found by Carville and Duret that the phenomena evoked by stimulating the motor areas persist, even after the destruction of the corpus striatum. Moreover, when the animal is deeply etherized, the excitability of the cortical regions is partially or wholly lost. ${ }^{2}$ Since the plysical conductivity of the gray nervous substance is not impaired by the anæsthesia, the loss of function must be due to the functional condition of this substance. More conclusive do the facts appear to be, which show that the nature of the motor reaction following upon the application of stimulus to the cortical substance is peculiar. Many observers have found that a stronger stimulation is necessary to bring about the same motor results after the cortical surface is removed; this is what we should expect on the theory of localization, but the reverse of what would be true if the effect of the current was transmitted unchanged through this surface. Then, too, Franck and Pitres ${ }^{3}$ hare shown that the effect of the electrical current is retarded in the gray matter; the difference of time, as dependent upon whether the stimulus is applied to the gray matter or to the white lying beneath, being about 0.015 second. This interval must be spent in evolving, under the influence of the stimulus, the distinct neural function which belongs to the gray matter. Finally, the excitation is apparently reinforced in strength by the functional activity of the cortical substance, since-as we have just seen-a stronger stimulation is needed to produce the same result after this substance is re-

${ }^{1}$ Ferrier, The Functions of the Brain, London, 18;6, p. $133 \mathrm{f}$.

${ }^{2}$ See Hitzig in Archiv f. Anat., Physiol., etc., 1873, p. 402.

${ }^{3}$ Archives de physiologie, 1875. 
moved ; such reinforcement is the peculiar property of the central organs.

It seems obvious, therefore, that experiments with electrical stimulation of the cortical surface demonstrate a special connection between certain more or less definitely circumscribed areas of that surface and definite groups of muscles; they also create a strong presumption that this connection is not merely anatomical or structural, but also functional.

$\S 16$. The second kind of direct experimental evidence is derived from observing the effects of extirpation. It is natural to argue that those areas of the brain, the loss of which is followed by the loss or disturbance of motion in definite groups of muscles, or by the loss or disturbance of any class of sensory impressions, are functionally related in a peculiar way to such muscles or organs of sense. But the application of this argument is encompassed with many difficulties. In the first place, it is impossible at each stage of the experiment-wlich often includes several days or months of observation-to know precisely what the condition of the brain is. Post-mortem examination of the brain reveals only what was the final effect of the experiment in destroying its tissues. The rise and fall of local or extensive inflammations, the progress of degeneration in the nerve-tracts and of abscesses resulting from the primary lesions, etc., cannot be followed by the experimenter in detail. Nor can he directly observe the formation and education of the tissue as it is called upon for an increase in the amount of its former functions, or perhaps for the discharge of functions partially new. As a rule, then, it is found that the effects of extirpation change from time to time; some of them are of first importance and cannot well be overlooked, and others are so delicate and minute as almost wholly to escape observation; some speedily pass away, others more slowly, still others perhaps not at all. The difficulties are, of course, especially great when we try to deal with effects upon the animal's sensory apparatus and his psychical world of sensations and perceptions. To tell whether an animal sees, hears, feels, smells, and tastes, or not; and to tell precisely in what sense it exercises these functions-whether, for example, its deficiency is "soul-blindness" in any of its various degrees-are not tasks which it is easy to perform, or about the correct performance of which one can indulge in a boundless confidence.

The demonstrative value of both kinds of experimental evidence -electrization and extirpation-is much lessened by the fact that it is almost wholly derived from the lower animals. Ethical con- 
siderations, which few investigators dare even occasionally to disregard, forbid that the living human brain should be made the subject of similar experiment. In order, then, to draw any safe conclusions from this evidence, it is necessary not only that the application of the principle of localization in general should be assumed, but also that some right should be gained to transfer to the human brain from the map of the cortical surface of the animal's brain, the so-called motor and sensory areas which have been determined by experiment. But it is not eren in all cases clear, precisely what convolutions or parts of convolutions of the human cerebrum correspond to those previously marked out on the brain of the animal. Moreover, in the effort to make any such transference of the argument from the animals to man, we meet again with the insuperable difficulty of forming a correct mental picture of the psychical life of the animals.

$\S 17$. The evidence from human pathology for the localization of cerebral function has a peculiar value; but it has also its peculiar puzzles and dangers. Such evidence is free, indeed, from the objections which arise against all attempts to carry the argument over from the cerebral hemispheres of the lower animals directly to those of man. Nature and human intercourse are less kind to this wonderful mass of nerve-cells and nerve-fibres than the electrodes and knife of the physiologist are compelled to be. Accident and disease destroy, either suddenly or progressively, the different areas of the cortical substance of the human brain. They have, in various cases, made such a variety of attacks upon it as to corer all the areas of both hemispheres. If, then, we had a large collection of cases in which the lesions were definitely circumscribed, or the progress made by the destruction of tissue was accurately recorded for every stage; and if we had also a correspondingly definite and accurate description of the motor and sensory disturbances occasioned by these lesions, we might perhaps be able to make a tolerably conclusive induction. But losses of brain-tissue, when caused by accident and clisease, have not the same circumscribed limits which can be observed by the knife or corroding acid of the physiologist. Lesions of the cortical areas entirely free from complication with lesions in the sensory and motor tracts below are comparatively infrequent. Cases of total destruction of any so-called "area" on both hemispheres, and of such area alone, rarely or never occur.

Furthermore, it is only by careful post-mortem examination that the precise extent of the pathological changes can be known ; this examination, at best, reveals simply the last state of the case. The reports of post-mortem examinations are also, as a rule, lacking 
in precision. On the other hand, the symptoms of motor or sensory disturbance are rarely described, from beginning to end, with sufficient accuracy of detail to be of great service. Many large losses of cerebral substance are followed by no sensory or motor disturbances which can be distinctly traced. In large numbers of cases where such disturbances arise, they in time pass almost or quite wholly away. For these and other reasons the best evidence attainable from pathological cases, when collected and sifted, appears surprisingly confusing and self-contradictory. Pathology has, therefore, furnished the common fund of cases from which the most diverse and even contradictory theories have drawn at sight their stock of so-called proof. It has been used as the careless and false witness upon which either party, and all parties to the suit, could call for precisely the testimony desired. An increase of information and care on the part of those who have opportunity for ante- and postmortem observation of such cases will doubtless, in time, cause pathology to yield much more assured results.

$\S 18$. The third kind of evidence to which the principle of the localization of cerebral function may appeal comes from comparative anatomy and histology. Comparative anatomy, however, gives us evidence of only the most general kind. Combined with experiment by electrical excitation, it shows that, on the whole, the higher the structure and intelligence of the animal, the more numerous and more definitely marked are the "excito-motor areas" which may be discovered on the hemispheres of its brain. Only traces, as it were, of such areas can be found upon the cerebral hemispheres of the frog or the pigeon; only a few areas can be doubtfully pointed out for the rat or guinea-pig. The indications are clearer and more numerous of localized cerebral function in definite centres of the brains of the rabbit and the sheep. But it is in dealing with the cerebral convolutions of the more highly specialized brains of the dog, and particularly of the monkey or the man-like ape, that the proofs of the theory become most abundant. While, then, the argument from all the other animals to man is uncertain and should be used only with great caution, the general drift of comparative anatomy encourages us to place the greater confidence in it, the more nearly the brain of the particular animal from whose case we wish to draw the inference resembles the brain of man. At the same time, the rash confidence with which the brain of the monkey has been mapped out in detail, and human pathology thereupon ransacked with the purpose of finding some warrant for copying this map upon the brain of the human species, cannot be too carefully avoided. 
Histology supplements and confirms the other evidence by showing that the structure and connections of different parts of the cerebrum are such as we should expect them to be, in case the functions of the parts were such as experimentation and pathology seem to have discovered. The modern arts of microscopy and photography have made possible an increasingly accurate knowledge of the intimate structure of the brain. Many great difficulties, however, still remain in the way of such perfection of this knowledge as will make it available as a secure foundation for a theory of the localization of cerebral function. At present the listology of the human cerebral hemispheres is not in a condition to take the place of a leader of physiological experiment and pathological observations. Its office is still rather that of rendering supplementary evidence in correction or confirmation of the evidence from the other two sources. Thus, for example, if Gliky's belief that he traced the nerve-tracts from the so-called motor centres of the cerebral hemispheres as they bend around the striate bodies and run into the crusta of the crura cerebri should be demonstrated, this fact would constitute an item of confirmatory evidence furnished by histology to experimental physiology and pathology, in favor of their general theory.

$\S 19$. According to the foregoing view of the nature of the three kinds of evidence available, it would seem that, in collating and estimating the combined proofs from them all, the following course of inquiry should be pursued. The indications of experiment upon the cerebral hemispheres of the animals-especially of those most closely allied to man in their cerebral structure-by the two methods of stimulation and extirpation, must first be gathered and carefully weighed. Only those conclusions upon which the two methods are found to yield substantially the same results should be selected for further testing. The instances of localization of cerebral function thus detected in the other higher mammals must then be allowed to suggest to pathology the questions it should undertake to answer with reference to man. In other words, experimentation with the other animals suggests and strengthens the hypothesis which human pathology must try to satisfy. But in undertaking to test such hypothesis, pathology must be both fair and comprehensire in its observations. All the accessible pathological cases must be sifted and those only selected to bring forward as evidence which have the definite nature, and have received the careful examination recorded in detail, that are necessary to make them of real value. The corrective or confirmatory evidence of histology must then, so far as possible, be summoned to aid in 
forming our final conclusions. It is not until all the kinds of proof unite with a large and substantial agreement, if not with an absolute uniformity, that we can feel the utmost confidence attainable in our results. If it be found that certain regions of the cerebral hemispheres of the higher animals are the only ones to respond when stimulated with movements in definite peripheral parts of the body, and that the injury of those same central regions alone, or chiefly, canses motor and sensory disturbances in the same peripheral parts; if it also be found that lesions of the corresponding regions of the human brain are alone, or chiefly, followed by similar motor and sensory disturbances, and that lesions of other regions alone are rarely or never followed by these same disturbances; and, finally, if it be found that these same cortical regions have in the human body a special anatomical connection with these same peripheral parts ; then we have reached the most conclusive evidence attainable for a theory that the cerebral functions are localized in the case of man. But precisely what is meant by such "localization " may still remain more or less a matter of dispute. We consider now a summary of the evidence according to the foregoing principles. 


\section{CHAPTER II.}

\section{THE LOCALIZATION OF CEREBRAL FUNCTION. [CoNTINUED.]}

\&1. Ox attempting to make an induction from all the three linds of evidence which may be adduced in answer to the question, whether the different functions of the cerebral cortex have special relations to its different localities, no other difficulties are on the whole so great as those which come from so-called "negative cases." These negative cases force the inquirer to undertake a detailed experimental and pathological examination. "That the cortex of the cerebrum, the undoubted material substratum of our mental operations," says Ecker, " is not a single organ, which is brought into play as a whole in the exercise of each and every psychical function, but consists rather of a multitude of mental organs, each of which is subservient to certain intellectual processes, is a conviction which forces itself upon us almost with the necessity of a claim of reason." But even the proposition that the brain is the "material substratum of our mental operations," is very far indeed from having the character of a rational necessity. The further proposition that the cortex of the cerebrum "consists of a multitude of mental organs," is an inadequate statement of a conclusion which, at the very best, we can adopt only as the result of a long series of complex and conflicting researches. In fact, considerable areas of the cortical surface appear, at first, not to have any immediate relation to any psychical function whatever.

The first general principle to be admitted in all attempts at a theory of the localization of cerebral function is, then, of a negative character. This principle is based upon the negative results of physiological inquiry. Considerable areas of the cortical surface do not respond with motor activities when stimulated. Considerable portions of the cortical substance may be extirpated or lost by disease without the destruction or appreciable disturbance of any motor, sensory, or more purely intellectual functions. To such an astonishing extent is this true as to throw temporary doubt not only over the whole theory of the localization of cerebral func-

' The Convolutions of the Human Brain, p. 1. London, 1873. 
tion, but even over the statement that the cerebral cortex, as a whole, is the only "material substratum" of mental operations.

§ 2. Attention has already been called (Chap. I., §11) to the fact that Longet, Flourens, and other great physiologists, considered the cerebral hemispheres to be active as a whole in all their functions, and this, partly, because they found them not irritable by the electrical current. The discovery of Fritsch and Hitzig in 1870 demonstrated that a part of the hemispheres of the dog, and a part only, gives sigus of being excited by the application of stimulus. This part they called "motor," and located, in general, in the fore part of the hemispheres; behind lay the region called "non-motor," because it gave no response on being stimulated." Even within this so-called "motor" region the early researches of these investigators pointed out only five spots of a small fraction of an inch in diameter (the electrodes were, as a rule, separated not more than 2-3 mm.) that could be more definitely related to the movement of certain groups of muscles; between and around these spots lay the much larger areas of negative result. Subsequent experiments added a few more such irritable areas to the map of the cerebral hemispheres of the dog. A large number of so-called centres, covering an increased amount of the cortical surface, have been pointed out by Ferrier and others on the cerebral hemispheres of the monkey. Fully half of this number, however, cannot be regarded as having anything like a demonstrable character ; and much fault has justly been found ${ }^{2}$ with many operators upon the brains both of monkeys and of dogs, for their lack of precision in experiment, and haste in drawing conclusions.

Experiments in extirpation also show that considerable areas of the cortical substance may be removed without perceptibly impairing any of the motor or sensory functions of the animal. Indeed, even when the loss of the cortical substance, thus artificially produced, extends over almost an entire hemisphere, or over a large portion of both hemispheres, the operation may not result (in the case of the dog, ordinarily does not result), in the permanent and complete loss of any specific function, motor or sensory. So true is this that one eminent observer, Goltz, has maintained, on the basis

1 Archiv f. Anat., Physiol., etc., 18\%0, p. 311.

'See, for example, Munk's strictures of Ferrier, Ueber d. Functionen d. Grosshirnrinde, Berlin, 1881, pp. 14 ff. (also p. 6 f. ; 36 f.-" "roh war operirt, roh beobachtet, roh geschlossen"). On the other hand, the charge of carelessness in experiment, and of illogical conclusions, is freely made against Munk himself, both by advocates of rival theories of localization, like Dr. Yeo and others, and also by opponents of all theories of localization, like Goltz, Löb, and others. 
of many experiments in extirpation, that it is chiefly the quantity of the cerebral substance destroyed, in large measure irrespective of the locality, which determines the nature and extent of the resulting psychical disturbances. The arguments of Goltz (as he himself admits) do not answer those urged for a certain kind and degree of the localization of cerebral function. But his experiments furnish a large number of facts which empbasize the negative character of many of the results of experiment. This fact is in itself undeniably unfavorable to any theory which would map out the eutire cortical surface into so-called centres or areas, to be considered as separate organs of particular psychical processes.

§3. The negative evidence from certain cases in human pathology is yet more astonishing and perplexing. At first sight it seems to suggest the conclusion that the mind can dispense, without impairment, with a considerable mass of brain-substance, no matter from what region it be subtracted. Many cases of large lesions of the cerebral hemispheres in man, with no resulting disturbance of the psychical functions, are recorded. ${ }^{1}$

Berenger de Carpi tells of a young man who had a foreign body of four fingers' breadth square driven into the substance of his brain until it was buried. Much of this substance was lost when the foreign body was removed, and more yet some thirteen days later. Nevertheless, the patient lived for a long time in the enjoyment of all his faculties.

Longet was acquainted with an army officer who had lost, by a wound in the parietal region, a large quantity of brain-substance; yet he remained mentally viracious and showed no other result of the lesion than a tendency to grow tired easily. The same authority communicates $^{2}$ the ease of an Italian whose skull was crushed in the right parietal region by a stone. So much of the substance of the brain was lost on the wound being dressed, and subsequently through a fall from his bed (on the eighteenth day) and through intoxication (on the thirty-fifth day), that the attendant physician calculated the lesion must have reached down nearly to the corpus callosum. The man, howerer, lived without any apparent impairment of psychical functions; but we note in this case a permanent laming of the limbs of the left side.

${ }^{1}$ See the list of such cases in Ferrier, the Localization of Cerebral Disease, London, 1878, pp. 25 ff.; Hermann, Handb. d. Physiol., II., ii., pp. 333 ff.; Brïcke, Vorlesungen uber Physiol., II., p. 57 f. Wien, 1884; and the works cited by the two former, especially Pitres, Lésions du centre ovale, Paris, 18.7.

${ }^{2}$ Recorded, however, by Quesnay. 
A remarkable case is narrated by Brücke, ${ }^{1}$ on the authority of a certain Dr. Kratter. By a blow from a stone on the parietal region of the skull, one Ivan Mussulin was thrown to the ground; but within two hours he recovered so that he himself went to the "praetor" and entered complaint against his assailant. For twenty days he lived in apparently full possession of his powers of motion, sensation, and intelligence; on the twenty-first day he suddenly diecl. The entire left cerebral hemisphere was found on examination to be a disorganized mass. It is to be noticed, however, that the autopsy did not take place until some eighteen hours after death, and that we have no good means of judging what the condition of the injured hemisphere was during the twenty days preceding his sudden death.

Remarkable instances of defective brains are also on reeord; for example, the case which Lallemand narrates of a person of normal psychical constitution in whose cerebrum the entire place of the right hemisphere was, after death, unexpectedly found to have been filled with a serous fluid. Here again, however, there had been lameness of the left side of the body from birth.

Extensive lesions without marked motor or sensory disturbances occur by far most frequently in the frontal lobes of the cerebral hemispheres. Yet similar negative cases are by no means infrequent also in the occipital and temporo-sphenoidal lobes. Trousseau narrates the case of an officer who was shot through the head in the middle of the frontal lobes, and who showed until death, which occurred from inflammation, no signs of any kind of paralysis. The work of M. Pitres ${ }^{2}$ contains a large collection of cases, in which the frontal lobes have been the seat of extensive disease, of softening, or of abscess, without any symptoms of laming whatever; in most of which, also, no disturbance of psychical condition was observed. That sudden extensive lesions may occur in this region without inducing sensory or motor paralysis, is shown in a marked way by the celebrated "American crowbar case." s By premature discharge of blasting powder an iron bar, three feet seven inches in length and one and one-fourth inch in diameter, was driven through the brain of a young man. The missile entered at the left angle of the jaw, and passed through the top of the head near the sagittal suture in the frontal region; it was picked up at some distance off, covered with blood and brains. The pa-

${ }^{1}$ Vorlesungen uiber Physiol., II., p. 57.

${ }^{2}$ Lésions du centre ovale.

${ }^{3}$ See the paper in the Am. Journal for Med. Sciences, by Dr. Bigelow, July, 1850, and the one read before the Mass. Med. So. by Dr. Harlow, June, 1868. 
tient, although for the moment stunned, recovered in a few minutes so as to ascend a flight of stairs and give to the surgeon an intelligible account of his injury. He lived twelve and a half years afterward, with no noticeable impairment of his sensory-motor powers. Examination of the skull showed that the substance destroyed by the bar must have been confined to the frontal region, with the possible exception of the tip of the temporo-sphenoidal lobe.

Boyer narrates the case of an epileptic child, that showed, however, no other abnormal nervous phenomena, whose entire temporal lobe on the left side was found to have been destroyed. Instances of extensive lesions in the occipital lobes, without any resulting sensory or motor disturbances, might also be given.

\& 4. It must be confessed, in the words of Exner, ${ }^{1}$ that the understanding of cases of this sort " is made more difficult rather than easier by recent researches." Nevertheless, a large amount of concurrent testimony from all three main sources of evidence proves that some theory may be framed in acknowledgment of a more definite localization of cerebral function. Such theory can be most clearly established with respect to the cerebral region especially concerned in the motor functions. This region is the one lying about the great central fissure, or fissure of Rolando; more precisely still, it embraces the gyrus centralis anterior, the gyrus centralis posterior, and the prolongation of the two on the median surface of the brain in the lobulus paracentralis. (Comp. Figs. 87 and 88). More definite localizations still, of smaller regions within the larger one-e. g., for the upper limbs, for the lower limbs, for the separate fingers, etc.-are more doubtful ; they can by no means appeal to the same amount of evidence as that at command of the more general induction.

$\S 5$. The evidence from experiments in stimulation indicates that we are to look for the so-called "motor areas" in the above-mentioned convolutions about the fissure of Rolando. The original experiments of Fritsch and Hitzig ${ }^{2}$ located the five motor areas as follows: The centre for the muscles of the neck (marked $\Delta$ in the figure) in the middle of the pro-frontal gyrus at the spot where its surface falls off steep; the centre for the extensor and adductor of the fore-limb, at the outermost end of the post-frontal gyrus in the region wear the end of the frontal fissure ( + in the figure); the

'In Hermann's Handb. d. Physiol., II., ii., p. 334.

${ }^{2}$ See Archiv f. Anat., Physiol., etc., 1870, p. 312 f.; comp. Taf. IX B. by Hitzig in the same Archiv for 1873, from which the accompanying figure is taken. 
centre for the bending and rotation of the same limb, a little farther back ( + in the figure); the centre for the hind-limb, in

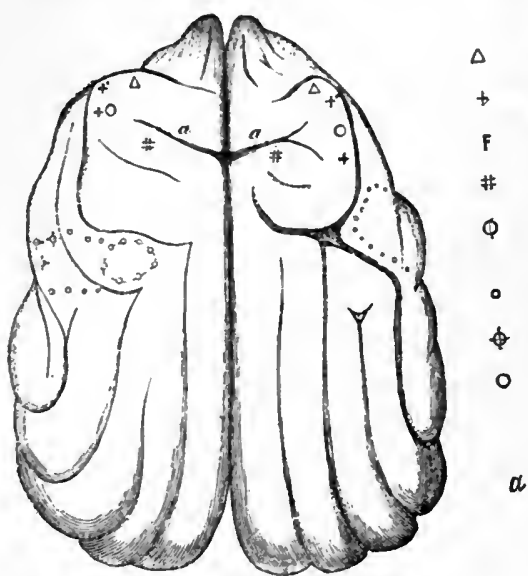

FrG. 83.-Hitzig's Motor Areas on the Cortex of the Dog. The left hemisphere belongs to one animal, the right to another; $a$, the sulcus cruclatus, around which the gyrus sigmoideus bends; $\infty$ ooo, area for the face. The other symbols are explained in the text.

a the post-frontal gyrus but toward the median line of the hemisphere and back of the preceding two centres (\# in the figure); the facial centre, in the midale part of the gyrus lying above the fissure of Sylvius ( $\phi-0$ in the figure). These experimenters found also that the muscles of the back, tail, and abclomen, were excited to contraction by stimulating points lying between those marked as above ; but they could not definitely circumscribe the cortical areas which were to be assigned to these muscles.

By reference to the chart of the numerous "centres of electrical irritation " which Ferrier " claims to have discovered on the cerebral hemispheres of the monkey, it will be seen that they are set close together in the two central convolutions (gyri centrales, called by Ferrier the "ascending frontal" and "ascending parietal ") and in the immediately adjoining parts of the frontal and temporosphenoidal convolutions. Thus, the centres $(2$, in part), $(3),(4$, in part), (5, in part), $(6),(7),(8),(9)$, and (10), are located in the

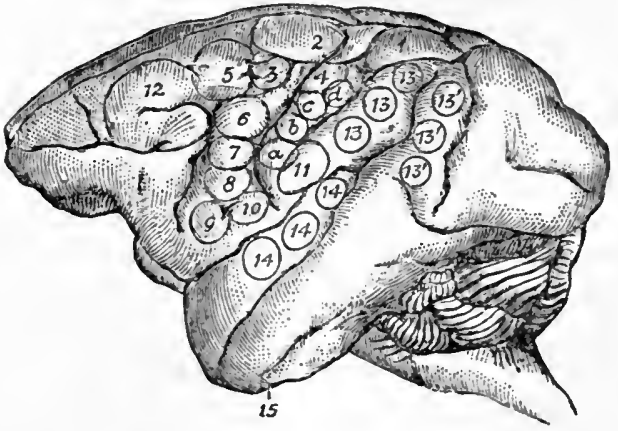

Fra. 84.-Areas on the Left Hemisphere of the Monkey, by stimnlating which Ferrier obtains notion in definite groups of muscles.

anterior central ("ascending frontal") convolution; (2, in part), (4, in part), (11, in part), and (a), (b), (c), (d), are placed on the poste-

${ }^{1}$ See The Functions of the Brain, pp. $141 \mathrm{ff}$, $149 \mathrm{f}$., and $305 \mathrm{f}$. London, 1876. In the second edition (1886), pp. $240 \mathrm{ff}$. 
rior central (" ascending parietal ") convolution; the centres(12) and (5, in part) are situated on parts of the superior and middle frontal convolutions adjacent to the anterior central; and (14) on the superior temporo-sphenoidal convolution.

Further and more recent information seems to render the experiments in the electrization of the cerebral areas of animals more available for use in confirming the general argument in behalf of some kind of localization of cerebral function in the case of man. Luciani and Tamburini, as well as other experimenters, have agreed with Hitzig and Ferrier in finding small, circumscribed motor centres on the cortex of the dog, monkey, rabbit, and other animals. Some experimenters (Bochefontaine and Vulpian, e.g.) claim to have discovered that the minute areas, at first excitable, after a time cease to be so ; and that other areas, at first not excitable, afterward become excitable-that is, a displacement of the excitable points takes place.

More recently still, it has apparently been discovered ${ }^{1}$ that $\mathrm{Ex}$ ner's view (to be explained subsequently) of the existence of "absolute" and "relative" motor fields in the case of man is probably applicable to the animals also. Paneth found that a number of minute areas (or spots) for each one of the several groups of muscles could be detected as lying in the larger "excitable zone" of the cortex. These areas, unlike the immediately surrounding ones, could be excited when cut around, but not when cut beneath; the fibres whose function it is to bring a definite group of muscles to contraction seem then to proceed directly from these cortical spots to the lower parts of the brain. A number of such belong to each muscle excitable; but only two general "fields" are distinguishable, within which all the isolated motor spots are located; one field is for the extremities, the other for the orbicularis palpebrarum. The former is situated in the posterior division of the gyrus sigmoideus. The minute areas for the different muscles of the extremities are sharply limited; they do not wholly cover each other; and those for any special muscle (the extensor digitorum of the forefoot, etc.) are of small extent in comparison with the field or zone which may be looked on as common to all the extremities. The excitability of the different muscles is not all alike; this Paneth explains by assuming that the number of nerve-elements assigned to each is not alike.

§ 6. Experiments in extirpation confirm, at least in a general way,

1 See the art. of J. Paneth, "Ueber Lage, Ausdehnung, nnd Bedeutung der absoluten motorischen Felder," etc., in Pflüger's Archiv, xxxvii. (1885) pp. $520 \mathrm{ff}$. 
the above-mentioned results of experiments in stimulation. The destruction of the substance of those cortical areas which respond to the current of electricity with the co-ordinated movement of definite groups of muscles, causes a temporary or permanent impairment of the functions connected with the same groups of muscles. In their first report Fritsch and Hitzig called attention to certain experiments of their own in removing from the cerebral hemispheres of two dogs the nervous substance of the centre which had already been fixed upon by them as that for the "right fore extremity" of the animal. These experiments they found confirmatory of the views derived from stimulation. The animals operated upon, when sitting or standing or running, used the right fore-leg unskilfully ; this part of the body, however, showed no marked diminution of sensibility under hard pressure. Other observers have since performed many similar experiments;-especially Ferrier on monkeys, and Goltz and Munk on dogs. Among them all no others are so carefully refined as are those of Munk.' But the very refinement of these experiments subjects them to more of distrust, in certain particulars.

§ 7. The earlier experiments of Munk were confined to the convex surfaces of the parietal, occipital, and temporal lobes of dogs; they consisted in removing clean-cut circular bits of the cerebral substance about three-fifths of an inch in diameter and one-twelfth of an inch thick-sometimes simultaneously, from the symmetrical areas of the two hemispheres, and sometimes with an interval between the two operations. Munk's general conclusion is stated as follows: If a line be drawn from the terminal point of the fissure of Sylvius vertically toward the falx cerebri, it will mark, approxiimately, the limits of two spheres that are sharply distinguished experimentally-namely, an anterior motor and a posterior sensory sphere. ${ }^{2}$ Extirpations in front of this line always occasion disturbances of motion, those back of it never so. More precisely, the cerebral convolutions of the dog may, according to Munk, be mapped out into the several spheres and regions indicated in the accompanying figure (Fig. 85). It will be noticed that three of these regions (namely, $C$ for the hind leg, $D$ for the fore leg, and $E$ for the head) correspond pretty accurately to the centres of stimulation fixed upon by Fritsch and Hitzig. Extirpations of the cortical substance in these regions, of only a few millimetres broad and not more than two deep, are regularly followed by definitely localized

${ }^{1}$ See his Gesammelte Mittheilungen aus d. Jahren 1877-80, in the book, Ueber d. Functionen d. Grosshirnrinde. Berlin, 1881.

${ }^{2}$ Munk, ibid, p. 11. 
disturbance of motion. For example, let the region $(D)$ be removed from the left cerebral hemisphere of a dog. At the end of from three to five days and after the fever from the operation has subsided, abnormal phenomena connected with the fore-leg of the opposite side will be observed. If any other limb of the animal than the right fore-leg be touched lightly, the dog will look quickly around;
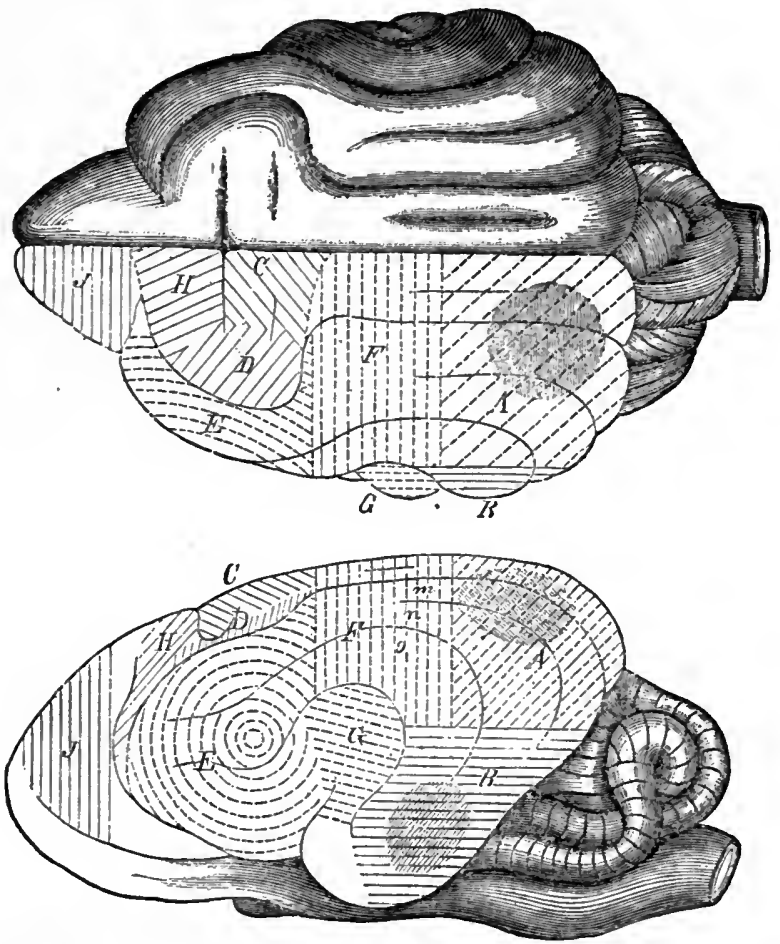

Fia. 85.-Areas on the Brain of the Dog. (According to Munk.) A, centre of the Eye ; $B$, of the Ear; $C$, of the sensations of the hind Leg; $D$, of the fore Leg; $E$, of the Head; $F$, of the Apparatus for protecting the Eye : $G$, of the Region of the Ear: $H$, of the Neck; $J$, of the Rump.

and if of a bad temper will try to bite the offending hand. $\mathrm{He}$ will also quickly withdraw any other limb when it is subjected to even very slight pressure. But hard pressure and pinching or sticking of the right fore-leg is either followed by no result, or else by mere withdrawal of the limb, as though in reflex motion, without any attention being paid to the attack. Noreover, this particular limb, unlike all the others, can be put into unnatural and uncomfortable positions-can be bent, stretched, set on the ground with 
the back of the foot down, etc.-without any resistance on the animal's part or any apparent disposition to remore it to the normal and comfortable position. According to Munk, the animal has apparently lost all mental picture of this one limb, and therefore all power to move it intelligently and voluntarily. If he has been accustomed, on call, to put the right leg into his master's hand, he will now respond with the left instead of the right foot to the same call. The dog no longer handles his food with the right foot. In running he slips on that foot. If he is drawn to the edge of a table and the right leg forcibly stretched out over it, he will allow the leg to hang down thus, although evidently aware of the dangerous position in which this places him. Such an animal can, however, still walk and run, using all four limbs. The " gross mechanism " of motion-to borrow Munk's phrase '-still acts as it did before ; but the so-called "cerebral " or intelligent quality in the management of this particular limb has been lost.

Gradually the phenomena which indicate impairment of cerebral function as related to the movement of the fore-leg diminish in magnitude. Less pressure is then necessary to secure the withdrawal of the injured limb; the dog is less surprisingly unskilful in its use. At the end of four or five weeks the more marked symptoms of his loss of function have probably disappeared; at the end of eight or ten weeks it may be difficult or impossible to distinguish his movements from those of a perfectly sound animal. If, however, the size of the pieces of cerebral substance taken from any of the so-called "motor regions" be somewhat larger than that indicated above, recovery is slower and more imperfect. In the opinion of Munk, if the extirpations are considerably enlarged the restitution of function is never complete.

$\S 8$. That explanation of the phenomena which regards the various cerebral regions, that seem somehow specially connected with motor activities, as true " motor centres,"-that is, as areas of the cerebral cortex that have for their peculiar function the initiating of definite motor impulse on occasion of the idea and volition to move definite portions of the periphery of the body,-is rejected by Munk. All the regions marked $C-J$, belong rather to what he calls the "feeling-sphere" of the cerebral hemispheres. It is an undoubted fact that the definite co-ordination of the limbs, from the higher cerebral centres, depends upon feelings of contact and pressure of the skin, and upon muscular feelings or so-called feelings of innervation. The effects of extirpating centres like $(C),(D)$, and $(E)$, is due, therefore, first to the sudden loss, and subsequently to

1 Ueber d. Functionen d. Grosshirnrinde, p. 47. 
the gradual restitution of these feelings, and of their corresponding mental representations, with respect to given groups of muscles.

Schiff ${ }^{1}$ agrees with Munk in the view that the real loss of function due to the extirpation of the above-mentioned cerebral regions is sensory rather than motor ; he considers that the impairment of the power of moving these parts is only an expression of the loss of the sense of touch in the same parts; in other words, it is tactile anæsthesia. He calls attention to the significant fact that an animal thus operated upon will freely allow parasites and insects to gather on that surface of the skin whose corresponding cortical area has been removed. Schiff also finds that the use which the higher apes
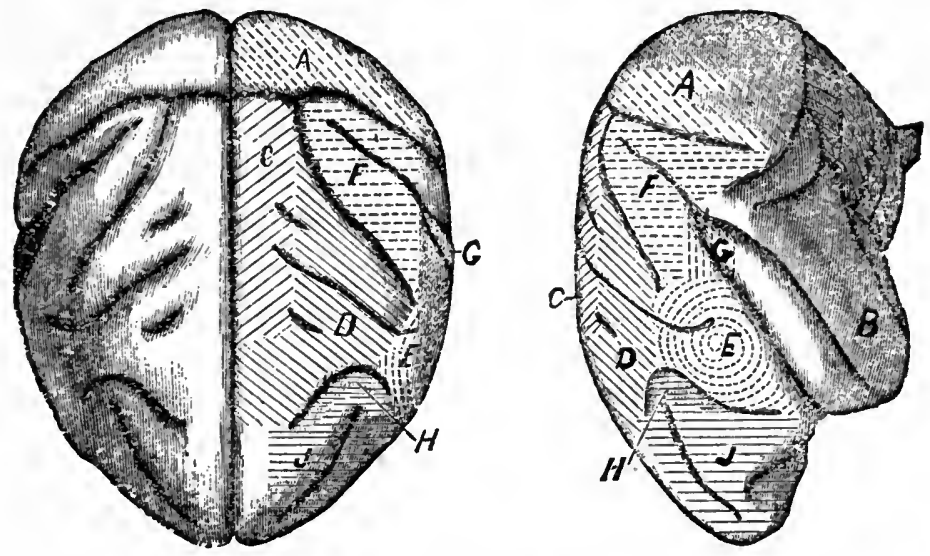

Fra. 86.-Areas on the Brain of the Monkey. (According to Munk.) The letters have the Bame reference as in tho preceding figurc.

make of their limbs for grasping the rounds of a trellis or ladder is not permanently impaired by removing to considerable depth the convolutions about the central sulcus, unless the trellis or ladder be turned at an angle of $60^{\circ}$ to $70^{\circ}$, so as to convert the animal's walking into climbing. Apparently the animal cannot climb because he is unable to form a mental picture of the next round so as to reach out and grasp it. Schiff therefore concludes, that " all motions are suppressed (by extirpating the cerebral substance) which, on being excited by the higher senses, receive a special supervision on the side of cerebral sense, in relation to direction, extent, and succession." $\mathrm{He}$ also asserts, in opposition to the conclusions of Goltz, that the injured animal never recovers the

'See Pflüger's Archiv, xxx. (1883), pp. $212 \mathrm{ff}$. 
powers it has once really lost; in other words, it is not possible to extirpate any of the centres, excitation of which produces a given motion, without effecting some permanent result.

\&9. The conclusions of Munk and Schiff undoubtedly have certain facts of experiment in their favor, but they can scarcely be said either to cover all the facts or to be wholly consistent with certain particular ones. Goltz ${ }^{2}$ agrees substantially with Munk in finding that destruction of the cerebral substance of the frontal lobe causes the animal to execute morements of the limbs of the opposite side in a coarse and unskilful manner. He also finds that the tactile sense is temporarily impaired, although by giving increased attention the animal is able to feel the slightest touch on any area of the skin. Indeed, deep and extensive lesions in this region may be followed by hyperesthesia. The muscular sense, on the other hand, seems permanently to suffer. Goltz's conclusions are squarely contradictory of all those which find any permanent laming of any muscle as the result of even the most extensive destruction of the cortical substance in the so-called "motor field." His theory lays more emphasis on the general impairment of intelligence which results from removing any considerable amount of the substance of the brain, from whaterer region it may be taken.

A still more recent investigator ${ }^{2}$ calls attention anew to the facts that an animal deprived of the "motor sphere" cannot use the extremities as hands; cannot hold the foot out on call, or push away the hand by which its chin is grasped, or stretch out the limb so as to grasp the dish containing its food. These phenomena imply, he thinks, some severance between the organ of will and the nerves which execute the will. The motor centres are to be limited, it is claimed, almost exclusively to the gyrus sigmoideus, and those for feelings of the skin and muscles to the region lying above the fissure of Sylvius.

On attempting to reconcile all the results of experiment upon the animals with one another, and with the facts of human pathology, it must be admitted that great difficulty is experienced; and even more difficulty when the effort is made to frame a consistent theory which shall cover them all. On the whole, however, it seems obvious that a certain region or sphere of the cortex of the brains of the higher animals is entitled to be called "motor" in a special sense; and that this region corresponds in a general way to

${ }^{1}$ See his article in Pflïger's Archiv, xxxiv. (1884), pp. 450 ff.

"Bechterew, art. "Wie sind die Erscheinungen zu verstehen die nach Zerstörung des motorischen Rindenfeldes an Thieren auftreten;" Pflüger, xxxv, (1885), pp. $137 \mathrm{ff}$. 
that which (as we shall soon see) pathology indicates as specially motor in the case of man. Stimulation of various minute areas in this region is followed by the movement of definite muscles of the body; extirpation of this region in its entirety, or in part, is followed by special disturbances of the motor functions of the animal. These disturbances are not of the kind which indicates so much a laming of any particular muscle, as a loss of cerebral, and so intelligent, quality in respect to the landling of the extremities. They probably imply more or less of all those various kinds of psychical disturbances and impairments of function, by some one of which exclusively the different investigators are wrongly inclined to account for the phenomena which they obserre. Extensive losses of cerebral substance in the motor region result in the loss of those tactile sensations and muscular sensations, by means of which the animal localizes and interprets the meaning of objects, and adapts the finer movements of its limbs accordingly. They also impair the porver to express the volition of the animal by motor impulses started, in accordance with the sensations and images of motion, in the appropriate area of the brain. Moreover, such loss of the powers of sensation, sense-perception, and skilful motion, necessarily implies more or less of loss of intelligence.

$\S 10$. It will always be difficult to designate precisely what factors in the animal's complex sensory-motor activities drop out as the result of the removal of a certain area of cortical substance from the brain of a dog or monkey ; and whether these factors are exclusively sensory or exclusively motor, rather than both sensory and motor. It is doubtful if enough can ever be known, concerning the mental life of the dog or the monkey, to determine confidently in this way the question of the localization of psycho-physical functions. The phenomena of human pathological cases indicate, however, that in man the corresponding general area of the cerebrum-that is, the convolutions on both sides of the central fissure and the lobulus paracentralss-is especially concerned in both sensory and motor factors for co-ordinated action of the limbs. Without adducing further confirmatory evidence from experiment upon other animals, we pass to the consideration of the evidence from human pathology. The results of experiments in stimulation and extirpation upon the lower animals are not to be transferred in toto, as a matter of course, to the human cerebrum; they are rather to be consulted as indicating the precise nature of the questions to be proposed to pathology, and of the answers to these questions which are antecedently probable.

From this point onward our chief reliance must be placed upon 
Exner's ${ }^{1}$ careful and scientifically classified investigations. The method pursued by this investigator is described at leugth by himself." Exner began, with true German thoroughness, by reading several thousand cases of cerebral disease which had been followed by post-mortem examination; the catalogue of works thus consulted by him occupies more than twenty pages. From all these cases he

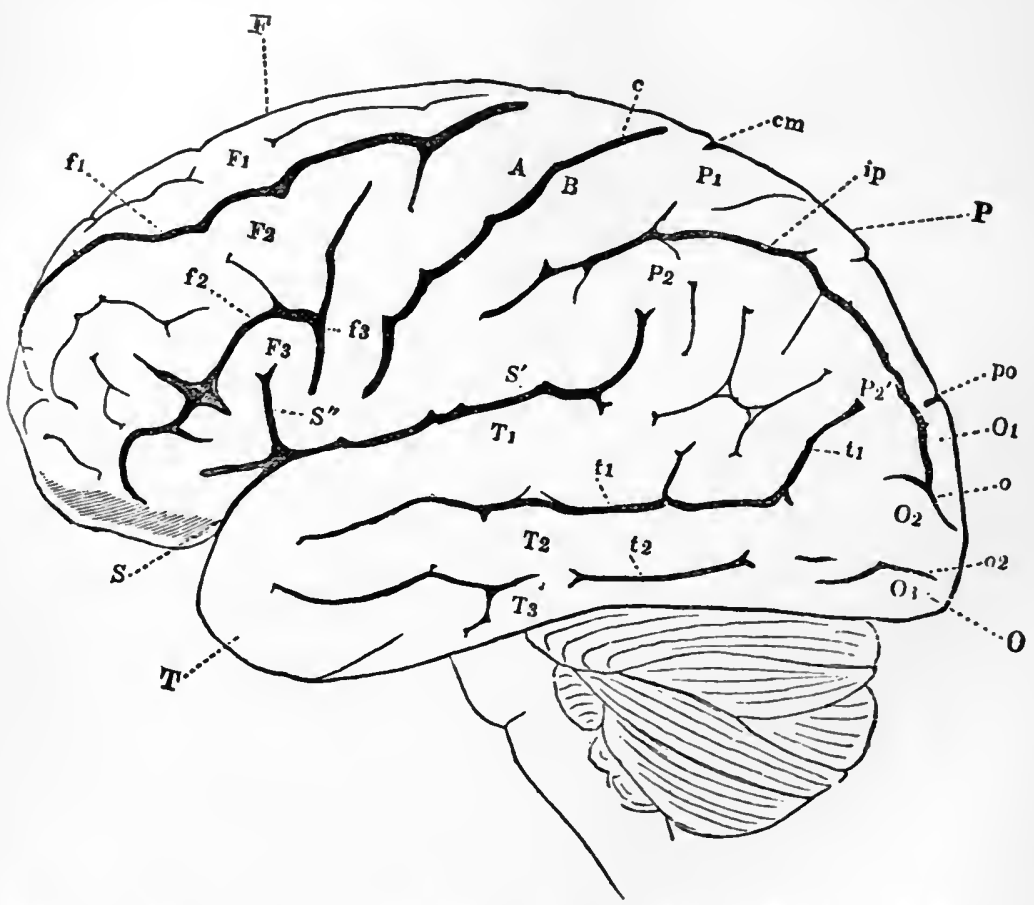

F1G. 87.-Lateral View of the Human Brain. (Schematic, Ecker.) F, frontal. P, parietal, O, occipital, and $T$, temporo-sphenoidal lobes, S, fissure of Sylvius. with $S^{\prime}$, the horizontal, and $\mathbf{S}^{\prime \prime}$, the ascending ramns; $\mathbf{C}$, suleus centralis; $\mathbf{A}$, anterior, and $\mathrm{B}$, posterior, central convolutions; F1, F2, F3, superior, middle, and inferior frontal convolutions; $\mathbf{f 1}$, sinperior, $\mathbf{f 2}$, infe. rior frontal sulci; 4 , sulcns pracentralis; $\mathrm{P} 1$, superior, and $\mathrm{P2}$, inferior parietal lobule; the latter, the gyrus supra-marginalis, and $\mathrm{P}^{\prime}$, the gyrus angularis; ip, sulcus interparietalis ; $\mathrm{cm}$ end of calloso-marginal fissure; 01. O2. O3, occipital convolutions; po, parieto-occipital fixsure; 0 , transverse, and 02 , inferior longitudinal sulcus; $T 1, T 2, T 3$, temporo sphenoidal con volutions: and $\mathrm{t} 1$, t2, tempero sphenoidal fissures.

then made a collection of such only as could safely form the basis of a scientific induction. The conditions of admittance into this collection were as follows: Both the history of the disease and the description of the post-mortem condition must be trustworthy, full,

${ }^{1}$ Untersuchungen über d. Localisation d. Functionen in d. Grosshirnrinde d. Menschen. Wien, 1881.

${ }^{2}$ Ibid, p. 6 f. 
and unambiguous; and there must hare been no other lesion than the one in the cerebral cortex, either elsewhere in the brain or in the spinal cord, to complicate the legitimate inferences. Only two exceptions to the latter rule were, for reasons peculiar to themselves, admitted. Nearly all cases in which symptoms indicative of diffuse meningitis occurred were also excluded. In this cautious way one

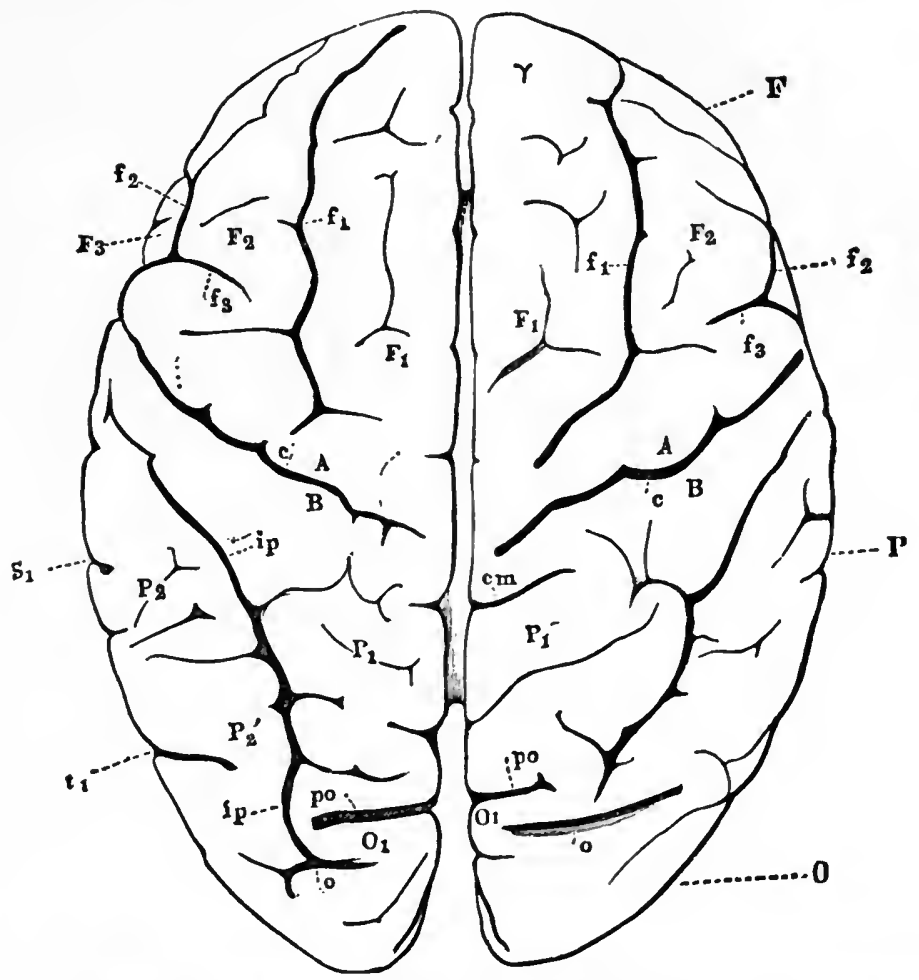

FiG. 88.- View of the Human Brain from Above. (Schematic, Ecker.) The letters have the same reference as in the preceding figure.

hundred and sixty-nine test-cases were secured from the thousands recorded. These test-cases were then tabulated on three sets of maps, according to the following methods of induction: (1) The method of negative cases, (2) the method of "reckoning per cent.," (3) the method of positive cases.

The methor of negative cases (if the number of such cases were large enough) would result in showing what regions of the cerebral hemispheres, if any, are not necessarily comnected with motor or 
sensory functions-both, or either one respectively. The charts constructed by this method would, accordingly, have only those convolutions and parts of convolutions left blank, or unmarked, in which no lesion had occurred that was not followed by some given kind of motor or sensory disturbances. The method of percentage was designed to show the amount of probability that a given small area of the cerebral cortex will be hit by disease, as it were, in case the lesion has been followed by a given kind of motor or sensory disturbance. For this purpose the entire surface of one hemisphere was mapped out into three hundred and

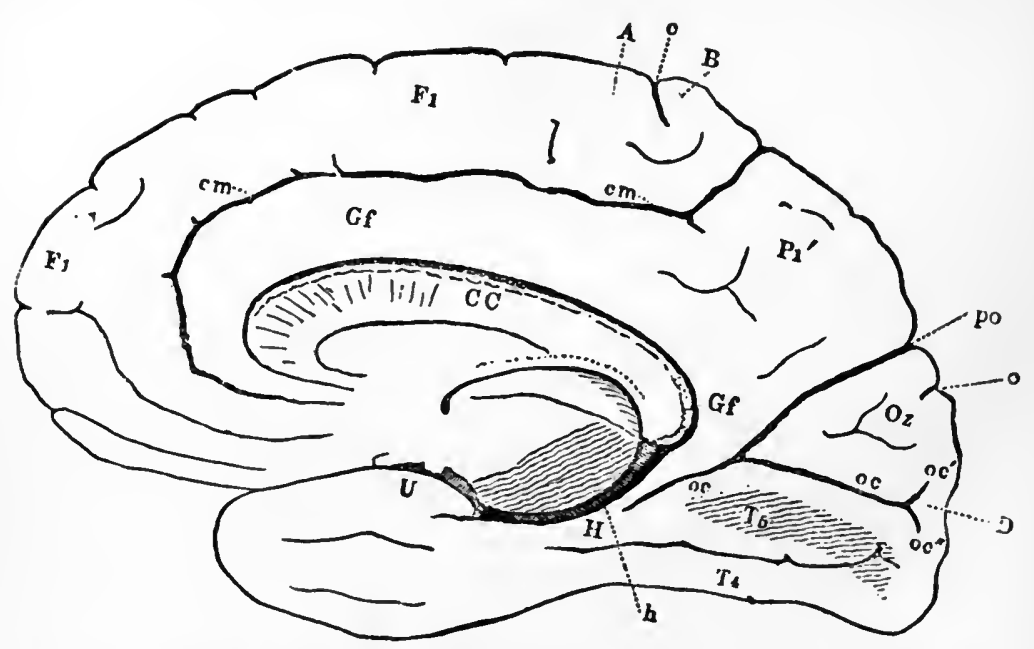

FIG. 89.-Median Aspect of the Right Hemisphere. (Schematic, Ecker.) CC, corpus callosum. Gyri : Gf, fornicatus ; H, hippocampi (with its sulcus, h), and U, uncinatus; $\mathrm{P1}^{\prime}$, praecuneus: $\mathrm{O}_{z}$, cuneus; oc, calcarine fissure, with its two rami, $\mathrm{c}^{\prime}$ and $\mathrm{oc}^{\prime \prime} ; \mathrm{D}$, gyrus descendens; $\mathrm{T} 4$, the lateral, and T5, the medial, gyrus occipito-temporalis.

sixty-seven quadrilateral areas, all small and yet of somewhat different sizes. As the different selected cases were recorded by painting the area of the lesions on this set of maps, the intensity of the color used would, of course, deepen in proportion to the certainty of a connection between that particular area and some par. ticular sensory or motor function. Thus a perfect black would indicate one hundred per cent. of cases in which a given quadrilateral was hit when a given disturbance of function had followed; pure white, nought per cent. of such cases. The third method (that of positive cases) is the one usually relied upon to prove (?) the theory of localization of cerebral function from pathology ; it is justly regarded by Exner as the least conclusive, as never of itself forming 
a basis for anything beyond conjecture. Its principle is the assumption that the region where the lesions connected with certain disturbances are most thickly crowded together, is the required cortical area with its specific function.

§11. The result of Exner's comprehensive induction from pathological cases, as based on all three of the methods just described, fixes almost beyond doubt the so-called "motor areas" of the human cerebrum. [For understanding Exner's induction, consultation of Ecker's charts, found on p. 276f., figs. 87, 88, and 89, will be found helpful.] The field of wholly "latent lesions"-that is, of lesions which are not necessarily followed by any disturbances of either sense or motion-covers a large part of the surfaces of both hemispheres ; it is not, however, precisely the same for them both. While Exner's collection of cases comprised 67 lesions of the right hemisphere, and 101 of the left, the absolute number of latent lesions was the same (namely, 20) for both hemispheres. The chances that a lesion of the right hemisphere will not be followed by any disturbance of function are, therefore, about fifty per cent. greater than the chances that the same thing will occur in the left hemisphere. On the right hemisphere the entire surface, with the exception of the two gyri centrales, the lobulus paracentralis, and certain small portions on the convex and inferior surfaces of the occipital lobe is latent. On the left hemisphere the latent region is of less extent. This result may be regarded as a restatement, on a basis of scientific induction, of the well-known fact that extensive lesions can occur in the frontal, temporal, and occipital lobes, without being followed by any sensory or motor disturbances. But it also confirms the impression that the portions of the cerebral cortex lying about the fissure of Rolando are entitled to be called "the exquisitely motor parts of the cortex."

Yet more precisely, the motor region on either hemisphere may be, according to Exner, marked out by the method of negative cases, and by the method of percentage of cases. The former method shows that, for the upper extremities, the corresponding cortical region on the right hemisphere is the lobulus paracentralis, the gyrus centralis anterior (with the exception of a small part of its lower end) and the upper half of the gyrus centralis posterior. The latter method further confirms the foregoing conclusion. It shows that the "absolute field" for the upper extremities - the field, that is, within which lesions are always followed by impaired motion of these extremities-covers quite completely the same parts of cerebral convolutions; while the "relative field," or portion in which more than fifty per cent. of cases of lesions are followed by similar 
disturbances, extends over the remaining half of the gyrus centralis posterior, the posterior third or half of the three frontal convolutions, the anterior half of the parietal lobe, and more of the neighboring median surface. Corresponding to the better motor education of the right arm is the fact that its motor region on the left hemisphere is more extended. Here the absolute field comprises the lobulus paracentralis, the three upper quarters of both gyri centrales, and the greater part of the upper parietal lobe. Portions of the median surface of the occipital lobe may also belong to this field. The relative field for the upper extremity on the left hemisphere includes the posterior half of the gyrus frontalis superior, almost the entire convex surface of the other frontal gyri, the parietal lobe at large, and the upper part of the occipital lobe.

More specific localization of cerebral areas, corresponding to the different parts of the upper extremities, can as yet be accomplished only with much less confidence and in a conjectural way. The method of positive cases seems to designate the gyrus centralis anterior as the special cortical area for the hand; with a probability that the area for the extensors of the hand lies in its middle part, and the area of the thumb somewhat below in the same gyrus.

$\S 12$. Exner's collection contained 75 cases of disturbances of motion in the lower extremities; 26 lesions being on the right, 49 on the left hemisphere. The methods both of negative cases and of percentage agree in indicating that the "absolute" cortical field of the left leg comprises the lobulus paracentralis, the uppermost third (as far as the lower end of the suleus frontalis superior) of the gyrus centralis anterior, portions of the corresponding third of the gyrus centralis posterior, and some small areas behind and below on the lobulus quadratus, - all, of course, in the right hemisphere. The "relative field" of the same limb on the same cerebral hemisphere includes both lower thirds of the central convolutions, the back parts of the frontal convolutions, the parietal lobules, and the upper portion of the occipital lobe. On the median surface of the brain, the posterior part of the gyrus frontalis superior and the anterior half of the lobulus quadratus belong to this field. On the left hemispliere the absolute cortical field for the right leg includes the lobulus paracentralis, the upper half of the gyrus centralis posterior, and most of the upper portion of the parietal lobe. A small lateral part of this lobe, and on the median surface the lobulus quadratus, and perhaps the cuneus, must be added to complete the relative field of this lower extremity. Exner does not consider it possible, as yet, to be more precise in designating the cerebral fields for the lower limbs of man. 
§13. On comparing with each other the foregoing conclusions, it is apparent that the absolute field for the upper extremities entirely covers the corresponding field for the lower extremities; but the gyrus centralis anterior and lower half of the gyrus centralis posterior belong only to this field for the upper extremities. The relative fields, too, for both arms and legs, have a similar relation in extent and intensity. There is considerably greater probability, therefore, that a lesion of a given size in the motor region will affect the arms than that it will affect the legs; indeed, the collection of Exner shows but one case in which the motions of the legs were disturbed and not those of the arms. This greater "sensitiveness" -if we may so speak - of the cortical region of the upper extremities, corresponds to the fact that their motion is more distinctively cerebral and intelligent than that of the lower extremities.

$\S 14$. In this same "exquisitely motor" region of the cerebral cortex, and in the most nearly adjacent regions of the frontal and parietal lobes, certain other cerebral fields corresponding to definite muscles or groups of muscles may be localized, conjecturally. By the method of percentage the cerebral area for those muscles to which the facial nerve is distributed may be rather indefinitely indicated as lying in the lower half of the gyrus centralis anterior, and lower third of the gyrus centralis posterior, on the right hemisphere ; while, on the left hemisphere, it appears to be more definitely fixed at a small strip that belongs to the gyrus centralis anterior, and lies between the places where the inferior and superior frontal gyri spring from this central gyrus, but nearer the first of the two. Both methods of induction apparently unite in indicating the cortical region for the tongue as lying where the middle and lower frontal gyri meet with the anterior central gyrus. In the nine cases of the collection in which the muscles of head and neck were affected, the lesions were all situated in one of the central convolutions; but a more definite localization within the limits of these convolutions does not appear to be possible. As to the localization also of the cerebral field for the muscles of the eyeball, including that for raising the upper lid, pathology is able only to say in a general way that this field appears to fall within the general motor area as thus far pointed out. Exner thinks it certain that the rectus internus muscle of one side, and the rectus externus of the other side, are innervated from the same hemisphere of the brain. This we should also argue from their ordinary physiological function.

$\S 15$. Positive cases of a nature to strengthen the foregoing induction as to the cerebral areas especially connected with the upper 
and lower extremities might be indefinitely multiplied. Especially interestiug are those where disuse, through accident or disease, of one of these extremities has been found, post-mortem, to have resulted in atroplyy of the corresponding cortical fields. That is to say, the cortical region, being unused on account of the loss of function in the peripheral member, has itself paid the penalty of all failure to exercise the normal functions; it has lost in size and strengtl. For example, atrophy of the upper end of the gyrus centralis anterior of the right hemisphere, and of its prolongation in the lobulus paracentralis, was in one case found to have resulted from the amputation of the left leg twenty years before death.

$\S 16$. The conclusions of other authorities as to the motor regions of the cerebral cortex in man-especially of Lépine, ' and of Charcot and Pitres ${ }^{2}$-as based on pathology, confirm those of Exner in the main, as well as also in some interesting particulars; any divergences arise almost wholly from the effort to make distinctions more nicely than the present condition of the facts will warrant. The most general conclusions of these investigators may be summed up as follows:" "The cortex of the cerebral hemispheres in man may be divided, functionally, into two parts ; motor and non-motor, according as destructive lesions do or do not cause permanent paralysis of the opposite side of the body." . . "The motor zone includes only the ascending frontal and ascending parietal convolutions and the paracentral lobule." It may be concluded then, as a well-established induction, that the convolutions on either side of the fissure of Rolando (the gyri centrales anterior and posterior) and the connected lobule on the median surface of the brain (lobulus paracentralis) are in the highest degree especially connected with the motion of the extremities of the body; that adjacent parts of the frontal and parietal lobes are thus connected in a less degree; that the cortical region for the arms lies, on the whole, anterior to that of the legs; and that, probably, the region for the hand is near the middle part of the front central convolution, and that for the tongue where the middle and lower frontal convolutions meet the front central. More precise localization of the motor functions of man must as yet be made with a lower degree of confidence. Beyond these general statements lies the undefined field of conjecture.

$\S 17$. It cannot be said that histology and comparative anatomy

${ }^{1}$ Localisation dans les maladies cérébrales. Paris, 1875.

${ }^{2}$ Localisation dans les maladies du cerveau, Paris, 1876; Revue mensuelle de Méd. et de Chir., 1877-1879; Etude critique et clinique de la doctrine dans l'écorce des hémisphères cérébraux de l'homme, Paris, 1883.

${ }^{3}$ See Brain, July, 1884, p. 270 f. 
much affect the strength of the argument for the localization of the cerebral motor regions, as derived from experimentation and pathology; whatever evidence they do furnish, however, is confrmatory of the conclusions reached above. In this connection reference may be made to the conclusion of Meynert," that the paths of the sensory nerves run more toward the occipital, and those of the motor nerves toward the frontal region of the cerebrum. The existence of nerve-cells of gigantic size, resembling those found in the motor region of the spinal cord, which Betz discovered in the motor regions of the cerebrum of the dog, the monkey, and of man, is an indication in the same direction. It should also be mentioned that the pathological researches of Pitres ${ }^{2}$ into the results of lesions in the medullary substance lying between the cerebral cortex and the basal ganglia, seem to show that such as occur in the frontoparietal portion of this substance cause paralysis of motion and degeneration of the motor tracts. Finally, the general structure of the cerebrum and the courses of its nerve-tracts, as already considered (Part I., Chapters II. and III.), are, in the main, accordant with the facts of experimentation and pathology.

$\S 18$. The remarkable degree of coincidence in locality which obtains among those circles whose extirpation is followed by disturbances of motion (disturbances due, in the opinion of Hitzig, to destruction of the physical basis of the animal's control over its limbs, but, in the opinion of Schiff, rather due to tactile anæsthesia) suggests the following question: Is not the cortical field of tactile sensation in the extremities of man coincident, in the main, or even in particular, with the field for the motion of the same extremities? An affirmative answer to this question would seem reasonable, even prior to experimental and pathological evidence. The sensory and motor mechanisms are, of necessity, most intimately connected, locally, in all the central organs. This statement is certainly true of the spinal cord and of the inferior parts of the brain. Moreover, in consciousness the sensations which guide the volitions in all the finer uses of the peripheral parts of the body are very promptly, and even almost inextricably, interwoven with the volitions. In walking, talking, handling a tool, or playing a musical instrument, to be unable to experience certain delicate sensations is to be unable to will the execution of corresponding nicely adjusted motions; whereas the appearance of the associated sensations may instantaneously call forth the requisite volitions. It would seem, then, that the cerebral mechanism for both the

1 Sitzgsbr. d. Wiener Acad., LX., heft iii., p. 455 f.

${ }^{2}$ Lésions du centre ovale. Paris, $187 \%$. 
sensory and the voluntary motor factors of these complex functions must be composed of elements having the closest local connection. Certain indisputable facts of pathology form, however, a stroug objection to such an identification of the cerebral fields of motor function and tactile sensory function. Many cases of motor disturbance occur without the disturbance of sensation in the same extremity; and cases of sensory disturbance without corresponding motor disturbance, although much less frequent, are by no means very rare. How, then, can these facts of pathology be reconciled with any hypothesis which locates in the same cerebral region the so-called "fields" for both classes of function?

The answer which Exner gives to the foregoing question is pertinent, but not wholly conclusive. No absolute cortical field for disturbances of tactile sensation in the extremities of the body can, indeed, be pointed out; that is to say, there is no portion of the cerebral cortex, lesions of which are invariably and necessarily followed by tactile anæsthesia, hyperresthesia, etc., in definite parts of the periphery. But that the entire relative field of sensations of touch in the extremities corresponds with that of the motor activities, is made highly probable by the method of positive cases. After excluding doubtful cases-in which the patient complained rather indefinitely of a feeling of " heariness" or " numbness," etc., in some area of muscle or skin-Exner's collection was found to contain 22 cases where marked disturbances of tactile sensations seemed clearly made out. Of these 22 cases no fewer than 16 were located wholly in the two central convolutions; and 3 of the remaining 6 extended for several millimetres into the same convolutions. Of those still remaining, the one farthest removed from the " exquisitely motor" region was in the gyrus angularis, and therefore in a portion of the relative motor field which has a considerable per cent. of intensity.

On the basis of so complete an agreement of the positive cases, Exner feels warranted in affirming that "the tactile cortical fields for the different divisions of the body coincide in general with their motor cortical fields." It is to be noted, moreover, that the percentage of the cases of disturbance of tactile sensations occurring on the right hemisphere is more than twice as large as that of the left. Sensibility seems, then, to be the predominating function of the right hemisphere, as motion is of the left. This fact, when taken in connection with the greater liability of the left hemisphere to be the seat of cerebral disease, accounts in part for the less frequent occurrence of sensory disturbances following lesions in this general area. Moreover, we are warranted in assum- 
ing that the cortical fields, in which the nerrous impulses occasioning tactile sensations are projected, are connected with each other, and with the ascending sensory tracts, in a very complicated way. The manner of this connection is doubtless different for the different areas of muscle and skin. Nor does it appear that the sensory areas are so well differentiated as the corresponding motor areas; although one case, at least, can be pointed out in which loss of sensation in the thumb and index-finger was the definite result of a lesion of the very limited cortical region already conjecturally assigned to these members. Finally, it must be remembered that those descriptions of pathological cases on which all our inductions have hitherto been based, are very liable to be faulty with respect to slight disturbances of sensibility.

It is a general conclusion, then, which is entitled to a large degree of confidence, that both the gyri centrales, the lobulus paracentralis, and the most nearly adjacent parts of the frontal and parietal convolutions, constitute a cortical region especially related to both the motor and the sensory functions of the extremities of the body.

The view of Exner concerning the nature of the motor area in man is, on the whole, greatly strengthened by the most recent conclusions of Luciani. This experimenter find $\mathrm{s}^{1}$ that total or partial extirpation of the "motor zone" in the dog and the monkey is uniformly followed, not only by motor paralysis, but also by cutaneous and muscular anæsthesia. The "motor" sphere and the " tactile" sphere are largely coincident in these animals; and "in all experiments upon the tactile sphere there was a manifest and constant crossing of the relations between the peripheral sensory fibres and their respective cortical centres." "What one calls 'motor zone' is the central focus of the large portion of the sensorial sphere visible on the external aspect of the hemisphere."

$\$ 19$. The testimony of the facts upon which reliance must be placed in the effort to localize the cerebral field for sensations of sight and hearing in man is by no means so satisfactory as the foregoing. Experiment upon animals by stimulation is of no direct value; it could at most only discover the cortical regions especially related to some of the motions of the eye or ear and their surrounding parts. Our conclusions from the method of extirpation also must always be somewhat uncertain, since we infer the sensations of the animal only by interpreting his motions into terms of our own selfconsciousness. It is not strange, then, that the leading experimenters differ irreconcilably in certain of their conclusions. There

${ }^{1}$ See an abstract of his results, in Brain, July, 1884, pp. $145 \mathrm{ff}$. 
is pretty general agreement at present, however, as to the localization of sight somewhere in the occipital lobe. Hitzig ' found that the removal of certain gyri in the posterior lobes of the dog produced blindness of the opposite eye, combined with a paralytic dilatation of its pupil ; stimulation of the same gyri produced contraction of the pupil. Ferrier ${ }^{2}$ claims that destruction (by cauterization chiefly) of the gyrus angularis of apes produces blindness of the opposite eye, and this loss of function alone; stimulation of the same region causes movements of the eye. He therefore considers this convolution as pre-eminently the cortical centre of sight. But Iunk, after numerous experiments upon $\operatorname{dogs}$, and some upon monkeys, locates the centre of sight above and behind the place assigned it by Ferrier-namely, in the upper and hinder part of the occipital lobe; the gyrus angularis, on the contrary, he makes the cortical region for the tactile sensations of the eye. Munk's experiments are so minute in carefulness, and his conclusions so based upon detailed analysis of the phenomena, that they perhaps deserve to suggest to pathology the exact form in which to put its inquiry. They are, undoubtedly, excessive, however, in the refinement to which they would carry the principle of localization.

\$20. Munk details the following among other phenomena which result from extirpating the region marked $A_{1}$ (see Fig. 85) from the brain of a dog. The animal thus operated upon is in a condition to which the name of "psychical blindness" (Seelenblindheit) is given; but it has suffered no other obvious impairment of its sensory or motor functions. By "psychical blinduess" is meant the inability of the dog to form those visual mental images or ideas which give it the meaning or interpretation, as it were, of its visual impressions. This includes the loss of the use of that portion of the retina which is necessary for distinct vision, and of the immediately surrounding retinal parts. If the region $A_{1}$ be removed from both hemispheres of the brain, when the animal has recovered from the inflammatory reaction, it will still move about freely, guiding itself by sight even under difficult circumstances. But it does not recognize by sight the dish from which it has been accustomed to take food or water, the companions with which it has formerly played, the man who has been its keeper, the threatening hand or

${ }^{1}$ Centralb. f. d. med. Wissenschaft, $18 \pi 4$, p. 548 .

2 'The Functions of the Brain, p. $164 \mathrm{f}$. In the second edition (p. $271 \mathrm{f}$.) Ferrier acknowledges that he was in error in localizing the visual centres in this gyrus to the exclusion of the occipital lobes. For a very telling criticism of this position of Ferrier, see Munk, Ueber d. Functionen d. Grosshirnrinde, p. $14 \mathrm{f}$. 
whip, the burning coal held before its face. It still retains its general intelligence and makes constant and diligent investigation into the objects by which it is surrounded. As time passes, it gradually learns to recognize again all these visual objects. The more complex and infrequent of the objects are the last in the process of recovery to receive interpretation. At the end of three to five weeks after the operation, the injured animal may be said to have recovered; its restlessness and curiosity have subsided in proportion to the progress made in the knowledge of visual impressions; it is itself" at last, its "soul-blindness" having departed. It may be shown, moreover, that this recovery consists in learning anew the meaning of visual impressions; or, in other words, in acquiring anew the stock of visual ideas that has been blotted out of the animal's mind by extirpating the cortical centre of sight. For if the dog be carefully kept, for a long time, from any given kind of experience-for example, from being struck with a whip or burned with a coal-it will give no sign of "psychical sight" in relation to these particular objects. More remarkable still is the fact that, according to Munk, ${ }^{1}$ in certain cases, after the extirpation of $A_{1}$, a single visual image or two-for example, the motion of the hand commanding the dog to hold out the foot-may be retained. Extirpations of the cortical surface on the occipital lobe in the regions marked A-that is, before, beneath, or in front and above, the sightcentre $A_{1}$-cause disturbances of sight in a less degree. Such phenomena Munk considers explicable by the hypothesis that, while a large part of the area of the occipital lobe is the seat of the perceptions (?) of sight, the risual images of memory are especially connected with the so-called sight-centre $A_{1}$. When, then, all, or nearly all, of the field of sight, in the widest sense, is extirpated from both hemispheres, complete and permanent "soul-blindness" results. The cortical projection-field corresponding to the entire retinas of both eyes, its accumulations of old visual ideas, and capacity for receiving new ones, has been wiped out.

Munk endeavors to establish a still more minute differentiation of function in the cortical field of sight as corresponding to the retinal field of sight. ${ }^{2}$ Each retina, he holds, stands for the most part in connection with the visual sphere of the cortex of the opposite side of the brain; only a small part-namely, the extreme lateral portion of the retina-is in connection with the cortical sphere of the same side. This lateral portion of the retina seems to be of different dimensions in different races of dogs. Further, the retina is

${ }^{1}$ Die Functionen d. Grosshimrinde. pp. 23, 34, $119 \mathrm{f}$.

${ }^{2}$ See the "Fünfte Mittheilung" of his Work, as cited before. 
projected, as it were, on the cortical field of vision in and about $A_{1}$, in such manner that its lateral area corresponds to the lateral area of the cortical sphere on the same side; its inner area to the median area of the cortical sphere on the opposite side; its upper area to the front area of the cortical sphere ou the opposite side; its lower area to the hinder area of the cortical sphere on the opposite side.

In monkeys, as well as dogs, Muuk finds that the sight-centre is not, as Ferrier at first supposed, the gyrus angularis, but rather the convex surface of the posterior lobes. Small circular extirpations, of not more than two-fifths or three-fifths of an incl in diameter, from this region are followed by disturbances of vision, and by these alone. If the whole convex surface of one lobe is extirpated, the animal has cortical blindness for those halves of both retinas that are on the same side as the lesion. If the convex surfaces of both posterior lobes are destroyed, the animal becomes entirely blind; no restoration of cerebral function subsequently takes place, unless some considerable parts of the edges on the upper surface of at least one lobe hare escaped destruction. The cortical projectionfield for the visual impressions of the monkey differs from that of the dog simply in having the lateral part of the retina, which corresponds to the cortical area of the same side, much more extended. Accordingly, extirpation of the lateral half of the left cortical sightcentre, and of the median half of the right cortical sight-centre, produces in the monkey total cortical blindness of the left eye.

$\S 21$. The searching examination which the views of Munk have received has resulted in throwing doubt over some of his alleged facts, and in discrediting several most important points in his hypothesis. This is true especially of the work of Lüb and Luciani, both of whom have gone thoroughly over the ground covered by Munk and come to conclusions dissenting from lim. The former ${ }^{1}$ has minutely investigated the effects of destroying Munk's visual centre $A_{1}$, and even his entire visual sphere in the case of dogs. He finds, contrary to Munk, that no blindness of the clear spot of vision in the opposite eye is produced eren by the most extensive lesion of this area; that losses of the cortical substance in the area bordering on the lateral part of the visual sphere (i.e., in Munk's auditory sphere) also produce disturbances of vision; that other disturbances of motion and intelligence also follow destruction of this area ; and that disturbances of sight may follow lesions in other than the occipital lobes, especially in the frontal lobes. This last conclusion agrees with the results obtained by other observers (Kriwotorow, Luciani and Tamburini, and especially Goltz),

${ }^{1}$ See articles in Pflüger's Archiv, xxxiv., pp. $67 \mathrm{ff}$. 
and must be accepted as correct. The more permanent disturbances which undoubtedly do follow injury of the occipital lobes are thought by Löb to be due to what is called a "homonymous lateral hemiamblyopia" (or weakness of the corresponding lateral half of the eye) on the opposite side. Munk's whole theory of "psychical blindness" as due to the extirpation of visual perceptions and images, and of recovery from such blindness as due to special education of the animal in forming new mental images, is rejected by Löb.

The admirable observations of Luciani also tend to disprove many of the particular conclusions of Munk, while at the same time showing how relatively important are the occipital lobes in respect to the cerebral and psychical elements of vision. Theselobes, together with the angular gyrus, are in a peculiar degree the region on which the animals are dependent for "psychical" vision - that is, for "discernment of things, and a right judgment concerning their properties aud their nature," by sight.

The foregoing general conclusions from experiment with the animals as to the especial importance of the occipital lobes for intelligent (or "psychical") vision are, on the whole, in accordance with the indications from human pathology. Even Löb testifies that after extirpating part of the occipital lobes he has never observed a mere motor disturbance without one of vision also; whereas after extirpating part of the parietal lobes he has never observed a disturbance of vision without a motor disturbance.

$\S 22$. The answer of pathology to the question, whether the cerebral field especially connected with visual sensations and ideas is the same in man as in the dog and the monkey, is not unambiguous. The method of negative cases, according to Exner, ${ }^{1}$ yields no certain results; no "absolute field" for vision can as yet be indicated on the cerebral cortex. The methods of percentage and of positive cases, however, point clearly to the occipital lobe as the risual field, and to the upper end of the first gyrus occipitalis (O1, in Ecker's charts; see p. 276 f.) as its most intensive portion. In six out of seven cases of disturbances of vision due to cortical lesion the seat of the lesion was here. The region of less intensity extends over both the first and second occipital convolutions, the cuneus, and the adjacent part of the lobulus quadratus. Confirmatory evidence may be found in the cases of several persons for a long time blind, whose brains have been found on post-mortem to be atrophied above the place where the parieto-occipital fissure emerges from the median surface upon the convex surface of the occipital lobe.

${ }^{1}$ Untersuchungen über d. Localisation, etc., p. 60. 
It should be said, on the other hand, that lesions of the occipital lobes are very frequently latent, and that extensive injuries of this cortical field in man are recorded which were followed by no marked disturbance of sight.

$\S 23$. Histology also has some evidence to contribute regarding the nervons connections of the retinas of the eyes with the cerebral cortex. The amount of crossing which the fibres of the optic nerve undergo in the optic chiasm has been the subject of much debate. It undoubtedly differs in different animals, and depends upon the structures of both retina and brain, and upon the relations of the two. The researches of von Gudden ' and others have tended to show that each optic nerve contains both a bundle of nerve-fibres that is crossed and one that is uncrossed, in the optic chiasm or beyond it, toward the cerebral connections of the nerve; and that the former bundle increases and the latter diminishes in size, on the whole, in the higher orders of animals as compared with the lower. Biesiadecki and others claim, on the contrary, that there is total decussation of the optic nerves in the monkey and in man, as well as the lower animals. Charcot ${ }^{2}$ has propounded a yet more elaborate scheme of decussation. In the case of man there is still doubt, therefore, how far-if at all-the retina of each eye is represented on the cortical surface of both hemispheres of the brain. That the cortical region especially concerned in the sensations, perceptions, and images of sight is in the occipital lobe, and especially on its upper convex surface, is a highly probable conjecture. But for the settlement of further details we must await the development of the evidence. In the work of this development, experiment with animals can only suggest the question which a more careful collation of a growing number of cases in human pathology will perhaps finally answer; meanwhile the evidence of histology may be used to confirm or modify the conclusions estab. lished, more or less conjecturally, on the basis of pathology. ${ }^{3}$

$\$ 24$. The localization of other sensory functions in so-called "fields" or "centres" on the hemispheres of man's brain-of hearing, taste, and smell-is even more doubtful. Little confidence can be placed in any conjectures thus far put forward. The temptation is naturally strong to suspect that those regions of the cortex unoccupied by such motor and sensory functions as we are able to

'Gräfe's Archiv f, Ophthalmologie, 1874, Abth. ii.; 1875, Abth. iii.; 1879, Abth. i.

"Le Progrès Médical, August, 1875.

"For a further description of phenomena and cases, and for a defence of his own views, see Ferrier, The Localization of Cerebral Disease, pp. $110 \mathrm{ff}$. 
locate should have the other mental phenomena assigned to them. In this way the entire brain appears to be made of some definite value and use. Convolutions which are located where they are unapproachable for purposes of experiment, and in which comparatively few cases of lesion occur, are peculiarly provocative of conjecture. In such fields of the cerebral cortex, theories of localization may roam at will. The auditory centre is assigned by Ferrier ${ }^{2}$ to the superior temporo-sphenoidal convolution; but the evidence adduced in proof--such as the pricking-up of the animal's ears, etc.-is highly unsatisfactory. The same centre is located by $\mathrm{Munk}^{2}$ at the region B1, for its greatest intensity, and with less intensity in the adjacent regions marked $\mathrm{B}$; but since the entire region on both hemispheres must be extirpated (an almost certainly deadly operation) in order that the animal may become wholly "soul-deaf," and since we have no sure means for ascertaining precisely to what deficiency we should ascribe the failure of the animal to respond intelligently to sounds, Munk's experimental proof is likewise unconrincing. Luciani, with much more probability, considers the "auditory sphere" to extend over the whole cortical area of the temporo-sphenoidal lobe, and probably also the cormu ammonis.

The centres of smell and taste are located by Ferrier close together in the subiculum and neighboring parts of the lower temporosphenoidal convolutions; the centre of touch in the gyrus hippocampi and hippocampus major. Munk, ${ }^{3}$ however, regards these centres of Ferrier as "phantasms." $\mathrm{He}$ is strongly inclined, on the basis chiefly of one well-differentiated case, to localize smell in the gyrus hippocampi. It is difficult to see how anything sufficiently definite for scientific purposes can be known as to disturbances of taste in a dog or a monkey. No adequate evidence is procurable as yet for an induction from human pathological cases in regard to the cortical fields of any of these so-called lower senses.

$\S 25$. To the foregoing remark a possible exception must be allowed for the sense of hearing. In this connection belongs the noteworthy localization of the cerebral functions concerned in the utterance and interpretation of articulate speech. The rarious deficiencies in the power of producing and interpreting articulate sounds, whether as spoken or written, which are due to lesions of the cerebral cortex, may be grouped together under the general

${ }^{1}$ The Functions of the Brain, p. $171 \mathrm{f}$. ; comp. The Localization of Cerebral Disease, p. $132 \mathrm{f}$.

${ }^{2}$ Ueber d. Functionen d. Grosshirnrinde, p. 22 f. ; 40 f.

${ }^{3}$ Ibid., p. 129. 
term "aphasia." For about a decade previous to the discoveries of Fritsch and Hitzig, in 1870, the facts which seemed definitely to connect the loss of speech with a certain region of the left cerebral hemisphere were nearly all to which any advocate of the localization of cerebral function could confidently appeal in behalf of his theory. As long ago as 1825, Boillnud located the articulation of words in the frontal lobes. Subsequently (1836) M. Dax maintained the proposition that "lesions of the left half of the encephalon are coincident with forgetfulness of the symbols of thought."

In treatises of the years 1861-1865, Broca first announced the substantially true discovery that the gyrus frontalus inferior on the left side of the cerebrum is especially concerned in using the power of speech. This circumstance he connected with the fact that men generally use the left hemisphere more than the right for the expression of thought with the right hand and arm, whether in writing or in the mechanical arts. The literal meaning of the statements made by Broca-such as that this part of the brain is "the seat of the faculty of articulate language" '-is, however, not simply inappropriate to the facts ; it is even absurd. There is no one "faculty" of language which can, in any possible meaning of the word, be regarded as having its "seat" or locality confined to some particular region of the brain. Speech involves, in a very complicated and large way, all the faculties; strictly speaking, then, it cannot be located, with all its attendant operations of self-conscious, rational mind, in any one cerebral area. But that the phenomena of aphasia show some special connection of certain cerebral centres with the complex process of apprehending and expressing articulate language, seems entitled to credit as an induction based upon a wide range of facts. Of course, in this particular attempt at localization of function, no real help can be derived from experiments upon the lower animals.

$\S 26$. The phenomena of various classes, among which the truly aphasic cases must be discriminated, vary all the way from those resembling the results of momentary inattention-such as that of the German professor who certified in writing, "A. B. has attended my remarkable lectures in chemistry with inorganic assiduity "-to the impairment and utter loss of speech in progressive paralysis with dementia. ${ }^{2} \quad A$ few of the more curious and instructive in-

${ }^{1}$ Sur le siège de la faculté du langage artícnlé, etc., Bull. de la Soc. anat., August, 1861 ; Du siège de la faculté du langage articulé dans l'hémisphère gauche du cerveau, Bull. de la Soc. d'anthropol, June, 1865.

${ }^{2}$ For the whole subject, see the great mouograpli of Kussmaul, in Ziemssen's Cyclopædia, xiv., pp. 581-875. 
stances furmish facts like the following: The aphasic patient may be entirely speechless, and yet understand what is said to him, and be able to write his wishes down on paper. Some thus afflicted retain the power to pronounce words of one syllable, but are obliged to resort to writing in order to communicate anything further. Others possess a small stock of words, which they make more serviceable with expressive gestures. Others, still, are simply able to speak "a few senseless, and often very extraordinary, syllables and words."

Among the surprising phenomena of the disease of aphasia, none are perhaps more so than those occasioned by the ability to utter certain syllables or words, when accompanied by an utter inability to put the same letters into slightly different combination. One patient, who could say "Bonjour, monsieur," tolerably well, could not pronounce the word "bonbon" at all. Another, whose vocabulary was almost entirely limited to the meaningless syllables, "cousisi," was quite unable to utter either "coucon" or "sisi." The celebrated case of the aphasic Le Long, reported by Broca, was that of a man confined to five words for his entire vocabulary. These words were, "oui, non, tois instead of trois, toujours, and Le Lo instead of Le Long." The first two and the last were used with their appropriate meaning; "tois" indicated all ideas of number whatever; and "toujours" was the word used when the patient could not express his meaning by gestures and the other four words. It appears, then, that Le Long could pronounce the $r$ in "toujours," but not in "trois," and the nasal sound in "non," but not in his own name. In another class of cases, the aphasic person ean utter only a few or no words spontaneously and correctly, but can repeat and write without difficulty words that are spoken before him. Such inability is sometimes called "simple aphasia of recollection." Different classes of words, as a rule, slip from the memory in succession, as it were. Proper names are most frequently forgotten; then substantives generally, and sometimes verbs, adjectives, pronouns, and all other parts of speech. "The more concrete the idea," says Kussmaul, " the more readily the word to designate it is forgotten, when the memory fails." Many cases of disease occur where the patient has lost the power mentally to find the appropriate words, although his power of articulation is unimpaired. Such disturbances of speech may, or may not, be accompanied by a corresponding impairment of general intelligence. This complication increases the difficulty of studying the phases of this disease.

Aphasia may also be accompanied by so-called "word-deafness"

${ }^{1}$ Ziemssen's Cyclopædia, xiv., p. 759. 
and "word-blindness." Persons thus aflicted hear words as confused murmurings, or see them as blurred images. The individual letters may be intelligently heard or read, but their combination has become unintelligible. The same thing sometimes happens with figures; as in the case of the accountant who could read the sum 766, figure for figure, but did not know what the figure 7 meant as placed before the two 6's. At other times the disturbance of speech takes the form of grammatical ataxy, as it were, or of verbal delirium-a medley of words, partly in themselves signifcant and partly unmeaning.

The agraphia, or inability to express thought in written language, which not infrequently accompanies aphasia, may be incomplete, or absolute and literal. Some patients, who have formerly been highly cultivated, become unable to produce a single letter with the pen. Others can write long rows of letters, but arrange them for the most part in meaningless fashion, with an intelligible word occurring here and there. In brief, the phenomena more or less closely connected with the disturbance called aphasia are exceedingly complex and various.

$\$ 27$. In the effort to classify so many complicated facts, and to distinguish among them such as are of truly cerebral origin, Exner ${ }^{1}$ makes the following distiuctions: First, there are cases where the understanding of the words is affected; and such loss may constitute the chief or the entire part of the aphasia. The patient can then hear and articulate, but the "acoustic image" of the word as the symbol of an idea has perished. In a second form of aphasia, the inability concerns the clothing of the result of thought (the idea) in words-whether for purposes of spoken or of written expression. In most such cases it is simply the appropriate word which is forgotten. In the third class of cases, the aphasic person can form the idea and select the word appropriate to express it, but cannot bring about those processes of central innervation which are necessary to initiate the expression. All these three forms may combine variously, and all may be connected with disturbances of speech which are not to be localized in the cerebral cortex, but which have their origin at some point in the extra-cortical nerve-tracts concerned in speech. The very elaborate analysis of Kussmaul leads him to make the following statement: "All disturbances of speech can be brought under two great classes, according as the connection between the conception and the word is impeded in the direction from the former to the latter or, vice versa, from the latter to the former. When the first happens, the

${ }^{1}$ In Hermann's Handb. d. Physiol., II., ii., p. 343 f. 
expression suffers; when the second, the understanding." By the last word, however, we must mean the " understanding" as applied especially to articulate speech. For aphasic persons are often very intelligent in carrying on the trains of thought necessary to success in games of skill, or in the expression of feeling in music; and if we accept, even with considerable allowances, the intelligent testimony of Lordat concerning his own mental condition when aphasic, they sometimes exercise the mind in abstract reasoning of a high order, even when unable to recall a single word appropriate for the expression of their thoughts.

In all true aphasia, then, the connection between ideas and articulate language is interrupted within the cerebral cortex. Is it possible to indicate any region of this cortex, lesions in which are regularly accompanied by aphasic symptoms? or, in other words, Can the function of articulate speech, so far as this consists in the ability to apprehend and successfully to will its acoustic and visual symbols, be localized in the cerebral hemispheres? In answer to this question it must be admitted that no absolute field for aphasia can be pointed out; that is, besides the region where lesions are connected in by far the greater number of cases with aphasic disturbances, other regions of the cerebral hemispheres only sometimes thus connected may be pointed out.

$\S 28$. In a large percentage of cases of disturbance of speech due to cerebral lesions, the posterior third of the third frontal convolution and the other regions bordering on the fissure of Sylvius (island of Reil, and immediately adjacent parts of the parietal and temporal lobes) are the seat of the lesions. Aphasia is far more frequently due to changes in the left than in the right hemisphere of the brain. Dr. Seguin, out of 260 cases, calculated the proportion of aphasias due to lesions on the left side, as compared with those due to lesions on the right, to be as $243: 17$ or $14.3: 1$. Such disparity is far too great to be attributed to the comparative frequency with which the left hemisphere in general is the seat of lesions. In Exner's ${ }^{1}$ collection of cases, out of 31 lesions resulting in aphasia, all but one were on the left hemisphere (in three cases, however, the right was also involved), and in that one the trouble was only temporary. Such facts have led to the theory that, in all but left-handed men, speech, like other motor functions, is chiefly left-brained ; remarkable cases of left-handed people who have become aphasic through lesions on the right hemisphere are actually recorded. ${ }^{2}$

\footnotetext{
${ }^{1}$ Functionen in d. Grosshirnrinde, p $51 \mathrm{f}$.
}

'See Kussmaul and his citations, p. $739 \mathrm{f}$. 
Of the left hemisphere, the gyrus centralis anterior and the adjacent conrolutions of the frontal lobe, but especially the posterior part of the third (lower) convolution, have much the highest intensity as seats of aphasic lesions. In 53 carefully collected cases by Lohmeyer, ${ }^{1} 50$ were on the left hemisphere, 24 in the lower frontal convolution, 34 in this convolution and neighboring parts, 13 in the island and adjacent parts, 6 in the island alone. Exner's collection, however, did not show that the "intensity" of the lower is any greater than that of the middle frontal convolution, or of the two upper temporal convolutions. This collection contained, moreover, five cases in which lesions were seated in the lower left frontal convolution without any resulting aphasia. Exner therefore justly concludes that the "cortical field" of speech, like the corresponding fields of all the motor functions, is really much more extended than has generally been supposed. He is himself, nerertheless, inclined to localize, yet more definitely, so-called "ataxic aphasia" in the third frontal convolution, "word-deafness" in the middle gyrus temporalis, and agraphia in the lower and front part of the parietal lobe; that is, in the neighborlsood of the motor region for the upper extremities. So specific localization can hardly, however, be safely based on the restricted number of cases which Exner considered.

Lohmeyer gives 2 cases of aphasia following lesions in the anterior portion of the frontal, 3 in the parietal, and 4 in the occipital lobe. Exner gives 3 cases in which the central convolutions were alone the seat of disease; 2 in which the temporal and parietal lobes were alone affected; 1 in which the only lesion was in the occipital lóbe. In the only sense in which the brain can be spoken of as the "seat of the faculty of articulate language," we must admit that the evidence confirms the following assumption of Kussmaul : "It is, a priori, probable that an enormous association-tract in the cortex has been assigned to speech, even though the keyboard of sound may be confined to the anterior cortical regions."

$\S 29$. The more ardent and positive advocates of the theory of locally specific cerebral functions find it exceedingly difficult to refrain from seating general intelligence, or the powers of perception, memory, comparison, etc., as applied to all the objects of cognition, in some particular so-called "field" or "area" of the brain. At present the frontal lobes offer themselves as the most convenient region for such pre-empting of the cerebral domain. The general propriety of considering the connection which undoubtedly exists between the central nervous mechanism and men-

'Archiv f. Klin. Chirurgie, XIII., p. 309, as cited in Kussmaul. 
tal phenomena, under any spatial terms whatever, will occupy our attention later on. It is enough at present to say that the experimental and pathological evidence do not warrant us in assigning such pre-eminence to the frontal lobes. Extensive lesions may occur in these lobes with little or no diminution of so-called general intelligence. On the other hand, small lesions in other regions of the brain are not infrequently productive of comparatively profound mental derangement or loss of function. Moreover, lesions localized in those areas of the cerebral cortex which have thus far been considered-namely, the parietal, occipital, and temporo-sphenoidal lobes-are, of necessity, connected with more or less impairment of intelligence.

There can be no doubt that the mental processes which we describe by the word "intelligence" are all closely related to the basic sensory and motor activities that are chiefly localized elsewhere than in the frontal lobes. An animal that is " soul-deaf" or "soul-blind" has, so far forth, an impaired intelligence. The same thing is eminently true of the man afflicted with aphasia in any of its severer forms. The loss of intelligence is not necessarily (or even probably) due to the partial destruction of that functioning of the hemispheres in general which results in intelligence; it is rather due to the fact that the support which all ideation receives from the audible and visible symbols of the idea-whether chiefly as respects its formation or as respects its expression-has become impossible. The impairment of any considerable area of tactile sensations, especially as localized in those parts of the body which are most used in perception through such sensations (e.g., the hand), also occasions a certain loss of intelligence. The restrictions which cerebral disease introduces into the number and nicety of the sensory and motor functions are, of course, much less important when they come upon minds already furnished, as we say, "with a stock of ideas." Still, even in such cases a basis of sensations and volitions constantly underlies, as it were, all the higher and pre-eminently intellectual mental processes.

$\S 30$. In spite of the evidence adduced, a few experimenters still either wholly reject the principle of the localization of cerebral function, or else urge arguments against carrying it out even with the limitations which the foregoing conclusions have observed. Among such experimenters the most prominent is perhaps Goltz. The method of extirpation practised by Goltz was that of wash. ing away the substance of the cerebrum by streams of water sent

${ }^{1}$ See especially his treatises as collected in the book, Ueber d. Verrichtungen d. Grosshirns, Bonn, 1881 ; and Pflüger's Archiv for 1876, 1877, and 1879. 
through orifices broken at selected places in the skulls of dogs. This method has the advantage of saving bleeding; it has the disadvantage of not definitely localizing the injury. Its author has applied it with great care and skill to a large number of animals, many of which he has succeeded in keeping alive for months, even after the removal of considerable areas from both hemispheres (in one instance the brain-substance, calculated to have weighed originally 93 grammes, had been reduced to 13). The principal conclusions drawn from his experiments by Goltz are adverse to the theories of localization held by Ferrier, Munk, Luciani, and others.

Goltz's conclusions may be summarized as follows. No impairment of intelligence follows the loss of a large amount of cortical substance from one side of the brain; but loss of any considerable amount of substance from both sides-whether in the frontal, posterior, or temporal lobes-produces a permanent impairment of all the functions, which corresponds in a general way to the amount of the loss. Every sense, and the intelligence of every sense, is thus weakened; for the cerebral elements of sense are impaired or destroyed (Hirnsehschwäche, etc.). For example, a dog which has been trained to give his paw on command loses the power to do so in consequence of such loss of brain-substance, and never regains it. It is not possible, by extirpating any amount of the substance of the cortex on either side or on both sides, to produce a permanent laming of any muscle of the body, or a total loss of sensibility in any of its parts. It is, however, possible, according to Goltz, by repeated removal of the cerebral substance on both sides, gradually to reduce an animal to a condition of almost complete idiocy-to an elaborate eating, drinking, and walking "reflex-machine." The removal of as much as 4 grammes from each hemisphere produces, as he calculates, a considerable degree of idiocy. No part of the cortex of the brain can, then, be called the exclusive organ or centre of intelligence or feeling; but the psychical functions of sensation, volition, ideation, and thought are connected with all of its parts. The quantity of the cerebral substance removed determines the amount of the general impairment of mental powers, instead of the locality from which the removal is made fixing the quality of mental impairment.

It must be admitted that the facts discovered by Goltz, and the conclusions which he reaches, seem at first strongly opposed to all localization of cerebral function. But they are not really so; nor is it quite correct even to say, as Foster ${ }^{1}$ does, that Goltz's results ${ }^{1}$ A Text-book of Physiology, p. 649. New York, 1880. 
are " absolutely opposed" to those of Munk. In fact, Goltz" himself asserts that destruction of the parietal lobes produces a greater permanent disturbance of feeling, and destruction of the occipital lobes a greater permanent disturbance of sight. In general, an animal operated upon in the two hind-quarters of the cerebrum is more stupid than one which has suffered loss of the fore-quarters; the former is duller of sight, hearing, smell, and taste ; the latter is duller in respect to skin-sensations. The effect of injury to the posterior parts of the brain is therefore much more marked in depressing the intelligence of the animal (as shown in sense-perception). Moreover, Goltz ${ }^{2}$ claims that he has never rejected the possibility of a localization of the functions of the brain. He confirms $^{3}$ the conclusions of Fritsch and Hitzig, by saying that he has often seen mechanical excitation of the parietal lobes produce motions in the limbs of the opposite side. His facts and arguments are rather directed against that form of the hypothesis of localization which seats the different functions in small circumscribed areas ${ }^{4}$ and then, when forced by facts, conceives of them as also capable of hopping about from one of these areas to another, like a bird from twig to twig in the branches of a tree. Furthermore, a detailed comparison of the experiments of Goltz with those even of Munk shows that the results of the two are in the main reconcilable; if only it be remembered that the former has not always precisely defined the areas of brain-substance removed, nor sufficiently taken into account the undoubted results obtained by others from definitely circumscribed lesions; and that the latter has, certainly, in many cases been more precise and confident than a fair view of all the facts will warrant.

§31. Three principles may be laid down as summing up the results reached by inference upon the basis of experiment with respect to the localization of function in the cerebral cortex. ${ }^{5}$ The first principle is to be accepted in the form of a general postulate derived from a study of the other parts of the nervous system, and confirmed on attempting to apply it to the cerebral hemispheres. It may be stated as follows : the different elementary parts of the nervous system are all capable of performing its different specific functions when, and only when, they have been brought into the proper connections and have been exercised in the performance of

\footnotetext{
1 Verrichtungen d. Grosshirns, pp. $114 \mathrm{f}$. and $150 \mathrm{f}$; and art. in Pflüger's Archiv, xxxiv., pp. $450 \mathrm{ff}$.

${ }^{2}$ Verrichtungen d. Grosshirns, p. 163. ${ }^{3}$ Ibid., p. 165. ' Ibid., p. 169.

'Compare the five general laws of central functions given by Wundt, Grundzuige d. physiolog. Psychologie, i., p. $224 \mathrm{f}$.
} 
those functions. This principle includes two important laws which, we know, hold good throughout the whole nervous mechanism, and which lie at the physical basis of important psychical facts and laws; they are the law of Specific Energy and the law of Habit. Different combinations of the elementary parts of the nervous system, forming composite parts or organs, have different values and functions in the general economy of the system. Every nerve-fibre, every element of an end-organ, or of a central organ, may be said to have a specific function, and to discharge that function in the exercise of a specific energy. As to how far the capacity for this specific energy is dependent upon the specific molecular structure of the elementary parts, we are only able to conjecture; but about its dependence upon the connections in which the elementary parts stand with each other, there can be no doubt.

Moreover, the elementary parts of the nervous system, inasmuch as they have the general adaptation necessary to the performance of nervous functions, can "learn" (so to speak) to perform the more specific of these functions-but only in case they stand in appropriate connections. The répeated action of the nervous elements in specific functions fits them the better to act in the same functions. The effect of the exercise of any function in the past may be "stored up" so as to increase the facility of the nervous structure to exercise again every similar function. Thus, different elementary parts of the nerrous system, if at first forced by circumstances to become active in a given way, may by repeating the activity gain a position of facility and value like that belonging to other parts whose so-called normal action lies in this particular way. This law of habit in the nervous system explains much of the behavior of the nerve-muscle machine, or of the decapitated frog, etc., under artificial stimuli ; it also underlies the theory of the sensory-motor effects attributed to centres in the spinal cord and basal ganglia; it throws light upon the physical basis of our experience in learning to walk, talk, play upon musical instruments, or handle tools, as well as upon the transmission from generation to generation of minute bodily characteristics. Both the law of specific energy and the law of habit undoubtedly apply to the functions of the cerebral cortex.

The remaining two of the three principles alluded to above may be said to follow from the first; they are the principle of localized function and the principle of substitution. The former asserts that, in the normal condition of the nervous system, all parts have not the same definite functions. Inasmuch as the functions of the different elementary parts necessarily depend upon the manner 
in which they are combined and connected, the composite parts or organs thus formed must also have certain normal functions. But such composite parts or organs have, of course, a clefinite locality ; hence the functions of the nervous mechanism must be more or less definitely localized. Nor can the principle be suspected of a disposition to stop short off and abdicate its authority, when we reach the region of the cerebral cortex. There is nothing in the structure of the cortex to show why the general law of differentiation of function should be inapplicable there. On the contrary, everything in both its anatomy and physiology indicates that the principle of localized function does apply, in some sort, to the cerebral hemispheres.

So-called "centres," or " areas," or "fields," of the cerebrum are in no case, however, to be regarded as portions of its nerrous substance that can be marked off by fixed lines for the confinement of definite functions within rigid limits. These areas are somewhat different for different brains of the same species; they widen when a heightened energy is demanded of them; their centres are neither mathematical points nor very minute collections of cells. They are not composed of elements which have, each one, a fixed and unchangeable value, and a definite function, as though the number of mental operations assigned to a locality needed to be precisely matched by the separate nerve-fibres and nerve-cells of the locality. Nor are these areas perfectly isolated localities; on the contrary, they obviously overlap each other in certain cases. According to the true statement of Luciani, "the single centres in the sensory-motor zone are so completely bound up with, and, so to speak, let into one another, that it is not possible to divide them with a clear and definite line, such as is the case when the cortex is incised and removed; so that in destroying a centre one necessarily eliminates a portion of the neighboring centres." Nerertheless, there is no doubt that the cerebral functions connected with the different sensations and motions of the peripheral parts of the body are not all alike exercised by all parts of the cerebrum. They are assigned specifically to those regions which alone have the proper structure and stand in the proper relations.

Furthermore, the functions of the cerebrum are not absolutely confined to those centres with which, under ordinary eircumstances, they are chiefly or wholly connected; in which, that is to say, they are localized. If such centres, for any reason, become incapacitated or relatively unfitted to perform their normal functions, the same functions may be performed by other areas of the cerebral cortex, provided these areas also stand in the proper connections. This 
is the principle of substitution. It is due to its working that animals subjected to experiments in extirpation, as a rule, so largely recover the powers of sensation or motion which they hare temporarily lost. It is on this class of phenomena that Goltz rests much of his argument. In the cerebral hemispheres, however, the principle of substitution does not overstep all limits, nor does it operate arbitrarily. The portions of the same hemisphere that are just adjacent to the so-called centres-the larger areas surrounding or contiguous to the smaller-and, on account of its bilateral structure, the corresponding portions of the other hemisphere, are in general those best capable of exercising such substitutive functions. It may be doubted whether these portions do not, in all ordinary cases, cover the entire limits within which the principle of substitution can act. Such substitutive functions improve under the law of habit to which the organs of the cerebral cortex are subjected.

The connections between the different cerebral areas and their functions are so complex and subtile that physiological science will need a long time to disentangle them; it may be doubted whether it will ever succeed in doing this completely. The connections among the phenomena of conscious sensation, volition, ideation, and thought are at least equally subtile and complex. Will psychology ever disentangle these connections?

The bearing of the subject on our conclusions concerning the nature of the mind and its connection with the body will be considered elsewhere. 


\section{CHAPTER III.}

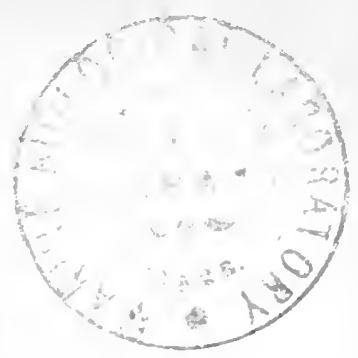

\section{THE QUALITY OF SENSATIONS.}

§1. THE world of ordinary experience consists of a great number of so-called "things" that are known to us by their distinguishing qualities. Although each one of these things is believed to be a separate existence, they are all perceived as having certain common characteristics, and as standing in certain relations to each other, of space, time, and action. It is with the things, their common qualities and mutual relations, that unreflecting practical life is chiefly concerned. But even without special reflection, everyone learns that his knowledge of such external objects depends upon the kind and degree of the effect they exercise upon his consciousness through the senses. Attention is thus turned from the things themselves to the sensations produced in us by their action. The variety of such sensations, at first bewilderingly great, is soon reduced to some order by a classification referring them to the different organs through which they come. Thus, certain sensations are received through the nose, others through the mouth, the ear, the eye, or the skin-especially as covering that part of the body (the hand) which is most active in touch. Smell, taste, hearing, sight, and touch are the five classes of sensation, as the grouping is made by the unprejudiced judgment of all.

A further rough and scientifically inadequate classification takes place among the sensations of the same sense. Those of smell, indeed, defy classification, whether popular or scientific. Among tastes, the most familiar are easily distinguished; such are the sweet, the sour, and the bitter. The two principal classes of sensations of sound are easily discriminated, as either noises or musical tones; the former are further classified as respects the character of the feeling which accompanies them, and the latter as high or low in pitch. The different more prominent colors-including black and white-are recognized by all persons of normal vision as modes of the sensations of sight; hence the colors commonly named, and the various so-called "shades" of these colors. That more than one class of sensations arise through the skin is shown 
by the popular use of the word to "feel." Things feel hard and soft, smooth and rough, as well as warm and cold. But things are also said to feel heavy or light. The feeling by which their weight is estimated, however, is only ascribed in a very indefinite way to the parts of the body that are chiefly concerned in passively supporting, or actively lifting, or pushing against their weight. The particular use of tactual feeling, as well as the general use of the muscular sense, in gaining this class of sensations is little noticed by ordinary reflection.

§2. All the sensations are also regarded as having some place in a scale of degrees of sensation; they are either strong or faint, or else lie somewhere between the two extremes. They are also habitually thought of as related in time, and as being connected with the motion in space of the objects that occasion them. Of the molecular action of their stimuli upon the end-organs of special sense ; of the hidden chemical, electrical, or other processes connected with the activity of the peripheral and central nervous system ; of the physiological, psycho-physical, and psychological laws under which the mind reacts in the form of simple sensations, and combines these sensations into the composite objects of sense; of all these and other similar matters, the unreflecting conception of sensation takes no account.

§3. It is obvious that the analysis of sense-percepts which suffices for working-day life will in no respect answer the demands of science. Its "common-sense" character is a distinct mark of its inadequacy. An adequate scientific treatment of this branch of Physiological Psychology requires at least four things : (1) to distinguish the simple sensations from those complex objects of experience with which alone our adult consciousness is familiar; $(2)$ to point out the varieties of quality and degrees of quantity which belong to these sensations, and to discover the laws which relate them to changes in the form and intensity of their stimuli ; (3) to show how the simple sensations are constructed by the mind into the socalled "presentations of sense" under mental laws of time-form and space-form; and (4) to indicate how far, if at all, the higher mental activities of association, memory, will, and judgment, may be brought under laws similar to those upon which the formation of these presentations of sense depends. It is upon these four heads of inquiry that modern psychology, as studied from the psycho-physical point of view, has expended most of its painstaking researches. Its success has been by no means complete. All these fields of inquiry still include many unanswered questions; all of them present the results of researches that seem in various re- 
spects conflicting. Yet it is precisely in these fields that modern psychology has achieved its most brilliant successes. It has thrown a flood of new light upon the essential nature and growth of human experience. It has profoundly influenced the current views on metaphysics. It has contributed important factors toward the solution of certain questions of interest to ethics and religion. It has given us a new point of view for renewing the ancient debate between Materialism and Spiritualism.

\&4. The distinctions with which scientific analysis begins are to a large extent received from ordinary experience. Some of the most essential of the distinctions are confirmed by the results of this analysis. They all, however, require to be carried farther and to be fixed with much more of accuracy than belongs to the impressions of common life. New distinctions also have to be introduced. For example, scientific investigation maintains the difference between sensations of smell and sensations of taste; but it points out what is not ordinarily apparent-namely, that certain results commonly referred to the latter sense really belong to the former. It also adds the sensations of the muscular sense to the classes popularly described; and it discriminates more clearly between two distinct kinds of sensation that have the skin for their organ-namely, temperature and pressure.

Psycho-physical science, moreover, accepts the common distinction between the quality and the quantity of the different sensations. But it describes with all possible accuracy the limits within which alone this distinction can be carried out. It shows that the quality and quantity of sensation are inseparably connected; that, as Lotze held (a view confirmed by von Kries and others), changes in quality can be distinguished from changes in intensity, with perfect confidence, only in the case of sensations of hearing. It is possible that even here the distinction is largely made on the basis of complex experience. Very intense sensations of heat and cold so far change their specific character as to tend to pass into each other, or, perhaps, to become submerged in a common tone of painful feeling. Minimum sensations of heat and pressure are difficult to distinguish from each other ; maximum sensations of pressure are likely to lose the cliaracteristic quality of touch and be displaced by sensations of pain. To treat scientifically of the quality of sensations requires, then, a large amount of the most careful analysis.

§5. It is essential, in the first place, to distinguish "simple sensations" from "presentations of sense," or those complex objects of consciousness which result from an act of mental synthesis 
on the basis of several simultaneous affections of sense. As respects developed experience, the simple sensation is a necessary fiction of psycho-physical science. Consciousness is scarcely more able directly to analyze a presentation of sense into those factors out of which it originated than it is to analyze a drop of water into its component oxygen and hydrogen gases. Simple sensations, therefore, are not objects which can be examined in the direct light of introspection. Yet they are factors which, as scientific analysis shows, actually enter into all such objects as can properly be spoken of under the term "presentations of sense." Any sensation which is absolutely unanalyzable with respect to distinctions of quality, and which, therefore, cannot be considered as consisting of component parts, is called simple. It is distinguished as a sensation from all other elementary forms of feeling or knowledge, by the relation which it sustains to the presentations of sense. A sensation, unlike the feeling of grief, of desire, or of weariness, etc., is a potential factor of a material object. Through the senses we know "things;" not, indeed, as though they appeared before the mind by immediate apprehension in the form of exact copies of extramental realities. But every sensation is an affection of the mind recognized as connected with an extra-mental reality, through the activity of the senses. Simple sensations are those elementary factors, themselves indecomposable, out of which the presentations of sense are composed. The objects of sense, however, do not have the character of mere compounds of simple sensations. Sensations must not only be associated and compounded, but also localized and projected without (that is, set in systematic relations of spaceform), in order to constitute the objects of sense.

$\S 6$. The foregoing remarks suffice to indicate, in a preliminary way, what is the nature and value of the psycho-physical investigation of sensation. We inquire, in the next two chapters, as to the Quality of Sensations. The inquiry, when conducted from the psycho-physical point of view, involves an answer to three questions : (1) What is the precise locality in the organism where the specific excitation which occasions each kind of sensation originates; and what is the nature of the action of the stimulus in producing such excitation? (2) What are the kinds of sensations which appear in consciousness as the result of the various excitations? (3) What are the laws by which the quality of the sensations is related to the kinds of excitation? Neither of these three questions can be answered completely. The investigation of the first is much restricted by our almost complete ignorance of those processes in the central organs that are in all cases the proximate internal 
stimuli or immediate antecedents of the sensations. Moreover, our knowledge of the intimate structure of the end-organs of sense, and of the nature of the physical processes which excite them, is still very incomplete. The detection of obscure but important differences in the qualities of conscious states of sensation is by no means easy; it requires great skill, strict and trained attention, and unwearied repetition of experiment. But these conditions of success have a great effect in altering the quality of the sensations themselves. Besides all this, remarkable idiosyncrasies not infrequently appear; and language can only imperfectly describe even the most common factors of the varied and living experiences with which science tries to deal.

In investigating the laws that define the relations between our subjective experience, called sensation, and objective phenomena in the shape of physical energy acting upon the nervous mechanism, there is often the greatest doubt as to what manner of laws are being investigated. They may be considered as purely physiological, or as psycho-physical, or as purely psychological. It is not strange, therefore, that different theories exist for accounting for all the more important groups of facts, depending upon the emphasis laid by different investigators upon the value of each of the three possible modes of explanation. The truth is, that each sensation is separated by a series of intricate physiological and psychical processes from the application of the stimulus in the gross, as it were, to the eud-organ of sense.

§ 7. The authority of one great law is involved, as a silent assumption, in all discussion of the quality of sensations. This law is known as the law of the Specific Energy of the Nerves. It has already been shown (Part I., chap. I., $\S 35$ ) that any such distinction of the kinds of nerve-fibres as denies their possession of common functions cannot be maintained. But the phenomena of sensation cannot be explained without a much more extended application of this law than has thus far been found necessary. Distinctions of quality in sensation depend upon the excitation of specific corresponding elements of the nervous system. That only the optic nerve is capable, when excited, of exercising the physiological function upon which sensations of light and color depend, does not admit of doubt; the same specific quality cannot be denied to the functional activity of the nerves of smell, taste, hearing, and touch. Moreover, in the end-organs of each of these senses, provision must be made for a further differentiation of function. What is the nature of the evidence, and what conclusions must be drawn from it, will be best appreciated at a later pe- 
riod in the discussion. Meantime we find ourselves obliged to assume the existence of some law of the specific energy of the nerves of special sense.

\& 8. Little of a scientific character is known concerning Sensations of Smell, considered as respects their quality. Anatomy, chemistry, and plyysics fail to furnish definite information on this point; experimental physiology as applied to the lower animals is, of course, unsatisfactory ; and the appeal to human consciousness asks for an analysis of which it is incapable. It has already been shown (Part I, Chap. V.) that the part of the mucous membrane of the nasal passages known as regio olfactoria contains the endorgans of smell; the specific stimulus of the organs in this region is applied as borne thither by the current of air, and almost, if not quite, exclusively in the act of inspiration. In order that any substance may act through the end-organs on the nerve (olfactorius) which is spread out in this region, it must either exist in gaseous form or else be raporizable under given conditions of temperature. The degree of temperature at which different substances become odorous therefore varies according to their physical characteristics. For example, arsenic, which at ordinary temperatures is inodorous, when raised to a dark-red heat is vaporized and the vapor excites an intense sensation of smell. Fluid bodies which give off an odorous reek, when brought in fluid form into contact with the mucous membrane of the regio olfactoria have no smell; if this membrane is soaked in fluid of any kind whatever, it loses for a time the capacity to be excited with olfactory impressions. E. H. Weber ${ }^{1}$ discovered that if the head be placed with the nostrils pointing upward, and the nasal passages be then filled with pure water, sweetened water, or a mixture of water and eau de Cologne, after these passages are emptied the sense of smell is, in all cases, temporarily lost; even when Cologne is used, with the exception of the instant at which the fluid is poured in, no odor can be perceived. Subsequent observers have confirmed the experiments of Weber. One investigator ${ }^{2}$ lost all sense of smell, even for acetic acid and ammonia, for a period of half a minute; another for five minutes, and the sense in its full acuteness did not return for nearly half an hour. Whether this effect of the fluid is due to impairment of the end-apparatus of smell by soaking it (so Valentin), or to the mechanical barrier which the layer of foreign substances interposes between the odorous particles and this apparatus (so Fröhlich), we cannot say ; it may be due to both causes. Contrary

${ }^{1}$ See Archiv f. Anat., Physiol., etc., 1847, p. 257.

'Fröhlich, in Sitzgsber. d. Wiener Acad., 1651, VI., p. 322. 
to the assertion of Wundt, ${ }^{1}$ that probably no gases or vapors, except atmospheric air and its constituents, are absolutely inodorous, so far as we have present information a number of gaseous and vaporizable substances are so; and no reason is known for such apparent exceptions to the rule.

\&9. The stimulus of smell is supposed to consist in certain exceedingly minute particles contained in the odorous gas or vapor which is drawn in with the current of air over the mucous membrane of the regio olfactoria. The question is as yet scarcely decided, whether other forms of stimulus, besides these odorous particles-mechanical, electrical, thermic, or so-called subjectirecan excite the sensation of smell. The older experimenters (Volta, Pfaff, Fowler, and Humboldt) failed to obtain any certain proof that the electrical current is an excitant of this sense. In one place, however, Pfaff speaks of a sensation resembling the smell of sulphur as caused by the application of electricity to the sensory passages of the nose. Ritter (in 1798) experimented by using bits of graphite and zine thrust into these passages, and also by holding one pole of a battery in the hand and placing the other in the nostril. In the latter way he thought that he excited a genuine specific sensation of this sense. He describes the positive pole in the nostril as producing an inclination to sneeze and a trace of a smell like that of "ammonia ;" the negative pole placed there does away with this inclination and produces a kind of "sour" smell. Such phenomena are probably, however, all to be assigned to the nerves of taste, touch, and common feeling. More recent investigations have done little to remove the reasons for doubt. ${ }^{2}$ The smell of phosphorus which is developed by the action of the electrical machine is probably due to the ozone set free; it is not a case, then, of the direct excitation by electricity of the sensation of smell. Some physiologists (notably Valentin) have observed that this sensation may be awakened by mechanical stimulation, such as strong vibration of the nostrils, violent sneezing, etc.; others have failed to produce this specific sensory effect in such ways. It does not appear that thermic stimulation will excite the sensation of smell.

Experiments to prove that subjective sensations of smell may be produced by injecting odorous substances into the veins of animals are very uncertain. Human pathological cases, in spite of the customary indefiniteness of the patient's testimony as to the nature of his sensory affection, show that compression of the olfactory nerve

\footnotetext{
${ }^{1}$ Grundzüge d. physiolog. Psychologie, i., 384; comp. von Vintschgau, in Hermann's Handb. d. Physiol., III., ii., p. 261 f.

${ }^{2}$ See Rosenthal, in Archiv f. Anat., Physiol., etc., 1860, pp. $217 \mathrm{ff}$.
} 
by tumors, etc., may produce sensations of smell. Disturbances of the central organs, such as occur in certain cases of insanity, may doubtless have the same result. The powerful effect which some odors have upon the brains of certain persons, so that nausea, giddiness, and other disturbances of feeling result, scarcely needs mention; it cannot all be resolved into mental associations connected with the sense-impressions.

$\S 10$. No approach can be made toward a scientific classification of the kinds of smells.' This specific sensation must, however, be carefully distinguished from the other forms of feeling with which it is most closely allied. Many supposed sensations of taste are really sensations of smell. Substances like ammonia and acetic acid powerfully excite the sensations of touch and common feeling through their action on the trigeminus as well as the olfactory nerve. Other sensations of touch and of the muscular sense are reflexly occasioned in such cases, and blend with the specific sensations of smell in the total mental result. But of all the attempts to classify the qualitatively pure sensations of this sense, none can be said to have any scientific value. The division into pleasant and unpleasant smells depends upon the idiosyncrasies of individuals; to some the smell of burning feathers, of assafotida, of valerian, or of rank cheese, is pleasant. Fröhlich's ${ }^{2}$ classification into those which excite merely the olfactory nerve, and those which call out other sensations reflexly through their action on the trigeminus, is purely physiological and not psycho-physical ; moreover, it does not apply to sensations of smell, as such. When we classify the sensations according to the objects which produce them-as practically we are obliged to do-we are not distinguishing the qualities of our feeling; the smell of a rose does not belong to a class of sensations as does a sour taste or the color red. No known principle will bring order out of the bewildering complexity of this sense.

Sensations of smell cannot, like those of pressure, hearing, and sight, be schematized or represented as standing in any definite local or mathematical relations to each other. Smells cannot be conceived of as having a scale of pitch, or triangle of color-tones. As Wundt" declares, the sensations of smell form "a discrete manifoldness which has an unknown arrangement."

\$11. The properties which any substance must possess in order to be odorous, and the nature of the action of the odorous particles

'For the entire subject, see von Vintschgau, in Hermann's Handb. d. Physiol., III., ii., p. 266 f.

${ }^{2}$ Sitzgsber. d. Wiener Acad., 1851, VI., p. 322 f.

${ }^{3}$ Physiolog. Psychologie, i., p. 386. 
upon the end-organ of smell, are wholly unknown-as much so now as when, more than a half-century since, Cloquet confessed the complete ignorance of the scientific world on these matters. A great variety of phenomena appear, but no known law has control of them. Some plants are odorous by day alone, others by night alone; still others only in the morning. Some plants have a smell when dried; others give off only a weak odor when dry, but a stronger one when moistened. Of course, the effect of any odorous substance depends upon the ease with which it may be vaporized, and the speed and extent of its diffusion through the atmosphere. Camphor, musk, and other similar substances are distinguished for their long-continued and far-reaching effects.

The discovery of Romieu, in 1756, that small bits of camphor on the surface of water have a rotary motion, has called out various investigations in the line suggested by this fact. Prévost subsequently (1799) observed that other odorous bodies liave a similar motion on the surface of water, and that a very thin layer of water on a perfectly clear plate or glass withdraws itself as soon as pulverized camphor is laid upon it. More recently still, Liégeois bas noticed the same phenomena, wholly or in part, exhibited by some two hundred odorous substances of either regetable or animal structure. Minerals, according to this observer, do not behave in the same way. Some of these odorous substances seem to inhibit or check the rotary motion in others. He concludes that we are justified in believing odorous substances to have the power, especially when in contact with water, of setting up a motion of these outside particles which distributes them through the atmosphere so that they reach the mucous membrane of the nasal passages. Just how they act upon the end-apparatus there it is impossible to say. The researches of Tyndall ' and others as to the influence which odorous particles of different substances have upon the capacity of the air to absorb heat may possibly be combined with the foregoing researches in a way to suggest some tenable hrpothesis touching the nature and action of the stimuli of this sense; but thus far, as has been said, we cannot go beyond a confession of ignorance.

$\S 12$. The condition of scientific attainment as to sensations of taste and their stimuli is only little better than that as to the allied sense of smell. The adequate specific stimulus for the nerves of this seuse consists in certain tastable substances; such substances, however, do not excite the end-apparatus unless they act upon it under definite conditions. Only fluid bodies, or such as are at least to some small degree soluble in a fluid or menstruum, excite

' Heat as a Mode of Motion, pp. 341 ff. New York, 1868. 
sensations of taste ; absolutely insoluble bodies are, without exception, tasteless. This fact may be due to the concealed position of the inner cells of the gustatory flasks, which is such that they cannot be reached by substances undissolved. By no means all soluble substances hare a taste. No known law regulates the relation between the solubility of bodies and their power to excite sensations of this class. It is disputed whether any of the gases are direct excitants of the end-organs of taste. The monograph of A. Stick ${ }^{2}$ maintains the tastable character of certain gases, on the ground that a stream of them, let fall upon the tongue when dry (so that they cannot well be absorbed by the saliva), produces the peculiar sensations of taste which these gases are known to possess. A stream of carbonic-acid gas, for example, when acting on the dry edge of the tongue, has a taste which is described as sweetish sour. It is diffcult, however, to secure such a degree of dryness of the tongue as will not leave a moist capillary layer; difficult, also, to exclude all the connected sensations of smell and common feeling. ${ }^{2}$

It is doubtful whether the sensation of taste can be excited by mechanical means; and there is no proof that heat can irritate the gustatory nerves. Certain authorities of the first rank have indeed described specific sensations of taste as mingled with the feelings which follow rubbing, pricking, and pressing the tongue. Henle observed a saltish taste to be excited by passing a current of air over the tongue; Wagner a bitter taste by pressing down the base of the tongue with the dry finger; Dr. Baly an acid or a saltish taste by repeatedly and lightly tapping the end of the tongue.

The long-debated question as to the electrical stimulation of this sense seems now to be decided affirmatively. ${ }^{3}$ It was discovered in 1752 that the application of two different metals to the tongue is followed by a peculiar sensation of taste. Volta recognized the fact that the effect of the metals is due to the electrical current called out between them. If the cathode is laid upon the upper surface of the tip of the tongue, a sensation is produced by the current passing out which is variously described as metallic, acid and metallic, or bitter and metallic, etc.; but if the anode is applied to the same spot, the sensation produced by the entering current is described as acid, or acid and metallic, or bitter and metallic. In the former case, not infrequently, a strong current is needed to pro-

\footnotetext{
${ }^{\lrcorner}$Ueber d. Schmeckbarkeit d. Gase, Berlin, 1857; article in Annalen des Charité-Krankenhauses.

"See von Vintschgau, in Hermann's Handb. d. Physiol., III., ii., p. $196 \mathrm{f}$.

${ }^{3}$ The whole question is discussed by ron Vintschgau, ibid., p. 181 f.; and Pflüger's Archiv, xx., pp. $81 \mathrm{ff}$.
} 
duce any sensation at all. Since the discovery of electrolysis, it has been objected that these effects are due to the decomposition of the fluids of the mouth and the consequent accumulation of free acid at the positive and free alkali at the negative pole; they are therefore not to be ascribed to the direct action of the electrical current on the end-apparatus of sense. Experiments by du BoisReymond, Rosenthal, ${ }^{1}$ and others have been directed toward answering this objection. The former showed that when a chain of four persons is arranged in such manner as to send a current of electricity through the tongue of one, the eyeball of another, and the muscles of a frog-preparation held by two of the four, the same current will cause simultaneously an acid taste, a flash of light, and a movement of the animal's muscles. Rosenthal discorered that, if two persons touch the tips of each other's tongues while one holds in a moist hand the positive and the other the negative pole, an electric current will cause the first person to have an alkaline and the second an acid taste. Still other experiments confirm the opinion that sensations of this sense may be directly due to electrical stimulation. Attempts hare been made to prove the possibility of exciting subjective sensations of taste by injecting tastable substances into the veins of animals; but the psychology of the subject has reaped no results from these attempts. Most of the alleged cases of such subjective origin are probably due to substances really brought to the tongue in the saliva. It is worth remarking here that sensations of taste rarely or never mingle in our dreams.

$\S 13$. The question whether a tastable substance excites precisely the same sensation when applied to all portions of the organs of taste is a difficult one to answer satisfactorily (see Part I., Chap. V., § 6). The tabulated results of different experimenters upon this question disagree considerably. Such disagreement is suggestive of idiosyncrasies of taste, and of doubt whether the different shades of the same class of sensations are either nicely discriminated or uniformly described by most persons. Descriptions which speak of the sensations as prickly, piquant, cooling, etc., show, of course, a combination of sensations of common feeling with those of special sense. The minor varieties of taste may be occasioned in a manner similar to that of the less important shades of color-sensations. It seems tolerably well established that sweet and sour are tasted chiefly with the tip of the tongue; bitter and alkaline with its roots. The experiments of two of the principal observers, Horn and Picht, agree in the conclusion that nearly all substances (even sugar) call

${ }^{1}$ Ueber d. elektrischen Geschmack; Archivf. Anat., Physiol., 1860, p. 217 f. 
out a bitterish taste when applied solely to the papille circumvallatce.

$\S 14$. Most of the different kinds of tastes admit of being considered as compounds of a few simple sensations of this sense with each other and with sensations of smell, touch, common feeling, and muscular sense. Many so-called tastes are really chiefly smells. Physiologists generally distinguish four principal classes of tastessweet, bitter, salt, and sour. Wundt ${ }^{1}$ adds to these four the alkaline and the metallic. But possibly the alkaline may be considered as a modification of the salt; and the metallic is probably a compound taste, although its analysis is by no means easy. The attempt has been made by Valentin and others to reduce this number to twothe sweet and the bitter. The sour is thus considered as not a pure sensation of taste, but as predominatingly a sensation of touch. Acids in concentrated form certainly bring into action the nerves of feeling; but in very dilute form they seem to excite purely the sensation of taste. The same thing is true of saltish substances. The bitter and the sweet are agreed by all to have the character of pure sensations of this specific sense. Powerful reflex sensations of the muscular sense are occasioned by strong stimulation of the nerves of the tongue, and these sensations blend with the specific sensations of taste. There is no satisfactory reason to be given for classing the sensation of nausea under the sense of taste.

The primary forms of taste are combined, in the greatest variety, with an indefinite number of shades under each of them. The hypothesis of four or more specifically different forms of the endapparatus corresponding to the primary forms of sensation-for example, "bitter-tasting" nerve-fibres, "sweet-tasting" nervefibres, etc.-offers, under the law of the specific energy of the nerves, an opportunity for explaining all the phenomena of this sense somewhat similar to that embraced by the so-called YoungHelmholtz theory of color-sensations.

\& 15. Concerning that in tastable substances which fits them to excite the end-apparatus of the gustatory nerves, or concerning the molecular action of such substances, we have no information whatever. No scale of stimuli, considered as differing in the rapidity of their vibration and corresponding to a scale of resulting sensations differing in pitch or tone, can be made out for sensations of taste. The great difficulties which accompany experiments upon this sense, and the fact that the most fundamental questions concerning its activities are still unanswered, place it in an unsatisfactory position only less hopeless than that occupied by the kindred sense of 'Physiolog. Psychologie, i., p. 382. 
smell. We have in the case of taste, however, the very great advantage of being able, at least loosely, to classify the sensations whose quality we are considering.

\$16. On passing to the consideration of sensations of sound much more help is received from the science of physics. But modern investigations, in the form in which they concern us, do not go back of the great work of Helmholtz, ${ }^{1}$ who made the entire field peculiarly his own. Since the first appearance of this work, the subject has also been greatly enriched by the original researches of Oettingen, ${ }^{2}$ Mach, ${ }^{3}$ Preyer, ${ }^{4}$ Hensen, ${ }^{5}$ Stumpf, ${ }^{6}$ and others. In speaking of the stimuli of these sensations, we are still compelled to refer chiefly to the vibrations of air, which are only remote excitants of the end-organs of this sense. Neither physics nor physiology has yet been able to fix the precise locality in the organism (the nervous structure of the cochlea) where the immediate stimulation of the end-apparatus takes place; or to tell what is the exact nature of its action. We are obliged, then, to confine ourselves in the main to considering a relation between the vibratory energy of the air and certain states of consciousness, without attempting to explain the nany intermediate links.

All sensations which arise in the mind by means of the irritation of the auditory nerve are called sensations of sound. The word "sound" is thus used by psychology for a wholly subjective affair, which has no more resemblance to those vibrations which physics designates by the same word than has the taste sweet to the unknown physical properties that produce it. The trained mind, or "trained ear," as we say, has indeed the power directly to analyze a compound musical sound into its constituent elements. But each of these elements is purely a sensation, a subjective affair. It carries in itself no token that it has been produced by vibrations of any kind; or that it sustains any numerical relation whatever to the vibrations of which some other sensation of sound is composed. We know nothing directly, through sensations, either of the struct-

${ }^{1}$ Die Lehre von d. Tonempfindungen als physiolog. Grundlage f. d. Theorie d. Musik, Braunschweig, 1st edition, 1862 ; 2d edition, 1865; 3d edition, $1870 ; 4$ th edition, 1878 .

${ }^{2}$ Harmoniesystem in dualer Entwicklung, 1866.

${ }^{3}$ Various contributions in the Archiv f. Ohrenheilknnde and elsewhere (especially the Sitzgsber. d. Wiener Acad.).

4 Ueber d. Grenzen d. Tonwahrnehmung, 1876; Sitzgsber. d. Jen. Gesellsch. f. Med., 1878 ; Akustische Untersuchungen, 1879.

${ }^{5}$ In Hermanu's Handb. d. Physiol., III., ii., pp. 3-142, and works by the same author there referred to.

- Tonpsychologie, Leipzig, 1883 (Vol I. only). 
ure of the ear or of vibrating strings and particles of air, or of the wathematics and physics of music.

Sounds are of two classes-tones, or musical sounds, and noises. The former are due to periodic motions of sonorous bodies; the latter to non-periodic. Noises are those sounds which, objectively considered, are wanting in the periodic regularity of stimulation which characterizes all musical sounds, and, subjectively considered, in the peculiar, pleasant modification of consciousness which the latter produce. But noises accompany almost all tones; and, conversely, tones may be detected by the trained ear as mingled with the noises of every-day life. No player of the riolin avoids all noise of scraping from the bow; no stroke of a workman's hammer, or slamming of a door, that does not start and catch up into itself some trace of musical tone. The interest of science has hitherto been almost wholly concentrated upon musical sounds, and little has been done by either physies or physiology toward the analysis of noises. It is characteristic of a noise, according to Helmholtz, ${ }^{2}$ that there is a quick and irregular alternation of different kinds of sensation of sound. This distinctive character can generally be detected "by attentive aural observation without artificial assistance." We can compound noises out of musical tones ; as, for example, by striking together all the keys of an octare on the piano. Hensen" distinguishes three "eategories of unmixed noises"-the "beats" or pulsations which disturb the purity of musical tones; the crackle, crack, or crash ; and hissing sounds. These three shade into each other, and, when mixed with different kinds and quantities of musical sounds, make up the noises which we hear on every hand.

$\S 17$. Musical sounds differ, not only in quality, but also in quantity or intensity of sensation as dependent upon the amplitude of the vibrations which produce them. With respect to their quality they are distinguished as either simple or complex, according as they result from one set of regularly recurrent (periodic) vibrations of a given number in a given unit of time, or result from a combination of two or more sets of such vibrations. The musical sounds of ordinary experience are complex. The blending of the simple tones into the complex tone is not so complete, however, that it cannot be at least partially analyzed directly by a trained ear. The complex sound, which results from this compounding of the contrasts or coincidences of several simple musical sounds, may be called by the term "clang"-in this meaning borrowed from the usage of the German. The quality of tones considered as

1 The Sensations of Tone, etc., p. $11 \mathrm{f}$. London, 1875.

${ }^{2}$ Hermann's Handb. d. Physiol., III., ii., p. 17. 
simple sensations is their pitch, which varies according to a scale of states of consciousness that are immediately apprehended and compared with each other, and that are discovered by objective methods to correspond to a scale of changes in the number of the vibrations of the waves which occasion them. The pitch of tones is therefore spoken of as "high" or "low," according to the place which we assign to the resulting sensations in this scale. Such place in the scale may be considered either with respect to the relation of any particular tone to the upper or lower limits of the scale, or with respect to the relation of the different tones to one another. "Clangs," or complex tones-the musical sounds with which we are made acquainted by all ordinary experience-have also a variable quality called timbre, or "color-tone ;" the timbre of the clang is dependent upon the pitch, number, and relative intensity of the simple tones which compose it. Thus a note having the same place in the musical scale (for example, $a$ of the oncemarked octave-440 vibrations) sounds differently, as we say, on the piano, violin, cornet, or when sung by the human voice. The pitch of the tone as produced by all these different methods is the same; but its color-tone is determined by the character of the over-tones which are blended with the fundamental tone.

$\S 18$. The pitch of tones depends upon the rapidity of the periodic vibrations (the number in a given unit of time-usually one second) which occasion then, or-what is the same thing-upon the length of the sound-waves: This class of sensations, however, has both an upper and a lower limit; that is to say, vibrations either below or above a certain number per second, or-what is the same thing-wave-lengths that are either shorter or longer than a given limit, produce no sensations of musical sound. The difficulty of determining these limits is great, because the intensity of extremely low or high tones has to be enormously increased in order that they may be heard at all; because the perceptions of the acoustic sense are so very blunt near the limits that the different seusations are almost certain to be confused; because distracting sensations of common feeling mingle in these ranges of tone with the sensations of sound, and because near the lower limits the over-tones-especially the octave above-become so strong as to be mistaken for the fundamental tones. On account of these difficulties the older investigators made numerous mistakes. Individual peculiarities are also very important in determining sensations of pitch. Some persons can hear tones below or above those audible to most others. Helmholtz ' thought that sensations of

1 The Sensations of Tone, p. 268. London, 1875. 
tone begin to cease when the vibrations fall below 34 per second; some tuning-forks of great size, which vibrated only 28 times per second, seemed to him, however, to have a trace of tone in the form of a "weak drone." Preyer ' found that while 14 vibrations produced no tone that he could hear, at 16 vibrations he was able to hear a tone; others could distinguish a musical sound only at 19 or 23 vibrations. The same observer experienced as a sensation of musical sound more than 40,000 vibrations per second ; Turnbull found that the majority of those with whom he experimented could not hear more than about 20,000 to 22,500 vibrations per second, and only one-a musician-heard 30,000 ; Despretz succeeded in producing with tuning-forks audible tones that had 32,000 vibrations. Blake thinks that persons with defective ear-drums are able to hear tones of higher pitch, reaching even 50,000 vibrations. Vibrations slower than 28 to 30 per second produce in most ears only a buzzing or groaning sound ; the more acute tones are unpleasant, or even painful, and finally inaudible to all ears. These results cannot be considered as very concordant or precise. They show, however, that the range of the average human ear is rather more than nine octaves, reaching from about $A_{2}$ of the subcontra octave ( $27 \frac{1}{2}$ vibrations per second) to above $c^{7}$ of the seven-times-marked octave (16,896 vibrations per second).

The following table ${ }^{2}$ gives the pitch of all the musical tones audible to the human ear, in the key of $\mathrm{C}$ major, on a scale in which $a^{1}$ is fixed at 440 vibrations. Only about seven of the rather more than eleven octaves of the table are, however, usable in music; these seven reach upward from $C_{1}$ of the contra, or from $A_{2}$ of the subcontra octave, to $b^{4}$-namely, the seven or seven and a half octaves of the modern piano.

\begin{tabular}{|c|c|c|c|c|c|c|c|c|}
\hline & $\mathrm{C}$ & D & $\mathbf{E}$ & $\mathbf{F}$ & G & $\mathbf{A}$ & B & \\
\hline 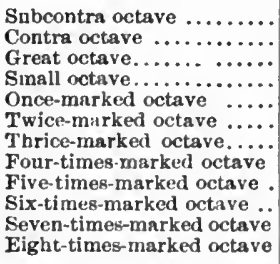 & $\begin{array}{r}16 \% \\
33 \\
66 \\
132 \\
132 \\
264 \\
528 \\
1,056 \\
2,112 \\
4.224 \\
8.448 \\
16,896 \\
33,792\end{array}$ & $\begin{array}{r}18 \% / 18 \\
371 \% \\
74 \% \\
148 \% \\
297 \\
594 \\
1,188 \\
2,376 \\
4,752 \\
9,504 \\
19,008 \\
38,016\end{array}$ & \begin{tabular}{|r|}
$20 \% / 6$ \\
$41 \%$ \\
$82 \%$ \\
165 \\
330 \\
660 \\
1,320 \\
2,640 \\
5,280 \\
10,560 \\
21,120 \\
42,240
\end{tabular} & $\begin{array}{r}22 \\
44 \\
88 \\
176 \\
352 \\
704 \\
1,408 \\
2,816 \\
5,1332 \\
11,264 \\
22,523\end{array}$ & $\begin{array}{r}243 / 4 \\
49 \% 6 \\
99 \\
198 \\
39 \% \\
792 \\
1,584 \\
3,118 \\
6,336 \\
12,672 \\
25,344\end{array}$ & $\begin{array}{r}271 \\
55 \\
510 \\
110 \\
220 \\
440 \\
880 \\
1,760 \\
3,520 \\
7,040 \\
14,080 \\
28,160\end{array}$ & $\begin{array}{r}3015 / 18 \\
6118 \\
1233 \\
24 \% \\
24 \% \\
495 \\
990 \\
1,900 \\
3,9400 \\
7.920 \\
15,840 \\
31,680\end{array}$ & 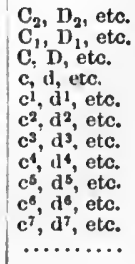 \\
\hline
\end{tabular}

${ }^{1}$ Grenzen d. Tonwahrnehmung, p. $23 \mathrm{f}$.

? Taken from Stumpf, Tonpsychologie, I., p. xiv., and giving the German scale ; the French fixes $a^{1}$ at 435 vibrations; the theoretical pitch in England gives 512 for $c^{2}$. 
§ 19. The sensitiveness of the ear to differences of pitch varies greatly with different individuals, and for the different octaves of the musical scale. Preyer found that unpractised persons, within the octaves from $c$ to $c^{3}$ (132-1,056 vibrations by the table, but 128-1,024 by the scale adopted for his experiments), distinguish a difference of from 8 to 16 vibrations as producing a distinct difference in the sensation of pitch. Extreme cases of deafness to differences in pitch are recorded; as, for example, that of the man ${ }^{2}$ who, in the middle part of the scale, could not distinguish an interval of less than a third, and, in the higher and lower parts, of less than a seventh. ${ }^{2}$ Persons insensitive to differences of a tone or half-tone, who are sometimes said "not to know one note from another," are by no means infrequently met with. Differences of the two ears of the same person, in the fineness of this kind of perception, are common enough ; in certain cases the difference may amount to a half-tone or more. Sensitiveness to pitch is generally capable of rapid cultivation, and may reach a high degree of perfection in persons who have what is called "a good natural ear" for musical tones, if the ear be also highly trained. Such persons may become able to discriminate differences in the sensations caused by changing the number of vibrations not more than a third of a single vibration per second, in the region of the scale between $a^{1}$ and $c^{2}$. In the octave from $b^{1}$ to $b^{2}$ more than 200 tones are distinguishable. But above and below this region the distinctions possible are less fine ; above $c^{5}$ even well-trained ears commit errors in identifying two notes that differ by 100 or even by 1,000 vibrations. It appears, then, that not only the musical quality of tones, but also the power of distinguishing differences in them, diminishes rapidly as we approach the upper and lower limits of the scale.

The fineness of the possible distinctions of purity of interval also differs for different individuals and for different intervals. The following table is compiled by Hensen s from data drawn from Preyer's investigations. The bracketed numbers of the first column indicate the proportion in which the vibrations of the different intervals stand to those of the fundamental tone; the quotient $n^{\prime}: n=i$, the variation from the pure interval which was found detectable in each case ; $V=$ the number of vibrations off from the pure interval which is the least distinguishable; and $S$ is the de-

\footnotetext{
${ }^{1}$ Reported by Grant Allen, in Mind, 1878 , p. $157 \mathrm{f}$.

${ }^{2}$ Comp. the lengthy and interesting discussion on "Individualität des Sinnes und Gedächtuisses für Tonqualitäten," in Stumpf, Tonpsychologie, I., pp. $262 \mathrm{ff}$.

${ }^{3}$ See Hermann's Handb. d. Physiol., III., ii., p. 114.
} 
nominator of the fraction which indicates the sensitiveness of the ear to the purity of each interval.

\begin{tabular}{|c|c|c|c|c|c|}
\hline INTERVAL. & $n$. & $n^{\prime}$. & $i$. & $V$. & $S$. \\
\hline Fourth (1.333). . & 187.58 & 251.23 & 1.3396 & 1.02 & 211 \\
\hline Fifth (1.5)....... & 167.68 & 251.23 & 1.4983 & 0.23 & 822 \\
\hline Minor Sixth (1.6)...... & 143.66 & 231.41 & 1.6108 & 1.19 & 148 \\
\hline Maior Third a & 139.60 & 163.68 & 1.2437 & 0.73 & 198 \\
\hline Major Third (1. & 139.62 & 175.53 & 1.2572 & 0.89 & 193 \\
\hline Minor Third (1.20). & 207.54 & 251.23 & 1.2102 & 1.90 & 117 \\
\hline Octave $(2.0)$. & 500.40 & 1,001 & 2.0004 & 0.13 & 5,000 \\
\hline Whole Tone (1.125).. & 215.15 & 243.51 & 1.1291 & 0.85 & 274 \\
\hline
\end{tabular}

Immediate judgment of absolute tone (as the $a^{1}$ carried in mind by musicians) is possible; judgment between two tones as to which is ligher or lower in pitch is also immediate, and may be exercised independently of everything except the two sensations themselves. The latter judgment is the common power of mind belonging to this sense ; the former is, as a rule, exercised only by skilled persons, and by them only very imperfectly. Experiments of Stumpf,' upon himself and three other musicians, showed that the mistakes in judgment of absolute tone amounted, in the lower region of the scale (from $C_{1}$ to $B_{1}$ ), to $15 \%-100 \%$ of the trials; in the midale region (from $a-g^{1}$, or from $g-e^{2}$ ), to $0 \%-70 \%$; in the upper region (from $g^{3}-f^{4}$, or from $f^{3}-a^{4}$ ), to $7 \%-80 \%$. Only one of the four persons experimented upon seemed to approach the point of infallibility. Judgment of absolute tone is, therefore, a different matter from that which makes distinctions in intervals or in the least observable differences of pitch, and is much more precarious.

\$20. Those psychologists appear to be in the right who claim that some power of the mind immediately to judge differences of quality in pitch, purely as such, must be assumed in order to account for the foregoing phenomena. ${ }^{2}$ Such judgment, however, may be, and ordinarily is, much assisted by auxiliary discriminations of other sensations which blend with those of musical tone. Among such secondary helps the most important are the muscular sensations which accompany the innervation of the larynx and other organs used in producing musical tones. For we ordinarily inner-

1 Tonpsychologie, I., pp. $305 \mathrm{ff}$.

2 On this subject, comp. Lotze, Medicin. Psychologie, pp. 265 ff., 480 f. ; Stricker, Studien über d. Association d. Vorstellungen, 1883, p. 2 f.; G. E. Müller, Zur Grundlegung d. Psycho-physik, Berlin, 1878, pp. $276 \mathrm{ff}$; and stumpf, Tonpsychologie, I., pp. 134 ff. 
vate these organs (at least in an inchoate and partial way) - that is, we sound the note to ourselres-when trying carefully to judge of its pitch. But the niceness of these muscular sensations is not great enough, even when most highly trained, to account for the discriminations of the "good ear." The trained musician can detect by ear a difference in quality between two tones of 400 and $400 \frac{1}{3}$ vibrations per second; but the most skilful singer-Jenny Lind, for example-scarcely succeeds in singing in quarter-tones. Moreover, the relative powers of larynx and ear by no means keep pace with each other in the same person. It should also be remembered that all our ordinary discriminations of musical sound apply to composite tones, or "clangs ;" in discriminating these we are aided by the color-tone, or tone-feeling, which belongs to each note as sounded by some sonorous body with whose peculiarities we are previously more or less acquainted.

It follows, then, that the judgment is supplied, by the varying qualities of musical tones, with the means for arranging them in a continuous series which may be symbolized by different positions assigned along an uninterrupted straight line. Of any three unlike tones, one must be, and only one can be, arranged as respects pitch between the other two. And whenever any two tones, as $m$ and $n$, are giren, another sliding tone, which begins with $m$ and ends with $n$, is possible. Moreover, within the bounds of our experience of tones, as we advance along the scale toward either the upper or the lower limit, we see no tendency in the qualities of the sensations to approach each other. In this respect the scale of sound-tones is wholly different from that of color-tones. There are not two ways, for example, of getting from $a^{1}$ to $c^{3}$ (one through $b^{1}, c^{2}$, etc., and the other through $g^{1}, f^{1}$, etc., around to $e^{3}, d,{ }^{3}$ and then $\left.c^{3}\right)$, as there are two ways of going from yellow to blue (i.e., through green and blue-green, or through violet, red, and orange). We speak, then, of the series of tones as a constant and infinite series; although, of course, no series of states of consciousness is really infinite, and although the upper and lower limits of the musical scale, as well as the limits of the least observable differences between two tones, are not constant but variable for different individuals.

The symbolism taken from relations of space, which we employ when we speak of certain acoustic sensations as "high" and of others as "low" in pitch, or when we distinguish so-called "intervals" between the tones as large and small, is strictly applicable only to the complex tactual, visual, and muscular sensations that accompany the acoustic. In sounding the lower tones with the 
voice the organs are depressed; in sounding the higher, they are elevated. Low notes hare a certain breadth and gravity which corresponds to the foundations of a spatial structure; as sensations they require more time to come into and depart from consciousness, as it were. A great intensity and slower tempo belong to the bass-riol than to the violin. We read up for the notes of highest pitch, and down for those of lowest pitch, in the written musical scale.

$\S 21$. We have seen that tones, like rays of light, come to us as compounded into "clangs;" these really composite tones being esteemed as single notes in ordinary experience. The nature of such composition determines the so-called "timbre," or "colortone," of the notes. Each sensation of a clang is a summing-up in consciousness of several absolute qualities of musical sound the stimulus which occasions this complex subjective state is a complex sound-wave made up of the contrasts and coincidences of several single waves that have the character of simple pendulum ribrations. The quality of each clang depends upon the form of this complex sound-wave. We need not consider in detail the physics and mathematics of such complex waves. It is enough to observe that those single tones whose vibrations stand in simple mathematical relations to each other, when combined into a clang, cause a peculiarly pleasant sensation; those whose vibrations stand in complex mathematical relations make, when combined, an unpleasant sensation. In an octave of the musical scale the eight different notes stand in the following ratios to each other. ${ }^{2}$

$$
\begin{aligned}
& \mathrm{C}: \mathrm{D}: \mathrm{E}: \mathrm{F}: \mathrm{G}: \mathrm{A}: \mathrm{B}: \mathrm{C}^{1} \\
& 1: \frac{9}{8}: \frac{5}{4}: \frac{4}{3}: \frac{3}{2}: \frac{5}{3}: \frac{15}{8}: 2 \\
& 8: 9: 10: 10 \frac{2}{3}: 12: 13 \frac{1}{3}: 15: 16
\end{aligned}
$$

That is to say, while the tone $C$ makes one vibration, $D$ makes nine-eighths, and $E$ makes five-fourths, etc. ; or while $C$ malies 8 vibrations $D$ makes $9, E$ makes 10 , etc. Of these relations in the number of vibrations the simplest is, of course, that of the octave, $1: 2$. The acoustic wares which constitute the stimuli of each complex sensation called a "clang," accordingly, also permit of being regarded as the summing-up of the waves of a fundamental tone and of certain partial tones belonging to the fundamental tone. These partial tones, or "over-tones," are called "the harmonics" of the "clang," or single compound tone.

$\S 22$. When two or more "clangs" are sounded together, the result is what is called either a "chord " or a "discord." The former

'For the mathematics and physics of tones, see Hensen, in Hermann's Handb. d. Physiol., III., ii., pp. 4 ff. 
is a pleasant, the latter an unpleasant, complex of sensations ; consonance and dissonance are thus spoken of as qualities of sensations of musical sound. Thus, if $c$ and $c^{1}$ are struck together upon a well-tuned piano, the combination of clangs is a chord, or harmonious musical 'sound; but if $c$ and $d$, or $c$ and $c \operatorname{sharp,~or~} c$ and its seventh, $b$ above, are simultaneously sounded, then the combination of tones is unpleasant. Cases of consonance and dissonance differ from those just considered under the term "clang" only with respect to the relative strength of the partial tones as compared with the fundamental tones: in the clang the over-tones are weak as compared with the one fundamental tone; but in the chord or discord the fundamental tones of the other clangs are, of course, strong, and stand in powerful relations of consonance or dissonance both toward the fundamental tone of the lowest clang and toward its partial tones. All the partial tones of the different combined clangs enter into the formation of the total result produced. According to the table already giren (p. 322), the Octare is the most perfect possible consonance $(1: 2)$; then the Twelfth $(1: 3)$, the Fifth $(2: 3)$, the Fourth $(3: 4)$, the Sixth $(3: 5)$, the major Third $(4: 5)$, the minor Third $(5: 6)$. With the relation of the Third we come upon the borders of dissonance ; indeed, the ancient Greeks and Romans considered the Third a dissonance, and aroided it in singing, because, as Helmholtz supposes, their ears were more sensitive to "beats" than ours. The consonance of the Sixth and that of the Fourth hare also been much disputed. The major Sixth and major Third are called by Helmholtz "medial consonances ;" the minor Third and minor Sixth, "imperfect consonances."

An analysis of the harmonics of these consonances yields the following results, ${ }^{1}$ which show the amount of coincidence belonging to the acoustic waves of the different tones when combined in a chord with a fundamental tone.

$$
\begin{aligned}
& \text { Octave }\left\{\frac{c^{1} g^{1} c^{2} e^{2} g^{2} b^{32} c^{3}}{c^{1} \mid c^{2} g^{2} c^{2}}\right.
\end{aligned}
$$

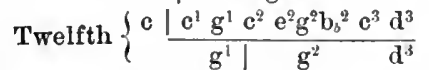

$$
\begin{aligned}
& \text { Fifth }\left\{\begin{array}{l|l}
c & \frac{c^{1} g^{1} c^{2} e^{2} g^{2}}{g} \\
\hline g^{1} d^{2} g^{1}
\end{array}\right.
\end{aligned}
$$

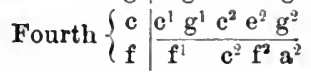

$$
\begin{aligned}
& \text { Major Third }\left\{\begin{array}{l|l}
c & e^{1} g^{1} c^{2} e^{2} \\
e & \frac{e^{1}}{b^{1} e^{2}}
\end{array}\right.
\end{aligned}
$$

The major Sixth is similar in the form of its harmonics to the major Third.

${ }^{1}$ Comp. Helmholtz, The Sensations of Tone, p. $281 \mathrm{f}$. 
Two psycho-physical causes for the characteristic feelings which belong to sensations of consonance and dissonance, respectively, may be assigned with more or less of probability. The first is that proposed by Helmholtz. ${ }^{1}$ The feeling of dissonance which is produced by sounding together two notes that differ only by a semitone is found to be increased when the difference in the pitch of the notes is still further diminished. Successive shocks called " beats" occur, less frequently but more decidedly and unpleasantly, as the pitch of the notes becomes more nearly the same. The feeling of dissonance is found to reach its height when the number of beats is about 30 per second. For example, if $b^{1}$ (495 vibrations) and $c^{2}$ (528 vibrations) are struck together, the number of beats is $33(528-495=33)$, and the dissonance is very strongly marked. In all marked dissonances such beats occur at the rate of from 20 to 40 in a second. The unpleasant effect in consciousness is analogous to that produced by all sudden and rapid intermission of stimulation; as, for example, the flickering of light or the scraping of uneven surfaces over the skin. The feeling of consonance is due to the absence of beats. In addition to Helmholtz's negative reason, Oettingen has proposed the positive one, that the pleasantness of harmony is due to what he calls the "tonicity" and "phonicity" of certain intervals and combined notes. "Tonicity" is the property of being recognized as a constituent of a single fundamental tone which is designated by the name "tonic." "Phonicity" is that property of a chord or interval which consists in the possession of certain partial tones that are common to all tones. The first of these qualities of harmony seems to ally the pleasure it yields to that which follows even the obscure and only half-conscious perception, as it were, of all relations, as such, between our sensations.

$\S 23$. In order that the physical apparatus of hearing may act as the organ of those wonderfully fine discriminations which belong to the most analytic of all the senses, it would seem that it must possess an outfit of end-organs with structure sufficiently minute to serve as a basis for a satisfactory development of "local signs." The number of the cells of Corti, and of their separate terminal auditory nerves, has been calculated by Hensen ${ }^{2}$ at about 16,400; by Waldeyer ${ }^{3}$ at 20,000 . It is doubtful, however, whether even this large number will suffice to account for that niceness of auditory discriminations which we have seen to be possible.

\footnotetext{
${ }^{1}$ The Sensations of Tone, p. $255 \mathrm{f}$.

z In Hermann's Handb. d. Physiol., III., ii., p. 115.

${ }^{3}$ Stricker's Gewebelehre, II., p. 954.
} 


\section{CHAPTER IV.}

\section{THE QUALITY OF SENSATIONS. [CONTINUED.]}

\&1. The analysis of the qualities of different Sensations of Sight is much more intricate than that of any of the other senses. They may all be described as sensations of color and light; but an indefinite number of colors is known to experience, and as many grades of the sensation of light. Moreover, the quantity of the white light which acts as stimulus upon the eye has an important effect upon the quality of the resulting color-sensation; in other words, the tone of the color is dependent upon the amount of white light which is mixed with the "saturated" spectral color. The size of the colored object and the resulting breadth of the sensation, as well as the intensity of the stimulus and the time during which it acts, also affect the quality of the sensation. Still further, the same stimulus produces different sensations as it falls upon different portions of a normal retina; while a considerable class of persons are color-blind, or incapable of certain kinds of color-sensations. The previous condition of the retina, and the relations between the contiguous portions when any considerable area of it is under stimulation, must also be taken into account. The fundamental laws governing sensations of sight can, therefore, be discovered only by excluding for the time many of those variable elements which, in fact, always enter into the determination of the exact quality of such sensations. Thus defining the first problem before us, we find that it may be stated in the following terms. What sensations result from the stimulation of a sufficiently small, but not too small, area of the most central part of a normal retina, for a given time, when it is not fatigued and the eye is at rest, and with neither too great nor too small intensity of a given kind of light? Such sensations may be called (though somewhat ineptly) normal sensations of color. When the foregoing question is answered we may go on to consider the most important variations possible on account of various forms of departure from the so-called normal conditions of sensation.

$\S 2$. The ordinary stimulus, the application of which to the eye gires rise to the sensations of sight, is light-or certain exceedingly 
rapid oscillations of luminiferous ether. Some forms of mechanical and electrical stimuli also produce the same sensations. Any violent shock to the eye, such as a blow upon the back of the head, may fill the whole field of vision with an intense light. The action of mechanical pressure of moderate intensity upon a limited part of the retinal elements may be studied by rolling the eyeball inward and using the fingernail, or a small, blunted stick, upon the outer surface of the closed lids. By such stimulation disks of light (called phosphenes), with darkly colored edges, are produced in the field of vision of the closed eye. Some observers have claimed that very strenuous exertion of the apparatus for accommodation occasioned in their eyes similar phenomena ("phosphenes of accommodation"). On making or breaking a weak electrical current sent through the eye, the entire field of vision is lighted up; the constant current also seems to excite the optic nerve. The quality of the sensations thus excited is found to depend upon the direction of the current through the nerve. When the current is ascending, the place where the nerve enters the retina appears as a dark disk upon a field of vision that is brighter than it, and of pale violet-color; when it is descending, as a bright bluish disk on a field of dark or reddish-yellow color. The retina has also a "light of its own" (Eigenlicht); for its nervous elements are rarely or never inactive, but have a continuous tonic excitation. Hence the most gorgeous and varied coloring is often seen when the eyes are closed in a darkened room. This normal light of the retina is not constant either in degree or in quality; both the form and the color of the different minute parts of the field of vision, as lighted by it, are very changeable. It may be said to have the rhythmic movernent of all tonic excitation. Such excitation is supposed to be due to chemical effects, wrought by the changing supply of blood, upon the nervous elements of the retina aud (perhaps, also) of the central organs of the brain. The peculiar action of the ascending and descending electrical current has been thought by some ${ }^{2}$ to be due to its catelectrotonic or anelectrotonic effect upon the central organs by way of the optic nerve. Aubert has estimated the retina's own light to be about equal (in his case) to half the brightness of a sheet of white paper when seen in the full light of the planet Venus.

$\$ 3$. The place where the light acts (and here, as is supposed, only indirectly through photo-chemical-and perhaps electro-motivechanges in the pigments of the eye) upon the end-organs of vision 230.

'See Fick, Physiolog. Optik, in Hermann's Haudb. d. Physiol., III., i., p. 
must be located at the back of the retina in the rods and cones (see Part I., Chap. V., §§ 18-22). The argument by which we have connected the analytic power of vision with the structure of this nervous layer may be carried yet further into details. It appears likely that each element of the structure-at least in some parts of the retina-should be regarded as an isolated sensitive spot, which corresponds on the one side to definite excitations from the appropriate stimuli, and on the other side to the smallest localized sensations of color and light. In order that two visual sensations may be seen as separate, yet side by side, in an object, two neighboring retinal elements must be excited by the stimulus. This implies that the breadth of retinal surface stimulated must be, at least, about that of the distance between two such elements. With this hypothesis the facts of histology and experimental physiology agree fairly well.

The degree of accuracy which sight can attain is dependent upon the size of the retinal elements directly affected by the light. ${ }^{\prime}$ Hooke observed that no one can distinguish two stars as two, unless they are apart at least $30^{\prime \prime}$; few, indeed, can distinguish them when less distant from each other than 60". E. H. Weber could not perceive as separate two lines whose distance did not cover at least $73^{\prime \prime}$ of the angle of vision ; Helmholtz puts the limit of his sharpness of vision at $64^{\prime \prime}$. The numbers $60^{\prime \prime}$, $64^{\prime \prime}$, and $73^{\prime \prime}$, in the angle of vision, correspond to a size of the retinal elements rarying from $0.00438 \mathrm{~mm}$. to $0.00526 \mathrm{~mm}$.; and this agrees very closely with the calculated breadth (by Kölliker) of the thickness of the cones in the yellow-spot-namely, 0.0045 $\mathrm{mm}$. to $0.0055 \mathrm{~mm}$. (0.000177 in. to $0.0002165 \mathrm{in}$.). If white lines be drawn on a dark ground so closely together as to approximate this limit of vision, they will appear, not straight, but knotted and nicked. This fact is due to the action of the stimulus on the mo-

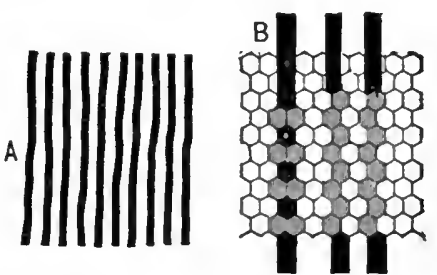

Fra. 90.-A shows the appearnnce of lines drawn very closely together, which is supposed to be due to their falling upon the nervous elements of the retina in the manner shown by $\mathbf{B}$.

saic of rods and cones, as seen by the accompanying figure (No. 90). The diminishing sharpness of vision as we move away on the surface of the retina from its most central area corresponds to the

1 See Helmholtz, Handb. d. Physiolog. Optik, Leipzig. 1867, p. 215 ff.; Fick, in Hermann's Handb. d. Physiol., III., i., p. 152 f.; von Kries, Archiv f. Anat. u. Physiol., Physiolog. Abth., 1882 (Appendix), p. 24 f. 
comparative paucity of the nervous elements which enter into the structure of the peripheral parts.

$\S 4$. Excluding consideration of those changes in the quantity, as such, of visual sensations which are produced by changes in intensity of the light, and confining our attention to what has already been defined as the normal action of the eye (comp. p. 325), we treat scientifically all the different sensations of sight when we describe (1) the ware-lengths of the different kinds of colored light, or pure color-tones, and (2) the relations in which the different colors stand with respect to the amounts of white (or colorless light) and saturated light (or light of pure color-tone) which enter into them. The foregoing distinctions in the quality of our color-sensations may be confirmed by an appeal to experience. Red is unlike yellow in "color-tone," and both are unlike blue ; but orange is more like either red or yellow than it is like blue, while violet is more like blue than it is like either yellow or red. Yet we distinguish colors of the same class (red, green, or violet) as being like or unlike with respect to their "brightness ;" and in respect of brightness, a certain shade of red may differ more from another shade of red than it differs from some shade of yellow, green, or blue. The brightness of a color is, scientifically speaking, dependent both upon the degree of saturation which the color possesses and upon the total intensity of the light.

§5. A color-tone is said to be "pure" or " saturated" when it is free from all admixture of other color-tones. Pure or saturated color-tones can be obtained only by use of the spectrum, which, on account of the different refrangibility of the different colored rays that compose it, analyzes the compound ray of white light into its constituent color-tones. By stimulating with different simple rays those nervous elements which have the same local situation at, or very near, the pole of the eye, we test the question whether each special color-sensation corresponds to a special physical construction of the stimulus. It is thus discovered that the compound ray of sunlight, so far as it stimulates the human eye, is made up of components formed by oscillations varying all the way between about three hundred and seventy billions and about nine hundred billions per second ; and that the color-tone of the sensation changes as the number of these oscillations changes. The following table ' exhibits these facts on the scale of Fraunhofer's lines, which mark those portions of the spectrum where its principal colors appear most obvious to the normal eye.

${ }^{1}$ Taken from Fick, Physiolog. Optik, in Hermann's Handb. d. Physiolog., III., i., p. 173 . 


\begin{tabular}{|c|c|c|}
\hline Name of the line. & $\begin{array}{l}\text { Number of vibra- } \\
\text { tions per second. }\end{array}$ & Wave-length in the air. \\
\hline 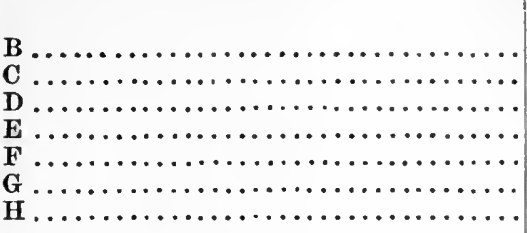 & $\begin{array}{c}\text { Billions. } \\
450 \\
472 \\
526 \\
589 \\
640 \\
722 \\
790\end{array}$ & $\begin{array}{c}\text { Millimeters } \\
0.0006878 \\
0.0006564 \\
0.0005888 \\
0.0005260 \\
0.0004843 \\
0.0004291 \\
0.0003928\end{array}$ \\
\hline
\end{tabular}

Rays of light which have a number of oscillations less than four hundred and seventy billions per second, so far as they affect the retina at all, occasion the sensation of Red; and this sensation does not vary essentially in quality when the oscillations are four bundred and forty to four hundred and sixty billions. But when their number increases beyond four hundred and seventy billions (C) the quality of the sensation changes rapidly, takes on a yellow tone (Orangeyellow), and finally, at about five hundred and twenty-six billions (D), corresponds to what we definitely call Yellow. This yellow becomes greenish as the oscillations increase in number, until they reach about five hundred and eighty-nine billions (E), when Green appears. (Changes from yellow to green occupy only a small zone in the spectrum.) The green in turn becomes bluish; at six hundred and forty billions (F) Blue begins to appear. From this point to seven hundred and twenty-two billions $(\mathrm{F}-\mathrm{G})$ the color-tones that lie between blue and violet are run through; beyond the latter number Violet comes to view.

The color-tones of the spectrum are, therefore, not sharply separated, but pass gradually into each other. The nearer together two colors are situated in the spectrum, the more nearly do they correspond in the quality of their sensations. Nor has the spectrum any sharply defined limit at either end, but passes gradually into black - more gradually at the violet than at the red end. The energy of the ultra-red rays, as measured by their physical and chemical action, is greater than that of the more highly refrangible rays. The fact that these rays do not excite visual sensations must, then, be due to the structure of the retina. The ultra-violet end of the spectrum has been made visible for a certain extent by experiment; ${ }^{\prime}$ it produces the sensation of a glimmer of lavender-gray color. Our inability to perceive these ultra-red and ultra-violet rays is not to be considered an imperfection of the eye, as Tyndall thought. It is rather purposeful, and of the greatest importance for vision; since, if these ultra rays were visible, the clearness of objects would 
be much disturbed by the chromatic aberration of the refracting apparatus of the eye. ${ }^{1}$

$\S 6$. Besides the foregoing distinctions of color-tones, the impression made by the green-yellow of the spectrum (D-E, and immediately about D) is by far the strongest; or, as we should say, this color is naturally the "brightest" of the spectral colors. From the region immediately around $\mathrm{D}$, the brightness of the color-tones diminishes toward both the red and the violet ends of the spectrum - at first quickly, then more slowly, and then more quickly again. Such a relation cannot be due to the spectrum as an objective affair; for if we measure by other physical means the amount of energy belonging to its different regions, we find that of the red rays (which are by no means brightest) to be strongest. We must,

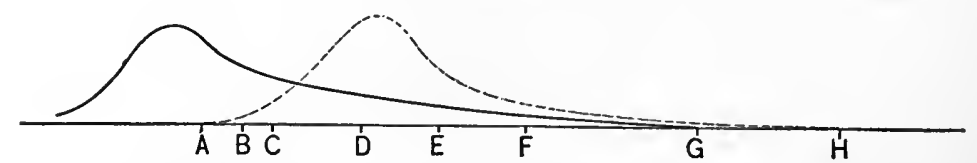

Fig. 91.- (From Fick.) The letters on the horizontal line stand for Fraunhofer's lines. The ordinates of the intermpted curved line show the bightness of rays as seen; the ordinates of the dark curved line, the intensity of the rays as measured by calorific effect.

then, seek an explanation in the structure of the retina, and conclude that it is peculiarly sensitive to stimulations by oscillations of about five hundred and fifty billions per second. The sensitiveness of the retina to slight variations in color-tone, as dependent upon differences in the wave-lengths of the stimulus, is also different at different portions of the spectrum. It is greatest in the green and bluegreen regions ( $\mathrm{D}$ and $\mathrm{F})$.

The following table represents both the foregoing laws. The numbers of the second and third columns show the relative brightness with which the different colors of the spectrum appear to the eye, as calculated by different methods and by two observers. It will be seen that the results agree substantially, though by no means perfectly. In the last two columns the letter sstand for Fraunhofer's lines, and the figures give the fractional variation in the wave-lengths which produces an observable variation in the color-tone for different regions of the spectrum. ${ }^{2}$

' See Fick, Compendinm d. Physiologie, 2d edition, p. 181 f.; and Hermann's Handb. d. Physiol., III., j., p. 181 f.

'See Helmboltz's Physiolog. Optik, p. $317 \mathrm{f}$; von Kries, in Archiv f. Anat. u. Physiol., Physiolog. Abth., 1882(Appendix), pp. 56-r6; Fick, in Hermann's Handb. d. Physiol., III., i., p. 174 f.; Mandelstamm and Dobrowolsky, in Archiv f. Ophthalmologie, XIII., ii., p. 399, and XVIII., i., p. 66. 


\begin{tabular}{|c|c|c|c|c|}
\hline \multirow[b]{2}{*}{ 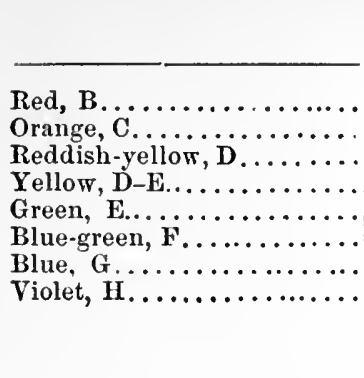 } & \multirow{2}{*}{$\begin{array}{c}\text { Fraunhofer. } \\
32 \\
94 \\
640 \\
1,000 \\
480 \\
170 \\
31 \\
5.6\end{array}$} & \multirow{2}{*}{$\begin{array}{c}\text { Vierordt. } \\
22 \\
128 \\
780 \\
1,000 \\
370 \\
128 \\
8 \\
0.7 \\
\end{array}$} & \multicolumn{2}{|c|}{ Mandelstamen and Dobrowolsky } \\
\hline & & & 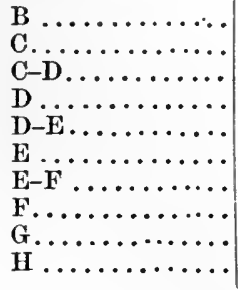 & 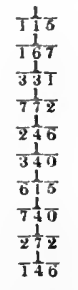 \\
\hline
\end{tabular}

§ 7. The colors of every-day experience, like its musical tones, are not simple and pure color-tones, such as are obtained by spectral analysis; they are composite. Inquiry must therefore be raised as to the effect produced in sensation from the co-working of two homogeneous rays of light upon the same elements of the retina under all the normal conditions to which reference was previously made. In pursuing this inquiry no direct assistance can be obtained from the discriminations of consciousness ; for sensations of color, unlike those of musical clang, cannot be mentally analyzed into their constituent elements. The science of optics makes us acquainted, however, with the following facts: When the wavelengths of the two colors mixed vary but slightly (a few billions of oscillations in a second) from each other, the color resulting from the mixture lies between, and may be recognized as a "shade" of, the colors mixed. By selecting for mixture color-tones that lie apart at all possible distances along the spectrum, an indefinite number of impressions of color may be obtained, which all differ from those obtained by the homogeneous colors. These mixed color-impressions, however, are not all different from each other; so that the number of the qualities of resulting sensations is far less than that of the compound physical processes which stimulate the retina. Their character depends both upon the place of the spectrum from which the simple color-tones are selected for mixture, and also upon the relative intensity of the ones selected. For example, if a ray of four hundred and fifty billions of oscillations per second (red) be mixed with one of seven hundred and ninety billions (violet), a new series of impressions of color (the purples) is attained by varying the intensities of the two. These impressions are more or less like red or like violet, according to the relative amounts of the rays of four hundred and fifty billions and of seven hundred and ninety billions which enter into the mixture. Moreover, there are found to be two ways of advancing by this process of mixing color- 
tones toward any one of the composite colors. Thus, we may pass from yellow to blue either through green-yellow, green, and bluegreen, or through orange, red, purple, and violet. The following table' is of interest in this connection. Where two colors are given as resulting from the mixture, the variation is to be understood as dependent upon the prevailing intensity of one of the two components.

\begin{tabular}{|c|c|c|}
\hline Components. & Tone of the color obtained by mixture. & $\begin{array}{c}\text { Degree of } \\
\text { saturation. }\end{array}$ \\
\hline Red and Yellow ............ & Orange. & Spectrai. \\
\hline Orange and Yellow-green........ & Yellow...... & Spectral. \\
\hline Yellow and Green............. & Yellow-green .. & Whitish. \\
\hline Yellow-green and Blue-green.... & Green ............. & Very whitish. \\
\hline Green and Cyanic Blue........ & Blue-green.............. & Whitish. \\
\hline Blue-green and Indligo.......... & Cyanic Blue......... & Spectral. \\
\hline Cyanic Blue and Violet.......... & Indigo............ & Spectral. \\
\hline Red and Yellow-green ......... & 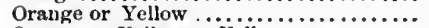 & Spectral. \\
\hline IRed and Green.............. & Orange or Yellow or Yellow-green...... & Whitish. \\
\hline Violet and Blue-green......... & Indigo or Cyanic Blne............... & Spectral. \\
\hline 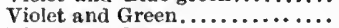 & Indigo or Cyanic Blue or Blue-green.... & Whitish. \\
\hline Violet and Orange............. & 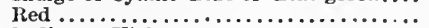 & Whitish. \\
\hline Red and Cyanic Blue.......... & 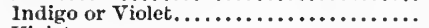 & Whitish. \\
\hline Red and Indigo .............. & Violet $\ldots \ldots \ldots \ldots \ldots \ldots \ldots \ldots$ & Slightly whitish. \\
\hline
\end{tabular}

§8. The number of colors distinguishable by the human eye is not easily stated with accuracy ; like the number of musical tones, it varies with different individuals. The usual number of seven fundamental colors, as fixed by Newton, with the intent of forming an octare in the scale of color-tones, has no sufficient claim to acceptance. Six of the seven-namely, red, orange, yellow, green, blue, violet-are indeed names in common use. But indigo, as an intermediate tone, or kind of semitone, between blue and violet, has perhaps no more real right to recognition than various other intermediate color-tones. Donders ${ }^{2}$ puts the number of color-tones distinguishable in oil-colors at one hundred; von Kries ${ }^{3}$ the recognizable number of spectral tints at about two hundred and thirty. But each of these yields different sensations of color according to the degree of its saturation or purity, due to freedom from admixture of white light. Another series of variations of sensation must be allowed for, which are due to differences in " brightness" or intensity. Introducing these two variable elements, von Kries calculates the number of distinctions of color-sensations, possible for all degrees of purity of tone and intensity of light, at

${ }^{1}$ Made according to investigations by J. J. Müller, and taken from Fick, in Hermann's Handb. d. Physiol., III., i., p. 190.

${ }^{2}$ Archiv f. Ophthalmologie, XXVII.

${ }^{3}$ Archiv f. Anat. u. Physiol., Physiolog. Abth., 1882 (Appendix), p. 58 f. 
about five hundred thousand to six hundred thousand. This number stands midway between the "many millions" of which Aubert speaks and the five thousand allowed by Donders. Herschel thought that the workers on the mosaics of the Vatican must have distinguished at least thirty thousand different colors.

§9. Experiment also shows that if certain color-tones with a given intensity are united on the retina, the result is a sensation unlike that of any other of the colors, whether pure or mixed. This sensation we call "white," and the two colors which by their admixture produce it are called " complementary." Complementary colors may be mixed upon the retina in various ways; either by allowing two spectral rays properly selected to be superimposed at the same spot, or by blending the reflected images of two colored wafers, or by blending the direct visual impressions of colored surfaces on a swiftly revolving top or wheel, etc. But however mixed, the resultant sensation is that of a so-called "white" color in which all trace of the constituent elements is lost. Following is a table of complementary colors : ${ }^{1}$

\begin{tabular}{|c|c|c|c|c|}
\hline Color. & Wave-length. & $\begin{array}{l}\text { Complementary } \\
\text { color. }\end{array}$ & Wave-length. & $\begin{array}{c}\text { Relation of } \\
\text { wave-lengths. }\end{array}$ \\
\hline Red. & 2,425 & Green-blue & 1,818 & 1,334 \\
\hline Orange & 2,244 & Blue.... & 1,809 & 1,240 \\
\hline Gold-yellow ... & 2,162 & Blue......... & 1,793 & 1,206 \\
\hline Gold-yellow... & 2,120 & Blue ......... & 1,781 & 1,190 \\
\hline Yellow ........ & 2,095 & Indigo-blue... & 1,716 & 1,221 \\
\hline Yellow.. & 2,085 & Indigo-blue... & 1,706 & 1,222 \\
\hline Green-yellow.. & 2,082 & Violet........ & $1,600\left\{\begin{array}{l}\text { and } \\
\text { less }\end{array}\right.$ & 1,301 \\
\hline
\end{tabular}

$\S 10$. If the foregoing facts and laws are held to be true of the "normal" connection between light and visual sensations, then various classes of circumstances must be taken account of as "abnormal," which, nevertheless, enter into all our daily experience with this sense. Indeed, the connection between stimulus and sensation is not the same for different individuals who possess substantially the same color-sensations; frequently the complementary colors for two different individuals are not precisely the same. Even the two eyes of the same individual often differ perceptibly in this regard. Important changes in the quality of the sensations, other than those directly ascribable to changes in the wave-lengths of light, take place when the intensity of the light approaches ei-

1 Taken from Helmholtz, Physiolog. Optik, p. 277. The numbers are given in hundred-millionths of a Parisian inch, and may be reduced to millimetres by multiplying by 27.07 . 
ther a maximum or a minimum. At the maximum intensities of the stimulus all sensations of color-tone cease, and even lomogeneous rays appear white. Previous to reaching this maximum, red and green pass over into yellow. At the minimum intensities of light every color-tone except the pure red of spectral saturation appears colorless when seen alone on a perfectly black ground. The different colors appear and disappear, as such, at different degrees of intensity of the stimulus-green, among them all, remaining visible in the weakest light. They all also change their tone as the light which falls on them diminishes; but it is scarcely possible to describe the law of this change, on account of the great difficulty of distinguishing color-tones in very weak light.

$\S 11$. Changes of color also take place when the time of the action of the light is reduced to a minimum. Sensations of saturated color can be produced by instantaneous illumination of the spectrum with the electrical spark. More time is needed, howerer, to produce these sensations with smaller intensities of the light. The different colors, even when of the same brightness, appear to require different amounts of time in order to reach the maximum of their effect-red, 0.0573 ; blue, 0.0913 ; green, 0.133 of a second. ${ }^{1}$ The tone of the color varies with the duration of the impression as well as with the intensity of the light. Very minute objects, too, appear of a different color on account of their size. In general, the larger the surface, the less the intensity of the light necessary to produce the sensation of any particular color-tone; the greater the intensity of the light, the smaller the surface which will suffice for such sensation. Fick ${ }^{2}$ has shown that the color-sensations derived from small distinct points support each other, as it were, in the same way as the contiguous points of a colored surface. For if we make with a fine needle a single hole (of about $0.6 \mathrm{~mm}$. in diameter) in a sheet of paper and look through it at colored paper distant some six and a half metres, the color of the paper cannot be distinguished. But if the number of holes be as many as sixteen, the color can be distinguished at the same distance, even when the holes through which we look are smaller. Subsequent experiment ${ }^{3}$ has shown that the smaller the distance between the single perforations, the greater the distance at which the eye can recognize colors through them. In general, then, two weak sensations, each of which belongs to one eye, may fuse together into one strong one.

${ }^{1}$ According to Kunkel, in Pflüger's Archiv, ix., p. 207.

'Pflüger's Archiv, xvii., p. 152.

${ }^{8}$ See Dobrowolsky, in Pflüger's Archiv, xxxv., p. $536 \mathrm{f}$. 
\$12. Very important changes in the visual sensations occur as dependent on the place of the retina which is stimulated. In this respect a great difference exists between the central and the peripheral parts. The entire field of this organ may be somewhat indefinitely divided into three zones-a central or polar, a middle, and an outer or peripheral. It is probably true that the peripheral parts of the retina produce no sensations which cannot be produced by stimulating the central zone.' But it is equally true that, under the same circumstances, the same stimulus produces a markedly different effect upon sensation when applied to different localities of the retina. Rays which, falling on the polar zone, produce the impression of red, yellow, or green, all make an impression of yellow when they fall on the surrounding zone (a few millimetres from the fovea centralis); and this yellow is so much the paler, the greener the impression on the polar zone. Rays which make on the polar zone the impression of blue or violet make on the outer zone the impression of blue; and this blue is so much the paler, the nearer the impression on the polar zone is to green. It follows, then, that whereas there is at the central zone an indefinite number of color-tones possible, this number is reduced to comparatively few impressions at the middle zone; while all color-tones gradually become indistinguishable and are lost on passing through the onter zone. These great changes in sensitiveness to color are not accompanied by similar changes in sensitiveness to colorless light; it even appears that regions of the retina distant about $30^{\circ}$ from its centre are more sensitive to light than is the polar zone.

A certain proportion of persons (perhaps one-twentieth or more) appear to have a defective structure of the retina, which may be described as corresponding in the polar zone to that of the normal retina in the middle or even the outer zone. Such persons are said to be "color-blind." The farther outward this imperfect condition of the retina extends, the nearer does the defect approach to total color-blindness. ${ }^{2}$ In most cases of this defect there is a partial or complete insensitiveness to the red rays; these rays are especially liable to be confused with the dark-green or the yellow. The spectrum is thus shortened at the red end. Cases of so-called violetblindness, as reported by Donders and Stilling, are much more rare and doubtful. In total color-blindness only shades of gray from

${ }^{1}$ See von Kries, Archiv f. Anat. u. Physiol., Physiolog. Abth., 1882 (Appendix), p. 90 .

${ }^{2}$ See Fick, Zur Theorie d. Farbenblindheit, p. 213 f. ; and in Hermann's Handb. d. Physiol., III., i., p. 206 f. 
white to black are visible. In general, the attempts to make out a spectrum for the color-blind are unsatisfactory, since we can only be sure as to what color-tones appear like or unlike to them; we cannot, on the contrary, be sure that their abnormal sensations are like any of our normal sensations-in other words, that what they see when red light falls on the retina corresponds to any of our colortones. The three or four cases reported where one eye of a person has been normal and the other color-blind are, of course, especially valuable; since they offer an opportunity to compare immediately the sensations of the normal with those of the pathological eye. These cases, according to von Kries ${ }^{1}$ show that the two fundamental colors to which the color-blind are reduced may be considered as either red and blue-green or greenish-yellow and blueviolet.

\$13. Important modifications of the normal action of the eye are also caused by the previous condition of the retina, or by the contemporaneous condition of parts of it contiguous to those on which the light falls. The former fact explains the phenomena of "inertia" and "exbaustion ;" the latter, the phenomena of "contrast." The reaction of the sense of sight is relatively very sluggish; or-in other words-the inertia of the eye is relatively great. This fact is undoubtedly due to the chemical nature of the stimulus which acts directly upon its end-organs. The light requires time in order to effect those photo-chemical changes on whose action upon the nervous elements of the retina our sensations of light and color depend. On the other hand, if we close the eyes after looking intently upon any bright object, the image of this object remains for some time, and only slowly fades out of sight. Such an image is called a "positive after-image," because its bright and dark lines and surfaces correspond to those of the original object. The delay which the sensations undergo, both in forming and in fading away, is said to be due to the inertia of the retinal structure. It is, of course, a law of all nervous excitation and action that it requires a certain amount of time for beginning and for changing its character.

White positive after-images (as Fechner, Helmholtz, and others have shown) pass quickly through greenish-blue to indigoblue and then to violet or rose-color. But "negative after-images" are due to the exhaustion of the retina. If the eye be intently fixed for some time on a small square of black lying upon a sheet of white paper, and then suddenly turned upon the white surface, a bright square appears, moves about with the eye, and slowly 1 Archiv f. Anat. u. Physiol., Physiolog. Abth., 1882 (Appendix), p. 152 f. 
fades away. If we look for a long time at a green surface and then direct the eye upon a white one, the latter appears for a moment to be of a red color. In general, the color of the negative after-image is such that, when combined with the color of the object, the two will produce white. In other words, the color of such an image is "complementary" of the color of the object. Such facts as the foregoing must in some manner be brought under the law which applies to all the elements of the nervous system, but especially to the end-organs and the central organs; these organs become wearied by continuous use, and require time for recovery of their suspended or diminished functions. Precisely how the application is to be made to the case of the retina is, however, a matter of the general physiological theory of vision which cannot as yet be stated with perfect certainty. The phenomena of exhanstion are among the most important for the formation of such a theory. Investigations in this direction have led to the discovery that none even of the spectral colors are perfectly saturated, since each of them can be made to appear more so by looking at it with an eye wearied by the complementary color. ${ }^{1}$ Red is most nearly saturated, blue and yellow next, and green least of all.

\$14. The different parts of the retina are interdependent in the production of sensation; or-to employ the statement of Wundt ${ }^{3}$ - "The sensation which arises throngh the stimulation of any given point of the retina is also a function of the state of other immediately contiguous points." Hence arise, in part at least, the phenomena of contrast, which are of two kinds-contrast of brightness and contrast of color-tone. The fundamental fact in the first class of contrasts is this : every bright object appears brighter with surroundings darker than itself, and darker with surroundings brighter than itself. These phenomena are explained by Helmholtz ${ }^{3}$ as deceptions of judgment, such as we are accustomed to in our estimates of distances. To this explanation, however, Fick," Hering, ${ }^{6}$ and others oppose strong and apparently conclusive objections. They would explain the same phenomena by the modifying influence of the excitation of one part of the retina upon the excitation of contiguous parts. Such influence does not always

${ }^{1}$ Comp. Helmholtz, Physiolog. Optik, p. 279 f. ; Exwer, in Pflüger's Archiv, i., p. 389 ; and see, especially, von Kries, Archiv f. Anat. u. Physiol., Physiolog. Abth., 1882 (Appendix), p. 115.

${ }^{2}$ Physiolog. Psychologie, i., p. 439.

${ }^{3}$ Physiolog. Optik, pp. 388 ff.

4 In Hermann's Handb. d. Physiol., III., i., p. $231 \mathrm{f}$.

${ }^{5}$ Sitzgsber. d. Wiener Acad., June, 1872, and December, 1873. 22 
take the form of depressing the excitability of the contiguous parts; on the contrary, stimulating certain elements for some time may finally involve contiguous ones in a secondary way. This fact they consider to be the true explanation of the spreading of a bright object on a dark background, whose after-image becomes a clear band of light around the dark image of the bright object. When colored instead of white light is used in experimenting under the law of contrast, phenomena similar to those of complementary colors are obtained. ${ }^{2}$ A small square of white on a surface of green, when covered with a transparent sheet of tissue-paper, appears as red on a surrounding surface of a whitish hue; on a red ground it appears as green, on a blue ground as yellow, and vice versa. There is the same dispute over these as over the other plienomena of contrast. Shall they be considered as cases of deception of judgment, or do they admit of a physiological explanation? Mere cases of deception they cannot well be. The theory which ascribes to each part of the retina an influence upon other contiguous parts is the most satisfactory form of a physiological explanation. But such physiological explanation seems to need supplementing by reference to induced conditions of the central organs, concerning the nature of which we are thus far almost entirely ignorant.

$\S 15$. It will readily be seen that a theory which shall satisfactorily account for the complicated phenomena of visual sensations is difficult to establish. Physiological optics will probably never be able to explain in detail the individual sensations of light and color. But each claimant to present such theory must, as Wundt ${ }^{2}$ maintains, account for the following four main classes of facts : (1) The subjective relations of the color-tones, and the fact that they may all be graded downward, as it were, into colorless light; (2) the law of the mixing of all the colors from three (or more) fundamental color-tones; (3) the phenomena of after-images; and (4) the phenomena of contrast. Among all the hypotheses litherto proposed to account for the quality of visual sensations, that brought forward by Young, and elaborated and applied by Helmholtz, is by far the most prominent. This hypothesis takes its point of starting from the undoubted fact that, by admixture of a few so-called fundamental color-tones, we can produce all the other colors, as well as the sensation called "white." There are said to be three such color-tones, because this is the smallest number which will account for the facts. Of these three, green must be one, since, in the spectrum of colors, this tone has no complementary color. Green

1 See Helmholtz, Physiolog. Optik, pp. $388 \mathrm{ff}$.

${ }^{2}$ Physiolog. Psychologie, i., p. 450. 
being fixed, the other two color-tones must be chosen from near the ends of the spectrum, and in such a way that, when combined with spectral green, they will produce white. Red (carmine-red, according to Fick) and either violet (so Young and Helmholtz) or blue (indigo-blue, Fick) best fulfil the required conditions. It is, then, assumed, by the Young-Helmholtz theory, that in every portion of the retina which is susceptible to color there exist three kinds of nerrous elements, the excitation of which separately would produce three distinct kinds of sensations; and that each kind of element is capable of producing only that kind of sensation which is peculiar to itself. It apparently follows that each of these three kinds of nervous elements has its special form of end-apparatus, the excitability of which differs from that of the others; that is to say,

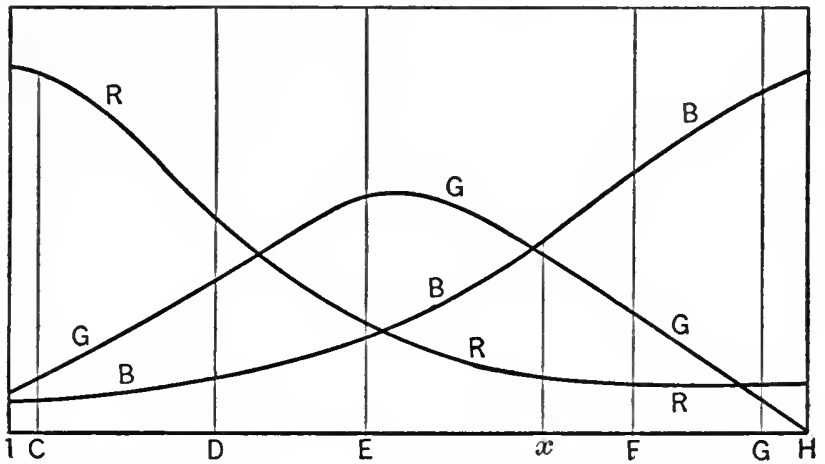

FIG. 92,-Diagram from Fick, illustrating the Young-Helmholtz Theory. (For explanation, see the text.)

there are fibres of red color-sensation, whose end-apparatus responds specifically to rays of small refrangibility ; fibres of green colorsensation, whose end-apparatus responds to rays of medium refrangibility; and fibres of violet or blue color-sensations, whose end-apparatus responds to rays of great refrangibility. We must suppose, however, since we cannot directly analyze into their components the sensations which appear in consciousness, that no one of the three kinds of elements is ordinarily excited alone. Erery actual sensation of color is therefore a complex affair, whose character is determined by the relations in which each one of the three intensities of excitation stands to both the others. In explanation of this assumption the following diagram is proposed." (See Fig. 92.) The curved lines $R, G$, and $B$ represent the three kinds of

1 Taken from Fick's Physiolog. Optik, in Hermann's Handb. d. Physiol., III., i., p. 198 ; comp. Helmholtz, Physiolog. Optik, p. 291. 
nerves sensitive to the three fundamental color-tones $-\mathbf{R}$ to red, $\mathbf{G}$ to green, $\mathrm{B}$ to blue (indigo). The curves described by them show the strength of the excitation exercised by the stimulus, corresponding to the colors of the spectrum, upon each kind of nerves. The perpendicular lines indicate the colors of the spectrum; and the way these lines cut the curves shows the relative strength of the excitation of each kind of nerves which is combined to produce these colors.

It should be gratefully acknowledged that the Young-Helmholtz theory affords a brilliant explanation of a great many of the phenomena of sensations of light and color. It is most successful with those that relate to the mixing of colors and to complementary color. The hypothesis cannot be said, however, to be wholly adequate and satisfactory. One of its most intelligent advocates (Fick) admits that it cannot explain the following cardinal fact: Every ray of light which, so long as it is confined to a moderate extent of the polar zone, makes the impression of a saturated color produces a whitish impression, almost devoid of color-tone, as soon as it is limited to an extremely minute portion of the retina. This is the very opposite of what the hypothesis would lead us to expect; for, according to it, extremely minute impressions on the retina ought to isolate the particular kind of fibres, and so yield the purest possible color-tone. The facts of histology seem rather adverse than favorable to the theory, although not much stress can be laid upon them alone. Moreover, it does not satisfactorily explain the facts of contrast of colors and of color-blindness. The most recent investigations seem to indicate that cases of color-blindness cannot be accounted for by dropping out one fundamental kind of nerve-fibres, as the Young-Helmholtz theory supposes.' Various other important objections are raised by its opponents (especially by Hering, Wundt, and others).

$\S 16$. In order to supply the alleged defects of the Young-Helmholtz theory of color-sensations, several other theories have been devised-notably those of Hering and of Wundt. The former ${ }^{3}$ differs from most other investigators in his view of the nature of the changes of sensation which take place as we, in experience, run through all the different shades of gray from white to black. All such changes Hering considers analogous to those alterations in the quality of our sensations that would be produced by passing

${ }^{1}$ See von Kries, Archiv f. Anat. u. Physiol., Physiolog. Abth., 1882 (Ap. pendix), pp. 134-153.

2 E. Hering, Zur Lehre vom Lichtsinne, Sitzgsber. d. Wiener Acad., 6 papers, $1872-74$. 
the eye over a surface on which the different color-tones almost insensibly shaded into each other. Hering, therefore, proposes six (or three pairs instead of three single ones) fundamental colortones-namely, black and white, green and red, blue and yellow. The changes which give rise to sensations of black, green, and blue are ascribed to the process of "construction" of a so-called visual substance ; those which give rise to white, red, and yellow are asscribed to the "destruction" of such visual substance. The three pairs of color-tones are thus made antagonistic rather than complementary. But the hypothesis of Hering appears to involve more uncertain assumptions, and to explain fewer facts, than the one it would displace. Moreover, the assumption that white, and its shades down to black, may be considered as color-tones, instead of alterations in the brightness of the true color-tones, is generally denied.

The theory of Wund $t^{2}$ emphasizes the difference in processes rather than in the kinds of retinal elements. It involves the following principles: (1) In every excitation of the retina two different processes are set up, the variations of which follow different laws; one of these is a "chromatic" process (which gives us color-tones), and is a function of the length of the waves of light; the other is "achromatic," and is also dependent upon the wavelengths, but varies only in intensity and remains in character the same. (2) The achromatic excitation consists in a "uniform photo-chemical process," which reaches its maximum at yellow and falls off toward both ends of the spectrum. (3) The chromatic excitation is a "polyform photo-chemical process," which changes continuously with the wave-lengths of light. The extreme differences of this length are such as to produce effects that approximate each other; while the effects of certain different intervening wavelengths are related in such a way that opposed phases of one and the same movement equalize each other perfectly. (4) Every process of excitation of the retina outlasts the stimulation for a certain time, and exhausts the sensibility of the nerve-substance for that particular form of stimulation. 'The positive after-images are to be explained by the persistence of the retinal excitation, the negative by exhaustion. (5) The difficult phenomena of contrast are to be explained by the general principle that all impressions of light and color are experienced in relation to each other. In other words, they fall under the general law of relativity.

$\S 17$. Von Kries ${ }^{2}$ has subjected all the principal theories of color-

${ }^{1}$ See Physiolog. Psychologie, i., pp. $450 \mathrm{ff}$.

${ }^{2}$ See Archiv f. Anat. u. Physiol., Physiolog. Abth., 1882, Appendix, pp. 1-178. 
sensations to a most searching criticism as considered in the light of all the facts. He naturally finds serious defects in them all, but arrives at the following highly important conclusions. The photochemical facts concerned in vision compel us to adopt a theory of component elements rather than one of changes qualitatirely alike and arranged in a continuous series. This would seem decisive against the theory of Wundt. Only by the aid of assuming the varied combination of such elements can we explain the phenomena

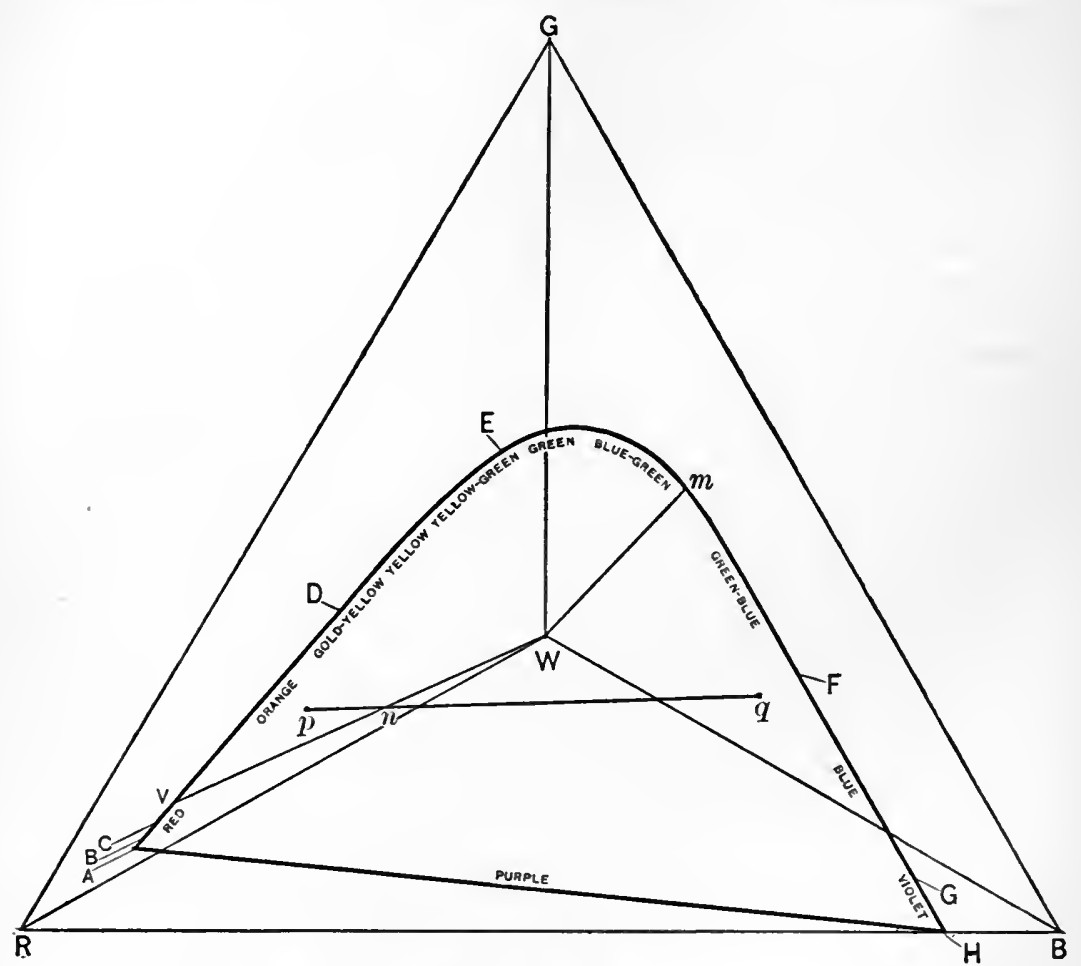

Fra. 93.-Color-Triangle, from Fick. (For explanation see text.)

of exhaustion. Three series of components are apparently requisite : one for the bright and dark, but colorless, sensations, and two color-tone series-a red-green series, and a yellow-blue series. White is, nevertheless, not to be considered as belonging to the three, since it corresponds to all the color-tones whenerer they reach a minimum of saturation. The processes corresponding to these three series of components may be located at different places in the nerrous apparatus of vision-either more centrally or more 
peripherally. The articulation and adjustment, as it were, of the three processes von Kries would assign to the central organs. And here we reach the extreme limits, not only of our assured knowledge, but also of our power to frame a plausible theory ; for it ap. pears that all theories must either leave certain important facts unexplained, or else make further assumptions concerning nervous processes-especially in the central organs of vision-of the existence and influence of which upon the sensations there can be no doubt, but of the precise nature of which we are completely ignorant.

$\S 18$. Much ingenuity and painstaking have been expended in devising some form of symbolism which should represent to the eye in geometrical relations the laws of the sensations of light and color. Obviously the sensations of this sense cannot, like those of hearing, be symbolized by the relations of points along a straight line. Color-tones, unlike musical tones, form a series of qualitatively different sensations that, at certain places in the scale, separate from each other with varying degrees of rapidity, and then toward the broken ends, as it were, of this scale, tend to approach each other again. Such relations are most successfully set forth by a triangle, which may be constructed as in the foregoing figure ${ }^{1}$ (93). In this triangle the different color-tones may be regarded as lying together along the curved line, from red to violet, and the difference in any two color-tones as measured by the angle which two lines make when drawn from the point $W$ through the points occupied on the curve by the two color-tones. For example, the difference between red and violet is less than that between red and green, as is indicated by the fact that the angle $R W H$ is smaller than the angle $R W G$.

By Fig. $94^{2}$ the relations of the color-tones as contrasting with, and complementary of, each other are rep. resented. Of the two concentric Fra. 91.-Scheme for showing the Rela. circles, each color in one corresponds

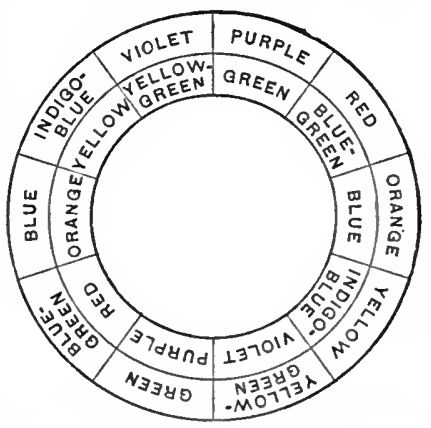
to the complementary color of the other. If the color inducing the contrast is represented by a segment of the inner circle, the coincident segments of the two circles represent the direction in which the induced change is moving, as it were. For example,

1 Taken from Fick, in Hermann's Handb. d. Physiol., III., i., p. 184.

${ }^{2}$ Taken from Wundt, Physiolog. Psychologie, i., p. 442. 
since the segment green coincides with purple, and red coincides with blue-green, green on a red ground is modified as it would be if blue-green were mixed with it ; and red, as it would be if purple were mixed with it.

$\S 19$. At least two specifically different forms of sensation-namely, Pressure and Temperature-have generally been admitted to have their organ in the skin.' The claims of various other kindred forms of feeling to be considered as primitive factor's of our sense-perceptions, arising from the activity of the skin as an end-organ of sense, are more doubtful. Sensations of motion, of innervation and weariness of the muscles, the so-called "common sensations" (or seusations of the sensus communis), the sensations of pain or pleasure, and those delicate shadings of sensation, as it were, which constitute the "local coloring" of all the feelings to which we assign a definite place in the fields of sight and touch, are all closely allied to sensations of pressure and temperature. But some of these forms of feeling-as, for example, the so-called sensations of motion and of the sensus communis - are undoubtedly complex modifications of certain simpler states of consciousness; others of them, as the sensations. of muscular weariness, of pain, of innervation, and "local coloring," may possibly have, in part, a central origin. As a rule, they lack the characteristic quality of being components of the "presentations of sense," as this quality belongs to all genuine sensations. Sensations of "local coloring" have, indeed, a most important part to take in the formation of the "presentations of sense ;" but they are, in the realm of touch and of muscular feeling, as infinitely and delicately varied (and even more difficult of description) as are the finest shadings of musical tones or color-tones.

\$20. A sixth sense, however, and a sixth organ of sensations must doubtless be recognized as constituted by the muscles and the various kinds of feeling which their action occasions. These muscular sensations, when combined with those of the skin, give certain complex feelings of motion on which the adjustment of the body to its environment is so dependent. The long-continued dispute concerning the presence of sensory nerve-fibrils in the muscles may be said to be settled affirmatively. ${ }^{2}$ Certain subjective phenomena cannot be accounted for by ascribing the so-called muscular sensations to feelings of central innervation, or by identifying them

I On the physiology of the skin, see Goldscheider, art. Neue Thatsachen über die Hautsinnesnerven, Archiv f. Anat. u. Physiol., Physiolog. Abth., Supplement-Band, pp. 1-104.

${ }^{2}$ See, especially, Sachs, in Archiv f. Anat. u. Physiol., 1874, pp. 175 f., 491 f., and $645 \mathrm{f}$. 
with the sensations of pressure through the skin. ${ }^{2}$ Bernhardt ${ }^{2}$ found that the degree of sensitiveness to different weights, when lifted by the foot or the finger, was little or not at all diminished by excluding all central innervation of the muscles through an act of will. The discrimination of differences of weight was not greatly impaired when the limb was bent by an induction-shock sent through the muscles instead of by motor impulses arising in the brain. The muscular sensations cannot, therefore, be due to such central activity. Investigation also shows that the muscular sensations supplement those of pressure in the skin in all our estimates of the position and motion of the limbs; these two are, therefore, not identical. Moreover, without assuming the existence and aid of such sensations we cannot account for that nice control of the muscles which, especially in the case of the eye, is so indispensable a prerequisite, not only for adjusting their action to the ends desired, but also for gaining an exact knowledge of the position and motion of objects in the outside world. The precise manner, however, in which the muscular sensations originate, through that stimulation of the sensory nerres which the contraction of the muscular fibre occasions, is as yet unknown. Nor can they easily be separated and classified into kinds, apart from the sensations of pressure with which they are in actual experience constantly allied. Their chief interest to psychology centres in the help which they furnish to the mind in forming its "presentations of sense."

$\S 21$. Sensations of Pressure are dependent upon the excitation of the sensory nerres of the skin through their appropriate endorgans. The excitation of the trunk of any of these nerves at some point along its course may produce the feeling of pain, but does not produce those definite sensations of pressure which we are able to localize so accurately and discriminate so nicely as to their degree. Precisely which of these end-organs are specifically related to sensations of pressure neither histology nor experimental physiology has thus far been able to determine (see Part I., chap. V., \$10). The ordinary stimulus of the end-organs of the skin active in these sensations consists in their compression or expansion by contact with some external object which either rests upon them or upon which they rest, or which is moved over or against them, or over or against which they are moved. Such stimulus may, of course, vary both in form and in degree. The quantity and succession of the sensations of pressure, as well as the manner in which they combine with one another and with sensations of the muscular sense,

${ }^{1}$ Comp. Funke, in Hermann's Handb. d. Physiol, , III., ii., p. 359 f.

2 Archiv f. Psychiatrie, III., p. 627. 
have a marked effect in determining their characteristic "tone" of feeling. In respect to quality pure and simple, sensations of pressure scarcely admit of a scientific classification. We localize them in the field of touch; we make an important use of them in connection with sensations of muscular origin, for constructing the field of vision and for giving to different objects their respective places in this field; but in ordinary experience we do not directly recognize kinds of the simple sensations of pressure as we do of tastes, smells, tones, and colors. A distinction is sometimes made between "light touch," or touch proper, and sensations of pressure or weight. But the distinction, so far as it leaves ont of account the muscular sensations, has hitherto been one only of degree and not of kind.

The more recent and thorough investigations of Goldscheider " have led him to distinguish two specifically different sensations which enter into what is ordinarily called the feeling of pressure. This distinction is based upon facts experimentally ascertained. If a very fine point of metal, wood, or cork, be touched lightly to the skin, it will be found to awaken a definite sensation, such as is entitled to be called a "sensation of pressure," only at certain minute spots in any given area of the skin. This sensation, when the pressure is very light, is described as lively and delicate, often accompanied by the feeling of being tickled. On increasing the pressure upon these same spots the sensations change their character somewhat, and become as though some small, hard kernel were pressed upon the skin ("Körniges Gefühl"). Between these distinctively "pressure-spots" it is not possible to excite by pressure the same characteristic sensation. Stimulation of the intermediate spots, on the contrary, produces a dull, indefinable, "content-less" sensation; and when the pressure is increased, a sense of being pricked or stuck. Both of these kinds of sensation, when the pressure is still further increased, pass over into painful feeling; but the character of the pain in the two is different.

The arrangement of the "pressure-spots" is analogous to that of the temperature-spots (to be described subsequently). They occur much more frequently in certain areas of the body than in others. They are placed in chains, as it were, sometimes more and some. times less thickly set. These chains ordinarily radiate from a kind of central point, and run in such directions as to form either circular, longitudinal, or pyramidal figures. Their direction is seldom identical with that of the temperature-spots. In the opinion of $76 \mathrm{ff}$.

'Archiv f. Anat, u. Physiol., 1885, Physiolog. Abth., Supplement-Band, pp. 
Goldscheider the spots of both kinds correspond to the terminal points of the nerve-fibres of two specifically different kinds of nerves distributed over the skin. But whereas all the area of the skin is well covered with such nerves as give us the general dull and indefinite feeling of contact, the nerves of the sensation of pressure are much more unevenly distributed. It need scarcely be said that, other things being equal, they are most numerous in the areas of the skin most sensitive to touch. The different pressure-spots themselves differ in sensitiveness; some are much more easily excited than others. The sensations themselves come under the
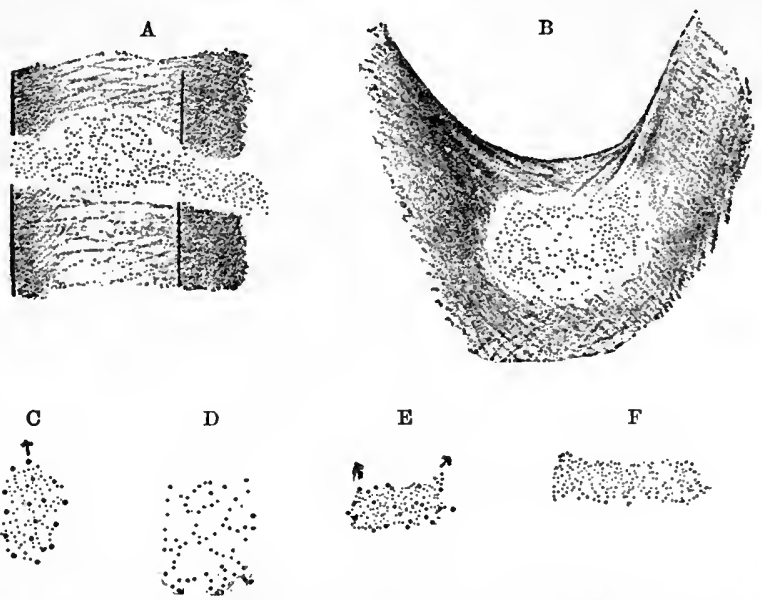

Frg. 95.-Arrangement of Pressure-spots (Goldscheider). A, dorsal and radial surface of the first phalan $x$ of the index finger; $B$, membrane between thumb and index finger; $C$, dorsal surface of forearm; D, back; E, Inner surface of forearm ; F, back of hand.

general laws of exhaustion, practice, etc., as these laws apply to the whole mechanism of sense.

The attempt has been made, on the other hand, to identify, in kind, sensations of pressure (especially those of light touch) and sensations of temperature. ${ }^{1}$ E. H. Weber observed that cold bodies resting on the skin appear heavier, and warm lighter, than they really are. A single silver dollar of the temperature of $25^{\circ}-19.5^{\circ}$ Fahr. appeared to be of the same weight as two dollars of the temperature of $98.5^{\circ}-100.5^{\circ} \mathrm{Fahr}$. Wunderli also argues the identity of these two classes of sensations on the ground that, if certain parts of the skin are lightly touched with cotton or slightly warmed by approaching $a$ heated surface to them, through a square opening in

'For a discussion of this question, see Funke, in Hermann's Handb. d. Physiol., III., ii., p. $320 \mathrm{f}$. 
a piece of paper laid upon the skin, the two sensations thus occasioned are frequently mistaken for each other. But Szabadföldi has much weakened the force of Weber's experiment by showing that small wooden disks when heated to $122^{\circ} \mathrm{Fahr}$. often feel heavier than those which are really larger but are not thus warmed. And Wunderli's observation at best holds good only for comparatively obtuse parts of the skin, especially the back. Moreover, if the same stimuli should serve to excite both the pressure-spots and the temperature-spots, this would not prove the identity of the two sensations.

Finally, the physiology of the sense of temperature re-enforces the indubitable testimony of consciousness, and leads us to the conclusion that from beginning to end-in the character of their stimuli, of their nervous processes, and of the resulting modifications of feeling - the sensations of pressure and the sensations of temperature are qualitatively distinct. They have in common only the organ in which their apparatus is located, and the fact that both kinds of sensations are constantly associated most intimately in time and space.

$\S 22$. Sensations of Temperature, therefore, form a second distinct species which have their origin in the excitation of the nervous end-apparatus of the skin. Whether their end-apparatus is locally the same as that upon the excitation of which the sensations of pressure are dependent, it has seemed until very lately impossible to say. But recent investigations (especially of Blix, ${ }^{1}$ Goldscheider, ${ }^{2}$ and Donaldson ${ }^{3}$ ) point unequivocally to the conclusion that certain definite spots of the skin, and these only, are susceptible to irritations of a kind to result in sensations of temperature. Such spots are insensible to pain (even the pain of temperature), and a needle can be run into them without being felt; they are probably also insensible to pressure. What is more remarkable still, the existence of "heat-spots" and "cold-spots"-or minute localities of the skin sensitive to heat but not to cold, and conversely-seems demonstrable. By using a machine which locates the stimulus micrometrically, the topography of the skin may be mapped out, and extremely minute spots indicated which respond to irritation with sensations of pain, of pressure, of cold, and of heat-respectively. These different kinds of sensation-spots appear never to be super-

${ }^{1}$ Zeitschrift f. Biol., 1884, XX., pp. 141 ff.

${ }^{2}$ Monatshefte f. prakt. Dermatol., 1884, III., Nos. 7, 9, 10 ; 1885, IV., No. 1 ; and art. in Archiv f. Anat. u. Physiol., 1885, Physiolog. Abth., SupplementBand.

${ }^{3}$ Research on the Temperature-sense, reprinted from Mind, No. XXXIX. 
imposed. They are not located alike on the symmetrical parts of the same inclividual, or on the corresponding parts of different individuals. An accurate mapping out of the different areas of the skin, with respect to their temperature-spots, is difficult; since experiment soon blunts the sense, and even the approach of a heated or cooled point raises or lowers the temperature over a considerable area. But, in general, such spots occur in lines that radiate from centres generally coincident with the roots of the hairs, in those
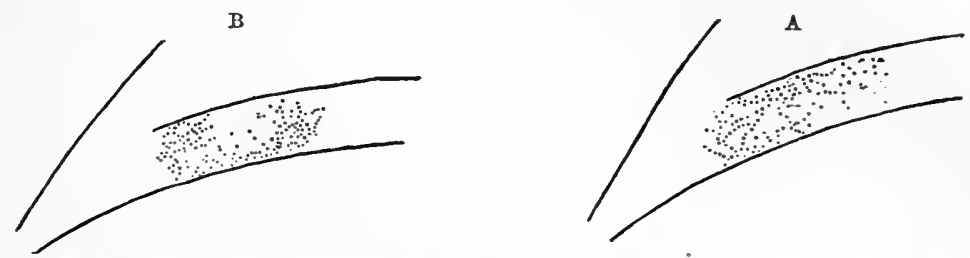

Fig. 96.-Arrangement of Temperature-spots. A, heat-spots; and B, cold-spots-from the palm of the left hand (Goldscheider).

regions of the skin where such appendages are found. These lines often run so as to cross each other, forming figures of various shapes, -triangles with rounded corners, etc. Heat-spots are, on the whole, less abundant than cold-spots ; but in parts of the body where the skin is most sensitive to either heat or cold the corresponding class of spots is relatively frequent. Temperature-spots may be divided into first-class and second-class (so Goldscheider) according to the strength with which they react on moderate stimulation. Some spots are roused only by excessive temperatures. The same object feels cool to one spot, ice-cold to another.

The electrical current when applied to these spots is thought to call out the corresponding specific sensations. Goldscheider considers that he has succeeded in exciting definite temperature-sensations by applying electricity to the trunks of the nerves distributed to certain areas of the skin. This would appear to be almost a demonstration that the nerves of this sense are specific, and of two kinds-nerves of heat-sensation and nerves of cold-sensation. Puncturing a temperature-spot also gives rise to temperature-sensations. The discriminative sensibility of the temperature-spots is found to be much finer than that of the tactile sensations. Everything which produces a change in the temperature of the skin acts, of course, as a stimulus for the sensations of heat and cold.

\&23. The above-mentioned discoveries as to the specific energy of the nerves and end-apparatus of the skin, interesting as they are, 
have not yet been completely brought into rational connection with our experience of temperature-sensations and our knowledge of the general laws of nervous action. It is obvious, however, that the principles of contrast, of relativity, and of exhaustion, must bear a large part in the explanation of all these sensations. Sensations of temperature have apparently a certain dependence on the temperature of the thermic apparatus itself. This law has been elaborated and defended in detail by Hering, ${ }^{2}$ in the following form: "As often as the thermic apparatus at any spot in the skin has a temperature which lies above its own zero-point we have a sensation of heat; in the contrary case, a sensation of cold. Either sensation is so much the more marked, or stronger, the more the temperature of the thermic apparatus at the time varies from the temperature of its own zero-point." By the "zero-point" of any part of the skin is meant the exact objective temperature which at that part wiil produce no sensation of either heat or cold. Such zero-point is, of course, different for different parts of the body, according as they are or are not exposed, and are or are not well supplied with arterial blood, etc. It also changes in connection with changes in the temperature of the surrounding air or of the bodies with which the skin is in contact. By this principle a great number of our ordinary sensations of temperature are explained by Hering. The finger and the nose are colder than the inside of the mouth, because they are exposed to radiation of their heat. On passing from a room of a given temperature into one of either higher or lower temperature we experience at first certain sensations of temperature while the zero-point of the thermic apparatus is becoming adjusted to its new surroundings. After such adjustment has taken place these sensations may cease-to be renewed in the reverse direction, however, on a return to the former surroundings. This adjustment has its limits; it is dependent chiefly upon the evaporation of the skin and upon the circulation of the blood.

If the surroundings are more than so hot or so cold, they may excite constant sensations of temperature. Among the inducements to sensations of heat at any locality of the skin, Hering mentions the following as prominent in our ordinary experience: All checking of the radiation of heat, while the blood-supply remains unaltered; all contact with a medium or object of higher temperature-and this according to the ease with which such medium or object parts with its heat; and all increase of heat in the skin

${ }^{1}$ In Hermann's Handb. d. Physiol., III., ii., p. 419 f.; and Sitzgsber. d.Wiener Acad., LXXV., Abth. 3, p. 101 f. 
coming from the interior of the body, as in the sudden hyperæmia which takes place in blushing. Inducements to sensations of cold are as follows: Increased convection of the heat of the skin by the surrounding medium, while the blood-supply remains unchanged (as when the wind blows over the hand or face, especially if the skin be moist) ; contact with objects which have the same (or even slightly higher) objective temperature as the surrounding air, but convey the heat from the skin more rapidly than it; contact with or proximity to objects colder than the skin; lessening of the interior warmth of the body-for example, by contraction of the blood-vessels which supply a given portion of the skin. Ordinary experience makes us familiar with many of the phenomena which come under all these cases.

The determination of the exact zero-point of different parts of the body is a matter of great difficulty. The rise and fall of the temperature of the thermic apparatus, in connection with that principle of exhaustion which applies to all the nervous mechanism, and especially to certain of the end-organs of sense, doubtless account (at least partially) in some way for the well-known phenomena of contrast in temperature-sensations. Weber showed that if the hand be held for a minute in water of the temperature $54.5^{\circ}$ Falnr, and then in water of $64.4^{\circ}$ Fahr., a sensation of heat will be felt for a few seconds, although the latter would have felt cold to the hand if placed in it at first. Moreover, if we hold one hand in moderately cold water, and dip the other repeatedly in the same water, the sensation of cold is stronger in the latter, although the temperature of the hand held in the water is really lower. But, according to an experiment of Goldscheider's, if one hand be left for ten seconds in water of the temperature of $104^{\circ}$ Fahr., and then both hands immersed in cold water, the warmed hand will feel the cold less distinctly than the other. This latter investigator, lowever, is inclined to dissent from Hering's theory, and return to the theory of E. H. Weber. Weber held that the rising of the temperature of the skin is felt as heat, and its sinking as cold.

After-images of temperature-sensations seem also to exist. But when a surface of the skin has been warmed or cooled, and the after-image has faded quite away, it is said that it can be called back by light mechanical irritation; this is especially true of sensations of cold. The phenomena of exhaustion are noticed in sensations of temperature. Our perception of the absolute degree of temperature, and of minute variations in its degree, is most acute for places in the scale lying close to the normal temperature of the skin. It would seem, on the whole, as though the phenomena 
of contrast of sensations of temperature, as well as of color, require for their satisfactory explanation a knowledge (possibly of the action of the central organs of the nervous system) which we do not yet possess.

E. H. Weber also showed that the amount of the skin which is stimulated has a marked influence on the quality of the resulting sensation. The temperature of the same fluid does not feel precisely the same to a single finger and to the entire hand. This experience is similar to that which has already been described in the case of sensations of color. It appears explicable in the case of the skin from what is now known about the existence of a certain variable number of heat-spots and cold-spots. In the same way, in part, may we explain the fact that smooth objects, which therefore come into contact with a larger portion of the skin-like leather, paper, wood, glass, and porcelain-appear colder to the whole hand even when they have the same objective temperature with it.

\$24. Nothing whatever is known as to the exact manner in which changes of temperature act upon the thermic apparatus to excite it ; the recent discoveries appear to make such action all the more difficult of conception and description. Since the terms "hot" and "cold" are in physics only relative, it is hard to see why absolutely different apparatus, with a distinct local position, should be used (as Goldscheider's discoveries indicate) for the sensations corresponding to each. Moreover, on Hering's hypothesis, how are we to account for the fact that heat-spots and cold-spots are in turn stimulated by the same objective temperature according to the rise and fall of the zero-point of the entire region of the skin? Possibly it may be found that certain chemical or electrical changes, dependent upon the increase or decrease of that mode of molecular motion which physics calls "heat," are the proximate stimuli of the two classes of end-organs of the temperature-sense. Goldscheider supposes that the difference in sensitiveness of different areas of the skin to temperature must be ascribed to the anatomical distribution of the heat-sensitive and cold-sensitive fibres, respectively. But he does not show us what kind of nervous contrivance would satisfy all the conditions which are imposed by the complicated facts of experience.

A. Herzen ${ }^{1}$ considers himself to have demonstrated, by pathological cases and experiment upon animals, that sensory impulses of cold, like those of touch, pass along the posterior strands of the spinal cord; and that the same region of the brain (Gyrus sigmoi-

${ }^{1}$ See Pflüger's Archiv, 1885, pp. $93 \mathrm{ff}$. 
deus) is the "centre" for both. Sensitiveness to heat can be retained, it would seem, after sensitiveness to cold has been lost.

\$25. In closing the subject treated in the last two chapters, attention is again called to the large amount and cumulative character of the evidence afforded by the special sensations, considered as respects their quality, for the law of the Specific Energy of the Nerves. It is impossible to account for the above-mentioned phenomena without carrying this law to a great length in its application to the special senses. We may not be able to affirm-as does Fick, ${ }^{1}$ for example-that two sensations are distinguishable as respects quality only in case they are occasioned by two individually different elements of the nerrous system. For we have seen that the quality of sensations depends upon their quantity, upon their relation to preceding and contemporaneous sensations, and upon considerations other than merely the one of what particular nervefibre or element of the end-apparatus was acted upon by the stimulus. Moreorer, there is no warrant for saying that identically the same nerrous apparatus cannot be excited variously according to the nature of the stimulus which acts upon it, or according to the combination with other parts of the system into which it enters for the time. It is obvious, however, that the differentiation of function, and the assignment to specifically distinct apparatus of particular nervous impressions corresponding to particular mental states, is carried to a great length in the special senses. In this differentiation of function it is not wholly or chiefly the nervefibres, as such, which should be taken into account; it is also the minute subdivisions of the end-organs of sense, and the comnections set up within the corresponding regions of the central organs. In accounting for those complex sensations which appear in ordinary consciousness, the law of permutations and combinations has, of course, to be considered. A vast variety of such sensations may be made up by changing the relations to each other of comparatively few simple elements. But in each of the senses our analysis, when carried to its utmost limit, leaves a number-in some of the senses very large-of simple sensations, which apparently must have their physical basis in the excitation of specifically distinct elements of the nervous mechanism.

The sense of smell apparently requires that the law of the specific energy of the nerves should be carried to such a length as almost to reduce it to an absurdity. Histology has discorered only one essential kind of olfactory end-organ, and that of comparatively simple structure ; and yet experience gives, as the result of its ex-

'In Hermanu's Handb d. Physiol., III., ii., p. 166. 
citation, a bewildering variety of sensations so specifically different as to baffle all our attempts to classify them. From the case of this sense an argument may then be derived which leads in either direction. It may be objected to the law that it is absurd to suppose a complexity of the end-organs of smell such as to correspond to each specific kind of olfactory stimulus with a specific sensation-for example, the smell of musk, or of sulphuretted hydrogen. It may be replied to the objection that, in the case of the ear, there are at least 16,000 or 20,000 distinct forms of auditory end-apparatus corresponding to the different musical tones; and it is therefore by no means impossible that the entire regio olfactoria may contain enough specifically different forms of its own peculiar end-apparatus to suffice for all the simple sensations of smell.

The sense of taste does not occasion so many difficulties in relation to the law of the specific energy of the nerves. It is thought possible by most physiologists to reduce all the sensations of taste to four, or at most six, different species. It is easy to suppose as many specifically different forms of the nervous apparatus corresponding to the different classes of sensations-sweet and sour, salt and bitter, alkaline and metallic. In spite of the fact that such a classification appears satisfactory to most authorities, experience is reluctant to confirm it. Many of the complex tastes, even when separated from their accompanying sensations of smell, are scarcely resolvable into combinations of the above-mentioned simple tastes. Into which of the six, for example, would experiment resolve the gustatory sensations which come from chewing a bit of chocolate, or of a nut from a black-walnut tree?

The strongest defence of the most extreme form of the theory of the specific energy of the nerves has hitherto been found in sensations of musical sound. Here we undoubtedly have a wide range of qualitatively distinct states of consciousness which are apparently dependent upon the excitation of a correspondingly large number of distinct nerrous elements. From sensations of sight, although many points of the prevalent theory are still obscure and unsatisfactory, a considerable force of evidence bearing in the same direction may be obtained. It seems almost certain that the numerous states of consciousness which result from stimulating the different nervous elements of the retina are due to combinations of a comparatively few kinds of such elements, each of which responds in a specific way to a special order of stimulus. Yet this is not precisely what the theory of specific energy seems to demand. For the different color-sensations all appear as simple and 
unanalyzable states of consciousness. None of them are twofold, as sensations. We are at a loss to say why, according to the theory of specific energy, each sensation should not result from the excitation of one, and only one, kind of nervous elements.

The recent discoveries as to the existence of pressure-spots, heat-spots, and cold-spots in the skin add important evidence to that already existing in favor of the law under discussion. It will further appear, when we consider the process of localization in the so-called "geometrical senses" of the eye and the skin, that the very possibility of such a process demands a strict and far-reaching application of the law of the specific energy of the nerves. Precisely how we are to state and limit this law, neither its opponents nor its adrocates have as yet been able satisfactorily to show. The exact expression of the theory waits for further evidence from experiment, although there can be little doubt that in its main features it is already secure. 


\section{CHAPTER V.}

\section{THE QUANTITY OF SENSATIONS.}

$\S 1$. Br an act of mental analysis, which all men readily perform, changes in the amount of sensation are distinguished from changes in its quality. This distinction obviously requires for its performance nothing beyond what is immediately given in consciousness. All sensations appear there as differing among themselves, not only with respect to the nature of the impression which serves to classify them into groups (as sensations of sight, sound, etc.), but also with respect to the degree in which each particular impression possesses the sphere of conscious attention and feeling. The best illustration of an alteration in the intensity of sensation, while its characteristic quality remains unaltered, may be derived from musical tones. The dying-out of a single note when the bow is drawn with decreasing force across the string of a violin, or a single key of the piano is struck and the pedal held, may be considered as a change in the quantity of sensation, while its quality is unchanged. A more complex case is the experience we have when approaching to, or receding from, a bell that is sounding or a steam-whistle that is blowing. Noises of a certain complex quality -such as slamming, hissing, grating, etc.-are continually described as very loud, moderately loud, or of weak intensity. So, too, when approaching a white or colored light, with our attention fixed upon it, we generally disregard almost wholly the changes in its color-tone which take place, and consider chiefly the changes in its intensity and apparent size. The pressure of different weights upon different parts of our shin is ordinarily regarded as the same in quality and as varying only in amount and locality. The same thing is true, in almost precisely the same way, with sensations of temperature. The thing we touch is called slightly cold or very cold, somewhat warm or hot, our attention being directed chiefly to the quantum of sensation which it calls forth. In other words, it is generally the same kind of pressure and temperature, with a varying degree of intensity, of which we are conscious. 
It is more difficult, however, even in the most indefinite way, to separate the quantities of our sensations of smell and taste from the changes in quality of the same sensations. A concentrated sweet or acid so strongly excites a variety of forms of feeling which mingle indistinguishably with the specific sensations of taste that we are compelled to attend to the very decided qualitative changes which are taking place. The increased intensity of the sweet or sour we may indeed speak of as "very" much of the same sensation which was excited in less degree by the diluted form of the stimulus; but we are more likely to regard it as constituting a complete change in the kind of taste. In the same manner, attention is forcibly directed toward the kind of sensation which results from increasing the quantity of any specific sensation of smell.

It is further obvious that the distinction which we make between changes in the quantity and changes in the quality of our sensations is to some extent applicable for comparing the sensations of different senses. And here the distinction, when applied to subspecies under certain specific forms of sensation, affords us a means of transition for such comparisons. Some yellows are bright and others dull; and the same thing is true of the reds and the blues. The sours, the sweets, the bitters, may be compared with each other as respects the degree of intensity which they possess. We may next, in a very indefinite way, compare the quantities of the sensations of the different senses as they appear side by side, or successively, in consciousness. We are ordinarily satisfied, however, with simply describing the varying degrees of intensity possessed by our different sensations as "weak" or "strong" (with or without the emphatic "very"), or as only "moderate." Thus we may judge that both the light which we see and the tone which we hear (either simultaneously or one immediately after the other) are, or are not, to be classed together under the same one of these three grades of intensity.

$\S 2$. That changes in the intensity of our sensations are not, in fact, independent of changes in their specific nature has already been proved (Chap. IV., \$ 4). Only in the case of musical tones are we able at the same time to attend carefully to both the quantity and quality of our sensations, and so discover with perfect confidence that the former is changing while the latter remains unchanged. Even in this case, since the tones which we ordinarily hear are composite, any considerable alteration of their intensity changes also their tone-coloring, through the alteration which it produces in the comparative intensities of the overtones. Any increase in the brightness of a particular color invariably changes its 
characteristic color-tone. A white of less intensity is not merely less white, but becomes a gray ; and by constantly diminishing its intensity white can be shaded through the different grays toward black, which is certainly not a feebler degree of the sensation of white. The same dependence of quality on quantity is true in all sensations of smell, taste, pressure, and temperature. It would be a mistake, howerer, on this account to consider "quantity" of sensations as only another name for shades of quality, or to deny that we can apply terms of measurement to these reactions of the mind upon the excitation of the nervous apparatus of sense. ${ }^{1}$ Scientific analysis confirms the distinction made by ordinary experience between "the way" we feel and "how much" we feel in any particular way.

§3. All descriptions of the changing intensities of sensations, when made on the basis of ordinary experience solely, leave the subject in a very indefinite and unscientific form. That a certain noise is louder or weaker than another of precisely the same kind, one may be quite ready to affirm; one may even be ready to say that one judges this noise to be about twice or three times as loud as the other. But when more precise estimates are demanded, one is obliged to hesitate before giving them. Is this musical tone ten (or a hundred) times as loud as the other; or is it only nine and nine-tenths (or ninety-nine and nine-tenths) as loud? Few would venture so nice an estimate with any confidence. Yet the case of sound is much more favorable than that of most of the senses for forming an exact judgment as to its intensity. It would be difficult under the most farorable circumstances to affirm that the sensation of the light $a$ is twice or three times as bright as that of the light $b$; or that of the shadow $x$ one-half or one-third as bright as $y$. The comparative intensities of different color-tones are yet more difficult to fix subjectively-even in the most indefinite way. This particular yellow may seem about as bright a color, of its kind, as does the red near it, of its kind. But the precise moment could not readily be told when the blue of the sky appears exactly twice as intense as the green of the grass. Still further, all estimates of the quantity of sensation approach the point at which they lose their meaning and tend to become absurd, when we compare, for example, sensations of smell or taste with those of pressure, temperature, or sight. We never say: The rose smells as sweet as it looks red; or the lemon is twice as sour as the sky is blue. And yet each qualitatively different sensation is assumed to have its place somewhere in that scale of intensities through which

${ }^{1}$ Comp. Stumpf, Tonpsychologie, I., p. $347 \mathrm{f}$. 
the different qualities may run; each may, therefore, be compared with every other, with respect to the general position which it occupies in its characteristic scale.

$\S 4$. All things to which terms of quantity apply admit of some kind of measurement and comparison with respect to their quantity. Sensations, to be sure, are not "things," but rather modes of the activity of mind, excited through the nervous mechanism of sense. Nevertheless, since, like material things, they admit of some application to themselves of the terms of quantity; and since they vary in their absolute and relative degrees of quantity, it is not strange that experimental science has endeavored to measure sensations, and to state laws for their comparison and mutual relations. The general question of the quantity of sensation involves an answer to two subordinate inquiries. Of these two the first concerns the limits within which the different sensations may rary in quantity, and yet remain sensations of the same sense; the second concerns the law of the relation which is maintained within the limits among the various sensations compared. But neither of these questions can be answered directly. Sensations cannot be kept constant in quantity, and measured by the direct application of physical standards, whether with a view to fix their absolute or their relative magnitude. They are all, however, under ordinary circumstances, connected with the action of different forms of physical energy upon the nervous system; that is to say, they are caused by the application of stimuli to the nerves, and the changes in the amount of the sensations are dependent upon changes in the intensity of the stimuli which occasion them. These stimuli admit of changes in quantity, which, theoretically at least, are measurable objectively, with more or less exactness. Resulting changes in consciousness can only be measured by attentive judgment, which directly discriminates the sensations as varying in intensity, and as being greater or less, one than the other, in the scale of impressions which experience has framed.

The problems of the measurement of sensation may then be stated as follows : (1) To determine how little and how much of each kind of stimulus will produce respectively the least and the greatest quantity of each kind of sensation of which the mind is capable, or to find the quantitative limits within which sensations of each sense are possible; and (2) to determine the law of the relation under which changes in the intensity of sensations, as estimated in consciousness, are dependent upon changes in the intensity of the stimuli.

$\S 5$. Unexpected and insuperable difficulties, however, stand in 
the way of a direct solution of either of the two above-mentioned problems, even in the modified form in which they were last stated. For, in the first place, it is only with respect to sensations of pressure and of the muscular sense that we can measure objectively the physical energies which act on the nerrous end-organs, with much approach to perfect exactness. ${ }^{2}$ The amplitude of the acoustic waves in the air which originate from a given source would indeed admit of exact measurement; but the modifications which these waves undergo before they reach the nerre-cells and nerre-fibres of the inner ear are so complicated as to make it impossible to calculate accurately the amount of the physical stimulus which is directly applied to the end-organs of hearing. The photo-chemical and thermic effects of light may be measured objectively. But this light is not the direct physical stimulus for the fibres of the optic nerve, or even for the end-organs of the retina; and we have no sufficient means for estimating the amount of those chemical changes in the visual substances, or pigments of the eye, which are supposed to be the immediate excitants of the terminal apparatus of vision. The case is yet more hopeless with respect to the senses of taste and smell; inasmuch as we do not even know what properties smellable and tastable substances must possess in order to influence the nerves of those senses. The objective measurement of the stimulus for sensations of temperature also is made difficult by the fact that its amount is dependent upon the zero-point of the skin itself, since this point is different at different times and for different areas of the entire surface, and is always difficult of precise determination.

Moreorer, could we measure with perfect exactness the intensity of the stimulus as it is applied directly to the appropriate endorgans of sense, our knowledge of the intensity of the necessary physical antecedents of the resulting sensations would be far enough from complete. How do the end-organs modify the quantities of the stimuli before they transmit their effect to the conducting nerve-fibres? Precisely how much further modification do these quantities receive in transmission to the central organs, at the hands of the conducting nerve-tracts? What are the laws which control the reception, diffusion, and modification of the different intensities of the transmitted nerre-commotions, within those parts of the nervous mechanism (the central organs), where they become the immediate occasions of the rise and change of sensations in the mind? These are questions to which we are absolutely unable to give any satisfactory answer.

${ }^{1}$ Comp. Wundt, Philosophische Studien, 1883, II., heft i., pp. $10 \mathrm{ff}$. 
§ 6. But if an exact objective measurement of the physical stimuli is intrinsically difficult, an exact subjective measurement of the sensations themselves is inherently impossible. Such subjective measurement can exist at all only in the form of a judgment which compares two or more sensations with a view to pronounce whether they are equal in intensity ; or, if unequal, which is the greater and which the less of the two. But we have seen that the ordinary estimate of the absolute strength of a sensation is able simply to assign to it an indefinite position in the scale of its kind. With certain exceptions, scientific analysis can do little to exclude the uncertainties of the ordinary estimate. These exceptions are all of the following kind: Where two sensations of the same quality are produced, either simultaneously on different corresponding areas of the same organ or successively (with the most favorable interval between) upon the same area, by amounts of stimulation that are very nearly or precisely equal, the attentive mind can discriminate the minute differences, or exact equality, of the intensities of these two sensations, with a great degree of nicety. The problem of measuring the quantity of sensations depends, therefore, upon obtaining the least observable differences in intensity for each kind of sensations, and for every point along the scale of degrees of intensity.

But in this connection another occasion for doubt and debate arises. Is "the least observable difference" of two sensations itself a constant quantity? The affirmative answer to this question is assumed by Fechner ${ }^{1}$ and all strenuous advocates of the law which he defends. It has even been argued that to hold another than the affirmative view involves a contradiction in terms. ${ }^{2}$ What can be meant, it is asked, by a "least observable difference" in intensity between two sensations, unless it be that this difference is a constant unit for the measurement of those sensations of the same kind which lie near the same point in the scale? If the difference is more than just observable, then of course it is not the least observable; if it is less, then it is not observable at all-that is to say, there is no change in sensation. But to this argument the following reply is pertinent: The "least observable difference" is not itself a mental entity or a mental state, that can be measured and used as a unit for measuring the quantity of other mental states. For example, if the addition of $n$ to the stimulus $S$ is the smallest amount that will produce such a change in the mental state $x$ as to cause it to pass over into $x^{\prime}$, which the mind recog-

' Elemente d. Psychophysik (1860), i., p. 54 f.; In Sachen d. Psychophysik (187\%), p. 45 f.; Revision d. Hauptpunkte d. Psychophysik (1882), p. 18 f.

${ }^{2}$ Comp. the first edition of Wundt's Physiolog. Psychologie, p. 294. 
nizes as having a greater quantity of sensation than $x$, such fact is to be stated and accepted as a mere fact; it does not follow, however, that we may conclude that $x^{\prime}-x=\Delta$, and that this $\Delta$ is entitled to a name ("least observable difference") and a rank among the mind's experiences by way of sensation. There are no sensations (whatever physical occasions of sensations may exist) except those that appear in consciousness ; ex hypothesi, there appear in consciousuess only $x$ and $x^{\prime}$, and no sensation whatever lying between the two in intensity. We judge, indeed, that the intensity of $x^{\prime}$, now present in experience, is greater than was the intensity of $x$, now remembered as an image of past experience; but $\Delta$ (or $x^{\prime}-x$ ) is a mere abstraction, a figment of the experimenter's brain, and not a real experience of the person with whom he is experimenting.

Moreover, if $\Delta$ were cupable in any case of being regarded as a unit of subjective measurement, it would by no means follow that its mental value is a constant. That $n$, or the amount of stimulus which must be added to $S$ in order to produce an observable change in the quantity of sensation, is not constant we know beyond doubt. For the different senses, for different individuals, for different degrees of the absolute stimulus (i.e., value of $S$ ), for different conditions of the organs of sense, this amount $n$ is constantly varying. The amount of $\Delta$ may also be held to vary, according to psychological changes in the means and power of mental discrimination, such as we have no way of measuring objectively. For we must again insist upon the fact that the real quantity of a sensation is not the same thing as the estimated quantity of the same sensation. The "least observable difference" would not, therefore, necessarily be the same as the least real difference, between two sensations." It is not the mind's custom to attend accurately to the changes in quantity of its sensations as such. Properly speaking, many considerable changes in our sensations, as we may judge by the guidauce they give to the bodily motions and the mental train, do not appear in consciousness with a label of exact quantitative measurement, as it were, attached to them.

It is therefore obvious, from the great difficulties which belong inseparably both to the objective measurement of the stimuli of seusation, and to the subjective measurement of the resulting sensations, that any law of their relation can have only an indefinite statement and a secondary value.

§ 7. Two methods of determining the lower limit, or minimum of stimulus producing a sensation, are possible. In the use of one method, a weak stimulus, but somewhat above the amount needed

${ }^{1}$ Comp. Stumpf, Tonpsychologie, I., p. 51 f. 
to produce a sensation is applied ; its intensity is then diminished by minute gradations until the exact point is reached and noted at which it ceases to produce any sensation at all. In the use of the other method a stimulus too weak to produce any sensation is first applied; its intensity is then very gradually increased until it begins to produce the smallest observable sensation. Both ways may be combined, and thus the "sensitiveness" of each organ of sense, and of each part of each organ, may be determined. Such sensitiveness increases, of course, in inverse ratio to the amount of stimulus necessary for producing any sensation at all, or for producing a sensation estimated as having a definite degree of energy. The effort to determine the lower limit of sensations of sight and of sound is embarrassed by the facts that the retina is always under excitation from the chemical changes going on in its pigments, and therefore has a certain quantum of so-called "light of its own," and that such a thing as "absolute stillness" cannot probably be secured for the ear. Total absence of sensation in the ear, could it be secured, would not be comparable to the black which we see with the eyes closed. ${ }^{1}$

The upper limit, or maximum amount of stimulus which the nervous organism can receive, cannot be determined experimentally. The use of excessive quantities of stimulus is not only too fatiguing but also too dangerous to the structure of this organism (for example, of blinding light upon the eye, stunning noise in the ear, etc.) to admit of successful experiment in this direction. Moreorer, the application calls out so much of those varied forms of feeling which are allied with all the specific sensations as to overwhelm the latter with the former. Very concentrated, sour, or bitter solutions, or very intense odors, are not simply tasted and smelled; they are also felt with all the adjoining parts of the body. Very strong light and very loud noise do not simply heighten the specific sensations of sight and hearing, they rather destroy them in a flood of painful feeling. We may affirm in general, however, that the "capacity" of each sense varies directly as the amount of stimulus which it can receive. The "circuit" or range of the sensations of each sense may then be said to be $\frac{\mathrm{C}}{\mathrm{S}}$ where $\frac{1}{\mathrm{~S}}$ stands for the measure of the sensitiveness, and $\mathrm{C}$ for the measure of the capacity, of each sense. ${ }^{2}$

\footnotetext{
${ }^{1}$ On the question whether absolnte stillness is possible, and whether the ear has any sensation comparable to the black of the eye, see Lotze, Medicinische Psychologie, p. 218; Volkmann von Volkmar, Lehrbuch d. Psychologie, 1884, I., p. 273 ; and Stumpf, Tonpsychologie, I., p. 380 f.

${ }^{2}$ See Wundt, Physiolog. Psychologie, i., p. 324.
} 
§ 8. There are three methods of deternining experimentally the least observable differences in sensations. These are called, (1) the method of least observable difference; (2) the method of average errors; (3) the method of correct and mistaken cases. Of the three methods, the first bears the name which suggests the real subject of investigation in them all. This method is divided by Wundt ${ }^{2}$ and others into tro-namely, the method of mean gradations of sensation, and the method of minimum changes of sensation. But these are really only two modes of applying one method. In the one case an attempt is made to form a scale of stimuli whose intervals correspond to equally large intervals in our estimate of the resulting sensations, by judging what amount of the stimulus produces a sensation (M) that lies exactly midway between two other sensations (A and O) separated by a clearly perceptible interval (hence $A: M:: M: O$ ). Between $A$ and $M$ another middle term, the sensation of magnitude $K$, may then be sought and found; and so on until the limit of observable differences is reached. This mode is, however, less comprehensive and fruitful than the second mode of applying the same principle. The "method of minimum changes in sensation" seeks directly to establish, all along the scale of intensities of the stimuli, that change in their strength which is just enough (and no more than enough) to produce a minimum change in sensation. Such minimum change may be conceived of as standing just on the "threshold" of our power to make distinctions in the degrees of strength with which our sensations are apprehended in consciousness. ${ }^{2}$

The "method of average errors" (2) begins by fixing upon some given sensation which is known to be caused by a given intensity of stimulus; the attempt is then made also to fix upon another stimulus, by means of the sensation it produces, as being exactly equal to the former. The trial results in a number of guesses that are more or less out of the way. By averaging all the cases of trial, the degree of sensitiveness to distinctions is discovered. In other words, the method attempts to determine, at each point along the scale and for each kind of stimulus, the differences in the strength of stimuli that are just below the amount necessary to make an observable difference in the resulting sensations.

In the "method of correct and mistaken cases" (3) minute additions or subtractions of the amount of stimulus are made, with the intent of seeing how many cases of right and how many of

I See Wundt, Physiolog. Psychologie, i., p. 325 f., and comp. his Philosophische Studien, 1881 , p. 8 f.

"Called "Unterschiedsschwelle" by Fechuer, Elemente d. Psychophysik, i., p. 242 . 
wrong guessing, respectively, will result for each of the different positions in the scale of the stimuli, and for each kind of stimulus. If, then, the proportion of the number of correct to mistaken guesses is kept the same for all points of the scale, the amount of change in the stimulus necessary for this may be held to measure the sensitiveness to differences which belongs to each of these points. Thus, let $n=$ the whole number of guesses, and $r=$ the number of right guesses; then $\frac{r}{n}=$ the sensitiveness to differences. But the positive value of this quotient being kept unchanged, the amount of stimulus added to or subtracted from the original amount will measure the sensitiveness to differences for all points of the scale. This method has been largely used and warmly defended by Fechner ${ }^{1}$ in experimenting with sensations of pressure. Much doubt has, however, been thrown upon the use made of it by this observer; and especially upon the propriety of reckoning the doubtful cases one-half to the right and one-half to the wrong guesses. ${ }^{2}$

A comparison of the above-mentioned methods shows that they are all simply different ways of measuring the sensitiveness of the mind to minute differences in the quantity of its sensations as dependent upon changes in the intensity of the stimuli. They should never be employed, therefore, without taking into account the fact that various other causes, besides such objective changes in the stimuli, always co-operate to determine the degree of this mental sensitireness. To eliminate these other factors from the calculation is by no means easy.

89. The one law which claims to be a scientific expression of the relations between changes in the intensity of stimuli and changes in the quantity of the resulting sensations is that known by the name of E. H. Weber. This observer originally used the method of least observable differences as applied to sensations of pressure and to the measurement of lines by the eye." "Weber's law" has been elaborated, confirmed by a vast amount of experiment, and defended as a psycho-physical principle of the widest application, by Fechner (in the works referred to, note, p. 361). The significant addition which Fechner has made to Weber's law consists in the assumption that all just observable differences are equally great. ${ }^{4}$

${ }^{1}$ Elemente d. Psychophysik, j., pp. 93-120.

'On this point see, especially, G. E. Müller, Grundlegung d. Psychophy. sik, p. $36 \mathrm{f}$. ; and Wundt, Physiolog. Psychologie, i., p. $330 \mathrm{f}$.

${ }^{3}$ Especially in articles on the sense of toncl, in R. Wagner's Handwörterb. d. Physiologie, III., ii. ; and Archiv. f. Anat., Physiol., etc., 1835, pp. $152 \mathrm{ff}$.

${ }^{4}$ On this point comp. Funke, in Hermann's Handb. d. Physiol., III., ii., p. 349 f. ; and Wundt, Philosophische Studien, II., Heft 1, p. 6 f. 
It is therefore also called "Fechner's law." As an empirical law it attempts to put into scientific form, on the basis of experimental investigation, the truth of ordinary experience-namely, our estimate of the difference in amount between two sensations is not directly proportioned to the real difference in their stimuli, but the latter must increase faster than does the former. For example, the difference in the intensity of the shadows cast by one and by two wax tapers is very perceptible in a dimly lighted room, but is altogether unobservable in open sunlight; or the strength with which two clocks tick can be discriminated with much nicety, but not the amount of noise made by two successive discharges of a cannon.

In other words, if we assume that the least observable difference in sensations may be regarded as a constant quantity, then, in order to produce this increase or decrease in the amount of sensation, the addition or subtraction of a much greater amount of stimulus is needed for the higher than for the lower portions of the scale. Weber's law undertakes to tell us how much greater such required amount of stimulus must be. It admits of statement in the several following ways: The difference between any two stimuli is experienced as of equal magnitude in case the mathematical relation of those stimuli remains unaltered; or, If the intensity of the sensations is to increase by equal absolute magnitudes, then the relative increase of the stimulus must remain constant; or, The strength of the stimulus must ascend in a geometrical proportion in case the strength of the sensation is to increase in an arithmetical proportion. ${ }^{1}$

${ }^{1}$ See Wundt, Physiolog. Psychologie, i., p. 335. For the detailed mathematical discussion and expression of Weber's law the reader is referred to the technical works, especially of Fechner and G. E. Müller. A simple statement of Weber's principle may be given as follows: Let $H=$ the intensity of the light of one-half of a white field $; \frac{\pi}{100}=$ the smallest fraction of stimulus added to $I I$ that will produce an observable increase in this intensity; and $H+\frac{H}{100}=$ the intensity of the other half of the same field. Then let $S=$ the sensation produced by $H ; S+s=$ the sensation produced by $H+\frac{H}{100}$; and $s$ will, of course, represent the so-called least observable difference at this point in the scale. We have, then, $H$ produces $S ; H+\frac{H}{100}$, or 18100 , pro-

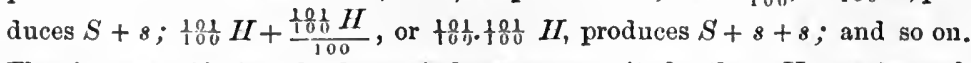
That is to say, if $s$ is to be kept of the same magnitnde, then $I I$ must be mul-

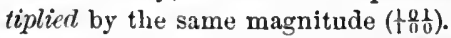

The three fundamental formulas which Fechner has employed to state and demonstrate the law are the following: Let $S$ be the magnitude of the sensation caused by the stimulus $\Sigma$, and $\Delta S$ a just observable increase in this sen- 
The empirical data upon which the adrocates of Weber's law rely are very numerous, but their value and trustworthiness are often much diminished by the fact that most experimenters have failed to isolate sufficiently the exact problem which it was desired to solve. Nevertheless, the data show that the law summarizes many facts reasonably well within a certain range of sensations lying near the middle of the scale of quantity. Near both the upper and the lower limits the law fails to prove applicable; even in the regions and under the circumstances which are most favorable it is only approximately true. Many fluctuations of unknown significance and origin occur in all the senses.

$\S 10$. In determining the least observable sensations of touch, the result is largely dependent upon the presence of muscular sensations also. It further depends upon the method in which the comparison is made ; for, as Weber discovered, an actually present sensation can be compared with the remembered image of one just past better than two present sensations can be compared. The interval of time and the locality of the organ have also a great influence. Most persons observe a stronger sensation of pressure when the weight is laid on the left than when it is laid on the symmetrical place of the right side. The same amount of surface must be covered, and the objects compared must have the same temperature, in order to secure trustworthy results of experiment. Weber found that, when the interval was fifteen to thirty seconds, under the most favorable circumstances, $14 \frac{1}{2}$ could be distinguished from 15 grammes, or $14 \frac{1}{2}$ from 15 ounces. That is, some persons can distinguish weights which differ as $29: 30$, by the sensations of pressure they occasion, when laid on the volar side of the last phalanges. By raising the weights the nicety of discrimination can be increased so as to be represented by the proportion $39: 40$.

sation which is caused by an increase of the stimulus $=\Delta \Sigma$. Let $C$ be a constant dependent on the valnes chosen for $S$ and $\Sigma$. Then $\Delta S=\frac{C_{\Delta \Sigma}}{\Sigma}$. Let it be further assumed that $\Delta S$ remains constant whatever values for $S$ and $\Delta \Sigma$ are assumed; then $d S=C_{\frac{\Sigma}{\Sigma}}^{d \Sigma}$, and by integration $S=C \log . \Sigma$, which is Fechner's "fundamental formula." But if the stimulus is just belowo the least observable amount, and be $=\mathbf{\Sigma}^{\circ}$, then substituting in the above formula we have $0=C \log . \Sigma^{\circ}$; from which Fechner derives formula No. 2 (the formula of measurement), namely, $S=C$ log. $\frac{\Sigma}{\Sigma^{\circ}}$, which means that the magnitude of the sensation is " negative," in case the stimulus sinks below the least observable $=\Sigma^{\prime}$. If two sensations $(S$ and $S$ ) are observably different, then $S-S$ $=C\left(\log . \Sigma-\log . \Sigma^{\prime}\right)$; this is called the "formula of difference," and means that the difference in the intensity of two sensations is proportional to the logarithm of the quotient of the magnitudes of their stimuli. 
By an extended series of experiments with weights ranging from 300 to 3,000 grammes Fechner ' employed the method of correct and mistaken cases to confirm Weber's law as applied to combined sensations of pressure and of the muscular sense. Some experiments were made with both hands; others with the right or left separately. The weight used to add or subtract was either 0.04 or 0.08 of the absolute weight. The results showed that the law held only approximately for all the series of experiments, and not absolutely for any one series. As calculated by G. E. Müller ${ }^{2}$ they give, instead of a constant quotient to express the degree of sensitiveness (as Weber's law requires), a quotient varying from ${ }_{4} \frac{1}{3} \cdot 6$ for weights of 300 grammes to $\frac{1}{73}$ for weights of 3,000 grammes. Nor can Fechner's effort to correct the variation, by introducing afterward a conjectural allowance for the weight of the arm itself, be considered successful. ${ }^{3}$ Biedermann and Löwit, by the method of just observable differences, obtained results departing widely from Weber's law." By experimenting with weights varying from 10 to 500 grammes they found that the sensitiveness to pressure rose with the increase of the weights from 10 to 400 grammes, and then fell off rapidly, as the following table will show:

Absolute weight.
Grammes.
10
50
100
200
300
400
450
500

Least observable difference. Grammes.

0.7

1.7

2.4

3.6

4.6

5.2

6.5

25.5

Quotient of sensitiveness.

7

7

$\frac{1}{16}$

$\frac{1}{29}$

$\frac{1}{4 x}$

$\frac{1}{86}$

$\frac{1}{6}$

$\frac{1}{17}$

$\frac{2}{69}$

$\frac{1}{20}$

The trustworthiness of these results is impaired, however, by the fact that no method, except the doubtful one of directing "attention" exclusively to the sensations of pressure, was employed to exclude the disturbing effect of the muscular sensations. The same observers concluded, also, that the fineness of the muscular sense, when isolated, does not vary according to Weber's law. They fixed it at $\frac{1}{2}$ for weights of 250 grammes, $\frac{1}{114}$ for weights of 2,500 grammes, $\frac{1}{98}$ for weights of 2,750 grammes.

That Weber's law does not hold good, near the lower limits, for

'Elemente d. Psychophysik, i., p. 183 f.

' Zur Grundlegung d. Psychophysik, p. 197.

${ }^{3}$ In Sachen d. Psychophysik, p. 198.

+ See Hering, Sitzgsber. d. Wiener Acad., LXXII., Abth. iii., p. 342 f. 
sensations of pressure, and of muscular innervation and movement, is admitted by all. The absolute sensitiveness of these sensations differs greatly, as has been shown (p. 346 f.), for different localities on the surface of the body. Aubert and Kammler found the lightest weight which produced a sensation of touch to be 0.002 gramme on the forehead, temples, and dorsal side of the forearm and hands; 0.003 gramme for the volar side of the forearm; 0.005 gramme for the nose, lips, chin, eyelids, and skin of abdomen ; 0.005-0.015 gramme for the volar side of the fingers; and 1 gramme for the fingernails and skin of the heel. This kind of sensitiveness has been thought to be chiefly dependent upon the number of the nervous elements present in the skin, its thickness, the character of its tension over the underlying parts, etc. ; but its variations are by no means parallel with those of the sharpness of the sense of locality. The foregoing and similar conclusions all need to be rerised in the light of Goldscheider's determinations of the pressurespots.

§11. Extraordinary difficulties accompany the attempt to apply Weber's law to sensations of temperature. As has already been seen (p. 350 f.), we do not know exactly what to measure-whether the rising and falling of the thermic apparatus, or its actual temperature in relation to its own zero-point - as constituting the quantitative changes in the stimuli. Even Fechner admits that Weber's law does not apply to the sensitiveness of the hand to changes in temperature when it is itself cooling off; but he thinks the law holds good approximately for degrees of warmth varying between $25^{\circ}$ and $37.5^{\circ}$ C. $\left(77^{\circ}-99.5^{\circ}\right.$ Fahr. $)$, if $18.71^{\circ}$ C. $\left(65.66^{\circ}\right.$ Fahr.) be taken as the zero-point. The assumption of this zero-point is, however, arbitrary. No general rule for the quantity of sensations of temperature can well be given except this : the skin is most sensitive to changes which lie near its own zero-point. In comparing two temperatures it is most favorable to nice discrimination that one should lie slightly above, the other slightly below, this point. The degrees of the thermometer between which the maximum of sensitiveness is attainable are given differently by different observers: By Nothnagel, $27^{\circ}-33^{\circ}$ C. $\left(80.6^{\circ}-91.4^{\circ}\right.$ Fahr. $)$; by Lindemann, $26^{\circ}-39^{\circ} \mathrm{C}$; ; by Alsberg, $35^{\circ}-39^{\circ} \mathrm{C}$; by Fechner, $12^{\circ}-$ $25^{\circ} \mathrm{C}$. - where it is so great as not to be easily measurable by a good quicksilver thermometer (about $\frac{1}{5}{ }^{\circ} \mathrm{Fahr}$.). Cold and heat alike, when applied for some time, reduce greatly the sensitiveness of the skin to minute changes of temperature; by heat it can be so dulled as not to distinguish alterations of less than $\frac{1}{2}^{\circ}$ or $\frac{3}{4}^{\circ} \mathrm{Fahr}$.;

'So Hering, see Hermann's Handb. d. Physiol., III., ii., p. 430. 
by cold it can be rendered insensible to changes measuring from $2^{\circ}$ to $5 \frac{1}{2}^{\circ}$.

We have already seen (comp. p. 348 f.) that the sense of temperature depends for its fineness upon the extent and locality of the surface excited. Weber found that water at $29 \frac{1}{2}^{\circ} \mathrm{R}$., in which the whole hand was immersed, seemed warmer than that at $32^{\prime}$ R., to a single finger. Nothnagel placed the following values upon the fineness of discrimination, for minute variations in temperature, of dif. ferent parts of the body : Middle breast, $0.6^{\circ} \mathrm{C}$; sides of the same, $0.4^{\circ}$; middle of the back, $1.2^{\circ}$; sides of the same, $0.9^{\circ}$; hollow of the hand, $0.5^{\circ}-0.4^{\circ}$; back of the same, $0.3^{\circ}$; parts of upper and lower arm, $0.2^{\circ}$; cheeks, $0.4^{\circ}-0.2^{\circ}$; temples, $0.4^{\circ}-0.3^{\circ}$. More recent investigations have shown that the table of sensitiveness for the different parts of the body must take account of the division of the temperaturesense into two species, and of the locality of the heat-spots and cold-spots in all such different parts. On the basis of experiment with areas of the skin whose topography with respect to the temperature-sense had previously been investigated, Goldscheider has given a lengthy statement ${ }^{2}$ of the sensitiveness of different parts of the body.

Thus he finds that the skin of the head is, in general, little developed for the sense of cold, and only in a few places for the sense of heat. The sensitiveness of the forehead to cold is intense, but to heat only moderate; that of the breast to cold moderate along the sternum, and elsewhere very intense, while to heat it is only moderate except near the nipples; that of the back everywhere very intense to cold, and only moderate to heat; while in all parts of the hand the intensity of sensitiveness to both cold and heat is alike.

In general, the skin in the median line of the body seems much less sensitive to changes in temperature than at its sides; and the number of thermic elements (according to Goldscheider, the distributory fibrils of the temperature-nerves), the thickness of the skin, etc., are determining factors.

$\S 12$. The possibility of executing or appreciating a musical passage in which the intensity of the successive notes is brought to a certain standard of memory, or in which these notes are nicely shaded so as to constitute a crescendo or a diminuendo, appears to depend upon applying to sensations of sound some law resembling that of Weber. It is partly by comparing such sensations with their images in memory that the singer or player reproduces certain

\footnotetext{
'See the Archiv f. Anat. u. Physiol., Physiolog. Abth., 1885, SupplementBand, pp. 60 ff.
} 
notes previously executed, with about the same stress of tone. ${ }^{1}$ Moreover, in order to shade the relative intensities of successive tones, our appreciation of their differences needs to be much greater for those that have a low degree of intensity. Many obstacles, however, stand in the way of determining either the lower limit or the least observable difference for sensations of sound. The general difficulty which belongs to investigating the intensity of sensations, even under the most farorable circumstances, is here enhanced by the facts, that the pitch and timbre of each clang have much to do with our judgment of its strength; that different ears differ so widely in their organic susceptibility, while the mind is peculiarly sensitive to changes of feeling and judgment connected with sensations of sound, and thus very weak sensations are vacillating and unsteady in cousciousness, and sounds appear and disappear in the ear while the degree of stimulus and of attention are unchanged; that the reflection and interference of the acoustic nerves, their distance and direction, and the absence or presence of " entotic" sounds, are so influential ; and, finally, that it is impossible to discover a sounding apparatus of definitely ascertainable and uniform intensity of action.

$\S 13$. None of the means employed for determining the amount of stimulus necessary to produce the weakest sensations of sound, or the least observable differences in such sensations, are entirely satisfactory. The method of listening to noises made by falling weights is rendered uncertain by the fact that the character, both of the weight and of the surface on which it strikes, has so much influence. Moreover, it is a matter of dispute whether the intensity of the stimulus is to be measured by the product of the mass into the height from which the body falls $(m \times h)$ or into the squareroot of that height $(m \times \sqrt{h})$. It is possible that neither of these measurements is exact. ${ }^{2}$ Assuming the former to be correct (noise $=m \times h)$, by using a sound-pendulum $\mathrm{A}$. W. Volkmann found that differences in intensity are observable when they stand in the proportion $3: 4$. Vierordt, on the other hand, concluded that the latter measurement (noise $=m \sqrt{h}$ ) is more nearly correct; and by assuming Vierordt's view, and using iron balls that fell vertically on a vibrating plate, Nörr ${ }^{3}$ attempted to fix a unit of measurement. This unit he made $=1,500$ milligramme-millimetres with the ear distant $50 \mathrm{ctm}$. from the source of the sound. Experimenting with sounds

${ }^{1}$ Comp. Stumpf, Tonpsychologie, I., p. 345 f.

${ }^{2}$ Comp. Wundt, Physiolog. Psychologie, i., p. 341 and note ; and E. Tischer, in Philosophische Studien, I., heft 4, p. $\tilde{5} 43 \mathrm{f}$.

'Zeitschrift f. Biologie, 1879, XV., p. 297 f. 
ranging in intensity from those a little above the least observable to those of unpleasant strength (1.71-524167.8 times the unit), and dividing the entire scale into 7 groups, within each of which about 1,000 experiments were conducted, he found that the proportion of right guesses to the entire number made $\left(\frac{r}{n}\right)$ remained approximately constant - that is, that Weber's law holds for sounds of varying intensity. A large, allowance, however, had to be made for relations of time; the percentage of correct guesses being about 8.7 larger when the sound of greater inteusity followed that of less intensity.

More recently, E. Tischer ${ }^{2}$ has apparently added some evidence to the validity of Weber's law by experimenting with an improved form of the method of Vierordt and Nörr. Keeping one of the two sounds to be compared at a constant intensity, he increased or diminished the other until from 4 to 6 successive correct guesses as to their relative value were obtained. But the fact that, when the second stimulus was diminished until certainty of judgment was obtained, very considerable unexplained variations from the results expected by Weber's law occurred leaves much doubt still hanging over the matter. In order to harmonize the conflicting results, the proposal has been made to introduce another function into the formula, noise $=m h$ or $m \sqrt{h}$. All the investigations, therefore, still leave the question of the applicability of Weber's law to sensations of sound in a rather uncertain condition.

Little or nothing has been accomplished by experiment to determine whether the same law applies to the intensity of musical tones. Among the various factors which enter into our judgment of the intensity of tones, the "color-clang" is especially influential." Absolute pitch and intervals of pitch are also very importaut. In general, tones and noises of a higher pitch, with an equal objective intensity of stimulus, are judged to be louder than those of a lower pitch. Künig showed that a tuning-fork of the pitch c must have about four times the amplitude of vibration required by one of the pitch $C^{\prime}$, in order to produce upon the ear the same effect from the same distance.

$\S 14$. The various attempts to determine the lower limit of sound for the liuman ear have not resulted in any precise statement. Schafhüutl, after experiments in as near perfect stillness as possible, at midnight, fixed the limit at the noise made by a cork ball of 1 milligramme weight (about $0.0154 \mathrm{gr}$.) falling from a height of 1

\footnotetext{
${ }^{1}$ Wundt's Philosophische Studien, 1883, I. . heft 4, pp. 495 ff.

' Comp. Stumpf, Tonpsychologie, I., p. 364 f.
} 
millimetre $(0.03937$ inch). Boltzmann and Töpler have reached results which Hensen ${ }^{1}$ considers to be as accurate as possible. By measuring the compression of the air at the end of an organ-pipe of 181 vibrations per second, they calculated that, even under circumstances not as favorable as possible, the ear responds with sensation to an amplitude in the vibration of the molecules of the air not more than $0.00004 \mathrm{~mm}$. at the ear, or about $\frac{1}{10}$ the wave-length of green light. The mechanical work done upon the ear-drum in a single vibration of such small intensity is reckoned at not more than $\frac{1}{343}$ billionth kilogrammetre; or only about $\frac{1}{1} \frac{1}{7}$ of that done upon the same surface of the pupils of the eye by a single candle at the same distance. These calculations indicate that the motions in the cochlea which excite the end-organs of sense are astonishingly minute-far too minute to be observed even by the microscope. Yet the sharpness of hearing may be enormously increased by disease.

\&15. Judgments of the intensity of sounds are dependent also upon practice, and upon other psycho-physical conditions such as determine the nicety of all judgments of quality. Small impressions of noise are apt to have their intensity underestimated; the inclination to do this has been attributed to the influence of our custom of withdrawing attention from them altogether under ordinary circumstances. ${ }^{2}$ The fact that sounds to which we become accustomed lose most of their intensity in consciousness must be explained chiefly under the same law of mental habit ; it canuot, on the other hand, be largely due to the physiological law of exhaustion.

$\S 16$. Attention was early called to the law of judgment in estimating the quantitative relations of sensations of sight, on account of its connection with astronomical observation. In the preceding century French physicists had already begun to investigate the sensitiveness of the eye to varying intensities of light. Bouguer, in answer to the question, What force must a light have in order to make a more feeble one disappear? placed the fraction of least observable difference in the intensities of two shadows at $\frac{1}{64}$. That the magnitudes of the stars are not to be classified according to their absolute brightness as determined by photometric observations was, of course, assumed by Sir John Herschel when he made the latter vary in the series $1: \frac{1}{4}: \frac{1}{8}: \frac{1}{16}$, while the former vary in the series $1: 2: 3: 4$. That the least observable difference in the intensity of two sensations of sight is absolutely much smaller for

'See Hermann's Handb. d. Physiol., III., ii , p. 117 f.

${ }^{2}$ So Stumpf, Tonpsychologie, I., p. 388. 
those of the lowest grade of intensity is a truth needed to explain many every-day experiences. For example, the finer gradations of shade in a lithograph or photograph are not lost when we take it from the open sunlight into a rather dimly lighted room; we can also observe them through smoked glass, if it be not too black. Through the same media we can measure rather delicate shades of brightness on the clouds. We observe, however, that in all such cases either too great or too weak intensity of the light destroys our power to distinguish the finest gradations of its intensity.

$\$ 17$. It has already been shown (p. 326) that the retina is never free from light of its own which has a varying intensity ; this fact greatly increases the difficulty of fixing accurately either the lower limit or the least observable difference of visual sensations. In the efiort to apply Weber's law to sensations of color, the laws of change in the quality operate to obscure the laws of change in the quantity of the sensations. Experiments with shadows for the sake of testing Weber's law were first conducted by A. W. Volkmann and others, under the direction of Fechner.' By measuring the distance to which a candle must be remored from an object in order that the shadow produced by its light might disappear in that of another candle of like intensity situated at a fixed near distance from the object, the quotient for the least observable difference was found to be I00. This quotient was also found to remain nearly constant for absolute intensities varying from 1 to 38.79 . If, lowerer, the light of the background diminished to 0.36 in intensity, marked rariations in the law occurred; the difference in the brightness of the two shadows had then to be greater than $\frac{1}{100}$ to be observable. Later experiments of the same observer yielded results less favorable to Weber's law. ${ }^{2}$ The quotient was found to vary from $\frac{15.6}{65}$ for weak intensities of light to $\frac{1}{95}$ for stronger intensities.

By using rotating disks and comparing the grayish circles made upon them when revolving rapidly, through the admixture of small black stripes with the white of their surfaces ("Masson's Disks"), Helmholtz ${ }^{3}$ found the medium value of the quotient of least observable difference to be $\frac{1}{13}$; this quotient is not constant, however, and increases, especially for sensations near the upper or the lower limit. By changing the method somewhat, Aubert obtained a variation of $\frac{1}{86}$ to $T^{\frac{1}{20}}$ in the degree of sensitiveness to differences in the brightness of lights, even when not going above the middle of the scale of intensity. Experiments with such intensities as lie nearest

${ }^{1}$ See Elemente d. Psychophysik, p. 148 f.

A. W. Volkmann, Physiolog. Untersuchungen, I., p. 56 f.

${ }^{3}$ Physiologische Optik, p. 315 f. 
the limits showed much greater departures from Weber's law. Just above the lower limit, an addition of even $\frac{1}{4}$ to $\frac{1}{3}$ to the stimulus might be necessary in order to produce an observable difference in the resulting sensation. Similar results have been obtained by Delboeuf, but, on the whole, more favorable to Weber's law than the results of Aubert.

A more accurate and carefully guarded series of experiments than any of the foregoing is recently reported by Dr. Emil Kraepelin. ${ }^{1}$ This experimenter used the method of just observable differences as applied to Masson's disks when looked at through gray glasses of varying intensity. The utmost care seems to have been taken to exclude disturbances from changes in the adjustment of the eye, retinal exhaustion, reflection of light from surrounding objects, etc. Three groups of experiments were conducted-one by daylight, one by candlelight, one by lamplight. Both eyes were experimented upon; and both directions of alteration in the intensity of the stimulus (stronger following weaker, and vice versa) were employed. Kraepelin concludes that for the unexhausted eye, with a good power of accommodation, the fraction which gives the least observable difference remains constant, while the intensity of the light varies between values of 1,000 and 9.61 of absolute intensity as fixed for his experiments. That is, within these limits the law of Weber holds good as expressing with closely approximating accuracy the results of experiment.

The experiments of Dobrowolsky ${ }^{2}$ and Lamansky ${ }^{3}$ with light of the different spectral color-tones shows that, with these sensations also, Weber's law holds approximately good for moderate intensities, but is subject to considerable variations as we approach the upper and the lower limits. The former used the method of comparing a white surface with one in which colored light had been mixed with the white. On changing the absolute intensity of the light between values of 1 and 0.0302 , only a slight variation in the quotient indicating the least obserrable difference of intensity appeared for the color red. This quotient was found, by the same observer, ${ }^{4}$ however, to be very different for different color-tones : thus for red, $\frac{1}{14}$; yellow, $\frac{1}{46}$; green, $\frac{1}{35}$; blue, $\frac{1}{13}$; violet, $\frac{1}{26 \frac{1}{8}}$. Lamansky and others have made the sensitiveness to changes in the intensity of color-tones greatest for green instead of violet; and have obtained other results different from those obtained by Dobrowolsky. $\S 18$. The minimum of the intensity of light appreciable by the

\footnotetext{
' In Wundt's Philosophische Studien, 1884, II., heft 2, pp. 306 ff.

${ }^{2}$ Pflüger's Archiv, xii., p. $441 \mathrm{f}$.

${ }^{3}$ Archiv f. Ophthalmologie, XVII., i., p. $123 \mathrm{f}$. "Ibid., XVIII., i., p. $74 \mathrm{f}$.
} 
eye under the most favorable circumstances was fixed by Aubert at $\frac{1}{300}$ of that reflected from white paper in the light of the full-moon. This result can only be considered as approximate. The individual factor in all such calculations must be held to be very large and variable; especially, perhaps, if we admit that there is a class of so-called "sensitives" to whom the ends of an electro-magnet when excited appear luminous, as Reichenbach's experiments seem to show. Weber applied his own law to so-called extensive sensations of sight. He showed that in judging of the comparative length of lines the least observable difference is, for each person, a tolerably constant fraction of the absolute length of the line with which the comparison is made. This fraction is different for different persons; and has a range from $\frac{1}{50}$ to $\frac{1}{100}$. Fechner' defends the validity of the law for liues of lengths varying between 10 and $240 \mathrm{~mm}$. ( $\frac{2}{5}$ to $9 \frac{1}{2} \mathrm{in}$.), with the eye removed from $1 \mathrm{ft}$. to $800 \mathrm{~mm}$. (12-32 in.). The lower limit for such cases has been fixed by A. W. Volkmann at lines of length from 0.2 to $3.6 \mathrm{~mm}$. It is obvious, lowever, that we are here not dealing with pure quantity of visual sensations, but with judgments of local relation which, in case the eyes are moved, have their basis, at least partly,,in our power to discriminate minute differences in the sensations of the muscular sense connected with such morements.

$\S 19$. The law of Weber can, of course, derive little or no support from sensatious of taste and smell. In the case of these two senses our knowledge of both series of quantities-of the intensity of the stimulus and of the amount of specific sensation which results from its application-is altogether too inadequate to admit of trustworthy comparison. We cannot measure forms of energy like those by which smellable particles and tastable solutions act on the end-organs of sense, until we have a unit of measurement and some information as to what the object is to which the standard should be applied. Nor can we compare amounts of sensations that are so largely matters of individual origin and capricious change, and that are so overlaid with other forms of feeling, as are the sensations of these senses. Moreorer, the element of time-both as respects the interval elapsing between the two sensations compared and also the order in which the sensations follow each other -is here a very important influence.

The intensity of taste depends upon a variety of circumstances besides the objective quantity of the stimulus. Among these circumstances is the extent of surface excited. Camerer ${ }^{2}$ found by

\footnotetext{
${ }^{1}$ Elemente d. Psychophysik, i, p 211 f.

${ }^{2}$ See Zeitschr. f. Biologie, 1870, VI., p. $440 \mathrm{f}$.
} 
experimenting with common salt in solutions of different degrees of concentration that the number of correct guesses increased almost in exact proportion to the number of gustatory papillæ upon which the solutions were placed. Certain mechanical and thermic conditions also have a great influence. Substances even in fluid form, when quickly swallowed, have little taste; pressing and rubbing against the gustatory organs, movement of the tastable matter in the mouth, increase the excitatory effect of the stimuli. It is doubtful whether this effect is due solely to the mechanical result of spreading the stimulus over the surface and urging it into the pores against the end-organs of the sense, or in part also to some direct physiological cause. The influence of temperature on the intensity of sensations of taste is well known. Weber showed that if the tongue is held for $\frac{1}{2}$ to 1 minute in very cold water, or in water of about $125^{\circ}$ Fahr., the sweet taste of sugar can no longer be perceived. Cold also destroys for a time the susceptibility to bitter tastes. Keppler 'endearored to test Weber's law by determining the sensitiveness to minute changes in the four principal kinds of taste; and arrived at a negative result. Fechner, however, considers that Keppler's experiments with common salt confirm Weber's law, and that his other experiments were not adapted to yield any assured result. We can only repeat the statement that other causes than mere increase in the quantity of the stimulus so largely determine the intensity of the resulting sensations as to discredit any arguments from the experiments either for or against applying Weber's law to sensations of taste.

$\S 20$. The experiments of Valentin ${ }^{2}$ and others, to determine how weak solutions of rarious substances will excite the end-organs of taste, are chiefly valuable as gratifying our curiosity. The figures are not to be accepted as exact, but as showing in general the extreme fineness of this sense, and the great difference of different substances in their power to excite it. Valentin found, for example, that 0.24 gramme of a solution containing 1.2 per cent. of canesugar excited the sensation of sweet; a solution containing $\frac{1}{2} \frac{1}{2} 6$ part of common salt was scarcely detectable; of sulpluuric acid $\frac{1}{100000}$ could be discerned, $\frac{100000}{100000}$ not; extract of aloes containing $90 \frac{1}{0000}$ could be distinguished from distilled water; $\frac{1}{3 \frac{1}{300}}$ of sulphate of quinine was plainly observable, and the observer thought he could detect a slight trace of bitter when the solution

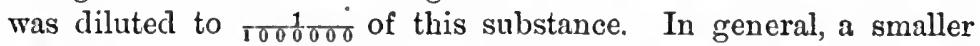
absolute quantity of stimulus, when in a relatively concentrated

'Pflüger's Archiv, 1869, ii., p. 449 f.

2 In his Lehrb. d. Physiol. d. Menschen, 2 ed., Abth. 2. 
solution, will suffice to excite the end-organs of taste. ${ }^{1}$ It will readily be seen that the minimum of some of these substances which will give rise to a sensation under the most favorable circumstances is exceedingly small.

$\S 21$. The intensity of sensations of smell is also largely dependent on other causes than changes in the quantity of the stimuli. The amount of sensation appears to be largely governed by the extent of surface excited; since it is greater when we smell with both nostrils, and with the current of inspiration which carries the exciting particles over more of the sensitive membrane. No assured results on this point, however, have yet been reached. Valentin supposes that a smaller number of odorous particles will excite seusation if presented in a concentrated rather than a dilute form. When the intensity of the stimulus increases beyond a certain point, the character of the resulting sensation changes-oftentimes from a pleasant to an unpleasant tone of feeling. All are familial with the fact that a large increase of some smells-for example, musk-does not give the same kind of sensation. This sense has a great degree of "sharpness," or power to be excited by small quantities of stimulus, as distinguished from "fineness," or power to distinguish minute variations in the sensations. It is undoubtedly different in different species of animals, as dependent upon unknown differences in their psycloo-physical constitution; but it is tolerably uniform among men where there is the same cultivation of it, and the same concentration of attention. It is well known that certain animals have an astonishing fineness of smell, and are able by it even to detect the individual variations that are quite imperceptible to man. Little value can be attached to the results reached by experiments to fix the least number of smellable substances which can excite the liuman end-organs of this sense. In general, we can say that incredibly small quantities of some substances will suffice. Valentin found that a current of air containing $\frac{1}{200000}$ of vapor of bromine excited a strong unpleasant sensation. Atmosphere polluted with even $\frac{1}{1700000}$ of sulphuretted hydrogen could be detected. It was calculated by this observer that $\frac{1}{2000000}$ of a milligramme of alcoholic extract of musk is about as little as can be perceived. The effect of constant over-excitement of the organs of this sense, in deadening their sensibility, is too well known to require illustration. No argument for or against Weber's law can safely be drawn from sensations of smell.

$\S 22$. A review of the preceding facts confirms what was previ' See Camerer's table in Pllüger's Archiv, ii., p. 322. 
ously said as to the unsatisfactory nature of the evidence adduced in proof of the principle which is thought to control the quantitative relations of our sensations and their stimuli. At best, Weber's law is only an approximately correct statement of what holds true of the relative intensity of certain sensations of sight and hearing, and, less exactly, of pressure and the muscular sense, when these sensations are of moderate strength, and other causes for variations in their intensity, besides objective changes in the amount of the stimulus, are as far as possible excluded. In general, it is true that the amount of matter pressing on the skin, or lifted by moving the arm or leg, as well as the intensity of the waves of light and sound acting on eye and ear, must increase much more rapidly than does the intensity of the resulting sensations, as estimated by comparing them with each other in consciousness. Within certain limits for the above-mentioned four kinds of sensation, the latter scale of quantities is ordinarily related to the former about as an arithmetical to a geometrical series. But other conditions than mere increase in the objective quantity of the stimulus largely determine its effect upon the resulting amount of sensation. Stimuli and sensations are not connected quantitatively in such a simple manner that we can measure one off in terms of the other; so much feeling for so much amplitude of wave-lengths, or work done on the end-organs by mechanical pressure. Numerous factors, some of which are individual and extremely obscure and variable, constantly mix with the purely quantitative relations between sensations and their stimuli.

$\S 23$. The value of Weber's law is so restricted, even as stating a general fact of experience, that it would seem scarcely necessary to discuss at length its higher significance. Three possible modes of explanation have all had their defenders; these are the physiological, the psycho-physical, and the psychological. The first of the three assumes that the physical construction of the nervous system, including chiefly the end-organs of sense and their central representatives and connections, is such as to supply the reason for this relation between the intensity of sensations and that of their stimuli. And certainly, if we were to make any assumption, it would be that the quantitative relation between the last antecedent molecular changes in the brain and the mental changes to which they give rise, is one of simple proportion; the more work done by means of the excitation in the appropriate cerebral centres, the more of physical basis laid, as it were, for a resulting quantity of psychical movement.

If, then, the sensations vary in quantity in an arithmetical pro- 
portion, while their external stimuli vary in a geometrical proportion, the explanation of the fact must be found somewhere in the chain of events between the external stimuli and the nerve-commotions set up as a result in the appropriate centres of the brain. And without doubt the explanation of so much as is true of Weber's law lies largely in physiological causes ; but our knowledge of the structure and function of the end-organs of sense, and especially of their cerebral representative elements, is so incomplete that no satisfactory statements can be made on this point. In all of the senses, the end-organs profoundly modify the intensity of the stimulus they receive. In the so-called chemical senses (smell, taste, sight) a profound quantitative modification takes place, even before the stimulus reaches the fibrils of the sensory nerve. In the case of the mechanical sense of hearing we cannot say how much of the effect stated in Weber's law may not have been gained even before the acoustic waves set agoing the nervous elements of the organ of Corti. As to profounder modifications in the same direction by reason of the interaction of different nerve-elements in the brain we are yet more ignorant. And although we can have little confidence in Wundt's " theory of an "apperception-centre" and its influence in accounting for Weber's law, we cannot deny the general assumption on which that theory is based.

The psycho-physical explanation of Weber's law is that adopted by Fechner. This explanation insists upon making the law one of the utmost generality and of the highest import as stating the relations between organic and spiritual activities. Although Fechner's view confessedly grew out of his speculation that body and mind are only two phenomenal aspects, as it were, of one and the same underlying reality, ${ }^{2}$ it has been defended by him with a great amount of mathematical science and experimental research. No other form of explanation, however, takes us so much into the regions of utter obscurity. Why the quantitative relations of body and mind should be such, and such only, that a geometrical series of changes in the one should invariably be represented by an arithmetical series of changes in the other, must indeed remain an ultimate mystery. And the experimental proof of Weber's law is as yet much too incomplete to make us ready to accept it as an ultimate psycho-physical principle.

The psychological explanation of Weber's law resolves it into a special case under the greater law of the relativity of our inner

'Comp. his Physiol. Psychologie, i., p. 351 f., and ii., p. 207 f.; also Philosophische Studien, 1883, II., heft 1, p. $31 \mathrm{f}$.

${ }^{2}$ In proof, see his Revision d. Hauptpuncte d. Psychophysik, p. $13 \mathrm{f}$. 
states. It is not so much, then, a law of the absolute quantity of sensations as dependent on stimuli, but rather a law of our apprehension in consciousness of the relation of our own feelings. In general, it may be said that every mental state has its value determined, both as respects its quality and its so-called quantity, by its relation to other states. It is the amount of change rather than the absolute amount of feeling which the mental apperception estimates. That the psychological explanation is needed to account for the facts there can be no doubt when we consider how important are the elements of attention, mental habit, power of acute discrimination, etc., in determining our estimates of the quantitative relations of our sensations. Estimates-that is, acts of the comparing judgment, are involved in the experience upon which reliance is placed for a demonstration of Weber's law. Further discussion of the significance and extent in application of the mental law of relativity will appear in other connections. The subject of the quantity of sensation as a matter of psycho-physical investigation is fitly closed with the following quotation from Wundt: "In the imperfect condition of cerebral physiology, we are not seldom in a position to recognize the psychological formulating of certain laws, the physiological meaning of which still lies in obscurity or belongs to the domain of hypothesis."

${ }^{1}$ Physiol. Psychologie, i., p. 352. 


\section{CHAPTER VI.}

\section{THE PRESENTATIONS OF SENSE.}

§1. Sensatrons are, primarily considered, modes of our being affected; but the objects of sense are known as real beings, which are assumed to exist independent of the affections of our minds, and to have their inherent qualities disclosed to us through the operation of the senses. There is a wide interval, however, betrieen our consciousness of being ourselves affected and the perception of "things" as having qualities resembling our mental states or revealed by them. This interval is filled, in nature, by the development of mind as conditioned upon its environment of sense-stimuli; it must be filled in psychological theory, by a description of the process of development. Physiological Psychology constructs such theory as much as possible on a basis of experiment to determine how the various steps in the mental development are related to the changes which the stimuli produce in the nerrous system, especially, of course, in the organs of sense. Upon this work of construction it has expended its choicest resources and utmost ingenuity. Its efforts are yet far from being completely successful. Many of the secondary principles, and even questions of fact, are still un. settled; no theory of perception that will account satisfactorily for all the admitted truths has hitherto been discovered. Nor is this lack of complete success surprising, when we consider how rapid and complex are the processes which combine to form the world of sensible objects; as well as how entire is the loss suffered by memory and consciousness of those details which served as a basis for the earlier and most significant stages of the development.

Nor should we fail to take account of the fact that the mechanism of both nerrous system and mind operates as rendered native to the individual by his inheriting the results of many ages of ancestral experience. The psychologist does not remember by what stages he first learned to see or feel the extended and external objects of sense. The child cannot describe the process to the psychologist; the child is farther from his own infantile experience in this regard than the philosopher is from that of the child. It is 
not even likely that, if the infant were endowed with the developed power of searching his own consciousuess, and of describing its contents, he could discover and impart what is needed in order to explain the process of his own mental development. In all stages of human growth the analyzable contents of consciousness represent only very imperfectly the nature of the basis upon which they rest.

$\$ 2$. Scientific analysis of the process of perception corrects in many particulars the so-called "common-sense" view. The conviction which everyone has on opening the eyes upon a landscape, for example, is undoubtedly that of being immediately impressed with a faithful copy of extra-mental reality. Some of the objects are seen as larger, others smaller, some in the foreground near by, and others more remote; but all have that solid, substantial character which makes them things as distinguished from the images of revery or dreaming. But it is precisely the acquired power thus to construct the landscape which psychological science tries to explain. The ordinary conviction accepts the apparent fact of an immediate and certain knowledge of these things through the eyes, as though it were matterof-course and needed no explanation. We must begin by removing certain assumptions obviously involved in the ordinary conviction.

The forms of being and happening in the world, outside of the body, furnish in themselves no explanation whatever of the presentations of sense. ${ }^{1}$ This is as true of the colored or smooth extension of an object as it is of its sweet taste or disagreeable smell. Whatever exists extra-mentally, so far as its pure existence goes, is of no account to the mind. It is only as so-called "things" act upon us, or -in other words - get themselves expressed within the mind, by causing changes in our mental states, that any theory of knowledge by the senses can make use of them. Centuries ago the popular feeling was framed into a doctrine that semi-spiritualized copies of the material realities enter the body through the senses and meet the mind somewhere within; or, that the mind itself, passing out through the openings of sense in semi-materialized form, embraces and so knows these realities. The time for all similar crude theories of knowledge by the senses ought, however, to have long gone by. And yet fragments or suggestions of essentially the same assumptions are still frequent enough.

What is true of all that exists and happens outside of the body is just as true of all the bodily conditions and processes. Strictly speaking, they can in themselves furnish no explanation for the rise and development of the presentations of seuse. Only mental factors

${ }^{1}$ Comp. Volkmann von Volkmar, Lehrbuch d. Psychologie, Cöthen, 1885, II., p. 1 f. ; and Lotze, Medicinische Psychologie, Leipzig, 1852, p. 325 f. 
can be built into mental products. The simple sensations are in themselves always psychical phenomena, and are to be referred, as modes of its being and action, to the subject called " mind." It is only when considered in this way that they afford, by their characteristic qualities and modes of combination, any explanation of the resulting knowledge of things. The image on the retina, for example, is a necessary physical condition of the clear vision of outside objects; it may also become an object for the inspection of another observer. But the retinal image never becomes a kind of inner object for one's own brain or mind. Nothing in its construction, in itself considered-that is, as independent of the system of localized sensations which result on the other or psychical sicle of the transaction-helps to explain the act of vision. What is true of the peripheral is also true of the central organs of sense. There is no image in the brain transmitted in exact copy from the retina by the optic nerve to its central nerve-fibres and nerve-cells; if there were such a brain-image, we should need another eye connected with a second brain and mind to read it. The mind is never to be conceived of as contemplating a spatial picture of its object formed somewhere within the cerebral substance.

Even more obvious is the worthlessness, for purposes of strictly psychological analysis, of all theory as to the precise spatial arrangement of the fibrils of sensory nerves within the skin or muscular fibre. That such fibrils exist in the muscles has apparently been demonstrated by Sachs and others (comp. Chap. IV., $\$ 20$ ) ; the nervous impulses occasioned in them, when conveyed to the central organs, are probably one main physical basis of those feelings of innervation, of being in the body, etc., which enter as essential factors into the spatial perception-field of our own periphery. ${ }^{1}$ But it is the muscular sensations, as modes of the affection of mind, which perform this office. We have nothing approaching an immediate cognition of the extended net-work of sensory fibrils in the skin or muscles; much less of the extended muscle or area of the skin. No copy in space-form of the various simultaneous or successive rubbings and stretchings of these peripheral fibrils is propagated to the brain ; and if it were, the mind could not be regarded as taking account of any of these neural processes.

A further negative statement may be made with entire confidence. The place at which each organ of sense is found in the periphery of the body, or the place at which any such organ is acted on by the stimulus, cannot of itself furnish a reason for the spatial perception of such place and for distinguishing it from other places near or

${ }^{\prime}$ See an article in Mind, by G. Stanley Hall, III. (1878), pp. 433 ff. 
remote. To suppose this is virtually to return to an ancient, discarded theory ; it is to regard the mind as well diffused through the extended body, especially over its nervous periphery; and as thus constantly sensing the condition of this periphery with respect to excitation, as well as the spatial relation of its various excited and non-excited parts. The locality where a stimulus is applied, except as this locality affects the mental coloring or qualitative shading of the sensations which result, is a matter of complete indifference to the mind.

$\S 3$. In contrast to all theories like those just rejected, the following positive affirmations are to be held firmly. Sensations, as the elements of so-called "presentations of sense" are psychical states whose place--so far as they can be said to have one-is the mind. The transference of these sensations from mere mental states to physical processes located in the periphery of the body, or to qualities of things projected in space external to the body, is a mental act. It may rather be said to be a mental achievement ; for it is an act which in its perfection results from a long and intricate process of development. The product of this act, the presentation of sense (or, considered objectively, the "thing" as known by the senses) has characteristics that do not belong to the simple sensations out of which scientific experiment and theory show it to be composed. The presentation of sense has "space-form ;" it is extended, and consists of an indefinite number of visible or tangible parts that are systematically arranged beside each other into a continuous whole; it is related with respect to position, magnitude, etc., to other similar objects of sense. Certain compound sensations-as, of light and color, of smoothness and hardness-are not regarded merely as psychical acts whose cause lies in the extended object; they are regarded as qualities of its surface, and appear to belong to it as forms of its objective being. Such a result, however, must be regarded as brought about by the action of mind, in forms and according to laws of its own.

The one characteristic which the presentations of sense possess, but which does not belong to the simple sensations that are their factors, is space-form. "Space-form" (whatever metaplysics may decide to be the nature, origin, and ralidity of our idea of space) must be regarded by psychology simply as the mental form of the presentations of sense. The problem which physiological psychology has to solve in this direction may then be stated as follows: On the basis of what combinations of physical processes of sense do the different resulting sensations come to be combined into presentations of sense under the new characteristic of space-form? 
$\S 4$. The most complete answer possible to the question just raised is obliged to recognize the following particular truths :

(1) A combination (or "synthesis," or "association") of two or more qualitatively different series of sensations is ordinarily-if not absolutely-necessary in order that presentations of sense in spaceform may be constructed. If our sense-perception were all by a single organ, or by a single activity of one organ, the objects of sense would largely, if not wholly, lose their present characteristics of position and extension in space. A series of sensations of one kind only, like the pure differences in pitch of musical tone, or of degrees of brightness and saturation of color-tone, or of pressure, temperature, or muscular innervation, is not adapted to form the material for constructing extended objects of sense.

(2) The characteristic differences in quality of the sensations of some of the senses, and so their adaptability to form graded series, are such as to fit these sensations for combination with other similar sensations into the presentations of sense under space-form; the sensations of other senses have not these characteristic differences and this adaptability. We may then speak of peculiarly "spatial series " of sensations, ${ }^{1}$ and of other series of sensations as nonspatial. By this term, however, it must not be understood that any sensations, as such (or quoad sensations), are extended in space, or can come by any process of theoretical manipulation to be endowed with extension.

The sensations of smell are manifestly not fitted to form a so-called spatial series; indeed, they are incapable of being arranged in any series at all. An experience consisting wholly of sensations of smell would have no elements from which to construct objects of sense. The same thing is true of pure sensations of taste and of sound. On the contrary, the various series of complex sensations that come through the eye and skin (including those of the muscular sense) are qualitatively adapted to enter into such relations to each other as shall give a ground in their combined existence for a perception of things. Accordingly the eye and skin are the socalled "geometrical senses."

(3) The locally different parts of the organ of sense-if this organ is itself to become known (as in the case of the skin), or if through its being stimulated at these parts an extended object outside of the body is to be perceived (as in the case of both skin and

${ }^{1}$ Comp. Volkmann von Volkmar, Lehrb. d. Psychologie, II., pp. 36 ff.; whose very interesting theory of the origin of space-intuitions can, however, no more be pronounced wholly satisfactory than the similar theory of Bain. 
eye)-must each have some mental representative in the sensations which stimulation of each calls forth. As places in material substance, or as parts of the organism, in themselves, they have no immediate significance for the mind. But a psychological equivalent or representative they must have. To this principle is due the fact that those senses are not "geometrical senses" in which we cannot vary, largely at pleasure, the locality to which the stimulus is applied. It is therefore assumed that every sensation, besides its general characteristic quality as sensation of this or that particular sense, must have a peculiar "local stamp," or shade, or mixture of quality, dependent upon the place of the organ at which the stimulus is applied; otherwise such sensation cannot serve as a factor in the construction of an extended object of sense. Such peculiar local stamp, or shade, or mixture of quality is a so-called "local sign." It is to Lotze that we owe the first elaborate theory of "local signs," and of their relation to the formation of the presentations of sense. The theory, as he truly says, is an indispensable assumption of every satisfactory account of perception by the senses. ${ }^{1}$ In what, precisely, these so-called local signs consist has been disputed by those who agree in holding that the explanation of the facts requires the assumption.

(4) Various stages in the process of elaborating the presentations of sense from the material of simple sensations must be recognized. Thus the construction of a retinal field of vision is a less elaborate work, both of the mechanism and of the mind, than the construction of that more objective field in which real things are seen with solid forms and set at varying distances. So, too, the knowledge of the things we handle-the fork, the tool, the penstands at a farther remove from the simplest perceptions of touch than does the discrimination of one area at the surface of the body as warmer or under more pressure than the surrouncling spots. Two noteworthy stages, or "epoch-making" achievements, in the process of elaborating the presentations of sense require a special consideration. These are "localization," or the transference of the composite sensations from mere states of the mind to processes or conditions recognized as taking place at more or less definitely fixed points or areas of the body ; and "eccentric projection" (sometimes called "eccentric perception"), or the giving to these sensations an objective existence (in the fullest sense of the word "objective") as qualities of objects situated within a field of space and in contact with, or more or less remotely distant from, the body. The law of eccentric projection is generally stated thus:

1 Medicin. Psychologie, p. 331. 
Objects are perceived in space as situated in a right line off the ends of the nerve-fibres which they irritate.

(5) The entire process of elaborating the presentations of sense presupposes for its explanation a constant activity of the mind in reacting, with sensations of different kinds, upon the stimuli which produce various forms of molecular disturbance in the nervous system ; and, furthermore, its activity in combining the sensations into the more complex presentations of sense, according to modes of behavior that belong to its own nature, as mind. This combining activity is best called "synthetic," or constructive. It may, indeed, always have a physical basis in some central organic combination of the neural processes resulting from stimulating, simultaneously or in the right succession, the different end-organs and areas of the end-organs of sense. About this, as a matter of scientific knowledge, we are almost wholly in the dark. On general grounds of our theory of the nervous mechanism we conjecture that it is so. But if this organic combination takes place in each instance as a physical basis for the psychical synthesis, the former does not do away with the latter. Obscure as the latter is, and doubtful as some of its elements and stages are, the former is more doubtful and obscure. ${ }^{2}$

Nor is the fact that this synthetic activity takes place to be concealed by ascribing the product to the so-called "association" of sense-impressions and of ideas. Ideas and sensations are not entities or real subjects of states; they are only particular phenomena. They cannot associate themselves; nor are they things which may be combined, after the analogy of material atoms, by the action upon them of neural conditions. The term "association," as applied in all theories of sense-perception, is only an inadequate expression for this same synthetic activity of the mind.

\$. It follows, then, that an analysis of the presentations of sense leads us to find our explanation of certain primary facts and results

"The word "synthesis" for this mental activity is employed and defended by Wundt (Physiolog. Psychologie, ii., pp. 28 f., 164 f., 177), who justly objects to the word "association" and the theories which have used the word, because of their concealment of the truth that the process imparts nevo properties to its product. He also calls attention (p. 175) to the fact that John Stuart Mill, a chief defender of the "association hypothesis." virtually admits the theory of a mental synthesis by using the term " psychical chemistry."

'Yet E. Montgomery, in Mind, 1885, pp. $227 \mathrm{ff}$. and $377 \mathrm{ff}$., speaks as though the indubitable and clearly understood portion of the whole psychophysical process were the nenral and organic part; and as though it were doubtful whether we have any right at all to refer the nature of the product to the nature of the subject whose product it is-namely, to mind. 
in the nature of the Mind itself. Physiological psychology can do much toward giving a descriptive history of the process in which these complex products have their rise. It can point out the elements which enter into the products-both the more primary and the derived-and can state the laws which regulate the process. But the forth-putting of these primary elements, and the modes of the activity which are called the laws of the process, must all in the last analysis be thought of as native to mind. It is in vain to object that to do this leaves the subject, ultimately, still shrouded in mystery. ${ }^{\prime} \quad$ As a matter of fact, the analysis of psycho-physical science does end in the recognition of ultimate mystery. This is no reproach to it; nor is it a failure or fault peculiar to it alone. All physical science, even, is obliged to accept the same result from its keenest analyses most vigorously pushed. For physical science always has to admit into its explanations the unexplained mystery of elements of physical reality which behave in certain ways-not simply because they are thus or thus circumstanced, but also because when they are thus circumstanced it is their nature so to behave. ${ }^{2}$

$\S 6$. The foregoing remarks indicate what is the correct position toward the two rival theories as to the nature and origin of presentations of sense. These theories have been named the "nativistic" (or intuitional) and the "empiristic " by Helmholtz, the " nativistic" and "genetic" by Wundt." Properly speaking, they are not two fundamentally different theories, but rather two tendencies which appear in the attitude assumed by two classes of observers toward the admission of certain alleged facts, or in the manner of explaining such facts as are admitted by all. These different tendencies are largely due to differences of position on certain fundamental philosophical questions, especially the question as to the reality and self-activity of Mind, which (however much the effort is made to avoid them) inevitably have their bearing upon the researches of physiological psychology. Thus influenced, the socalled "Nativistic School" is inclined to depreciate the explanations

\footnotetext{
${ }^{1}$ Sully thinks it an objection to Wundt's view that it "burdens us with the mystery of what may be called a psychical form of spontaneous generation" (see Mind, 1878, p. 192). But it is one chief merit of Wundt that he frankly acknowledges the mystery and knows where to locate it. Doubtless he would rejoice with us all, if Sully, or any other investigator, could push analysis further.

2In the article just referred to (Mind, 1878, p. 184) Sully speaks of Lotze as "burdened with survivals of Herbart's metaphysics." We fail to see why any recognition of the reality and self-activity of mind should be considered as such a "burden" by certain English psychologists.
}

${ }^{3}$ Physiolog. Optik, p. 435.

- Physiolog. Psychologie, ii., p. 23. 
offered by the other school as to how and why the presentations of sense come to have the character they really bear; the advocates of this school prefer to emphasize the intuitional and underived activities of the mind.

The so-called "Empiristic School," on the other hand, is inclined to give little or no place to the mind's native intuition; it prefers to fill the gaps in the explanation as based on experiment, - with probable conjecture and hypothesis. It often aims to show how what we call "mind," and popularly look upon as immediately conscious of the reality of things by virtue of its native right and power, is itself rather the result of a genesis induced by the activity of things through the nervous system. The one school is inclined to look upon the space-form, which presentations of sense possess, as the mind's form, in some large sense native to it and not to be explained as the result of a development. The other is inclined to look upon space-form as wholly a form which "things " have come to acquire, and which will be fully explained when science has described the empirical process by which solely this acquisition is gained for them. Inasmuch as the chief difficulties of a theory of perception have hitherto been found in accounting for the construction of visual space, the one party in the controversy has insisted, as a rule, on the native power of the mind in and through the retinal image directly to intuit space of two, or even of three, dimensions; the other has denied this power, and has laid great stress upon the necessity of motion, with its equipment of double images and of graded muscular and tactual sensations.

\& 7. Certain principles adopted both by the empiristic and by the nativistic school have their undoubted rights; and no satisfactory theory of sense-perception can be framed without admitting them. There can be no doubt that the presentations of sense which so largely constitute our every-day adult experience are not direct results of untrained organic and mental activities; they are not simple intuitions dependent solely on the native and inherent powers of the mind. With whatever speed and certainty they are formed, and however the impression they make is characterized by a perfect "immediateness," they are really extremely complex products, involving not only the organic habit of the species and individual peculiarities of mind and body, but also the acquisitions of experience through memory, attention, association, and so-called "instinctive inference." All this is as true of the picture of a single object when seen by instantaneous illumination, or of the unhesitating localization of a burning or cutting pain in some area of the skin, as it is of the most deliberate judgment about the distance of a mountain. 
On the other hand, however far the "empiricist" may succeed in resolving these "intuitions" of sense into more nearly primitive elements, and however minutely he may describe the processes and laws of their development, he will never succeed in withholding from the mind itself the ascription of all its so-called native powers. The elements (the simple sensations) reached by his most complete analysis must always be considered as reactions of the mind upon the stimulation of the nervous centres through the end-organs of sense ; they all imply a native disposition and ability of the subject of the sensations. The nature of the process in which what is simple and homogeneous becomes complex and heterogeneous, and acquires the added characteristics of so-called space-form, can never be regarded otherwise than as due to the constructive and synthetic action of the mind. And both theories must alike admit that the nature of the elements and of the synthetic process is conditioned at every step upon the action of the central nerrous mechanism as sensitive and excited through stimulation of the end-organs of sense.

The only satisfactory course in considering this subject of per-' ception by the senses is, therefore, perfectly obvious. The different simple sensations which enter as elements into the presentations of sense, the method and laws of their combination, the correlations of mind and nervous mechanism involved in the process, must all be pointed out as fully as the present condition of psycho-physical science will admit. But the existence of unexplained mysteries, the fact that original and underived activities of the mind are necessarily assumed, the truth that the entire complex process is to be ascribed-however occasioned or conditioned-to the mind, must also be admitted. Especially must we aroid all attempts, whether avowed or concealed, to account for the spatial qualities of the presentations of sense by merely describing the qualities of the simple sensations and the modes of their combination. It is position and extension in space which constitutes the very peculiarity of the objects as no longer mere sensations or affections of the mind. As sensations, they are neither out of ourselves nor possessed of the qualities indicated by the word "spread-out." As objects of sense, they are both ont and "spread-out." No manipulation of their mental qualities and values can fully explain how it is that when combined they acquire the new peculiarity of their space-form. If it should be found that any system of simple sensations is probably originally given as localized, it would be necessary to acknowledge this, too, as an inexplicable matter of fact. Science cannot explain, from previous experience, an event in ex- 
perience which is so fundamental as this. Such an event constitutes an unsolvable datum of nature, so to speak. And if the further question be asked, Of the nature of what? the only possible answer must be, Of the nature of the subject of the experience, of the Mind.

88. Before proceeding to iilustrate and confirm in detail the five principles already laid down, several questions raised by the mere statement of these principles require an answer. And first: What are those characteristic differences in quality which the sensations belonging to some of the senses possess, and which adapts them to combine into presentations of sense under space-form ? In other words, what kinds of sensations are fitted to constitute a so-called "spatial series?" Plainly, it is not necessary that those elements of the complex objects of sense, which make the objects appear to be composed of parts set together side by side, should themselves be immediately known as side by side. The mosaic of nerrous elements (rods and cones) "set side by side in the ninth layer of the retina is a physical pre-condition of the extended visual object which the mind has when the retina is excited. According as the irritation spreads over this mosaic the extent and shape of the object are determined. But the extension of the object is not a copy of the extension of the retinal nerve-expanse with its minute parts set side by side. For example, the object under ordinary circumstances has no gap corresponding to the blind-spot; and the nervous elements, on whose excitement the appearance of the extended object depends, belong to the retinas of two eyes, and are therefore not side by side at the periphery; nor do histology and physiology warrant us in assuming that the elements in the brain corresponding to the retinal elements are set exactly side by side there. Moreover, the different parts of the object appear side by side as the result of motion of both eyes with its resulting museular and tactual sensations. The nervous elements whose irritation induces these latter sensations are not locally contiguous to the elements of the retina whose excitation produces the sensations of light and color; yet the two kinds of sensations combine to form one extended object.

In brief, we have no reason to assume that any two kinds of sensations require, in order to combine into one object, that the excited nervous elements which form the physical bases of them both should be set precisely side by side in the brain. If they werewe may ask-what good would it do? if they were not, what harm? Let the locally different sensation-elements of the light-and-color series be represented by $a, b, c, d$, etc.; and those of the muscular 
and tactual series by $a, \beta, \gamma, \delta$, etc. How shall we arrange the two series so as to form within the brain a physical basis for one object with its parts all set in visible spatial order? Shall this be accomplished by interpolating one of the latter series between every adjoining pair of the former series-thus $a, \alpha ; b, \beta ; c, \gamma$; etc. ? What is really necessary is that both series of sensations, if they are to be combined into one presentation of sense, shall be capable of clearly and reciprocally determining each other as series of sensations. They must both have, that is to say, the common qualities and mutual relations of a "spatial series."

§9. Of the qualities which characterize spatial series the following are the most important: Series of sensations of like quality, which are adapted to combine into extended objects of sense, must admit of easy, rapid, and frequent repetition in varying order of arrangement. An order of sensations in time, however varied and frequently repeated, can never of itself account for an order of parts in an extended object set side by side in space. But the character of the ordering possible for the simple sensations does determine whether or not they can become elements of such an object. If a portion of the body be moved, as, for example, a finger, an arm, a leg, or the bending of the back - a graded series of sensations, due to the varying quality and quantity of strain upon the different muscles, joints, etc., is the result. This series is composed of individual compound sensations that shade into each other with no apparent interruption, each of them having a certain value and position in consciousness. In adult experience the series is rapidly concluded, and instantaneously interpreted as a whole. The individual members of the series are then scarcely, or not at all, distinguished in consciousness; that is, as sensations, many of them largely or wholly drop out. But they may be reproduced in a measure by slowly moving a limb in any direction, and endearoring to pay strict and exclusive attention to the succession of feelings which results. Every motion of each limb, from about the same position $a$ to about the same position $m$, relative to the whole body, with similar energy, speed, and other concomitant circumstances, yields a nearly identical series of sensations $(a, \beta, \gamma, \ldots \mu)$. Other motions of different limbs, or differing otherwise (in energy, speed, point of starting or of conclusion, etc.), yield series differing in the value and ordering of their individual members. What is true of the muscular sensations that result from the movement of the limbs is also true of the accompanying sensations of the skin, such as arise from

' On this subject comp. the remarks of Volkmann von Volkmar, Lelirb. d. Psychologie, II., pp. $36 \mathrm{ff}$. 
changes in its tension, etc. These sensations, however, largely blend with the series of muscular sensations so as to be nearly or quite inseparable in consciousness. The same thing also holds good of the series of tactual sensations (sensations of light pressure or touch proper) developed by moving an object over the skin, or by moving a tactile organ (especially the hand) over an object at rest. The muscular and tactual sensations which result from motion of the eye hare the qualities of a graded spatial series.

Accordingly, senses like those of the eye and hand, which have organs capable of rapid and precise motion, are equipped with a peripheral mechanism adapted to the production of so-called spatial series of sensations. The succession of sensations of light and color which accompany the movement of an object in the field of vision, or of the glance from one object to another, are also of the kind favorable to forming a spatial series. Slight changes of colortone and brightness, as the different parts of the surface of the same object come successively in review, or more abrupt changes on transition from one object to another, characterize the effect of such movement. In all these cases the rate of the sensations is important. Either too slow or too rapid movement of the organ will not yield a spatial series of sensations. Moreover, such series are capable of repetition, not only forward, as $\alpha, \beta, \gamma, \delta, \ldots . \mu \mu$, or in inverse order, as $\mu, \lambda, \kappa, \ldots . \quad \beta, \alpha$, but also in an endless variety as an intersecting net-work of sensations.

$\S 10$. It has been claimed that all the foregoing qualifications belong to musical tones, and yet hearing is not a "geometrical sense." In reply, it must be admitted that the foregoing are not the ouly qualifications possessed by sensations of spatial series. Yet in regard to these qualifications alone, the sensations of hearing bear no comparison with those of the eye, skin, and muscles. Few persons have more than a very imperfect and infrequent experience with series of musical tones. Such series, when arising in consciousness, ordinarily have little variety, and are rarely or never repeated in changed order of arrangement with a recognized qualitative value to the members of the different series. Few persons have heard frequently more than half a score of tunes; fewer still have had any considerable number of the notes composing the strains of these tunes repeated in recognizably inverted or otherwise varied order. Most of our experience with sounds is that of sudden shocks of noise which occur to interrupt the continuous flow, as it were, of sensations of the eye, muscle, and skin.

In close compection with the foregoing stands also the fact that the ear is not, like the eye and the hand, a movable organ; nor is 
the irritation which its nervous elements experience graded according to the varying extent of the surface affected, except as the noise becomes so loud or acute as to occasion decided forms of muscular sensation and of common feeling. Compared with the almost unceasing call made for attention to sensations of sight and touch, the experience of men with sensations of musical sound (the only sensations of hearing which easily admit of being arranged in any sort of a graded series) seems meagre and trivial indeed. Music is, therefore, of the nature of an indirect recreative and æsthetic acquaintance with things, rather than of a necessary direct and practical acquaintance with them.

$\S 11$. The second class of qualifications which must be possessed by a spatial series of sensations secures their habitual combination with other series, also of a spatial kind. They must be in nature comparable and associable with each other, and, in fact, simultaneously experienced by the mind. In singing a musical scale a series of sounds is accompanied by another series of muscular and tactual sensations occasioned by the use of the vocal organs; both series may be produced in inverse order by singing the same scale backward.' Yet these two simultaneous series of qualitatively different sensations do not combine into a succession of extended objects of sense. For this fact there are several obrious reasons. Of the three principal kinds of sensations involved-namely, sensations of musical sound, sensations localized in the organs with which the sounds are executed, and sensations localized in or near the organs with which the sounds are heard-only the last two belong to spatial series. The first of the three, however, is not a spatial series. It has not been accustomed to give us direct knowledge of any part of our own body; nor does it combine with either of the other two so as to form an object of sense. We know, indeed, not only that we are singing the scale with the vocal organs, but also that we are at the same time hearing it with the ear. We know both these facts, however, through sensations of muscle and skin that have already become inseparably associated and localized in our own body.

On the contrary, from the dawn of consciousness onward through all the development of experience, series of sensations of light and color are constantly accompanied by, and combined with, other series of tactual and muscular sensations of the eye. So, too, the different series of sensations that arise from the irritation of the nerves in muscle and skin are, of necessity, habitually combined. In forming the field of touch, the fact that certain parts of the periphery of the body so frequently come into contact with other parts ' Comp. Lotze, Medicin. Psychologie, p. 382 f. 
is of the highest significance. Two series of complex sensations, corresponding to the terms "touching" and " being touched," are thus brought into juxtaposition, as it were, in consciousness. This "juxtaposition" in consciousness is not itself, of course, a spatial juxtaposition; the former is, however, the necessary pre-condition of the latter.

$\$ 12$. The third characteristic of the spatial series of sensations is the possession of a system of local signs. By a "local sign" we understand that peculiar shading or mixture of quality which belongs to sensations, otherwise qualitatively similar, on account of the locality of the organ at which the stimulus is applied. Further as to their nature and origin it is not easy to give a satisfactory account. While accepting the general theory of Lotze as to the existence of such local signs, his more specific view as to what they are must be held subject to doubts. The local signs of sight Lotze conceived of in the following way. ${ }^{2}$ In addition to the same sensation (for example, red, $r$ ) which each color produces at all places of the retina, it produces also an accessory impression $\alpha, \beta$, $\gamma$, etc., for each of its different places $a, b$, or $c$, etc. The existence of such accessory data must be assumed, or the spatial differences and relations among the retinal impressions could not be "compensated for" by corresponding non-spatial and merely intensive relations among the impressions in the soul.

We can only conjecture, however, in what the accessory impressions consist. Lotze's conjecture is that, since we involuntarily and by reflex motion of the eye fixate its most sensitive spot upon every especially luminous point, and in order to do this must rotate the eye through an arc $P E$, or $R E$, etc., according to the position from which it takes its start in the fixation of $E$, a great number of series of changing "feelings of position" are developed corresponding to each arc (thus, $\pi \epsilon, \rho \epsilon$, etc.). When, then, both $P$ and $l i$ are simultaneously stimulated, although with equal intensity and so that actual rotation of the eye cannot take place orer both of the opposed arcs $P E$ and $R E$, the stimulation of $P$ and $R$ reproduces the series of feelings of position belonging to each (respectively, $\pi \epsilon$ and $\rho \epsilon)$. Thus there comes to be connected with every excitation of the places $P$ and $R$ the mental presentation of the magnitude and qualitative peculiarity of a series of changes which consciousness "would have to experience in order that these exci-

\footnotetext{
${ }^{1}$ The most mature expression of Lotze s theory is to be found in Outlines of Psychology, Boston, 1886. p. 51 f.; and Metaphysik, Leipzig, 1884, iii., chap. 4 ; the earlier, in Medicin. Psychologie, Book ii., chap. 4; and article, Seele n. Seelenleben, Wagner's Handwörterb. d. Physiol., 1850, III., 1. Abth.
} 
tations might fall upon the place of clearest vision." The physical basis for this is to be found in the cerebral connection between the sensory and the motor nerves; it is the excitation of the latter at their central endings which gives to every color-impression its local character. ${ }^{2}$ The local sign of sight is, then, a kind of feeling of tendency to motion, mentally reproduced ont of the associated series of impressions that have previously accompanied the movement of the eye in fixation from one position to another. The sign consists in awakening definite "motor tendencies," " or, rather, "associated feelings of movement." 3

Various objections to Lotze's hypothesis may be brought forward. To speak of "accessory" or "adjunct" impressions as attaching themselves to the chief impressions of color-tone seems unfortunate. Indeed, Lotze himself conceives of them rather as blending in one mixture of feeling with the principal sensation. The colortone itself does indeed change with the position upon the retina where the stimulus falls. ${ }^{4}$ But the change is always a change into another color-tome, which is, so far as it is color-tone at all, capable of reproduction at any one of innumerable other points of the retina. The question then recurs as to the origin and nature of the individual members of the series of original so-called "feelings of position" ( $\pi$ to $\epsilon$, or $\rho$ to $\epsilon)$. Are they feelings which arise from the changing condition of the muscles of the eye, or of the skin surrounding the eyeball, when the eye is in motion? Or are they specific shades of feeling which naturally belong to each of the nervous elements of the retina, and which are awakened by stimulating these points without any dependence on the motion of the eye?

$\S 13$. Several riews are possible as to the nature of the local signs of the skin. It may be held that they'are not qualitative differences at all, but differences in the intensity and time-course of the tactual sensations. Von Kries and Auerbach, ${ }^{5}$ however, have shown that locality on the skin is much more quickly discriminated than even considerable differences in the quantity of sensations. We can tell the point where we are touched easier than the point where the amount of pressure is increased. Again, it may be held that the local signs of touch are qualitative differences of sensation de-

'Medicin. Psychologie, p. 360.

${ }^{2}$ So in the earlier view, Medicin. Psychologie, p. 340.

${ }^{3}$ Comp. Sully, in Mind, 1878, p. $181 \mathrm{f}$.

'Bain makes the odd mistake of supposing that both Lotze and Wundt regard this series of changes in color-tone as constituting the local signs of vision. The Senses and the Intellect, p. $397 \mathrm{f}$. (note). New York, 1879.

${ }^{B}$ Archir f. Anat. u. Physiol., Physiolog. Abth., 1877, p. 351 f. 
pendent upon the modifications which the stimulus undergoes on account of the changing character of the skin with respect to tension, nature of the substance of muscle, tendon, and bone over which it is stretched, etc. But experiment shows that stimulation of the skin by electricity, and in such a way that these influences could have no appreciable effect, is immediately localized. Finally, it may also be held that the local signs of the skin are qualitative differences of sensation peculiar to the different nervous elements existing in different parts of this organ of sense. They are the direct result, that is, of the mind's reaction upon the specific energies of the nervous elements as called out by the stimulus. This is, of course, to fall back upon the ultimate mystery involved in the original nature of that reaction which the mind makes as dependent upon the locally individual nervous elements being stimulated. It is to surrender explanation at this point, and accept what takes place as mere datum of fact.

$\S 14$. In view of all the evidence, it would seem that the general theory of local signs must be constructed in somewhat the following way: Within certain limits, which it is impossible for science as yet definitely to fix, the irritation of the different nervous elements of certain organs of sense gives rise to sensatious which differ in the shading of their quality according to the locality in the organ at which the elements are situated. This is probably true of both peripheral and central areas of the total organ. It is true of the latter areas as dependent on the excitation of the former. The simultaneous irritation of several locally related elements of the organ (and the irritation is seldom or never confined to a single element) results, then, in a certain mixture of feeling dependent upon the number and local relation of all the elements thus simultaneously irritated. For example, the color-tone of the complex sensations aroused by irritating together the retinal elements $\alpha, \beta, \gamma$, $\delta$, etc., differs from that aroused by irritating the elements $\gamma, \delta, \epsilon, \zeta$, etc. The same thing holds true of locally related nervous elements of the skin. How much of the local coloring is due, on the physiological side, to differences in structure and how much to differences in processes, how much to peripheral elements and how much to central nervous connections, it is impossible to say. Each of the spatial series of sensations is characterized by this shading of its elements. We must, therefore, hold that every sense which is the medium of space-perceptions has a system of local signs of its own. ' The theory is thus opposed to that of Bain ${ }^{2}$ and others of

' Comp. Funke, in Hermann's Handb. d. Physiol., III., ii., p. 408 f.

2 The Senses and the Intellect, pp. 73 ff., 348 ff. New York, 1879. 
the Association school, who are inclined wholly or largely to reduce all the local signs to mere symbols of associated differences in the muscular sense. They are thus made to become mere signs of signs of "Things."

Not only each "geometrical sense," but also each of the "spatial series" of sensations arising through the total operation of that sense, consists of members that have a local coloring peculiar to the series. Thus the spatial series of tactual impressions produced by moving an object from $a$ to $d$ on the hand differs from that produced by moving it from $a$ to $n$; that belonging to movement from $a$ to $d$ on the hand from that belonging to movement from $m$ to $x$ on the back. The series of muscular sensations developed by raising one pound differs, with respect to the color-tone of its members, from that developed by raising two pounds, with the hand; both differ from the series belonging to the raising of the same weights with the foot. Every series of muscular and tactual sensations produced by moving the eye also depends upon the direction and amount of its motion; the series of local signs of the retina depends upon the direction and amount of the motion of the object over the retinal field.

But another important consideration remains. The local signs of the different spatial series which frequently combine in the operation of the same organ must necessarily modify each other. Hence there arise admixtures of feeling dependent upon the combined specific energies of the nervous elements simultaneously excited, with a given amount of energy and with given relations to preceding conditions. Thus it comes about that the place where we locate a visual object does not depend merely upon the place where its image falls on the retina, but also on the feeling of the position of the eye as indicated by its muscular and tactual local signs. In other words, the real and complete "local sign" (that which signifies the locality of the object to the mind, as it were) is the whole complex of feeling which is combined from the local signs of the different spatial series that are acting together. We define the local sign, then, as that mixture of feeling which gives to the sensation its peculiar coloring, and is dependent upon the combined result of exciting the nerves of a given locality of the organ.

$\S 15$. It may be objected that the foregoing theory of local signs is too elaborate and artificial to be in fact true. But the nervous elements themselves are indescribably numerous and varied; and so is, without doubt, the total complex of feelings which results from their activity. Reflective consciousness finds itself baffled in its effort to catch and fix, for purposes of analysis, all the various 
shadings of sensations which actually appear in adult life. Yet it is characteristic of this adult life that its very development presupposes the loss from consciousness of many discriminations of shades of feeling on which the development is itself based. The adult does not, and cannot, recall the complex of sensations by which he learned to talk; nor ean the skilful player of the violin reproduce the "tact" with which he experimented among the innumerable muscular and tactual sensations concerned in producing a required musical tone. Let anyone, however, who imagines that the limit can be easily fixed for the peculiar shadings which sensations ordinarily classed together really possess, spend a half-hour, with one hand lying motionless across the other, in the endeavor to pick out all the different color-tones of tactual feeling which he can localize in both hands.

The dependence of even developed experience upon these local signs in consciousness, for its apparently instinctive localization of sensation, may be faintly manifested by various experiments. Select two portions of the body that are in structure and function most nearly identical, and whose local signs will accordingly be such mixtures of feeling as, when brought into consciousness, will be most difficult to leep separate. The corresponding points on the tips of the two middle fingers, perhaps, fulfil these conditions best. With closed eyes rub these points gently together, concentrating the attention, as much as possible, solely upon the sensations thus produced. The tactual sensations may thus be made to fluctuate in locality from one finger to the other; at times they appear almost to lose their objective character, and to resemble musical tones heard without consciousness of the direction from which they come, or of the extent and locality of the bodily affection through which they come. A similar nearly complete detachment of sensations of light and color may be secured by closing the eyes, letting all after-images die away, suppressing the tendencies to motion of the organ, and directing attention, as much as possible, solely to the quality of our affection. Color seems then to be felt (but nowhere in particular), rather than seen as localized in space-form.

$\S 16$. 'The most noteworthy stages, or "epoch-making" achievements, in the process of elaborating the presentations of sense, have been declared to be "localization "and "eccentric projection." The first, primarily, gives us the knowledge of our own body, mainly by passive sensations of touch ; the knowledge of our own body which comes through sight is by eccentric projection. We immediately feel the peripheral parts of the body as the places where the sensa- 
tions are localized; we see some of the same parts as projected in space before our eyes. Oljects that are not a part of ourselves are given to us as projected eccentrically, either by touch through their being in contact with the skin and occasioning sensations of muscular exertion, or by sight as having distance in its field of vision. Localization and projection are not to be regarded as two phases of one and the same process; we do not first have the presentations of sense as parts of the periphery of our bodies, and then, on further experience, push them beyond this periphery, either to an infinitesimal distance or to one remote. Localization and eccentric projection are rather two processes, largely unlike, which go on contemporaneously and are set up chiefly on the basis of different classes of sensations.

Where two parts of the sensitive skin of our own bodies come together the conditions for both of the above-mentioned processes are fulfilled. Accordingly, one part has localized in it those complex sensations which make us aware that this part of our body is touching something; the other has localized in it those sensations which make us aware that this part is being touched by something. Which of the two parts shall be regarded as touching, and which as being touched, depends on various considerations. Those members of the body which are most used in active touch are generally known as touching, and the less active parts as being touched. For example, if with closed eyes the forehead be moved across the stationary tip of a finger, the latter will appear to be the active organ of touch. Comparatively insensitive areas of the skin are less likely to be presented to the mind as touching other more sensitive parts; callous spots, indurated surfaces, etc., seem, as a rule, to be touched. Parts of the body which lose all sensitiveness come to be regarded as external things. If the tip of a finger of normal sensitiveness be brought into contact with the callous tip of the corresponding finger of the other hand, the former will be known as touching and the latter as being touched. The direction of attention often determines the strife, as it were, between the motifs to localization and those to eccentric projection. We ordinarily strive to gain knowledge of the qualities of some outside object, rather than of the condition of our own periphery with respect to the sensations localized in it; the attention is therefore directed to those series of sensations which form the basis of eccentric projection, even when some part of our own sensitive organism is the object known. But sensations which are accompanied by obtrusive feeling of some kind furnish superior grounds for localization. We locate pains, pricks, severe pressure, sensations of creeping, and tickling, in the body. In gen- 
eral, then, a strong tone of feeling with the sensation favors the process of localizing; tonelessness of sensation favors the process of objectifying.'

A system of localized sensations, gained chiefly by pressure of the skin and muscles, and accompanied by a strong tone of feeling, gives us the primary field of the body as known to touch. Certain points of starting, as it were, must first be fixed in the process of localizing ; this process then goes on by relating all other localized sensations to these points of starting. But by eccentric projection, the system of muscular sensations of movement and the system of visual sensations are combined to develop our perceptions of objective space with its three dimensions. The sensations of touch are subsequently projected into a space thus originally constituted by combined muscular sensations and visual sensations. The eye and hand in motion, therefore, project their extended objects into a space which they develop themselves; while the ear and the nose project their perceptions into a space which they are compelled to assume on the authority of the other senses.

The foregoing principles must now be illustrated and confirmed by a brief statement of facts which relate to the formation and development of presentations of sense by a synthesis of simple sensations. Attention will, for obvious reasons, be directed almost exclusively to those presentations of sense which come through the eye and skin, including in both the influence of muscular sensations.

$\S 17$. Perceptions of Smell differ only in fineness, duration, and accompanying tone of feeling ; they have no size or shape, no spatial properties of any kind. They cannot even be said to be localized. Fineness of smell, or power to make minute distiuctions in quality, and so infer the presence or direction of an object previously known to excite such quality of sensations, differs greatly in different species of animals and in different individuals of the same species. The exploits of some animals give ground for the conjecture that every species, and even every individual, has an odor of its own. ${ }^{2}$ The direction and nature of the object which causes the sensations are judged by variations of intensity on turning the head, or on approaching or receding from the object. Sensations of smell are known to come through the nose, by localizing there the accom-

\footnotetext{
${ }^{1}$ Compare Volkmann von Volkmar, Lehrbuch der Psychologie, II., p. $126 \mathrm{f}$.

${ }^{2}$ See the articles of Dönhoff, in Archiv f. Anat., Physiol., etc., Physiolog. Abth , p. 750 f. (1874); and Jäger, in Zeitschr. f. wissensch. Zool., xxvii., p. 319 f. (1876).
} 
panying muscular and tactual sensations with their strong tone of feeling. This is readily done, since we draw the air through the nostrils and feel its double effects in producing the two classes of sensations. As to the simultaneous influence of two smells, little is known beyond the statement of Valentin, that the stronger overwhelms the weaker. The power of discrimination may, of course, be cultivated in this sense as in every other. ${ }^{1}$

$\S 18$. Most of the remarks just made as to perceptions of smell apply also to Perceptions of Taste. Sensations of taste, however, are much more closely connected with those of touch; since the tongue is a chief organ of active touch. It is the tactual and muscular sensations, and not the purely qualitative affections of taste, which are localized in the mouth. Concerning contrast and compensation of tastes, little is known which does not belong to ordinary experience. Valentin ${ }^{2}$ alleges that when a sour mass is laid on one half, and a bitter mass on the other half, of the root of the tongue, the predominating taste may sometimes be determined by our choice. It is well known that certain tastes compensate each other, as it were, in experience, without any chemical equivalence of their properties. The sugar neutralizes the acid of the lemonade, not in the ressel that contains the mixture, but in the nervous system of him who drinks it. Brücke holds ${ }^{3}$ that the neutralizing of one sensation of taste by the other takes place in the brain. The sensation of bitter is especially difficult to cover or neutralize.

$\S 19$. Perceptions of Hearing next demand consideration. More difficulty accompanies the effort to establish the proposition that sensations of sound are not directly localized, but are projected in a space constituted chiefly by the eje and the hand, through complicated indirect inferences. Such a proposition is, however, undoubtedly true. The localizing of the area of the body which serves as the organ of the sensations of sound, the knowledge that we hear with the ear, is accomplished chiefly through those sensations of shock to the muscles and skin of the region which come from loud and massive or piereing sounds. Sensations of sound originating through excitement within or very near to the ear itself are called "entotic." A great part of such sounds, if not all of them, are transmitted through the tympanum. Perceptions combined of such sensations may be located either within the ear or at some dis-

${ }^{1}$ On the whole subject see von Vintschgau's monograph in Hermann, Hand b. d. Physiol., III., ii., pp. $225 \mathrm{ff}$.

${ }^{2}$ Lehrbuch der Physiol. d. Menschen, etc., Abth. ii., p. 308 (second edition).

${ }^{3}$ Vorlesungen über Physiol. (ed. 1884), ii., p. 262. 
tance from the body, according to previous associations and opportunities for judgment. The sound produced by the vibration of the adjoining muscles, and heard as a low musical tone when the fingers are pressed in the ears (especially if the teeth are tightly set together), is located in the head by the help of its accompanying sensations of other kinds. The same thing is true of the crackling noise sometimes produced by yawning, or of the whirring occasioned by the passing of the blood through the neighboring large bloodvessels. In the same way we learn to hear the beating of our own hearts, or the noise of air in our respiration. But the click of the valves of the internal organ may, when experience gained through tactual and muscular sensation fails us, be located in the watch under our pillow; just as the singing or ringing "in the ears" produced by quinine, or cerebral excitement otherwise occasioned, may be located in a cricket supposed to be upon the sill of the open window. In certain pathological cases the power to distinguish between entotic sounds and those having an external origin is almost wholly lost.

$\S 20$. We can orientate ourselves in space with reference to external sounds with great speed and considerable precision, but as an acquired art differing in different individuals and dependent upon attention and previous experience. E. H. Weber thought that we tell the direction of sounds by the help of the feeling of the swing of the ear-drum; and instanced, in proof, that this eccentrie projection is hindered by filling the external passage of the ear with water. When using both ears and moving the head freely in space, we undoubtedly determine the direction of sounds by differences in the intensity of the sensation dependent upon changes in the relative position of both ears. Rayleigh ${ }^{1}$ found that, in a quiet place under favorable circumstances, the direction of a word or letter uttered in a natural voice could be given with considerable accuracy; that of a musical tone much less accurately. The direction of a sound from a tuning-fork could not be given when it was held either behind or before, but could be given if the fork was held to the right or to the left. It is said that the conducting of an intermitting current from a telephone through both ears causes a perception of tone localized in the median plane of the head.

Such facts as the foregoing introduce us to the theory of "acoustic shadows," or of the amount of "covering" power which the sound produced by the waves of a given intensity entering one ear would have upon the sound produced by waves of a different

$$
{ }^{1} \text { Nature, XIV., p. } 32 .
$$


intensity entering the other ear. It does not appear to Hensen, ${ }^{1}$ however, that the prompt and accurate localization of direction possible to some ears can be wholly accounted for by the theory of acoustic shadows. Some other form of feeling, possibly connected with the remarkable arrangement of the semicircular canals, may blend with the estimate of differences in intensity to form a basis for judgment. Von Kries and Auerbach found that the promptness with which the direction of the noise from an electric spark can be localized depends upon its relation to the circuit of the head. ${ }^{2}$

Our perceptions of the absolute distance of sounding objects are entirely dependent upon our knowledge of the quality and quantity of the sounds ordinarily proceeding from them; they are, that is to say, not presentations of sense, but indirect estimates as to the objective cause of the sensations immediately experienced. It has been claimed that a change in the relation of the partial tones to the fundamental tone, dependent upon the remoteness of the place of origin of the compound clang, aids our estimate of distance by sound.

$\S 21$. An account of the process by which a Field of Touch is constructed, and extended objects are known as in contact with the skin at definite points or areas of it, must begin by enumerating the data which the mind has for such activity. The most important of these data are indicated by certain facts as to the fineness of the so-called "sense of locality" belonging to the skin. E. H. Weber first established a rule for measuring the degree of this fineness accurately; he also mapped out the entire field of the surface of the body into areas differing greatly in their fineness. ${ }^{3}$ For a measuring instrument he used the two points of a pair of dividers, blunted so as to prevent the sensation of being pricked; the principle of measurement was that the minimum distance apart at which the two points, when touching the skin of any region, are felt as two localized sensations is the measure of the sensitiveness to local distinction of that region. The following table gives some of the results of Weber's experiments; the figures indicate the number of millimeters ${ }^{4}$ apart which the points of the dividers were when the given area of the organ was just able to distinguish them:

1 In Hermann's Handb. d. Physiol., III., ii., p. 136.

${ }^{2}$ Archir f. Anat. u. Physiol., Physiolog. Abth., 1877, p. $331 \mathrm{f}$.

${ }^{3}$ Annot. Anatom., vii., p. 4 f. ; Wagner's Handwörterb. d. Physiol., III., Abth. ii., p. $529 \mathrm{f}$.

${ }^{4}$ The numbers were given by Weber in Parisian lines; in the table they are taken from Wundt, Physiolog. Psychologie, ii., p. 7, who has reduced them to even millimeters. 
Tip of the tongue $. . \ldots \ldots \ldots \ldots \ldots \ldots \ldots \ldots \ldots \ldots \ldots \ldots \ldots \ldots \ldots, 1$

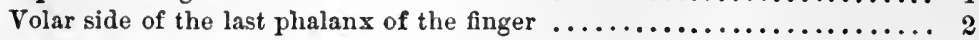

Red part of the lips.................................... 5

Volar side of the second and dorsal side of the third phalanx of the finger. 7

White of the lips, and metacarpus of the thumb................... 9

Cheek, and plantar side of the last phalanx of the great-toe............. 11

Dorsal side of the first phalanx of the finger..................... 16

Skin on the back part of cheek-bone, and forehead................. 23

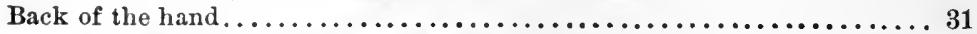

Knee-pan, and surrounding region.......................... 36

Forearm, lower leg, back of the foot near the toes ................ 40

Skin of the nape, and of the back in the five upper cervical vertebra.... 54

Skin of the middle of the back, and of the upper arm and leg........ 68

Weber also found that the fineness of the sense of locality is greater in a transverse than in a longitudinal direction, on both arms and legs. On these surfaces of the skin the "sensation-circles," or areas within which the minimum distances of the dividers' points are felt as two points, have an elliptical shape, with their long axes up and down. That the size of the sensation-circles, or the fineness of the sense of locality, largely forms the basis for our judgments of the position, number, and magnitude of the localized sensations in the field of touch may be shown by a simple experiment. If the points of the dividers be separated somewhat less than is necessary in order to distinguish them as two on the cheek just in front of the ear, and then (the distance apart of the points remaining unchanged) be slowly moved until one point rests upon the upper and the other upon the lower lip, to a person blindfold, and unprejudiced by knowing what is to take place, the point first felt as one will appear to become two, and then the two recede from each other continually as the parts with a finer sense of locality are traversed. The same experiment may be tried upon any other part of the body. It appears, therefore, that the mental representation of the magnitude of the distance between two impressions varies in inverse proportion to the real magnitude of the smallest perceivable distance, on any given area of the skin. The same principle holds good when all the space between the impressions is filled up, as it were, so as to make a continuum of localized sensations. Thus Weber found that the circular form of a tube of only $1 \frac{1}{2}$ Parisian line in diameter could be recognized by pressure on the tip of the tongue; while on the skin of the abdomen the diameter of the tube must reach $3 \frac{3}{4}$ inch before its form was recognizable. Our estimate of the length of lines of pressure marked out by laying rods upon the skin follows the same principle.

\&22. Other important discoveries as to the skin's so-called 
"sense of locality" have been made since those of Weber. Valentin has called attention to the fact that enormous individual differences exist in the fineness of this kind of perception; some persons are not more than one-fourth as sensitive as are some others. The relative degree of fineness belonging to different areas of the skin remains, however, approximately the same in different persons. A. W. Volkmann ${ }^{1}$ showed the remarkable effect of exercise upon the cultivation of the sense of locality. After fixing the value of the least perceivable differences of locality for a number of small areas in the field of touch, Volkmann found that each successive series of experiments with each area increased its fineness of perception, until within a few hours twice the original degree of fineness could be reached. The growth in perceptive skill of the skin was slower at first for areas not ordinarily used for touch; quicker for those accustomed to daily use. The improvement ceased at a certain limit, and was soon lost by disuse, so that a few months out of practice served to reduce the acquired tact of any area to its original condition. A most surprising discovery of this experimenter was, that the practice exclnsively of a member of the body on one side resulted in improving the fineness of touch of the corresponding member of the other side. Thus, if the smallest perceivable distance for the tip of a left finger was, to begin with, 0.75 line, and that of the corresponding place on the right finger, 0.85 , practice with the left finger exclusively reduced the distance for both fingers - for the left to 0.45 line, and for the right to 0.4.

It is well known that the blind, who have no spatial series of sensations or presentations of extended objects by the eye, attain by exercise a high degree of fineness for certain space-perceptions of the skin. ${ }^{2}$ In the case of those who have sight, the most movable and discriminating organs of the skin-such as the tips of the fingers-are capable of being cultivated to great delicacy of touch; but Funke ${ }^{3}$ dicl not succeed, even by an education lasting an entire month, in reducing the obtuseness of the skin of the back between the shoulder-blades and in the lumbar region more than by about one-fourth.

$\S 23$. The explanation of Weber's "sensation-circies" of the skin has been the subject of much debate. It is natural at first to assume that each entire circle is provided with one and only one nerre-fibre, whose terminal expansion corers the circle, and whose excitation is represented in consciousness by a sensation of a spe-

'Berichte d. Sächsischen Gesellschaft d. Wissenschaften, 1858, p. 38 f.

${ }^{2}$ Comp. Czermak, Sitzgsber. d. Wiener Acad., XVII., Abth. ii., p. 563 f.

'See Hermann's Handb. d. Physiol., III., ii., p. 382. 
cific value. Doubtless certain anatomical differences in the nervefibres of the skin, and certain corresponding physiological differences in their function, must be assumed as the basis of every theory to account for the skin's sense of locality. But Goldscheider's experiments show that a number of pressure-spots must be recognized within each sensation-circle, and each pressure-spot at least should have a sensory fibre. Moreover, every point within each sensation-circle is itself sensitive (however large the circle may be), and the limits of none of the circles are fixed as would be the expanse of a single nerve-fibre distributed orer them. Still further, different individnals differ greatly in the size of these circles (and we cannot well suppose a corresponding difference in the number of sensory nerves of the skin), and practice suddenly and greatly diminishes the area covered by a single circle. It must at least be admitted that "the smallest perceivable distance is not a direct measure for the diameter of the sensation-circle." ${ }^{\prime}$

Weber himself assumed that sensation-circles always contain a number of isolated nerve-fibres ; and that, in order to have the impression of two localized sensations, several unexcited fibres must exist between the two excited. The number of these unexcited fibres serves the mind as a kind of means for the approximate measurement of distances on the skin. Other advocates of Weber's explanation have spoken as though the brain could somehow become conscious of the unexcited fibres lying between the two excited ones, and so derive a support for its judgment from their number. ${ }^{2}$ Of course, all attempts to explanation which assume the mind's knowledge of the condition of the minute. subdivisions of the nervous elements are wholly futile and illusory. Wundt ${ }^{3}$ correctly calls attention to the fact that the differences in the so-called sensation-circles of the skin are simply a special case under the general psychological laws of the least observable differences in sensations ; only in this case the differences are not pure differences in intensity, but rather differences in the complex color-tone of the quality of sensation. In other words, the sensation-circles represent the local difference between the points at which stimulus must be applied to the skin in order to produce enough of difference in the color-tone of the resulting sensations to make them observable by the mind. These local signs of the skin, as the organ of touch proper, like all local signs, are complex mixtures of feeling belonging to different localities; as such they are dependent, not only

${ }^{1}$ So Funke, in Hermann's Handb. d. Physiol., III., ii., p. $392 \mathrm{f}$.

${ }^{2}$ Comp. Bernstein, The Five Senses of Man, p. 31 f. New York, 1876.

${ }^{2}$ Physiolog. Psychologie, ii., p. 10. 
upon original, anatomical, and physiological differences, but also upon other peculiarities of the individual, upon habit, and upon association with each other and with other spatial series of sensations of the skin.

$\S 24$. Difficulty has been found in assigning a conclusive reason why the different areas of the skin should differ so greatly in the fineness of their capacity for making local distinctions. In the view of Lotze, ${ }^{1}$ this difference is chiefly due to the varying character of the areas of the skin, with respect to richness in nerve-fibres, thickness and so sensitiveness, support and tension according as the skin is stretched over underlying soft or harl parts-fat, muscle, tendon, bone, etc. Doubtless all such influences enter into the determination of that mixture of feeling which characterizes the local signs of the skin. The theory suggested by Vierordt, ${ }^{2}$ on the basis of experiments made by himself and his pupils, should also be mentioned. This investigator concluded that the fineness of the sense of locality belonging to any area of the skin increases in direct proportion with the distance of that area from the axes about which it is rotated. The relative fineness of the organ's local sense is a function of its mobility. Thus an uninterrupted increase of the power of localization exists in the arm from the acromion to the tips of the fingers; an increase of its movableness, on the whole, also exists. If a value of 100 be assigned to the power of discrimination exercised at the acromion, 151 will represent that of the upper arm, 272 that of the lower arm, 659 of the hand, 2,417 of the thumb, and 2,582 of the tips of the fingers. In estimating the relative movableness of these different parts, it should be remembered that they not only all move in an enlarging circuit from the shoulder-joint downward, but that each of them from the elbow-joint downward has its special increased circuit and more numerous forms of motion.

But even if Vierordt's law could be strictly demonstrated for every portion of the body, its meaning would have to be translated into other terms in order to be of any real service to psychology. It is therefore suggested by Funke ${ }^{3}$ that the increased power of discrimination which belongs to the more movable areas of the skin is really due to the superior facility which they thus have for exercise ; it therefore falls under the law of habit. Furthermore -as we have occasion to remark concerning many similar function's

\footnotetext{
${ }^{1}$ See Medicin. Psychologie, p. $405 \mathrm{f}$.

2 Pflüger's Archiv, 1869, ii., pp. 297 ff. ; and Zeitschr. f. Biologie, VI., VII., IX., X., XI.

${ }^{3}$ Hermann's Handb. d. Physiol., III., ii., p. 384.
} 
of the mind in correlation with the nervous mechanism-the effect of acquired habit is not limited to the experience of the individual ; it belongs also to the race. The superior fineness of local sense in some parts of the body may therefore be regarded as largely native to the individual.

$\S 25$. The view which must be taken of Weber's "sensation-circles," and of the entire subject of the localization of areas of pressure on the skin, has been largely changed by the recent experiments of Goldscheider ${ }^{1}$ and others. We have already seen (p. 346 f.) that this experimenter distinguishes, more carefully than has hitherto been done, the sensations of pressure from other closely allied sensations coming through the same organ. The finest point, when it touches a " pressure-spot," produces a sensation of pressure, and not one of being pricked; but touching other spots does not produce a sensation of pressure at all. It must be held, then, that the sensations produced by laying a single blunted dividers' point upon the skin, as in Weber's classical experiment, are really very complex, and are composed of the sensations from several pressurespots blended with other sensations from the rest of the same area not covered by the pressure-spots. The fineness of discrimination possible in any area of the skin depends, then, upon how all the points irritated stand related to the specific pressure-spots. Goldscheider finds that only when two irritating points touch two pressure-spots are they felt as two. But when one of the points touches a pressure-spot, and the other touches some place in the contiguous area of skin which is free from such spots, the two points are not both felt; in this case only the one resting on the pressure-spot is felt.

Moreover, the impression of being doubly touched may be excited by the points when lying much nearer together, in case they rest upon pressure-spots that belong to two different chains of such spots than when both spots belong to the same chain. This is to say, pressure-spots thus located have a high degree of sensitiveness. Still further, the minimum distance required to produce a sensation of being touched twice is surprisingly small, when one of the touching points rests upon a pressure-spot from which the chain radiates or at which it makes a sharp bend.

The table of minimum distances at which two points can be felt as two, when the exact nature of the area of the skin on which we are experimenting is knowu, and everything made as favorable as

${ }^{1}$ On this subject, see Goldscheider, Archiv f. Anat. u. Physiol., Physiolog. Abth., 1885, Supplement-Band, pp. 1-104; especially, p. 84 f. 
possible, consists of numbers very much reduced from those of Weber. Following are some citations from Goldscheider's table :

\begin{tabular}{|c|c|c|}
\hline Part of the body. & $\mathrm{mm}$. & Part of the body. \\
\hline Back & $4-6$ & Back of hand $\ldots \ldots \ldots \ldots \ldots$ 0.3-0.6 \\
\hline Breast & 0.8 & I. and II. phalanges (volar). . $0.2-0.4$ \\
\hline Forehead. & $0.5-1.0$ & I. and II. phalanges (dorsal).. $0.4-0.8$ \\
\hline Cheek. . & $0.4-0.6$ & Upper leg............... 3.0 \\
\hline Nose and chin..... & 0.3 & Lower leg............. $0.8-2.0$ \\
\hline Upper and lower arm & $.5-1.0$ & Back, and sole of foot.. \\
\hline
\end{tabular}

From the foregoing data it would seem to follow that, as the construction and relation of the chains of pressure-spots differ in the different areas of the body, so will our sense of locality change. The number, sensitiveness, and direction in the chains of these spots determine the sensitiveness of a given area. Moreover, our perception of the size and shape of objects in contact with the skin depends upon the same conditions. This can be shown in an astonishing way by comparing the apparent direction which the outlines of any small body moved across the skin seem to assume with the way the pressure-spots are located in the different areas through which it is moved. If the curve of the chain of pressure-spots, for example, bends in the reverse direction from that of the outline of the body moved, the effect may be to make this outline curve irregularly or even straighten it out.

It need scarcely be said that Goldscheider regards the true explanation of these phenomena to lie in the anatomical distribution of the specific nerves of sense in the different areas of the skin. However this may be, it is certain that our sensations of pressure are primarily " punctiform," and afterward massed into a tactual continu$u m$; and that what we primarily know is not the extended object as such, but our sensations of pressure which are afterward objectified.

$\S 26$. Closely connected with the foregoing is the difference in power of different parts of the skin in giving to the mind data for discriminating the fact, the amount, and the direction of motion in contact with the body. Upon this point the experiments of G. Stanley Hall ' are of special interest. These experiments seem to show that we are more likely, when in doubt, to judge motion on the surface of the limbs to be up rather than down their axis; on the breast, the shoulder-blades, and the back, the tendency is to judge motion to be toward the head. The discriminative sensibility of the skin for motion is much greater than that for separate touch, as determined by Weber's experiments. Tbus, while at

1 Motor Sensations on the Skin, by Professor G. S. Hall and Dr. H. H. Donaldson, in Mind, October, 1885, pp. $557 \mathrm{ff}$. 
least a distance of $25 \mathrm{~mm}$. betrieen the dividers' points was needed on the volar surface of the right arm, in order to perceive them as two points, botly the fact and the direction of motion could be discriminated at an average distance of between 6 and $7 \mathrm{~mm}$. In judging the rate and distance of motion over the skin the liability to error is always great; but, as a rule, distances rapidly traversed are judged to be relatively shorter than the same distances more slowly traversed. Inasmuch, however, as the judgment of motion on the left arm was expressed by reproducing the rate and distance with the right hand, ${ }^{1}$ we have a double liability to error involved in regulating the muscular movement of this hand by means of its series of muscular and tactual sensations.

Hall found the motor sensibility of different parts of the surface of the skin to be different; but the differences do not appear to correspond to those belonging to Weber's sensation-circles. The average distance, in millimeters, which a metallic point of $12 \mathrm{~mm}$. in diameter could move over the skin at a rate of $2 \mathrm{~mm}$. per second before a judgment of direction could be formed was found, for one subject of experiment, as follows: forehead, 0.20 ; upper arm, 0.40 ; forearm, 0.44 ; shin, 0.60 ; palm, 0.74 ; back, 0.85 . Motion can be produced so slowly as not to be discriminated at all, even when the body in contact has really moved from 6 to 12 centimeters. It can also be produced so rapidly as to make it impossible to tell when it begins and when ends. Heavy weights seem to move faster than light ones going at the same rate; but here other sensations are called out by the deep pressure, and combined with those of contact. Hall concludes that heat-spots and cold-spots traversed by the moving body are of great service in judging motion and its direction on the skin; the cold-spots more than the heat-spots, "because of the fainter sensation and wider irradiation" of the latter.

Further experiments with a travelling metallic point that carried the stimulus of an electrical current over the surface of the skin showed an astonishing diversity of sensations developed at different points of the area thus traversed. Points of cutting pain, "thrillpoints," "tickle-points," "acceleration-points" (or places where the rate of motion seems suddenly to increase without any real change in the speed of the moving metal), "blind-points" (or spots where all impression of contact is momentarily lost), are all to be differentiated. Yet the sharp differentiation of these sensations is rendered difficult by the fact that the rarious linds are so impacted and run together, in a tangle of sensation. The experimenters also speak as though many dermal sensations may thus be partially dis-

${ }^{1}$ Mind, October, 1885, p. 564 f. 
entangled, for the description of which language furnishes no adequate terms. All these facts agree exceedingly well with the theory of local signs already proposed. These dermal signs are complex "mixtures" of feeling, which give to each discernible locality a characteristic local stamp. The fact that our sensibility to motion is so much greater in each area of the skin than our susceptibility to the distance of stationary points accords with the same theory. Our ability to localize the dermal sensations is dependent upon the degree and rate of the changes in the color-tone of these sensations. Hall is undoubtedly right in holding that, by moving the touching surface over the surface touched, we do not simply multiply, but also diversify, our data for filling up the dermal blind-spots and judging the nature of impressions.

$\S 27$. The localizing of sensations of temperature in the skin is, in principle, the same as that of sensations of light-pressure or of motion. The former, however, are in all our ordinary experience interwoven with the latter ; they therefore have the help of the latter in getting a place assigned to them in the periphery of the body. Recent researches, already referred to (Chap. IV., §22), demonstrate the fact that the relative number and arrangement of heat-spots and cold-spots is different for different areas of the skin. Goldscheider ' has experimented to determine how far apart the heat-spots and cold-spots must be, respectively, in order that two of them, when stimulated, may be fell as two. Both kinds of sensations are localized, not as points, but as minute warm or cold drops in contact with the skin. By the following table, which gives the minimum distances for different areas of the body, it appears that the sense of locality connected with the cold-spots is about twice as fine, as a rule, as that connected with the heat-spots. The distances are given in millimeters.

\begin{tabular}{|c|c|c|}
\hline Part of the body. & Cold-spots. & Heat-spots. \\
\hline 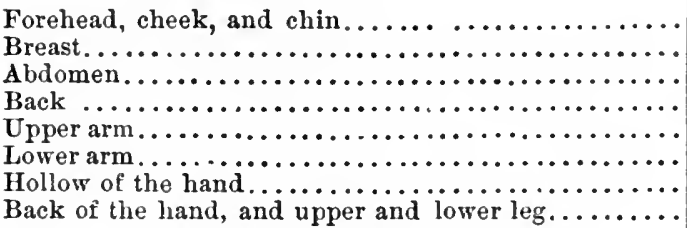 & $\begin{array}{l}0.8 \\
2.0 \\
1-2 \\
1.5-2.0 \\
1.5-2.0 \\
2-3 \\
0.8 \\
2-3\end{array}$ & $\begin{array}{l}3-5 \\
4-5 \\
4-6 \\
4-6 \\
2-3 \\
2-3 \\
2.0 \\
3-4\end{array}$ \\
\hline
\end{tabular}

'Archiv f. Anat. u. Physiol., Physiolog. Abth., 1885, Supplement-Band, pp. $70 \mathrm{ff}$. 
Some basis seems to be laid in the foregoing facts for a system of local signs of the skin, that consist in a mixture of color-tones of temperature-sensations. Yet sensations of heat or cold, in themselves considered, differ chiefly, if not wholly, in intensity. In themselves, therefore, they are not well fitted to constitute a so-called "spatial series" of sensations. If, for example, a certain area of the skin be stimulated simultaneously by both heat and cold, at points too near together to be distinguished by touch, the result is weither a modification of one seusation by the other nor a localizing of the two sensations as lying closely side by side.' A wavering of perception rather takes place, similar to the strife of colors in vision ; the experience is as though the skin were being touched with a single body alternately hot and cold. Klug also found that the least observable distance between two points touching the skin at the same time depends upon their temperature relative to that of the skin. The medium value of this distance is reached when these points have a temperature of $20^{\circ}-40^{\circ} \mathrm{C}$. $\left(68^{\circ}-104^{\circ} \mathrm{Fahr}.\right)$; it diminishes on either raising or depressing their temperature greatly above or below the zero-point of the skin. The fineness of our sense of locality, as well as of our sensitiveness to motion (comp. $\S 25$ ), is increased by exciting sensations of temperature up to the point where pain intervenes. But the localizing of these sensations is primarily dependent, to a great extent, upon their connection with localized sensations of touch. If we bring two parts of the skin, that differ considerably in temperature, into contact-for example, a cool hand and warm forehead, or a cool hand and a warm one-it is often difficult by strict attention to the sensations of temperature alone to tell which part is cooler, which warmer. The difficulty is doubtless largely due to the fact that each part which feels the temperature of the other is also changing its own temperature in the direction of the temperature of the other. It is therefore induced to feel itself, as it were, as being of the temperature of that other. A confusion of the data for judgment, accordingly, takes place. Any localization of the sensations which occurs under such circumstances is largely depen "ent upon secondary considerations, and especially upon the direction of the attention.

We judge of depth by sensations of temperature, indirectly, and through our ability to remove or change the intensity and locality of these sensations by changing the position of the body in space as related to what we know to be hot and cold bodies or surrounding media.

'See Czermak, Sitzgsber. d. Wiener Acad., March, 1855, p. 500; confirmed by Klug and others. 
\$28. The specific sensations of the muscular sense constitute another spatial series which combines with the foregoing in the localizing of areas at the periphery, and of external objects as projected in space and yet known as in contact with the body. Indeed, it is upon this particular system of local signs that the mind is chiefly dependent for its data-other than the visual-in the synthetic construction of its presentations of bodies that stand related to each other in three dimensions in objective space. Three principal theories have been held as to the nature of the so-called muscular sensations: (1) So far as they are not tactual, they are to be resolved into "central feelings of innervation," which differ only in intensity and not in specific quality, and which result from the changes, initiating movement of the bodily organs, that take place in the brain as correlated with impulses of the will (so Wundt, and others) ; (2) they are not specific sensations, but are due to interpretations of those feelings in the skin which originate on account of its changes of position, tension, etc., as the underlying muscles are moved (so Schiff, and others); (3) they are specific sensations dependent on a specific nerve-apparatus of sensé, which has its endorgans in the muscle-fibre, and which is excited by the contraction of the latter in a manner dependent upon the kind, amount, and direction of the muscular movement taking place (so Bell, Weber, Funke, and others).

We have already given certain reasons for rejecting the first two and accepting the last of the foregoing views (see p. $344 \mathrm{f}$.) ; other reasons will be mentioned subsequently in discussing the so-called "feeling of innervation" or of "active energy." The muscular sense, like all the other senses which contribute to our presentations of objects extended in space, appears to hare its own system of local signs. The muscular sensations are qualitatively (and not merely quantitatively) different, according to the combination of the muscles moved, and according to the exterision over the muscular area of the stimulus imparted to the sensory nervefibres situated in the muscle by the changing condition of the latter as it contracts and relaxes. The series of sensations - with all the qualities of rapid and nice gradations which belong to "spatial series" of sensations-called out by moving one limb differs from that called out by moving another limb. At each step in the flexing of the leg-for example-the color-tone of the muscular sensations has a specific quality and value as a local sign, in our consciousness, of the position of the member. The same thing is true of the bending arm, back, or single toe or finger. These sensations are intimately, and even inextricably, combined with the 
spatial series of specifically dermal sensations; but in themselves they have a different quality, and are not localized simply at the surface of the body. As the extent of the circuit of motion gone through by any limb increases, or the intensity of the strain becomes greater, the quality of the mass of resulting muscular sensations is perpetually changing. These sensations are, accordingly, localized orer a broader area of the body and deeper in its substance, as it were. Everyone knows what-new mixtures of sensation are produced in consciousness by calling into vigorous exercise the unused more deeply lying muscles of the body. Bain ${ }^{1}$ has discussed these seusations at great length and with commendable acuteness. But the apparent assumption that these particular sensations can, by being associated, acquire of themselves the quality of extension in space, and the accounting for all our other perceptions of spatial qualities and relations as merely secondary and symbolic of the associated muscular sensations, are in plain contradiction of established psychological facts and principles.

The muscular sensations also assist the more strictly tactual in discriminating locality for all cases where the pressure upon the skin exceeds a certain small degree of intensity. In strong contact or heavy pressure the sensory nerves of the underlying muscle are excited; we have the feeling, not simply of being touched, but also of being pressed. The combination of these two spatial series gives to the mind a doubly constituted system of local signs; hence, as the experiments of G. Stanley Hall ${ }^{2}$ show, our judgment of direction of motion is quicker as the weight resting on the skin is increased up to the limit where other disturbing sensations intervene. The superior discriminating power which any member of the body has when permitted to move-that is, to call forth familiar series of muscular sensations-is largely due to the help which the local signs of this system render to the mind. When the particular member (the hand) which is capable of the nicest tactual discrimination is also permitted to move over an object freely, and to acquire abundant data from all the sources described above, we have fulfilled the most advantageous conditions for the utmost nicety of knowledge possible to "touch," in the widest meaning of the word.

\& 29. It is unnecessary to illustrate in further detail the process by which the mind, with its native synthetic activity, and with the help of qualitatively different sensations, constructs its field of

\footnotetext{
1 The Senses and the Intellect, especially pp. 57-100, 336-348, and 364398.

${ }^{2}$ Mind, October, 1885, p. 567.
} 
touch. To multiply instances would neither explain the ultimate mystery which enters into the processes of "localization" and " eccentric projection" by touch, nor add materially to our comprehension of known psycho-physical principles. The muscular sense may probably be said to have the leading position in the development of the perception of spatial objects and relations, so far as attainable without the aid of sight. Perceptions of the magnitude, distance, and primary spatial qualities - such as the extension and inertia-of material things are largely dependent upon associated sensations of the muscular sense, although these perceptions cannot be said to be mere compounds of such sensations with secondary and symbolic sensations of other kinds added to them. The activity of the hand, as it moves over various surfaces of the body, either. touching them itself or carrying with it something with which these surfaces are touched, early combines with the different series of muscular sensations other spatial series of tactual sensations.

The localization of certain points in the area of the body which are of marked local characteristics, and frequently recurrent in experience, is the first achievement in constructiug the field of touch. To these landmarks, as it were, other points or areas, subsequently discovered, are referred. One hand learns to know the other; the right hand chiefly explores the left arm and side and the upper right leg; the left hand, the right arm and side and the upper left leg. The finger-tips, especially of the right hand, have an office similar to that performed by the yellow-spot of the retina; they are the centre or hearth of clear perceptions of touch. But in order to bring them to their object they must be moved; through this motion fresh combinations of muscular and tactual sensations result.

$\S 30$. But long before the entire field of touch has been constructed with any considerable approach to completeness, the eye has already explored those parts of the body which are open to its inspection. It learns first to know the hand, which nature keeps constantly in motion before it. As objects rest on the hand, it notes the place where they rest ; with its perceptions of sight certain combinations of tactual sensations thus become associated. As the hand moves over other objects, or especially over the other parts of the body, the eye marks its successive progress; combined sensations of muscular and tactual kind are thus associated with each position of the hand and with each area of the body which it touches. Very early in the development of a normal experience the eye comes to be the leader and critic of the discriminations connected with the muscular and tactual sensations. Its power of rapid movement over its total field, and its delicate judgment on account of the 
finely shaded complex local signs which it calls forth with a comprehensive simultaneousness, give a great superiority to the organ of vision as a geometrical sense. The results of such superiority it constantly places at the disposal of the more slowly moving and less delicate sense of touch. For this reason, one born blind can never attain the same quality (of "comprehensive simultaneousness") for his spatial intuitions and ideas of spatial relations; even the field of touch, in spite of the greater refinement which the muscular and tactual sensations of such an unfortunate person acquire through use, cannot possess this quality as it is imparted by the eye.

The familiar experiments of trying to estimate the size, shape, and relation of objects, the amount and direction of motion, etc., when blindfold, show our dependence upon the organ of sight. It must not be forgotten, however, that the discriminations possible through the muscular and tactual sensations alone are wonderfully exact; and that in certain circumstances touch has sight at a disadrantage, as it were. Thus the player on the violin who should adjust his spacing of the strings by the sensations of the eye, with the unaccustomed and unfavorable perspective made necessary by its position in relation to the left haud, would not attain the art of making true and pure tones.

\$31. Among the most complex perceptions of which the skin and muscles by their combined action are capable are the so-called "feelings of double contact." It is largely by means of these feelings that skill is acquired in the use of tools, weapons, and musical instruments. In these cases the process of projection goes so far that we seem to feel the object with which the implement is in contact, not so much in the hand (the feelings of contact being located there), by the external means of the implement, but rather as ourselves being in the implement and using it as a sentient part of the organism. The carver in wood feels his chisel move through the stuff he is shaping, and guides it as unerringly as he would his finger, so as to lay it with a given degree of pressure upon a given spot. We are all familiar with the experience of feeling the ground we are about to tread, with a cane or other stick. If the fingers be lightly brushed over the hair when it stands out from the head, it will be difficult to localize the sensations of pressure at the scalp rather than in the hair. We feel the touch of our finger at the end of the tooth, where the contact takes place, instead of where the sensory nerves really receive the stimulus and convert it into a nerve-commotion

The mauagement of the implement is, of course, really made 
possible by delicate changes in the shades of feeling called out by its changing pressure upon the nerves terminating in the skin and muscles of the hand, and by the accompanying feelings of strain and of effort that result from the movement of the arm which carries the hand. These feelings are aroused by the end of the implement which is in contact with the body, and are primarily localized in that part of the body; but they are felt through a more artificial and elaborate process of localization, as though directly dependent upon the other end of the implement. Upon the rsthetic and pleasurable uses of these feelings of double contact Lotze ' has remarked at length.

At this point the further discussion of the development of our presentations of sense in general must be arrested, in order to consider more in detail the activities of the other great "geometrical sense."

${ }^{1}$ See Microcosmus, i., pp. 586 ff. Edinburgh, 1885. 


\section{CHAPTER VII.}

\section{THE PRESENTATIONS OF SENSE. [CoNTINUED.]}

§1. THE application of the general principles which control the development of our presentations of sense to the particular case of the eye has many peculiar difficulties. The physiological psychology of visual perception is, therefore, a much controverted and very obscure domain. This fact is doubtless in part due to the amount of experimenting and speculating which has been bestowed upon it. For here, as elsewhere in scientific research, one chief result of extended examination is to raise unanswered inquiries. Peculiar difficulties, however, are intrinsic in the case of the eye. These are due to the great complexity of its native activities, and to the speed with which it reaches a generous maturity of development. Nature has equipped this organ with superior means for furnishing to the mind a variety of data, as respects both quantity and quality, for the nicest discriminations; it has also provided it with such constant stimulation as to cause it to acquire an incomparable facility. But the character of its structure, functions, and development is such as to make experiment difficult in a way to disentangle the simple factors from those complex forms into which the synthetic activity of the mind has constructed them.

$\S 2$. It is affirmed by one authority' that no less than eight different data, or motifs, are used in monocular vision by the adult for perceiving the third dimension of space and of visual objects in space. These are the changes with respect to (1) extent and (2) clearness, of the complex of the sensations of color and light, as dependent on distance; (3) the perspective elevation of the bottom of distant objects above the horizon; (4) the covering of known distant objects by those placed nearer; (5) the alterations of light and shadow on the curved surfaces of the object, according as they are nearer or more remote; $(6)$ the perspective contraction of the retinal image; (7) the change of the visor angle in proportion to the distance of the object; (8) the muscular sen-

\footnotetext{
' Volkmann von Volkmar, Lehrb. d. Psychologie, II., p. 84.
} 
sations of the accommodation of the eye. To these eight data, two others at least must be added for binocular vision ; namely (9), the stereoscopic double images, and (10) the sensations arising from convergence of the axes. These ten sets of variable experiences may be combined, of course, in an almost infinite variety of proportions.

Moreover, it is not improbable that we shall have to admit still: other data as entering into the complex perceptions of sight. The tactual, as well as muscular, sensations which accompany the movement of the eyeballs in their sockets are not ineffective in giving grounds for judgment in certain cases. The question must also be raised : Do not the visual sensations themselves have a certain local coloring directly dependent upon the nervous elements of the retina which are excited by the stimuli? If we answer this question affirmatively, we shall have a system of local retinal signs as constituting one of the most primary of the spatial series of sensations entering into the space-perceptions of this sense. And after all this cataloguing of data, the dispute as to the existence of series of sensations of innerration that have a central origin and differ only in intensity as directly dependent upon so-called acts of will (so Wundt) remains unsettled.

Several of the data just enumerated, however, are plainly of only secondary rank and value; they do not necessarily enter into every preception of a visual object as such. What does seem necessary to the most elementary form of risual perception may be stated as follows: Sensations of light and color, differing in intensity and quality, but simultaneously present in consciousness, must be systematically arranged with reference to each other by being localized with the help of retinal signs, and associated with other spatial series of muscular sensations that arise from accommodation of the eye and from its motion. The complexity of the combinations arising in the normal use of the organ of vision is, of course, increased by the fact that there are two eyes, and, therefore, two retinas with their systems of retinal signs, two images of each object, and two sets of motions. But the two eyes are (as we shall see subsequently) in a certain sense to be regarded as one eye-certainly as constituting one organ of vision. So that, even when one eye is closed, the other does not see what it sees without being influenced by the closed and relatively inoperative part of the one organ. The constancy with wlich the eyes act together explains, in part, why they are one organ as the two hands are not; but the frequency with which we voluntarily suppress the activity of one eye by closing it explains, in part, why they are not one organ as are the two nostrils or the two ears. 
§ 3. Could we select an adult human being who had never seen, and proceed to develop his visual perceptions, experimentally, in the direct order of their complexity, we might possibly rely upon his description of his experience to solve certain problems that now seem unsolvable. We should wish, before either eye had been moved when open, to excite the nervous elements of a small area of one stationary retina, and to ascertain how far the sensations of light and color thus excited could be said to have any strictly "local" arrangement with reference to each other. We should then wish to try the effect of combining with these sensations other spatial series, consisting of miscular sensations and arising from the accommodation and motion in its orbit of the same eye. Finally, the intricate process of putting the two eyes together-both open and both moving - might be studied in detail. At present, however, it is quite impossible to say what the experience of the subject of such experiments would be. The testimony of the few blind persons whose eyes have been couched is so meagre and unsatisfactory, on account of its failure to comply with the conditions of scientific investigation, that it can be used only to confirm conclusions arrived at on other grounds. Nothing remains, then, but to employ the data which physiological optics has secured, in order to make a theoretic reconstruction (confessedly imperfect and doubtful) of the process that nature is all the while successfully completing. In this effort we naturally follow the order of nature, so far as possible; we begin with the sinplest conceivable case, and proceed from it to the explanation of the amazing complexity which really belongs to our apparently simple daily experience of vision with two trained eyes. This is substantially the course followed by Wundt, ${ }^{2}$ who finds three things to be considered in explaining the developed perceptions of sight: (1) The retinal image of the eye at rest, and the motifs which it furnishes; (2) the single eye as moved, and the influence of these movements ; (3) the conditions furnished by the existence and relatious of the two eyes exercising their functions in common. But, in reality, from the very beginning of its activity the eye is in motion, and acts as a clouble organ.

Corresponding to the three sets of considerations just mentioned, we may speak of three fields of vision which are to be constructed in the order of their complexity. They may be called, respectively, the retinal field of vision, the field of monocular vision, and the field of binocular vision. In the "retinal field of vision" we mean to include only such a perception-or mental spatial arrangement

\footnotetext{
${ }^{1}$ Physiolog. Psychologie, ii., p. 62.
} 
of sensations of color and light as points lying side by side-as would be presented through the excited expanse of nerrous elements constituting the retina of one motionless eye, in case there had been no previous vision with both eyes in motion. The field of monocular vision, when completely constructed, includes all that can be seen with one eye as the result of its experience, dereloped, but unaided by the other eye. The field of binocular vision includes all that can be seen by both eyes. The first two socalled "fields of vision" are, strictly speaking, fictitious and theoretically constructed in order to explain the process by which the mind reaches the construction of the third and last. Indeed, the question may be pressed, whether we can speak of a purely "vetinal field of vision," and whether the excited mosaic of nerrous elements on which the image is formed, without aid from muscular sensations of the eye, could furnish any presentations of sight.

$\S 4$. The most nearly original experience of sensations of light and color which can be easily produced for adult observation is gained by closing and blindfolding both eyes, and then keeping them as motionless as possible. Let time enough be allowed for all the after-images, both positive and negative, to die wholly away. Nothing is then seen but a small and undefined expanse or massive aggregate of related color-sensations, which we will call the "retinal field;" it might almost be said that this is felt rather than seen. Such "vision" (?) of a certain continuum of sensations cannot be said to be either localized or projected in space, as a whole, and by the eye alone. When we speak of it, for example, as "in front of " the upper part of the body, we introduce terms that are derived from experiences of touch. Now, without moving or uncovering the eyes, let the head be turned to the right or to the left, and the expanse of color-sensations will move in the same direction; or, if we turn the face upward, the retinal field seems above us; if downward, then it seems to sink toward our feet. But each position of this field, as a whole, is entirely determined by the fact that the customary muscular and tactual sensations assure us of the posture of the head with reference to the rest of the body. Such localization is accomplished chiefly by sensations in the neck. So far as sight alone is concerned, the entire expanse of color-sensations cannot be said to be perceived as anywhere in space.

The "retinal field" has no clearly defined limits, or boundarylines; it may be described rather as having its expanse of sensations distinguished by a shifting, graded transition into a region of no-sensations. This fact is, of course, due to the constantly changing activity of the nervous elements of the retina. Yet the sensa- 
tions which are massed in the foregoing experience constitute a true spatial expanse; they are not simply recognized as differing in color-tone, or brightness or intensity of effect, but as having true local distinctions, and as being arranged into a system of points of color and light lying side by side. In other words, the different sensations do not fall together in consciousness so as to resemble the one sensation of smell produced by irritating simultaneously a number of fibres of the olfactory nerre; nor are they simply analyzable into several qualitatively different factors, as is the complex sensation of a musical clang. They are presented as spatially systematized, as a true perception of an extended object. The "retinal field" may, then, be said to be extended in two dimensions; and the minima visibilia which compose it all have local relations to each other. It cannot properly be said, however, to have depth (as Stumpf ${ }^{1}$ and Hering ${ }^{2}$ hold that it does) ; for the different colored points are not projected as different in distance, nor can we be said to look into the colored space thus presented before the mind. It is true that the expanse of the retinal field is not like that of a darkly colored wall or curtain placed in front of the eye. But the quasi-appearance of depth is due to constant change in color-tone and brightness of the minute portions of the field, which has an effect somewhat like that we get on looking at a very dense mist of particles differently colored and drifting. In other words, the secondary and derived data give to it an appearance which we have learned to associate with the perception of deptll.

\$5. Further experiment, however, with this so-called "retinal field" serves to show how complicated its apparently simple character really is. In the first place, even this field is the result of the combined action of the two retinas. If, with both eyes closed, a "phosphene" (see p. $\mathbf{1 9 5}$ f.) be produced in either eye by pressing upon its ball, the colored circle will be located in the corresponding part of the field; but the character of the entire field, as formed by the activity of both retinas, will be changed. It is, of course, impossible to suppress the action of one retina, and thus examine a monocular "retinal field," as it were. But it may easily be shown that, even in vision with one eye open and in motion, the character of the whole field of vision is under the influence of the retinal activity of the closed eye. Let one of the eyes-both hitherto closed and motionless-now be opened. Immediately a

${ }^{1}$ Ueber d. physiolog. Ursprung d. Raumvorstellung. Leipzig, 1873. Stumpf holds that "Space is just as originally and directly perceived as quality" (p. 115).

2In Hermann's Handb. d. Physiol., III., i., p. 572 f. 
picture of all the objects falling within the field of monocular vision appears before us; each object seen with its position, magnitude, and spatial relations determined according to the laws of visual perception. This monucular field seems bounded on one side (the left side if the right eye is opened, the right side if the left) by the rather dim outline of the nose and lower line of the forehead. What has become of the retinal field of the closed eye? It has been submerged or overwhelmed by the field of the open eye, on account of the latter's stationary and clearly defined images and strong arrest and fixation of attention. But if a character to arrest and fix the attention be given to the field of the closed eye, it may be made in turn to overwhelm that of the open eye. This can be accomplished by producing strong "phosphenes" in the former. On pressing the closed eye brightly colored circles are presented in the corresponding part of the field; and by using sufficient pressure the objects seen as projected in space by the open eye are drowned in a shower of minute, vivid sparks.

The "retinal field" has its character determined also by associated muscular sensations dependent upon the movement of both eyes. It will be found impossible to make any definite area of this retinal field, which lies much to the right or left, to the upper or lower part, of its centre, a matter of regard without detecting slight movements of the eyes according to the direction in which the attention is to be fixed. The value of muscular movements in this case cannot consist in their enabling a clear image of objects situated in different relations to the eye to be formed on its retina ; for with closed eyes no change is occasioned in the retinal images by motion of the eyes. The conclusion, then, is, that certain muscular sensations constitute an indispensable part of the data for localizing objects even in the retinal field-at least such as are only a slight distance from its centre. Moreover, it will be found that the extent of this entire field and its prolongation, as it were, in any given direction are dependent upon the accommodation and motion of the eyes. The explanation of this fact can be found only in the same truth, namely : The perception of localized areas in the two-dimensioned "retinal field" of the closed eyes is dependent upon the revival of associated muscular sensations.

$\S 6$. The foregoing facts undeniably afford considerable support to the "empiristic" theory of visual perception; but they do not show that the considerations it brings forward are entirely conclusive. They do not even prove the truth of Wundt's statement: ${ }^{1}$

'Physiolog. Psychologie, ii., p. 69. 
"Our sensations of light do not immediately possess spatial form." After excluding all the factors which combine into our ordinary presentations of sight-such as double images, accommodation, convergence of the axes of the eyes, and secondary helps by way of shadows, perspective, elevation, etc. - a certain spatial quality still remains to the simplest sensations of color and light which we are able to reproduce. It will naturally be objected that these sensations are the reactions of a mind that has had a long previous experience in localizing visual sensations by means of just such helps as the foregoing. The question then recurs: Is the fact that the sensations of light and color, which are produced by the simultaneous excitation of many nerrous elements of the retina, appear as locally distinct (even when the eyes are closed and motionless) an otherwise unexplained datum due to an original activity of the mind under the law of the specific energy of these nerrous elements; or is it a result of acquired experience, to be explained by the revival of images of previously associated impressions obtained when the eyes were both open and moving? To take the former position is to adopt, so far forth, the nativistic theory of visual perception; to take the latter is to espouse the empiristic opinion. Either position has its difficulties. The former seems to us, however, nearer to the ultimate truth.

$\S 7$. That the sensations of light and color occasioned by stimulating different elements of the retina have a different value in consciousness, and that the recognition of this value, and the presentation of the sensations as locally separate and arranged into a spatial system, is native to the mind, may be argued from the following among other reasons: The peculiar mosaic structure of the retina is obviously the fundamental cause for the pre-eminence of the eye as a "geometrical sense." It has already been shown (Chap. IV., \& 3) that each element of this structure may be regarded as an isolated sensitive spot, which corresponds, on the one side, to individual irritations from the stimuli, and, on the other, to the smallest localized sensations of light and color. But the latter part of this statement could not be true unless each of the elements in this nervous mosaic had a certain peculiar representative value in conscionsness. In other words, sensations of light and color are localized in part, at least, by means of the specific local quality which belongs to the result of the different points in the retina being simultaneously irritated. The very construction of this organ, as well as the correspondence between its construction and the nicety attained in its use for local distinctions, indicates that the spatial quality of our visual percepts depends upon its specific functions. 
Moreover, unless the series of light- and color-sensations had an original spatial character, it is difficult to see how they could combine with the other spatial series of the eye into perceptions of extended colored objects. It is difficult to see what advantage they would then have over the series of musical tones varying in pitch. Still further, it is as impossible to prove experimentally as it is to make seem true to consciousness that the arrangement of the points of light and color which appears before us with closed and motionless eyes is only the residuum, as it were, of past sensations of a muscular kind. Such an appeal to consciousness could not be made, indeed, with any confidence, if scientific analysis were able to show that the color-sensations can be perceived simultaneously, as a system of points lying side by side, without having the characteristics of a spatial series. But in view of our inability to do this, we only account for the facts of consciousness by admitting what the very structure of the organ suggests, and what general psychological theory seems to confirm, when we hold that spatial perception, at least in germinal form, is native to the mind as a synthesis of the qualitatively different sensations which result fron stimulating simultaneously the retinal mosaic of nervous elements.

The foregoing view is very different from that which assumes that we have an immediate knowledge of the retinal image; or that a knowledge of the direction from which the light falls upon the retina is an unresolvable intuition of the mind. ${ }^{\prime}$ To such mistaken statements it is a sufficient reply to show that the subjective image (or mental presentation) of the object does not correspond either to the image on the retina or to the real object as it is otherwise known to exist in space. The mental presentation, for example, has no blind-spot; it is a different representation of the real object from that offered by the retinal image, with more inaccuracies than belong to the latter as seen by an observer looking at it from without. ${ }^{2}$ To the question, then, whether sensations of light and color would have space-form if they came only from an excited but motionless retina, and were uncombined with other sensations of a spatial series, we can give only a tentative and partial answer. Doubtless the "presentations of sense" formed by combining such sensations alone would be indescribably different from those to which we now ascribe visual space-form. An animal with a single immovable expanse of nervous elements susceptible

'See Le Conte, Sight, pp. 85 f. and 105. New York, 1881. Le Conte is obliged to admit, however, that this "law of direction" is sometimes opposed to the "law of corresponding points" (p. 258).

${ }^{2}$ See Wundt, Physiolog. Psychologie, ii., p. 68. 
to irritations from light could not be said to have what we call "vision." But, on the other hand, the spatial quality which belongs to the visual sensations of man cannot be all resolved into muscular and tactual sensations of eye and hand ; these sensations, quoad sensations of light and color, do have the quality which insures their arrangement in consciousness in spatial order. This fact is due to the working of the law of the specific energy of the nerrous retinal elements in connection with the native activity of mind in synthesizing these sensations. The law as applied to the eye is essentially the same as that already demonstrated for the skin; the activity assumed as native to man is not essentially different from that ascribed to the lower animals in the use of their senses. That this tact for the individual has been largely won by the development of the race is a proposition to which our attitude is determined by more general conclusions. But physiological optics cannot account for the phenomena of vision without assuming both the original exercise of this tact and the theory of local retinal signs as data hitherto unresolvable by its analysis.

$\S 8$. Whatever may be thought of the foregoing assumptions, it is certain that ordinary adult visual perception involves the motron of the open eye-monocular vision of one eye and binocular of both. The sensations which accompany such motion must be coinbined with sensations of light and color to make the complete presentations of sight. The consideration of the simplest case requires that we should recur to the physiology of the eye. Only one small spot in the retina (the so-called "forea centralis," see p. 183), is capable of giving a perfectly clear image of an object. When, then, we clesire to see an object clearly, we bring its image upon this spot and fixate it there. That point of the object to which the centre of the retinal area of clearest vision corresponds is called the "point of regard" (or "fixation-point"). In ordinary vision, then, the eye constantly changes its point of regard, and so brings successively upon its most sensitive area the images of the different points of its object.

The different changes of position in the point of regard are accompanied by sensations of motion and strain; they are accomplished by the six muscles of the eyeball. This wandering of the point of regard over an object may be considered as accomplished by rotating the eye upon a pirotal point, or "centre of rotation," by motions that have different axes of rotation. The centre of rotation is, however, only theoretically a point, but is really an interaxial space. It has been variously located for normal eyes at about $13.45-13.73 \mathrm{~mm}$. behind the cornea, and 1.24-1.77 
$\mathrm{mm}$. behind the middle of the optical axes. Of such axes of rotation, three are especially to be distinguished-an antero-posterior, a vertical, and a transverse. A line drawn from the centre of rotation to the point of regard is called the "line of regard;" since each eye has its own centre of rotation, there are, in vision with both eyes, two lines of regard. A plane passing through these two lines is called "the plane of regard" (or "plane of vision ").' In the "primary position" the head is erect and the line of regard directed toward the distant horizon. The plane passing through the lines of regard of both eyes in this position is the "primary plane of vision." In this position for most eyes, however, the line of vision is inclined somewhat below the horizontal plane.

Starting from the primary position, one set of positions are suc-

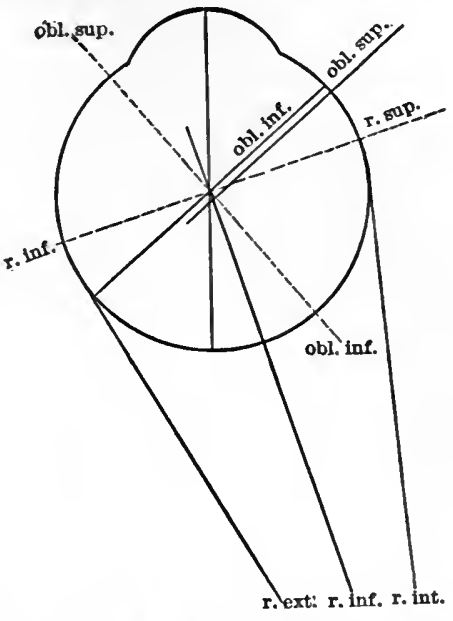

FIG. 97.-Dingram of the Attachments of the Juscles of the Eye, and of their Axes of Rotation-the latter being shown by dotted lines. The axis of rotation of the rectus, externns, and internus, being perpendicnlar to the plane of the paper, cannot be shown. cessively assumed by moving the eye upon its transverse and vertical axes. When the eye rotates round the former, the line of regard is displaced either above or below; it thus makes a varying angle with the line corresponding to its first direction, and this is called the " angle of vertical displacement" (so Helmholtz), or the "ascensional angle." When it moves about the vertical axis, the line of regard is displaced from side to side, and forms with the median plane of the eye a varying angle called "the angle of lateral displacement." In passing from the primary position to the foregoing secondary position no rotation of the axis itself occurs. Another order of positions is assumed by an apparent rotation on the anteroposterior axis, combined with lateral or vertical displacements; this movement results in bringing the eye to an oblique position, and is really a torsion of the eye. The angle which the plane of regard makes with the transverse plane measures the amount of torsion, and is called the "angle of torsion."

' For the detailed theory of the movements of the eye, see Hering, in Hermann's Handb. d. Physiol., III., i., chaps. 9-11; Helmholtz, Physiolog. Optik, $\$ \Im 27-30$; and Wundt, Physiolog. Psychologie, ii., p. 72 f. 
\$ 9. The various morements possible for the eye in all the directions just described are accomplished by the combined pull of the muscles of the eye as summarized in the following table ${ }^{1}$ (for the muscles and their position, see Fig. 49 f., p. 174) :

\begin{tabular}{|c|c|c|}
\hline Number of muscles active. & Direction of line of regard. & Muscles acting.. \\
\hline $\begin{array}{l}\text { One } \ldots \ldots \ldots \ldots \ldots \ldots \\
\text { Two } \ldots \ldots \ldots \ldots \ldots \ldots\end{array}$ & $\left\{\begin{array}{l}\left\{\begin{array}{l}\text { Inward } \ldots \ldots \ldots \ldots \ldots \\
\text { Outward } \ldots \ldots \ldots \ldots \ldots\end{array}\right. \\
\left\{\begin{array}{l}\text { Upward } \ldots \ldots \ldots \ldots \ldots \ldots \\
\text { Downward } \ldots \ldots \ldots \ldots \ldots\end{array}\right. \\
\text { Inward and downward. } \\
\text { Outward and upward }\end{array}\right.$ & $\begin{array}{l}\text { Internal rectus. } \\
\text { External rectus. } \\
\text { \{ Superior rectus. } \\
\text { Inferior oblique. } \\
\text { \{ Inferior rectus. } \\
\text { I Superior oblique. } \\
\left\{\begin{array}{l}\text { Internal rectus. } \\
\text { Superior rectus. } \\
\text { Inferior oblique. }\end{array}\right. \\
\left\{\begin{array}{l}\text { Internal rectus. } \\
\text { Inferior rectus. } \\
\text { Superior oblique. }\end{array}\right. \\
\left\{\begin{array}{l}\text { External rectus. } \\
\text { Superior rectus. } \\
\text { Inferior oblique. } \\
\text { External rectus. } \\
\text { Inferior rectus. } \\
\text { Superior oblique. }\end{array}\right.\end{array}$ \\
\hline
\end{tabular}

$\S 10$. The law which seems to govern all the eye's movements of torsion-or combined movements sideways, and either up or down -was conjectured by Listing, whose name it bears, and elaborated by Helmholtz. Listing's law is stated by Helmholtz ${ }^{2}$ in the following terms: "When the line of regard passes from its primary position into any other position, the torsion of the eye (as measured by the angle of torsion) in the second position is the same as if the eye were turned about a fixed axis standing perpendicular to both the first and the second positions of the line of regard." The same principle is stated in different language by Wundt:" "All morements of the eye from its primary position take place about fixed axes, each of which at the point of rotation stands at right angles to the plane which is described by revolving the line of regard; and all of these axes lie in a single plane, at right angles to the primary position of the line of regard, at its point of rotation." The orientating of the eye, then, for every possible position of the line of regard, may be referred to a constant standard. Concerning one important matter in the carrying out of Listing's law, there is a direct conflict of view between authorities. According to Helm-

'Given by Beaunis, and to be found in the Encyclopædia Britannica, ninth ed., VIII., p. 825.

${ }^{2}$ Physiolog. Optik, p. 466.

${ }^{3}$ Physiolog. Psychologie, ii., p. 79 f. 
holtz,' when the plane of vision is raised, lateral displacements to the right produce rotation of the eye to the left, and lateral displacements to the left produce rotation to the right; when the plane of vision is depressed, lateral displacements to the right produce rotation to the right, and vice versa. But according to $\mathrm{Le}$ Conte, ${ }^{2}$ in elevation of the visual plane the eyes both move and rotate to the right or to the left; in depression of this plane, motion of the eyes to the right is accompanied with rotation to the left, and motion to the left with rotation to the right.

More detailed statement of the laws of the eye's motion in vision is not necessary for the purposes of physiological psychology. It need only be noted that the construction of the field of monocular or binocular vision is a synthetic mental achievement dependent upon the varying sensations which result from the wandering of the point of regard over the outline of an object. Starting from its primary position, the eye may come around, as it were, by a variety of circuitous paths, to the fixation of any particular point of its object. In the pursuit of these paths it develops various series of muscular sensations that have spatial qualities and are fitted to combine with the spatial series of light and color sensations. Thus the field of vision necessarily has the same form as the surface orer which the point of regard can be made to wander. Its construction is a progressive synthesis of the mind, stimulated and guided by means which consist in varying states of consciousness, chiefly dependent upon the local coloring of the two sets of sensations thus far described.

\$11. Certain important consequences follow as to the relation between the lines of the extended and objective "thing" and the lines of the retinal image, as affording the mind data for the spatial ordering of the sensations that arise from stimulating the nervous retinal elements and nerve-fibres of the muscles of the eye. Both the general form of the field of vision and the relative position of the objects in it are determined by the movements of the eye. The rule is, that only those objects which are seen by direct vision (their images lying in the line of regard when the eye is in its primary position) appear in their actual place; objects indirectly seen appear in the place which they would assume if their retinal images were transposed to the point of regard and its inmediately surrounding points. ${ }^{3}$ It follows, further, that all lines lying outside of the vertical and horizontal meridians of the retina, in order to be seen straight, must be really bent; and all really straight lines in

1 Physiolog. Optik., p. 463.

"Sight, pp. 173 ff. ; and American Journal of Science and Arts, xx. (1880), pp. $83 \mathrm{ff}$.

${ }^{3}$ Comp. Wundt, Physiolog. Psychologie, ii., p. $90 \mathrm{f}$. 
such positions are seen bent. This fact may be proved in various ways. If a sheet of white paper, having a black dot in its centre to serve as a point of regard, be held at right angles to the line of vision, with the eye in its primary position and constantly fixed upon this point, thin, straight slits of black paper outside of the two meridians will appear bent. Or if the after-images left on these meridians of the retina by light falling through narrow and straight slits be studied when torsion of the eye takes place, these after-im.

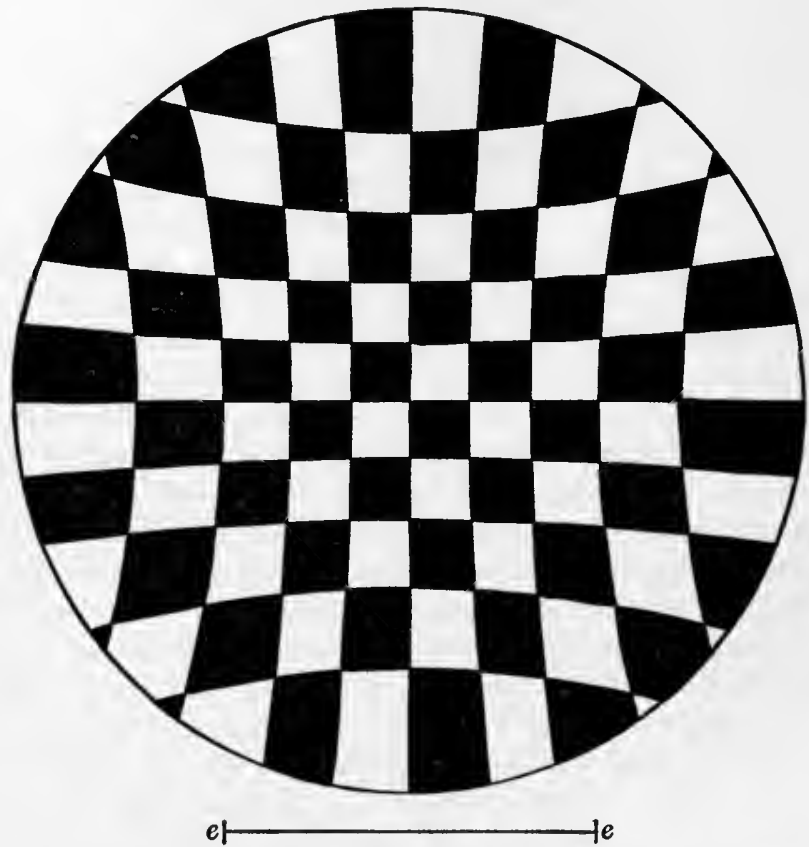

Fig. 98 (From Hering, after Helmholtz). - With the eye at the distance $e-e$, and fixated upon the centre, the hyperbolic lines which limit the black and white surfaces show the so-called "right lines" of the ficld of vision.

ages will themselves be found to suffer torsion.' Such images, received upon the vertical meridian of the eye when it is in its primary position, lean to the right, thus / , when the risual plane is elevated and the eye moved to the right; but when, with this plane elevated, the eye is moved to the left, the vertical image inclines to the left, thus $\backslash$. With depression of the visual plane, the inclination of the after-image is reversed. The image of a perfect rectangular cross is distorted as follows by different torsions

\footnotetext{
${ }^{1}$ See Le Conte, Sight, pp. 164 ff.
} 
of the eye: Upward and to the right, $f$. ; upward and to the left, $f$; downward and to the right, $f$; downward and to the left, $f$. The connected results of all the possible torsions of the eyes in curving the lines of the field of vision is illustrated by the accompanying figure $(98)^{2}$; the study of this figure, as it appears at various distances, from arm's length to contact with the nose and forehead, is an instructive exercise. The dependence of the field of vision upon the positions and motions of the eye is one principal source of the errors of this sense.

$\S 12$. Besides the help from muscular sensations due to movements of the eye in fixing its point of regard, account must be taken of those which result from accommodation of the eye (for the mechanism of accommodation, see p. 177 f.). As says Helmholtz : ${ }^{2}$ "There can be no doubt that anyone who has much observed his own changes of accommodation and knows the muscular feeling of the effort belonging to them, is in a condition to tell whether: when he fixates an object or an optical image, he is accommodating for a great or small distance." There is scarcely greater doubt that the significance of this change of muscular feeling would not be realized as indicating a third dimension of space, were it not combined with sensations belonging to the use of both eyes in conjunction with the organs of touch. Even adult judgment of distance, by accommodation alone, is extremely imperfect. Wundt ${ }^{3}$ experimented to determine the niceness of this judgment by regarding a black thread, stretched vertically against a white background, with one eye through an aperture in a shield. He found that almost nothing could be told in this way as to the absolute distance of the thread. Its relative position, however, could be discriminated with considerable accuracy by changes in accommodation ; and, as might be expected, with more accuracy when the apparatus was called into more active operation by approach of the object toward the eye. Helmholtz ${ }^{4}$ found that he required a stronger accommodation to see a red stripe clearly through a tube than was necessary to see one of blue.

1 Taken from Hering (after Helmholtz), in Hermann's Handb. d. Physiol., III., i., p. 537.

${ }^{2}$ Physiolog. Optik, p. 633.

${ }^{3}$ Beiträge zur Theorie d. Sinneswahrnehmung, 1862, pp. 105-118.

${ }^{4}$ Ibid., p. 634. 
$\S 13$. But all the achievements possible to a single eye, when open and in motion, would not avail to produce the presentations of sight as our ordinary experience is familiar with them. Strictly monocular rision is for the most part a fiction of science. What we can see with one eye, after experience in binocular vision, depends upon what we have been accustomed to see with both eyes. Indeed, what we see at any instant with one open eye depends, in part, upon the position, motion, and retinal condition, of the other and closed eye. A theory of binocular vision, lowever, requires the consideration of two sets of data in addition to those already enumerated. These are the existence and relations of the two retinal images, and the relations and laws of the binocular morements of the eyes.

The fact that two eyes are ordinarily active, and that there are, therefore, two images of the object, is a fact of the first importance for the theory of visual perception. Each eye is in itself, indeed, a complete optical instrument; each has its own point, line, and plane of regard, and movements of rotation, torsion, and accommodation. The two eyes, however, act normally as one instrument ; and yet they cannot be regarded as mere duplicates. The theory of binocular vision, then, considers the two eyes acting as one. For the purposes of such theory it is not important what shape the two

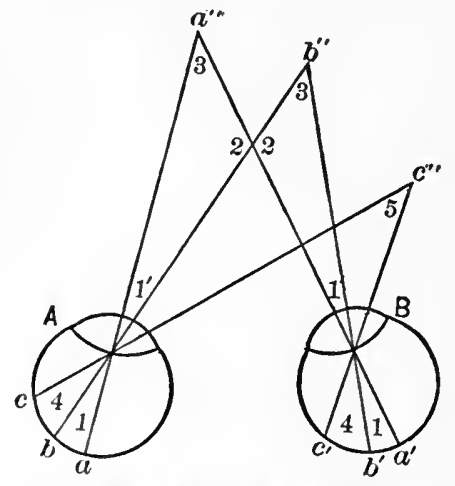

FIG, 99,-Diagram to illustrate the theory of corresponding retinal points. The im. ages of objects at $a^{\prime \prime}$ or $b^{\prime \prime}$ or $c^{\prime \prime}$ will fall on corresponding points of the retina$a$ and $a^{\prime}, b$ and $b^{\prime}, c$ and $c^{\prime}$-and be seen single. retinas are regarded as having; they are usually taken as surfaces with the curvature of the inside of a sphere whose centre lies at a point where all the lines of direction intersect." It may be assumed, to begin with, that this point of intersection is the same for accommodation to all distances of the object. If the two retinas were perfectly symmetrical all the nervous elements which compose the mosaic of each one might be regarded as situated at points identical with those occupied by the nervous elements of the other. In other words, the surfaces of the

two retinas might be regarded as capable of being perfectly superimposed. Upon such retinas, when the eyes were parallel, each single point of an object would have its image formed upon two "iden-

' See Hering, Physiolog. Optik, in Hermann's Handb. d. Physiol. , III., i., p. $349 \mathrm{f}$. 
tical "points of the two retinas-upon points, that is, whose position would be mathematically the same with relation to the centre of each retina.

But the retinas are not symmetrical, and the physiological centre is not the true mathematical centre ; moreover, the eyes, to be of use, must act together in other positions than that called "primary." A distinction must then be made between corresponding points and identical points; the former are such as are found by experiment actually, as a rule, to act together and to combine their images when simultaneously stimulated. If the eyes be fixated upon any very remote object without apparent magnitude-for example, a star-the points of two retinas upon which its image falls when it is seen as single are "corresponding." One image then exactly covers the other. But in certain cases the points of the retinas which customarily act together do not so act; points not exactly corresponding sometimes cover each other, and points usually corresponding sometimes fail to cover each other. Hence, a distinction may be made between corresponding points and "covering points;" the latter term being used for those points whose impressions, in each individual case of seeing, are actually referred to one and the same point of the object." The two points of regard of the two eyes are in all cases identical, corresponding, and covering.

Scarcely more than a reference to previous elaborate attempts to determine the corresponding points of the two retinas is necessary for our purpose. ${ }^{2}$ Experiment shows that considerable reciprocal substitution takes place among the different points of both retinas. The eyes of most persons, if not of all, are both structurally and functionally incongruous. When the lines of regard lie parallel in the plane of the horizontal meridian of the two retinas, the vertical meridians do not correspond. A vertical meridian of the left eye, with its upper end inclined to the left, may be conjoined with a vertical meridian of the right eye that has its upper end inclined at about the same angle to the right. The image of a line which lies on these meridians thus inclined, appears in the vertical horizon of the field of vision and divides it into a right and a left half.

$\S 14$. That objects are ordinarily seen as single when their images are formed on corresponding points of the retinas, and otherwise as double, may be shown by many familiar experiments. ${ }^{3}$ If we hold

\footnotetext{
${ }^{1}$ By Wundt, Physiolog. Psychologie, ii., p. $122 \mathrm{f}$.

${ }^{2}$ Here see Hering, in Hermann's Handb. d. Physiol., III., i., pp. 355 ff.: and Helmholtz, Physiolog. Optik, pp. $695 \mathrm{ff}$.

${ }^{3}$ See, especially, the ones described by Le Conte (Sight, pp. 92 ff.), from which the immediately following are taken.
} 
a finger before the eyes and look, not at it, but at the wall or the sky; or if we point it at some distant object, and keep our eyes

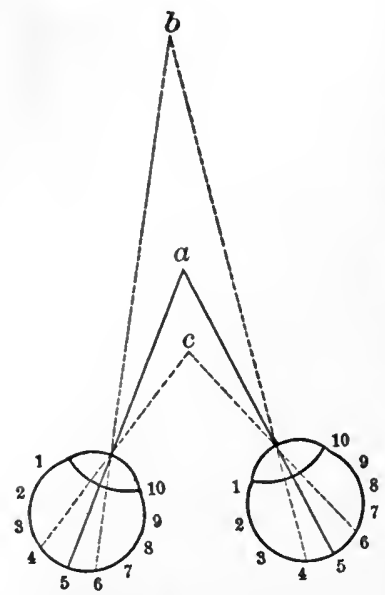

Frg. 100.-Diagram to illnstrate phenomena of donble vision. If the image of the point $b$ fall in one eye on 6 , and in the other on 7 , the distance of the two images seen will equal that hetween 6 and 7 . If the image of $a$ fall on 5 and 5 , it will be seen single, bnt if the image of $b$ fall on the left eye at 6 , and on the right eye at 4 , it will appear double. steadily fixed on the object-two transparent images of the finger, rather than one solid finger, will be seen. Many persons may have difficulty in seeing the two images, but none will fail to notice their transparent character. Under these circumstances the wall, sky, or distant object, may readily be seen through the finger. By experimental methods the images of a single object may be dissociated, and what is really one be seen as two; on the other hand, images coming from two objects may be combined upon corresponding points, and thus what is really two be seen as one. It needs only a little skilful pressure upon one eyeball to create for us the double of each one of a group of friends, and to see one body partially through the transparent image of another. If two objects very similarfor example, the two forefingers-be held a little way apart at about a foot distant and against a clear sky, three like objects, one solid and two transparent, may be made to appear by combining the two middle images and dissociating the two on the outside. Two systems of regularly recurring similar objects-such as a regular small pattern of carpet or wall-paper, or the diamond-shaped spaces of a wiregrating-may have all their images combined by slipping them, as it were, simultaneously to one side. There is, then, a double-seeing of what is really single and a single-seeing of what is really double; but the latter is much rarer than the former, and seldom occurs except when brought about for purposes of experiment.

$\S 15$. It is obvious that the relations of the two images of an object cannot remain unchanged when the eyes are moved from their primary position. When the eyes are converged upon an object, the images which are formed on the central spots of the two retinas, by rays coming from the point of regard, are exactly identical and corresponding; the object in this case is therefore seen absolutely sin. gle. Points of the object lying near to the point of regard in any direction, and thus having their images formed close to the centres of 
the two retinas, are also seen single. For the points of the retinas on which the images are then formed, although not strictly identical, are corresponding; that is, they have habitually acted together in seeing objects single by binocular vision, and the slight incongruousness of the two sets of images is disregarded, as it were, by the mind. But all objects lying nearer or more remote than the point fixated by the eyes are liable to be seen double; for their images do not fall on corresponding points of the re. tinas. Objects lying below or above, or to one side or the other, of the point of regard, do not, as a rule, have their images formed on corresponding points; they may, therefore, also be seen double. Some of these points, however, which occupy positions below or

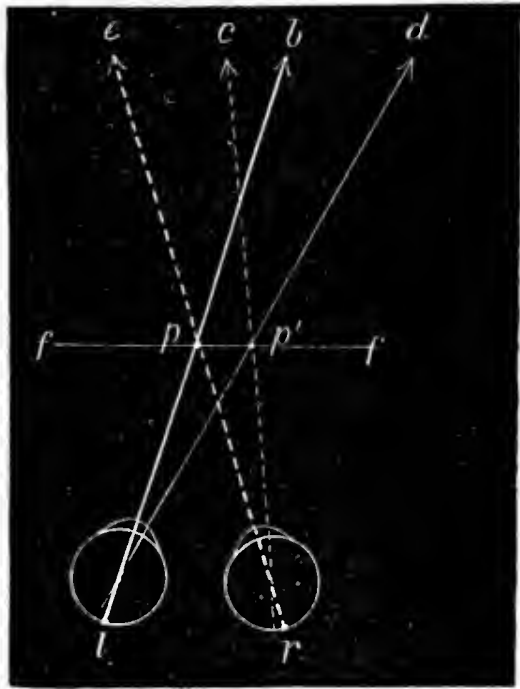

Fia. 101 (From Hering). $-f f$, the sash of the window, and $p$ the black spot fixated. On the left line of vision $b b$ lies a distant object, and on the right line $r e$ another object. The images of $b$ and $e$, as well as the image of $p$, fall on the place of direct vislon and, therefore, on corresponding points of the two retinas.

above, to the one side or the other, of the point of regard, are seen single. The sum of all the points which are seen single while the point of regard remains the same is called the horopter.

There has been a great amount of calculation, experiment, and discussion, to determine the exact nature of the horopter. It has been held to be a surface (plane or curved), a circle, a line, a number of disconnected points. Its calculation as a matter of mathematics is unsatisfactory, for the really corresponding points of any two retinas are not to be determined by mathematics. Experiment is made exceedingly difficult by the indistinctness with which we see objects that do not lie near the point of regard. No conclusions regarding the nature of the horopter are, perhaps, on the whole, more trustworthy than Meissner's. ${ }^{1}$ They are thus summarized by Le Conte. ${ }^{2}$ With the eyes in the primary position, the horopter is

${ }^{1}$ Beiträge zur Physiologie d. Sehorgans, Leipzig, 1874; and Archives des Sciences, iii. (1858), p. $160 \mathrm{f}$.

${ }^{2}$ Sight, p. 204 . 
a plane perpendicular to the median line of sight. For all nearer points in the primary plane, it is a line which dips toward the observer with an inclination to the visual plane, increasing with the nearness of the point of regard. When the plane of vision is turned upward, the inclination of the horopteric line increases; when the plane is turned downward, the inclination of the line decreases until it becomes zero at $45^{\circ}$, and the line expands into a plane. The plane of the horopter, then, passes through the point of regard perpendicular to the median visual line. With these conclusions the careful experiments of Le Conte himself correspond in the main; but Le Conte considers that the inclination of the horopteric line remains constant, and that its surface, when the horopter becomes a surface, is curved instead of a plane.

$\S 16$. The existence and relation of the two images in binocular vision is of the greatest importance for all perception of solid objects set at varying distances from each other. It is largely by their help that binocular perspective and stereoscopic vision are explicable. But all such elaborate and complex presentations of visual sense require for understanding them certain considerations concerning binocular movements of the eyes. In binocular movements the laws of parallel motion hold good only for the case when the eyes, being in the primary position, are both turned equally in the same direction. But in fixating the point of regard for the two eyes for a near object the eyes move in opposite directions, so that the lines of vision may converge upon the object. In convergence the eyes rotate on the optic axis in opposite directions. ${ }^{1}$ Since divergence of the eyes in visual activity is, in all ordinary cases, impossible, there are three customary indissoluble conjunctions of motion which belong to the eyes as under control from the central nervous organism ; these are, right and left together, up and down together, or turning symmetrically inward. In lowering the plane of vision, as well as in fixating the point of regard upon near objects, convergence naturally takes place; in elevating this plane or in looking upon distant objects, the converging lines of regard diverge toward a parallel position. Convergence may be "symmetrical" or "asymmetrical;" in the former case the two lines of regard are turned inward at equal angles and the point of regard is kept in the median plane of vision; in the latter case the point of regard is outside of the median plane, and either the two eyes are turned at unequal angles inward, or else one is turned inward, and the other, at a smaller angle, outward. Both kinds of convergence are possible at different angles of the elevation of the plane of vision.

${ }^{1}$ See Le Conte, Sight, p. 178 f. 
Listing's law does not hold for movements of the eyes in convergence. $^{1}$ The principal points at which this law is abrogated for converging motion of the eyes are stated thus by Le Conte : ${ }^{2}$ When the right eye mores to the left in convergence, it rotates to the right instead of to the left as in parallel motion; so the left eye rotates to the left when turning inward. Whereas in parallel motion the torsion of the eye increases with the angle of the depression of the plane of vision, in convergent motion it decreases to zero at $45^{\circ}$. These facts doubtless result in imparting variety of local coloring to those sensations of strain, etc., which are produced in the two kinds of motion of the eyes, and which serve the mind as local signs in its synthesis of extended visual objects.

Changes of accommodation naturally accompany the changing convergence of the eyes for near objects, and the resulting sensations enter into the spatial series out of which the presentations of visual sense are constructed. In the alteration of the indices of refraction, and in the contraction of the pupils, the eyes act together under the influence of motor impulses from the central nervous organs.

\$17. An effort to see, and a corresponding fixation of the attention upon the object lying at the point of regard, are implied in the convergence of the eyes. The eyes of new-born children and eyes that are recently couched after long-continued blindness move, as a rule, in parallel lines. ${ }^{3}$ Arrest of attention brings the two eyes into use as one organ, and this necessitates the turning of the lines of vision of both so that they shall meet at a common point where lies their common object. It follows, also, that the sensations accompanying innervation of their muscles so as to produce convergence are of capital importance in the construction of the most elaborate and intelligent risual presentations. According to Wundt, these "feelings of innervation" are the direct expression in consciousness of the cerebral changes that accompany the initiating of motor impulses in the central organs. They differ only in intensity or amount. It is by the "feeling" of this amount, as it were, that our knowledge of the size and distance of the object seen in convergence is obtained. Wundt's view ${ }^{4}$ of the nature and origin of the feelings of innervation, however, is unsatisfactory.

In the opinion of Hering, the innervation of both eyes is equal,

${ }^{1}$ Comp. Hering, in Hermann's Handb. d. Physiol., III., i., p. 497 f.; and Le Conte, Sight, pp. $177 \mathrm{ff}$. ${ }^{2}$ Sight, p. 190.

${ }^{3}$ Comp. Donders in Pflüger's Archiv, xiii., p. 383.

- See Physiolog. Psychologie, ii., p. 118 f.

' Physiolog. Optik, Hermann's Handb. d. Physiol., III., i., p. 519 f. 
however they are moved with relation to each other. Eren when the movements of the two are unequal, the law holds ; for each eye is then under the influence of two innervations, one of which is directed toward turning both eyes right or left, and the other toward turning them inward or outward. As a result, in one eye the two innervations would support, and in the other eye oppose, each other-thus bringing about a compensation. In this way the will guides its pair of horses in either direction by a pull upon one rein. The imnervation for accommodation is also supposed to be in like manner bilateral and uniform. Whatever view may be taken of the foregoing theories as to the distribution of central innervation to the two eyes and as to the origin of so-called "feelings of innervation," there can be no doubt that the mental representatives of the different areas passed over and positions reached, in both parallel and converging motions, are important factors in constructing the presentations of sight.

$\$ 18$. By the various helps already described, stereoscopic vision and the seeing of things in perspective are made possible. To one eye acting alone and without previous experience, only one of the spatial series possible could, in any event, serve as a suggestion of depth; this is the series of muscular sensations accompanying the accommodation of the eye to near distances. How little such sensations of themselves can accomplish, even at the end of years of experience in binocular vision, the experiments of Wundt make obvious (already alluded to, p. 433). Our localization of objects by one eye, with respect to the third dimension of space, is confessedly very imperfect even under the best of circumstances. It is probable, then, that the field of monocular vision is directly known only as a plane, and that all immediate perception of depth depends upon the existence of double images and muscular sensations derived from the movements, especially in convergence, of the two eyes.

The stereoscopic and perspective vision which takes place, with apparent immediateness, even when one eye is closed, is therefore really mediate and indirect; it is accomplished solely by secondary means of varying intensities of light and color, changes in apparent magnitude, etc., on the basis of associations gained by using both eyes and the hand. Accordingly, it is easy to reduce all the objects seen in the field of monocular vision to one depthto flatness outlined on the same plane-by cutting off these secondary helps and withdrawing attention as much as possible from the influence of judgment based on experience. By nearly closing one eye while the other is wholly shut, objects really 
situated at different distances from the head may easily be made to appear as patches of light and color blended, indistinguishably to the visual perception, with other patches of the retina's own light. That is to say, when the results of experience in interpreting the secondary signs of the third dimension are withdrawn, the field of monocular vision becomes as purely two-dimensioned as is the "retinal field."

$\S 19$. There is no doubt that the double images, and the muscular sensations resulting from binocular movement, furnish motifs for the immediate perception of the distance and solidity of objects. In other words, these two spatial series are most important data for constructing visual presentations of objects having the third dimension. It is more doubtful just how this service is rendered. Stereoscopy has made the fact familiar, that the two images of each object are different as furnished by the two eyes. ${ }^{1}$ The right eye sees the object farther around on its right side, the left eye on its left. Every small portion of a solid object, as seen in binocular vision, provided it lies a little way out of the point of regard, instead of consisting of two exactly similar sets of lines which might be superimposed, consists of two sets of minute curves that are partial images of its lines and are different for each eye. The constant and uniform objects of sense which appear through the use of both eyes result, therefore, from uniting a great number of varying partial images of these objects due to simultaneous excitation of both retinas. In some manner or other the perception of solidity is substantially aided by the combination of these partial images.

Furthermore, in ordinary binocular vision, our perception of the solidity and distance of objects is accomplished largely by motion of the eyes which successively unites and separates the double images of the objects seen. In viewing all objects of any size, whether near or distant, we may readily become conscious of the fact that we are engaged in sweeping over the field of vision with a moving point of regard. Even when we suppose the eye to be looking at a single point, with a perfectly fixed regard, it is actually making short and rapid excursions in one direction and another around this point. How difficult it is to keep the organ of vision perfectly motionless, anyone knows who has tried to hold steady one of the floating specks (muscee volitantes) situated in, and projected in the

${ }^{1}$ For the study of the theory of stereoscopy as a matter of optics, the reader is referred to treatises on this science; a brief allusion to the fact is enough for our purpose, which primarily is, of course, to illustrate the psychology of visual perception. 
air before, this organ. Such facts strengthen the theory of Brücke ' and others, that we gain our perception of depth by running the point of regard back and forth with a varying degree of convergence to the axes, and so combining successively the different parts of the two pictures as seen by the two eyes.

But that motion is not necessary for stereoscopic vision with adult eyes is proved by what is known as "Dove's experiment." A field composed of different solid objects stationed at different distances in space, or of two stereoscopic pictures, may be seen in perspective when illuminated by the light of an electric spark. Since the duration of this spark is perhaps not more than $\frac{1}{2 \operatorname{li}_{0}} \overline{00}$ sec., it is plain that no change of convergence, or running back and forth of the point of regard, has time to take place. It is asserted by Le Conte ${ }^{2}$ that the interpretation of the double images depends upon the fact that such images of any object are different according as the object lies nearer or more remote than the point of regard. In the latter case, the double images are called "homonymous," and are united by less convergence; in the former case they are called "heteronymous," and are united by greater convergence of the optic axes. Now the observer knows, "instinctively and without trial," whether greater or less optic convergence will be necessary to unite the double images; and accordingly refers the homonymous images to objects beyond, the heteronymous images to objects this side of, the point of regard. But the question arises, How does this so-called "instinctive" knowledge come? It can scarcely be by way of a native insight into the distinction between homonymous and heteronymous images, as such; or through any seeing of both retinal images by the mind's eye, ${ }^{3}$ as it were. Since what is needed to unite the images is motion of the eye, and since the mind has always been accustomed to associate sensations of motion with the double images of binocular vision, it is impossible to aroid the conclusion that instantaneous binocular vision, like monocular vision, of solidity and distance, is secondary and wholly dependent upon previous experience acquired with both eyes in motion.

$\S 20$. Localizing of the third dimension is, accordingly, much more secure in binocular than in monocular vision; and judgments of distance are assisted greatly by movements of both eyes. If

1 Archives des Sciences, iii. (1858), p. 142.

${ }^{2}$ Sight, p. 151 ; and Am. Journal of Science and Arts, ii., 1871, p. 425.

3 To say that "each eye, as it were, knows its own image, although such knowledge does not emerge into distinct consciousness," is in plain contradiction with all the fundamental laws which psychology has to propound concerning the nature of visual perception. 
no other motif for seeing depth of space is present, according to Hering ' the following law seems to hold: All the lines or points whose images lie, with a given position of the point of regard, in the vertical horopter, appear clearly defined on a surface which is either plane or slightly cylindrical, and all the lines or points lying this side of the surface of the vertical horopter and whose images have a "crossed disparateness" (that is, the left one of the double images belongs to the right eye, and the right one to the left eyemaking them "heteronymous"), appear in front of the surface; while those lying beyond the horopter and whose images have an "uncrossed disparateness" (that is, the right image belongs to the right eye, and the left image to the left eye-making them "homonymous"), appear behind the surface on which whatever lies in the horopter is seen. But, as we have already learned, interpretation of the double images for the stationary eyes is an acquired art, which is dependent upon previous association of the retinal signs of both eyes with muscular sensations arising from the innervation and movement of the eyes. It is also in perfection of practice dependent, as all stereoscopic vision is, upon the so-called "secondary" means of such vision.

\$ 21. All stereoscopic rision, or vision of perspective for remote objects, requires, in order to secure any considerable accuracy, the larger use of "secondary helps." Five or more classes of such helps may be mentioned. Vision, as accomplished by such means, is often called judgment in distinction from immediate perception. This should not be held to imply that activity of the mind in association and discernment is not involved in all the presentations of sense. The distinction lies between such a synthesis of the sensations into objects of sense as is inseparably connected with all normal binocular vision, and such other seeing (or judging) of the spatial properties and relations of remote objects as depends for its accuracy upon changing aspects of these objects. The increased necessity for secondary helps when the objects of vision are remote arises largely from the fact that the mind loses the data (or motifs) that accompany strong couvergence and accommodation of the normal eye for near objects. Changes in the tone and intensity of the muscular sensations are comparatively slight on passing from vision of objects 20-40 feet distant to vision at infinite distance. On the contrary, such changes are relatively great on converging the eyes to alter the point of regard from a distance of 20-40 feet to one of 5-6 inches ; still greater on increasing the convergence for still nearer vision. Hence the increased necessity, in vision of 'distant 'Physiolog. Optik, Hermann's Handb. d. Physiol., III., i., 400 f. 
objects, for other secondary helps to take the place, as it were, of the diminished value of the primary data or motifs of the eye.

$\S 22$. The principal secondary lielps of stereoscopic vision and vision of perspective are the following: ${ }^{1}$ 'The course of the limiting lines of the objects in the field of rision determines our perception of their distance and form as lying in the third dimension of space. In looking at a building, we connect together into vertical, horizontal, or curved wholes, the successive fragments of the images of its lines as the eyes are swept along in the requisite directions. If these lines become confused in distinctness, or changed into directions that are contrary to our previous experience of how the parts of a building appear to the eye, we are liable to errors in perception. When the bottom lines of a distant object are covered, its distance and shape in the third dimension become uncertain to the eye. Mountains that tower behind each other seem to lie in one surface, provided the presence of other secondary helps, such as atmospheric perspective, etc., is excluded. The parts of unshaded geometrical figures drawn on a plane where the course of their outlines does not define the matter to us as fixed in one way, may often receive two or more iuterpretations; they may thus actually appear as subject to change from a nearer to a more remote place in space. The same arrangement of lines may appear

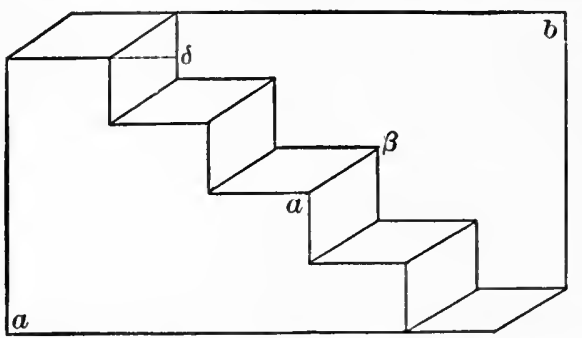

Fig. 102 (From Wundt). $-a$ can be made to appear either nearer or farther off than $b$. either as a staircase or a portion of an overhanging wall (see Fig. 102). The same angle of a polygon may be made to seem either the nearest or the most remote. Indeed, the whole stereoscopy of certain figures may thus easily be reversed. But if the course of the limiting lines of an object for-

bids more than one interpretation of the relations of its parts in the third dimension of space, then the object must be seen as interpreted in that one way. Objects of known size and shape are seen as nearer or remote, according to the manner in which the parts cover each other and are covered by each other. The contour of an object, then, is one determining factor of its stereoscopic appearance (see Fig. 103).

${ }^{1}$ Comp. Wundt, Physiolog. Psychologie, ii., p. 145 f.; and Helmholtz, Physiolog. Optik, pp. 622 ff. and 766 ff. 
Mathematical perspective, or the size of the angle of vision which is covered by near and far objects respectively, is one of the most important secondary helps of stereoscopic vision and vision of perspective. In this way objects of known size are seen as placed at a distance necessary to give them their apparent size. The street appears narrower and more distant, the houses lower and more remote, in the upper part of its visual picture. Parallel lines, like the tracks of a railway, appear to converge from us more and more toward a point; the same thing is true of the sides of the table or box at one end of which we are standing, or of the walls of the
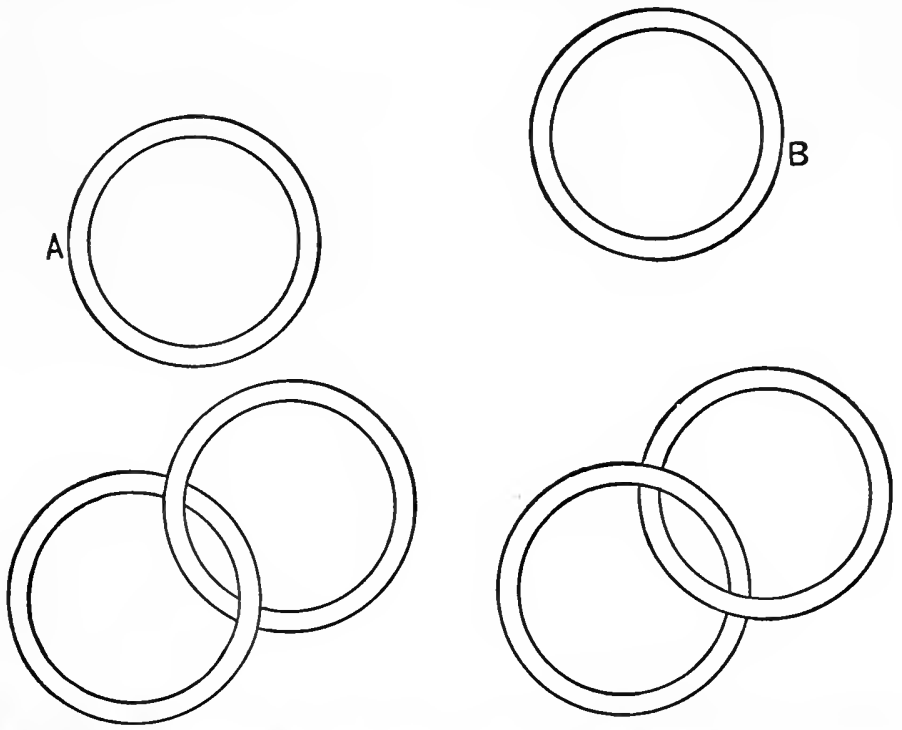

Fte. 103 (From Wnndt).- The two rings $A$ and $B$ may be stereoscopically combined in either of the following ways-according as the vertical or horizontal contonrs prevail.

room. For although the perspective of visual experience is rery different from true "mathematical perspective," the latter affords to the former one of the secondary helps.

More distant objects are also, on account of the amount of atmosphere through which the rays of light reflected from them have to pass, more dim in outline and of changed shades of color. Such alterations in the character of the image furnish another of the secondary helps of our vision of perspective. Accordingly, things are seen nearer in a clear atmosphere, more distant in one less clear. This is sometimes called "aërial perspective."

The size and direction of the shadows also furnish data for the 
perception of the distance and shape in the third dimensions of visual objects. In the morning and evening light, when all shadows are lengthened, the objects of the landscape appear more distant from us and from each other. The direction of the shadows of different objects with relation to each other and to the source from which the light comes is also an aid to vision of perspective. The arrangement of the lights and shadows is by far the most important means for determining the relative position in space of different parts of objects like intaglios or medallions. A change of the arrangement of the lights and shadows of such an object, so as to substitute the one for the other throughout, converts an intaglio into a medallion or bas-relief, and vice versa. A medallion, placed near a window, but shielded from its direct light, and lighted from the other side by reflection from a mirror, has its relief reversed.

$\$ 23$. Other secondary helps to stereoscopic vision and vision of perspective are derived from experience in a still more inclirect way. Within certain limits we see what we know to be in the field of vision; but, on the other hand, we are not infrequently compelled to see what we know cannot be there. The account of such phenomena depends upon laws of association and reproduction, the physical basis for which is exceedingly obscure. Since the ultimate psycho-physical processes take place in the brain; and since the central processes come under the law of habit and are in part determined by the tendencies embedded, as it were, in the structure and customary functions of the central mechanism; the influence of changes in the peripheral organs of vision, the shape and clearness of the retinal image, etc., cannot always deter-

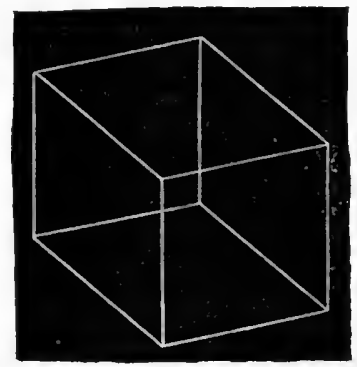

Fia. 104.-First one, then the other corner of the figure may be drawn forward, partly at will. mine just what the presentation of sight will be. Many retinal images admit of two or more interpretations-which interpretation will be chosen depends upon a variety of circumstances that perhaps cannot all be accurately defined. The few lines drawn upon the blackboard, or employed by the skilful etcher, cause us to see what is not, but rather ought to be, in the image formed upon the retina. Anyone accustomed to studying the effect of the colored points and outlines which appear in the image seen with closed eyes by the retina's own light, knows how apparently lawless is the interpretation given to this image. This is especially true when attention is somewhat relaxed-as, for exam- 
ple, on sinking into revery or sleep. Much of the "stuff" out of which the usual phenomena of dreans are made, may be suggested and controlled by the condition of the "retinal field." In all these cases, only a sharper attention and more objective view of things is needed to dispel the illusion and make us aware how scanty is the schema, as it were, out of which, by association and reproduction, we have constructed our presentations of sense. Similar experiences not infrequently occur even with open eyes by day, in the dimly lighted room, or in the obscurer nooks and recesses of vision on the street. In this way numberless ghosts and apparitions have been most perspicuously seen. The face of a friend whom we know to be thousands of miles distant may look at us from the window of a house; it is only after persistently trying to interpret the appearance in accordance with our knowledge that we finally succeed in resolving the face into some chance combination of lights and shadows, of window-sash, curtain, or other objects.

$\$ 24$. Phenomena like the foregoing recall once more the general office of experience in determining the existence and character of particular presentations of visual sense. We have seen that the strife between the two rival theories of the origin and development of sense-perception concerns the relative amount of what is to be counted "native," on the one hand, or accredited to a process of "learning" how to perceive on the other hand.

The analysis of the mind's data or motifs has made it apparent that the influence of experience through the association and reproduction of its past forms is very great over the presentations of sense. ${ }^{1}$ The mind sees, not simply according to the objective character of so-called " things," nor simply according to the retinal images as connected with sensations of motions, but also according to its custom in seeing. When, therefore, its habits are broken up for the time, its interpretation of the sensations, as well as its synthesis of them into recognized objects of sense, is liable to be disturbed. Various experiments impress this truth in a vivid way. For example, let one regard, with one eye, the reversed picture of a landscape or the photograph of a friend turned upside down after covering up nearly all of it except the face. The effect of the pseudoscope, or optical instrument, which, by exchanging the two stereoscopic pictures, changes convex into concave, and vice versa, when applied to a complicated scene of landscape, streets, etc., is very bewildering. The data with which the mind has been wont

' Comp. the chapter, Der Einfluss der Erfahrungsmotive auf die Localisi. rung, in Hering's Physiolog. Optik, Hermann's Handb. d. Physiol., III., i., pp. $564 \mathrm{ff}$. 
to deal may all be given, and the sensations localized according to the laws of stereoscopic vision, but the relation of the parts is inexplicable out of any previous experience. Similar effects are produced by the telestereoscope, ${ }^{1}$ or optical instrument which enables us to see a larger portion of a distant object than is possible with two ordinary eyes, after the fashion of a pair of optical organs in the sides of a gigantic head. Individual peculiarities of localizing, such as are acquired by the practice of some trade or art, are also accounted for under the principle of influence from experience over those elements of reproduction that determine what object of sense shall be constructed out of the various sensational data at command. Indeed, all our estimates of visual size, shape, and distance, as well as our "errors of sense," can be understood only in the light of this same general principle.

$\S 25$. Not only what we know, but what we choose, has an influence-often a determining one-upon what we see. This is true, not simply because we can at will, within certain limits, decide the area of the field of vision over which the point of regard shall move, as well as the parts of this area upon which it shall be fixated, but also because we can regulate the amount of attention which shall be given to visual impressions and the manner of the distribution of attention over the various parts of these impressions. Furthermore, it often lies with us to say how we will interpret the data, and so see the complex product resulting from the act of mental synthesis. This is especially true of geometrical figures in outline, as in the cases already referred to under another head (\$22).

$\S 26$. With the use of the foregoing data, and under the guidance of past experience, we judge of the spatial extension and relations of lines, angles, and solid bodies, of their shape, size, distance, and relative situation. The position of lines and angles affects our estimate of their magnitude ; under this principle many errors of sense originate. Distance and size are, of course, so related that they vary inversely, and when one is known the other is immediately or readily judged on the basis of such knowledge. But the size of the visual object is measured by the magnitude of the visor angle covered by its image, or the relative extent of the retinal surface simultaneously excited by the rays of light reflected from the object. This is called its " apparent magnitude." The real magnitude of any object is its size as related to certain fixed standards of measurement formed on the basis of generalizations from the use of both eye and hand. Distance, apparent magnitude, and real magnitude, are therefore con-

\footnotetext{
${ }^{1}$ See Helmholtz, Physiolog. Optik, p. 646 f., for an account of these two instruments.
} 
nected as three factors of one problem proposed by each presentation of sight. Given the apparent magnitude and the real magnitude of an object, we judge of the distance according to our experience of how large an object of such size appears at an assumed distance. The remote spot on which a human figure is standing seems nearer or farther away according as we know the figure to be that of a man or that of a boy. Distance and apparent magnitude being given, the real magnitude of the object is judged as that which it would need to have in order to appear so large at the given distance. When one of the two necessary data is lacking, no judgment can be formed except upon the basis of other secondary helps, such as aërial perspective, etc. Thus, no common standard for estimating the distance of the sun or moon being given, their size appears different according to the place where different observers are inclined to locate them, or according to the standard of comparison made necessary for the time by their position. These bodies ordinarily appear to some persons no larger than a saucer, to others larger than a large cart-wheel. When the sun sets behind a tree, the size of the spreading of whose branches is fairly well known, it may be enormously magnified by being seen to fill its branches entirely.

$\$ 27$. When the eye is in motion, as in all ordinary vision of objects not very minute and very near, the number, duration, and intensity of the spatial series of sensations called forth by the motion determine our estimate of the outline-form, magnitude, and distance of the objects. ${ }^{2}$ Every spatial series of sensations contributes the larger magnitude to the object the greater the number of members which enter into the series. For this reason the same extension of a line or surface when broken up into parts by intersecting lines appears larger than when perceived as an uninterrupted whole. The repetition of similar figures in architecture, upon walls, columns, etc., takes advantage of this effect.

The intensity of the sensations of a spatial series, and of the act of attention necessary to comprehend them in one whole as a presentation of sense, also has an influence on the size of the object. When the morements of the eyes are made with lamed or tired muscles, the size of the thing perceired by them is increased. When the function of one of the muscles (for example, the rectus extermus) is impaired, so that the circuit of the eye in a giren direction is shortened, objects lying at any position in the field of rision, as seen by the eye moving in the shortened circuit, are located where they would have been if the same intensity of muscular sen-

${ }^{1}$ Comp. Volkmann von Volkmar, Lehrb. d. Psychologie, 1885, II, pp. 99 ff. 
sation had been necessary to bring them to this position with a normal function of the muscles. A patient with paralysis which prevents turning the eye more than $20^{\circ}$ will locate an object actually lying only $20^{\circ}$ from the median plane much farther to one side. Such a patient will reach beyond when he tries to grasp the visual object. The increased size which is given to objects that are parti-colored or mottled, and so have an interrupted surface and furnish greater difficulty to perception of them as wholes, may be due to both the foregoing causes. Volkmann von Volkmar ${ }^{1}$ calls attention to the fact that both monotony and variety may, under the working of these principles, be productive of the same effect in magnifying the size of an object. For the size of any visual surface is usually estimated by the application of some standard of measurement selected from the field of vision. The frequent repetition of this standard creates the impression of vastness ; and the absence of any standard to apply, or a vague, unsuccessful effort to find a standard, may produce the same impression. Monotonous areas of unbroken snow, and stretches of streets crowded with forms of men and animals, both seem of great extent.

The amount of time through which the spatial series of sensations endure has also an influence on the magnitude of the objects perceived through those sensations. It is as enduring in time that the changing qualities and quantities of sensation which belong to the perception of any complex object are expressed. The length of the time-course, as well as the degree of the intensity of the spatial series of sensations, may be interpreted as extensive magnitude of the perceived object.

$\S 28$. The laws which control our estimates of visual magnitudes are psychological, and apply to all the action of the mind in constructing its sense-data into the presentations of sense. Yet more elaborate mental activities, such as take place when the distance, size, and contour of visual objects are deliberately estimated and expressed in terms of an accepted standard, of course imply more of dependence upon skill acquired through experience.

The degree of fineness with which clifferences of distance and magnitude can be seen, under the most favorable circumstances, is limited by the least observable differences in the members of the spatial series of sensations which compose the visual objects. Of such series, those most capable of exceedingly fine differentiation are the local retinal signs and the muscular sensations accompanying convergence of the eyes for near distances. It is difficult 'Lehrb. d. Psychologie, II., p. 101 f. 
to assign the exact proportion of help which these two series render in making the finest possible distinctions of visual magnitude. Hering ' denies that any help is obtained from muscular sensations, or "feelings of innervation," in comparing the size of two minute objects near by, and assigns all the work of furnishing such data to the "spatial sense of the retina." Lotze, ${ }^{2}$ who admitted the assistance of muscular sensations, nevertheless held that the fineness of the distinctions possible anong them is not sufficient to support our ordinary judgments of the size, distance, and direction of objects. Wundt ${ }^{3}$ and others claim that it is by gradations in the socalled "feelings of innervation" alove that we make the most accurate of these estimates; they deny that any "spatial sense" (in Hering's meaning of the words) belongs to the retina. The evidence seems to favor the view that both the muscular sensations and the local retinal signs furnish data for all nice discrimination of visual extension.

The particular degree of accuracy with which minute differences in the distance and magnitude of visual objects can be perceived varies greatly, according to different positions of the eyes and the object, the amount of light, practice, etc.-and all these, as connected with individual peculiarities of structure and previous function of the organs of sense. That such estimates fall to some extent under Weber's law-in other words, that the least observable difference in the length of visual lines and surfaces is relative and not absolute-lias already been shown (Chap. V., § 18). Chodin found the relative value of the least observable difference, with a variation of the absolute vertical distance from 2.5 to $160 \mathrm{~mm}$., to be as follows when the lines lie in the same direction:

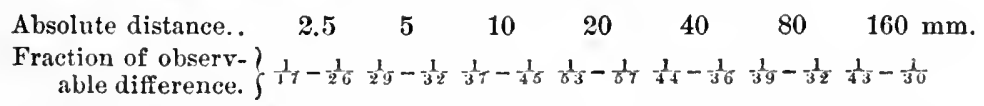

The fineness of ocular judgment is greater for horizontal distances.

The measuring power of the eye is much less accurate when the distances compared lie in different directions. In particular, points lying at a vertical distance of $20 \mathrm{~mm}$. are estimated as equally far away with those lying at a liorizontal distance of $25 \mathrm{~mm}$." Most estimates of direction and distance are comparatively inaccurate when only one eye is used. A vertical line drawn at right angles to

' Hermann's Handb. d. Physiol., III., i., p. $533 \mathrm{f}$.

2 Medicin. Psychologie, $3 \& 4 \mathrm{f}$.

${ }^{3}$ Physiolog. Psychologie, ii., pp. 85 ff.; comp. i., pp. 375 ff.

${ }^{4}$ So Wundt found, Physiolog. Psychologie, ii., p. 96. 
a horizontal appears bent to monocular vision; its apparent inclination is variable, and was found by Donders ${ }^{1}$ to vary between $1^{\circ}$ and $3^{\circ}$ of the angle within a short time.

Helmholtz ${ }^{2}$ experimented to determine the accuracy of the binocular perception of depth by trying how small a deviation from a perfectiy straight line could be detected in a wire bent at one point so that its two halves formed an extremely obtuse angle, when the wire was looked at both in and out of the horopteric line; he also employed for the same purpose three nails, the heads of which could be very slightly displaced from a straight line. Under the most favorable circumstances, he found that a displacement of a nail by a change of its distance corresponding to $60 \frac{1}{2}$ seconds of the angle of vision, or $0.0044 \mathrm{~mm}$. variation in the position of the retinal image, could be detected. The latter distance corresponds so well to the calculated size of the retinal elements (see p. 327) as to form an argument in favor of the theory that estimates of size and distance are dependent upon the local signs attached to the excitation of these elements.

But, on the other hand, it is claimed that Weber ${ }^{3}$ showed the muscular sense of the eye to be one of the finest of micrometric apparatuses, since a distinct muscular sensation is attached to a displacement of the most sensitive spot of the retina of not more than $\frac{1}{521}$ of a Parisian line. By experimenting with a black thread stretched orer against a white wall and moved in the median plane toward and from both eyes, which looked at it through a horizontal slit in an upright board, it was found possible to detect changes in distance of $3.5 \mathrm{ctm}$. at an absolute distance of $180 \mathrm{ctm}$., and changes of $1 \mathrm{ctm}$. at an absolute distance of $60 \mathrm{ctm}$.

$\$ 29$. The data or motifs already described are the foundation, also, of our perceptions of motion, and of our estimates of its direction, speed, and extent. It need scarcely be said that all such perceptions and estimates are relative; they imply the existence of some point which may be regarded as fixed, and the application of a standard of measurement. For perceptions of motion by the eye, the point of regard when the organ is in the primary position furnishes the means of orientating ourselves and of placing the different things of vision in their right relations to us and to each other. Suppose the body and head to be erect, and the cyes motion-

${ }^{1}$ Archir f. Ophthalmologie, XXI., iii., p. $100 \mathrm{f}$.

${ }^{2}$ See Physiolog. Optik, p. $644 \mathrm{f}$.

${ }^{3}$ In the Ber. d. sächs. Gesells., etc., for 1852, p. 130; cited by Volkmann von Volkmar, Lehrb. d. Psychologie., II., p. 56.

${ }^{4}$ See Wundt, Physiolog. Psychologie, ii., 94 f., and the reference there. 
less and looking into the distance with the lines of vision parallel ; the perception of motion may then arise in either one of two ways. Of these, by far the most frequent is the change of relative position of an object in the field of vision which is occasioned by its morement. What is necessary, however, is simply the successive stimulation of continuous points or areas of the retina with images that are sufficiently similar to be perceived as one object. The perception of motion may also be produced by the successive stimulation of the same points or areas of the retina with inages that are too dissimilar to be regarded as one object. One may thus see motion when neither the eyes nor any external objects are really moved. It is in the latter way that the colored points of the images formed by the retina's own light, when the eyes are closed and motionless, seem to be in constant motion.

The direction and amount of motion perceived with the eyes is measured off upon the entire field of vision in accordance with previous experience and by means of the data already described. With the eyes at rest, the retinal local signs, or space-values belonging to the retinal elements, furnish the only primary data ; secondary helps, and associated ideas of muscular sensations which have been by experience found necessary to follow objects in motion, complete the perception.

It is assumed, in cases like the foregoing, that no sensations indicating motion of either the organ of vision, or the head, or the whole body, complicate the problem. But ordinary perceptions of motion are gained with the eyes in motion out of the primary position. When the eye and the object both move in such a way that the point of regard remains fixed on the object, our perceptions of motion, and estimates of its direction and magnitude are dependent upon muscular and tactual sensations occasioned by the eye's changes of position. We know from experience what kinds and intensities of sensations are produced by keeping the point of regard fixed on an object which is moving at about a given rate in a given direction. If any of the links ordinarily belonging to this chain of conscious experiences drop out, our measuring instrument fails us either partially or completely. The head, too, is invariably turned when we are watching an object that is moving in any direction other than straight forward or away from us along the line of regard. The sensations originating in the action of the muscles and skin of the head and neck thus enter into our computation; they must have such a value in consciousness as to inform us about how far the head has gone from the position with which it started, in order to fixate the moving object. According to 
Helmholtz, ${ }^{1}$ the ordinary movements of the head in vision follow the same principle as that followed by the eyes in movement; that is to say, the head turns from its primary position on an axis that is approximately parallel to the axis of the simultaneous rotation of the eyes. But Hering ${ }^{2}$ asserts that a difference between the laws of the motion of head and eyes is of essential significance for our perception of space. However this may be, it is certain that the position and motion of the head, as known by its muscular and tactual sensations, must be taken account of in all ordinary visual perception of motion. The same thing is true of the position and motion of the entire body. Many of our errors of sense, or false perceptions of motion-its existence, direction, rate, and amountare dependent upon the principles of judgment governing such data of sensations. We are peculiarly liable to error in all cases where the motions of our own bodily organs are passive; in such cases we do not have the ordinary motifs, or data, at our command.

Objects are perceived at rest, either when, our organs of vision being themselves at rest, the images of the objects do not change their position in the field of vision, or when sensations of motion occasioned by moving these organs are such and so great as we know by experience correspond to (or compensate for) the changes in the position of their images which are occasioned by their actually remaining at rest. But whenever we look with moving eyes upon a number of objects arranged in fixed position with relation to each other, a conflict between two sets of data really takes place. The result with respect to our perceptions of motion may depend upon which of the two is chiefly effective in arresting attention. When the eyes are brought from the parallel position, which they assume in vision of remote objects, to convergence upon some near object, the two fields of view belonging to the two eyes rotate in opposite directions, while the middle visual line maintains its position in the median plane. ${ }^{3}$ Ordinarily we do not perceire this rotary motion of the two fields of vision, but consider the field as one and stationary and ourselves as changing our point of regard in it. By attention, however, we may see that the external objects, although they really continue at rest, appear to move as the relations of their double images are changed. So, also, when the eye or head or body turns in either direction, in order that a new object may be brought under regard, it is possible either to perceive or not to perceive the entire field of objects sweeping by ; which

1 Physiolog. Optik, p. 486.

2 In Hermann's Handb. d. Plyysiol., III., i., p. 495.

${ }^{3}$ See Le Conte, Sight, p. 229. 
of the two happens depends upon the direction in which attention is drawn. When strictly attending to the phenomena, we cannot well fail to regard everything as moving in the opposite direction from that in which we know the organ of vision to be turning.

$\S 30$. The principles already laid down also suffice to explain most of the ordinary "errors of sense," as well as certain extraordinary experiences of a somewhat different kind. The right to speak of errors of sense has been questioned. It has been claimed that such errors belong rather to judgment, and that sense pure and simple caunot err. The claim is based upon a misunderstanding of the nature of perception. A very obvious difference exists, indeed, between a mistaken estimate of the distance of a mountain through extraordinary clearness of atmosphere and the seeing of a square of white paper as green on a red ground, or as yellow on a blue ground. But the latter is surely an "error of sense," or sensation, in as pure form as such error is conceivable. That sense cannot err is true only in case we speak of unlocalized and unprojected sensation, regarded as not predicating anything beyond itself. In all presentations of sense a certain psychological judgment is involved; for all such presentations imply association of impressions discriminated as similar or dissimilar, and a mental synthesis which is dependent upon attention and the interpretation of certain motifs or data according to past experiences. Clear vision is always mental interpretation.

The attempt to assign the relative amount of blame to sense and to intellect, in cases where our presentations of sense do not represent objective relations of things, assumes an ability to make distinctions which we do not possess. Moreover, the distinction, when made as the objection would have it, will not hold. Innumerable experiences contradict the statement that immediate sense-perception cannot err. When one sees (with no power to see otherwise) a gigantic human form through the fog, or projected against the scenery of a stage, and yet judges that this form is only of usual size, the error is not one of judgment, but just the reverse. Errors of sense are only special instances where the mind makes its synthesis unfortunately, as it were, out of incomplete data, instantaneously and inevitably interpreting them in accordance with the laws which have regulated all its experience. As Lotze has remarked, "The whole of our apprehension of the world by the senses is one great and prolonged deception." Objects of sense are in no case exact copies of ready-made things which exist extra-mentally just as they are afterward perceived, and which get themselves 
copied off in the mind by making so-called impressions upon it; they are mental constructions. In the special case of sight we have seen that, in every particular-in its elements, its mode of construction, its laws of change-the field of vision is a subjective affair. The case is in no respect essentially different, whether our presentations of sense are so-called errors or true images of things. In both cases the same data and laws of the use of these data maintain themselves. Errors of sense, however, are distinguished from hallucinations, because the former result from the activity of an organism which is normal in structure and function, while the latter do not.

$\S 31$. The errors of visual perception are almost innumerable; they may be classifed in part, however, according as they fall under some one or other of the before-mentioned principles. Such errors may be called "normal," because they are committed in accordance with principles which regulate the ordinary activity of the mind in making its synthesis by the help of the sense-data or motifs furnished to it through the excitement of the organism. Deceptions of this class really result, then, from the fidelity of both mind and nervous system. Certain errors of sense, for example, are special examples of the working of the laws which regulate the correspondence of the two images in binocular vision. Thus, near objects erroneously appear double when the eye is adjusted for distant vision, distant objects when it is adjusted for near vision; solid things are seen through other solid things; relations in space in general are perceived different from the reality; and all according to the law of the correspondence and non-correspondence of the two retinal images. Accordingly, the inquiry, Why is vision single when it is performed witl two eyes? can demand and receive only one answer. A chief condition of the single vision of solid objects is that they shall be seen with two eyes. Whether anytling whatever is seen as two or one does not depend, primarily, upon its really being either two or one, or upon the existence of one or two retinal images of it (as though such images were directly perceived); it rather depends upon the appropriate data of sensations being furnished to the mind for completing its mental synthesis of the object. The two eyes being simultaneously affected in a certain way, these data are supplied. What is one is seen as one, and what is two is seen as one, and what is one is seen as two-all in essentially the same way.

A still larger class of errors of the visual sense falls under the laws which regulate the smallest observable differences in the muscular sensations as related to the mathematical perspective of 
lines, angles, and surfaces. ${ }^{1}$ Reference has already been made to the working of this principle in our ordinary perceptions of the visual magnitude, contour, and distance of objects. Vertical distances are regularly perceived as larger than equally large horizontal distances. On trying

.FIG. 105.

to draw a cross with limbs of equal

$\mathrm{HHHHH}$

FiG. 106.

length one is apt to get the vertical dimension too small; exact squares are likely to appear higher than their

breadth. When comparing magnitudes in the upper part of the field of vision with those in its lower part, one is likely to overestimate the former. The upper and lower half of a letter " $\mathrm{S}$ " or a figure " 8 " appear of nearly the same size; but when they are inverted (" $\mathrm{s}$ " and " 8 ") the difference in the

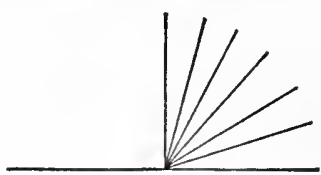

Fig. 10\%. size of the two halves becomes magnified. Under the same principle-in part at least-may those errors be brought which are
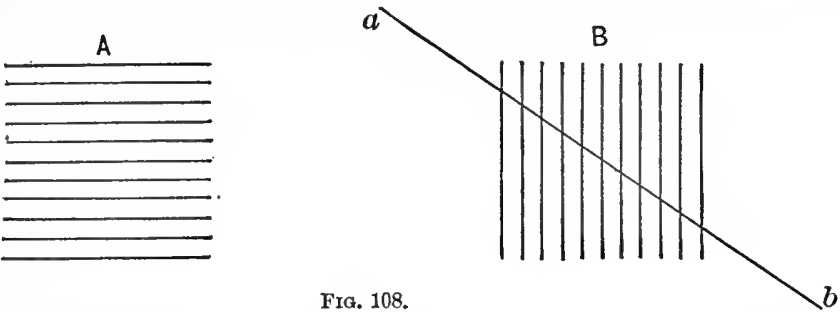

determined by the way in which the field of vision is filled up. If the horizontal distance between two points be exactly half filled with a line, this line will appear longer than the remaining empty space. A square intersected by parallel horizontal lines appears elongated upward, but one intersected by parallel vertical lines appears elongated sideways. If one of the two right angles formed by drawing a vertical perpendicular to a horizontal

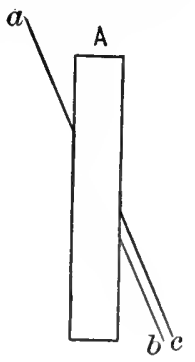

FIG. 109.

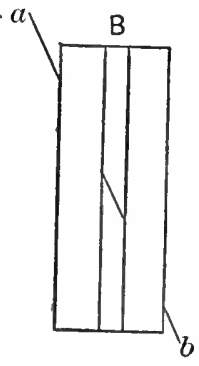

line be filled with several lines diverging from the point of the an-

${ }^{1}$ Comp. Wundt's discussion of such cases, Physiolog. Psychologie, II., pp. $92 \mathrm{ff}$. 
gle, the angle thus filled will appear the larger and the perpendicular will seem bent. For essentially the same reason, when two unequal angles together make $180^{\circ}$, the obtuse angle appears rela-

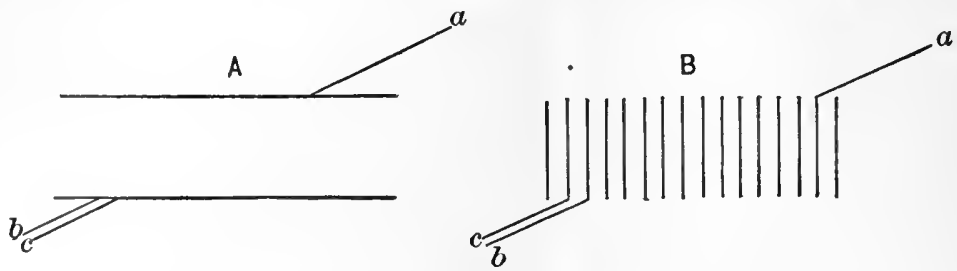

Fra. 110.

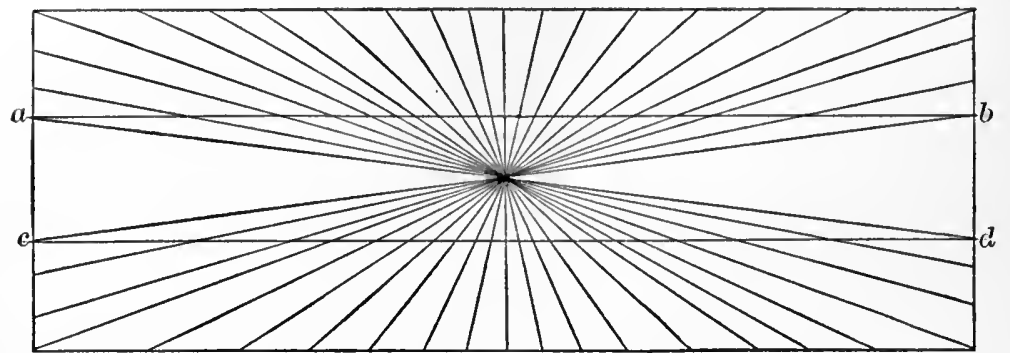

Fic. 111.
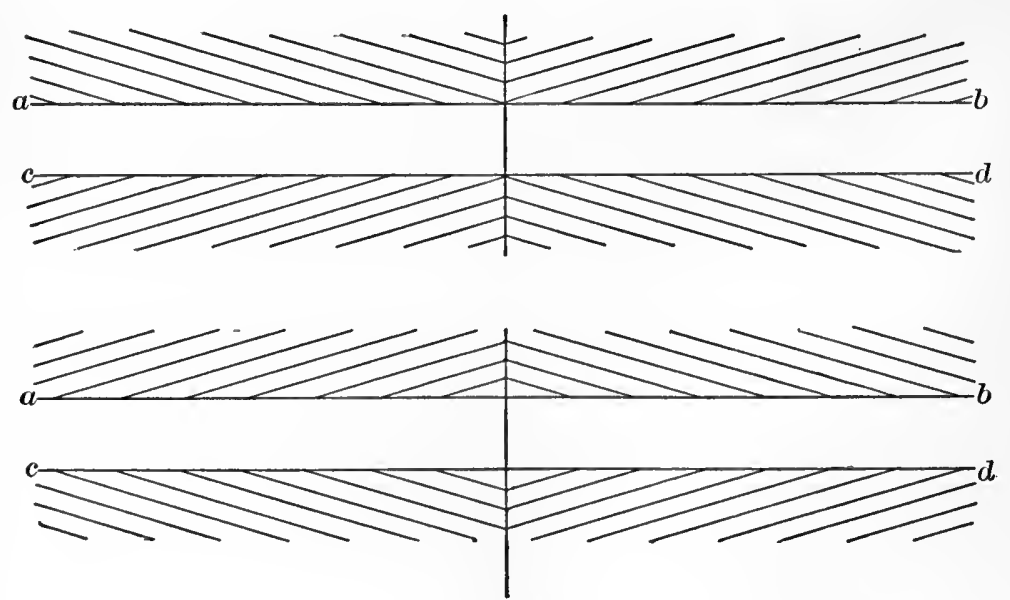

Fı,. 112.

tively too small, and the acute angle relatively too large. Many surprising errors of sense result from the varied applications of this principle. (See the Figs. on p. $457 \mathrm{f}$.) 
§32. The influence of experience, which often corrects what would otherwise be an instinctive interpretation of the data furnished to the mind, is at other times the cause of errors. If the data will at all permit it, we incline to perceive any object as we know that similar objects are usually perceived. Such errors of sense as result from the vision of distant objects through secondary helps are too well known and frequently remarked upon to require extended treatment. All the pleasant illusions of art-in architecture, drawing, and painting-are obliged constantly

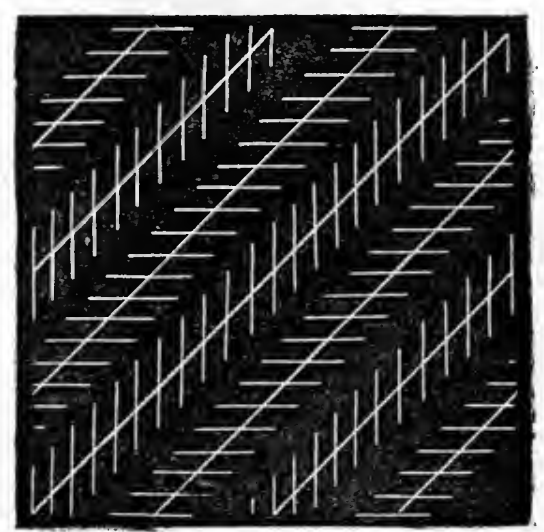

FIG. 113. to take them into account. The very relations of light and shade, the conjunction, separation, and covering of lines and surfaces, upon which reliance is ordinarily placed for perception of fact, may be employed by either nature or art to compel us to perceive what is contrary to fact. Painting is successful according to the skill it displays in furnishing to the eye its customary data so as to entice it to regard things as other than they really are. That its success is so good need not surprise us, when we remember that the mind has never anything more than these same data out of which to construct its objects of sense and to make its various judgments concerning them. The many errors in our perceptions of motion may, for the most part, be explained in the same way. It matters not whether the data for such perceptions are furnished by actual changes in the relative position of things in external space, or whether the same sensations arise through changes confined to the organs of sense. Past experience has great influence in all this domain. We incline for this reason, when two objects are changing their relative position, to perceive the smaller of them as in motion; we also over-estimate the speed of small bodies in motion, and under-estimate that of large bodies.

$\S 33$. Some errors of visual perception differ from the foregoing in that their explanation seems to be due to cerebral activity under other laws as yet unknown to us. We have already seen (Chap. IV., $\S 14$ ) that the phenomena of contrast of colors must be referred 
to certain inexplicable activities of the central organs as related to our sensations or states of consciousness. The same thing is apparently true of those errors of sense which occur in connection with the strife and prevalence of contours, and the binocular mixing and contrast of colors. If a well-defined image of some contour, such as a sharp-marked limit between two differently colored surfaces, be formed on one retina, and on the corresponding points of the other the image of a uniform-colored background, then only the former will be visible. This is called the "prevalence of contours." But if the contours of the images of two differently colored objects run on the retina so as to cross only in one place, then sometimes one color and sometimes the other will prevail and get itself perceived at that place. This is called "the strife of contours." If two squares of red paper and two of blue, all of equal size and brightness and without any distinguishing marks, be laid side by side at equal distances, and their images then combined, the color of the middle one of the binocular images will at first be sometimes redder and sometimes bluer than that of the two side images, but in no case exactly like either of them. By steady looking it is said to be possible to mix the colors of the two objects in a binocular image which is reddish blue (or violet)." This is called "the binocular mixing of colors." If such a deception can be secured, it is manifest that the mixing of colors on which it depends must take place in the brain, and not upon the retinas of the two eyes. If a white stripe be placed upon a black surface and divided into two images, the right one of which is formed by looking at one half through blue glass, the left by looking through gray glass, then the right image will be seen blue, but the left will be seen yellow. This is called " binocular contrast of colors."

The peculiar perception of luminosity is due to a struggle between the two fields of vision which results, not in combining the black images of one field with the white images of the other so as to produce an equal tint of gray, but in a rapid alternation of the two. Very smooth bodies, when they reflect the light perfectly, do not appear luminons. But when the surface of such bodies-as, for example, the surface of a sheet of water-becomes ruffled by ripples, it becomes luminous. The perception of luminosity may be produced by combining two stereoscopic pictures of an object which are alike in contour, but one of which is black with white lines where the other is white with black lines. Two such pictures not combining to produce an equal tint of gray over the

${ }^{1}$ So HIering asserts, Physiolog. Optik, in Hermann's Handb. d. Physiol., III., i., p. 592. Binocular mixing of colors has been denied by some authorities. 
whole surface, the images of the separate points on the two retinas enter into a struggle with each other ; and the rapid alternation of the prevalence, first of one and then of the other, gives rise to the appearance of luminosity.

Such phenomena as the foregoing seem to require a reference to certain unknown processes in the central organs as a physical basis for the psychical experience. Some experimenters claim that in these cases of contrast they are able to see either color at will by giving attention, first to the image on one retina, and then to that on the other. The words in which this claim is couched, however, afford no explamation of the phenomena; for, we repeat again, the mind does not see the image on the retina, and cannot direct special attention to it. It can only attend to this or that feature of the "presentation of sense," which is in every case a subjective affair. But the very question that we are unable to answer concerns the reason why the presentations of sense are constructed as they are in such cases; the reply, so far as any reply can be giren, must be, that such data or motifs furnished by the spatial series of sensations as we cannot connect with known laws of the peripheral organs of sense must be referred to unkuown laws of the central organs of the same sense. Apparently this truth holds good of certain optical illusions of motion. The fact that a steady succession of images (as in the case of watching a fall of water), passing over a particular region of the retina for a long time, sometimes ceases to be perceired as a motion, and that the image of a stationary body on the same retinal region may appear to be moving in the opposite direction, has been explained by "Thomson's law." This law refers the phenomena to the principle of fatigue. Recent investigations, however, seem to show that the explanation is incorrect. They bring out the remarkable result that the same elements of the retina, when stimulated simultaneously, may give rise to impressions both of motion and of rest. For this result some unknown law of cerebral action would seem to afford the only possible explauation. ${ }^{1}$

$\S 34$. The fact that things are seen upright and in correct relations horizontally, by means of data furnished through inverted retinal images, as well as all illusions and errors that are connected with this normal fact, implies yet more maturity of experience. Why do we see the upper part of the object by means of the lower part of the retinal image, and vice versa? and why do we see the right side of the object by means of the left side of the retinal image, and vice versa? Such questions have often been propounded as psychological puzzles of special difficulty. The only answer possible fol-

' See Journal of Physiology, iii., p. 299 f. 
lows, obriously, from the foregoing principles. Strictly speaking, we neither see the external object nor the retinal image; the field of vision is a subjective affair, and is like neither of these two. The presentation of visual sense is normally dependent upon the retinal image for the data from which it is constructed; the image is dependent upon the external object for its formation by rays of light reflected from the object and converged upon the nervous elements of the retina. The different parts of the object as seen are primarily localized simply with reference to each other by means of local retinal signs and of muscular sensations produced by motion of the eyes. But as yet the field of vision has no locality in objective space; no part of it can be said to be either up or down, either right or left. The use of such terms of position implies an association of localized sensations of sight with those of touch and of the muscular sense, in giving us a picture of the relation of the different parts of the body to each other, and of the entire body to the ground, the sky, and the various parts of surrounding objects. When the eyes are moved downward, the lower parts of the body and objects situated on the ground successively come into the field of vision; when the eyes are mored upward, the near ground and lower parts of objects successively disappear from the field of vision, and remoter or higher objects come to view. Seeing objects to the right or to the left is accomplished by motion of the eyes in the corresponding direction. Right is the direction in which the right hand is placed from the middle of the body ; left is the direction in which the left hand is found. The massive feelings of touch and muscular sensation keep us informed of the general relation of our bodies to the earth and to objects on its surface. The head is the upper part, or part farthest away from the ground; the feet are the lower part, or members of the body in contact with the ground. Thus we come to use terms for localized sensations of sight which, in this use of them, have no primary reference whatever to the field of vision in itself considered.

$\S 35$. The nature of the "sense-data" which the mind has at its disposal for constructing its presentations of sense, and the psychophysical laws which are followed in the process of construction, have been explained in such detail that little need be added concerning the development of visual perception. Visual space presents itself to us as a coherent complex of sensations of light and color systematically arranged. The arrangement implies certain native activities of the mind in connection with and dependence upon the action of the nerrous organism; but it also implies an immense influence from experience. It is extremely difficult, if not 
wholly impossible, to distinguish with confidence the limits which must be drawn between what is native and what is learned. The seeing of colors is undoubtedly a far more simple and primary act than the seeing of colored objects as situated in relation to each other in objective space. A colored surface, or a system of colorsensations related to each other as side by side in space-form, results in experience from the weaving together of several spatial series of sensations. Such a surface may theoretically be conceived of as presented to the mind through the activity of the nerrous elements belonging to the retina of a single motionless eye. The motifs or data which the mind would have for constructing such a surface must be found in the series of sensations of light and color as rarying in intensity and quality according to the locally distinct nerrous elements which are simultaneously excited. The evidence seems, on the whole, farorable to the assumption that some indefinite picture of visual space might be gained wholly through the excitation of a motionless nervous mosaic (like the retina) sensitive to light.

But visual space, as experience makes it known to us, requires binocular vision with moving eyes. The firm spatial connection of all the parts requires that a system of lines of direction shonld be fixed, prescribing the objective points at which the sensations produced by exciting together the different pairs of the covering points of the retina must appear in visual space. To establish such spatial connection, both eyes must move in their conjoined action as a single organ of vision. By this action the field of binocular vision is built up in an order of experience which, on the whole, consists in the successive mastery of more and more complex problems. For the process of learning to localize, the one centre-the point of starting and the goal of return-is the spot of clearest vision of the retina (the yellow-spot), to which the point of regard in the object corresponds. With the point of regard fixed in the primary position of the eye, the first and most essential means is gained for orientating objects in the field of vision. The meridians, horizontal and vertical, and the locations of different points in the surface of the field of vision thus presented to the mind, afford the comparatively simple problems furnished by the primary position. In this way a central point, determining lines, and finally a continuous surface are fixed, to which may be referred all the directions and locations of the binocular points and lines of regard in the secondary positions of the eye.

The motifs or data which give to the mind its guidance in achieving its more difficult tasks are the spatial series of muscular 
and tactual sensations which are caused by the motions of the eye for parallel turning, for accommodation, and for convergence in near vision. The general principle is, that by motion the relatice space-values of the retinal elements are not changed; but their absolute values-that is, the complex which is formed by combining all these muscular and tactual sensations with the local signs of the retina-are ehanged in equal sense and measure. What moving the eyes does for the retinal images, moving the head and body does for the presentations of sense as constructed in binocular vision; it alters the absolute values of the complex of sensation as related to objective space, while lieeping the relative values belonging to the different positions of the eyes unchanged.

The visual perception of depth involves a later and more complex training from experience than the perception of two-dimensioned extension. To solve the problem of depth, binocular vision with moving eyes, and its resulting combination and separation of the double images of objects, seems necessary. The existence and assistance of those secondary helps, which are so important in perceiving the solidity and distance of objects, imply a further development of experience. In all these advances, however, the course of acquisition is not in separate straight lines that run parallel or converge, as it were. More complex experience, when obtained, modifies what is really more simple and primary. What we see in monocular vision with an open eye, and even what we see with both eyes closed and motionless, depends upon what we have learned to see with both eyes in varied movement and availing themselves of all possible secondary helps. It also depends upon what we bave learned to know of the nature and probable position and shape of manifold objects of which the eye has already attained the mastery.

$\S 36$. Finally, brief mention must be made of the connections which are constituted, in the development of our perception of objects as having the qualities and relations of space-form, by the joint action and mutual assistance of eye and hand. With the sense-presentations of one of these senses the images of objects as known by the other become most intimately related. It is a misuse of terms, however, and involves the entire subject in confusion, to speak of this joint product as a "sense-perception." It is rather to be spoken of as a mental image or concept. The visual presentation of an object-as, for example, a ball, a pen, a table-may recall its tactual presentation. We readily interpret one into terms of the other-sight into terms of touch, and touch into terms of sight. But all the perceptions, as such, of spatial properties and relations, whether gained by eye or hand, are kept quite distinct 
and separable in the mind. No such synthesis takes place between the spatial series of the one sense and the spatial series of the othersense as takes place between the spatial series of the same sense. And all the properties and relations of bodies as known in spaceform are given by each of these senses. The view which makes the sense of sight dependent upon the sense of touch and the muscular sense for the construction of its spatial objects is erroneous. While feeling the pen, we can image how it would look; when seeing it, how it would feel. We can image how much exertion would be required to reach a mountain which appears to the eye so far away, or how a mountain would look at a clistance of so many miles as measured by the exertion required to walk there. But the true presentations of the visual objects and tactual objects do not mix in one combined perception. They unite only in one image or idea of the object.

$\S 37$. Interesting experiments have been conducted to determine the degree of accuracy with which perceptions of distance by sight can be translated, as it were, into terms of the tactual and muscular sense. Some of these experiments show the amount of harmony which can be obtained between optical localizing and localizing with the finger. Helmholtz ${ }^{1}$ made use of a vertical thread which he tried to locate, as seen in monocular vision, by litting it with a pencil's point; Donders, ${ }^{2}$ of a very small induction-spark, which was to be touched with the index-finger. The result of 50 experiments, made for distances along the same line of regard varying between 60 and $610 \mathrm{~mm}$., when only the spark itself was seen in perfectly dark surroundings, showed that the distance was overestimated 34 times, under-estimated 12, estimated right 4 times. The greatest errors were +35 and $-34 \mathrm{~mm}$.; the mean error $10.6 \mathrm{~mm}$. When the surroundings were visible and the electrodes seen with open eyes, the eyes then closed, and the finger reached to the estimated distance, the greatest errors were +30 and -12 $\mathrm{mm}$., and the mean variable error $9.8 \mathrm{~mm}$., for distances from 80 to $630 \mathrm{~mm}$. The exact localizing of the point of regard in terms of touch is more difficult the farther the object is removed and the less assistance is had from secondary helps. Localizing in the same way when the object lies out of the line of regard is still more inaccurate. In 29 experiments, where the spark to be localized was flashed at a distance of $210-600 \mathrm{~mm}$. to one side of this line, the greatest errors were +120 and $-68 \mathrm{~mm}$., with a mean error of about $34 \mathrm{~mm}$. 
The problem of comparing the judgments of linear extension made by the eye, the hand, and the arm, and of determining their relative accuracy, has recently been examined, experimentally, at considerable length by J. Jastrow. ${ }^{2}$ His method was to present a definite length, varying from $5 \mathrm{~mm}$. to $120 \mathrm{~mm}$., to the retina, the skin (by application of a pair of points, or by motion of a single point), to the forefinger and thumb (by being held between the two), or to the arm when in free movement and guiding a pencil to express its estimate. The subject of experiment was required to get a clear perception of the given distance by one of these organs (called, in such case, the "receiving sense"), and then either simultaneously or successively express this perception through the same or some other one of these organs (the "expressing sense"). In this manner it was discovered that, if the eye is both receiving and expressing sense, small lengths will be under-estimated and large lengths exaggerated, the point where no error is made being at about $38 \mathrm{~mm}$. ; whereas, if the hand is both receiving and expressing, small lengths will be exaggerated and large lengths under-estimated, the "indifference-point" being at about $50 \mathrm{~mm}$.; but the arm exaggerates all lengths within the limits of the experiments. When, however, the eye expresses and the other organs receive the impression, all lengths are greatly under-estimated; but if the hand is the expressing sense, all lengths are greatly exaggerated. The arm as expressing sense exaggerates all lengths received by the eye, and under-estimates all received by the hand.

The relative accuracy of the three senses, whether receiving or expressing, or both, stands in the order of eye, hand, arm-the hand being only slightly better than the arm. The degree of confidence felt in the estimate mado is naturally greatest where the accuracy is greatest. Inasmuch as "the expressing sense gives the characteristic properties to the curve of error," " the question arises whether all the phenomena cannot be accounted for by a special application of the law of habit in connection with the normal action of the sensory apparatus. Each sense, when expressing the estimate, tends to approximate it in size toward those dimensions which it is most accustomed to judge accurately.

All the foregoing results show plainly that the interpretation of visual distance in terms of the tactual and muscular sense is a matter of complex experience, and is not usually more than very imperfectly attained. It bears little comparison with the nicety of

${ }^{1}$ Art. on The Perception of Space by Disparate Senses, in Mind, October, 1886, pp. 539-554.

${ }^{2}$ Ibid., p. 549. 
the spatial perceptions belonging to each one of the two senses concerned when interpreting its own specific data in corresponding terms, as it were.

$\S 38$. In closing this subject, the one psychological truth of preeminent value which has been most obviously demonstrated should be stated again. Perception is the result of an extremely complex activity of the psychical subject, Mind; it involves the synthesis of a number of sense-data according to laws that are not deducible from the nature of the external objects, or of the physiological action of the end-organs and central organs of sense. An analysis of these data themselves is not sufficient to explain perception. The descriptions of Physiological Psychology can do no more than enumerate these data, show their dependence on external stimuli, and the value which they have as motifs for the perceiving subject; and then understand the laws of this synthesis as the permanent modes of the behavior of the psychical subject. The object of sense-perception, the presentation of sense, is not an extra-mental entity made up outside of the mind and borne into or impressed upon it through the avenues of sense. It is a mental construction. The field of vision is a subjective aftair, and so is the field of touch. The same psychical subject which reacts upon the stimulation of the nervous organs of sense in the form of sensations, by its activity in synthesizing these sensations, constructs the objects of sense. The fundamental fact is the presence and activity of the subject, known as Mind. 


\section{CHAPTER VIII.}

\section{TIME-RELATIONS OF MENTAL PHENOMENA.}

§ 1. "Presentations of sense" appear in consciousness, not only as having spatial qualities and relations, but also as occurring either simultaneously or successively as respects Time-form. The clearest experience of the manner in which our sensations are located in this framework of time, as it were, is gained by attention to the successive notes of a melody, or to the rhythm of risual or muscular impressions which accompanies a regularly recurrent motion of some member of the body. What is true of the presentations of sense is also true of all mental phenomena, of the reproduced images of sense, of pure creations of fancy, and of thoughts. All have that form of occurrence and relation which we call "Time."

Physiological Psychology, however, can no more give an ultimate explanation of this time-form which belongs to all mental phenomena than of the space-form which objects of sense acquire as the result of a mental synthesis. Experimental science cannot explain "time." Nothing is accomplished toward comprehending the origin of the mental representation of time by indicating the speed, number, and order of the various series of conscious experiences. Successive presentations of sense or successive ideas do not of themselves constitute a mental presentation or idea of succession. The idea that $a$ follows or precedes $b$ is not the idea of $a$ nor the idea of $b$; neither is it the idea of $a+b$ or of $a-b$. Experimental science can explain the order of succession; but in doing this it implies the idea of succession, and this idea is not itself a succession, or an order of succession, or a compound of successive ideas. ${ }^{1}$

Many thousands of experiments have been made (since the work of Donders in 1868), with the use of the most complicated and delicate machinery, in order to fix the amount of time required for the various processes, both nervous and mental, which are the conditions of our conscious life. These experiments have succeeded in bringing many interesting facts to light. But the laws thus established beyond all reasonable question are remarkably few ; more-

${ }^{1}$ Comp. Volkmann von Volkmar, Lehrb. d. Psychologie, II., p. 11 f. 
over, they are nearly all merely restatements in more definite form of familiar generalizations. That a kind of sluggishness or inertia, which the stimulus must overcome, belongs to all the senses, and that they often continue to act, when once roused, after the exciting cause is withdrawn; that different sensations following each other too quickly tend to confuse or destroy each other; that no one can see or think more than about so rapidly, but that this rate varies with different individuals and with the same individual at different times; that it takes more time to perceive or think where the objects are complex, and are either too small or too large or too closely alike; that it takes time to will or choose, less time to act when we know what to expect, and more time to move, in response to a particular sensation, some part of the body which we are not accustomed to connect with that sensation; that practice increases the speed of our mental and bodily action, and that fatigue and certain drugs diminish it-all these statements are matters of common observation.

$\S 2$. It is not necessary to describe the construction of the machines which have been used in experimenting upon the timerelations of mental phenomena, or the methods of using them employed and commended by different observers. The general problem is in all cases essentially the same-namely, to produce certain definite impressions upon the organs of sense, to secure a definite result in the form of motion of some part of the body as a sign that the impressions have been received (and, perhaps, interpreted and mentally combined), and to measure with extreme accuracy the interval between peripheral stimulation and resulting motion.

The electrical current is ordinarily used to mark both the instant when the external sense-stimulus acts on the organ and that when the resulting motion occurs. The stimulus may consist in the flash or crackle of an electric spark, the appearance of one or more colors or figures, or letters or words, the sounding of a bell or a falling ball, etc.; the motion may be with the finger pressing a key, or the foot or hand closing or breaking a circuit, or the vocal organs calling into a tube, etc. The one difficult matter which marks the success or the comparative failure of any series of observations is the arrangement of the experiments and their tabulated results so as to analyze the different elements of the complex process inrolved. Such experiments need to be repeated many times upon the same individual, so as to eliminate the variable factors of bodily condition, attention or distraction of mind, practice, etc. ; they need also to be repeated with many individuals, so as to calculate upon the so-called personal equation. 
§ 3. The interval between the instant when the external stimulus begins to act upon the end-organ of sense and the resulting movement of some member of the body has been called "physiological time" by Hirsch and others, and "reaction-time" by Exner. The latter term is preferable. Reaction-time is "simple" when all the elements which tend to complicate the processes involved in the reaction, and so to lengthen the time required by it, have been as far as possible eliminated. Reaction obtained in response to a single sensation of known quality, the instant of whose appearance is expected, by executing a single natural and easy motion, best fulfils the conditions of simplicity. It is therefore requisite, for all experiments of this sort, that the average simple reaction-time of each individual experimented upon shall be determined; and also the effect of practice, exhaustion, and other influences upon this interval. But even the simplest reaction-time is, of course, a very complex affair.

Donders ${ }^{1}$ distinguished no less than twelve different processes as entering into "physiological time" (or simple reaction-time)and this without interpolating any purely psychical elements, as occupying separate periods, into the entire interval. The analysis of Exner ${ }^{2}$ is more pertinent to our purpose. Exner finds seven elements in all reaction-time: (1) An action of the stimulus on the end-organ of sense preparatory to excitation of the sensory nerve ; (2) centripetal conduction in this nerve; (3) centripetal conduction in the spinal cord or lower parts of the brain ; (4) transformation of the sensory into the motor impulse; (5) centrifugal conduction of the impulse in the spinal cord; (6) centrifugal conduction in the motor nerve; (7) setting-free of the muscular motion. Of these seven factors, however, the fourth is most interesting to psychology. It may properly be called "psycho-physical" as distinguished from more purely physiological time. The other six elements (with the exception of the first, on account of difficulties inherent in the experiments) have been determined with some degree of definiteness (see Part I., chap. iii., on the speed of nervous processes). It is, then, theoretically possible to ascertain the amount of these six and subtract them from the entire reaction-time; the remainder would be the interval occupied by the central cerebral processes (that is, by No. 4). Thus Exner ${ }^{3}$ assumes 62 meters per second as the probable rate of conduction in both sensory and motor nerves; and in the spinal cord, 8 for the sensory and

1 Archiv f. Anat., Physiol., etc., 1868, p. 664.

${ }^{2}$ See Hermann's Handb. d. Physiol., II., ii., p. 271.

3 Ibid., p. $272 \mathrm{f}$. 
11-12 for the motor process. He thus calculates that about 0.0828 sec. is the "reduced reaction-time," or interval occupied within the cerebral centres in transforming the sensory into motor impulses-in the special case of reaction from hand to hand, where the whole reaction-time is 0.1337 sec. The uncertainties of all such calculation, however, occasion the demand for other methods of determining the strictly "psycho-physical " portion of reactiontime.

§ 4. "Psycho-physical time" (No. 4 of Exner's seven processes) is analyzed by Wundt ${ }^{2}$ into three psycho-physical processes: (1) Entrance into the visual field of consciousness, or simple perception; (2) entrance into the point of clear vision with attention, or apperception (attentive and discerning perception); (3) the excitation of the will, which sets free in the central organ the registrating motion. Obviously, the mental processes are here all conceived of after the analogy of sight. Consciousness is regarded as a field of vision ; objects enter it and are at first only obscurely and indefinitely perceived, as are those visual objects whose images enter the field of the eye at the sides of the retina. Time is required for the objects to arrive at the spot of clear vision-the fovea centralis of consciousness (Blickpunkt) - where discerning attention is bestowed upon them and they are apperceived. When they are apperceived, further time is required to get up the corresponding molecular motion in the motor areas of the brain. All three foregoing processes are psycho-physical-that is, they comprise physiological processes in the central organs and simultaneous corresponding changes of consciousness occurring in time-form. There is no good reason to suppose that the mind occupies time for its own processes which is separate from and-as it were-thrown in between the physiological processes. Indeed, all the evidence is contrary to such an liypothesis.

Wundt has made an elaborate defence of his positions with regard to the nature of psycho-physical time. He and his pupils have attempted more definitely to characterize the cerebral changes which correspond to each of the mental elements of (1) perception, (2) apperception, and (3) will. His figure of speech, which likens all changes of conscious states to those produced by moving an image over the retina to the spot of clear vision, may be accepted as helpful to the imagination; it must not be forgotten, however, that it is still a figure of speech. The fact of which it takes account is, that all clianges of consciousness require time in order to define themselves with their maximum of clearness and intensity. The

' Physiolog. Psychologie, ii., pp. 220 ff. 
position that the mental forms of perception, apperception, and will, are exactly simultaneous with corresponding cerebral processes, and that mental states are not to be regarded as forming themselves in a separate time, as it were, on top of these processes, may also be admitted in a provisional way. It is probable theory, however, rather than demonstrated fact. Accordingly, the first problem of psychometry is, to determine the simple reaction-time, and from it to find the three factors of psycho-physical time-namely, perception-time, apperception-time (or discernment-time), and willtime.

§5. Any psycho-physical theory of the time-relations of mental phenomena requires that account should be taken of the inertia of the nervous system. As composed of moving molecules, it necessarily requires some time to be started by the action of a given stimulus, then reach its maximum of activity in a particular direction, then subside into a negative condition with respect to this direction (called "Anklingen" and "Ablingen" of the nervous excitement, by the German investigators). This statement follows as a necessary assumption from the physical nature of the nerrefibres and nerve-cells, since inertia is a property of every material mechanism. It is difficult, however, to justify the assumption experimentally, or to fix the exact amount of time consumed by the inertia of different parts of the nervous system. Experiment demonstrates no stadium of latent excitation for the motor nerve, such as is about ${ }_{10}^{1} \sigma$ sec. for the muscle when electricity is used. The case is different, however, with the end-organs of sense. They do exhibit a certain sluggishness, and this is one reason why only so many sensations in a given unit of time can be produced by their successive irritation.

The result of the inertia of the end-organs, as determining the number of separate excitations of which they are capable in a second, varies for the different senses. The nerve-endings of touch probably exceed all others in the promptness with which they respond to stimulus and then return to a relative equilibrium. But the number of separate sensations of this sense which can be produced during a given interval depends in a remarkable way upon the quality and intensity of the stimulus, the place where it is applied, etc. The results of different experimenters therefore differ widely. Preyer thought that 27.6-36.8 nervous shocks (per second) of the skin fused into one continuous sensation; but Valentin put the limit at 480-640, and von Wittich ${ }^{1}$ succeeded in distinguish-

1 For his remarks on Preyer's experiments, see the article in Pflüger's Archiv, ii., pp. $329 \mathrm{ff}$. 
ing about 1,000 separate excitations in this unit of time. Hearing can receive nearly as many separate sensations in a second as can touch. The noise of the electric spark has been heard with one ear only, as separate sensations, at intervals of 0.00205 sec.; but hardly or not at all at intervals of $0.00198 \mathrm{sec}$. The number of possible sensations of sound may then be placed at about 500 per second. Mach,' however, by using the click from a revolving toothed-wheel, claims to have reduced the interval to $0.016 \mathrm{sec}$. The interval is increased to about 0.064 sec. when the same auditory impressions are heard by both ear's. E. H. Weber noticed that we can tell whether two watches are ticking exactly together much better when both are held near the same ear than when one is held at each ear. Far fewer musical tones than noises can be heard in a second ; and, indeed, a number of vibrations, occupving a considerable fraction of a second, must be secured before the sensation of tone is established, as it were.

The smallest interval for sensations of sight, when the two stimuli act on the same place of the retina, is still greater. In ordinary daylight, rotating disks whose surface is part white and part black become gray (that is, the sensations fuse) when they attain a motion of about 24 per second. It can be told which of two images of electric sparks that are $0.011 \mathrm{~mm}$. apart on the retina occurs first, if the difference in the time of their occurrence is $0.044 \mathrm{sec}$. If the two sparks are seen as one with an apparent motion, its direction can be distinguished when the two ends of the line of motion are only $0.014-0.015 \mathrm{sec}$. apart. But if one stimulus strikes the forea centralis and the other a point of the retina $6 \mathrm{~mm}$. off, the smallest interval for distinct perception is increased to 0.076 sec. $^{2}$ Within certain limits these intervals are independent of the intensity of the light, when it falls on the retina near its centre ; but (comp. p. 334) the intensity and quality of the sensations are connecterl with the time during which the stimulus acts. The law for the "timecourse" of such retinal excitations has been stated and defended by Fick, ${ }^{3}$ as known by the name of "Talbot's principle:" If any place of the retina is periodically excited with light of given intensity, for a certain time $a$, and then left unexcited for a time $b$, and if the time $a+b$ is less than about 0.04 sec., then the sensation becomes continuous, with a strength corresponding to the excitation $\frac{a}{a+b}$.

1 Sitzgsber. d. Wiener Acad., LI., p. 142.

${ }^{2}$ Comp. Exner, in Hermann's Handb. d. Physiol., II., ii., p. 256 f.; and Sitzgsber. d. Wiener Acad., LXXII., p. 156 f.

${ }^{3}$ Archiv f. Anat., Physiol., 1863, p. 739 f.; and Hermann's Handb. d. Physiol., III., i., p. 212 f. 
If the inertia of the eye for the different color-sensations were greatly different, we would see objects differently colored according to the time that the rays from them were acting on the retina. That the different parts of the spectrum do actually require slightly different intervals of time to reach the maximum of their excitation has been shown by Kunkel.' Equally bright light, as before stated (p. 334), attains its maximum effect, for red rays in about 0.0573 sec., for green in $0.133 \mathrm{sec}$, for blue in 0.0916 sec. With the same color-tone, the greater the brightness the quicker the maximum effect is reached. Thus for three degrees of brightness the time for red rays is $0.0573-0.071 \mathrm{sec}$; for green, $0.0699-0.133$ sec. ; for blue, $0.0916-0.102$ sec. Accordingly, the spectrum may be reduced in size and number of color-tones by diminishing the duration of the action of the light which forms it.

The measurcment of the smallest interval for sensations of smell and taste cannot be made with satisfactory exactness on account of the nature of the stimuli of these senses. Little is known which goes beyond ordinary experience concerning after-tastes analogous to the after-images of the eye. One experimenter (Bidder) thought that the sensation continued after the tongue had been so carefully dried off that no particles of the tastable substance were left remaining; but of this we can scarcely be sure. It may be that certain substances leave their after-taste because their tastable particles are dissolved later; or because their effect, being weaker, is at first suppressed by particles of stronger quality. ${ }^{2}$

\$6. When the successive sensations are of different senses, the "smallest interval" between them, and so the number possible in a second, varies still more. The following table ${ }^{3}$ exhibits the results obtained by several different observers :

Between two sensations of sound (electrical sparks)............ 0.002

Between two sensations of light (direct electrical excitation of same

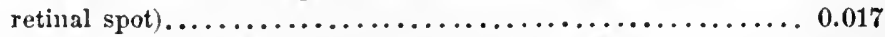

Between two sensations of tonch (impact on finger-Mach) ....... 0.0277

Between two sensations of light (at forea centralis, by optical images). 0.044

Betreen $t$ wo sensations of light (at periphery of retina, by optical images) 0.049 Betreen sensation of sight and sensation of touch (sight following)... 0.05 Between sensation of sight and sensation of hearing (sight following).. 0.06 Between two sensations of noises (each heard by one ear) ........ 0.064 Between sensation of sight and sensation of touch (sight preceding)... 0.071 Between two sensations of light, one at the periphery and the other at the centre of retina .......................... 0.076

Between sensation of sight and sensation of hearing (sight preceding)... 0.16

'Pflüger's Archiv, ix., p. 206 f.

'Comp. von Vintschgau, in Hermann's Handb. d. Physiol., III., ii., p. 221.

s By Exner, in Hermann's Handl. d. Fhysiol., II., ii., p. 262. 
$\S 7$. The way that the intensity of sensations of light rises to a maximum, continues there, and then falls off through exhaustion of the retina, in time, has been represented by Fick $^{1}$ with the use of the accompanying figure (No. 114).

$\S 8$. The point of starting for determining experimentally all the problems which concern the durations and relations in time of mental phenomena is gained by fixing the "simple reactiontime." This is found to vary for different persons, for the differ-

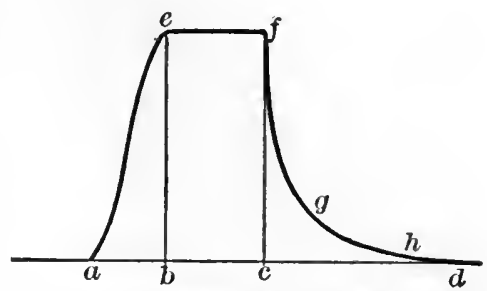

F1G. 114.-Cnrves showing the Rise and Fall of the Intensity of Sensations of Light-the abcissas measured along $a-d$ representing the time.

ent senses, and under different conditions of expectation, attention, habit, etc. In its very simplest form, the question may be stated as follows : How long an interval will elapse, under the most farorable circumstances, between the instant when some end-organ of sense is stimulated and the instant when motion follows as the result of recognizing the fact, in consciousuess, that such stimulation has taken place? In this form the three elements of psycho-physical time (perception, discernment or apperception, and choice) are supposed to be reducible to one-namely, to simple perception. This supposition is, of course, true only in case that, by practice in reacting upon an expected sensation in one definite way, the cerebral sensory-motor processes have attained the highest possible rate of speed, and the time ordinarily occupied in deciding what to do, and in starting the voluntary motor mechanism, has been reduced almost, or quite, to zero. ${ }^{2}$ The entire process then becomes reflex, simply the sensory central part of it being represented by a conscious act of perception. To shorten the reaction-time as much as possible, the subject of the experiment must know what place of the sensory organism is to be hit by the stimulus, and about when to look out for it; he must also be called upon to react, in one and the same easy and natural way, in all cases, as soon as he knows that he is hit at all.

The following table ${ }^{3}$ gives the mean values of the reaction-time "from hand to hand" (one hand being hit by the electrical current

'Hermann's Handb. d. Physiol. III., i., p. 216.

${ }^{2}$ Comp. Wundt, Physiolog. Psychologie, ii., p. 226 f.

${ }^{3}$ Taken from Exner, in Hermam's Handb. d. Physiol., II., ii., p. 263; the two sets of numbers indicate values which were found in two series of experiments. 
and the other reacting, for example, to press a key), as determined by various observers:

Helmholtz. Hirsch. Kohlrausch. Won Wittich. Exner. Vintschgan. Aon Kries and $0.12 \%$ \% sec. 0.1733 sec. $\} 0.1697$ sec. 0.153 sec. 0.1276 sec. 0.1087 sec. 0.117 sec. 0.12495 sec. $\{0.1911$ sec. $\} 0.169$ sec. $\} 0.16$ sec. $\{0.12 s 3$ sec. $\{0.1560$ sec. $\} 0.146$ sec.

The last two experimenters developed certain interesting results. They found the reaction-time, when the stimulus was applied to the middle finger, to be for Kries 0.117 sec. and for Auerbach 0.146 sec.; but when applied to the back of the hand, to be for Kries 0.119 sec. and for Auerbach 0.147 sec. But the hand being about $16 \mathrm{ctm}$. nearer the brain than the middle finger, its reaction-time should have been some 0.004 sec. shorter instead of longer, as a matter of physiological time. Exner found that the reaction-time, when the forehead is stimulated, is greater than when the stimulus is applied to the hand. Bloch found the same thing true when the nose is stimulated. The intercerebral relations, taken in connection with the law of habit, probably account for the foregoing facts.

The value of the reaction-time also changes when the character of the stimulus is changed on which the subject of experiment reacts. This fact is made apparent by the following table :

\begin{tabular}{|c|c|c|}
\hline $\begin{array}{l}\text { Optical stimulus. } \\
\text { Sec. }\end{array}$ & $\begin{array}{l}\text { Acoustic stimulus. } \\
\text { Sec. }\end{array}$ & $\begin{array}{l}\text { Stimulus of tonch. } \\
\text { Sec. }\end{array}$ \\
\hline . 0.200 & 0.149 & 0.182 (hand). \\
\hline Hankel . ........... 0.225 & 0.151 & 0.155 \\
\hline Donders............ 0.188 & 0.180 & 0.154 (neck). \\
\hline Von Wittich ......... 0.194 & 0.182 & 0.130 (forehead). \\
\hline Wundt........... 0.175 & 0.128 & 0.188 \\
\hline Exner ............. 0.1506 & 0.1360 & $0.12 \% 6$ (hand). \\
\hline Anerbach ........... 0.191 & 0.122 & 0.146 \\
\hline Von Kries . . . . . . . . . . 0.193 & 0.120 & 0.117 \\
\hline
\end{tabular}

We conclude, then, that under the most farorable circumstances the reaction-time can scarcely be reduced to $\frac{1}{10}$ of a second, while it rarely rises much above $\frac{2}{10}$ of a second.

\& 9. It has been argued that the apparent difference in the reaction-times of different senses is due to difference in the intensity of the stimuli applied. Increasing the strength of the stimulus decreases the reaction-time in all the senses; but we have no very good means of measuring stimuli of one sense in terms of another sense. It has been proposed ${ }^{2}$ to reduce them to a common standard by referring the sensations to the point where they barely reach the "threshold of excitation" (Reizschwelle) ; that is, where they

'Taken from the article of von Kries and Auerbach, Archiv f. Anat. u. Physiol., Physiolog. Abth., 1877, p. 359 f.

" By Wundt, Physiolog. Psychologie, ii., p. $223 \mathrm{f}$. 
are just perceptible in consciousness. In this way the mean result for sound (0.337), light (0.331), and touch $(0.327)$ are found to be almost exactly the same. It has further been argued that the speed of perception and the duration of psycho-physical time are the same for all the senses. On the contrary, there seems good reason to suppose that the reaction-time of sight is necessarily longer than that of hearing or touch, on account of the photochemical nature of its more immediate stimulus. One observer (von Wittich) has even gone so far as to conjecture that the speed of conduction in the optic nerve is less than that of the other nerves of sense; it is rather to be concluded, however, that the latent time of the sensory end-apparatus, and of the cerebral processes by which sensory impulses pass over into motor impulses, ${ }^{2}$ is different.

$\S 10$. The effect of increasing the intensity of the stimulus, in diminishing the reaction-time, has been studied by Wundt for sensations of sound occasioned by the fall of a hammer or ball-with the following result :

\begin{tabular}{|c|c|}
\hline Height of hammer. & $\begin{array}{r}\text { Reaction-time. } \\
\text { Sec. }\end{array}$ \\
\hline $1 \mathrm{~m}$ & 0.217 \\
\hline & 0.146 \\
\hline 8 millime & . . 0.132 \\
\hline 16 millimeters & $\ldots 0.135$ \\
\hline
\end{tabular}

Height of ball. $\quad$ Reaction-time. Sec.

2 centimeters ......... 0.161

5 centimeters ......... 0.176

25 centimeters .......... 0.159

$5 \tilde{5}$ centimeters ......... 0.094

This effect is obtained, of course, only within certain limits ; for the sound must not be so loud as to startle and confuse. Thus, also, when the length of the electric spark which stimulates the retina is increased the reaction-time is diminished. Exner ${ }^{2}$ found that, while it was $0.1581-0.1502$ sec. for sparks $0.5-1 \mathrm{~mm}$. in length, it was $0.1479-0.1384 \mathrm{sec}$. for those $2-5 \mathrm{~mm}$. long, and diminished to $0.1229 \mathrm{sec}$. for those of $7 \mathrm{~mm}$.

The reaction-time is also diminished by indicating the instant at about which the sensation may be expected, through some preceding signal. The interval for the sound caused by a ball falling $25 \mathrm{ctm}$., which without signal was $0.253 \mathrm{sec}$., was reduced by $\mathbf{a}$ signal to $0.076 \mathrm{sec}$; and when the fall was $5 \mathrm{ctm}$. the interval was reduced by the signal from $0.266-0.175$ sec. $^{3}$ In order to secure this effect, however, the interval between signal and impression should be nearly constant, and not so long as to overstrain atten-

${ }^{1}$ Comp. von Kries and Auerbach, Archiv f. Anat. u. Physiol., Physiolog. Abth., 1877, p. 359 f.

' Hermann's Handb. d. Physiol., II., ii., p. $269 \mathrm{f}$.

${ }^{3}$ See Wundt, Physiolog. Physiol., ii., p. 238. 
tion or prevent the carrying of a definite mental image of this interval.

The detailed investigations of Berger, ${ }^{1}$ recently published, announce the following conclusions: (1) The reaction-time increases in inverse ratio to the intensity of the stimulus, and so much the faster the nearer we approach the "threshold" (or lower limit) of the stimulus; (2) discernment-time is related to alterations in the intensity of the stimulus in the same way as simple reaction-time; and (3) will-time is independent of the intensity of the stimulus.

$\S 11$. When the quality of the impression to be expected is known, but its intensity is unknown, the reaction-time is increased. The increase is greater if the alternation of intensities is very irregular. This fact is exhibited by the following table: ${ }^{2}$

I. Uniform change of intensity.

II. Irregular change of intensity. Sec.

Loud sound............. 0.116

Feeble sound............. 0.127

Loud sound............. 0.189

Feeble sound.............. 0.298

By suddenly intercalating a feeble sound in a series of loud noises the reaction-time may be prolonged to 0.4 or 0.5 sec. It is also greatly lengthened when the impression is wholly unexpected by the subject of the experiment being taken off guard, as it were ; in such a case, also, it may reach 0.5 sec. As might be expected, it takes longer to react in an unnatural and unaccustomed way. It requires more time to react with the foot than with the hand; a mean reaction-time from eye to foot was found by Exner to be 0.1840 sec.

$\S 12$. The reaction-time for the sense of taste varies in dependence upon the part of the tongue to which the stimulus is applied, and upon the character of the gustable substance. It also varies greatly with different persons. Von Wittich fixed it at 0.167 sec. from tongue to hand, by using the sour taste which the electrical current excites. The reaction-time for sugar on the tip of the tongue varied, for three different persons, from 0.1639 to 0.3502 sec.; and for quinine, for two persons, from 0.2196 to 0.993 sec. For the root of the tongue, it was found to be $0.552 \mathrm{sec}$. for sugar, and 0.502 sec. for quinine. ${ }^{3}$ Little has been done to determine the reaction-time of smell. Some have maintained that it must be much longer than the reaction-time of sight and hearing, and even reach several seconds; others have held that, although slower than these

1 Wundt's Philosoph. Studien, 1885, III., heft i., pp. 38 ff.

2 Wundt, Physiolog. Psychologie, ii., p. 241 f.

${ }^{3}$ Von Vintschgau and Hönigschmied, in Pflüger's Archiv, x., 1 ; xii., 2; xiv., 3. 
senses, smell has probably a reaction-time of only a fraction of a second. The latter view seems more recently to have been confirmed by the experiments of Moldenhauer, ${ }^{1}$ who obtained the following among other figures: Oil of mentha, $0.203-0.362$ sec. : oil of bergamot, $0.212-0.374$ sec. ; camphor, $0.226-0.492$ sec. ; musk, 0.319 sec. Taste and smell are much more subject to change in the length of the reaction-time through individual peculiarities of the exciting substances and of the subjects of experiment, than are the senses of hearing, sight, and touch.

§ 13. Having obtained the mean reaction-time for the different senses under varying circumstances, the method of investigation requires that it should be determined how much the reaction-time is increased by increasing and complicating the psycho-physical elements. One principal question to be answered by this method is the following: How much time is required for " apperception," or clear discernment of perceived objects in the central point of consciousness under different conditions? This question has been very patiently and fully investigated, at first by Donders, and since by many observer's, especially by von Kries and Auerbach. ${ }^{2}$

Donders ${ }^{3}$ and his pupils were the first to examine in detail the speed of psychical processes, with a view to determine how long it takes to recognize one of two or more different presentations of sense; and also how long to solve the dilemma of choosing one of two means for making the reaction. For example, in one series of experiments the eye was suddenly stimulated with either red or white light (the subject of the experiment not knowing which to expect), the signal for the former to be given with the right hand, the signal for the latter with the left. In another series of experiments, the quality of the light or sound was to be recognized before reaction, but reaction was to take place only in case a particular one of the two sensations was recognized; in case the other sensation appeared in consciousness, no reaction was to take place. In other words, discernment of the presentation of sense was to be followed by the choice between reacting in a prescribed way and refraining from reacting at all. In still other experiments the stimulus consisted of a "vocal clang" called into a "phonautograph" by one person, and the reaction consisted of the same clang repeated by another person; or, again, the recognition of one or more letters seen was signalled by a movement of the hand. By such methods

1 Wundt's Philosoph. Studien, I., heft iv., p. $606 \mathrm{f}$.

${ }^{2}$ See their article in Archiv f. Anat. u. Physiol., Physiolog. Abth., 1877, pp. $297-378$.

${ }^{3}$ Archiv f. Auat., Physiol., etc., 1868, pp. 657-675. 
Donders made the mean reaction-time of five persons, for discernment between red and white light, with choice of the hand by which to react, to be $0.154 \mathrm{sec}$; the minimum, $0.122 \mathrm{sec}$; the maximum, 0.184. The mean reaction-time for two letters, with discernment and signal by calling them out, was found to be 0.166 sec.; when the number of letters was increased to five, the mean reaction-time rose to 0.170 . It took 0.180 sec. to discern and repeat a rocal clang when known, 0.268 sec. when unknown. With the method of reacting only on one clang and keeping silent when others were heard, the mean reaction-time varied from 0.201 to $0.284 \mathrm{sec}$. The investigations of Donders made obvious the fact already stated (p. 475 f.), that the natural connection between the sensation and the peculiar means chosen for reaction is of influence in determining the interval. Donders assigned $0.039 \mathrm{sec}$. to the psychical process of the development of a presentation of sound in his own case, and a little less to the formation of a decision of will.

\$ 14. Another and ingenious method of determining the time required for " apperception" was proposed by Baxt ;" it was based upon the principle of the inertia of the senses, especially of sight. Suppose the question raised, How long must an image act on the optical apparatus in order to occasion a clear presentation of sense? It may be answered by discovering how quickly after a given impression another stronger one must follow in order that the latter may orertake the former, and quench it-as it were-before it reaches the focus of apperception. Let then some image which requires discernment to interpret it-as the image of several letters, or of a simple geometrical figure-be thrown upon the retina, and let this image be succeeded after a brief interral by the image of a bright white disk ; then, if the interval be less than a certain time, apperception (or clear vision, with discernment of the significance of the image) will not take place at all. Baxt found that the time necessary under these circumstances for a presentation of risual sense depends upon the intensity of the second excitation; it increases as this intensity increases. It depends also upon the complexity of the apperception required; to recognize three letters required only about half the time necessary to recognize five or six. With an interval of $0.0048 \mathrm{sec}$. between the two excitations, the first appeared as scarcely a trace of a weak shimmer; with an interval of 0.0096 sec., letters appeared in the shimmer-one or two of which could be partially recognized when the interval increased to 0.0144 sec. When the interval was made $0.0192 \mathrm{sec}$, the objects were a 'See Pflüger's Archiv, iv., pp. 325 ff. 
little more clearly discerned; at 0.0336 sec. four letters could be well recognized; at 0.0432 sec., five lettters ; and at 0.0528 sec. all the letters could be read.

$\S 15$. The method of Baxt was rejected by vou Kries and Auerbach $^{1}$ as unsuitable to answer the question most interesting to psycho-physical researches; because it includes as an inextricable factor the time used up in the peripheral nerves. Besides, we have no means of estimating just to what stadium a psycho-physical process must have advanced when it becomes impossible for a strong succeeding sensation to overwhelm it. These observers preferred, therefore, the method employed by Donders, and especially in the form (called "Donders' C-method") in which the subject of experiment reacts in one prescribed way or else refrains from reacting at all. By this method they endeavored to answer the questions : "How long time passes after the occurrence of a stimulus of sight before I know what color it has; and how long before I know at what place in the field of sight I experience it," etc. ? Their results may be summed up in the following table." [The numbers give the time assigned by them to the discernment involved in the various processes performed-that is, the psycho-physical time exclusive of all solution of a dilemma by the will. This time is found by subtracting the simple reaction-time, or time necessary for reacting when no discernment is required, from the whole time required for the process including such discernment.]

\begin{tabular}{|c|c|c|}
\hline & Auerbach. & Von Kries. \\
\hline & Sec. & Sec. \\
\hline $\begin{array}{l}\text { Discernment of the direction of light. } \ldots \ldots \ldots \ldots \ldots \ldots \ldots \\
\text { Discernment between two colors. }\end{array}$ & 0.011 & 0.017 \\
\hline 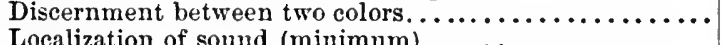 & 0.012 & 0.034 \\
\hline Localization of sound (minimum) ...... & 0.015 & 0.032 \\
\hline Discernment of tone when higher..... & 0.019 & 0.049 \\
\hline Localization of sensations of touch .... & 0.021 & 0.036 \\
\hline Localization of distance by sight... & 0.022 & 0.030 \\
\hline Discernment between tone and noise. & 0.022 & 0.046 \\
\hline Judgment of intensity of sensations of touch (strong) & 0.023 & 0.061 \\
\hline Discernment of tone when lower.... & 0.034 & 0.054 \\
\hline Judgment of intensity of sensations of touch (weak)..... & 0.053 & 0.105 \\
\hline Localization of sound (maximum) ......... & 0.062 & 0.077 \\
\hline
\end{tabular}

$\S 16$. Various interesting discoveries were made during the course of the experiments which resulted in preparing the foregoing table. For example, it was found that the simple reaction-time for $A$. (Auerbach), when stimulus was applied to the middle finger or

${ }^{1}$ See Archiv f. Anat. u. Physiol., 1877, Physiolog. Abth., p. 298.

' Ibid., p. 346 f. 
back of the hand, was $0.146 \cdot 0.147 \mathrm{sec}$; and for $\mathrm{K}$. (Kries), 0.117-0.119 sec. But, as the table shows, when discernment was required of the two observers, the reaction-time of $K$. was relatively so much increased as to make his discernment-time greater than that of A. The result of practice in discernment was found to hold good for other areas of the skin than those in experimenting upon which the practice was gained. For discernment among three places (middle finger, back of hand, and middle of lower arm), the order being unknown and only one to be reacted on-the mean interval required was for A. 0.028 sec., and for K. 0.050 sec. ; further practice, however, reduced this interval to about the same as that required for two places.

Discernment between two intensities of the sensation of touch was found to be very uncertain and difficult. Many more false reactions followed the attempt to tell whether the dorsal side of the last of the phalanges of the middle finger was being hit with the weaker or the stronger of two stimuli than occurred in the attempts to localize tactile sensations. The discernment-time, when reaction followed the stronger stimulus, was $0.016-0.034$ sec. for A, and $0.05-0.07$ for $K$; ; when reaction followed the weaker stimulus, the discernment-time was $0.035-0.069 \mathrm{sec}$. for A., and 0.089-0.114 for K. The character of our judgments of intensity is, perhaps, dependent on the steepness, as it were, with which the curve rises in consciousness; but, however this may be, it appears that we discern how and where we are affected with a sensation more promptly than about low much we are affected.

When discernment between two simple tones of different pitch is required, the reaction follows the one of higher pitch more promptly. Thus the discernment-time, under such circumstances, was for A., 0.015-0.044 sec., and for K., $0.043-0.11$; but, if reaction followed the tone of lower pitch, the discernment-time for $\mathrm{A}$. was $0.03-0.059$ sec., and for $\mathrm{K}$. 0.045-0.092. To discern tone from noise, when reaction followed the tone, A. required $0.015-0.023$ sec., and K. 0.036 -0.055 ; when reaction followed the noise, A's discernment-time was $0.017-0.025 \mathrm{sec}$, and K's, 0.045-0.047. The reaction-time diminishes as the pitch rises; for very high notes it nearly reaches the limit required for hearing the noise of the electric spark. The explanation for these experiences requires reference again to the fact that some 15-20 vibrations are necessary to start the organ so that the sensation of musical tone can be received at all.

The simple reaction-time for sensations of sound remains nearly the same for all changes in the angle by which the locality of the sound diverges from the median plane between the two ears. But 
the time required for discerning the locality of the sound varies greatly for the different sizes of this angle. Thus the discernmenttime for locality, as to right or left, varied for Auerbach and Kries as follows :

\begin{tabular}{|c|c|c|c|}
\hline & Angle $120^{\circ}-35^{\circ}$. & Angle $35^{\circ}-26^{\circ}$. & Angle $26^{\circ}-11^{\circ}$. \\
\hline 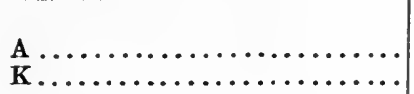 & $\begin{array}{c}\text { Sec. } \\
0.020 \\
0.013\end{array}$ & $\begin{array}{c}\text { Sec. } \\
0.033 \\
0.122\end{array}$ & $\begin{array}{c}\text { Sec. } \\
0.120 \\
0.153\end{array}$ \\
\hline
\end{tabular}

The discernment-time required for localizing the direction of a spark by direct vision varied for $\mathrm{A}$. from 0.005 to $0.025 \mathrm{sec}$., and for K. from 0.006 to 0.029 sec. ; by indirect rision, for A. from 0.008 to $0.028 \mathrm{sec}$, and for $\mathrm{K}$. from 0.007 to $0.028 \mathrm{sec}$. For localizing distance, A. required 0.019 to 0.027 sec. of discernment-time, when the object arose in front of the fixation-point, and $K .0 .027$ to 0.035 sec. ; but A. required 0.019 to 0.029 sec., and K. 0.021 to 0.036 sec., when the object arose behind this point.

$\S 17$. Various strong objections have been raised to the validity of the results reached by the observers last mentioned. For example, Richet' thinks that an interval so small as their "discernment-time" (about $0.03 \mathrm{sec}$.) is below the limit of the experimental error. But the constancy of the results obtained by such a large number of experiments with reference to the mental peculiarities of the two subjects of experiment, and to the different kinds of mental processes involved (discernment of locality, quality, quantity, etc.), is too great to allow of the results being summarily rejected. The criticism of Wundt ${ }^{2}$ as applied to the method employed (the so-called "Donders' C-method") is much more pertinent. This veteran experimenter considers that the psycho-physical time required to discriminate between two or more possible presentations of sense, and then react only in case one of them is apperceived, cannot all be allotted to discernment-time. For an element of volition, or will-time, is as truly involved in the decision whether to react or not to react as in the decision between two modes of reaction.

With a view, then, to analyze these elements more perfectly, and so determine the exact duration of "apperception," in the sense in which the word is used by Wundt, another method of experiment has been employed by Friedrich. ${ }^{3}$ In this method the subject of experiment is warned when to expect one of two or more colors

See Revue philosophique, VI., p. 395.

2 Physiolog. Psychologie, ii., p. 251 f.

${ }^{3}$ See Wundt's Philosoph. Studien, II., heft i., pp. 39 ff. 
to be discerned, but does not know which one to expect; only one way of reaction is employed for all cases; and the judgment of the subject is left to determine just when he clearly discerns, or " apperceives," the object. The time of apperception is then found by subtracting the simple reaction-time (or time necessary to announce the sensation without discernment) from the entire reaction-time thus obtained. In this way the interval for apperception was fixed at 0.030 to $0.185 \mathrm{sec}$. for white, in the case of Wundt, and 0.044 to 0.146 for black; in the case of Tischer, 0.046 to 0.112 sec. for white, and 0.021 to 0.061 for black; and in the case of Friedrich himself, 0.042 to $0.084 \mathrm{sec}$. for white, and 0.019 to 0.064 for black. The mean duration of apperception, as derived from all the experiments with two color-sensations, was 0.086 sec. for W., 0.047 for T., 0.050 for $\mathrm{F}$.

$\S 18$. The time required for discernment increases, of course, when the other conditions are kept as nearly as possible the same, but the number of objects is increased among which the discernment is required. For example, Friedrich found that when four (instead of two) colors-black, white, green, and red-were interchanged in an unknown order, both the reaction-time as a whole and the duration of apperception were increased. The latter for black as one of four colors was, when averaged for several series of experiments, 0.081 to 0.141 in Wundt's case, 0.021 to 0.105 in Tischer's, 0.076 to 0.197 in his own. With red as one of four colors the duration of apperception was 0.049 to 0.247 for W., 0.024 to 0.124 for T., and 0.066 to 0.234 for F. Experiments were also instituted with a view to determine how long it takes to discern composite perceptions of sight.' For this purpose printed figures of $6 \mathrm{~mm}$. high and $3.8 \mathrm{~mm}$. broad were employed, ranging from one to six placesthe reaction being in the form of calling the number constituted by the figures as arranged when displayed. The mean time required for apperception by the three subjects of experiment is shown by the following table: [The figures at the head of the columns indicate the number of places which the numbers had in the different series of experiments; the letters, the subjects of experiment; the figures under the head, the seconds of apperception-time.]

\begin{tabular}{|c|c|c|c|c|c|c|}
\hline & 1. & 2. & 3. & 4. & ธ. & 6. \\
\hline 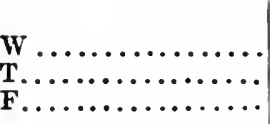 & $\begin{array}{c}\text { Sec. } \\
0.344 \\
0.290 \\
0.320\end{array}$ & $\begin{array}{c}\text { Sec. } \\
0.361 \\
0.380 \\
0.346\end{array}$ & $\begin{array}{c}\text { Sec. } \\
0.354 \\
0.493 \\
0.344\end{array}$ & $\begin{array}{c}\text { Sec. } \\
0.459 \\
0.709 \\
0.481\end{array}$ & $\begin{array}{c}\text { Sec. } \\
0.573 \\
0.849 \\
0.670\end{array}$ & $\begin{array}{c}\text { Sec. } \\
0.817 \\
1.197 \\
1.043\end{array}$ \\
\hline
\end{tabular}

1 Wundt's Philosoph. Studien, I., heft i., p. 53 f. 
It will be noticed that the reaction-time for numbers of three places is not much greater than that for numbers of one place; but when the complexity is increased to four places, the reaction-time is suddenly and largely increased. This fact is probably due in part to the habit of grasping numbers mentally in periods of three places each. It is not certain, however, that the apperception-time as calculated by this method is trustworthy ; for, although the subject of experiment reacts, and then notifies the number discerned, the act of discernment may really be completed after the reaction has taken place. Horeover, a certain time for accommodating the eye must be allowed ; especially in the case of numbers of five or six figures each. This time, in the opinion of one critic, may be placed at 0.166 to $0.186 \mathrm{sec}$. A more recent investigator concludes that the true discernment-time in a composite perception is possibly so short as to occupy only a few hundredths of a second. For numbers of one to three places the interval between perception and apperception is 0.015 to $0.035 \mathrm{sec}$. This conclusion accords with and enforces that arrived at by the careful experiments of vou Kries and Auerbach.

Cattell ${ }^{2}$-by assuming that perception-time and will-time are abont equal, and thus dividing into two parts the remainder obtained by subtracting the simple reaction-time from the whole time including both discernment and choice-estimated the perceptiontime for light, of B. at $0.030 \mathrm{sec}$, and of himself at 0.050 . It took longer, of course, to discern between two colors. Between red and blue discernment required about 0.038 sec. for B., and 0.054 for $\mathrm{C}$. If one color among ten was to be discerned, the time rose to 0.105 for B., and 0.117 for C. The time required to discern one letter from all the others was found to vary somewhat for the different letters; but the legibility, or comparative accuracy of the quick discernment, varied still more. In this sense the letter $\mathrm{W}$ is about four times as "legible" as $\mathrm{E}$. The discernment-time for a simple picture of a familiar object was found to be about the same as that for a color, and shorter than that for a letter or a word.

$\S 19$. We have already seen (p. 480) that Donders assigned a little less time $(0.036 \mathrm{sec}$.) to the operation of will in setting free the required impulse when a dilemma was presented to it than is required for discernment between two objects of the visual sense. The duration of "will-time" (the third element of psycho-physical time) has since been investigated at greater length by Friedrich, Buccola, Merkel, and others. The first of these experimenters

\footnotetext{
${ }^{1}$ Tigerstedt, in Zeitschrift f. Biologie, 1883, XIX., p. 42 f.

'See Mind, July, 1886.
} 
determined the time required for simple choice by finding how much the reaction-time is increased when the subject of experiment must decide whether to react or not. ${ }^{1}$ For example, let the sensations of black and white follow each other in unknown order, it being understood that only white is to be followed by reaction. The interval for simple choice was thus found by Friedrich to have a mean value of $0.152 \mathrm{sec}$. for W., 0.184 for T., and 0.183 for himself ; it lay, therefore, between $\frac{1}{8}$ and $\frac{1}{5}$ sec. If choice was required, however, between the two hands-reaction with one hand being the sequence of the appearance of one color, and with the other hand of the appearance of the other color-the interval assigned to this element of psycho-physical time increased to 0.188 sec. for W., 0.330 for T., and 0.287 for $\mathrm{F}^{2}$

Merkel ${ }^{3}$ has perhaps investigated most thoroughly the timerelations of activities of the will. The question he proposes for answer is the following: How long does it take, under different circumstances, to set free a voluntary impulse? He assumes that, in all cases where reaction follows discernment between two or more impressions, some "will-time" is present, although the amount of this time may become so minute as to escape detection. The method of Merkel was suggested by Wundt. The simple reaction-time $(R)$, or time required when the nature of the stimulus is known and the mode of reaction fixed the same for all cases, is first found. The reaction-time required to discern clearly one of two or more impressions, and announce the fact in some one way previously determined upon $(R d)$, is next found. Finally, the reaction-time is found for cases where there is involved, in addition to discernment, a choice between one or more ways of reacting, or between reacting and not reacting $(R d w)$. The difference $R d-R$ is then held to give the "discernment-time;" the difference $R d w-R d$ is held to give the "will-time." It is with the latter that we are now concerned. For his experiments Merkel used figures of about $13 \mathrm{~mm}$. altitude placed $250 \mathrm{~mm}$. distant from the eye. To determine the reaction-time, including discernment $(R d)$, but not including choice, reaction is ordered invariably with the same finger. To determine reaction-time, including choice $(R d w)$, reaction is ordered with some one finger of either hand previously assigned to each number. Will-time is then found by subtracting the reactiontime in the former case from that in the latter $(R d w-R d)$. The

' Friedrich, in Wundt's Philosoph. Studien, I., heft i., p. $57 \mathrm{f}$.

: Only a few experiments of this kind were tried; the numbers are those given by Merkel in his article.

${ }^{3}$ Article in Wundt's Philosoph. Studien, II., heft i., pp. 73-127. 
results showed that while the simple reaction-time for the different fingers of both hands does not differ greatly, the difference in willtime for the different fingers is much more marked. The latter difference is greater among the fingers of the right than of the left hand. For ten persons experimented upon, the mean interval. required for setting free a definite reaction, with a choice between two possible courses, varied from 0.024 to $0.155 \mathrm{sec}$. This interval increases for every additional course possible until, in case the subject of the experiment is required to select one of his ten fingers with which to react on receiving an impression corresponding to that finger, the will-time becomes 0.298 to $0.448 \mathrm{sec}$.

Very interesting individual differences in the speed of decision attained under different conditions of complexity are brought out by Merkel's experiments. 'This fact may be shown by plotting the curve of the will-time of each individual. In general, it was found that the individual differences increased as the complexity of the choice required was increased from one to five places, and that they then fell off, being least at nine or ten places. That is to say, different individuals differ much more markedly in the speed with which they can choose one of two or five than one of nine or ten different possible ways of reaction. Merkel's value for will-time when the choice lies between two courses ( $R w=0.024$ to $0.155 \mathrm{sec}$.) may profitably be compared with that given by Buccola for choice between motion and rest with discernment of color-tone and locality $(0.028 \mathrm{sec}$. and 0.066 sec. respectively), or with that given by Tischer ${ }^{1}$ for choice between motion and rest (for nine persons, 0.052 to $0.179 \mathrm{sec}$.) or for choice between two symmetrical motions $(0.033$ to $0.179 \mathrm{sec}$.).

$\S 20$. A careful survey of the statistics and discussions furnished by different experimenters shows that it is not as yet possible to analyze with perfect confidence the different elements of psychophysical time. Wundt seems justified in holding that will-time is necessarily involved in the choice between motion and rest. But, on the other hand, von Kries and Auerbach appear to have reduced this time almost or quite to zero, by practice and by arranging their experiments under the most favorable conditions. By eliminating will-time they have, perhaps, found about what is the least possible interval required for simple acts of discernment. There is other evidence (from the phenomena of rhythm, etc.), however, to indicate that successive acts of discernment may attain a higher rate of speed than is possible for successive acts of will.

\&21. Investigation has also been directed toward determining how far series of events in consciousness correspond, as regards ${ }^{1}$ See Wundt's Philosoph. Studien, I., heft iv., p. $533 \mathrm{f}$. 
time, to the series of excitations which occasion them. Something may thus be accomplished toward fixing the time-rate of consciousness, as well as the interval which it is possible for the mind to appreciate with the nearest approach to perfect accuracy. In all the - preceding experiments the subject of them is uniformly aware that a pause, as it were, takes place between the excitation and the reaction; this pause he is able to estimate with much accuracy, and so to tell whether his effort to react promptly has been more or less successful. Exner ${ }^{1}$ states that in 39 cases of reaction from eye to foot, which had a mean of 0.184 sec., the reaction was always felt (with a single exception) to be "too slow" when it reached 0.1994 sec., and pronounced "very good" when it fell below $0.1781 \mathrm{sec}$.; its time was therefore estimated within about 0.01 sec. There is abundant proof, however, that the speed and duration of our sensations, as estimated in consciousness, do not precisely correspond with the series of stimulations of the organ of sense. Indeed, under certain circumstances very remarkable errors may occur in our estimate of both the rate and the interval of our mental acts.

To show the fact and amount of the error which takes place when we are called to intercalate an excitation of any kind in a series of impressions, Wund $t^{2}$ devised the following experiment: An indicator is kept moving at a uniform rate over a graduated scale, and so situated that the place of the needle can be clearly seen at each instant of time. The action of the same clock which moves the needle causes a sound at any moment, but in such a way that the subject of experiment does not know when to expect it. With what position of the needle, now, will the sensation of sound be combined? Will the sound be heard exactly when it occurs as indicated by the needle; or later than its real time ("positive" lengthening); or earlier than its real time ("negative" lengthening)? The result shows that one rarely hears the sound without either positive or negative displacement of it ; but most frequently the lengthening is negative-that is, one believes one hears the sound before it really occurs as measured by the indicator.

$\S 22$. Vierordt, " after experimenting upon our power to reproduce the interval as heard between two sensations of noise, concluded that very small intervals are regularly overestimated and greater ones underestimated. The minimum of error in estimating intervals-the duration that corresponds most perfectly in our consciousness to the real duration as measured by objective methods

'Hermann's Handb. d. Physiol., II., ii., p. $273 \mathrm{f}$.

${ }^{2}$ Physiolog. Psychologie, ii., p. $264 \mathrm{f}$.

${ }^{3}$ Der Zeitsinn. Tübingen, 1868. 
-he placed at 1-1.5 sec. More recently (1881) Kollert ' published the results of experiments instituted with a view to determine the accuracy of our sense of time. Suppose that one metronome is marking off time, by the sound it makes, with a normal interval $=\mathrm{t}$. Another metronome is at the same time at work with an interval $=9$, that may be made to vary with different experiments, and that is set as either equal to or slightly greater or less than $t$. Let $T=$ the time in our consciousness which is equivalent to $t$, and $\Delta=\mathbf{a}$ constant representing the mean error which is made in estimating the relations of the two intervals ( $t$ and 9 ); that is, $\Delta=$ $t-9$. Let $\vartheta_{1}=$ the intervals of consciousness just observably smaller, and $9_{2}=$ those just observably greater, than the normal time (t). Then the following table shows how $\Delta$ varies as $t$ varies; $d_{1}$ giving the experiments in which the interval of the variable metronome was smaller, and $d_{2}$ those in which it was greater, than $\mathrm{t}\left(\vartheta_{1}-\mathrm{t}=\mathrm{d}_{1}\right.$, and $\left.\vartheta_{2}-\mathrm{t}=\mathrm{d}_{2}\right)$.

\begin{tabular}{lrrr}
$\mathrm{t}=$ & $\mathrm{d}_{1}=$ & \multicolumn{1}{c}{$\mathrm{d}_{2}=$} & \multicolumn{1}{c}{$\Delta=$} \\
0.4 & -0.018 & +0.090 & +0.036 \\
0.5 & 0.044 & 0.098 & 0.026 \\
0.7 & 0.044 & 0.055 & 0.005 \\
0.8 & 0.073 & 0.060 & -0.006 \\
1.0 & 0.107 & 0.063 & 0.022 \\
1.2 & 0.206 & 0.074 & 0.066
\end{tabular}

It will be noticed that, according to these results, our sensitiveness to minute differences of time varies for different intervals so that it is greatest at $0.7-0.8 \mathrm{sec} .(\Delta=0$ at about $0.755 \mathrm{sec}$.); while it falls off quickly for intervals less than this, and more slowly for intervals longer than this. Kollert confirms Vierordt's statement that times above this most favorable time are estimated too small, and those below too large. He also concludes that, as the normal time increases, our sensitiveness to minute differences of diminution is lessened, while our sensitiveness to minute differences of increase grows greater.

The same method as that employed by Kollert has been applied by another observer ${ }^{2}$ to intervals longer than $0.4-1.5$ sec. Using intervals of 1.8-8.0 sec. for the normal time, the sensitiveness of our estimate of minute differences was still found to diminish as the normal time increases; thus the mean error of all the individuals engaged in the experiments $(\Delta \mathrm{m})$ increased from 0.0792 0.0879 sec. for intervals of $1.8-2.0 \mathrm{sec}$. to $0.5988 \mathrm{sec}$. for intervals

'Wundt's Philosoph. Studien, I., heft i., pp. $78 \mathrm{ff}$.

${ }^{2}$ Estel, in Wundt's Philosoph. Studien, II., heft i., pp. $37 \mathrm{ff}$. 
of 8.0 sec." $^{1}$ Estel reaches the conclusion that "our ideas of time, like our other sensations and ideas, are essentially conditioned upon past impressions belonging to the same domain of sense; and a short time makes the one next succeeding appear longer, while a long time shortens still more the succeeding shorter time." A more recent investigator ${ }^{2}$ places the interval which can be reproduced with greatest accuracy at $0.53-0.87$ sec.; but reaches the conclusion that, with all other intervals, an error is made which is plus for those above, and minus for those below, this so-called indifference-point. This conclusion would seem to need re-examination, since it is exactly the opposite of that of preceding investigators. 'The difference in result may, however, be due to difference in the method of experiment, which in the one case consisted in noticing the least observable difference in two series of intervals, and in the other consisted in catching, mentally, a given interval, retaining and reproducing it.

$\S 23$. In this connection should be mentioned the results of interesting "studies of rhythm" undertaken by G. Stanley Hall and J. Jastrow." These observers experimented to find the degree of accuracy with which successive clicks having a constant interval can be counted. It was found that persons most successful were able to count 2-4 clicks with perfect accuracy, when the interval between them was $0.0895 \mathrm{sec}$; but if this interval was diminished to 0.0523 sec., they could not be sure of more than two clicks. When the number of clicks was increased to 45 , with the longer of these intervals, the most successful estimates were 42 and 43 ; with the shorter interval, for the same actual number, the best estimate was 32 and the worst was 17 . The conclusion is thus reached that " counting requires a series of innervations, if not of actual muscular contractions," and that "attention discriminates sensation much more rapidly than the will can generate impulses." If, then, the interval between the acoustic sensations is less than the reduced reaction-interval (or time necessary for starting successive impulses of innervation) between ear and tongue, some of the sensations will drop out of consciousness as a result of the blending, as it were, of the later afferent with the earlier efferent stage of the complex process. The time-sense for series of mental phenomena is then different for different classes of these phenomena. The rate of sensation may considerably surpass the rate of motor impulses; "we do not realize how far the fastest counting falls short of the

${ }^{1}$ Estel, in Wundt's Philosoph. Studien, II., heft i., p. 43.

${ }^{2}$ L. 'T. Stevens, in Mind, October, 1886, pp. 393-408.

${ }^{3}$ Mind, January, 1886, pp. 55 ff. 
fastest hearing." The same observers found that the most rapid possible rate of pronouncing the names of letters was greater than that of counting them; the former being 0.248 sec. per letter for 50 letters, and the latter 0.283 sec. The reasons for this difference are apparently to be found in the fact that counting involves a severer strain on the attention and more complex processes of association and discernment in order to give to each number its right name and place in a series of numbers.

$\S 24$. We are thus led to consider certain researches for determining the reaction-time of yet more complex mental processes, such as involve reproduction of composite images of memory and the association of ideas. The mean duration of association-that is, "of the time which is required for the reproduction of an image of memory by some apperceived presentation of sense"-has been investigated at great length by Trautscholdt ${ }^{1}$ under the direction of Wundt. For purposes of experiment the following elaborate classification of the possible kinds of association was adopted. All associations are either (I.) External or indirect, or else (II.) Internal or direct. The former are such as are induced by the habit of perceiving objects together-simultaneously or successively-in space or time, without any interior relation between them; the latter are such as imply kinship in common properties or other inherent relations. External associations may, then, be either simultaneous, as in the case of the parts of a single presentation of sense or of the coexistence of independent presentations ; or they may be successive, as in the case of repeated impressions of sound, of sight, and of the other senses. Internal associations may either be such as involve the relation of ranking one mental object below or above another in terms of genus and species; or they may follow relations of co-ordination, as similar or contrasted ; or they may follow relations of dependence, as of cause and effect, means and end. To determine the time required for a "word-reaction" ( $W r)$, the experimenter spoke aloud some word at the instant that he pressed down his key; and the person reacting indicated the instant at which he apprehended the word. The time required for such reaction was found to have the following mean value for the four persons experimented upon : For W., 0.303 sec.; for B., 0.285 ; for H., 0.280 ; and for T., 0.173. ${ }^{2}$ The time required for the discernment of a single word is obtained by subtracting from these numbers the simple reaction-time $(R)$ for each individual $(W r-R)$. The result obtained

${ }^{1}$ Experimentelle Untersuchungen über d. Association d. Vorstellungen, in Wundt's Philosoph. Studien, I., heft ii., pp. 213-250.

"Philosoph. Studien, I., heft ii., p. 236. 
gare, for W., a "discernment-time" of 0.107 sec.; for B., of 0.177 ; for $\mathrm{H}$., of 0.137 ; and for $\mathrm{T}$., of 0.057 . In experiments to determine the duration of association, the person reacting does not press his key until the instant when the reproduction of some idea called forth by hearing the word spoken has fully taken place. For example, on hearing the word "zero" the idea of "infinity" may arise in the mind ; or " market-place" as associated with "market," "portfolio" with "letter," etc. The mean reaction-time for such association, both before and after subtracting the time for wordreaction alone, was as follows:

\begin{tabular}{c|c|c|c|c}
\hline & W. & B. & H. & T. \\
\hline Association-reaction $(A r) \ldots \ldots$ & 1.009 & 1.037 & 1.154 & 0.896 \\
Association-time $(A r-W r) \ldots$ & 0.706 & 0.752 & 0.874 & 0.723 \\
\hline
\end{tabular}

The mean duration of association for all the subjects of experiment (excluding one for special reasons) is, therefore, placed at 0.727 sec. It takes, that is to say, about $\frac{3}{4}$ of a second of psychophysical time to recall a familiar idea associated with a word we hear. This association-time was most prolonged in certain cases where the result may be looked upon as odd and unexpected, or where a pause would seem to have taken place through hesitation between several ideas simultaneously suggested. For example, the association-time which elapsed between the word "pious" and the idea "God-fearing" was 1.132 sec.; between "throne" and "ling," 1.437 ; between the German word "Sieg" and "a person of this name," 1.626 ; between "Karl" and "August," 1.662. The minimum of association-time was reached with ordinary words where the associated ideas were such as all individuals are likely to have in common. Thus, from "gold" to " silver" required only 0.402 sec.; from "storm" to " wind," 0.368 ; from "clear" to "dark," 0.507 ; from "north" to "south," 0.502 ; from "duty" to " right," 0.415 ; etc.

$\S 25$. Trautscholdt" also found-as we should expect-that judgments involving subsumption, or the definition of the word heard, required more time than mere association, when they were at all complex. But when very simple, the minimum time for such judgments was about that of association; and the mean value of "subsumption-time" (0.766) differed but very little from the mean value of "association-time." Thus it required 1.403 sec. to judge that a "ray" is a "form of the motion of light;" 2.023 to judge

' Philosoph. Studien, I, heft ii., p. 245 f. 
that "fame" is a "form of the ascription of praise ;" and 1.899 to judge that "art" is an "æsthetic activity" of man ; but only 0.391 sec. to recognize a "mast" as a "part of a ship," and 0.469 to identify "egro" and "cell."

The time necessary for multiplying, in the head, two numbers of one figure each has also been investigated by von Vintschgau. ${ }^{1}$ The psycho-physical processes involved in this achievement are necessarily some what complex. The influence of association must vary largely, according to the number of times we have previously made the same or any very similar calculation. For example, the whole process involved in answering the question, How much is $1 \times 1$ ? is different from that involved when the numbers are $8 \times 12$; much more different when they are quite unfamiliar-as, e.g., $76 \times 89$. The order of the numbers is not indifferent; as a rule, reaction is quicker and more correct when the smaller number precedes. The mean reaction-time for multiplying two numbers (1 to 10) was 0.211 sec. for $L$., 0.207 for $P$., and 0.259 for V., when reproduction was with the finger; and 0.200 for L., 0.252 for P., 0.248 for V., when reproduction was with the lip. Of this, 0.049 for $\mathrm{L}$. reproducing with the finger, and 0.096 with the lip; 0.051 for $P$. with the finger, and 0.082 with the lip; and 0.098 for $\mathrm{V}$. with the finger, and 0.087 with the lip-are calculated to have been due to the cerebral processes involved in the calculation. As might be expected, any great increase in the speed was found to be accompanied by an increase in the number of mistakes.

Cattell, ${ }^{2}$ after objecting on good grounds that the results of the laboratory are always too artificial and often too incorrect to "give the time it takes a man to perceive, to will, and to think," attempted to do away with "involved methods and complicated apparatus," and in simpler fashion determine the time we usually require to see and name an object, such as a letter or a color. $\mathrm{He}$ concludes that to see and name letters requires from $\frac{1}{3}$ to $\frac{1}{5} \mathrm{sec}$. for each letter, and to see and name words that do not make sentences requires $\frac{1}{4}$ to $\frac{1}{2}$ sec. When the words are connected into sentences it requires only about one-half as much time to name them - the rate at which one can read varying from 0.138 to 0.484 sec. for each word, according to one's degree of familiarity with the language. Single letters can be named more rapidly when several are in view at the same time; nearly all persons are helped by having as many as three, and most persons by having as many as four

${ }^{1}$ See Pflüger's Archiv, xxxvii., pp. $127 \mathrm{ff}$.

'See Mind, January, 1886, p. 63 f.; and Wundt's Philosoph. Studien, II., heft iv., pp. $635 \mathrm{ff}$. 
or five, letters in view at the same time. That is to say (using Wundt's figure of speech), the time of perception, or field of consciousness, covers the time of apperception, or clear spot of consciousness; while one presentation of sense is at the focus of consciousness, several others may be coming toward the focus from the background of consciousness. The second letter in view shortens the time of apperception about $\frac{1}{4} \overline{0} ;$ the third, $\frac{1}{60} ;$ the fourth, Ito

$\$ 26$. Finally, the closing remark of the foregoing paragraph introduces the inquiry into the "circuit of conscionsness"-that is, the number of impressions which can exist within the field of conscious perception at any one time? This question has been much debated on abstract or metaphysical grounds touching the nature of the soul. Since the soul is one and simple-it has been claimedthere can be before it but one object at the same instant of time; but since all its knowledge is relative, the claim las also been made that at least two impressions are always contemporaneous in consciousness. Hamilton,' on the contrary, concluded, from observing his own mental activities, that the circuit of cousciousness could embrace as many as six or seven distinct simultaneous impressions. It is evident that only an appeal to facts can decide such a dispute; it is also evident that the manner of appeal should be more precise and scientific than the one proposed by Hamilton.

Experiments have therefore been instituted to determine the "circuit of consciousness," by finding how many regularly recurrent successive impressions of sound, for example, can be so far united into one mental image as to have their likeness or unlikeness to another similar series clearly discerned. The method of experiment employed by Dietze ${ }^{2}$ was the following: The stroke of a pendulum, heard at regular intervals, was employed as the stimulus. After a single stroke as a warning, a series of successive strokes was given, which was begun and ended by the sounding of a clockbell simultaneously with a stroke of the pendulum. Arother series of strokes of the pendulum followed immediately upon the sound which announced that the first series was closed. The second series was ended by simply stopping the pendulum. A fixed number of strokes constituted the first series; a variable number (either one more or one less than the first series) constituted the second. The question proposed for answer was: How many impressions of sound can be received in the first series and the relation of the second series to the first (as equal, greater, or less) be accurately discerned

' Lectures on Metaphysics, p. 165. f., and elsewhere. Boston, 1860.

${ }^{2}$ Article in Philosoph., Studien, II., heft iii., pp. $362 \mathrm{ff}$. 
without counting? The effect upon the subject of such an experiment may be described as that of sending through the focus of consciousness a train of impressions, in regular succession, from the obscure regions of perception at which they enter to the obscure regions at which they depart from the field of consciousness. An image of the whole field of consciousness, with the line of march of the impressions, must be formed in order accurately to compare two series like those described. The result of such experiments showed that the number of successive impressions which can be comprised within the circuit of consciousness depends upon the rate with which they succeed each other. The most favorable interval was found to be $0.2-0.3 \mathrm{sec}$; ; with this interval most of the subjects of experiment attained a high degree of accuracy for even as many as ten or twelve impressions.' Individual differences were marked, however; thus, for one subject, the time of 0.21 sec. interval was too great for the maximum of accuracy, and $2.0 \mathrm{sec}$. was so large as to prevent his having any satisfactory apperception of even a single impression. The manner of uniting the impressions was also found to have a great effect upon the circuit of consciousness. When the process of apprehension was allowed to have a rhythmic form of grouping the impressions, the number possible in a single field of consciousness was increased. Without grouping, 16 was the maximum even number, 15 the maximum odd number, attained. Rhythmic grouping raised this number to 40 , or a little more, as the maximum even number, and to 35,37 , and 39 as the maximum odd numbers. It is possible, then, to apprehend a larger even number than odd number of impressions in a single circuit of consciousness. Certain numbers, perhaps those most familiarly grouped in experience, seem also to have the preference over others.

\$27. In all experiments to determine the time-relations of mental phenomena, the effects of practice and attention in diminishing psycho-physical time, and of fatigue to increase it, are made apparent. Certain special experiments have also been instituted to show how illness, old age, and drugs operate upon the speed of psycho-physical time. Thus Merkel ${ }^{2}$ found that the will-time necessary for choice between two motions was reduced by practice, for three subjects of experiment, from 0.080 sec. to 0.050 , from 0.097 to 0.0535 , and from 0.098 to 0.062 , respectively. For choice among

${ }^{1}$ See Dietze's statement, Philosoph. Studien, III, heft iii., p. 386, and a note, p. 384, correcting the erronous conclusion drawn by M. Ribot (German Psychology of To-day, p. 278, New York, 18\$6).

"Wundt's Philosoph. Studien, II., heft i., p. $110 \mathrm{f}$. 
five and ten possible motions, the effect of practice was yet more marked : thus, with fire possible choices, the will-time of one person was reduced by practice from 0.239 sec. to 0.083 ; and of another, with ten possible choices, from 0.358 to 0.094 . For each single day's series of experiments, the time diminished faster at first than subsequently; but, in many cases, more distinctly on the second than on the first day of experiment. Kries and Auerbach ${ }^{1}$ discovered that, after a short time, further practice has no influence on sim. ple reaction-time; but some practice is necessary to give any reliable value to such time. The effect of practice on discernment-time is very different. In the experiments upon the localization of tactile sensations, the discernment-time for $A$. was at first as great as 0.064 sec., or eren 0.117 , but afterward fell to a mean of 0.021 . Discernmenttime continues to decrease by practice, after all diminution of simple reaction-time has ceased; it is also transferable to other regions of the same sense. Trautscholdt ${ }^{2}$ found that practice for fourteen days reduced the "word-reaction-time" of one subject from 0.309 sec. to 0.149 ; association-time was found to be sensitive to practice in a much smaller degree. The effect of attention must also always be taken into account. It was, apparently, by guarding this carefully, and by practice, that Kries and Auerbach succeeded in obtaining such small values for discernment-time. The effect of distracting the attention was observed by Wundt, ${ }^{3}$ who introduced disturbing sensations of the same or of a different sense into series of regularly recurring impressions. Thus, the mean reaction-time for a weak impression of sound was lengthened from 0.189 to 0.313 sec., by a disturbing noise ; and for a strong impression of sound, from 0.158 to $0.203 \mathrm{sec}$. The mean reaction-time for sight of an electric spark was increased from 0.222 to 0.300 by a disturbing noise occurring simultaneously.

All the experiments also make obvious the great influence of individual peculiarities. But this influence may not be in the direction in which it would most readily be supposed to lie. For example, Exner ${ }^{4}$ found that, of two young men, one of whom had a very lively temperament and the other not, the former had much the longer simple reaction-time $(0.3311$ sec. as compared with 0.1337$)$. The reaction-time of a man of seventy-seven, taken from the almshouse, was at first 0.9952 sec. ; this was, however, reduced by practice to $0.1866 \mathrm{sec}$. The enervation produced by a hot summer's day, or

${ }^{1}$ Archiv f. Anat. u. Physiol., Physiolog. Abth., 1877, p 361 f.

'Wundt's Philosoph. Studien, I., heft ii., p $237 \mathrm{f}$.

${ }^{3}$ Physiolog. Psychologie, ii., p. 243.

4 Hermann's Handb. d. Physiol., II., ii., p. 268. 
the exhaustion of a sleepless night, or bad news, etc., increases the reaction-time. A small quantity of wine, slowly drunk, decreased the reaction-time; but a larger quantity increased it from 0.1904 sec. to 0.2969 , although the subject of the experiment considered himself to be reacting more promptly than usual. Coffee begins to decrease the reaction-time at 20-25 minutes after it is taken, and continues this effect for about 2 hours. Subcutaneous injections of morphine delay ${ }^{1}$ it; but this effect does not last long unless the injection is repeated. Obersteiner found that a subject whose reaction-time in the first stage of paralysis was 0.166 sec., gradually lost control of himself until, in the last stages when experiment was possible, the interval was $0.281-0.753$. Buccola, who has experimented upon idiots, imbeciles, epileptics, etc., finds that the duration of perception is lengthened in all these cases, with the exception of some forms of abnormal excitement.

$\S 28$. On summing up the results of all the experiments hitherto made in psychometry, we can only reiterate what we began by saying: Experimental research does not explain the origin or nature of our idea of time and its relations, nor has it succeeded in establishing many new principles of great moment for psychology. It is, however, a vigorous and promising branch of psycho-physical study. It has placed upon a scientific basis, and defined in accurate mathematical terms, many of our ordinary impressions as to the time-relations of mental phenomena. The attempt to analyze psycho-physical time seems to show that its various elements of simple perception, apperception, or clear discernment, and volition, occur in the order named, and yet ordinarily overlap each other, as it were. Practice and attention, under the most favorable circumstances, may reduce either one nearly to zero. In this way, simple reaction-time becomes most nearly equal to purely physiological or reflex time; reaction-time, with discernment, is almost reduced to simple perception-time; and the duration required by will-time for solving a dilemma is wellnigh eliminated.

Conclusions as to the existence and intercerebral relations of nervous centres of apperception and volition would be premature, and probably misleading, in the present state of this science. Nor have we as large hopes as to its ability "to solve many of the old problems" in the future as have been expressed by some of its enthusiastic students.

'See von Vintschgau and Dietl, in Pflüger's Archiv, xvi., pp. $316 \mathrm{ff}$; and Exner, in Hermann's Handb. d. Physiol., II., ii., p. 270 f. 


\section{CHAPTER IX.}

\section{FEELINGS AND MOTIONS.}

\& 1. From this point onward the study of Physiological Psychology is compelled to content itself with opinions much more indefinite and uncertain even than those to which we have already become accustomed. Theories of the localization of cerebral function, of the quantity and quality of sensations and their combination into presentations of sense, and of the time-relations of mental phenomena, admit to a considerable extent of experimental tests. But the feelings and their physical basis elude the efforts made to subject them to the conditions of a strictly scientific investigation. The same complaint may be justified concerning all opinions as to the physical basis of the higher intellectual operations, and as to the effect of age, temperament, sex, and race, upon the character and development of the mind. No attempt whatever will be made to conceal the meagre, obscure, and doubtful character of the evidence upon which our conjectures must be based. Indeed, on these matters nothing but the greatest caution is fitted to inspire confidence; the supreme wisdom is not iufrequently a frank confession of ignorance or uncertainty.

About our "Feelings" so-called-their nature, origin, relation to a physical basis and to sensations and ideas-we know remarkably little. Nor has any classification of the feelings hitherto been made which is entitled to command general assent. The reason for this fact is not difficult to discover. By their rery nature, the phenomena are obscure, indefinite, and yet extremely variable and multiform. They are also connected with our sensations and ideas in such a way as to make all separation in fact quite impossible. The psychology of the feelings, as studied from the introspective point of view, has therefore always been peculiarly unproductive of assured results. The fact that their physiological conditions are laid so largely in obscure, rapid, and infinitely varied changes within the central organs, such as cannot be either directly observed or indirectly subjected to experimentation, increases the difficulties of the subject. What is the nature of feeling? How do 
the different feelings differ, and what elements have they in common? Under what conditions do we have sensuous feelings; and under what conditions are these feelings pleasant or unpleasant? Is feeling ever perfectly indifferent; is there a zero-point of feeling? How are the feelings related to the quality and intensity of physical stimuli? What is the physiological basis (if any exist) of the higher rsthetic, moral, and religious feelings?" ${ }^{2}$ These and other similar questions may be asked of psycho-physical science with little satisfactory result.

\& 2. Many diverse views have been held as to the essential $n a-$ ture of feeling. These views may, for the most part, however, be classified under two heads: They are either such as emphasize the dependence of the feelings on bodily conditions, and so resort to physiological explanation of their origin and nature; or else they are such as emphasize their dependence upon relations that obtain among the so-called "ideas," or purely mental states and products of the mind. The extreme form of one of these two theories holds that feeling is always merely a consciousness of a certain condition of the nervous elements. The extreme form of the other leads to the position from which all feeling is regarded as a sort of secondary consciousness of the "furtherance " or " hindrance" of one idea by another. The principal real ground for the former theory lies in the fact that certain conditions of the nervous elements under stimulation are, as a rule, followed by painful, and certain others by pleasurable, feeling. The latter theory is based solely upon the truth that certain mental states called "ideas" are, as a rule, accompanied or followed by corresponding modes of being affected which hare the characteristic tone of all feeling-that is, are either agreeable or disagreeable. Neither of these views, however, serves to define the essential nature of feeling, since to feel is as simple and fundamental an operation of mind as it is to know. Feeling can never be stated in terms of knowledge. Inasmuch, then, as all definition is only the expression of an elaborate and complex form of knowledge, the nature of feeling is not capable of being defined; it must be felt. When, then, this nature is defined as consisting in some relation to physical sensation or to mental images, it is deprived of the very characteristic which makes it to be feeling rather than sensation or idea. Both theories, however, have succeeded in stating certain conditions or antecedents of the reaction of mind in the way of feeling.

$\S 3$. The foundations for a physiological theory of the feelings

${ }^{1}$ Comp. Horwicz, Psychologische Analysen, i., p. 21 f. 
were laid by Lotze, 'with that blending of scientific caution and psychological insight which characterize most of his work. He distinguished the feelings, as mental conditions of pain or pleasure, from sensations as indifferent elements of our percepts of things. Yet, in fact, sensations are always, or usually, colored with feeling; and analysis is therefore obliged here to distinguish in theory what coexists in fact. Feelings are of two kinds: "sensuous," as coming from bodily impressions; and "intellectual," as flowing from the relations of ideas. Pleasurable feelings always arise from the coincidence, and painful from the opposition, between the effects of the stimulus and any one of those conditions to which the regular expression of the bodily or spiritual life is attached. Yet even this statement is not true without making further explanations and limitations. For something bitter may be harmless, or even beneficial; and acetate of lead may be sweet and pleasant, but deadly. More precisely, then, "feeling is, in general, only the measure of the partial and momentary concord between the effect of the stimulus and the conditions of rital activity." ${ }^{2}$ Lotze was far too keen a psychologist, however, to suppose that in laying down this law he was explaining the nature of feeling as a secondary and derived form of consciousness. He has himself vindicated its right to be regarded as primitive, and not deducible from either sensations or ideas." But even in the way in which he understood his own theory, much doubt may be thrown upon its truth.

It is admitted by all that certain intensities of nearly all forms of stimuli are both productive of painful feeling and also antagonistic to the vital conditions of the organism. Undoubtedly, also, suffering is both an indication and a cause of abnormal and injurions action of the nervous mechanism. But that the feeling of pain measures the degree of this antagonism, or that everything found at the time disagreeable is in any degree demonstrably opposed to the vital welfare of the organism, cannot be assumed; and the alleged law even seems incompatible with the individual peculiarities which characterize what is agreeable or disagreeable to the senses of different persons. The excessive stimulus of the surgeon's knife is not rendered any more really in accord with the conditions of the vital activity of the organ to which it is applied by the fact that anrsthetics prevent the pain which would otherwise result. An excessive and immediately injurious stimulation of considerable portions of the body may be accompanied by a large amount of positive

\footnotetext{
${ }^{1}$ Medicin. Psychologie, pp. $233 \mathrm{ff}$.

2 Ibid.

${ }^{3}$ See Metaphysic, p. 474, Oxford, 1884; and Microcosmus, i. p. 177 f., Edinburgh, 1885.
} 
pleasure or, at most, with very little pain, while a small and quite harmless degree of another kind of stimulation may result in great discomfort. Attention, association, and control of the will have also much to do with determining the subjective state which is connected with any given relation between the effect of the stimulus and the conditions of vital activity.

§ 4. More recent attempts to give a general physiological law for the phenomena of pleasurable and painful feeling can scarcely be said to be any more satisfactory. It is true, as Bain ${ }^{2}$ declares, that "a very considerable number of the facts may be brought under the following principle, namely, that states of pleasure are connected with an increase, and states of pain with an abatement, of some, or all, of the vital functions." But other facts in no small number cannot be brought under this principle. It is not a difficult task for the physician to abate all the vital functions of the patient, even down to or beyond the line of danger, with the immediate result of producing pleasure rather than pain. After objecting to Bain's statement as being "too vague," etc., Grant Allen ${ }^{2}$ declares the true principle of connection to be the following: "Pleasure is the concomitant of the healthy action of any or all of the organs or members supplied with afferent cerebro-spinal nerres, to an extent not exceeding the ordinary powers of reparation possessed by the system." Esthetic pleasure he prorisionally defines as "the subjective concomitant of the normal amount of activity, not directly connected with life-serving function, in the peripheral end-organs of the cerebro-spinal nervous system." ${ }^{3}$ Now, that pleasure is the reflex of healthy and unimpeded activity is an old psychological truism; and that we are dependent upon impulses propagated in the sensory nerves of the cerebro-spinal system for sensations, pleasureable or painful, of muscular, organic, or more special sort, scarcely needs statement as a newly discovered law of "physiological resthetics." Nothing, howerer, could well be more "vague" than the limit fixed by the words "to an extent not exceeding the ordinary powers of reparation possessed by the system." Does the man whose powers of nerrous reparation are extraordinarily great necessarily find his quinine any the pleasanter? The statement that pain is a warning of danger from excessive or abnormal activity of the nervous system must, of course, be accepted as summing up a large number of facts; but there are other facts not easily brought under such statement. Moreorer, until we have some objec-

${ }^{1}$ The Senses and the Intellect, p. $281 \mathrm{f}$.

'Physiological Esthetics, London, 1877, p. 21.

3 Ibid., p. 34. 
tive means of determining what is the "normal amount" of function in any tissue, the alleged law that pleasure is "the subjectire concomitant" of such amount is of little or no value. The whole subject is left in that indefinite condition which invites us, on the one hand, to consider pain as the proof that the function of the nerrous system which occasions it is destructive; and then tells us, on the other hand, that the essence of the pain is in its being the subjective concomitant of such function.

$\S 5$. But if purely physiological theories of feeling do not succeed in defining its uature, or in stating the relation between the action of the nerves and the pleasureable or painful tone of the feeling, the success of the second class of theories is no greater. Of this class the views of Nahlowsky, ${ }^{3}$ as set forth in his interesting monograph, are perhaps the best example. This author begins by drawing a sharp distinction between sensation and feeling. The "tone" of sensations as pleasant or unpleasant he would not call by the term "feeling;" such tone is rather that which gives us "the how" " "Wie") of the sensation, and depends for its pleasant, or unpleasant character upon whether the effect of the stimulus furthers or inhibits the functions of the vegetative life. Even those states of consciousness which are constituted from various elements due to stimulations of the nerves at various quarters not definitely localized, and ordinarily called "common feeling," Nahlow. sky would define as "common sensations." Pain also is a sensation and not a feeling. But feeling, according to this author, is neither tone nor quality of sensation ; though it may be an elevation or depression of mind produced indirectly by the sensations. Feelings, properly characterized, comprise all the conditions resulting from the simultaneous existence of ideas in the mind, which either support or interfere with each other. In the former case, they are agreeable; in the latter, disagreeable. They are, then, secondary conditions of mind, dependent on ideas, recognized as not of bodily origin, but as having a content of a mental rather than physical order. Hunger, thirst, weariness, shivering, etc., are sensations ; sympathy love, gratitude, reverence, admiration, etc., are feelings. Accordingly, feeling is defined as " the immediate consciousness of the momentary rising or depression of one's own psychical activity" (that is, of the movement of the ideas). Even to the affections this theory would deny a place among the kinds of feeling-for the former spring from the latter under the mediating influence of organic effects.

The foregoing view of the nature of feeling is adhered to sub-

'Das Gefühlsleben, in seiner wesentlichsten Erscheinungen u. Bezügen, 2d ed. Leipzig, 1884. 
stantially by many others, especially by the followers of Herbart. Thus Drbal ${ }^{1}$ holds that feelings are not primitive states of mind, but result from the reciprocal action of ideas-if this ideating activity is one of reciprocal inhibition, the feeling which is the becoming conscious of the inhibition is unpleasant ; if the activity is one of reciprocal combination, the conscious feeling of this fact is pleasant. Feeling in general is therefore the immediate consciousness of the rising or falling of one's power of ideating, as it were. Beneke, ${ }^{2}$ also, considers that two mental images excited belong to every feeling; of these, one is that which is felt, and the other is one "against" which the first is felt. Feeling and no-feeling, or this or that feeling, can therefore attach itself to one and the same image. Volkmann von Volkmar, in his great work, ${ }^{3}$ considers feeling as the consciousness of the process of ideation itself as distinguished from consciousness of this or that idea, and it is conditioned upou some resistance being offered to the process. Feeling is, then, no one proper idea, to be placed in conjunction or classed with others. It is rather a becoming conscious of the degree of tension, as it were, which characterizes the process of ideation at each particular moment. The condition of the origin of a feeling is, then, the existence of two simultaneous opposed ideas. Their coexistence occasions a state of tension ("Spannung"), as it were, and this state gives way as one idea triumphs over the other. The type of simple feeling may be illustrated by the condition in which the mind finds itself when listening to harmonious or discordant musical sounds.

$\S 6$. The theory which makes feeling a derived consciousness dependent upon the relations of the ideas as furthering or checking each other is unsatisfactory. It cannot be admitted, to begin with, that feeling is a secondary or derived form of consciousness. No form of mental activity is more primitive and unanalyzable than feeling; none is earlier in the development of mental life." Before the infant has localized the different sensations, and combined them into percepts of the different parts of its own organism, the consciousness of being affected in a given way, either pleasurable or not, must predominate. Other forms of feeling-of desire, uneasiness, comfort, etc.-are inseparably connected with its first states of consciousness; they belong to its inherited impulses and

${ }^{1}$ Lehrbuch d. empirischen Psychologie, 2d ed., pp. 200 ff: Wien, 1875.

${ }^{2}$ Lehrbuch d. Psychologie als Naturwissenschaft, pp. 170 ff. Berlin, $187 \%$.

${ }^{3}$ Lehrbuch d. Psychologie, II., pp. $298 \mathrm{ff}$.

+ This view of the feelings is maintained by Horwicz, and developed at length, polemically, by Lotze (Horwicz, Psychologische Analysen, i., p. 168 f.; Lotze, Medicin. Psychologie, p. $235 \mathrm{f}$; and references already cited in note p. 500). 
instincts, and are only later definitely related to the appropriate ideas. The primary formation of self-consciousuess is quite as truly connected with self-feeling, pleasurable or painful, as with the process of ideation in constructing the concept of "me" and "not-me."

Moreover, although we are to distinguish sensation from feeling, we must regard the feeling which inseparably accompanies sensation as feeling, strictly speaking, and not as tone of sensation; or, in other words, the tone of every sensation, as either pleasurable or painful, is given to it by the feeling which accompanies and blends with it. The sensation, as having a certain quality, quantity, and locality, is capable of being built into a "Thing" which the mind perceives as vot-itself. But the feeling, the pleasurable or painful tone of the sensation, is always recognized as purely and simply a way in which the mind is affected. To refuse to speak of sensations and emotions, with all their complicated physical basis, as belonging at all in the realm of "feeling," is to restrict the use of the word unwarrantably. The Herbartian theory commits in this matter the mistake which it is guilty of committing repeatedly; it regards the "ideas" as realities that have in some sort a substantial existence, and can do something by way of furthering or hindering each other. But ideas are themselves nothing more than mental products that exist only when and so long as the mind acts with a definite degree and kind of energy. In determining the kind and degree of this ideating energy, the previous action and habit of the mind by way of feeling is quite as influential upon the mode of feeling as is the manner of its ideating energy. Finally, this theory wrecks itself upon the denial of all that which the physiological theory maintains and establishes. The two theories, then, supplement and correct each other; but even when combined they only tell us in part what are the physical and mental conditions under which feeling arises.

$\S 7$. The truth appears to be as follows: Feeling is an original and underived form of consciousness, or mode of the operation of conscious mind. It can neither be defined by, nor deduced from, sensation or ideation. To know what it is to feel, the highest intelligence of itself would be incapable. Such knowledge comes only from having felt. Feeling accompanies all mental experience, both that of sensation and of the higher intellectual processes. It undoubtedly has a certain physical basis; and certain laws may be stated which discover some of the relations that hold good between conditions of the nervous system and resulting conditions of feeling. Certain other laws may be laid down which partially define 
the relations existing between the purely intellectual and the feeling activities, or reactions, of mind. These two sets of laws give us the physical and the intellectual conditions of different tones of feeling respectively. But no one law has yet been discovered which corers all the facts of relation, either between feeling and the bodily states or between feeling and the ideas. Nor is it likely that any such law exists to be discorered. Manifold relations, as determined by heredity, individual peculiarities, association, attention, etc., always exist, and contribute to the complex result.

\& 8. The various attempts to establish fixed classes of the feelings can scarcely be pronounced more satisfactory than the attempts to define their nature. Very great difficulties stand in the way of such a classification, of which the following are most important: The phenomena are themselves very obscure, changeable, and multiform ; they are also inextricably associated with the phenomena of sensation and ideation. Moreover, the theory held by any inquirer as to the origin and nature of the feelings is pretty sure to determine his classification of them. For example, two before-mentioned authorities (Nahlowsky ${ }^{\prime}$ and Drbal ${ }^{2}$ ), as a result of the "ideational" theory, divide the feelings into (1) such as are dependent on the form of the course of ideas, sind (2) such as are conditioned by the content of the ideas. Besides these simple classes of feelings, one of these writers speaks of certain "mixed feelings," that are feelings of oscillation and change. Under the "formal" feelings, or such as" are dependent on the form of the course of representation, the other writer finds four classes-namely, $(a)$ feelings of expectation and impatience, $(b)$ of hope, anxiety, surprise, and doubt, $(c)$ of tedium, and $(d)$ refreshment and work-all according to the aspect of the ideas in time-form. Four kinds of "qualitative" feelings are also distinguished-these are the intellectual, the rsthetic, the moral, and the religious-according as the ideas exciting the feelings refer to truth, beauty, morality, or religion. But the foregoing attempts and all similar attempts at classifying the feelings lay a false emphasis upon the dependence of certain feelings on mental representation; they thus overlook all those considerations on which the physiological theories of feeling rightfully insist.

But, on the other hand, the classifications made under the influence of the physiological theory are even more unsatisfactory. In their desire to reduce all the phenomena of human feeling under some one physical "law," so called, they bring the higher forms of

1 Das Gefühlsleben, p. 44 f.

${ }^{2}$ Lehrbuch d. empir. Psychologie, p. 205 f. 
feeling into a much closer and more complete connection with the feelings of sensation than the facts will warrant. Thus, with Grant Allen, the resthetic feelings are "the cumulative effect of many infinitesimal physiological factors," " which differ from the pleasures and pains of sensation chiefly in the fact that the activity of the end-organs in them is "not directly connected with life-serving function ;" all the different tastes of different individuals, their varying "perceptions of beauty and ugliness," are then boldly stated to be "depending on the structural variations of the nervous system."

Horwicz's more profound theory as to the nature of feeling leads him to a more satisfactory classification of its forms. The variety of feeling, he holds, is dependent on the natural organic variety in the activities of the soul. ${ }^{3}$ Thus we derive (1) the sensuous feelings, or such as depend on the different qualities of the sensations of the special senses and of common feeling; (2) the cesthetic feelings, or those agreeable or disagreeable forms of consciousness which correspond to the mental images of perception and imagination; (3) the intellectual feelings, which correspond to the theoretic interests called out by the higher forms of thinking; (4) the moral feelings, or those which correspond to the relations of desire and will. The development of these feelings in varying relations to each other gives rise to various mixed or complex forms. Certain moods and characteristic affections result from the combined tone, color, and rhythm, of the simple feelings, and the strength with which the physical organism reacts. Higher feelings, or "feelings of feelings," unfold themselves; these are dependent upon the complex relations of society as organized in its several existing forms.

A recent writer in Mind, ${ }^{4}$ after criticising all previous attempts at classification of the feelings, proposes an exceedingly elaborate substitute for them all; but this substitute is so burdened with uncouth terminology, is founded on so many false or doubtful psychological assumptions, and involves so many artificial distinctions and cross divisions, that it is little likely to meet with general acceptance.

\$. Another and insuperable difficulty in the way of a strict classification of the feelings is the fact that they are actually, and as peculiar conditions of consciousness, unclassifiable. In other words, no principle of classification can be suggested which will undeniably apply to them all. For example, if we classify them

\footnotetext{
' Physiological Esthetics, p. 42.

- Psychologische Analysen, ii., p. 82 f. 4 Mercier, July and October, 1884, and January, 1885.

2 Ibid., p. 34.
} 
into pleasurable or painful, we indicate in this way only a quality of tone which itself constantly varies in dependence on more permanent characteristics. Besides, it is not easy to demonstrate that feelings are never indifferent (neither agreeable or disagreeable) in tone. If we classify according to the anatomical part or physiological function of the nervous system which chiefly gives conditions to the feelings, we can carry our classification only a little way without resorting to unwarrantable assumptions. Incleed, there are grounds for supposing that the feelings are of central origin-that is, have their physiological basis in those regions of the nervous system that have thus far almost wholly eluded scientific research. If we classify according to the relation of each feeling to other activities of the soul (as Horwicz does), we encounter the facts that sense, will, and intellect, doubtless all enter into all the activities connected with our developed feeling; but that the measure of the degree in which they are influential upon feeling is so uncertain and changeable as to render it unfit to serve as a basis for classification.

No hard and fixed line can be drawn about the different so-called classes of feelings. Feeling, with its color-tone of pain or pleasure, enters into all conscious life. The rsthetic feelings cannot be separated from the sensuous; for example, the feeling which accompanies the sensation of a musical chord, or of the color purple, may be classed under either head. Nor can the intellectual feelings be separated from the resthetic ; the perception of harmony of colors and sound is inseparably connected with æsthetic and sensuous feeling, and the latter is intensified or otherwise modified under the intellectual laws of contrast, change, habit, and higher association. Even the feelings which we call "moral," on account of their connection with will and desire, often have an indefinite part of them so combined with feelings located in the bodily organism, or so dependent on its functions for their quantity and quality, that a strict separation becomes impossible. Love is seldom or never so purely ideal as not plainly to involve in itself feeling of sensuous and rsthetic sort; hate not mixed with anger, and so supported on some elements of that physical basis which underlies the latter, is hard to discover in real life. All psychological analysis that would extend to the point of establishing fixed classes of the psychical activities is difficult; but in the special case of the feelings, the character of experience is such as to make strict classification impossible.

Accordingly, in treating of the feelings from the physiological point of view we shall content ourselves with selecting certain ex- 
amples from the current classes which admit of being thus treated most successfully. Such are, obviously, the so-called sensuous feelings, the so-called common feeling, certain resthetic feelings, certain of the feelings known as emotions or affections, and certain feelings connected with the functions of will-especially the feeling of effort.

$\S 10$. All feelings are characterized by tone, strength, rhythm, and content. " Their content is determined by the ideating activity with which they are directly connected, or to which they are related ; and this content may be simple, as is the case with the feeling connected with the presentation of a colored surface (for example, purple or green), or complex, as is the case with the sentiments of patriotism, loyalty, and religious derotion.

Feelings, like all other mental phenomena, occur under timeform; they are, in general, rhythmic in character, and change in respect to content, tone, and intensity, with a movement marked more or less distinctly by the quality of periodicity. Their rhythm, with respect to content, is, of course, determined by the recurrence of changes in the ideating activity as dependent especially upon attention and the laws of association. Feelings of sadness or joy, comfort or discomfort, may come around again in consciousness, as it were, according to the rhythmic movement of the sensations which occasion them. Sometimes an alternation of tone takes place, which carries the mind back and forth by the point of indifference (or lyppothetical zero-point of feeling) betreen agreeable and disagreeable sensations, or ideas of the same kind. Thus we are sometimes forced to say that we do not know whether a certain combination of colors, or quality of taste or smell, is pleasing to us or not ; in such a case feeling seems to more rhythmically back and forth between a slightly pronounced tone of pleasure and a slightly pronounced tone of pain.

The intensity, too, of feelings rises and falls alternately in dependence upon the rhythmic movement of the nervous processes and of the train of ideas. No feeling is kept at a long continuous level with respect to its vigor and pitch of strength. The law of quickly alternating exhaustion and repair of the nervous elements underlies, to a large extent, this rhythmic movement of the intensity of the feelings. This is one of many proofs which go to show that the conditions of the end-organs and of the central organs (comp. p. 108 f.) are determinative of the tone and strength of feeling. Even when we are strictly attending to our painful feeling, the toothache is not a perfectly uniform and steady strain; even when we are doing our best to abstract attention from the

' Comp. Volkmann ron Volkmar, Lehrb. d. Psychologie, II., p. 311 f. 
pain, we succeed only intermittently. But the course of the ideas must also be taken into account as influencing the rhythm of feeling. As our sensations or mental images become more clear and vivid, the feelings attached to them gather strength; as the former become more obscure and feeble, the feelings also die away in consciousness.

\$11. The tone of all feeling is either one of pleasure or of pain (using these words in their widest possible meaning). The feeling of pleasure and pain is probably the most general, most simple, and earliest psychical process. That almost all feelings are characterized by some positive tone-or, in other words, are not absolutely indifferent to us-there can be no question. Is it agreeable or disagreeable, at least in some slight degree and in some more or less indefinite manner? is an inquiry which we can pretty readily answer with respect to nearly all our sensations and ideas. The question has been debated, however, whether this is necessarily true of all our feelings. Is there any such thing as completely "neutral" feeling, or feeling that is in no respect or degree either agreeable or disagreeable to us? Neutral or indifferent feelings were recognized by Reid, but disputed by Hamilton. ' Bain asserts it as undoubted that "we may feel, and yet be neither pleased nor pained," and that "almost every pleasurable and painful sensation and emotion passes through a stage or moment of indifference." ${ }^{2}$ Wundt ${ }^{3}$ argues, on theoretical grounds, that pleasure and pain, as tones of feeling belonging to sensation, are conditions which may be regarded as on different sides of a zero-point, or point of indifference lying between them. It does not follow, however, that, because the mind passes in time from feeling of one positive tone (pleasure) to feeling of the opposite tone (pain), it must, therefore, at some instant be in a state of feeling that has no tone and lies between the two. The curve plotted to represent the rise and fall of feeling is a material line; it cannot be at one time below, and at another above, the abscissa-line, without at some single point (the zero-point) coinciding with it. But it does not follow that, because such a curve is a picture of the plienomena of fecling in one respect, it is so in all other respects. The question whether there is any zero-point to the tone of feeling can only be answered by an appeal to consciousness; and this answer, like all others given to similar appeals, is likely to contain dubious and conflicting elements. It is quite certain that one can pass from a high state of pleasure to

${ }^{1}$ Hamilton's Works of Thomas Reid, p. 311. Edinburgh, 1854.

2 The Emotions and the Will, p. 13.

${ }^{3}$ Physiolog. Psychologie, i., p. 465 f. 
one of intense pain without any interpolated neutral feeling. For example, if while one is viewing a beautiful landscape one is stung by hornets, the condition of quiet massive pleasure nay be converted into one of great physical suffering without any intervening feeling of indifference. We incline, then, to agree with Sully: in affirming that every feeling is either pleasurable or painful in some degree. "We apply the name 'feelings," says Lotze," "exclusively to states of pleasure and pain, in contrast with sensations as indifferent perceptions of a certain content."

\$12. Various questions may be raised as to the physical apparatus, the nerrous elements and processes, for pleasurnble or painful feeling, which cannot be answered satisfactorily. Are there special nervous elements-whether end-organs, or nerve-fibres, or nerve-tracts and centres in the central organs-which must be excited in order to give rise to the feeling of pain? If the apparatus for feeling is the same as that for the sensations to which the feeling gives its color-tone, do the feeling and the sensations imply different processes in these same elements as the physical basis on which they rest? Is not pleasure, rather, the result of a normal and moderate amount of process in these elements; and pain the result of a process in the same elements whose amount has been increased so as to be destructive or injurious to the tissue involved? Lotze ${ }^{3}$ raised these questions, and answered them with the opinion, somewhat doubtfully expressed, that sensation and feeling are due to two forms of processes in the same nerrous elements, and that there is no need of assuming special organs of feeling, whether peripheral or central. Probably the prevalent view hitherto has been, that the same apparatus of end-organs, conducting nervetracts, and central areas, which on moderate excitement produces the simple sensations of pressure or of temperature, or the more complex sensations of tickling, shuddering, etc., produces the feeling of pain when irritated with increased intensity. Such a view would apparently have also to hold that muscular sensations have the same physical apparatus as do feelings of muscular weariness or exhaustion ; and, perhaps, that cardialgia and hunger are due to modifications of the action of the same nerves of the stomach. But from the psychological point of view it is as certain that sensations of pressure or mere temperature are unlike the feeling of pleasure produced by gentle rubbing or by comfortable warmth, or the pain

${ }^{1}$ Outlines of Psychology, p. 449, New York, 1884; and comp. Volkmann ron Volkmar, Lehrb. d. Psychologie, II., p. $311 \mathrm{f}$.

' Outlines of Psychology, p. 73. Boston, 1886.

${ }^{3}$ See Medicin. Psychologie, pp. 245 ff. 
that comes from heavy pressure or burning, as it is that sensations of light are unlike those of musical tone.

Besides the obvious difference which the results of exciting it have in consciousness, there are other and physiological reasons for doubting the complete identity of the nervous apparatus of pleasurable and painful feeling with that of the sensations with which the feeling is allied. The facts upon which Schiff and others support the view that nerrous impulses resulting in pain travel by more or less distinct paths along the spinal cord have already been stated (Part I., chap. III., §32). The most recent experiments seem to show that the end-organs of temperature, pressure, and pain are locally separable in the different minute areas of the skin (Part II., chap. IV., \$ 21 f.). Pathological results indicating the same separation of the nervous elements of feeling also deserve a brief mention. In certain cases the sensibility of the skin to pain is lost (a condition called "analgesie" by Beau, and "analgie" by Lotze), while its sensibility to touch is not weakened or is even increased. The reverse condition also sometimes occurs. "Analgie," as occasioned by pathological states of the spinal cord due to lead-poisoning, was noticed in many cases by Beau. This loss of sensibility to pain can hardly be explained by any change in the activity of certain end-organs common both to touch and to painful feeling. What impairment of function could possibly result in destroying the sensitiveness to strong mechanical and thermic excitations, such as ordinarily occasion great pain, while the response by way of sensations of touch to much feebler excitations remains undiminished?

The same argument would appear decisive against identifying, locally, the central nervous processes which result in sensation with those which result in feeling. In certain stages of narcosis, produced by ether or chloroform, the patient is able to perceive the slightest contact with the skin, but feels no pain even when the same area is treated severely. Moreover, in some cases of tabes dorsalus, a constant difference seems to exist in the time at which the sensations of pressure and the feelings of pain, simultaneously excited at the endorgan, arise in the mind. If the patient is pricked with a needle, he will instantly feel the contact, and the pain only one to two seconds later.' The case of the eye, which responds with sensations of light and color when the optic nerve is moderately excited, and with the painful feeling of being blinded when the stimulus is increased, is not perfectly clear. For cases of amaurosis are on

${ }^{1}$ See Funke, in Hermann's Handb. d. Physiol., III., ii., p. 297 f.; such phenomena have been especially discussed by Osthoff, Die Verlangsamung $d$. Schmerzempfindung bei Tabes dorsalis, Erlangen, 1874. 
record where the painful feeling persisted after the eye had lost all power to distinguish light. It has been argued, therefore, that while the specific sensations of light and color are due to the irritation of the optic nerve, the excitement of feeling indicates a simultaneous irritation of part of the trigeminus.

We are compelled, then, to confess that the localizing of the nerrous apparatus, and the nature of the plyysiological processes which form the physical basis of painful and pleasurable feeling, require further investigation. The tendency of the evidence, however, is toward a theory which assigns to feeling a more or less separate mechanism of end-organs, conducting nerve-tracts, and central areas (or at least of nervous elements in the central areas). But how such a theory will reconcile itself with the other familiar facts which appear, obviously, to make the tone of feeling depend upon the degree of intensity which the nervous processes attain, it is impossible to predict.

\$13. One kind of feeling, which has the tone of pleasure or pain belonging to all feeling, is undoubtedly of central nerrous origin; this is the so-called sensus communis, or "common feeling." "Such feeling may have more or less of content of one kind or another, according to the state of perception and ideation with which it is combined. Nervous impulses of indefinite variety and the most manifold peripheral origin are constantly pouring in, as it were, upon the cerebral centres-each one contributing some element to the characteristic tone of consciousness. The resulting feelings are modes of our being affected which are not converted into definite presentations of sense, or referred to a particular part of our own bodies. The effect of changes in the minute blood-vessels and other capillaries about the nerve-endings, the presence of impurities in the blood, the condition of the lower cerebral centres, the action of the heart and lungs and other internal organs, and the connection of the sympathetic with the cerebro-spinal nervous system, are all felt in this way. Moreover, inasmuch as few (if any) sensations are without some tone of feeling, while many sensations are exceedingly heterogeneous in their elements, and not clearly referred to the place of their origin, a melange, as it were, of obscure bodily affections is readily formed.

Sensations in themselves heterogeneous may also be brought into a temporary relation by the partial identity of their source of excitation, and of the nervous connections in the central organs. It is also always a very important question, how the more obscure

' Comp. Funke, Der Tastsinn u. d. Gemeingefühle, in Hermann's Handb. d. Physiol., III., ii., pp. 289 ff. 
and mixed bodily feelings stand related to the mind's course of ideation, to attention, association, etc. This relation often determines whether such obscure impressions shall be definitely objectified or not; whether they shall not rather run together in the dark stream of common feeling. Let anyone suspend for an instant a train of interesting thought, which has up to the moment been interrupted only by certain obscure feelings of uneasiness, and such one will be: able instantly to select and localize in the cramped chest, or oppressed limbs, or tired organs of special sense, most of the sensations whose painful tone has thus colored the stream of common feeling. Separation from localized sensations is, then, the chief negative characteristic of common feeling. ${ }^{1}$ Under its different. principal forms we may distinguish different total results, according to the general relation in which the being aware merely that we are affected in an agreeable or disagreeable manner stands to the being aware of what affects us in this manner. Thus we sometimes feel well or ill, elevated or depressed, without ability to assign these feelings at all definitely to the physical organism, either as perceived or imaged, or to any reason in the train of ideas. At other times the general impression of being in the body, for some greater or less amount of either weal or woe, is emphatic; we feel ill all over, or seem to enjoy the coursing of the blood through every artery and vein, as though mentally present in the extended tissues.

$\S 14$. According to Stricker, ${ }^{2}$ information derived from the peripheral nerves consists of either sensations or feelings ; the latter. implies self-reference, which may be of two kinds. If this reference extends to the whole sensorium, and so to the whole organism, the feelings are called " common" or " collective;" under certain circumstances they appear as fixing the mood of our conscionsness. Some of the organs, in their sound condition, have no organic feelings; others of them undoubtedly largely determine the character of our common feeling by their condition, tension, action, etc. If, now, we extend the sensations of touch so as to include all the obscurely localized organic and muscular sensations, we fecl the necessity of distinguishing such sensations from what we have called the "common feeling."

One characteristic which the sensations have as sensations, strictly speaking, concerns the method of their excitation. In general, the stimulus must affect the nerves through the specific end-organs of sense, in order to give rise to a proper sensation. E. H. Weber sought to prove that, whenever nerves are irritated, not through the

${ }^{1}$ Comp. Lotze, Medicin. Psychologie, p. 278 f.

${ }^{2}$ Studien über d. Bewusstsein, p. $17 \mathrm{f}$. Wien, 1879. 
end-organs, but along the trunk, the irritation gives rise to feelings of pain instead of sensations. One experiment for this purpose consisted in dipping the point of the elbow into ice-cold water; when the sensation of cold is at once located in the skin, and the feeling of pain arises as soon as the stimulus has penetrated to the trunk of the nerve lying beneath. But other experiences seem to show that tones of common feeling may be indirectly excited, which are characterized by the massing of a great number of minute and obscurely localized sensations of touch. For example, the prick of a needle is felt at a given point as a circumscribed pain. The tickling from a fenther, confined to a small surface, may be regarded as consisting of complex sensations of light pressure, with no fixed locality for each one, but localized in general at about such a spot. But the tickling may be continued until a general tone of painful feeling is developed, which quite overwhelms all localized sensations. These phenomena may be considered as agreeing with the other phenomena to show that common feeling is due to widely extended and complex conditions of the central areas, in which the results of a large number of separate peripheral stimulations may unite so as to lose all their individual character, although each one contributes something to the common result.

$\S 15$. There are feelings so connected with the operation of the organs of sense as to be called feelings of sensation. A certain tone of feeling (a third element, as distinguished from its specific quality and intensity) belongs to most sensations. We are scarcely warranted, however, iu asserting that every sensation, as such, possesses some tone of feeling.' The question whether every sensation has some feeling must be distinguished from the question whether every feeling is of either painful or pleasurable tone. The tone of the feeling of sensations is the agreeable or disagreeable affection of consciousness which they often carry, as inseparably connected with them. The particular tone belonging to any sensation is, to a large extent, dependent on its intensity. The laws of this dependence have been ingeniously conjectured by Wundt. ${ }^{2}$ Sensations of moderate intensity - that is, of intensity below the point at which the minimum of painful feeling begins-are usually pleasurable. The feeling of pain rises in intensity, from the point where it begins, as the intensity of the stimulus increases. The curves which represent the increase of feeling and the increase of sensation by no means correspond. It is assumed by Wundt that the maximum point of pleasure lies about the so-called "cardinal value" of the sensation,

${ }^{1}$ As Wundt does, for example, Physiolog. Psychologie, i., p. 465.

${ }^{2}$ Physiolog. Psychologie, 1., p. 469 ; comp. p. 360. 
or place where the sensation ceases to increase in simple proportion to the strength of the stimulus. The amount of pleasurable feeling is also dependent on the element of time. It is thought to reach a maximum at about the point where the strength of sensation is the most favorable for accurate discernment of the objective stimulus.

As to the dependence of the tone of feeling belonging to a sensation upon the quality of the latter, it has been held ' that no sensation is absolutely pleasant or unpleasant irrespective of its intensity. Even then, however, it would have to be admitted that qualitatively different sensations differ greatly in the amount which is consistent with an agreeable tone of feeling. It is, of course, with regard to the organic sensations, and the special sensations of touch, smell, and taste, that the relation between tone of feeling and the quality of sensation is most apparent. Doubtless large allowance must be made in all cases for individual peculiarities of organism, association, etc. Probably, also, the disagreeable tone of feeling which almost universally attaches itself to certain qualities of sensation, however moderate or unobtrusive their intensity, is largely explicable on the principle of heredity. But, taking matters as they stand in present experience, it is impossible to maintain that the tone of feeling is not directly dependent on the quality of sensation. This is a question upon which only consciousness can pronounce. All degrees of some tastes and smells are disagreeable to most persons. Bitter is a distinctive species of the quality of gustatory sensations; but the pleasure which some persons have in greater or less degrees of it is, as a rule, acquired. It is true that some substances, whose odor in large quantity is disagreeable, become tolerable, or even pleasant, when the smell from them is faint. But this faint smell is not the same, but a distinctly different quality; oftentimes it could not be immediately recognized as coming from the same substance as that which emitted the strong odor. Discordant sounds are, in all degrees of intensity, naturally unpleasant; and so most witnesses would pronounce certain complex sensations of the skin (as of creeping, prickling, etc.).

$\$ 16$. Characteristic mixtures of feeling-some of them scarcely describable-seem to be attached inseparably to different kinds of sensations. This is obvious when we consider the marked difference in the way we are affected by major and minor chords, by successive tones having different musical intervals (for example, the diminished third, etc.), and by the characteristic clangs of different musical instruments. Writers upon this part of musical theory may disagree as to the precise significance of the violin, clarinet,

'So Wundt, Physiolog. Psychologie, i., p. 470. 
cornet, or hautboy, with respect to the tone of feeling belonging to each; but they can scarcely deny the fact of a marked difference. Goethe ' called attention to the change in spiritual tone, as it were, which harmonizes with what the eye sees when looking upon the world through different-colored glasses. Here, again, the precise equivalent, or value, in terms of feeling, which the different colortones possess, may be a matter of dispute; but the fact that the tones of feeling change with the color-tones is beyond dispute. That feelings of soberness or gloon go with black, of excitement with red, of cheerfulness with light green, of cool quiet with dark blue, of intense sensuous pleasure with saturated purple, would probably be admitted by most persons. Fewer would agree to describing the tone of feeling belonging to dark yellow or spectral orange as one of "suppressed excitement," or to brown as one of "perfectly neutral mood." "

$\S 17$. The character of the disagreeable or painful feeling belonging to different classes of sensations also differs with respect to the nature of its attachment to a recognized physical basis. Unharmonious colors produce in us a feeling of mild dissatisfaction, which appears as almost wholly of a spiritual kind. Discordant tones cause more of physical suffering; and disagreeable smells or tastes create a widespread sense of organic discomfort. Pains in the skin and interior organs, however, may take a character of intense bodily anguish, which is distinctive of no other qualities of sensation, and which is capable of submerging all sensation, as such, in a flood of painful feeling. ${ }^{3}$

\$ 18. The tone of sensuous feeling is also dependent upon the total condition of consciousness as determined by attention, mental habit, association of the feelings among themselves and with the ideas, control of the will, etc. Such feeling is, therefore, largely a secondary element of experience, which arises through certain acquired effects of the sensations as connected with previous activities of the mind. But concerning the physical basis of the feelings, in this aspect of them, we know nothing whatever; and the subject is not as yet one with which physiological psychology can successfully deal.

§ 19. The consideration of the affections and the emotions, or passions, involves at least three important particulars: (1) The characteristic feeling which distinguishes each; (2) its relations to the train of ideas, and the changes induced by it in the ideas; (3)

${ }^{1}$ Farbenlehre, $\$ 763$.

${ }^{2}$ Comp. Wundt, Physiolog. Psychologie, i., p. 477.

${ }^{3}$ Comp. Lotze, Outlines of Psychology, p. $75 \mathrm{f}$. 
the relations to the different bodily organs, and the reflex effect of the changes in these organs upon both the feelings and the ideas.

Each of the various affections or passions is characterized by a peculiar feeling, whose tone is either agreeable or painful, whose intensity admits of various degrees, and whose content is determined by the mental representations to which it has become attached. Each may be considered as having its rise, psychologically, in some form of blind, instinctive impulse that needs to be connected with a mental image of the object which experience has related to it as corresponding to the impulse. The germ of the impulse is the natural susceptibility of having desire awakened by an appropriate stimulus, and the capacity of forming by experience the idea which corresponds to, or gratifies, the impulse. ${ }^{1}$ Impulses may be described as of two kinds-craving, or attraction, and repulsion. When the feeling, which as mere impulse is blind with respect to the object of gratification, becomes connected in experience with ${ }^{*}$ appropriate presentations of sense or mental pictures, the basis for an affection or passion has been laid. Thus the germ of anger and hate is found in that instinctive impulse of repulsion which is produced by all unpleasant resistance of effort, or painful excitement of the nervous system. Sudden and intense irritations-as the striking of one's hand against the table, the slamming of a door in one's ear-tend to arouse the feeling of resentment. The affection of the child for the mother ultimately becomes far more than the feeling of comfort it has in her arms or at her breast; but the former is cradled and nursed in the latter. By varied associations, impulses of attraction or of repulsion become developed into a great variety of affections, emotions, and passions, characteristic of the different manifold relations in which the sentient soul finds itself standing toward things and persons.

$\$ 20$. All emotional forms of feeling are accompanied by abrupt and marked changes in the character and time-course of the mental train. Such changes may be regarded as standing in the relation both of cause and of effect to these feelings. Some impression with which strong feeling has become associated is made upon the mind; the result is a transitory interruption of the mental equipoise. This constitutes in part the justification for the saying that from mere feeling to affection is a "leap." " As a rule, the effect of any sudden and surprising impression-perception of some object of sense, or remembered image-is to start the flow of emotion. Thus anger, fear, desire, avarice, take men "off their guard;" the

${ }^{1}$ Comp. Wundt, Physiolog. Psychologie, ii., p. $33 \mathrm{f}$.

${ }^{2}$ Comp. Nahlowsky, Das Gefühlsleben, ete., Einleitung. 
feelings of such kind that are started by a given mental impression themselves produce a confusion of the mental train. But, on the other hand, this very disturbance of the mental train is itself productive of a new phase of feeling, such as is associated with the particular ideas that in confused and hurried throngs rush into consciousness, as well as with the general state of consciousness considered as one of haste and confusion. The physical basis of this state is laid in the extraordinary condition of excitation that exists within the central organs-the ideo- and sensory-motor centres of the cerebral hemispheres.

$\S 21$. But the wonderful, characteristic effect which these forms of feeling produce upon certain of the vital organs is the most noteworthy peculiarity of all affections, emotions, and passions. Upon this point science has far less than we could wish of information reaching beyond the observations of ordinary experience. Of such information, perliaps the most important concerns the influence exerted through many groups of muscles, from the central organs, upon the vaso-motor system. The effect of shame, fear, or anger, for example, upon the circulation of the blood is matter of common remark. But some grow pale and others red, when angry. In 1854, R. Wagner investigated the effect of fear upon the heart of a rabbit. A blow on the table near the animal was found to cause its heart to stand still a short time, and then resume beating with accelerated frequency of stroke. Subsequent investigations have made obvious the general effect of emotion upon the curve indicating the blood-pressure. The effect produced upon the pulse of a dog by hearing the sudden cry of another dog depends for its character upon whether the vagus nerves are cut or not; but even after their severance a marked effect of this kind is still manifest. ${ }^{1}$ The great influence of these forms of feeling upon all the action of the capillary vessels, upon the secretions, etc., and upon the respiration to retard, or accelerate, or make it irregular, is of the same order. That care and anxiety disturb nutrition, that pain and sorrow cause the tears to flow, that fear and love and anger act upon the abdominal organs, is generally recognized. The effect is sometimes seen in suddenly innervating, and sometimes in depressing, one or more of the bodily organs; or in both innervating and then depressing them, in certain well-recognized cases. On the basis of such facts, Kant suggested a division of the affections into "sthenic" and "asthenic." But many forms of feeling, as they run their

${ }^{1}$ This subject has been investigated by Conty and Charpentier, by Cyon, Heidenhain, and others; comp. Exner, in IIermann's Handb. d. Physiol., II,, ii., p. 289 f. 
course, become by turns sthenic and asthenic. Strong emotions or passions of all kinds tend to destroy the nervous mechanism; "the sthenic kill by apoplexy, the asthenic by laming the heart." " Unusual tension or relaxation of certain groups of muscles characterizes all these forms of feeling.

The marked effect which certain feelings have upon particular organs of the body is complemented by the fact that such organic effect has in turn a marked effect upon the feelings. The organic disturbances advance step by step to form the physical basis of a rising tide of emotion, and then fall off with equal pace as the tide of emotion subsides. The organic changes are not merely an expression of the mental ; they are its material cause and support. Professor James has emphasized these facts with great skill and in an interesting way. ${ }^{2}$ The effect upon the emotions and passions of putting the muscles or other organs of the body into certain conditions, which is so remarkable in all cases of hypnotism, is also undoubted in what we ordinarily consider normal states of body and mind. "What kind of an emotion of fear would be left, if the feelings neither of quickened heart-beats nor of shallow breathing, neither of trembling lips nor of weakened limbs, neither of goose-flesh nor of visceral stirrings, were present, it is quite impossible to think. Can one fancy the state of rage and picture no ebullition of it in the chest, no flushing of the face, no dilatation of the nostrils, no clinching of the teeth, no impulse to vigorous action, but in their stead limp muscles, calm breathing, and a placid face?" In view of the foregoing facts, Professor James propounds the thesis, that the "bodily changes follow directly the perception of the exciting fact, and that our feeling of the same changes as they occur is the emotion." s

To neglect, however, that element of feeling in every emotion which is immediately attached to certain perceptions and ideas would be quite as faulty as to neglect the elements which are only reflexly blended with the complex of feeling on account of the condition into which the bodily organs are thrown. The relation between perception and feeling as a psychological fact is as certain and immediate as any relation ean be. What the physiological basis for this connection is we do not know ; but there is every reason to suppose that it is, at the same time, direct and of the nature of reciprocal influence between the nerrous elements and areas of the

1 Wundt, Physiolog. Psychologie, ii, p. 330.

Mind, 1884, IX., p. 188 f.

${ }^{3}$ But since its author seems scarcely to have taken this thesis seriously, it may be thought superfluous even to object in brief to it (see p. 205). 
cerebral centres; as well as indirect, through disturbances produced by perceptions and ideas within the remote bodily organs. The influence of perception upon the feelings in the form of strong emotion is partly, but not wholly, through the skin, muscles, bloodvessels, organs of respiration, and viscern. At the same time the characteristic tone which strong emotions have is largely colored by the sensuous and common feelings occasioned by the disturbance of the organs. When even the feelings called astlietic, or intellectual, or ethical and religious, are vehemently aroused, an "emo. tional" equality is imparted to them from the same source.

\$22. By mental " moods" is ordinarily understood those collective conditions of the mind which are characterized by some fundamental tone, but without any special feelings accompanied by clear consciousness of their inducing causes. The principal elements that enter into such moods consist of ill-localized sensations arising from the internal organs-especially due, perhaps, to disturbed or depressed cerebral function ${ }^{2}$ - and a throng of half-reproduced feelings and ideas, or of vague single feelings, such as undefined foreboding, anxiety, fear, etc. 'Since these elements belong to the somewhat permanent equipment of the mind (at least until a marked and lasting change in cerebral condition and the train of ideas is brought about), their prevalent tone is characteristic of different persons; whereas the emotions and passions run their course quickly, and give a color to the personality rather by the suddenness and frequency with which this kind or that, respectively, is present in consciousness. But mental moods also may be characterized by emotions or affections of a low and lingering tone-pale and faded specimens of the type, as it were.

$\S 23$. Of all the so-called "higher feelings" (æsthetic, intellectual, ethical, religious), it is only certain elementary forms of æsthetic feeling concerning whose peculiar physical basis we have any assured information. All these feelings, however, when they reach a certain degree of intensity, tend to assume an emotional character. They then come in part under the considerations which have already been urged as applying to the emotions in general. A large portion of the strong feelings of admiration for scientific objects, discoveries, laws, and personalities, or of religious fervor, aspiration, and devotion, or depression, is reflex; it rests upon the physical basis of effects that are produced in the muscles and vital organs--especially the organs. of secretion, respiration, and circulation. But these facts do not explain or annul the other class of facts, which leads the judicious investigator also to emphasize the spiritual origin of such feelings

${ }^{1}$ Comp. Stricker, Studien über d. Bewusstsein, p. 63. 
considered as complex reactions of the mind in view of the presence in consciousness of certain classes of ideas. So far as such spiritual reactions by way of feeling have any even conjectural physical basis peculiar to them, this basis must be sought for in the central organs of the nervous system. How far such a basis really exists, and in what it consists, we have as yet scarcely a right to imagineso complete is our ignorance.

$\S 24$. The oesthetic feelings arise and develop chiefly in connection with presentations of sense, or with the remembered or created mental images that represent objects of sense. In their elementary form, therefore, they plainly have a physiological side which admits of scientific treatment-although they have received such treatment far less than could be wished. Many interesting facts and certain partial generalizations called laws-having most application to the lower classes of pleasurable feelings through the organs of smell, taste, and the skin, when viewed in the light of the hypothesis of evolution-are given in the work of Grant Allen on "Physiological Esthetics." But even the most elementary æsthetic feelings cannot be considered as on a par with the sensuous feelings, or as mere aggregates of such feelings. ${ }^{2}$ The tone of feeling which characterizes the sensations furnishes a material, as it were, for genuinely asthetic feeling; but the latter always implies also the working of certain intellectual laws, and a union of the simple feelings of sensation under time-form and space-form. Assthetic feelings, then, may be said to spring from the manner of the combination of sensuous feelings; time and space furnish the framework in which they are arranged. Hearing is the principal sense for combining sensuous feelings so as to produce æsthetic feelings under time-form, and sight under space-form. The development of even the elementary but genuine cesthetic feelings by other senses than the eye and ear is extremely limited. The agreeable and disagreeable feelings which come through sensations of smell, taste, and touch are for the most part sensuous, rather than strictly æsthetic.

Hearing, as pre-eminently the time-sense, has two forms of resthetic feeling-harmony and rhythm. The nature of the complex sensations which produce the feeling of consonance and dissonance has already been discussed. Harmony is determined by the coincidence of certain partial tones belonging to different clangs simultaneously sounded. The feeling of harmony is colored by the peculiar way in which the combination of the clangs occurs. The principal difference of this sort is that which obtains beween major

1 See pp. $30 \mathrm{ff}$.

${ }^{2}$ On this point, see Wundt, Physiolog. Psychologie, ii., p. $179 \mathrm{f}$. 
chords and minor chords; in the former the different clangs are perceived as firmly beld together by the fundamental clang, while in the latter the coincident overtone performs the same office less obviously. The one is productive of agreeable rsthetic feeling satisfied; the other of such feeling left unsatisfied-a feeling of longing. When, then, the one form of feeling becomes very intense, it may involve the pain of over-excitement; the other, when intensified, stirs a kind of agreenble pain of unrest. In musical time it is the periodic nature of the excitation, with a change in the individual presentations of sense, which produces the pleasurable rsthetic feeling.

Two or three regularly recurring impressions, having the same or a different content of musical sound, are combined into a series; certain members among the whole number are then accentuated, in order to form the different series that constitute the various kinds of musical time. All musical time, fundamentally considered as respects its rhythm, is either two-time or three-time. The difference in the feelings which respond to these two classes of musical rhythm is obvious in a pronounced form, in the funeral march, on the one hand, and the waltz, on the other. In general, it is the harmony of music which gives direction to its feeling, and the rhythm which determines the rise and fall of feeling. Thus waves of different kinds of feeling are made by music to pass over the soul. ${ }^{1}$

The elementary resthetic feelings which come through sight lead to the consideration of the resthetic effect of visual form. Such effect can be considered only very imperfectly from the physiological point of view. In one important particular, however, pleasurable æsthetic feeling is directly dependent upon the combination of the sensations, with their accompanying tone of feeling, under the laws of the mechanism of vision with both eyes in motion. Beautiful form is determined by the course of the limiting lines ; and limiting lines, in order to have the effect of arousing agreeable ${ }^{*}$ xsthetic feeling, must accommodate themselves to the physiological and psycho-physical necessities of the eye when in motion. These necessities thus determine both the direction and the extent of the limiting lines. Lines of slight curvature, not too far continued in one direction, best comply with such necessities. Lines of very sharp curvature, or lines continued too long in one direction, do not produce a pleasing æesthetic effect. So also must the main lines of a building lie in horizontal or vertical directions, preferably in the

${ }^{1}$ For a treatment of æsthetic feeling in music, comp. Wundt, Physiolog. Psychologie, ii., pp. 180 ff. 
former direction. But long oblique lines-for example, from a lower right-hand to an upper left-hand corner of a building-are scarcely tolerable. The ease with which the eye sweeps the lines, in order to make that synthesis of successive similar presentations of sense in which every perception of a line consists, is plainly a determining factor in all these cases.

The resthetic effect of visual form is also determined by the way in which the form is constructed, through repeating similar or unlike simple shapes and combining them into a totality. By this means a feeling of pleasure akin to the feeling of musical rhythm is excited by the successive impressions which occur periodically as the eye, with a nearly uniform movement, sweeps the entire field. In horizontal directions, the law for the arrangement of the parts is that of symmetry of the simple parts; in vertical, rather the law of asymmetry. Certain proportions between the connected parts, and between the whole and the parts, are favorable to the development of rsthetic feeling. The rule, that the whole of a presentation of sight shall be to the larger part as the larger part is to the smaller part, has been called "the golden diameter" $(x+1: x:: x: 1)$; since the proportion thus determined has been supposed to be par. ticularly farorable to pleasurable rsthetic feeling. Ease of the mental apprehension with which the relations in proportion of the different parts are presented is favorable to agreeable resthetic feeling.

$\S 25$. But all the foregoing rules, and all others similar, are applicable to the rsthetic feelings of form rather as coming under the general class of intellectual feelings. That change in degree or kind of activity, recognition of similarity or contrast, and mental apprehension of a law or principle as expressed in the presentations of sense, determine the agreeable character of our intellectual feelings, is recognized by psychologists ${ }^{1}$ generally; but as to the physical basis of mental facts of this order we are almost completely ignorant. It is not unlikely, however, that the effects of monotony and change upon the feelings of an intellectual order are connected with the same law of the exhaustion of the nervous elements as applied to the cerebral areas which we know to hold good in other parts of the nervous system.

$\S 26$. The only other class of feelings which admit of consideration from the physiological point of view is the so-called "feelings of effort, or of innervation." These feelings are especially connected with all the motions of the body considered as furnishing

${ }^{1}$ See the judicious remarks of Sully, Outlines of Psychology, pp. $457 \mathrm{ff}$. New York, 1884. 
information concerning its position and the condition of tension or strain to which its parts are subject; as well as furnishing, through revived mental images of such feelings, the means for reproducing voluntarily the required definite modes of motion. Such feelings also have a great psychological interest on account of their obvious connection with the development and consciousness of acts of will. The dispute as to whether they are of central or peripheral origin, and as to that in which their precise nature consists, has already been alluded to (pp. 344 and 415).

That we have a "feeling of effort" is a fact, as says Professor James, " "consecrated by the institution of the word effort, and its synonyms exertion, striving, straining." The nerrous process which occasions this feeling the great physiologist Müller ${ }^{2}$ considered to be purely central, and to consist in the discharge from a motor centre into the motor nerves. This view has since been widely adopted by physiologists ; it has also been used-especially by Bain and Wundt-as an essential factor in a theory of sense-perception, as of chief importance in accounting for our experience of solid resisting objects of sense and of whatever belongs to the inertia of matter in general. On the contrary, it has been maintained that the feeling of effort, over and above what is purely "moral" (as in the effort to remember to make a decision, etc.), is a complex of afferent sensations " coming from the tense muscles, the strained ligaments, squeezed joints, fixed chest, closed glottis, contracted brow, clinched jaws, ${ }^{3}$ etc."

Of the two views above mentioned, the latter has by far the most in its favor. The argument from the consciousness of effort which we may have when we intensely make believe use any limb, but do not actually move it (as, for example, the pulling of a trigger with the forefinger of an extended hand), has been answered by Ferrier: "This observer calls attention to the fact that the feeling of effort in such cases is due to keeping the glottis tightly closed, and actively contracting the respiratory muscles. If we try, however, to make believe exert ourselves without actually contracting the muscles of the limbs, and at the same time keep breathing regularly, we shall not experience the slightest trace of the feeling of effort, no matter how hard we try. This feeling, then, when the glottis is closed and the respiratory muscles are tense, is due to centripetal impressions coming from the parts thus innervated.

The argument from the feelings of effort which determine our

1 The Feeling of Effort, Anniversary Memoirs of the Boston Soc. of Nat. Hist., 1880, last monograph. $\quad$ : Physiologie d. Menschen, II., p. 500.

'See James, Feeling of Effort, p. 4. The Functions of the Brain, p. 222 $f$. 
localization of objects has been presented in the most convincing way possible by Helmholtz and Wundt, as applied to the case of partial paralysis of the external rectus of one eye. Inasmuch as the patient feels (so von Graefe ${ }^{2}$ showed) that he has moved his lame eye much farther than he really has, the inference is drawn that this exaggerated feeling of effort must originate in central motor impulses which have followed upon the fiat of the will. This argument, however, neglects to notice what goes on in the otherand sound eye. Since this eye, unlike the lame one, continues its motion until the limit of motion and its corresponding condition of peripheral strain is reached; and since, as Hering ${ }^{2}$ has shown (comp. what has already been said, p. 439 f.), both eyes are innervated by one common act, and their motor apparatus is to be regarded as functioning as one organ - the feeling of effort is probably due to afferent sensory impulses occasioned by the condition of the sound eye as well as of the other eye. ${ }^{3}$ Moreover, the more critically we examine those cases which occasionally occur, where, on account of paralysis causing anæsthesia, the sense of position of the limbs is impaired or lost, the more conclusive does the evidence appear against the theory that the feeling of effort is of purely central origin. For, in general, it seems that, while the power of voluntary motion remains unimpaired, if the sensations which have a peripheral origin are impaired or lost, the various feelings of effort connected with the accomplishment of a given amount of motion, or with the act of holding any member of the body against the pull of gravity, are disturbed or disappear." As far as the evidence regarding this obscure subject reaches at present, the feeling of effort

${ }^{1}$ Handb. d. gesammten Augenheilkunde, VI., p. $18 \mathrm{f}$.

'See Hermann's Handb. d. Physiol., III., i., pp. $512 \mathrm{f}$. and $520 \mathrm{f}$.

${ }^{3}$ See James, Feeling of Effort, p. $10 \mathrm{f}$.

${ }^{4}$ See a recent paper on "Le Sens musculaire et les Sensations mnsculaires," by E. Gley, in the Revue Philosophique, 1885, pp. $601 \mathrm{ff}$. In this paper the resnlts of the investigations of M. Maguin, conducted upon paralytics in the Hôpital de la Pitié (Comptes rendus, Mars 1884, i.), are appealed to as confirmatory of the view that the stretching and rubbing of skin, ligaments, joints, etc., enter into our so-called feelings of effort. M. Demeaux has reported the case of a woman who could move her limbs, but could not tell whether they were moved, or in what direction, or how far. No fewer than three similar cases were reported by French physicians in the year 1885. The same view of the complex peripheral origin of the feeling of effort seems also to be favored by the experiments of M. Bloch, who tried placing his hands symmetrically on a screen with two leaves covered with paper, divided into small squares, under the guidance of the so-called muscular sense. The testimony of persons who have lost their limbs, as to whether they can produce any feeling of effort by the fiat of will to move the lost member, is conflicting. In all 
must be held to be complex, and so is akin to other forms of common feeling; its constitueut elements are the various obscurely localized sensations, with their characteristic tones of feeling, which arise in the condition of skin, muscles, ligaments, joints, etc.

$\S 27$. The feeling of effort is closely connected in experience with the changing positions of the members of the body, and its consideration therefore fitly introduces that of the bodily motions. As concerns their relation to the phenomena of mind, these motions may be divided into two great classes ' - namely, such as are not demonstrably connected with antecedent changes in the states of consciousness, and such as, in addition to their physical conditions and causes, require that their explanation should take account also of preceding states of consciousness. The former are to be regarded purely as activities of the physical mechanism, ${ }^{2}$ and are either automatic or reflex. Autonatic motions are such as, without any corresponding idea or fiat of will, originate from inner excitations of the central nervous system; the reflex are those in which the central excitations resulting in motion are traceable to the action of sensory nerves which have been peripherally excited. It is extremely difficult to distinguish between automatic and reflex motions, and scarcely less so to distinguish between the automatic and the impulsive. Indeed, while it is true, on the one hand, that reflex and centrally co-ordinated movements form the basis upon which all our developed life of voluntary motion takes place, it is also true, on the other hand, that the more complex co-ordinated movements are themselves originally voluntary motions which have, as it were, become habitual and so dropped out of consciousness into a statical and mechanical way of taking place. The sensations and ideas of motions may then be said to tend constantly in two directions-either toward consciousness or out of it. It is by means of these processes in two directions that all our learning of complicated movements of the body, of feats of dexterity and skill -learning to handle tools, to play on musical instruments, etc.takes place. The interest which psychology has in the automatic

such cases, however, it is probable that the alleged feeling of effort is to be looked for in the actual condition of strain into which some existing part of the body is thrown-especially the apparatus of respiration (see Bastian, British Med. Journal, 1869, p. 461).

${ }^{1}$ Comp. Wundt, Physiolog. Psychologie, ii., p. 400 f.; and Lotze, Medicin. Psychologie, p. 286 f.

2 The mechanism of the bodily motions has already been treated at length: for the nerve-muscle machine, see pp. $104 \mathrm{ff}$; for reflex motion, see pp. $132 \mathrm{ff}$; for automatic motion, see pp. $147 \mathrm{ff}$; for the sensory-motor areas of the cerebrum, see p. $267 \mathrm{ff}$. 
and reflex motions is chiefly on account of their relations to motions which are actually preceded by conscious ideation and volition.

$\S 28$. Such motions of the body as require us to take account of antecedent or accompanying states of consciousness, in addition to the connections of the physical mechanism, are either impulsive or voluntary. But this distinction is one which admits of such a great variety of degrees shading into each other, that, although it is valid and necessary in principle, it cannot be carried through in practice with any considerable precision. By an impulsive motion we understand a motion which, without a conscious fiat of will, follows upon certain ideas and excited states of feeling. The motif of the impulsive movement lies, then, in some form of feeling that determines will one way without any proper choice. If we speak of such motions as volitional or voluntary, it must be understood that we are referring to activities of will of a lower order, psychologically considered, than those which come into play in all cases of conscious choice. Impulsive motions are, in general, more quickly accomplished than are voluntary motions; because the reactiontime is shortened through will-time proper having been dropped out (comp. chap. VIII., \& 19 f.).

\$29. All voluntary movement has its basis laid, so to speak, in impulsive movement, and in the reactionary effect which the latter has upon the conditions of reflex and automatic bodily activities. As laid in this basis, voluntary motions imply a development of intelligence and will. The infant finds itself equipped with a bodily mechanism which, under the influence of external and internal stimuli, is kept excited to unceasing activity of the peripheral members. This activity results in certain sensations and feelings of effort, in the manner previously described. The tone of these states of consciousness is one of either pleasure or discomfort, under those laws of relation between the nervous mechanism and conscious feeling which can be only imperfectly stated; and which, when most perfectly stated, can only be accepted as ultimate matters of fact. By nature the nerrous mechanism is so arranged that certain other bodily motions of peripheral origin are started on occasion of the pleasant or painful feeling, and these motions are adapted to enhance the feeling if pleasant and to relieve it if painful. The feelings thus become further connected with the ideas of the motious that modify them; yet the mechanism of the motions is not to be regarded as originally dependent upon the ideas, but rather as originated in connection with the feelings of pleasure or discomfort and naturally adapted to secure an increase of the one or a diminution of the other. 
The voluntary movements of the body, accordingly, presuppose the impulsive, and yet they reach far back into the obscurity of the earlier development of consciousness. Strictly speaking, they imply the presence in consciousness of two or more different or conflicting ideas of motion, one of which rather than the others is realized as a sequence of an act of conscious choice. They imply, thenas has already been said-a considerable development of the mental activities of ideation and volition. Moreover, those movements which are ordinarily called voluntary are really so only with respect to certain of their elements; they all also contain elements which must be classed as reflex, centrally co-ordinated, and impulsive. The term "voluntary" fitly lays the emphasis upon the conscious act of choice; and this, in turn, implies ideas of various possible forms of bodily motion gained by previous experience with the correlated states of conscious feeling and conditions of the body as giving rise to or modifying these states.

The voluntary motions, therefore, constitute the highest class of motions, both because their conditions include all those which belong to the other classes, and other conditions besides, and also because of their more direct connection with the development of certain mental phenomena of supreme psychological interest and importance. To move any part of the body voluntarily requires the following particulars: (1) The possession of an educated reflex-motor mechanism, under the control of those higher cerebral centres which are most immediately connected with the phenomena of consciousness ; (2) certain motifs in the form of conscious feelings that have a tone of pleasure or pain, and so impel the mind to secure such bodily conditions as will continue or increase the one and discontinue or diminish the other ; (3) ideas of motions and positions of the bodily members, which previous experience has taught us answer more or less perfectly to the motifs of conscious feeling; (4) a conscious fiat of will, settling the question, as it were, which of these ideas shall be realized in the motions achieved and positions attained by these members; (5) a central nervous mechanism, which serves as the organ of relation between this act of will and the discharge of the requisite motor impulses along their nerve-tracts to the groups of muscles peripherally situated.

As to the first and second of the foregoing particulars, nothing further need be said; and as to the definite nature of the physical basis which underlies the connection of ideas of motion, fiat of will adopting one idea, and the starting outward of the right motor impulses, our ignorance is almost complete. It is more than probable that we cannot will the movement of muscles, of the results of whose 
actual movement in the induced motion of the limbs we have acquired no idea from previous experience. The mental images of the various feelings of motion and position which have been acquired in the past are our guides in realizing again the same motions and positions of the limbs. To say I will, refers to the future. But we can never " will" motion in general-motion, that is, of no particular members of the body, and without specific quality, direction, and velocity of the motion. That certain nervous processes in the central organs form a physical basis for the mental phenomena of ideation and fiat of will there is sufficient ground for believing. The phenomena of reaction-time show that interrelated cerebral activities of more and more complicated sort are implied in the increased time required for completing the mental actions of representation and choice between two members of an alternative. It would be a great mistake, however, to regard the mind as having before it the cerebral machinery, all nicely laid out, together with the acquired art of selecting and touching the right nerrous elements in order to produce the desired motion, as a skilful player of the piano handles his key-board. The mind has no native or acquired knowledge of the different ideo-motor areas of the cerebrum. Even less can we regard the mind, acting under the form of energy of will, as bringing some stress to bear upon the right centres of the brain, and thus setting them in motion by laying its own hand to them, as it were. The activity of which we are directly conscious under the term "to will" is a purely psychical activity; it is marked by no transition of force from the spiritual realm to the material molecules of the nervous structure. The feeling of effort, which seems to us to accompany the active putting-forth of will, is itself a resultant of mixed sensations that have a peripheral origin. The whole description of such transactions of voluutary motion as are constantly occurring-for example, when we rise to close the window, take the pen in hand to write, etc.-is as follows: We desire to have something done; mental images of the bodily motions and positions involved in this doing arise in the mind; the fiat of will goes forth adopting one of them, and villing it, as we say; an order of nature which has correlated this fiat with certain cerebral changes, but of which we know nothing whatever directly, and little through the most searching investigations of science, runs its course, and the transaction which we have ideated and willed takes place.' The mind can represent the ideas in consciousness, and issue the fiat of will; it can do nothing more. Science can ouly conjecture at

1 This view of the subject has been repeatedly enforced by Lotze; see, especially, the Microcosmus, i., pp. 283 ff. Edinburgh, 1885. 
present what then takes place. It is to its advancing theory of nerve-physiology, ${ }^{1}$ and of the localization of cerebral function, ${ }^{2}$ that we must look for more light on the question-What happens in the brain when the fiat of will issues in consciousness?

$\S 30$. Reflection on the foregoing principles makes it obvious that the different concrete motions of ordinary experience cannot be assigned with confidence to this or that class exclusively. In the life of the infant we can trace a general progress from an almost exclusive predominance of reflex and automatic motions, through the impulsive, to more and more of the voluntary. But even in the infant's case no hard and fixed lines can be drawn between the various classes of motions. It is impossible to say how much of the constant movement of its legs and arms is reflex, how much automatic. It is also doubtful how far and how long the winking of the eyes, the grimaces of face accompanying the stimulating of the tongue, the starting at sound, etc., are reflex rather than impulsive. The same thing is true of its earlier cryings, mimetic and imitative movements, and various ways of thrusting out and drawing in its limbs in a purposeful way. Nor can the earlier voluntary motions be confidently distinguished from the impulsive.

This line of inquiry is especially interesting with respect to the beginnings of articulate speech. A tolerably regular transition from the sounds in which the earliest emotions express themselves to the deliberate formation of words and sentences makes it impossible to tell precisely when the child assumes control of its organs of speech. But our difficulties with the unclassifiable phenomena of infantile life do not seem so strange when we reflect upon the fact that the complicated bodily motions of adult life partake at one and the same time of all the four above-mentioned classes; and that precisely the same motions may pass rapidly out of one class into another. The person, for example, who is balancing with a pole on a tight-rope, or dancing to music, is involved at once in motions which correspond to all four of these principal classes; and a quick change in circumstances may make any one of the four more prominent than another. So perfectly may the nervous mechanism be trained to its work that it may continue to play the violin in an orchestra after the player has lost consciousness. Yet the rise and fall of feeling usually serves as a guide to the artist, so that impulsively his bowing draws nearer the bridge in the crescendo, and nearer the key-board in the diminuendo, passages. If he plays false or out of time, the sight of the leader's baton, or his own

${ }^{1}$ Comp. Part I., chaps. III. and VII.

${ }^{2}$ Comp. Part II., chaps. I. and II. 
sensations, may decide him to the fiat of will which changes the spacing with the left hand or the bowing with the right arm.

$\S 31$. The origin and nature of those motions of the body that are specifically expressive of certain ideas and feelings constitutes one of the most interesting fields of inquiry. It is a field, however, in which comparative psychology, by dealing with the facts of animal life under the theory of evolution, is particularly successful; whereas Physiological Psychology, strictly speaking, has little to communicate. This little has been summarized by Wundt' under three general statements or principles-namely, the principle of the direct alteration of innervation, the principle of the association of analogous sensations, and the principle of the relation of motion to the presentations of sense. Under the principle of the direct alteration of innervation are placed those facts which show that strong emotions exercise an immediate reaction on the central parts of motor innervation in such a way that many groups of muscles are lamed at once, and others are excited to tense action followed by exhaustion. Hence the tremblings of limbs and organs of speech, the changes in the blood-vessels and capillaries connected with secretion, the paling of fear, the reddening of anger and shame, the erect hairs under the influence of terror, etc. The principle of the association of analogous sensations emphasizes such facts as imply that sensations having a common tone of feeling are most easily combined, and then operate mutually to strengthen each other. Under this principle come the mimetic movements of mouth and nose expressive of disgust or pleasant tasto, the posturings of the tongue in connection with ideas of sweet or bitter, the expressive condition of the muscles due to certain sensations of the skin, etc. The consideration of the third principle-that of the relation of motion to the presentations of sense-brings before us the question of the origin of all the gestures and pantomimic action not accounted for under the two foregoing principles. Gestures with eyes and head and limbs, indicative of extension and relations in space; the arrangement of the muscles and slin of the countenance, and the motions of the eyes under the influence of care, expectation, and reflection; the angles of the lines about the mouth and the openings of mouth and nostrils when weeping or laughing, etc., all belong under this principle. But the physiology and psychology of the comic, the science of physiognomy, and of articulation in expressive speech, although properly coming in this connection, lead into descriptive anatomy and the theory of rsthetics much beyond the limits necessarily set to our investigation.

' Physiolog. Psychologie, ii., chap. 20. 


\section{CHAPTER $\mathrm{X}$.}

\section{PHYSICAL BASIS OF THE HIGHER FACULTIES.}

§ 1. Av ardent advocate of "Psychology without a soul" affirms" that " the study of abstract concepts (time, number, etc.) falls outside the province of physiological psychology, and has been made incidentally only." To be sure, this author has previously ${ }^{2}$ anticipated the time when the science of mind will succeed "in determining the (physical) conditions of all mental action, of whatever sort, as well of pure thought as of perception and movement," will -in brief-be "entirely physiological." It is not necessary to inquire how these two sentences can be reconciled. But, undoubtedly, at present the statement of fact is far better founded than the anticipation. It is not easy to predict how far psycho-plysical science will be able to push its discoveries in the future; or just where it will meet those insuperable barriers which surround all fields of human inquiry. It is perfectly safe, however, to affirm of all the plienomena of the so-called "higher faculties" of mind what M. Ribot says of the study of abstract concepts-they still "fall outside the province of physiological psychology." Certain difficulties are so obviously intrinsic and essential to the very nature of the facts with which this science attempts to deal when approaching these faculties that we cannot see how they will ever be successfully met.

$\S 2$. The foregoing conclusions apply most obviously to the formation of abstract concepts, the conducting of trains of reasoning, the exercise of choice, and the activities of the creative imagination in artistic production, scientific discovery, or mechanical invention. They apply only less obviously to the higher resthetic, ethical, and religious feelings; although we have already pointed out certain facts and laws which connect such feelings with a physical basis. We are also almost as much at a loss how to be "scientific" (strictly speaking) in our treatment of the phenomena which suggest some kind of physical basis for the action of will

${ }^{1}$ See M. Ribot, German Psychology of To-day, p. $306 . \quad$ New York, 1886.

2 Ibid., p. 15. 
- especially in the direction of attention for the apperception of objects of sense, and for the control of the train of ideas or the movement of the bodily organism. The same thing is true of the phenomena of memory, whether considered as involving retention merely or reproduction as well. All the attempts hitherto made to explain or deduce consciousness, either in general or in the particular phase called self-consciousness, from cerebral functions and activities, have been quibbling and wholly unsatisfactory. Yet there are indubitable proofs of the dependence of consciousness for its existence and modes upon the cerebral centres.

The inquiry after the physical basis of the mental phenomena usually classed as "higher" is, therefore, although peculiarly interesting, peculiarly unproductive of assured results. We may suspect that there exist in the nervous elements of the gray matter of the cerebral hemispheres inherited and acquired peculiarities of molecular constitution and of dynamical combination, which, if we could only get at them, would throw a flood of light upon such mental phenomena. But after all, to speak soberly, we are obliged to admit that the very existence of such peculiarities is still almost wholly a matter of conjecture; while the request for precise and verifiable information as to their nature, and as to the laws which connect them with undoubted facts of consciousness, can only be met by evasion, confession of ignorance, or poetizing and declamation under the garb of science. ${ }^{2}$ Physiological Psychology has a right to its own hypotheses; it has, however, no right to introduce myths about the genesis and marriage and "erethism" of nervecells, and speculation as to nerve-fibres dynamically inclined, into the domain of either physiological or psychological laws.

$\S 3$. The only safe method of arriving at the few probable conclusions attainable concerning the subject of this chapter is, accordingly, the following: The points of starting and the guides as to the way must, in nearly every case, be taken from introspective psychology. In studying the higher mental phenomena, physiological psychology is obliged almost wholly to adopt, as the only direct path open, the non-physiological method. Here, at any rate, we start from that which appears to us as terra firma. We know what it is to attend, to choose, to remember, and to reason-in short, to be conscious in some of the many modes or phases of consciousness. Moreover, whatever may be said in disparagement of the

${ }^{1}$ It is only by such terms as " poetizing" that we can truthfully characterize the greater part of what is said, for example, by M. Luys, in his work on The Brain and its Functions; this, while admitting the skill and brilliancy with which the author treats his own interesting conjectures. 
"old psychology," it cannot fitly be denied that it has most thoroughly and subtly analyzed the phenomena of judgment, memory, and choice, as these phenomena appear connected with each other in the flowing current of our conscious life. The result of such analysis has been secured in the laws of logic, of the association of ideas, etc., and in the various doctrines of the will and its relations to motive and conduct. In fact, all study of these mental phenomena from the physiological point of view is compelled to accept in some form the conclusions of a study of the same phenomena from the introspective point of view. For example, the reproduction of ideas under the so-called laws of association is a general fact of consciousness; in the attempt to explain this fact according to psycho-physical causes we are obliged to rely upon the results reached by the introspective psychology. The application to mental phenomena of uncouth terms derived from the physical sciences - such as "agglutination," "agglomeration," "cohesion," "organic phosphorescence," "histological catalepsy," etc.-has simply the effect of repeating certain psychical facts and laws in a less appropriate way, without adding an item of information regarding the real nature of their physiological basis. Ideas, or states and products of consciousness, cannot-speaking literally-cohere, or become agglutinated or agglomerated; and we need some better proof than mere declamation to show that these states and products depend upon any physical processes resembling agglutination, phosphorescence, or catalepsy of the nerve-cell. Physiological psychology is obliged, then, to accept certain conclusions of the psychology of self-consciousness; otherwise it has no motif or guide in its investigation of the higher mental faculties.

But while our conscious psychical experience of the higher mental activities is so far obvious as to make that side of the subject capable of scientific statement, our knowledge of the physiological processes connected with those activities is in precisely the opposite condition. Over and over again the confession has been forced from us that-strictly speaking-a scientific physiology of the cerebral hemispheres does not yet exist. We can only dimly conjecture what takes place in the nerve-elements of the cortex of the cerebrum as the physical basis of conscious sensation and perception. The molecular physics, or general nerve-physiology of the nerve-muscle machine-the simple peripheral nerve with muscle attached-is in a very unsatisfactory state. A science for the vast complex of nerve-cells and nerve-fibres which exists in the gray matter of the brain proper is at present scarcely a matter for even hopeful anticipation. Faint and doubtful guesses, more or less 
intimately connected with general principles of molecular physics and physiology of the nerrous system, are all that can appear in the name of such a science. But the very business of physiological psychology is to connect together under general laws the mental phenomena, on the one side, and the ascertained facts of physiology, on the other side. In this case, we are tolerably equipped with information as to the former; we have little but unverifiable assumption to take the place of the latter. In attempting the inquiry into the physical basis of the higher faculties (the physiological psychology of rolition, memory, conception, etc.), no other course is open but to accept the facts of consciousness, and then speculate as to how they may, perhaps in part, be accounted for by a conjectural extension of certain physical and physiological facts to the cerebral hemispheres. This procedure certainly cannot be called "science;" it is, however, the only one open instead of a confession of complete iguorance.

$\S 4$. The mental phenomena of the higher order, concerning whose physical basis conjecture is most plausibly supported by a number of related facts, may be divided into two great classes. One of these covers the phenomena of Will, in the forms of attentive perception and the effort determining the extent and character of the field of consciousness; the other covers the phenomena of Memory, whether considered as the retention or the reproduction of ideas. Certain conjectures as to the physical basis of both these kinds of mental activities are in good degree warranted by the principles discussed in the foregoing chapters.

The physiological basis (so far as such basis can be said to exist) for those mental phenomena which appear in consciousness as "acts of will" is laid, in general, in that power of automatism which is concentrated, so to speak, in the nerve-cells of the central organs. Automatism, or the power of originating motions which cannot be explained as due to external stimuli, is indeed in some sort a property of all living protoplasm ; but in that elaborate differentiation of structure and function which the human body exhibits, the nerve-cells of the central organs have absorbed this power and become distinctively automatic. To them chiefly does it belong to initiate within themselves the molecular changes which are necessary to keep the body, both as a whole and in its several parts, adjusted to the changes of its environment. It is sometimes said that "an amœba has a will of its own." Our only right to speak in this manner is derived from the fact that many of its formal changes seem to arise from within, and are quite inexplicable under any known laws of merely reflex motion. If we raise the inquiry 
whether such automatic changes of its molecular structure are accompanied by anything which corresponds to what we call conscious volition, it must be admitted that we are quite unable to answer such an inquiry. We can easily imagine the amœba, however, to have a consciousness of an "act of will" as an accompaniment of each automatic change in the arrangement of its molecules. A large part of man's activity in the control of his bodily organism, we know, is unaccompanied by any conscious volition. Such unconscious but purposeful activity belongs to the spinal cord and to the lower cerebral centres, which act both reflexly and automatically under the laws of acquired skill and of habit. In this way many even of our so-called voluntary movements really take place. But some sudden emergency-as, for example, the sight of a threatening object, a change in the character of the soil on which the pedestrian treads, the parting of a rein in the rider's bandmay call for a succession of distinct and intense acts of will. And, ordinarily, mild and rather obscure volitions connected with the movement of the body intermingle with the succession of sensations and ideas which compose the principal material of consciousness.

In all such cases as the foregoing we have reason to suppose that, either through external or internal stimuli (either through sensory impulses coming in along the centripetal nerve-tracts or as started by changed conditions of blood-supply), the nerve-cells of the cerebral hemispheres are called upon to exercise their peculiar functions. Such functions we may well believe are always both reflex and automatic; that is to say, the nerve-commotions which issue from the cells are dependent for their intensity and character both upon the excitations coming to them from without and also upon their own internal molecular structure and conditionespecially as respects the blood-supply. Accordingly, it must be held that volitions, or acts of will in consciousness, do not have their physical basis in any special organ or area of the brain. There is no special organ of will. All the central organs have preeminently the property of automatism. But since, in the case of man at least, it is only on occasion of a certain kind and degree of activity of the cerebral hemispheres that what takes place in the nervous system has any corresponding expression in consciousness, the plyysical basis for acts of will in general is the automatism of these hemispheres in general.

\& 5. An act of will, however, is always an act of some special kind. There can be no volition to motion in general, but only a volition defined and limited to the movement of certain limbs, or of the 
trunk including the limbs, with a certain direction and degree of motion. Thus also every act of will for the control of the mental train, or for the apperception of an object of sense, through concentrated attention, is defined by some particular mental state or modification upon which it is directed. We have seen good reason to believe that certain areas of the cerebral cortex are especially connected with certain corresponding sensory-motor activities (comp. Chap. II. throughout). In the same areas, then, the physical basis is laid for those acts of will that are concerned witl the corresponding activities. The acts of will which have to do with the movement of the upper and lower limbs, for example, imply the special activity of the cerebral areas on either side the Fissure of Rolando; those acts of will that have to do with the movement of the organs used in articulate speech are especially related to the areas lying about the lower part of the Fissure of Sylrius-the posterior third of the lower frontal convolution, etc. We have no sufficient ground for locating in one circumscribed spot the plyysical basis of such acts of voluntary attention as concern the different presentations of sense and the images of memory derived from them. The case is not as though the mind made a transit, as it were, from some special seat of intelligence and will, to contemplate with attention and pronounce upon the complicated sensory impressions which have arrived and been elaborated in the particular sensory areas; or as though it travelled from adjacent parts to lay its grip upon the right motor areas when sensation or desire indicated to will that certain groups of muscles should be innervated. Whenever an act of will takes place, then at the cerebral area which corresponds to the particular nature of the act (namely, the will to attend to this object of sense, or to start in motion that limb) the particular molecular changes arise in the nerve-cells which are correlated with such mode of consciousness.

\&6. As to the relation in time which is maintained between the conscious act of will and the particular form of automatic cerebral excitation which we have called its physical basis, it is not possible to pronounce with confidence. But there is no good reason to suppose that the conscious mental act is interpolated as an independent element of time, so to speak, among the physiological processes. The flow of consciousness from obscure sensation to perception and clear attentive discernment, then to the act of decision between two or more possible forms of appropriate movement, and, finally, to the issue of the right fiat of will, all keep pace with the corresponding physiological processes in the cerebral areas. As to the exact nature of these processes, and as to how they fur- 
nish necessary conditions to the mental movement, there is no information to be imparted.

$\S 7$. The problem is complicated when our consciousness becomes one of deciding to which of several presentations of sense or images of memory we will to direct the attention. "Concerning the physiological processes," says Exner, " " from which we abstract the conception of attention we know absolutely nothing." This is true even when attention seems determined or forced upon us by causes over which the mind has no control; it is, of course, more obviously true when the mind is conscious of deliberation and choice. The attention which directs to the single object and heightens the clearness of our perception, converting it into an "apperception," may properly be spoken of as an act of will; but starting from the point of view of consciousness, it must be admitted that, in the majority of such activities of apperception, there is no consciousness of choice - the will is determined in one way. This is equally true of the attachment of attention to certain particular images of the mental train, as that train is conducted along under the laws of association. Most things which we clearly perceive, or feel with any decided pain or pleasure, or which are vividly brought before the mind as images of memory and imagination, we cannot help attending to. The sudden flashing of a light, the passing of a bright object across the field of vision, the occurrence of a loud noise, or of a fainter one with a character that interests us, the smells in the atmosphere and the taste of our food, the sensations of the internal organs and of the skin, when sufficiently intense-all these compulsorily draw after them the attention. They get themselves perceived by an impulsive and involuntary act of will. So, too, do the revived images of memory, in ordinary circumstances where the perception of external objects is relatively suppressed, appear to force themselves upon the attention.

In view of the foregoing familiar facts of consciousness, we may conjecture that when the cerebral centres are not preoccupied, as it were, with contradictory forms and phases of nerve-commotion, certain processes set up within them, whether due to external stimuli or to changes in the blood-supply, are necessarily followed by the phenomena of conscious attention. Even when these centres are largely thus preoccupied, similar changes may be rapidly forced within them, by the action of some very strong excitation from the end-organs of sense, or from some connected cerebral centre. Hence the shock of surprise which sudden and vehement impressions create. In all such cases of forced attention the resulting tone of feel-

'In Hermann's Handb. d. Physiol., II., ii., p. 283. 
ing in consciousness is different from that which prevails when the choice to attend is being deliberately maintained or persistently revived. The motifs of much of our activity of will in attention, therefore, plainly lie in that state of the cerebral centres which is compelled by the intensity of the stimulation they receire (either from external or internal stimuli). ${ }^{\prime}$ If there were no other phenomena of will than those of forced attention, it would be necessary to admit the probability that all the mental activities are purely mechanical and absolutely dependent upon the action of the nervous system under the exciting influence of stimuli.

\$ 8. Certain phenomena of will in the form of attention suggest conclusions of a different order from the foregoing. Taking our point of starting again from consciousness, we know by a manifold experience that the different degrees of clearness with which we perceive objects or apprehend the images of memory implies a graded application of attention. The grading of this application of attention is by no means always determined solely by the intensity of the stimulus, so far as we can measure such stimulus. It is a principle of wide reach, that-to quote the words of Wundt- " the degree of apperception is not to be measured according to the strength of the external impression, but according to the subjective activity by which the consciousness is applied to a definite sense-stimulus." The subjective activity which applies the consciousness, as it were, to this or that presentation of sense or image of memory, rather than to some other, is an activity of will; and the effect of the activity is seen both in heightening the attention as directed to the object, and also in adapting the attention to the particular object upon which it is directed. Accompanying this twofold control of attention, and indeed forming the very basis upon which it rests, when deliberately exercised, is the consciousness of "choice"-the activity of will in deciding the direction and amount of attention bestowed upon one object among several in the field of consciousness. Percepts and ideas do not move from the various obscurer parts of the field of consciousness into the focal point by virtue solely of a momentum belonging to them as such; they are placed and kept there by an act of will. This must be admitted as an indubitable fact of consciousness, whether or not the physiological correlate or so-called explanation of this fact can be discovered or even conjectured.

Many indisputably valid phenomena, both those accessible to ordinary observation and those discovered by special experiment,

${ }^{1}$ Comp. Wundt. Physiolog. Psychologie, ii., pp. 387 ff.; and Stande, Der Begriff d. Apperception in d. neueren Psychologie, Philosoph. Studien, I., Heft ii., p. $194 \mathrm{f}$. 
illustrate the foregoing principle. By an act of will attention may be heightened and accommodated to the object, with a marked influence upon apperception and the association of ideas. Upon this - point we have to recall facts already mentioned. The effect of a voluntary increase of attention upon the reaction-time is to diminish it, of distracted attention to increase it or destroy its value altogether. ${ }^{1}$ If, simultaneously, the ear is stimulated by the periodic strokes of a bell, and the retina by regularly recurring electrical sparks, the attention will naturally be directed to the former; the image of the latter will then be located only very obscurely in the flow of consciousness, and the time of its occurrence may scarcely be noticed at all. We incline to attend to the stronger of two excitations of sense ; to yield to the inclination depresses the weaker still further-perhaps below the plane of conscious perception. But within certain limits we attend where we will. We also incline to attend to objects lying in the point of regard of the field of rision, but we can will to attend to objects lying in the outward portions of this field. ${ }^{2}$ The voluntary direction of attention in this case determines the apperception of these objects to the neglect of those lying in the more favorable parts of the field. We can attend to the field of vision of one eye, neglecting the other, as skilful microscopists do. We can see by voluntary attention the otherwise invisible double images. It is claimed by some experimenters with the "conflict of colors" in binocular vision, that, by the direction of attention, when a green image is formed on one eye and a red upon the other, they can see either at will, or at will can combine the two.

Experiments with instantaneous illumination by the electrical spark also demonstrate in a marked way the effect of attention. Objects which under ordinary circumstances are without great effort seen, either as stereoscopic or as double images, can also be seen in both ways by the electric spark, according to the direction of attention. The first impression is ordinarily stereoscopic ; but if a pause of 10 sec. be allowed for the after-images to die away, the experimenter can at will see the double images, although the point of fixation and the influence of the light remain absolutely the same. $^{3} \quad$ The effect of attention in analyzing composite musical

' See the table of Obersteiner, Brain, I., p. 439, to show the fluctuations of the reaction-time of a person reacting while an organ was playing in the same room. The normal reaction-time of the person being 0.100 sec., it rose to 0.148 , and even 0.215 , while the instrument was heard, and fell to 0.095 and $0.08 \pi$ during pauses in the playing.

'See Helmholtz, Physiolog. Optik, p. 740 f. $\quad{ }^{3}$ Ibid., p. 741. 
clangs into their elements is equally marked. ${ }^{1}$ In addition to a previous acquaintance with the character of such over-tones as are to be expected and analyzed out of the clang, the analysis can take place in no case without a "certain undisturbed concentration of the attention." That changes in the clearness of perceptions take place in dependence on the changes in the degree of attention is a matter of the most ordinary experience. On waking gradually from sleep our surroundings become less and less obscure to the senses of eye, ear, and skin, as the grade of voluntary attention in apperception progressively rises. On casting the glance casually upon a landscape seen through a window, its objects are, at first, scarcely perceived at all; by gradual increase in the intensity of attention (changing the casual glance into a steady look) these objects become apperceived more and more clearly. The voluntary concentration of attention (comp. p. 446 f.) often dissolves an error of sense or changes the entire appearance of the visual object. On the other hand, a great strain of attention may lead one to anticipate an expected impression of sense, and perceive its occurrence before it has actually taken place. It may also cause other illusions, as when, on expecting eagerly the stroke of the clock, some weaker sound may be mistaken for it. ${ }^{2}$

\$9. Voluntary attention directed toward the images of memory has also a marked effect upon their character and duration in consciousness. In certain cases it may impart to them the vividness of presentations of sense, although the power to bring this about differs greatly for the different senses and in different individuals. By an effort of will the player of a musical instrument can cause himself to feel again the revired images of the muscular and tactual sensations which accompanied a particular exercise of his skill. The hearer of some impressive musical air may voluntarily set it running, with its variation of tone and rhythm, through his mind's ear, as it were. Not to speak of hallucinations and visions, most men see sights in dreams, and even in reverie, that closely approach the intensity of the presentations of sense in the waking state and in broad daylight. Artists in forms of art involving a special susceptibility and activity of some one or more of the senses, are, of course, gifted with a specialized creative energy of imagination. Particular images of memory may be seized upon at will, as it were, and the attention so concentrated upon them as to impart to them much of the strength which their originals enjoyed.

Moreover, the effect of attention upon certain images of memory

${ }^{3}$ Helmholtz, Die Lehre von den Tonempfindungen, etc., p. 84 f.

'See Fechner, Elemente d. Psycho-physik, ii., p. $491 \mathrm{f}$. 
is such as apparently to localize them anew in the organs by which these originals were formed. To try to revive a melody as distinctly as possible produces a sense of strain (a feeling of being innervated) in the region of the ear. We recall sounds, especially if we recall them vividly, with the organs of hearing. The prolonged effort to recall or inage colors or visual forms tires the visual organs; the impression is as though the recollection or imagination were accomplished in and through these organs. The violin-player remembers and goes over the solo he is to play, not only in his ear, but in his arms and fingers. Upon such power of reproduction the power of new production depends. Indeed, we may say that "the activity of voluntary sensuous attention largely consists in a voluntary reproduction of earlier conditions of sensations." ${ }^{1}$ It has even been claimed that vivid representative images of color-sensations may be followed by the corresponding negative after-images. ${ }^{2}$

$\S 10$. Concerning the physiological basis of the phenomena of voluntary attention, little is known. Apparently, part of the effect must be due to the changed condition which is brought about in the end-organs of sense when especially innervated and so prepared for receiving the stimulation appropriate to them. To this fact is due the peculiar feeling of strain in the organ of attentive apperception, or of vivid reproduction of the image of memory. But the chief effect of attention is realized in the altered condition of the cerebral centres. It is only obvious, according to Exner, ${ }^{3}$ that we have to do with changes in the central mechanism set up by act of will, and that these changes vary quantitatively and concern the circles of our psychical activity. Moreover, the point of attachment, as it were, for the attention is found only after the impression of sense has been elaborated to a certain degree. We cannot voluntarily attend without perceiving, at least obscurely, that to which (presentation of sense or image of memory) attention is to be directed. Still further, fluctuations of the cerebral activity are constantly occurring; for, as every one knows who has experimented with himself (for example in determining reaction-time), it is impossible to keep attention on a perfectly steady stretch with respect to its object. Waves of consciousness in connection with these fluctuations of attention rise and fall. ${ }^{4}$ In the most successful reactions the attention is effectual in producing and main-

${ }^{1}$ Comp. G. E. Müller, Zur Theorie d. sinnlichen Aufmerksamkeit, Leipzig, p. 89.

${ }^{2}$ Wundt, Vorlesungen über Mensch u. Thier, I., p. $38 \%$.

${ }^{3}$ In Hermann's Handb. d. Physiol., II., ii., p. 283.

${ }^{4}$ Comp. Fechner, Elemente d. Psycho-physik, ii., p. 452 f. 
taining a state of strained expectation, in which the occurrence of the expected stimulation sets the motor mechanism off without, and even in spite of, a separate act of will. In such cases the cerebral centres liave apparently been thrown into an exalted and explosive state of irritability. We all know that very little suffices to set the muscles agoing when the mind is on the stretch. It is not unlikely that the effect of attention is felt in depressing certain cerebral areas not intimately connected with the production of the particular image of memory or presentation of sense, as well as in heightening the activity of others that are thus connected. ${ }^{1}$ The phenomena, at any rate, imply an increased difference of excitability and conductivity for their specific forms of nerve-commotion in the different cerebral areas. Under the influence of attention the cerebrum has become more susceptible for certain impressions, less so for certain others. ${ }^{2}$ Stored energy of the nerve-cells is being rapidly called forth. Concentrated voluntary attention implies a large amount of work being done in the cerebral hemispheres. We recognize this fact in the accompanying feelings of strain and in the subsequent feelings of brain-exhaustion. The subject of experiment to determine the reaction-time under concentrated attention often, though sitting quiet, sweats profusely.

$\S 11$. What happens when two different excitations, arising either from conflicting presentations of sense, or conflicting ideas, meet in any single region of the brain? We can only answer this question with vague conjecture. The phenomena of the conflict of colors in binocular vision seem to imply that either the more intense of the two may prevail over the other, or the two may both persist and interpenetrate as it were. In certain cases voluntary attention may determine which event shall ensue. The sugar sweetens the acid of the lemonade, not in the vessel which contains it or on the tongue which tastes it, but in the brain. According to the conjecture of Wundt, ${ }^{3}$ the frontal regions of the cerebrum are the "bearers (Träger) of the physiological processes which accompany the apperception of the presentations of sense." In order, then, that the process set up in any cerebral region by an excitation of the organs of sense connected with it may result in clear discernment of an object, certain physiological processes must be conducted from the frontal regions to that region. Wundt's conjecture is plausible, at least it gives the frontal region something to do, and answers in part the inquiry why so much of the cere-

${ }^{1}$ See G. E. Müller, Zur Theorie d. sinnlichen Aufmerksamkeit, p. 52 f.

2 See Exner, in Pflüger's Archiv, xi., p. 428.

${ }^{3}$ Physiolog. Psychologie, i., p. 218. 
bral substance should seem merely negative as respects the phenomena of sensation and motion.

$\S 12$. Nothing thus far said, and nothing of scientific value which physiological psychology has to offer, throws any clear light on the problem of the "freedom of the will." When M. Luys, " for example, maintains that to imagine "we think of an object by a spontaneous effort of mind is an illusion," and that, in fact, the object is only forced on us by the cumning conjurer, the brain, "because the cell-territory where that object resides has been previously set vibrating in the brain," he is controverting a plain and universal dictum of consciousness by his private and unverifiable hypothesis on a question of cerebral physiology where experts and novices are alike ignorant. Physiology neither disproves nor verifies the postulate of free will ; accordingly, this postulate must be raised and discussed upon other grounds. Metaphysics and ethics cannot properly dictate their facts and conclusions to the science of physiological psychology; but, in turn, this science cannot properly dictate to metaphysics and ethics the conclusions which they shall draw from facts of consciousness, by giving out its myths and fables in the garb of well-ascertained history of the cerebral processes.

$\S 13$. Consciousness, or the having any form of sentient life, in distinction from being in a condition of dreamless sleep or swooning, and Self-consciousness, or the recognition of the states of consciousness as states of the ego or subject of them all, are intimately connected with the phenomena of will. By the amount and speed of the energy expended in attention we measure in large degree the extent and intensity of consciousness. The stir of feeling through the presentation of some object of sense, or through some idea, causes us, either voluntarily or involuntarily, to rouse ourselves to what is then recognized as a wider and higher energy of conscionsness. But inasmuch as consciousness is the condition of all internal experience whatsoever, we cannot deduce or explain the essential nature of consciousness from other forms of such experience. ${ }^{2}$ For the same reason we cannot define consciousness.

Concerning the physical basis of consciousness little can be added to what has already been said concerning the physical basis of the various forms of consciousness. Consciousness is never consciousness in general-never an activity or state that may be separated from the individual states and processes of consciousness. In the case of man, the cerebrum is apparently the sole, as it cer-

1 The Brain and its Functions, p. 254.

2 Comp. Wundt, Physiolog. Psychologie, ii., p. 105 f. 
tainly is the chief, organ of consciousness (comp. pp. $249 \mathrm{ff}$.). By calling the cerebrum the "organ" of consciousness, however, little more is meant than that the constitution and processes of the nervous matter of this organ are related in the most immediate and special way to all mental phenomena, and that what takes place in material elements outside of the cerebrum (including the elements of the other portions of the nerrous system) has an effect upon consciousness only in case it gets itself represented, as it were, in the corresponding cerebral processes. As to a special organ of consciousness in the brain-that is, a cerebral area where the mind comes to consciousness-it is not proper to speak.

Accordingly, the physical basis of the different forms of consciousness is laid in those cerebral areas which have been found to be-or, though still undiscovered, actually are-especially connected with these forms. But if the question is further pressed as to the physical basis for the activities of self-consciousness, no answer can be given or even suggested. From its very nature that marvellous verifying actus of mind in which it recognizes itself as the subject of its oẉn states, and also recognizes the states as its own, can have no analogous or corresponding material substratum. It is impossible to specify any physiological process representing this unifying actus; it is even impossible to imagine how the description of any such process (in case we knew what to attempt to describe) conld be brought into intelligible relation with this unique mental power.

In general, concerning the physical conditions of consciousness, it is known that they are dependent upon the character and amount of the blood-supply. To stop this supply is to put an end for the time to consciousness; to impede or corrupt it is to depress and to disturb consciousness; to alter its character is to affect, more or less promptly and profoundly, the character of consciousness. The character of the circulation in the cerebrum largely determines the nature of the phenomena of consciousness. Quickened circulation here accelerates or agitates the circuit and time-rate of consciousness; slower circulation diminishes and inhibits them. It has been alleged by Mosso that certain changes in the relative circulation of the human body occur when the attention is occupied intensely either with external impressions or with psychical work. Such obserrations are not as yet extended and accordant enough to command unhesitating assent to the details of their results.

$\S 14$. The other group of so-called higher mental phenomena which admits of the most of probable conjecture regarding the nature of its physical basis comprises Memory, as retentive and re- 
productive, and the laws of the Association of Ideas. The experience of consciousness is one of a constantly changing succession of states. The rise and fall in voluntary or involuntary attention, and the change of its direction, are accompanied by a continual alteration of the phases and of the circuit of consciousness. Of these shifting mental states certain ones bear the peculiar mark of a claim to represent previous states of consciousness, in some regard and to some extent similar to themselves. The image of memory is itself a product, a phase, of present consciousness; it is not itself of the past, and yet it claims, by virtue of its essential character, to stand for the past. This claim can, of course, in no instance be verified by carrying the consciousness back to that past; we are never able by attentive apperception to compare the image of memory with its alleged original and thus make sure of the validity of the claim. Experience also teaches us that the mental images do not come and go wholly at random and irrespective of the characteristic content of such as are most closely connected in time. That these images are associated in time is a part of the fundamental fact of memory; mental states are not states of memory without some more or less definite localization of the ideas thus presented to the mind with reference to its past.

Further examination of the particular character of the ideas which most frequently occur simultaneously, or in closest succession, has given rise to the assumption that the images of memory are associated in a regular way. Hence those general facts of psychology called the "laws of the association of ideas." From the phenomena of memory and reminiscence, as experienced in the consciousness of the individual, arises the belief that the objects of past experience are retained in the mind, and that they suggest each other (at least ordinarily) in some orderly way. But properly speaking, the "retention" of states of consciousuess, whether of ideas or of presentations of sense, is not a faculty or power of mind. To ask, Where is the idea I once had, or the object $I$ once saw, between the time of the original experience and the time of recall, is to ask a question that can have only one answer. Such idea or presentation of sense is nowhere, for it does not exist in any sense of the word whatever. Both presentation of sense and image of memory are transitory phases of consciousness, each perishes with that phase of consciousness in which, and as which, it has its existence. It is the power of recall solely which induces us to speak as though the mental object were retained or kept in the Mind. It is only in the facts and laws of conscious reproduction that any trace of the activity of mind, as memory, is to be found. Much 
"cerebration" may be unconscious; there may be considerable periods of complete unconsciousness in the daily life of every individual, as there certainly are such periods occasionally in the lives of some individuals. But of unconscious retention or reproduction of ideas as an activity of mind, there is none.

$\S 15$. Both ordinary observation and experiment in reaction-time indicate that the speed with which the images of memory vanish depends upon a variety of circumstances-such as individual peculiarities, intensity and frequency of the repetition of the original impression, condition of the cerebral centres and of the consciousness at the time of this impression, etc. The thousands of faint impressions which enter into every-day life seem quickly to vanish, without leaving a trace behind in either body or mind. But that these impressions do linger for a time in memory, as we say, or are reproducible in consciousness under the form of images of memory, there can be no doubt. For example, if, while one person is intently counting the lines of a page or adding a column of figures, another near by makes some slight motion, the image of the motion in the mind of the former may be recalled within a few seconds of the occurrence; a little later, however, such an image may hare so completely vanished that the observer will declare the motion was not noticed at all. ${ }^{1}$ The viridness of fresh images of memory may be so great as even to make it difficult to distinguish them from true presentations of sense. But even in the case of impressions made clear and strong by the strenuous concentration of attention, the vividness of the image of memory diminishes at first very quickly. Even in such a case the so-called "primary" image of memory may not last beyond a few minutes; while in cases where there is little attentive apperception this image vanishes in a few seconds. ${ }^{2}$ Let a line of given length be regarded for a brief time, then removed, and after a varying interval the effort made to recall its image so as to compare it accurately with another line of nearly the same length. It will be found that the clearness of the image of memory, which quickly falls off at first, falls off afterward more slowly, and finally approximates more nearly to a stationary condition. Lotze ${ }^{3}$ has insisted upon the caution that we should not confuse the clearness of ideas with their intensity; the idea of the most intense brightness is not intensely bright. Yet we cannot agree with Lotze in the opinion that the ideating activity, when applied to the recall of sensations, does not differ in intensity

\footnotetext{
1 See Exner, in Hermann's Handb. d. Physiol., II., ii., p. $281 \mathrm{f}$.

${ }^{2}$ Comp Fechner, Elemente d. Psycho-physik, ii., p. 491.

${ }^{3}$ Outlines of Psychology, p. $28 \mathrm{f}$.
} 
as well as in clearness. Besides the difference in the clearness with which two persons, for example, remember a mosaic of colors, as respects all the details of what particular colors were arranged in what particular order, there is also a difference in the strength which the various revired images of the colors have in the two consciousnesses.

$\S 16$. That the mental phenomena which lead us to speak of the retentive power of memory have a physical basis there can be no doubt. This conclusion is warranted by the nuture of the phenomena themselves, by the impossibility (already alluded to) of conceiving of a permanent modification of unconscious mind, and by all that we know of the principles of biology in general and of human physiology in particular. Every sensory impulse, and every combination of such impulses, must produce changes both in the end-organs and in the central organs; and although these changes vanish, so far as their effect in the corresponding phenomena of conscious mind is concerned, they nevertheless cannot fail to leave the organs in different condition from that in which they found them. As a matter of course, the effect of stimulus upon every end-organ of sense consists in the production of molecular changes, which, on account of the principle of inertia as applied to such organ, continue for a time after the stimulus has been removed. Of this fact the existence of the after-images on the retina is the most notable example. The successive stages passed through by the after-images, both positive and subsequently negative, are themselves indicative of a series of molecular changes set up by the action of the stimulus. But the effects of stimulus must also be felt in the production of molecular changes in the central organs, in the nerveelements (especially the nerve-cells) of the cerebral hemispheres, if the sensory impulses are to result in conscious sensation and perception. Experience would then lead us to infer, further, that each combination of sensations produces changes in the cerebral hemispheres which outlast the action of the stimulus upon the end-organ of sense. A study of consciousness, simply as consisting of changing sensations and perceptions, might appear to indicate that the after-forms of molecular changes themselves die out and leare the end-organs and cerebral centres in precisely the same condition as before. But the formation of habits of perception and motion, the plienomena of conscious mental reproduction, and the general principles of molecular science as applied to the nervous mechanism, suggest and enforce another view.

Certain experiences in the use of the senses show that molecular activities induced in the end-organs by stimulation may, under certain circumstances, persist much longer than we are at first inclined 
to suppose. For example, a study of the after-images left by strong impressions on the retina shows that traces of them recur again and again, even several minutes after the eyes have been closed. Prolonged work with the microscope will cause the images seen in its focus to "live in the fundus of the eye" so that, after several hours, shutting the eyes will cause these images to reappear with great distinctness. Of a similar kind is our experience with sounds-the rattle of the railroad-car after a long journey, the impressive cries or words to which we have listened, the successive notes and chords of the musical composition heard at a concert, seem to be repeated in the ear for hours after the primary sensations have subsided. According to Dr. Moos, ${ }^{1}$ after long musical séances the sounds persisted for fifteen days in one patient; and a professor of music was accustomed to hear over again the notes sounded, for several hours after each lesson. After startling and impressive experiences with different kinds of sense-perceptssounds, sights, etc.-it is not an uncommon thing for the same bodily affections to recur with such vividness as to make it almost impossible at the instant to distinguish them from fresh experiences of the same kind.

Moreover, all those inherited and acquired unconscious habits of motion, with which the study of the nervous mechanism has already made us familiar, imply that the effects of repeated stimulations persist in the molecular constitution and tendencies to molecular change of the nerrous substance of the central organs. The puppy which has inherited a brain and spinal cord embodying the habits of his race, and the trained gymnast or skilled player on a musical instrument, alike illustrate this principle of stored and organic experience as pertaining to the elements of the nervous system. The general principle of molecular science, which finds numerous examples both in inorganic chemistry and in biology, compels a similar conclusion as to the physical basis of memory. The established practice of photography depends upon the fact that a plate of dry collodion, after being briefly exposed to the sun's rays, retains for weeks, in the darkness, the effects of the indescribably delicate changes which have been brought about in it. The "latent image " contained in it may be revived by proper treatment. The phenomena of phosphorescence, also, show that the impressions of the luminous undulations persist in certain bodies for a considerable time after these undulations themselves have ceased acting. Niepce de Saint-Victor ${ }^{2}$ has shown that such undulations may be

${ }^{1}$ So Luys, The Brain and its Functions, p. 136.

¿ Comptes-rendus de l'Acadómie des Sciences, xlv., p. 811 ; and xlvi., p. 448. 
" to some extent garnered up in a sheet of paper," ready to be revealed at the call of special reagents. Inasmuch as the nervous system consists of an inconceivably complicated and delicate molecular mechanism, every element of which may be regarded as a highly complex molecular structure, it may well be expected to accomplish much more wonderful results than the plate of dry collodion or sheet of paper, in the way of storing up for future demand the results of the impressions made upon it. We may even go so far as to say that the retentive power of this molecular mechanism is perfect; that it never loses entirely the effect of any inpression once made upon it.

$\S 17$. In view of such considerations as the foregoing it has been proposed by some writers to regard memory simply as one phase of the general biological fact, as a particular form of synthesis "of one of the primordial properties of the nervous elements." Conscious memory is then considered as "a phosphorescence of the nervous elements" plus consciousness; and this power of these elements itself is to be called a power of "unconscious reminiscences" (M. Luys) or an "organic memory" (Hering and M. Ribot). But, while admitting the general fact of molecular science, and the application of it to the phenomena of habit in the nervous mechanism as contributing something to the description of the physical basis of conscious memory, it is wise to refuse to use such terms as the foregoing. For these terms are not needed to state the facts; of themselves they lend nothing to the desired explanation, and they are liable to lead to serious confusion. "Organic" memory, or the habitual mode of the behavior of the nervous system, together with that tendency to reproduce the mode which belongs to all habits, when minus consciousness, is not memory at all ; it bears, indeed, no resemblance to memory. " Unconscious" reminiscence, regarded as a function of material elements, is not reminiscence at all.

Moreover, when we inquire as to precisely what constitutes this wonderful power of conserving the results of molecular changes induced by the action of stimuli, which the nervous system possesses, we find it impossible to give a wholly satisfactory answer. The most plausible answer consists in inferences touching the lighly probable application of certain biological laws to the special case of the nerrous system, regarded as furnishing a physical basis for the phenomena of memory. The general fact from which these inferences take their point of starting is undoubted; the entire nervous mechanism must be regarded as a vast system of interrelated elements (nerve-fibres and nerve-cells), each of which must also be 
regarded as a system of interrelated molecules. The excitation and propagation of nerve-commotion consists in producing and continuing changes in the atomic structure and mutual relations of these molecules. In order to account for that " bent," direction, or ten. dency to act in a certain way, which all habit of the nerrous system presupposes, the internal molecular alteration of the nervous elements, especially of the individual nerve-cells, has to be assumed. But the power of propagating their kind belongs to these individual nerve-cells; and it is likely that all the essential principles of heredity and evolution apply to the exercise of this power in the case of these cells.

The biological laws which control the nutrition of living organisms also have an application to the nervous elements. The exercise of any of the nerve-cells or groups of nerve-cells of which the end-organ or the central organ is composed tends to enlarge them by appropriation of the nutriment brought to them in the bloodsupply. Such nutrition, however, will necessarily be dependent, for the special type which must characterize its manuer of building, upon the acquired molecular character of the cells that build the new material into themselves. And when the cells, thus enlarged and molecularly altered according to the character and amount of their exercise, multiply themselves, their offspring of new cells will necessarily come under the general principle of heredity in its application to all living cells. Accordingly, three things must be taken into the account when considering what has been called (ineptly, as we believe) "organic memory" namely:(1) The enlargement of the single cells or fibres of which the organ is composed; (2) the multiplication of these elements so that new cells and fibres originate under the laws of heredity; (3) the internal molecular alteration of the nerve-cells and nerve-fibres. ${ }^{1}$

$\S 18$. Furthermore, it is certain that the unity and continuity of the nervous system is such, even with respect to its individual cells, that alterations in one group of elements involve alterations in other groups. Indeed, it is much more difficult to predict where such sympathetic alteration will end than to affirm that it certainly must begin and proceed to considerable lengths. The phenomena of aphasia, for example (see chap. II., $\$ \$ 25 \mathrm{ff}$.), indicate how many and intricate are the ways in which those elements of the central organs must be internally connected and related that constitute the physical basis of the memory as retentive and reproductive of the ideas and symbols of articulate speech. The phenomena occurring

\footnotetext{
' See the Vortrag of F. Hering, Ueber d. Gedächtniss als eine allgemeine Function d. organisirten Materie. Wien, $18 \% 6$.
} 
in other diseases than aphasia, and in so-called "freaks" of memory, clearly indicate the same truth. But no attempt to bring these phenomena under any strictly scientific formulas has hitherto been rewarded with any considerable success. By continual correlated action on the part of groups and areas of nervous elements more or less remotely situated, such elements become in some sort associated together; being thus associated together, they tend to act together for mutual helpfulness and modification (that is, to intensify, inhibit, or characteristically alter each other), whenever either one is in any manner roused to yield up the energy it has in store.

The physical basis of memory as retentive is therefore laid in the habit, or acquired tendency, of the elements of the nervous system-both as respects the molecular constitution of the indiviclual elements, and also as respects the association of groups of these elements more or less distant from each other. Each element of this system, especially in the more significant of its central organs, may be considered as a minute area intersected by an indefinite number of curves of different directions and orders; thus a molecular commotion in any such area may, according to its character and point of greatest intensity, run out into the system along any one of these many curves. In every such small fragment " the whole curve slumbers," although the microscope of the histologist cannot detect the full significance of the fragment or distinguish it from similar fragments of other curves intersecting each other in the same area. ${ }^{1}$

$\S 19$. The nature of the physical basis of memory considered as reproductive, under the so-called laws of association, is even more purely conjectural than that of memory considered as retentive. To speak of an excitation as imprinting itself upon the cerebral cells, and "perpetuating itself in them in the form of persistent vibrations," or to imply that mental reproduction is only the weaker "echo" of these vibratory conditions, to persist in which is the mysterious property of all the nervous elements-as does M. Luys " -is neither good physics nor good psychology. The nature of nerve-commotion, so far as we know anything about it, is not such as fitly to be described by the word "vibrations;" and that the forms of nerve-commotion, even if properly described by this word, do not "persist" within the cells, there is every reason to believe. Much more unobjectionable is the language used by $\mathbf{M}$. Ribot ${ }^{3}$ to describe

\footnotetext{
${ }^{1}$ A figure of speech adapted from Hering, Ueber d. Gedächtniss, etc., p. $15 \mathrm{f}$.

${ }^{2}$ See The Brain and its Functions, p. $147 \mathrm{f}$.

${ }^{3}$ Diseases of Memory, p. $26 \mathrm{f}$.
} 
the conjectural physical basis of the same psychical phenomena. According to the hypothesis of this authority, "determinate associations," or "dynamic affinities," are formed among the nervous elements by their acting together; by repetition these affinities may become as stable as are the primitive anatomical connections. Such "dynamical associations have a much more important part to play in conscious memory than in organic memory." A rich and extensive memory is not a collection of impressions (for all such terms as "impression," "imprint," "registration," etc., are inapplicable to the case), but an accumulation of those dynamical associations that are "very stable and very responsive to proper stimuli." The recurrence of some ideas rather than others, as started by this or that sensuous impression or other phase of conscionsness, would then depend upon the character, number, and strength, respectively, of the different " dynamical associations."

$\$ 20$. According to the physiological theory of memory, forgetfulness, or loss of memory, is to be accounted for as the result of the process of dissolution. As says M. Ribot:" "To live is to acquire and lose ; life consists of dissolution as well as assimilation. Forgetfulness is dissolution." A large amount of such "forgetfulness" must then be considered as indispensable to the exercise of memory ; for if all the alterations of the intermolecular constitution of the nerre-cells were alike conserved and propagated, and if all the dynamical associations among the different more or less remote groups of these cells were equally stable, there could be no basis laid for specific and characteristic reproduction of the images of memory. The survival of any of these associations involres the dissolution of many others. Under this general fact of the conditions of forgetfulness the phenomena of those sudden losses and disturbances which lesion of the cerebral substance often produces must be brought. Temporary forgetfulness or disturbance of memory may be assumed to be connected simply with such functional derangement of the cerebral centres as interferes with the working of the customary "dynamical associations" among the nervous elements of which these centres are composed, or with which they are regularly connected.

$\S 21$. No good ground exists for speaking of any special organ or seat of memory. Every organ-indeed, every area and every element-of the nerrous system has its own memory. This view belongs to the very essence of every theory which considers conscious mental reproduction as only one form or phase of the biological fact of "organic memory." We might properly speak, then, of the

'Diseases of Memory, p. 61. 
memory of the end-organ of vision or of hearing, of the memory of the spinal cord and of the different so-called "centres" of reflex action belonging to the cord, of the memory of the medulla oblongata, the cerebellum, etc. But if only the cerebral hemispheres are specially and directly related to the phenomena of consciousness, then it is only the organic memory of these bemispheres which can be spoken of as the physical basis of our memory. For only the molecular constitution and dynamical associations of the nervous elements of this organ can immediately determine the character of conscious mental reproduction.

Much of the foregoing language arouses a protest against such a misuse of psychological terms. The fact that repeated action under stimulation of the nerve-cells of the cerebral cortex results in a modification of their molecular constitution, and in the establishment of certain tendencies to associated action among them, is doubtless a biological fact. It is, perhaps, most important in laying the physical basis, in determining the physical antecedents and concomitants of memory; but it is not in any sense a fact of memory. It is no more fitly called "organic memory" than are the molecular alterations produced by generations of use in the wood of an old Cremona. The changes in nerve-cells are indeed far differently related to memory from the changes that take place in the molecules of the violin; but it is only the addition of consciousness to the whole transaction that gives us any right to characterize it by the word "memory."

\$22. Just as there is no such experience as that of willing in general, so there is no such experience as that of remembering in general. The image of memory always possesses certain characteristic features; and if it be an image representing the percept of some one of the special senses, its features are determined by the nature of the percept which it represents. There is sound reason for the customary form of sipeech which recognizes a good or bad " memory of the ear," of the eye, etc. Such phrases might fitly be extended to all the forms of sensation and perception; and, indeed, to all the mental experiences capable of being represented by the images of memory. It would be equally fitting to speak of a good memory of the fingers, of the tongue, of the larynx and other organs of speech, etc. Inasmuch as the sensations which arise in, and the movements which are imparted to, all these peripheral portions of the body have their representatives in certain cerebral areas, the physical conditions of the images of memory (the physical basis of the different kinds of memory) are undoubtedly laid iu these same areas. There is no one place where memory, par excellence, 
is at home in the brain; or from which it rules the different organs of expression by making involuntary or voluntary sallies forth, as it were. Yet the memory of any one thing or event involves so many complex and closely related activities of mind-and doubtless also of the brain-that it is impossible to tell how far weakness or disturbance set up at any single point may succeed in spreading itself.

As to the physical basis for characteristic weaknesses or excellences of memory (such as the inability or marked ability to remember names, or dates, etc.), and for those apparent freaks of memory which some emotion or bodily disturbance may produce, little can be affirmed with confidence. Where the mental peculiarity extends to rather a large range of subjects, such as come under one special sense, or under one of the more general forms of the operation of one sense, the natural constitution or acquired condition of the particular organs involved may be assumed to be peculiar. The phrases, "a good ear" for music, "a good eye" for form, color, proportion, or whaterer is visible, have doubtless both a psychological and a physiological significance. But what reason should exist in the brain why some particular date or name should repeatedly slip away beyond the power of attention to recall it (Forbes Winslow tells of a man who, after a fever, lost all knowledge of the letter $F$ ), while other dates or names in which we hare had little interest cling so as to make it difficult to be rid of them, even conjecture fails to make evident. That there $i s$ such physical reason, however, the phenomena of aphasia, and of other diseases of memory, as well as the results of experimentation upon animals for the localization of cerebral function, all seem strongly to indicate. The pbysical reason for those times of general depression or exaltation of conscious memory, with which almost all persons are familiar, is less difficult to assign. Such reason is to be found chiefly in the changes of character and quantity suffered by the blood-supply of the cerebral areas-especially in their effect upon the extremely sensitive nerve-cells which abound there.

All calculations as to the possibility of representing all the individual ideas and images of memory by one or more nerve-cells and nerve-fibres each, we regard as wholly useless-whether the number of nerve-cells in the cerebrum be, as Meynert calculates, $600,000,000$, or even many more, as Lionel Beale supposes. Everything which psychology teaches as to the character of the mental phenomena, and everything which plyssiology teaches as to the nature of the cerebral functions, discourages the puerile attempt to connect separate mental images or ideas with isolated nerve-cells as their product. 
\$23. It will doubtless occur to thoughtful readers that nothing which has thus far been said concerning the physical basis of will and memory is, in any true sense of the word, an explanation of these mental activities. In what sense physiological psychology can be said to explain any mental phenomena we shall consider elsewhere. But in the particular case of memory, for example, none of the relations conjectured as probably existing between the molecular constitution and dynamical associations of the cerebrum, on the one hand, and the facts of conscious experience, on the other hand, even on the supposition that these conjectured relations were all demonstrated facts of psycho-physical science, would amount to anything approaching the character of an explanation. For none of these physical conditions immediately concerns the very mental activity which constitutes the essence of conscious mem. ory. What is explained, if anything, is simply why I remember one thing rather than another-granted the mind's power to remember at all. This power is a spiritual activity wholly sui generis, and incapable of being conceived of as flowing out of any physical condition or mode of energy whatever.

The truth of the position just taken may be enforced (among other considerations) by certain conclusions which resulted from our psycho-physical study of perception. In the study of perception psycho-physics can do much toward a scientific explanation. It can tell what qualities of stimuli produce certain qualities of sensations; it can suggest a principle relating the quantity of the stimuli to the intensity of the sensation; it can investigate the laws under which, by combined action of various excitations, the sensations are combined into presentations of sense; it can show how the time-relations of the sensations and percepts in consciousness correspond to the objective relations in time of the stimulations. But for that spiritual activity which actually puts together in consciousness the sensations, it cannot even suggest the beginning of a physical explanation. Moreover, no cerebral process can be conceived of which-in' case it were known to exist-could possibly be regarded as a fitting physical basis for this unifying actus of mind. Thus also, and even more emphatically, must we insist upon the complete inability of plysiology to suggest an explanation for conscious memory, in so far as it is memory - that is, in so far as it most imperatively calls for explanation.

Any example of an act of memory will serve to illustrate the foregoing truth. Let it be supposed that one has looked for a few seconds steadily at certain pickets of a fence standing in the open sunlight. On closing the eyes, the strong positive after-image of 
the object remains for some seconds presented to the mind; this positive after-image is then succeeded by a succession of negative after-images. When these latter have subsided, one can still recall the image of the section of the fence seen some minutes since; one can recall the same image the next day, or the next week, or after an indefinite length of time. But a very marked difference exists between that which is before consciousness in these cases of so-called recollection and that which was before consciousness while the impression of sense was going though its various phases of dying away. Physiological explanations, having reference chiefly to the action of the nervous elements in the retina, may be given as to why the after-images are produced, and as to why they have the order of succession which actually belongs to them. Other plysiological explanations, having to do chiefly with assumed activities of the cerebral nerrous elements, may attempt the problem, why the image of memory is fainter than the original impression of sense, and why this image rather than some other is represented at a particular time. But all such psycho-physical explanation does not touch, does not even approach, the real mystery of memory. The positive after-image of the pickets of the fence is not the same as the percept which preceded it; nor is it the same as the negative afterimage, or the images of memory, which follow it. Yet all these images are regarded by the mind as similar to the original object; indeed, as standing for it. How can they be regarded as similar to one another, and to their common original, when no two of them are at the same instant before consciousness in order to be compared together there? The very essence of the act of memory consists in the ability to say: This after-image is the image of a percept I had a moment since; or this image of memory is the image of the percept I had at a certain time-I do not remember precisely how long since.

It would, then, be quite contrary to the facts to hold that, when the image of memory appears in consciousness, it is recognized as belonging to a particular original percept on account of its perceived resemblance to this percept. The original percept does not exist, and will never be reproduced. Even more palpably false and absurd would it be to hold that any similarity of the impressions or processes in end-organs or central organs explains the act of conscious memory. Consciousness, of course, knows nothing of such similarity in impressions and processes; knows nothing even of the existence of nervous impressions and processes. Moreover, we could never know two impressions or processes that are separated in time to be similar, without implying this same inexplicable 
act of memory. It is a fact of consciousness, on which all possibility of connected experience and of recorded and cumulative human knowledge is dependent, that certain phases or products of consciousness appear with a claim to stand for (to represent) past experiences to which they are regarded as in some respect similar. It is this peculiar claim in consciousness which constitutes the essence of an act of memory; it is this which makes memory wholly inexplicable as a mere persistence or recurrence of similar impressions. It is this which makes conscious memory a spiritual phenomenon, the explanation of which, as arising out of nervous processes and conditions, is not simply undiscovered in fact, but utterly incapable of approach by the imagination. When, then, we speak of a physical basis of memory, recognition must be made of the complete inability of science to suggest any physical process which can be conceived of as correlated with that peculiar and mysterious actus of the mind, connecting its present and its past, which constitutes the essence of memory.

$\$ 24$. We decline to enter upon the discussion of a special physical basis for the mind's power to form generalized concepts, to combine the elements of past experience into the creations of invention and art, to discover laws, and to reason about a world of reality assumed to exist extra-mentally, or about the nature and order of the phenomena of its own consciousness. There is absolutely no scientific ground on which to place such a discussion. A physical basis of the logical faculty, so far as it is a subject of either knowledge or conjecture, is laid in those general processes of the nerrous system that are correlated with the elementary forms of mental activity upon which the bigher forms are built, as it were, or which they presuppose. Particularly important is the function of articulate language in serving as a support for the logical processes. But that which is peculiar to all these forms of psychical activities, and which causes them to be spoken of as higher and more distinctively spiritual faculties, does not, as such, admit of being made the subject of psycho-physical researches. The attempt to deal with this subject psycho-physically only leads to a tedious and meaningless repetition of the phrases and statements which the psychology of consciousness correctly employs; but nothing is made the clearer by repeating words that are only applicable to psychical phenomena in connection with conjectures concerning related physical phenomena. "For all the higher spiritual faculties," says Lotze," "which consist in judgment of the relations of given conceptions, we neither know how empirically to

${ }^{1}$ Outlines of Psychology, p. 141 f. 
demonstrate a definite bodily organ, nor should we know how to conceive precisely what, that is of any use, such an organ could contribute toward the solution of the most essential part of the problem-that is, the pronouncing of the judgment itself. It is conceivable, on the other hand, that these higher activities might presuppose the complete and clear representation of the content about which the judgment is to be passed, and, consequently, also the undisturbed function of those organs which contribute, first, to perception by the senses; then to its reproduction and combination with other perceptions; and, finally, to the appropriate attachment of feelings of value to each of them." 


\section{CHAPTER XI.}

\section{CERTAIN STATICAL RELATIONS OF THE BODY AND MENTAL PHENOMENA.}

§1. The intimate relation between the constitution and functional changes of the bodily structure, on the one hand, and the character and course of the phenomena of consciousness, ou the other hand, is most easily made obvious by such alterations of experience as are connected with the use of the organs of sensation and motion. These alterations are usually sudden. Where, on the contrary, the relation of body and mind is stationary, or subject to only very slow changes, it is far less obvious; it may be, indeed, completely hidden from our observation. The relation is not for this reason, however, any the less certain and profoundly influential. Indeed, it is just those physical conditions which are part of the unchanging equipment of our lives that most surely, though most stealthily, determine the development of conscious experience. These are the influences of whose very existence we are for the most part unaware, and over the effect of which we lave comparatively little or no control. Within certain limits, one can determine the character and number of the excitations that fall upon the end-organs of sense, and the resulting changes in the movable parts of the body; one can also regulate to some extent the succession of images of memory and fancy, and so the character and intensity of the feelings and emotions that possess the field of consciousness. But one cannot determine one's own age, or sex, or race, including parentage and prenatal and infantile environment; nor can one choose one's temperament. Yet how pervasive, mighty, and enduring are the unobserved influences that flow into the conscious life of the individual from age, sex, race, and temperament!

The bearing of the foregoing remarks might be enforced by many illustrations. No one is in need of technical information to assure him that the character of his consciousness is every instant dependent upon whether his eyes are closed or open. That we hear with the ear, feel both the roughness and smoothness and 
also the heat and coolness of objects with the skin, taste with the mouth, and smell with the nose are matters of experience belonging to each moment of our work-a-day life. The pleasures and pains of sense irresistibly demonstrate the dependence of our mental states upon the condition of the body. Other common experiences, although not arresting attention in the same obtrusive fashion, nevertheless tend to confirm the same impression. The disturbances of consciousness which follow the altered bodily condition of sleep are too much an affair of daily experience wholly to escape attention. The question, Why are dreams so queer? taken in connection with our observation of the abnormal state of the sleeper's body, is necessarily answered in a way further to emphasize the general relation between bodily and mental states. Almost everyone has also particular times of experience when he is forced into the admission that the physical system is to be blamed for the altered conditions of his mental life. The loss of a night's rest renders attention to work impossible on the following day; a slight fever sets the train of memory's images and fancy's creations into accelerated and altered movement, or throws it into wild confusion; a settled melancholy comes as the obvious result of chronic dyspepsia. In these ways the popular impression that the body dominates the mind, and that bodily conditions determine our feelings and thoughts, is strongly corroborated.

But other phenomena constantly tend to confirm the other popular impression, that the mind dominates the body and makes it the servant of its feelings and thoughts. In the ordinary estimate, all performance of physical work by the body, when accompanied by the feeling of effort, is an indisputable proof of the immediate influence of the mind over the body. It is even more than this; it is a proof that the conscious self, the ego, is a source of physical energy, which pours forth, as it were, into the limbs and braces them to the appointed task. The thought of the aged but vigorous French philosopher, who insisted upon remarking how well he carried his legs, rather than upon how well they carried him, accords accurately with the popular impression. All the customary language about looking, listening, recalling, etc., as distinguished from mere seeing, hearing, and happening to remember, also enforces the same impression. The average man or woman, whose life is one of constant toil, is led to say with about equal frequency-"My limbs are tired, and $I$ must stop trying," or " $I$ am tired, but my limbs must be made to go on with their work."

Other phenomena of an abnormal kind tend to confirm still fur- 
ther the above-mentioned vague popular impression. Among them may be mentioned the wonderful cases of so-called diseases of memory or of will, and of double personality or other alienations from normal self-consciousness. In such cases strange alterations of the modes of the mind's behavior-alterations which appear to involve the suspension or reversal of some of those mental laws and activities which we are accustomed to consider among the most fundamental-seem to be connected with certain alterations of the bodily organs. The effect of certain drugs, through the body, upon the feelings and mental train gives other occasions for insisting upon the dependence of the phenomena of consciousness upon the state of the body. On the other hand, hallucination, hypnotism, and the yet more obscure phenomena of so-called " mind-reading," as well as all the phenomena to which modern Spiritualism appeals, present the question to us afresh from a somewhat different point of view.

§2. It requires more reflection upon wider experience to originate and confirm the impression that all the characteristic experiences of the individual are built upon a solid and enduring lasis of common relations which universally maintain themselves between certain types of physical constitution and activity and certain corresponding types of the character and action of the mind. The development of the child is ordinarily regarded, whether from the physical or the psychical point of view, in an isolated and disconnected way. It is perhaps noted that he has grown so much taller or heavier, or that certain external features of the body are becoming relatively more pronounced; it is also noted that he is learning to walk, to talk, to take an interest in certain things before unnoticed, and to remem. ber what he has been taught. But the close relations between the bodily changes of advancing years and the mental development of the child are not (at least until the age of puberty is reached) apt to be made the subject of careful observation. In somewhat the same way does ordinary reflection deal with the question as to any relation between the physical and the mental peculiarities of the sexes. It is common enough to note that boys and girls do not, even in selecting and conducting their plays, act precisely alike. That the former are, as a rule, taller, heavier, coarser, than the latter is patent to all observers. But that the absolute and relative development of all the organs of the male and female is different, and that certain sexual peculiarities of perception, feeling, thought, and action are constantiy related to this difference, is something which few-if they even suspect it-take any pains accurately to remark or describe. Moreover, while almost all agree that the psy- 
chical life of the adult male and female is distinguished by sexual peculiarities, there is the widest diversity of opinion as to the precise nature and range of these peculiarities.

When the inquiry concerns characteristics of both mind and body belonging to race and ancestry, the answers given by different observers seem to lose all claim to strictly scientific quality. The Frenchman does not describe himself as the Englishman describes him ; and neither one of the two can be expected to agree with the Russian as to what are the peculiarities that characterize this last type of the human species. The history of the discussion regarding the kinds and significance of so-called "temperaments," and even regarding the very existence of temperament, shows clearly how uncertain is this entire field of research.

Perhaps the most remarkable instance of firm conviction concerning the general fact that intimate relations exist between mind and body, accompanied by the utmost vagueness concerning the precise nature of the basis of such relations, may be derived from the ordinary views as to heredity. It is constantly being remarked of children that they resemble some one of their ancestors in one or more physical characteristics. But this remark is scarcely more frequent than the corresponding one with respect to mental constitution or mental idiosyncrasies. Of course the obvious implication is, that we are to look to the laws of heredity for an account of the origin of both classes of qualities ; in other words, botlı physical and mental qualities are regarded as inherited. Further than this admission ordinary reflection upon experience with facts of this order does not lead most men. It is obvious, however, that even the loose popular impression must be explained, if at all, by insisting upon much more numerous and intimate relations between body and mind than the impression would seem at first to imply. For how can ancestral characteristics be transmitted, unless they are potentially carried over in those living cells from the two parents which actually fuse together in the production of the new life; or else are also due to the prenatal conditions that control the nutritron of the infant's body before it separates from the body of its maternal ancestor? But to admit this is to insist upon the profoundest connection between the molecular structure and dynamical associations of the elements of the physical organism and the development of conscious life. It is even to insist upon the mysterious fact that the character of the conscions life is determined in no small degree by the statical peculiarities of the organism.

§3. In general, it may be said, then, that while no doubt exists 
in the popular impression as to the dependence of the mental life upon the age, sexual differences, and inherited ancestral qualities of the bodily organism, the greatest uneertainty and vagueness exist as to the nature and extent of such dependence. When this kind of inquiries is brought to the tests of science, it is found that all the evidence confirms the positive part of the ordinary impression; but it cannot be said that any substitute for the uncertainty and indefiniteness of the popular estimate has yet been found. The reasons for this failure lie in the very nature of the subject. No guidance by the immediate evidence of consciousness is possible in determining the nature of this class of psychical phenomena. What it is to will, to remember, or to reason, each one trusts himself to know as a matter of lis own inner experience. But the inquiries, How are the mental peculiarities of the different ages, or of the two sexes, to be distinguished from each other? or, How does a person of this or that race or temperament think, feel, and act differently from a person of another race or temperament? are plainly not subjects for an appeal to consciousness. Few questions can be raised, for example, about which a wider diversity of view is likely to be evoked, than the question as to how man and woman differ mentally. Yet this question must be answered, if we are to have an answer to the further inquiry concerning the correlations between sexual differences of organism and sexual mental differences.

The difficulty of simply getting at the anatomical and physiological facts necessary for an induction is scarcely less unmanageable. A great amount of careful measurement and a vast array of statisties are necessary even to tell how human beings differ in the most external features, at different ages and as between the two sexes. Certain data with respect to the height, weight, relative size of the different external members of the body, and of the brain, are obtainable ; but other data equally desirable are as yet unattainable. Coneerning the nature of the physical basis of temperament and of personal idiosyncrasies we are wholly in the dark.

There are therefore many gaps and deficiencies in both the physical and the psychical series. But where the members of both of any two series to be compared are in this condition, the laws of their relation eannot be pointed out. Nothing remains, then, but to guide ourselves as best we may by general observation of the psychical facts, and by use of such few statistical results as are available. On few points will precise conclusions be found attainable. But the one conclusion of greatest value concerns the main 
point called in question-and this is the general fact of the correlated action of the bodily organism and the mind as the subject of the phenomena of consciousness.

$\S 4$. Certain facts of general import, concerning the height, weight, comparative growth of the members, size of the brain and organs of sense, etc., which characterize the different Phases of Life may be relied on with considerable confidence. The structural and physiological development of the prenatal human being has been investigated with more or less success by embryology (comp. Part I., chap. VI.) ; but scarcely any trustworthy data exist for a comparative psychology of the fotus. It cannot be held that its sentient life keeps even pace with the formation and growth of its bodily organs-not even of those which, like the brain and the end-organs of sense, are most intimately connected with the phenomena of consciousness. ${ }^{1}$ Large or elaborate structures, such as the lungs, the eyes, the ears, etc., are formed under morphological conditions and influences with which we are only very imperfectly acquainted, without any corresponding psychical development. The brain at birth is apparently little different from the same organ a few weeks later; but at this later period an important psychical advance has been made through the activity of the endorgans of sense. This psychical advance must be represented in the cerebral areas by the formation of such molecular changes and dynamical associations of the nervous elements as constitute the physical basis of memory considered as a retentive and reproductive power.

It is a reasonable conjecture that the psychical life of the unborn child consists wholly of sensations of pressure and temperature, for the most part exceedingly transient and disconnected, occasioned by the stimulus of its changing conditions and positions in the womb of the mother. Such a low grade of mental experience -if, indeed, we are to speak of prenatal "consciousness"-can as little be accurately represented by any conscious state of the human adult as can the experience of the animals to which the structure and functions of the body of the foetus, in succession, bear more or less of resemblance. About such a matter it is safest to refuse to speculate. About one principle, however, there can be little doubt - many of those structural and physiological factors which form the most important and intimate foundation for the spiritual functions are secured only indirectly in the central organs through the cultivation giren to these organs by the use of the end-organs of sense.

${ }^{1}$ Comp. the article of A. W. Volkmann, in Wagner's Handwörterb., I., p. 563 ; and the strictures of Lotze upon it, Medicin. Psychologie, p. $546 \mathrm{f}$. 
According to Soltmann' and others, stimulation of the cerebral areas, considered as "motor" by Hitzig, in new-born animals, does not produce the usual localized movements (comp. chap. II, $\S 5 \mathrm{f}$.). The use of his hand by the child, the use of his organs of speech, etc., educates his brain. So that the dependence of mind on brain is not-whether with respect to the life before or after birth-merely direct and simple, but also indirect and complex.

§5. Chaussier considered that the growth of the foetus in length for the six months preceding birth is regular, and that it averages about $54 \mathrm{~mm}$. a month. The mean height at birth of 100 infants of both sexes, measured in Brussels, was found to be $0.501 \mathrm{~m}$. for the boys, 0.491 for the girls, ${ }^{2}$ or about 193 and $19 \frac{1}{3}$ inches respectively. The most rapid growth of the child takes place in the first year after birth; this amounts to an average of about $2 \mathrm{dcm}$. (7.87 in.). The growth of the second year is about half of that of the first year; that of the third year about one-fourth. From the fourth or fifth year until the age of puberty the annual increase of height is nearly regular, and amounts to some $56 \mathrm{~mm}$. During or shortly before this period a sudden rise in the curve of growth occurs; but after this period the rate continues to diminish until the age of about twenty-five, when the full height may be regarded as attained. In most cases, however, a slight increase takes place between this age and fifty, after which a decrease goes on-especially in extreme old age. The average height attained by the human being is an effect of race, of climate, of conditions of living and work, etc. For nine hundred persons, measured in Brussels, ranging from nineteen to thirty years of age, the mean was 1.6648-1.6841 m. ${ }^{3}$ The average height of eighty stuclents, at Cambridge, was $1.768 \mathrm{~m}$. To express the facts by the fraction of the whole height previously attained which the growth of each year amounts to-for the first year it is about $\frac{2}{5}$; for the second, $\frac{1}{3}$; for the third, $\frac{1}{12}$; for the fourth, $\frac{1}{14}$; for the fifth, $\frac{1}{15}$; for the sixth, $\frac{1}{1 \overline{8}}$; etc.

$\S 6$. The weight of the newly born infant is said by Quetelet, ${ }^{4}$ as a rule, to remain about stationary, or even to diminish a little, for some seven days after birth ; it then, like the height, grows with its maximum rapidity during the first year of life. Like the height, also, the weight at birth varies according to parentage, prenatal

\footnotetext{
1 Centrblt. Med. Wiss., 1875, p. 209.

'See Quetelet, Physique Sociale de l'Homme, II., p. 13 f. Paris, 1869.

${ }^{3}$ See Quetelet, Ibid., II., p. 19.

${ }^{4}$ Ibid., II., p. 81 f.
} 
conditions as respects nutrition, etc. The average weight of 119 infants weighed at birth, in Brussels, was found to be 3.055 kilo., or $6.735+$ lbs. aroirdupois. With this the number 3.059 kilo., given in the "Dictionnaire des Sciences médicales," agrees very closely. A year after birth infants of both sexes hare, on the average, tripled their weight. Six years more are necessary to double the weight attained at the end of the first year ; and thirteen more to quadruple it. At about the age of nineteen the mean weight of. both sexes is nearly that of old age. The maximum weight of the male is attained, as a rule, about forty ; that of the female; somewhat later. At this time the weight is about twenty times that of the infant at birth. At sixty the weight, like the height, begins to diminish. Quetelet' has attempted to establish the empirical law that, during the period of development, the square of the weight at different ages is, on the average, as the fifth power of the height; while for fully developed individuals of both sexes the weight is as the square of the height.

§ 7. The proportions which exist among the different organs and members of the human body are of interest in this connection. These proportions vary greatly for the different ages of life, but remain nearly the same for all individuals (not obriously deformed) of the same age. The parts least subject to any departure from the normal type are the most essential parts. The height of the head at birth is about one-lialf that attained on complete development-or an average of about $111 \mathrm{~mm}$. (4.37 in.). It attains about $154 \mathrm{~mm}$. by the end of the first year, and $173 \mathrm{by}$ the end of the second ; its growth of the first two years $(62 \mathrm{~mm}$.) is, therefore, more than all the subsequent growth up to complete development (when it is, on the average, $228 \mathrm{~mm}$.). The developed head is about $\frac{1}{6}$ to $\frac{1}{8}$ of the leight of the entire body. The back of the infant, however, has at birth only about $\frac{1}{3}$ its subsequent length; the arm, $\frac{1}{4}$; the leg, up to the place of bifureation, only about $\mathrm{g}^{2}{ }^{2}$ The foot of the infant (which will probably never afterward appear in its natural form and proportions) is about $\frac{1}{7}$ of the length of the body. This member has naturally, for all ages and both sexes, about the same length as the head. The hand is about ${ }_{3}^{1}$ of the length of the entire body. Unlike the head, the limbs grow rapidly after the second year; especially are they lengthened at the expense of their transverse dimensions at the age of puberty, when the bony framework is outstripping the muscles, as it were. The

\footnotetext{
${ }^{1}$ Physique Sociale, II., p. 92 f.

'See Quetelet, Anthropométrie, ou Mesure des Différentes Facultés de l'Homme, pp. 45 f., 194 f.
} 
following table 'shows the relative weight of several internal organs in the infant at birth and in the adult:

\begin{tabular}{|c|c|c|c|}
\hline \multirow{2}{*}{ Organ. } & \multicolumn{2}{|c|}{ Percentage of body-weight. } & \multirow{2}{*}{$\begin{array}{l}\text { Ratio of the two, } \\
\text { the infant taken } \\
\text { as } 1 \text {. }\end{array}$} \\
\hline & Infant at birth. & Adult. & \\
\hline 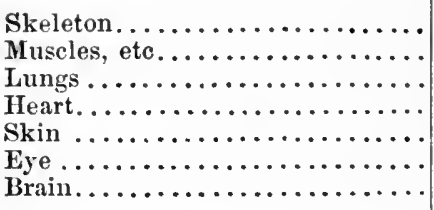 & $\begin{array}{r}16.70 \\
23.40 \\
2.16 \\
.89 \\
11.30 \\
.28 \\
14.34\end{array}$ & $\begin{array}{c}15.35 \\
43.10 \\
2.01 \\
.52 \\
6.30 \\
.028 \\
2.37\end{array}$ & $\begin{array}{c}26 \\
28 \\
20 \\
15 \\
12 \\
1.7 \\
3.7\end{array}$ \\
\hline
\end{tabular}

$\S 8$. A survey of the physical changes which take place in dependence upon the age of the human being shows that most of them are only indirectly connected with the development of the mind. The connection is, however, scarcely less strongly marked and important on that account. The changing size and weight of different members of the body, both absolutely and relatively, gives conditions to the life of sensation and motion; and it is by actual use of these members in sensation and motion that the development of the mental powers of discernment, memory, and will takes place, and all the linowledge of the spatial qualities and relations of things is acquired. The metabolic activities of the infant are much more pronounced than are those of the adult; and much of this metabolism is directed toward the ends of construction. To make the rapid growths of the first years, a great amount of food, representing a great amount of potential energy, must be converted into living tissue. More rapid metabolism is also demanded by the necessity of keeping up the normal temperature of the infant's body, which is slightly warmer $\left(.3^{\circ}\right)$ than the body of the adult, and which loses heat much faster on account of its extremely vascular skin. The heart of the infant is, relatively to its bodyweight (see table above), considerably larger than that of the adult, and the whole circuit of the circulatory system is traversed much quicker (in about 12 seconds, instead of 22). Accordingly, the heartbeat is more frequent-namely, about $130-140$ per minute, falling off to about 110 in the second year and to about 90 in the tenth year. The respiration is also more frequent-it being about 35 per minute at first, 28 in the second year, and 26 in the fifth. The brain and organs of sense are relatively very much larger in the

${ }^{6}$ See Vierordt, Grundriss d. Physiologie (5th ed.), p. 605. 
infant than in the adult, and accordingly grow less rapidly in early life.

Everything in the infant indicates, therefore, a mobile, flexible, changeable condition of the bodily organs, with a relatively large development of the most important parts of the nervous mechanism. Such a condition is significant of a paucity of bodily and mental habits; the lines of the habitual action of the mechanism, the character and number of the dynamical associations among its elements, have not yet been rigidly marked out and firmly fixed as they subsequently are. But the advanced development of the brain and end-organs of sense is significant of the potentialities, as it were, rather than of the actual experience of the babe. There is difficulty in tracing accurately the course of the earliest mental development, if by "mental" we intend to designate the phenomena of consciousness. The eyes of the child during the first days of its life are seldom open for any length of time. Preyer $^{-1}$ asserts that some newly born children move the eyes with associated and coordinated movements, others not; but there is no fixation of eyes such as indicates an act of will in attentive regard until much later, and then in such way as to show a gradual unfolding of the power of attention.

All newly born children are deaf; the temporary deafness is caused by lack of air in the tympanum previous to respiration. Great individual differences exist as respects the age at which children give unmistakable tokens of having sensations of sound. It was not until the first half of the fourth day that one investigator was satisfied his child could hear. From the conditions under which the foetus grows we might suppose that the sense of touch, as regards both pressure and temperature, would be well developed in the infant. The reflex excitability of the different regions of its skin is, however, inferior to that of the adult, and only gradually approaches it under the influence of constant cultivation. According to Preyer, ${ }^{2}$ it is highly probable that the sensations of sweet, salt, sour, and bitter are distinguishable from birth. Taste may then be said to be "instinctive" with it as with other young animals. There is more doubt about sensations of smell; according to some these are not experienced earlier than from four to eight weeks, but according to others they belong to the first days of the child's life. It is, of course, largely in connection with the unfolding of the activity of these organs of sensation and motion that its entire mental development takes place.

§. After full maturity has been attained, and the period of ${ }^{1}$ Die Seele d. Kindes, p. 25 f. Leipzig, $1882 . \quad{ }^{2}$ Ibid., p. 76. 
decline for the bodily powers has begun, the mental powers also are, as a rule, less aggressive and acquisitive, or eren begin to decline. But the period of the more immediate dependence of the latter upon the sensory and motor activities of the bodily organs has passed ; the lines of spiritual as well as of corporal habit have become firmly drawn, and both mechanism and mind may be said to contain a great amount of stored experience; judgment is trained, and less liable to sudden action under the assaults of various forms of impulse. If, then, no sudden accident or slow decay impairs the cerebral centres, the fullest and most impressive maturity of the mental powers may arrive and continue years after the activities of sense and motion are past their prime.

$\S 10$. That the two sexes differ in many ways, as respects both physical and mental characteristics, is an almost universal impression. As to what are the mental characteristics of either sex a wide difference of opinion undoubtedly prevails. But the statistics of certain plyysical characteristics of the sexes are tolerably complete. Besides the more obvious bodily differences of man and woman, the two sexes differ from birth in average height, weight, physical energy, proportion of parts, relative development of organs, frequency of pulse, respiration, etc. They also differ in many other subtler and less obrious characteristics. As we have already seen (p. 566), the height of the male infant at birth slightly (about $0.01 \mathrm{~m}$.) exceeds that of the female. The excess increases, but not with perfect regularity, until full maturity is reached. At this time the height of the man may be given as $1.467-1.890 \mathrm{~m}$. (about $4 \mathrm{ft}$. $11 \mathrm{in.}$ to $6 \mathrm{ft}$. 4 in.) ; that of the woman, $1.444-1.740 \mathrm{~m}$. (about $4 \mathrm{ft}$. $10 \mathrm{in}$. to $5 \mathrm{ft} .10 \mathrm{in}$.). The curve of the growth of the two sexes from birth onward runs somewhat differently; although up to the age of four or five the difference is scarcely perceptible. All the sexual differences are, of course, least pronounced in the earliest years of life. For these years the proportions of height remain about as 1 to 0.988 ; at complete development they are as 1 to 0.937 , or about as 16 to 15 . But at sixteen or seventeen years of age the growth of girls is relatively as far advanced as is that of boys at eighteen or nineteen. Between five and fifteen years the former make an annual growth of about $56 \mathrm{~mm}$., the latter of about $52 \mathrm{~mm}{ }^{1}$

$\S 11$. The relative weight of the two sexes raries in somewhat the same manner, but not precisely, as their height. Of 119 infants weighed at birth, in Brussels (63 males and 56 females), the average weight of the males was 3.20 kilo. (7.05 lbs. avoirdupois); of the latter, $2.91 \mathrm{kilo}$. $(6.42 \mathrm{lbs}$.). At the same age the male is, as a rule,

\footnotetext{
${ }^{1}$ See Quetelet, Physique Sociale, II., p. 15 f. ; and Anthropométrie, p. 176 f.
} 
heavier than the female; but although the boy is born heavier, and in his earliest years makes a larger gain of weight, at about twelve the two sexes have nearly the same average weight. The limits of weight for persons normally formed are about 49.198.5 kilo. (108-217 lbs.) for man, 39.8-93.8 kilo. (98-207 lbs.) for woman. Woman attains her maximum weight several years later than man.

The relative proportion of the bodily parts is different for the two sexes. At about the age of four or five the sexual differences in this regard become more observable. The bony framework of the boy is relatively prominent, and the outlines of the limbs become more clearly traced in a way to conform to agile and strong movement. Roundness of limbs and amplitude of flesh concealing the framework are more characteristic of the girl. At the age of puberty these and other similar differences suddenly become more strongly marked. Careful measurement of many individuals who have attained the development of the adult shows certain noteworthy statical differences of sex. The head, which is contained about 7.4 times in the entire height of the man, is contained only 7.2 times in the height of the woman; it is, then, relatively a little longer in the latter. The chest of the adult male is more developed. The length of the arms stretched out is about 1.045 of his height; of the female, only 1.015. The relative length of the legs is greater in the man. The circumferences of the different parts of the body are also relatively different in the two sexes. The relative step is as 1,000 to 1,157 ; and the weight of the brain as about 1,272 to 1,424 (see chap. I., § 4).

There are also marked differences between the sexes in the formation of the pelvis, and in the part of the body on which the centre of the line of length of the entire body falls. The costal mechanism of respiration differs. The girl of five breathes with her ribs as does the adult woman. The pulse of woman is quicker in abont the same proportion as her height is less. The physical energy of which the male is capable, whether as measured by lifting weights, by pressure with the hands, or other ways of producing a measurable mechanical effect, is much greater than that of the female. This follows, of course, from his larger brain and skeleton, and from his superior equipment of muscles. Before puberty the difference has been estimated as expressed by the ratio $3: 2$; after that age it is greater, and is measured by the figures $9: 5$, or is perhaps double. The average boy of nine or ten can support his own weight for some time with his hands; the girl cannot. The average man can, by using his disposable energy, lift some 154 
kilo.; the woman scarcely half as much. ${ }^{1}$ The metabolism of the female, whether measured by respiratory or other excreta, is not only absolutely, but relatively less; her blood is not only less in quantity, but also of lighter specific gravity, and contains fewer red corpuscles. ${ }^{2}$ The woman is more inclined than the man to be "hyperresthetic" (in the physiological meaning of the word); this involves a tendency to many forms of cramping of the muscles, to sudden secretions, to the wide spreading of stimulation so as to involve a considerable number of the bodily parts. ${ }^{3}$ Many of the woman's sensations are less sharply discerned as to their qualitative content, but stir up accompanying forms of feeling with more energy.

$\S 12$. In the description of those mental characteristics of sex which undoubtedly exist, and which are dependent upon or connected with the foregoing physical characteristics, a great diversity of view prevails. We cannot enter into the details of the discussion. It is plain, however, that the greater bulk of those nervous and muscular masses which are involved in the conscious life of sensation and motion both implies and necessitates great differences in the development of this life. But judgment and decision are also involved in the conscious life of sensation and motion; they are dependent upon that life for the amount, direction, and lower or higher order of their development. The superior strength of the chest, shoulders, and hips of the male, in lifting and moving heavy burdens, and the fitness of body and legs for walking firmly and running swiftly, cannot fail to produce a marked consciousness of ease, elasticity, and security, both of posture and of movement.

Other important sexual differences, consisting of variations in the kind and amount of feeling-sensuous, resthetic, intellectual, moral-and especially of the so-called emotions, are undoubtedly connected with the existence and development of those organs specifically characteristic of sex. The differences in circulation, respiration, metabolism, etc., are also the cause of characteristic differences in sentiment and feeling. Especially important, and even determinative, is the man's larger mass of nervous matter in the cerebral centres. In active energy, whether as given out on sudden call or in the form of sustained endurance of the strain of labor, and in all pursuits and achievements requiring such energy, the woman (however much she may seem to be superior in the passive endurance of pain, etc.) can never compete successfully with the mau.

1 Quetelet, Anthropométrie, p. 359 f.

2 Foster, Text-book of Physiology, p. 713.

${ }^{3}$ Lotze, Medicin. Psychologie, p. 559 f. 
Other mental differences closely related to the more obvious ones, and largely dependent upon them, are less obvious and easy to demonstrate. Our purpose will be served sufficiently by citing, concerning such differences, a few points from Lotze, ${ }^{1}$ who has treated the whole subject briefly, but with much insight and caution. In Lotze's opinion, woman naturally adapts herself more easily to new conditions of life; while acquired habits have a stronger hold on man. Her characteristics involre a mixture of the sanguine temperament and the sentimental stage; while varieties of education conceal more of native qualities. This would seem to imply a greater molecular immobility and stronger dynamical associations among the elements of mau's organism. The intellectual capacity of the sexes, Lotze thinks, differs chiefly or solely in so far as special emotional interests prescribe the course of the intellectual life. It is characteristic of masculine philosophy to analyze striking phenomena ; it is characteristic of woman rather to hate analysis. Masculine thought depends upon the conviction that whatever is most great and beautiful in the world has its mechanical conditions; masculine effort upon a profound reverence for general principles. The faith of woman is that no general principle or form has an independent value, but that this value belongs to the living reality founded upon such principle; the sentiment of the feminine mind is devout toward completeness. The notions of the two as to spatial and mathematical relations, and their perceptions as to the nature of the concrete realizations of the ideas of space and time, are markedly different. In seeking for some physical basis for these and similar differences-in case their existence be once assumed-we are forced to admit that any such known basis can be at best only indirectly related to the differences themselves. The general truth holds, however, that certain intellectual differences are intimately, and even necessarily, connected with certain emotional differences; and that the latter plainly have, in many cases, their ground in the organic differences of the two sexes.

$\S 13$. The different intellectual and emotional characteristics of the different races, and the relations of such characteristics to definite variations of the bodily type of each race from that of our common humanity cannot be discussed without raising even more obscure and doubtiul inquiries. If external influences of soil, climate, food-supply, character of the prevalent civilization, etc., have any observable influence upon the type of the bodily form-and of this there can scarcely be a doubt-and if the laws of heredity are to be allowed the scope and influence which belongs to them, the

1 Microcosmus, ii., p. 39 f. Edinburgh, 1885. 
existence of both physical and mental characteristics of race must be admitted. The popular impression confirms the assumption of anthropological science. But there are few subjects concerning which statistics and impressions are both more incomplete and more unsatisfactory.

According to Quetelet's conclusions, the proportions of the average human body are such as to render it the type of manly beanty; and the limits of these proportions are the more fixed and unchangeable the nearer we approach to perfection. In the special features of height, weight, and relative form, rather than size of the organs, certain differences appear which belong to different peoples and races. Each people may be said to have its peculiar type ; and among each people such type exists, not only in fact and determinable by scientific means, but as vaguely established in the general appreciation. According to Quetelet, ${ }^{1}$ the principal proportions of the human figure vary very little among different races of men. "The real differences which the races present appertain to characteristics which the eye seizes better than the compasses; in order to establish them firmly, an appreciation of minute differences is required, and a tact that presupposes a long experience in such researches. One can see the difficulties with which phrenologists meet in making numerical estimates of the characteristics of the skull; nothing precise can be formulated in this regard" (comp. chap. II., § 4). This conclusion of Quetelet is formulated in view of careful measurement, not only of many individuals from the modern European peoples, and of certain selected cases among the North American Indians, the Chinese, and the Kaffirs, but also of Egyptian mummies, of Greek statues, and of other means for ascertaining the proportions of ancient man.

$\S 14$. Few impressions are more firmly fixed than this, that different individuals (at least among all the more highly civilized peoples) possess, each one, a characteristic "natural disposition." Such disposition constitutes a predominating tendency to feel, think, and act in certain forms rather than others among the nany that are conceivable. The conviction that the disposition of the individual is innate and inherited, rather than the result of training or environment, is doubtless due to the fact that it appears with considerable strength in childhood, and generally maintains itself under great alterations of circumstances, and against effort, to the close of the individual's life. The so-called "disposition" can, indeed, be greatly modified, and even seem wholly chruged; but such modification is invariably made at the expense of greater energy

'Anthropométrie, p. 323. 
than is required to form and break those habits which are acquired differently in different individuals after birth. Moreover, the modification is often one of expression and power of control rather than of disposition.

Patent facts like the foregoing have given rise to the theory of Temperaments. Curiously enough, the number four has usually been chosen as sufficient to designate the kinds or types of native disposition, the varieties of temperament. The attempt has also often been made to connect the different temperaments with a bodily basis. As in several of the foregoing inquiries, so in this, our reliance is mainly placed upon the correctness of certain widespread but vague impressions. It is impossible to classify the temperaments with the use of methods required by strict scientific induction. The individual can judge of his own temperament only by remembering his actions and the states of consciousness connected with them. But upon such a point memory, and eren the immediate recognition of consciousness, are but little trustworthy. Few things are more common than for the indiviclual quite to misconceive and misinterpret his own mental states and tendencies. On the other hand, we have no means of judging the temperament of others except by their action-using the word action in its broadest signification. A large part of such judgment is unaroidably misjudgment. But notwithstanding all the doubts and uncertainties which attach themselves to the subject of temperaments, those who are carefully observant of their fellows will continue to believe that important and determining natural differences exist among them.

$\$ 15$. Some of the older treatises on psychology contained elaborate discussions of the doctrine of temperament, in which many well-observed facts and shrewd conjectures were united with no little fanciful speculation. This is to some extent true of the treatment (on the whole admirable) given to the subject, for example, by Dr. Leopold George. ${ }^{1}$ According to Dr. George, the four temperaments are defined by the nature of the interior relation which exists between perception and the affections of the mind. Thus the greater the mind's wakefulness to impressions, the greater is also its susceptibility to the feelings of pleasure or pain which are attached to the impressions. The "sanguine" temperament is distinguished by strength in this interior relation. But the greater the attention given to the objects before the mind, the greater are the emotions of hope or fearful expectation which the objects excite; and from the emphasis being laid, as it were, on this relation the

'Lehrbuch d. Psychologie, pp. 125-151. Berlin, 1854. 
the applicability of the conception of temperament to orders, families, and species of other animals as well as to man. $\mathrm{He}$ also makes the penetrating observations that Pessimism generally rests upon an individual peculiarity of temperament; and that the true art of life consists in not having one temperament, but in combining them all. "One should be sanguine amid the petty sufferings and joys of daily life, melancholy in the more serious hours of life's more important events, choleric toward impressions that fetter one's profounder interests, phlegmatic in the execution of the resolves that have been reached."

Lotze's ${ }^{1}$ treatment of the doctrine of temperaments is more extended than Wundt's, but no less eautious and suggestive. Varieties of temperament, as of all other innate natural capacities, appear to be most marked under the conditions of an advanced civilization. By the term " temperaments," according to Lotze, we understand: "(1) The differences, in kind and degree, of excitability for external impressions; (2) the greater or less extent to which the ideas excited reproduce others; (3) the rapidity with which the ideas vary; (4) the strength with which feelings of pleasure and pain are associated with them; (5) finally, the ease with which external actions associate with these inner states themselves." The ancient fourfold division of temperaments is approved by Lotze —as, indeed, it must be by all who advocate intelligently any theory upon the subject. The sanguine temperament is distinguished by great rapidity of change and lively excitability. This indicates a permanent excess of the general capacity for reciprocal excitement among all the different psychical states, and an excessive sensitiveness of the soul to all external stimuli. It is natural in children and uncivilized tribes; it is, on the whole, advantagreous to the beginnings of culture, and prevents the establishment of narrow notions and attachment to ideas acquired accidentally. But adults who are strongly marked by this temperament make the impression of immaturity, of being "grown-up children." For the temperament usually called "melancholic" Lotze prefers the term sentimental. This temperament is distinguished "by special receptivity for the feeling of the value of all possible relations," but is indifferent toward bare matter of fact. Here a lively appreciation of the harmonies and discords of surrounding objects may be combined with little inclination for hard work; a great variety of resthetic feeling, of imaginative activity, may go with theoretical vagueness and the disturbance of an established sense of duty by

${ }^{1}$ Microcosmus, ii., pp. 24 ff., Edinburgh, 1885; Medicin. Psychologie. 560 f. ; Ontlines of Psychology, p. 137. 
this æsthetic feeling. The sentimental temperament shows itself in science among those who "spend their ingenuity in constantly devising some new dress for the knowledge they have acquired;" and in art by dealing with "isolated lyric movements of emotion," without being capable of grasping them and bringing them together into a coherent whole. It is distinctive of youth and, in its most pleasant form, of those who retain a youthful disposition on into the later and the latest years of life.

The marks of the choleric temperament are "one-sided receptivity and great energy in single directions." It is therefore distinguished by diminished susceptibility to excitement, but great force and endurance in reaction when feeling has once been aroused. Its fine effect is an apparent moral steadiness of character; its uncomely eflect is obstinate and narrow perseverance in a path once entered upon, even when reasons exist for deviating from or abandoning it. Its time of most natural development is in adult manhood; but its occurrence in a notable way even among children shows it to be one of the native dispositions of the mind. Finally, the phlegmatic temperament, which is the natural temper of advanced age, is distinguished by slightly varied and slow, but not necessarily weak, reactions. Sluggishness in youth and equanimity in old age may both result from the action of this adjustment of the feelings and impressions to external stimuli and the train of ideas.

$\S 17$. The permanent common features of the foregoing views as to the nature of temperament illustrate sufficiently the real truth of the case. The doctrine as a whole is one which in its main principles is undoubtedly required by the most wide and varied observation. On the other hand, the differences in the details with which the different descriptions are filled out show the uncertainties which belong to every attempt to elaborate it. Common impressions, producing a common play of feeling and regulating the train of associated ideas, belong to all individuals. But in each individual there is something characteristic as to the mode, the intensity, the speed with which these impressions arise when the stimulus acts, then combine with one another, and so provoke feeling or regulate the ideas. In a more or less definite way, all men generalize the various individual examples and form them into classes which have necessarily lost that variety and minuteness of peculiarities that characterizes the individual and have been conformed to some idea of a type. No real individual perfectly expresses such a typical idea. But especially in those conditions of civilization where the expression of individuality in a varied and impressive way is pos- 
sible most individuals are recognized as conforming more nearly to some one rather than another of these types.

$\S 18$. As to the exact nature of the physical basis of temperament nothing is known. The influence of abnormal bodily conditions, and of certain diseases, to produce or alter the disposition of the mind in a manner resembling temperament would seem to indicate that the original constitution of the brain is not the principal determining factor. The nature of the excitation which external stimuli produce upon the end-organs of sense, the strength of the resulting reactions in the form of common feeling, the habitual condition of the internal and visceral organs and the coloring they impart to common feeling, seem to be of prime importance in determining the temperament. Further than this it is difficult to be more specific, even in conjecture. The fact that the different periods of life are apt to be characterized by a predominance of one of the four temperaments is not an argument against the physical nature of the basis of temperament in general. Certain changes in the nature, speed, and strength of the reactions derived from the endorgans and the internal organs of the trunk necessarily accompany the early development, riper maturing, and decay of the bodily powers. These cannot fail to have a great, though indirect, influence upon the activities of the cerebral centres. But where so much already said is so uncertain, we refrain from adding further conjectures.

§ 19. What has litherto been developed in detail respecting the relations which maintain themselves between the structure and activity of the nerrous mechanism and the phenomena of consciousness may now be summarized in somewhat the following way. We seem warranted in insisting that the following five great groups of correlations between body and mind are always maintained during the mind's conscious existence :

1. The quality and intensity of the sense-element in our experience is correlated with the condition of the nervous system as acted on by its appropriate stimuli. That the precise character and amount of our sensations are dependent upon what and how much of various forms of physical energy acts upon the organs of sense there is scarcely need to say. But the phenomena which demonstrate the effect of attention upon the sense-element itself prevent us from regarding the relation as only one-sided and simple. The true state of the case is never represented by considering the sensations as mere passive inpressions depending solely upon 
the kind and degree of the action which the stimuli exert. These sensations depend also on the condition of the mind at the time the stimulating effect of the excited sensorium is realized in consciousness, and in terms of consciousness. To represent the mental condition of attention as itself simply and absolutely dependent upon the condition of the centres of the brain is to cover up much of our ignorance concerning the relation of body and mind with a scanty stock of conjecture in cerebral physiology. There are many facts to countenance the reverse statement-the condition of the centres of the brain depends upon the state of the mind with respect to attention. For the present we content ourselves with this expression: The sense-element in our experience is constantly correlated with the condition of the nervous system as under excitement from its appropriate stimuli.

2. The combination, whether simultaneous or successive, of our conscious experierces is correlated with the combination of the impressions made, from whatever source, upon the nervous organism. That the number and form of the different sensations composing any presentation of sense is dependent upon the number and quality of the different excitations of the nervous system which combine in such presentation there ean be no doubt. So, too, does the order and time-rate of the phenomena of consciousness depend upon the order and time-rate of the separate excitations of the nervous system. But no object of sense can be considered merely as a compound of the elements of sensation entering into it; nor is the nature of the mental product to be derived from the physical laws according to which the different stimulations modify, support, supplement, or inlibit each other. A mental synthesis, an activity that combines under different laws from those which govern the putting together of stimuli of various wave-forms and degrees of intensity, must take place in order that one object of sense may be constructed out of several sense-elements. Again, the order in succession and time-rate of the conscious states is not a mere copy of the order and time-rate of the impressions made upon the nervous system. It is simple matter of fact that mental education in the making of those syntheses which take place in all acts of perception is necessary in order to see or touch extended "Things," as distinguished from merely having visplal and tactile sensations. Whatever special form of activity in the cerebral centres is assumed as the physical basis of this mental aet of synthesis, it is by no means certain that such cerebral aetivity does not as truly depend upon the mental act as the mental act depends upon it. Here again, at any rate, the word "correlation" seems best adapted to 
express the connection between the physical basis and the mental phenomena.

3. Those phenomena of consciousness which we designate as "menory" and "recollection," as well as the play of the reproduced images of representation in general, are correlated with the molecular constitution and tendencies, and with the so-called "dynamical associations," of the elements of the nervous system. It is not necessary to repeat in this connection what has already been said in proof of the fact that these elements furnish, in part, the necessary conditions of conscious acts of memory; and, on the other hand, that the enumeration of certain physical conditions throws no light upon what is peculiarly mental in the phenomena. To say that memory depends on the condition of the cerebral centres emplasizes the relations involved in one class of facts; to say that memory depends upon the conscious act of attention, both to the original object and to its reproduced image, presents the relations involved in another class of facts.

4. The course of thought, and all the higher forms of self-conscious experience, are correlated with the condition of the cerebral centres. The dependence of these conscious mental activities upon the quantity and character of the blood-supply in the brain, and upon the integrity and unimpeded activity of its tissues, cannot be called in question. Of the exact nature of this dependence we can form only a very inadequate picture; and we have no means whatever of subjecting this dependence to a rational explanation. But, on the other hand, many of our experiences would just as certainly lead to the conclusion that the condition of the cerebral centres depends upon the higher forms of self-conscious experience. This is true of the results of all our voluntary acts accompanied by conscions discernment and choice of one among several possible courses of action. The facts of which we are sure in such cases are these : An idea of something to be done, an idea of the means (the parts of the body to be movel, and the sensations and feelings of effort which are associated in experience with such movement), a fiat of will, and a result in sensations and perceptions showing that the movement has been accomplished. The actual movement we trace back, under the guidance of physiological facts and laws, to the starting of some form of nerve-commotion in the requisite motor areas of the cerebrum. But unless we stoutly, and from mere prejudice, refuse to acknowledge a possibility of the mind exercising any influence upon the body, we are warranted in saying that this nerve-commotion in the cerebral motor areas depends upon the preceding ideas ending in the fiat of will to exe- 
cute a certain form of external motion. At this stage of the discussion, however, we prefer to use the vague term " correlation" to indicate the mutual connections between physical condition and this class of so-called higher mental phenomena. It has already been made clear that such connections are here much less patent, direct, and susceptible of being stated in the form of general laws than are those of the first two classes.

5. The statical condition of the body (by which we mean all those inherited peculiarities of the organism, the sexual and tribal bodily characteristics, the corporal constitution as dependent upon age, which change only slowly and within narrow limits, or do not change perceptibly at all) and the general tone or coloring of conscious experience are correlated. Upon this obscure subject we may (at least at present) wisely decline to take either one of two extreme and indefensible positions. It cannot be said to be required by the facts that all the phenomena of consciousness should be regarded as strictly predetermined by the constitution, environment, and independent action of the corporal elements. The really convincing argument for all such thorough-going organicism is the wish to have it so. At another extreme stands the fanciful philosophy which considers the mind as the builder of the body-as in some way fashioning to its own inherent constitution and uses the organs of the physical mechanism. This conclusion, also, we must decline to accept without further testing. All the facts, however, do obviously impress upon us the conclusion, how pervasive, intimate, varied, and profound are the mutual relations-the correlations-of the physical mechanism and the phenomena of consciousness. Any further speculation as to the real nature of this connection, and as to the nature of the subject of the mental phenomena- of the Mind-must be reserved for the succeeding part of our inquiry. 


\section{PART THIRD.}

\section{THE NATURE OF THE MIND.}





\section{CHAPTER I.}

\section{THE FACULTIES OF THE MIND, AND ITS UNITY.}

§ 1. UP to this point in our psycho-physical investigations we have been content to speak of the mind simply as the "subject" of the phenomena of consciousness. In other words, the plienomena of consciousness may with equal propriety be spoken of as mental phenomena; for they are phenomena of, or appertaining to, what all men indicate by the subject "I" (the ego) when describing their different conscious experiences (comp. p. 3 f.). This indefinite and provisional recognition of the mind as an existence to which different states, or conditions, or modes of activity, may belong without destroying its unity, suffices for a simple description of the constitution and activities of the nerrous mechanism, and of the relations between it and the phenomena of consciousuess. But psychology, even when pursued from the physiological point of view, can scarcely be satisfied to push its inquiries no farther into the nature of mind. Psychology, from whatever point of view it is pursued, aims to perfect a science of mind. Like every other science, it strives to discover the essential nature of that which it investigates.

Pains must iudeed be taken to avoid substituting words for things, abstractions for realities. By the "essential nature" of mind we mean just that nature which is duly inferred from the phenomena as essential to their rational explanation. But there are especial and unique reasons why psychology should not willingly desist from renewed attempts at such rational explanation. Is the subject of the phenomena of consciousness-the so-called Mind-entitled to be considered as having unity and reality in any defensible meaning of the words? Is it not peculiarly entitled to be considered as a real being, with a permanent and essential nature of its own? It is impossible for human reason not to attach the greatest interest and importance to these ultimate psychological inquiries. A sentient and rational life, without any self-interest in the examination of its own permanent characteristics, and of the grounds upon which it rests, would be an absurdity. 
\$2. Various objections may be raised against allowing considerations like the foregoing to apply to the researches of that branch of psychology which is called "physiological." It may be claimed that the rational explanation of the mental plenomena belongs to Metaphysics rather than to Physiological Psychology. To a certain extent the force of such objections must be admitted. They are not of a nature, however, to debar us from the inquiries that are to be raised in the following chapters. On the contrary, the result of the discoveries made by starting from the point of view held by experimental science is such as irresistibly to urge upon us some of these very inquiries. For example, it has been shown beyond doubt that the construction of presentations of sense requires the activity of both body and mind, considered as standing in peculiar relations to each other with respect to the conditions which they furnish for the spatial relations and spatial properties of these presentations. But do body and mind themselves exist in spatial relations; and may the latter be spoken of as having spatial properties? In other words: In what sense can we localize mind in the body, or speak of the body as the seat or organ of mind? Moreover, as we observe the two classes of phenomena (the organic and the mental) the impression is inevitable that in some sort they keep pace with each other in the order of development.' This fact unavoidably raises an inquiry as to the relation of the mind to the body with respect to its origin and destiny.

As a matter of fact, moreover, it is found that those who are most inclined to complain at the introduction of any " metaphysical " inquiries into the discussions of physiological psychology are quite as apt as others to give grounds for the same complaint against themselves. They themselves rarely escape the charge of having a so-called metaphysical theory of the soul to maintain. With such complainants, moreover, it is often from the very beginning a foregone conclusion what the general nature of that theory must be. They decry metaphysics and advocate a "psychology without a soul." Yet they hold, as an unalterable but unverifiable assumption, that psychological phenomena must not be so discerned and interpreted as to seem to require for their explanation an existence called "a soul."

$\S 3$. In order to arrive at any satisfactory conclusions regarding the essential nature of the Mind, it is plainly necessary that we should take our point of starting from a consideration of mental phenomena. For these are the very phenomena for which an account is to be given; and there is no safe way of concluding what is the nature of any reality, or even of determining whether any 
assumed reality actually exists, except by considering the phenomena which are attributed to it. The questions, how far mental states and mental changes are explicable by referring them to antecedent or concomitant states and changes of states in the nervous system; and how far such mental states and changes require us to assume the existence of some other real being than the molecules of the brain and spinal cord-cannot even be properly approached without a clear knowledge of what these states and changes in themselves are. But the only way to know what mental phenomena, as phenomena, in fact are, is through observation of such phenomena by the method of introspection. We must then begin this particular part of our general discussion by changing for the time our point of view.

Much fault has been found of late with the failures of the socalled "old" psychology. It has often been explained at length that these failures were largely due to its wrong method; and, as is well known, its method was almost exclusively the method of introspection or self-consciousness. The exclusive use of this method resulted in confining the efforts of psychology very largely to the rather barren task of classifying the different kinds of mental activities, and of discussing what so-called "faculties" must be assumed to belong to the mind in order to account for so many kinds of activities. Now, classification of phenomena is certainly one important part of the work of every science ; nor should it be forgotten that much of the more recent progress in psychology is due to previous painstaking observations of mental phenomena-resulting in their classification-from the purely introspective point of view. Classification, however, is not explanation ; and the "faculties" into which the "old psychology" divided the mind were too often mere names that repeated the bare fact of the observer having succeeded, to his own satisfaction, in classifying the phenomena. It is demanded, however, in order to make real progress in psychology as a science, that the correlations, under precise and definite laws, of the mental phenomena with one another and with the events which happen in external nature shall be ascertained. Nevertheless, even after adopting this view of the problem we cannot dispense with the method of introspection; for we have no other way of ascertaining what are the phenomena that require explanation. If the further question be raised, What is the real nature of that subject of the mental phenomena popularly spoken of as the mind? we surely cannot approach the answer to this question without calling attention to the nature of the phenomena themselves.

So far as the necessities of the present discussion are concerned, 
it may be said that there are two lival and contrary ways of replying to the general inquiry into the nature of the Mind. One of these denies that, in order to account for mental phenomena, we need assume-much less are able to prove-the existence of any reality other than the material substance of the living and active nervous system (especially, or wholly, of the brain). The other, on the contrary, claims that no explanation of mental phenomena is possible without referring them to a non-material or spiritual entity as the real subject or ground of them all. Both of these ways of explanation admit of various modifications. The former, as held by its different advocates, has used different termus to set forth the relation in which it believes that the phenomena of consciousness stand to the states and activities of the brain. The latter, also, has by no means always beeu self-consistent in its adrocacy of the unique and independent character of the subject of mental phenomena. Even the power of immediately penetrating in consciousness the secret of its own interior nature has sometimes been claimed for the mind. The former of the foregoing views, in whatever particular shape it may occur, has customarily been regarded as essentially the "materialistic," and the latter as the "spiritualistic," theory of the human mind. A third view, which regards both the so-called "brain" and the so-called "mind" as merely phenomenal aspects of some one reality that is like neither, but manifests itself in botll, requires for its discussion so much of subtle metaphysics, and is so foreign to all the scientific material with which we have thus far been dealing, that it is for the present passed by with a bare allusion.

$\S 4$. In the remaining part of our discussion we shall be chiefly occupied with considering which one of the two theories just stated best accords with all the facts. These facts, which are to test the theory, are facts of the nervous mechanism, and of the correlations between this mechanism and the phenomena of consciousness. The question before us may then be stated in the following provisional form: Do the phenomena of conscionsness require for their explanation nothing more than a statement of those changes in the material mechanism with which they are obviously correlated; or do they also require the assumption of one real and non-material being as the subject and ground of them all? To repeat a remark already made, the approach to this question must be through the introspective study of mind; for only such study can tell us what the phenomena of consciousness actually are.

$\$ 5$. It is so obvious as scarcely to need or admit of debate, that mental phenomena are not identical with the changing conditions 
and activities of the nervous system. However our states of consciousness may be related to the states of the brain-even if the former are absolutely and without exception dependent upon the latter-the two are certainly not the same. What the exact states of the brain are with which any of the mental states are correlated we know only very imperfectly and by remote conjecture. But so far as we do know anything about the particular molecular activities of the central nervous system which are most directly connected with the phenomena of consciousness, they do not differ essentially from other molecular activities of this system not thus connected with consciousness. The chemical constitution and structural form of the nerve-fibres and nerve-cells of the brain do not differ from those of the spinal cord in any such respect as, of itself, to account for the difference in the relations in which the two stand to conscious mental states. They do not so differ even from the molecules which enter into the living plant or animal of much lower species, mentally, than man. It is a surprise, from which scientific investigation can never recover, to find that the connection between our: sensations, mental images, and volitions and the peculiar material constitution and functions of the cerebral mass of nerrous matter should be so intimate as it undoubtedly is. The foregoing fact shows that it is quite impossible to regard the two classes of erents - the molecular changes of the central nerrous mass and the happenings of our conscious experience-as one and the same.

All plysical events are modes of motion-alterations in the relations of the material atoms or masses to each other in space. This is as true of the human brain as it is of the clod of the valley. Its atoms cannot be conceived of as doing anything, so long as they remain material atoms, that does not essentially consist merely in changing their relations in space to other material atoms. This is the activity which chemistry supposes to be continually taking place as the work of nutrition and depletion in the nervous centres accompanies the process of thought; this is what, as general "nerve-physiology" rightfully conjectures, occurs when any form of stimulus acts upon the afferent nerve through the end-organs of sense, and corresponding states of sensation arise in the mind. But the conscious process of thinking is not the change in the chemical constitution of the nervous mass; the conscious sensations are not the wave-like movements of nerve-fibres and nervecells. It is not simply true that to identify these two kinds of phenomena-phenomena of the motion of material atoms and phenomena of change in mental states-is difficult for the average mind, but attainable by the scientific observer; it is rather true 
that no mind can frame any intelligible idea of what could be meant by identifying the two.

Moreover, the history of investigation shows that a man may be highly trained, both in the observation of the phenomena of the animal body and of his own self-conscious mind (for example, Aristotle), without even suspecting the important relation which exists between the latter and the cerebral mass. Indeed, there is no distinction which all men are compelled to make more clearly than that between their own conscious states and the changing conditions, by way of motion, of the masses and molecules of matter. All theory which assumes the possibility of identifying molecular motions of brain-atoms with the shifting forms of mental experience, or attempts to set forth the peculiar nature of the latter by simply stating the conjectured laws which control the former, in. creases the general confusion which tends to surround the whole subject.

\$6. Furthermore, it is not easy to see what conld possibly be meant, that is worth serious consideration, by speaking of the phenomena of consciousness as the product of the brain. By the word "product" we ordinarily understand the new form into which a material substance has been thrown by the action upon it of some machine or mechanism. Thus we call certain secretions of the body the "product" of the tissnes where they are secreted in somewhat the same way as that in which we speak of the products of the field or of the loom. The function of the living molecular mechanism of which certain tissues consist is exercised in producing from the pabulum brought to them by the blood the secretions of gastric juice and bile. To speak of mental states and processes as the "product" of the nervous mass of the brain, in any sense of the words corresponding to that which we rightly apply to the various secretions of the body, involves us at once in the grossest absurdities. The secretory product of the brain is the fluid found in certain of its cavities ; its nutritive product, so to speak, is the new nerrous tissue which is constantly being formed from the blood by that activity of reproducing its own kind which this tissue has in common with other living tissues. But this fluid and these newly produced molecules or nerve-corpuscles in the brain are in themselves no more like mental processes, and no nore to be identified with such processes, than are the tears that flow from the tear-ducts or the pus that exudes from a wound. In so crass a way of speaking it is difficult to distinguish what can properly be meant by comparing-under the term "product"-the relation of conscious sensation and thought to the brain with the relation of 
the gastric juice to the stomach or of the pancreatic juice to the pancreas.

There is another and more plausible use of the word "product" to describe the connection between the nervous matter of the brain and the phenomena of consciousness. When a system of material molecules is acting under relations to each other which are determined by their constitution, arrangement, and environment, we may speak of the constantly new relations which they assume as the product of their previous constitution and arrangement, and of whaterer influences act upon them from material molecules outside of the system (the environment). Thus the functional activity of the nervous centres, the complex and interacting nerve-commotions of the brain, might be regarded as the product of the matter constituting these centres. This manner of speaking has certain marked advantages. It emphasizes the merely mechanical point of view. It insists upon the valid assumption that the account of every change that arises in the material particles out of which the brain is composed must be sought for in the previous constitution and arrangement of those same particles as acted upon by stimuli either external or internal to the whole brain-mass.

Let us suppose, for the sake of illustration, an incredible increase in our powers of observation. Let us suppose that it were possible with the microscope to discern the exact chemical constitution of every molecule of the nervous substance of the brain, to watch the motion of all the atoms composing these molecules as chemical changes take place or as wares of nerve-commotion in infinite rariety move hither and thither among its countless nerve-fibres and nerve-cells. All this would be in itself absolutely nothing more than an expansion under the eye of the observer of what he sometimes sees in somewhat grosser form-for example, in the amœba-and of what he infers is constantly taking place in every kind of nervous tissue. The status of the system of moving molecules at each instant is to be explained-so far as explanation is possible-from its status at the preceding instant, in connection with any influences brought to bear upon it from the outside. Moreover, all such outside influences, so far as they are of a physical sort, are nothing but modes of the molecular activity of other material particles. Looked at in this way, the product of the brain is the molecular activity of the brain. That is to say, the function of this unique system of molecules is to be constantly in motion, in the form of activity which we have already examined as "nerve-commotion."

But if the foregoing statement be admitted, how does it help us in the least to understand the phenomena of consciousness regarded 
as the product of the brain? In order to hold that mental phenomena are related to the substance of the brain, in the same way as that in which the nerve-commotions or molecular changes are related to the same substance, we must identify mental phenomena with molecular changes. But we have already seen that it is impossible to identify the two classes of phenomena, as phenomena. The phenomena of nerve-commotion may be regarded as the product of the nervous mass in which they occur; that is, they may be attributed to the constitution and arrangement of the molecules which compose this mass, as showing what the mass can do. But the phenomena of consciousness cannot be regarded as the product of the same nervous mass in any similar meaning of the word "product."

\$. Yet another unsatisfactory way of regarding the relation between the brain and conscious mental phenomena requires a more detailed and careful consideration. Everything in our previous examination has tended to show that the molecular changes which go on in the brain, whether they are occasioned by afferent impulses that originate in the application of stimuli to the endorgans of sense, or by the modified amount and quality of the bloodsupply, are not only regular antecedents, but true causes of what takes place in the mind. All the second part of this treatise was occupied in pointing out the differcnt classes of such relations between physical antecedents and consequent mental phenomena. [The objections which are ordinarily urged against speaking of physical changes as the cause of the phenomena of consciousness will be raised and answered later on.]

It is plain to every unprejudiced mind that, in some valid sense, changes in the condition and activity of the substance of the brain are specially related to certain of the shifting phenomena of conscious mental life. From this admission, which is enforced by the entire study of Physiological Psychology, the temptation is strong to proceed at once to the completion of an apparently simple and comprehensive theory. This theory claims that all mental phenomena, whatever their varied characteristic shading, have exact equivalents, as it were, in specific forms of the nerve-commotion of the living brain. Every such phenomenon, therefore, is only the manifestation of what has previously taken place, or is simultaneously taking place, in the physical molecular structure of the nervous centres. To employ a figure of speech-for every state and action of the so-called mind in consciousness, that collection of nervetibres and nerve-cells which we call the brain exacts a payment in some special kind of coin. For example, with the molecular 
changes in the substance of the brain which may be designated $A$, $\mathrm{B}, \mathrm{C}, \mathrm{D}$, etc., the mental states called $a, \beta, \gamma, \delta$, etc., are uniformly and necessarily joined; and with the combination of molecular changes which may be described by $\mathrm{A}+\mathrm{B}+\mathrm{C}+\mathrm{D}$, etc., the mental states $a+\beta+\gamma+\delta$, etc., are as uniformly and necessarily joined. When the molecular changes recur in a fainter or modified form, as $A^{\prime}, B^{\prime}, C^{\prime}, D^{\prime}$, then there must be a recurrence of the corresponding mental states, only in fainter form, as $a^{\prime}, \beta^{\prime}, \gamma^{\prime}, \delta^{\prime}$. Finally, it is without exception true-so this theory holds-that nothing happens in the mind by way of conscious sensation, presentation of objects of sense, ideation, reproduction of mental images, and higher æsthetic feeling or intellectual processes or choice, which does not find its only real explanation in the equivalent changing states of the nervous system.

$\S 8$. Our first impression on considering the foregoing way of accounting for mental phenomena is that of a certain surprising audacity. The theory, standing on a sleuder basis of real fact, makes a leap into the dark which carries it centuries in advance of where the light of modern research is now clearly shining. Physiological Psychology, as we have been compelled to regard it, has been seen to be encompassed with difficulties at every step; and some of these difficulties appear absolutely insurmountable. It has achieved its greatest triumphs in giving a physical and physiological explanation of the variations in the quantity and quality of sensation, and of the time-rate of the simpler mental processes. But even in these domains of greatest achievement it is found that almost everything needed for an exact science of the relations of the molecular changes in the substance of the brain and the changes in states of consciousness is lamentably deficient. In the first place, little progress has been made in framing a theory of the nature of the physical changes themselves. Physical science is not as yet able to deal with the phenomena of nervous action, as shown even by a single living nerve with a muscle attached when acted upon by any one form of external stimuli ; how much less, then, with that vast complex of nerre-fibres and nerve-cells which constitutes the human brain! As to purely physical explanations of the variations in the quantity of seusations we are also in great doubt. No adequate means exist for measuring accurately the changes in the amount of all the stimuli which act on the end-organs of sense. We have less knowledge of the laws which regulate the amount of excitation set up in these organs by changes in the amount of stimuli; and scarcely any real knowledge at all of what molecular changes take place within the central organs when the afferent nerves have stirred 
them to their characteristic action. Hence Fechner's laws, considered as merely empirical statements of relations between the amounts of certain external stimuli and our judgments under extraordinary conditions as to how much we are affected thereby, are readily disputed. But considered as setting forth the essential relations which exist between the physical changes in the brain and the intensities of the resulting mental changes they are quite indefensible. Moreover, the amount of our being affected, the quantity of the sensation which results from the application of physical stimulus, can be determined only by the judgment of the same consciousness which is affected with the sensation. But judgnent itself is a form of mental phenomena for the essential part of which no physical equivalent can be discovered or even conceived of.

What has just been said concerning our inability to give a complete physical explanation of variations in the quantity of sensations applies with equal force to their qualities and time-relations. But even if the whole field of sensation, as respects the amounts, kinds, and order in time of its phenomena, were already covered by such purely physical explanation as refers them wholly to changes in the molecular condition of the brain, the above-mentioned theory would by no means be established. For in investigating the correlations which undoubtedly exist between the nervous mechanism and the phenomena of consciousness, it is found that some of these phenomena imply activities of the mind which do not admit, in any sense of the word, of being thus correlated. For an example upon this point, we may refer to what was said (Part II., chapters VI. and VII.) as to a mental synthesis being implied in the formation of all presentations of sense. "Things" are not mere loose aggregates of sensations. They are the results of mental synthetic acts, the laws of which cannot be attributed solely to the various ways in which the physical molecules of the brain are made to move by the action of stimuli reaching them. Let it be admitted that, with increased information, we should find the scale of varieties in the kinds of mental phenomena called sensations corresponding, point for point, to the scale of varieties in the manner of the motion of the waves of nervous excitation. Let it also be admitted that the scale of the changes in the intensities of mental phenomena, that are of the same kind, corresponds with equal exactness to the scale of the changes in the vibratory swing of these waves. Let it be still further admitted that, whenever any presentation of sense occurs, there exists a kind and amount of various excitations simultaneously effected in the brain which corresponds exactly to this par- 
ticular presentation of sense. The mere congeries or common occurrence of such sensations, as the necessary result of the excitations of the brain, does not constitute a real "Thing." Each "thing" implies, not simply a vast number of moving physical molecules, on the kind and amount of whose motion the phenomena of conscious sensation are dependent, but a uniting energy and a unity in mind. Fifty million molecules, even when they are highly complex and unstable phosporized compounds, gyrating in the most wonderful fashion with inconceivable rapidity, certainly do not constitute one thing. They do not, then, by molecular constitution and activities, even constitute a physical basis which is conceivable as a representative or correlate of one thing. Each molecule among them all, even in order to be conceived of as being itself one such thing among the other millions of more or less similar but not identical molecules, is dependent upon this same synthetic activity of the mind.

$\S 9$. Still further, the study of metaphysics shows us that certain assumptions, which are not of a sensuous character, or verifiable at all by an appeal to the sensations, enter into every presentation of sense. No such presentation of sense consists of a mere putting together of indiridual sensations. Whatever account one may choose to give of the nature and origin of this belief, there can be no doubt that all men do believe that the "things" they perceive are neither bare groupings of mental phenomena nor forms of the molecular motion of a nervous mass; men believe that things are real existences set in space outside of their own bodies. Things are known as real; they are supposed to have attributes; they act on each other and on us who observe them; they exercise force; they are extended and movable in space, and continue uninterruptedly through more or less of time. To be all this is necessary in order to be a "Thing." Now, the assumptions which enter into the popular belief may be regarded as all true or all false, or as partly false and partly true, in the form in which men ordinarily hold them. But not one of them is capable of being justified, or in any way accounted for, by an enumeration of the sensory states into which consciousness is thrown by the action of the stimuli on the nervous system. Much less is it accounted for by reference to certain hypothetical wave-like motions in the substance of the brain. That such wave-like motions occur we have no doubt. That the changes in the quantity and quality of the sensations are related to, and dependent upon, the intensity and the kind of these motions is a most reasonable conjecture. That the motions which are correlated with the presentations of sense differ in kind or de- 
gree from those which are correlated with mere images of imagination may also be true. It is true, furthermore, that our perceptions and ideas of the extension and motion of things are dependent for their characteristics in great measure upon the structure of the physical organism. But it is impossible to conceive of any form of molecular motion which could serve as the physical basis or physical representative of any of those metaphysical assumptions which enter into all knowledge of things. What kind of nervous action can be the equivalent of an unchanging conviction or belief in the reality and true causal energy of all things both visible and invisible? What splitting up of the chemical constitution of the molecules of nerrous substance, or difference in the character of their agitations, can be conceived of as analogous to, or serving as true cause of, the distinction which is involved in our speaking of each Thing as though it were a substance with attributes?

Reference to what has already been said (Part II., chapter X.) concerning the impossibility of assigning a physical basis to the mental operations of voluntary recollection, with its recognition of similarity, to attentive choice, and to all the discrimination which underlies the work of the intellect proper, will furnish further occasion for distrusting the above-mentioned theory. For example, the new process of physical excitation, which serves as the "basis" -so to speak-of any image of memory, may be similar to the process which served as the basis of the original presentation of sense. But the mental act of recognizing the similarity of the object before the mind to one no longer before it, and yet of distinguishing the former from the latter as characterized by the time in which it occurs, does not admit of being conceived of under any analogy to such physical processes. The same thing may be said of consciousness in itself considered, and so of every mental phenomenon considered as being what it actually is-a phase of consciousness. But to make clear this aspect of the case requires that we should resume the consideration of the so-called "materialistic" theory of the relations between mental phenomena and nervous substance from a slightly different point of view.

$\S 10$. There can be no doubt that the popular and wellnigh universal belief regards the subject of the mental phenomena as a real, non-material, and permanent being. This belief also undoubtedly regards this subject as one indivisible being, a "unit-being." In other words, the prevalent conception of the Mind is that of an existence which is spiritual and is a unity in some unique sense. The many objections which have been raised against the belief may be divided into two classes-one of which may be called 
metaphysical, and the other physiological or physical. The metaphysical objections arise, in part, from the difficulty which is felt in defining what is meant by "reality," "spirituality" (or nonmateriality), and "unity" in that strict sense in which these terms are thought to apply to the mind. The consideration, both of these objections and of that way of considering the facts which escapes them as far as possible, will be for the present postponed. But the other class of objections arises from the very facts with which it is the special business of the science of Physiological Psychology to deal. It may be summarily stated in the form of questions like the following: What kind of permanent reality can belong to a being whose essential characteristic of having various states of consciousness can be temporarily laid aside when the brain sleeps or is deprived of its blood-supply; or can be wholly lost when certain nervous centres are subjected to permanent pressure or destroyed by disease or the surgeon's knife? How can nonmateriality be affirmed of phenomena which-so far as we can trace them at all-exist only in immediate dependence upon a certain chemical constitution, structural form and arrangement, and functional activity of material atoms? How can the claim of being the highest unity be made for that which exists at all only as it is in a constant flux ; which, indeed, is possessed of its one characteristic activity of being conscious, only on condition that it divides itself into subject and object and experiences a constant change of the forms in which it is conscious?

In spite of such objections from the physiological point of view as are the foregoing, the popular assumption, when freed from its crudities and interpreted intelligently, may be shown to be the only one compatible with all the facts of observation. It may be shown that it is demanded by these facts. On the contrary, the attributing of mental phenomena to the substance of the brain (with or without including the rest of the nerrous substance) does not satisfy the facts of observation. The relation between brain and mind is not such that the former can be considered as a real being, of which the phenomena of the latter may be regarded as activities. Another real being must be assumed to exist as the subject of the mental phenomena-a being with a nature quite unlike that of material molecules.

$\$ 11$. The phenomena whose relation to the molecules of the nervous system is in dispute are phenomena of conscrousness. Nothing in regard to these phenomena is more impressive, upon first subjecting them, as such, to introspective observation, than their surprising complexity in unity. Or we may rather say, the way in 
which all states of consciousness, however different they may be with respect to characteristic quality or origin, are attributed by the conscious subject to one subject as his own states is the most surprising of all facts. This fact underlies all the truths and laws into which psychology inquires, whether starting from the physiological or from some other point of view.

It has been customary for psychologists to classify all the mental phenomena under a few so-called "faculties" of the mind. The objection has often been made to such classification that it tends to confuse or destroy a just appreciation of the unity of mind. A more obvious objection to the ordinary psychological classification is perhaps this, that it fails to take due account of the rast complexity of mental phenomena. For-it should never be forgottenmental phenomena are always, primarily considered, nothing more than events. This is true of sensations and perceptions, with all their objective reference, as well as of acts of imagination and of so-called pure thought. The yellow of the watch, the red of the rose, which I see, are modes of the affection of my consciousness. Excluding for the present all reference to any metaphysical assumptions, these colors are simply events in consciousness. The real processes outside of consciousness - the objective existences and events - to which the events in consciousness are referred by science are in no sense similar to the events of consciousness themselves. What the real process outside of consciousness is-whether it consist of rapidly vibrating waves of ether, or of photo-chemical changes in the tissues of the retina, or of nerve-commotions propagated along the optic nerve and in the upper occipital lobe of the brain-we know only by doubtful inference from certain conscious affections of our own to certain material existences assumed to exist out of consciousness. Whether the right to make these inferences be disputed or allowed, there can be no dispute over the statement that the phenomena of conscious vision are not copies of any of these external events. What is true of colors is also plainly true of smells, and tastes, and sounds. These sensations are all erents in consciousness. Recent researches into the nature of the sensations which come through the excitation of the skin show that these also are, primarily considered, mental events of various kinds; such as having the feeling of cold, or of heat, or of pain, or of pressure, or of motion, etc.

Moreover, the modern experimental view of the way in which the sensations are localized and synthetically combined to form the presentations of sense shows that the latter also must be considered as being, primarily, mental events. Of course there is a sense 
in which it is absurd to say, not simply, "Perceptions of things are always merely mental events ;" but also, "Things themselves are merely mental events." It has already been shown that certain assumptions enter into all our perceptions and conceptions of socalled "things ;" but that even the attempt to account for these assumptions, by assigning them to any conceivable form of a physical basis in the brain, leads to absurdity. Still there is also a sense in which "things," that can certainly never be any different with respect to their known qualities from the way in which they appear to us when perceired, are mental events. Sensations are mental events ; the discriminating, combining, and localizing of sensations are mental events. Things to us are never more than discriminated, combined, and localized sensations, plus the metaphysical assumptions to which reference has been made.

$\$ 12$. Reflection upon facts like those just stated leads us to wonder at the enormous complexity of mental phenomena. For the purposes of the practical life we are warranted in regarding the surrounding world as composed of a limited number of material existences that undergo little or no change from day to day. But this way of regarding the objects of experience does not at all satisfy the demands of psychological inquiry. Such inquiry considers the knowledge of external nature, as well as of what is recognized as pertaining most strictly to the world within, to involve an uncersing change in the activities of consciousness. Indeed, with the limitations already referred to, we may say that the entire world, so far as it is our world, consists only of these changing states. It follows, then, that the first truth of which we have to take account is the following: Our entire conscious existencewhether regarded simply as being ourselves affected in a certain way or as having a purely objective experience of the existence and qualities of so-called Things-is a continually shifting succession of individual mental activities, no one of which is exactly like any other or is to be considered as a mere repetition of any other.

It would be acknowledged by all that a mental state of pain on account of toothache is different from a state of pleasure in smelling a rose; that the sensation of yellow is unlike the sensation of red, and that these sensations, in common with all sensations of color, are unlike those of touching a cold piece of iron or of hearing a musical tone. All the practical activities of men are based upon the conviction that the individual things with which they deal differ from each other-not simply in respect to the gualities they have, and the degree in which they severally possess these qualities, but also as separate real beings differ. But the truth 
now under consideration reaches further than this common acknowledgment. Strictly speaking, from the point of view taken by an analysis of consciousness, the same so-called "thing" is never in experience twice the same. Every time that it exists before the mind, as a presentation of sense, it is constituted anew by an activity of the mind. Unless various localized sensations and remembered images of sensations are "synthesized" under the laws which govern such kinds of mental phenomena, there can be no presentation of sense; and the only "thing" which has immediate existence for us is the presentation of sense. Accordingly, the variety of the mental phenomena, when we begin the attempt to classify them, appears as great as that of all the individual acts and states of consciousness, whether those acts and states have reference to comparatively unchanging beings outside of consciousness or not.

Even a description of the different kinds of mental phenomena which psychological science proposes to explain would be impossible, if the foregoing truth were not merely one side of the whole truth. For what is infinite in variety and always clanging its kind cannot be described. There could then be no science of the mental phenomena, no classification of the states of consciousness, or explanation of them by relating them to each other and to the physical basis on which they are supposed to rest.

$\S 13$. Observation of the phenomena of consciousness, however, shows us that they are plainly classifiable, and that the authority on which this classifying reposes is immediate and indisputable. For the phenomena of consciousness are directly recognized in consciousness as like or unlike with varying degrees of similarity and dissimilarity. They are assigned by everyone possessed of a developed experience to this or that class, without any question that the act of classification is legitimate and correct. The number of distinguishable colors, or kinds of visual sensations, is indeed indefinitely large; and so is the number of sensations of musical tone. The power to make all the necessary discriminations in these sensations varies greatly with different persons. It is perfectly proper to say that the number of kinds of the states of consciousness arising through the senses of sight and sound varies greatly in different individuals. There are many more colors and tones for some men than for others. The qualities belonging to the sensations which follow excitation of the skin are also diverse; and, in the case of sensations of smell, the means of classification for each individual are limited chiefly by the number of the different smellable things with which he has happened to come in contact. 
It must also be confessed that the popular classifications of the states of consciousuess, as based on conscious experience itself, is not infrequently erroneous. So far as the sensations are concerned, this is partly due to the fact that they are ordinarily referred to some thing or to some part of the body, which is only approximately correct, and yet correct enough for practical purposes. The taste of the onion is no less clearly distinguished from other states of sensation arising through the mouth because the subject of the taste does not know that his sensations are in fact largely sensations of smell. Because one believes that one tastes the pepper instead of feels it with the tongue, one has lost none of one's power to make clear discriminations in the domain of consciousness. Nor is this power taken from the color-blind, although the length of their color-spectrum is shortened so that they have a less extended domain of sensations of this order within which to discriminate.

It appears, then, that likenesses and unlikenesses in the phenomena of consciousness, and an immediate "awareness"-so to speak-of these likenesses and unlikenesses, or direct discrimination by consciousness of the quality of the states of consciousness, as such, are involved in that classification of the mental phenomena which all men make. If the classification becomes more refined and elaborate, it at no time ceases to repose on the same foundation. All classifications of mental phenomena which have any other foundation, or which introduce any other consideration than the simple one of how $I$, the conscious subject, am affected, are not really classifications of mental phenomena as such. Such alleged classifications may indeed tell us much that scientific inquiry desires to know about the origin or correlations of the mental phenomena. They may assume to say what special part of the external organ of sense, or what special area of the brain, is more directly concerned in producing the physical state which precedes or causes the mental phenomena. They may demonstrate what kind of physical stimuli result, respectively, in exciting such and such states of consciousness. But they tell nothing whaterer as to what the phenomena really are, whether like or unlike, or as to how they may be ranged and rated together. They are classifications of the causes, or occasions, or physical excitements of the mental phenomena ; they are not classifications of mental phenomena, as such.

\$14. Moreover; the principles which regulate the formation of classes among the conscious states of the mind are very different from those which regulate the proposal to classify the elements of the physical basis of these states, or the molecular activities of these elements. To be sure, the Young.Helmholtz theory of vis- 
ual sensations proposes to distinguish red, green, and yellow as the three kinds of retinal elements; and the opto-chemical theory of vision speculates as to the classes of pirments connected with the seeing of various colors. It has been held by some physiologists that the two main parts of the inner ear (vestibule and cochlea) correspond to the two principal classes of sensations of sound (to noises and musical tones); or even that the vibration of an individual nerrous element in the organ of Corti represents, in some sort, each definite one among the many states of consciousness that constitute our hearing of a melody. Sensations of feeling may be classified as of peripheral or central origin, in reference to the place from which the nervous impulses occasioning them are supposed to take their rise. But one need not be deceived by all this. The "red" retinal elements are not themselves red ; and if they did appear of this color to external observation, it would not in the least degree help us the better to understand what is the quality of that state of consciousness which we call the sensation of red. The different nerrous elements of the organ of Corti may be capable of being arranged in a scale ; if so, they are classified as longer or shorter, and as arranged in space one above the other. But this classification is in no real respect like that which we make when we arrange the states of consciousness called musical tones in a scale of their pitch.

In general, the physical elements of the nerrous system are divisible into classes according to their chemical constitution-as, for example, the phosphorized and the non-phosphorized proximate principles of the brain ; or, according to their structural form, into nerve-fibres and nerve-cells of a great variety of shapes, sizes, etc. ; or, according to their physiological function, into afferent and efferent, reflex or inhibitory or automatic. But no such principles of division can be carried over to the mental phenomena. They have absolutely no applicability to these phenomena, as such. We cannot introduce distinctions of chemical constitution, structural form, and physiological function into sensations and ideas, into feelings and thoughts and acts of will. A phosphorized thought, or a stellate feeling, or an afferent thought, are phrases that have no meaning.

Nor should we be any better able to apply the principles by which we classify the different factors of the physical basis of consciousness to the phenomena of consciousness itself, in case we had a much more minute acquaintance with those factors. Let it be supposed that psycho-physical science should so far advance as to be able to tell precisely what kinds of molecular activities of what nervous elements are correlated with all the various classes of 
mental phenomena. The latter would still remain precisely such as they are in kind; they would still have to be classified, if at all, on the ground of their likenesses and unlikenesses as directly known in consciousuess. If it should be discovered that the molecular agitation of a particular group of fibres and cells in the organ of Corti is necessary in order to produce the sensation of the musical note $a^{\prime}$, and that this agitation resembles that which somewhat similar fibres and cells in the retina undergo preceding the sensation of a particular shade of green, the two sensations would then be no easier to classify together than they are now. Both stand near the middle of their respective scales. In that respect it might be said that the sensation of the musical tone $a^{\prime}$ is like the sensation of green. This point of likeness is really, however, one that appertains solely to a physical quality or function in certain bits of nerrous matter more or less directly connected with the physical antecedents of the sensations; it cannot be conceived of as appertaining to the sensations themselves.

$\$ 15$. Considerations derived from the theory of the quantity of stimuli and their resulting intensities in the states of consciousness might be urged in the same direction. The variations in the amount of the molecular processes within the nervous system are all measurable and classifiable, if at all, on principles with which physics is familiar. The excitation in the nervous system always consists simply of so many molecules, having such a chemical constitution, moving so much in such a direction with relation to each other. But the most nearly related mental phenomena are feelings, and judgments comparing feelings, such as can be expressed only somewhat as follows: "Now that shade of red seems to me a little more intense ;" or, "now I think something has been added to the sensation of pressure;" or, "I should call this note the major third of the other." In general, we may say that, quantitatively considered, the changes in the nerrous mechanism are all precisely alike as to kind-that is, are all that mode of motion called "nervecommotion "-and admit of being measured off by a common physical standard or unit of measure. But the mental phenomena are classifiable only as a succession of states, that are continually changing their quality and have no quantitative measure which we can separate from these changes of quality. Nerve-commotions can be conceived of as added and subtracted, switched to the right or to the left, concentrated, distributed, dissipated. But, strictly speaking, the resulting mental phenomena admit of no treatment of this sort. Every sensation exists, if at all, as an inclivisible qualitative state of consciousness; it caunot even, as such state, be retained to 
be compared with some other succeeding state, in order to decide whether it is more or is less in respect to quantity. Without memory and comparison, which are complex activities of mind different from the mere having of sensation, we could not affirm of any mental state that it is more or less than another.

Still further, other forms of mental activity which have an undoubted reality do not admit of even the loose application to them of terms of quantity which is proper when speaking of sensations and feelings. A "weighty" judgment and a " high" ideal are not to be measured by standards that admit of correlation with the increasing or diminishing swing of nervous molecules. Yet if those classes of the phenomena of consciousness which these words imply have, as such, any plyysical basis whatever, this basis must consist in some form of nerve-commotion as to the quantity of which an exact measurement is conceivable. If, however, a wide swing of the molecules were found to go with a good, sound, clear judgment, and a contracted swing to be the physical cause of narrowness of mental apprehension, such a fact would not help us in any regard the better to revise the Aristotelian classification of the syllogism. Reasoning, as a mental activity, would be deductive or inductive, analytic or synthetic, as before, after making the discovery that the one process is connected with a continual diminution of the cortical arens over which the nerve-commotions spread themselves, and the other with a noble diffusion of such commotions over an ever-widening expanse of the brain.

$\S 16$. It is an undoubted fact, however, that mental phenomena admit of a fairly satisfactory arrangement into classes. The arrangement can be made only upon the basis of their likenesses and unlikenesses as known in consciousness and by consciousness. No other arrangement of these phenomena, as such, is possible-none that is not founded upon the same ultimate facts thus distinguished. All attempts at an arrangement of them by other principles, and from other points of view, result in the classification of something else (such as the physical antecedents, causes, or concomitants) than the phenomena themselves. It is not strange, then, that the "old psycliology" won its principal triumphs, by the method of introspection, within the field of classification. The crude beginnings of a physiological psychology in the phrenology of Gall and Spurzheim were obliged at that time to accept from introspective psychology its division of the mind's activities into so-called "faculties." In its more scientific and experimental form at the present time physiological psychology is just as dependent as ever upon introspective psychology for a classification of the mental 
phenomena. The introspective science of mind has already arrived at very geveral agreement upon this point.

It is very generally agreed that all the mental phenomena are classifiable under three great heads-into phenomena of knowing, phenomena of feeling, and phenomena called acts of will. The distinction of the two latter classes of phenomena is, indeed, a matter established in comparatively recent times. A certain number of investigators, who use the method of introspection, still venture to affirm that acts of feeling in the form of desire are not to be distinguished as differing in kind from acts of will. In their view, so-called acts of will are resolvable into phenomena of feeling. But the opinion of the great majority of students of psychology is decidedly in favor of adhering to the threefold division of the mental faculties. There is also a large amount of agreement as to the sub-classes that fall under these three-at any rate, with the exception of the varied efforts made to deal with the very complex phenomena of feeling in its many forms. The more recent attempts (made especially by certain English writers on psychology) to depart from the accepted classifications of the "old psychology" have attained little or no valuable result. These attempts have introduced into psychology a great number of uncouth terms, derived largely from false analogies of physical science, which tend to represent the case as though sensations could be weighed or measured or compounded like the nervous shocks which cause them, and as though ideas could become "agglutinated" or "agglomerated" like globules of mercury or minute particles of water. Such attempts, however, have thrown no light on the uature of the mental phenomena, or on the question of their correct classification; they have not really succeeded in supplanting or discrediting the classifications of the "old psychology." Nor is it at all likely that the principal classes into which all mental phenomena are now thrown will ever be changed. The "faculties" of the mind-however the term is to be understood-will remain the same. At all events, if a change should be made in these divisions, such change could only be accomplished by the method of introspection.

$\S 17$. The question may now be raised: How are we to account for facts like the foregoing? In attempting an answer to this question, the great significance of the facts themselves should be recalled. It has been shown that mental phenomena cannot be conceived of as identical with the molecular motions of the nervous mass; and that the fundamental relations between the two cannot be expressed by the statement, the phenomena of consciousness are the product of the brain-in any meaning of the word "product" 
which can be clearly defined. Nor can the theory be accepted that every mental state and process has its exact equivalent, with respect to all its factors, in some antecedent or concomitant state or process of cerebral nerve-fibres and nerve-cells, and that therefore all mental phenomena are to be directly and exclusively referred to these physical structures as their sole subject or ground. Moreover, it has been found necessary to admit that mental phenomena, as such, can be classified only by introspection; and that the principles on which the classification must be based differ from those applicable to the nerrous substance, while the actual classes discovered by the only available method do not correspond to any of those with which physies and physiology make us familiar. And yet there is no insuperable difficulty in classifying the phenomena of mind. Introspective psychology furnishes us with a classification, on the whole, tolerably satisfactory

What, then, is the fair inference from all these facts with respect to a decision between the two theories of mind which were previously proposed? (See § 3.) Plainly, such inference favors looking toward some other subject or ground of the mental phenomena than the nervous substance of the brain. Its result commends substantially the same view as that held by the great majority of mankind. We shall state and explain this view, however, in different terms from those employed by this majority.

The phenomena of human consciousness must be regarded as activities of some other form of Real Being than the moving molecules of the brain. They require a subject or ground which is in nature unlike the phosphorized fats of the central masses, the aggregated nerve-fibres and nerve-cells of the cerebral cortex. This real being, thus manifested immediately to itself in the phenomena of consciousness, and indirectly to others through the bodily changes, is the Mind. To it the mental phenomena are to be attributed as showing what it is by what it does. The so-called mental "faculties " are only the modes of the behavior in consciousness of this real being. We actually find, by the only method available, that this real being called Mind behaves in certain perpetually recurring modes; therefore, we attribute to it certain faculties. The mental faculties, then, are not entities that have an existence of themselves; nor are the individual behavings of the mind (the so-called "ideas") existences that can become "agglutinated" or "associated" or "compounded" in any way. They are not divisions of the mind; nor are they powers of the mind, if by this word be meant some permanent recognizable reality, stored up in a spiritual subject, or attached to it or inherent in it, after 
the analogy of the relation of plyysical forces to their subjects, the atoms. The faculties of the mind are the modes of the behavior, in consciousness, of the mind. And the very nature of the classifying acts which lead to their being distinguished is explicable only upon the assumption that a real being called mind exists, and is to be distinguished from the real beings known as the physical molecules of the brain's nervous mass.

That the subject of the states of consciousness is a real being, standing in certain relations to the material beings which compose the substance of the brain, is a conclusion warranted by all the facts. That the modes of its activity in consciousness are correlated under law with the activities of the brain-substance is a statement which Physiological Psychology confirms; one upon which, indeed, it is largely based. It will be our task, in a subsequent chapter, to consider under what general terms such correlation may best be expressed. All pliysical science, however, is based upon the assumption that real beings may have an existence such as is sometimes called "independent," and yet be constantly related to each other under known or discoverable laws. If this assumption could not be made and verified, all the modern atomic theory would stand for notling but a vain show of abstractions. Upon what grounds of reason or courtesy-we may inquire at this point-does Materialism decline to admit the validity of similar assumptions as demanded by mental phenomena?

$\S 18$. The foregoing view of the mind and its faculties is greatly confirmed by another consideration. Consciousness has a certain remarkable unity. If the complexity of mental phenomena is bewilderingly great, the unity of consciousness is striking and unique. Many disputed questions may be raised touching the essential nature of this unity, the means we have for recognition of it, and the inferences which may legitimately be drawn therefrom. Some of these questions will be merely alluded to at this point, and their further consideration postponed until later on.

All developed forms of consciousness involve an attribution of the present particular state of consciousness to a subject of the state. It is for this reason, as has already been remarked, that we express each state of our consciousness by saying: " $I$ am in such or such a condition;" "I feel thus and so;" " $I$ see, or hear, or smell, or taste, or think, or plan," etc. Such language-and all language designed to describe our mental phenomena is such-plainly shows that some kind of distinction is made by everyone between the state and the "ego" which is the subject of the state. It is no adequate explanation of this fact to say that by the subject, in all 
these sentences (the " $I$ " to which the states are attributed), we mean to denote a mere concept of myself formed upon the basis of past experience. Such a concept may indeed be formed. Its completeness and correctness furnishes an excellent test of the amount of development attained by each individual in self-knowledge. Few individuals, however, would be found able to give a statement, at all satisfactory to themselves or to others, of just what characteristics are to be considered peculiar to their "self-hood." We do not, then, merely designate the "self" when we thus constantly refer to the "I" which is the subject of each state. Our knowledge that the state is our state, or that we are in this definite individual state, is perfectly clear and immediately conclusive with reference to all the experience we have or can remember.

The clearness and immediateness of the reference which we are continually making of our states to the subject of them all is in marked contrast with the obscurity and indirect nature of the concept we are able to form as to what manner of persons we are. Moreover, all the ability we have to frame a concept of the "self" is dependent upon this constant, primary, and incxplicable fact of a possible reference of each state to the subject of the state (the "I"). To explain this reference, we have to assume that it has ahready been made; we have to assume it in each attempt at explanation. We nay express the absurdity of the effort to think or imagine ourselves out of the reach of this form of all consciousness by asking ourselves such questions as follow: How can there be a pain, a sensation, a thought, an act of will, that is not somebody's pain, sensation, thought, or act of will? What is a state of consciousness considered as separable, or actually separated, from a subject of such state? That is to say, no state of consciousness can even be conceived of that does not involve this same reference. There may, indeed, be great doubt whether some of the lower animals ever make any such reference. It may be that the amœba or the oyster can have a sensation that is not, quoad sensation, the conscious state of the amœba or the oyster. As to this we cannot say. But we can say that if such a so-called sensation is possible for any animal, it is impossible for us to imagine it. We cannot imagine what we cannot bring under the unchanging forms of our own consciousness.

The force of the foregoing remarks is not destroyed by calling attention to the fact that the attribution of the state of consciousness to the subject of the state, to the "I," is by no means continually being made. It is plain that some distinction must here be drawn between being conscious and being self-conscious. The 
crowd intently watching a tragedy in a theatre, or a conflagration, is certainly not unconscious, but is rather in an exalted state of consciousness; on the contrary, he who is intently watching the spectacle is not at all, or is only in a slight degree, self-conscious. There are considerable periods of every day when, so far as we can remember, we liave been "conscious" (not being in profound slumber or having fainted away), but with little or no reference in consciousness of its activities to the subject of them all. Nevertheless there can be no doubt that we are capable of this reference; that it is found to be involved in every mental state just so soon as we seek to determine the factors of such state; and that to recognize its being there is essential to any explanation of the nature of mind.

\$19. All the different mental phenomena of an individual must be regarded as states of one consciousness; they are all said to occur in the unity of consciousness. There can be no doubt that every person (with the exception, at most, of certain rare cases of socalled double consciousness) attributes all the forms of his consciousness to one and the same subject. This is what is meant by saying that he regards them all as his states. We cannot conceive of ourselves as dubitating whether some particular pain or pleasure, or act of memory or of imagination or of will, present in consciousness, is to be ascribed to our ego or not. We cannot attribute any such state to some other than our own ego. It is true that in certain cases of disease or lesion of the brain an abnormal condition in this respect seems to occur. The one person sometimes seems to pass back and forth between two mental lives, which are so distinct from each other that they may well be said to belong to two personalities. But reflection upon these abnormal cases only makes the stronger and clearer our conviction as to the unity of consciousness. Living two seemingly distinct mental lives is not possible without its being assumed that each one of the two is lived in the unity of its own consciousness. This would be as true of twenty distinct lives, if they followed each other in the case of any individual as the result of disease of the brain, as it is of two such lives.

It is not at all surprising that the fact of the primary unity of consciousness should be inexplicable ; for it is itself the fact implied and assumed in all attempts at explanation of other mental facts. Were this the line of thought necessary to introduce at this point, it might be shown that all the unity possessed by "Things" is dependent upon the unity of consciousness. Without memory and judgment there can be no perception of Things. But the bind- 
ing force of memory is dependent upon this unity. We cannot remember that which has not in some form or other been previously present in consciousness, in our own consciousness, in one and the same consciousness as that in which the remembered image is now present. We cannot judge except by uniting two terms in one consciousness.

Of course, all language as to the unity of consciousness, when carefully examined, turns out to be figurative, and to have no meaning except as interpreted over from entities and relations of a material sort into terms of consciousness. By the "unity of consciousness" it cannot be meant that consciousness is some kind of an entity which remains one and unchangeable throughout, like those atoms which physical science has supposed to constitute the whole world of material reality. It will be found, however, that no conception can be formed of the unity which is supposed to belong to the atom without involving in it the unity of consciousness. We can, indeed, picture to ourselves a very little bit of extended matter, barely risible under the highest powers of the microscope, which never changes its shape or color, etc., and which always behaves itself in exactly the same way under precisely similar circumstances. But this mental picture would itself have any unity belonging to it only as it existed in the unity of consciousness. It is this unity which makes each "Thing" to be one thing; it is this unity which imparts to all else that is one whatever unity it may have.

When, then, we speak of the unity of consciousness we mean, first of all and chiefly, to call attention to the following primary fact of experience : All states of consciousness involve a reference of the state to an "I," as the subject of the state; and, in spite of the constant change of states which goes on, so that in reality the same state never recurs, and even the same thing is never twice known, all the states are understood to be states of one and the same subject. This reference and this understanding enter into all our experience; they give conditions to experience and make it possible. Whatever changes experience may be conceived of as undergoing, they, as conditions of all possible experience, must be conceived of as remaining. To ask us to try to imagine a mental state or act not involving this reference and understanding, with respect to the unit-subject of consciousness, is to ask us to try to be conscious and unconscious at the same time. The "I" may become unconscious; that is, the phenomena of consciousuess in that connected development which characterizes the individual may cease to exist. But phenomena of consciousness cannot be con- 
ceived of as occurring without being referable to some one subject as its modes or states.

$\S 20$. Metaphysics, presuming upon its intimate relations to the "old psychology," has doubtless often made an unwarrantable use of the facts above mentioned. It has often declared that we have an immediate and indubitable knowledge of the mind as one and the same real being in all acts of consciousness. The facts have been interpreted as though the case stood as follows: I have the power to look within inyself, and by thus looking I can discern what I really am. I immediately know (that is, know by the introspective act of self-consciousness) that " $I$ " am always, however my states may change, one and the same real being. I am a real, selfidentical entity; and if asked how I know that I am all this, my appeal is to the indubitable evidence of the act of self-consciousness.

The foregoing metaphysical statement of the case is by no means obviously correct; we believe it, on the contrary, to be exaggerated and incorrect. In thus overstating the case, there is liability that the case itself will be lost. Consciousness carries with it no immediate knowledge of any real and self-identical being-not even of that real being which we call Mind and, with good reason, assume to exist as the ground or permanent subject of mental phenomena. Metaphysics is the science which treats of those assumptions that underlie all of our experience with what we call "reality." But it treats of assumptions or beliefs such as we find do actually and inevitably enter into all our experience. The real existence of "Things," whether of the masses of matter we daily test by the senses, or of those hypothetical beings called atoms which physical science requires in order to account for the plienomena, depends upon such assumptions. If it be admitted that we cannot be immediately conscious of ourselves as real unit-beings, we are no worse off than we are with respect to our belief in the existence of any of the socalled real beings of which all men suppose the world to be composed.

It can also be shown that the case of the mind or soul, with respect to its unity as a real being, is made no better by admitting that an immediate consciousness of ourselves as such unit-beings is possible. For let it be supposed that by concentrating all my attention upon the present state of consciousness I most clearly and indisputably discern myself as one real being, forming the ground of that state. Let it be supposed that every half-hour in the day I repeat this mental act. It would still have to be assumed, as somewhat altogether out of consciousness, that the real being discerned 
in any one of these acts of introspection is one and the same real being as that discerned in all the rest. A real unit-being that should last only while the difficult act of concentrated introspection was taking place would be of no value to serve as a self-conscious mind. In fact, such a nnit-subject of the individual state would have no claim to be considered as a real being at all.

$\S 21$. The grounds on which depenids the assumption, that the subject to which all the phenomena of consciousness are actually referable is one real being, will be considered more in détail at another point. For the present we merely adopt the assumption, provisionally, as much more probable than any which accounts for our conscious reference to such subject, by enumerating certain possible relations into which the masses and molecules of the brain may be thrown as conditions of the empirical unity of consciousness. That there is such empirical unity of consciousness there can be no dispute. Dispute itself would assume it. It scarcely admits of more doubt that all physical theories to account for this unity are wholly unsatisfactory. We know, indeed, certain of the physical conditions and concomitants of consciousness. If oxygenated blood is shut off from the cerebral substance, consciousness disappears. If the blood has floating in it certain drugs, or products of the combustion of tissue, consciousness is disturbed. If certain cerebral areas are injured or eradicated, the psycho-physical basis of certain forms of consciousness is altered. Still, all this does not seem to bring us a step nearer a satisfactory physical account of the unity of consciousness. The molecules of the brain are infinitely numerous; they are made up into structural forms of indefinite number and variety; the kinds of the relations they assume toward each other are indescribably many. Consciousness, so far as we know, has no special centre or seat within the cerebrum; and if it had, the constituents and activities of that centre would have to be exceedingly manifold and complex. Now, all this is precisely the opposite of what we should expect of a physical structure which should be called upon to exhibit the phenomena of many conscious states, all referable to one subject. No help toward solving the problem is derived from calling attention to the fact that the different portions and elements of the brain are all interconnected. The connecting structures only add still further to its multiplicity and complexity of elements. It would be easier to conceive of an atom as becoming conscious than the cerebral cortex.

$\$ 22$. But surely the assumption that one real being is the subject of these states, which are certainly all referable in conscious- 
ness to one and the same subject, is not an impossible one. On the contrary, it is the most natural assumption ; it is the assumption instinctively made by men in general. Notwithstanding the difficulties which encompass it as soon as we attempt to define it, or to test the ground on which it rests, we shall find that it is defensible and valid.

We conclude, then, from the previous considerations: The subject of all the states of consciousness is a real unit-being, called Mind; which is of non-material nature, and acts and develops according to laws of its own, but is specially correlated with certain material molecules and masses forming the substance of the Brain. 


\section{CHAPTER II.}

\section{THE DEVELOPMENT OF THE MIND.}

\$ 1. A Distrnctive feature of modern science is its endeavor to satisfy inquiry into the nature of the objects of its investigation by a detailed description of their development. In answer to the inquiry what a thing $i s$, we are invited to listen to an account of how it became what it is. Indeed, the universal process of "Becoming" has been almost personified and deified so as to make it the true ground of ali finite and concrete existences. There can be no doubt as to the great fruitfulness and value of this historical and genetic way of studying everything. Any complex existence is certainly far better understood after it has been not simply analyzed into its present component parts, but has also been traced back to its most nearly primitive and undifferentiated stages. The history of the egg explains the bird even more than the nature of the bird explains the egg.

Both of the two subjects, with whose correlations Physiological Psychology deals, require for their most satisfactory understanding to be studied by this genetic method. The structure of the nervous system appears in a new light, when regarded as the result of a process of evolution. Beginning with the unimpregnated ovum, by propagation of cells of living protoplasm, by segmentation of larger sections of these cells, by proliferation of cells and separation into layers, the one portion of the germ from which the mechanism of nerve-fibres and nerve-cells is to unfold itself becomes differentiated from the other portions. By vital processes kept up through nutrition and resulting in the growth of some areas beyond others, and by mechanical influences at work to crowd forward here or push back there, to fold and tuck and cause to dip or curve, etc., this epiblastic portion develops the system of end-organs, central organs, and connecting tracts of nerves.

Psychology, also, has felt strongly this modern impulse. It has been forced to confess that its real task is but begun when it has, by introspection, examined and classified the phenomena of adult conscious life. All the mental phenomena undoubtedly have a 
truly vital connection. Those of the present have their roots in those of the past. The so-called faculties of the mind are neither hard and fixed lines drawn to exclude from internal relation the various modes of its behavior in consciousness, nor are they kinds of activities that spring up, full-formed at once, at different intervals in its entire history. Although we can never reproduce in adult self-consciousness the forms of the earliest stages, we can show that these forms differed greatly from those taken in this adult self-consciousness. We can show that the earlier forms must have been much the simpler. For example, an analysis of the presentations of sense shows that the "things" of developed experience are resolvable into certain elements of sensation which the mind has learned to localize. In other words, perception is a result of development ; for " things" are not ready-made products existing, as they appear, outside of the mind, but resultants of mental activities that have to be performed anew so often as the things appear. It is in the evolution of the mind that we find our means for understanding its true nature. Moreover, the characteristics which distinguish one mind from another are to be understood as largely resulting from the order and relative prominence of different activities in the development of each.

$\$ 2$. So far as the connection of mental phenomena with the increasing complexity of the nervous activities, and with the stored energies and hardening habitus of the nervous elements, affords any explanation of the development of the mind, we have already said all that is necessary. The growth of the mind in the acquirement and arrangement of sensations, in the recalling of ideas, in the forming of judgments about objects of sense, etc., is plainly dependent upon the evolution of the bodily members. But the nature of the relation which exists between the mental phenomena and the nerrous mechanism, so far as this can be learned by studying the development of both, furnishes us with another question. Upon this question, also, the same conflict of view as that to which we have already drawn attention may arise. On the one hand, the attempt is made to refer all the so-called development of the mind to the evolution of the substance of the brain, under purely physical and mechanical causes. This attempt, then, denies that any real unit-being called the Hind needs to be assumed as undergoing a process of development according to laws of its own. It cannot be disputed that many facts of experience tend to strengthen such an attempt.

There is a general correspondence, with respect to the complexity and quality of the work done, between the different stages in 
the derelopment of both body and mind. Nerrous system and mental condition are both immature in infancy; both develop with great rapidity in early childhood, and then more slowly on into adult life; both-it is claimed-remain comparatively statiouary through the period of man's highest maturity ; and as old age advances, both keep pace in their decline. Moreover, cases of arrested development of brain are cases of arrested development of mind. Idiots are frequently microcephalic; many of them have brains weighing less than thirty ounces. Degeneracy of the tissues of the cerebral hemispheres is commonly connected with increasing degeneracy of the mind. As the tides of molecular nerve-commotion rise and fall in the nerrous mass, so rise and fall the tides of mental vigor. A temporary increase of cerebral action, caused by a glass or two of wine, is expressed in the form of mental phenomena by a heightening of imagination, a quickened flow of ideas. What need, then-it is asked-of assuming any permanent subject of what we regard as mental development, other than the mechanism of physical molecules with its evolution under the control of physical law?

On the other hand, all attempts to account for the orderly increase in complexity and comprehensiveness of the mental phenomena by tracing the physical evolution of the brain are wholly unsatisfactory to many minds. We have no hesitation in classing ourselves among this number. Those facts of experience which show a correspondence in the order of the development of the body and the mind, and even a certain necessary dependence of the latter upon the former, are, of course, to be admitted; but they are equally compatible with another view of the mind's development. This other view has the additional advantage that it makes room for many other facts of experience which are very difficult of reconciliation with any materialistic theory. On the whole, the history of each individual's experience is such as requires the assumption that a real unit-being (a Mind) is undergoing a process of development, in relation to the changing condition or evolution of the brain, and yet in accordance with a nature and laws of its own.

$\S 3$. That the development of a real non-material being is implied in the history of the mental phenomena of each person may be argued on two principal grounds. In the first place, it may be shown that the stages and laws of the development of mind do not fully correspond to those which are observed on tracing the evolution of the nervous system. It may also be shown that certain elements necessarily enter into the development of mind, which have nothing like them, or strictly correlated with them, in the evolu- 
tion of the material mechanism. One real being may be dependent on other beings for its starting, as it were, and for certain factors that enter into its growth or furnish the indispensable conditions of its growth. It may thus receive the form and direction of its development, in large measure, from these other beings. And yet this fact gives us no right whatever to refuse to such a being all title to take rank among other real existences with a complex nature of its own. No existence loses or impairs its claim to reality by being dependent for its development on other existences. The mind, on the contrary, most indubitably establishes such a claim, becanse the stages and laws of its unfolding, and some of the factors which necessarily enter into this unfolding, are peculiar to itself (sui generis).

§4. That the words, "development of the Mind," stand for a real process, there can be no reasonable doubt. The sum-total of the conscious experience of each individual is something far more than a mere series of states of consciousness. No difference in degrees under the same kind can be conceived of which is greater than the difference between the most mature and highly developed mental performances and those inconceivally simple activities with which the mental life begins. So far as the character of the phenomena of conscionsness is concerned, the mind of the adult Newton or Kant is much farther remored from the mind of the infant Newton or Kant than the latter is from the mind of one of the lower animals. There is much more which is companionable and mutually intelligible between the adult man and his dog than between the adult man and his newly born child. The latter is, however, raised at once above the most intelligent animal when we consider the possibilities of its mental development. What the human being is cannot be at all adequately described without considering the nature and limits of that process of becoming which belongs to it.

There is no doubt, also, that the incomparable improvement of the mental processes which distinguishes the adult from the infantile human being is a true development. Each stage of this improvement is dependent upon preceding stages. The changes are all in some sort according to a plan. Thus the life of every individual's mental experience is capable of being made into a history. A certain tolerably uniform order in the relative development of the different faculties is discernible. At first the senses are awakened to a lively and varied activity; then memory and imagination become more prominent; and, finally, judgment and the reasoning powers assert their sway. Gradually, things become known and conduct 
shaped under principles which are assumed to have a universal validity as so-called general laws. The history of the mental life of every human being, from the cradle (or even from its embryonic existence) to the grave, has all these characteristics of unfolding itself in a regular order, in which all that comes at all comes in due sequence and acknowledged dependence upon what has preceded. This is the very essence of a true development.

§5. Can the development of the mind be explained as merely the resultant or expression of the physical erolution of the nerrous system-this system being regarded as situated in the rest of the bodily environment, and surrounded by the more extended environment of the world of active physical energies outsicle? Against an affirmative answer to this inquiry stand many facts and laws of all such mental development. In spite of what must be said concerning the striking correspondence between the evolution of the bodily organism and the development of the mental powers, it must be held that there are marked divergences as well. At certain epochs of life the erolution of the brain seems to stand far in advance of the mind; at others, the mind appears to have overtaken and passed by the stage reached by its physical substratum. During a long period of life the growth of mental powers is constant and solid, while the growth of the physical basis has nearly ceased, and such changes as are taking place in it appear quite inadequate to serve as correlates for the mental growth. Moreorer, the most distinctly typical features in the derelopment of the mind remain the same when malformation or disease or accident have largely changed the physical evolution of the brain.

$\S 6$. We hare no sufficient means for deciding how far the mental life of the human embryo keeps pace with its organic evolution. We do not eren know beyond doubt that the embryo has a mental life, in the only tenable meaning of the words-that is, a life of conscious states. But it is probable that its antenatal movements are not all purely reflex, and neither accompanied nor directed by conscious sensation, feeling, and volition. The mental life of the embryo, if it exist at all, can hardly be more than an irregular and fitful succession of the lowest and least complex mental phenomena. Taste, smell, hearing, and sight are, of course, not to be thought of as entering into such a mental life. Touch, as we understand the word to express the localized sensations of pressure which arise through the practised organ of the skin, is scarcely more likely to belong to the human embryo. Obscure feelings arising from changes in its relation to the surrounding tissues and fluids of the mother, or from disturbances in its own internal organs, and result- 
ing equally obscure feelings of innervation, as its limbs are moved, must constitute the great part of its experiences. As yet there is no experience, properly so called ; no perception of things, no feeling of self, no discrimination of ego and state. Yet long before the child is born it possesses a wonderfully elaborate nerrous mechanism, far surpassing in its grade of evolution the nervous system of the most intelligent adult animals. Previous to birth this nerrous mechanism must also be constantly in action in a highly complicated way ; it is engaged in supervising the processes of nutrition, and in the reflex and automatic activities which are expressed by the changes of the child's position within the womb of the mother. The mind, however, is as yet unawakened ; this is not because the nervous mechanism is not complex and active enough to serve as the physical basis of a rich mental development, but because the kinds of sensation-visual, tactual, auditory, etc. which start and furnish and direct this development have not yet been supplied. The mental life cannot then be said to have kept pace before birth with the evolution of the brain, or with its distinctive activities. On the contrary, it is far behind the stage already reached by its physical support. It waits to be aroused and set to its own work of combining and interpreting those sensations which are to serve as its chief means of early culture.

For the first few weeks of infancy the same relation between the relative development of the body and soul of the child is maintained. Both are subjects of a rapid growth, but the former is still much in advance of the latter. The newly born infant is, in respect to the condition of its nerrous system, much the most highly organized and fully equipped of all young animals; but as judged by the number and quality of its rolitions and perceptions, many other young animals are less stupid and insensate. If we may represent its mental condition by anything conceivable through the adult imagination, the human infant is in a dreamless sleep occasionally interrupted by instants of unlocalized and unmeaning sensations.

The cavity of the infant's tympanum is filled with a fluid, the place of which is only gradually taken by the air. Sensations of sound, if they arise at all, must be at first only occasional and faint. Binocular movements of the eyes in the direction of bright objects take place early ; and it is through sensations of light and color that the first activities of the mind in perception are aroused and controlled. But for some weeks there are only sensations and impressions, without true perceptions; there is as yet no knowledge of any "Thing." This earliest relation of mind and brain, with respect to the degree and rate of their development, is not 
favorable to any form of the materialistic theory. It rather favors the view that the mental plrenomena belong to another principle than any material substratum. The dependence of the mind on the brain is indirect and through the sensations (chiefly of sight and touch) which must be furnished to the mind as the primary factors in its development. The halt in the development of mind at first, and its distinct backwardness with respect to the relative stage it has reached, are due to a lack of such sensations as have the characteristics of spatial series (see Part II., chap. VI.), and so are able to stimulate the mind, and afford it the requisite material for the construction of true presentations of sense.

\& 7. Within a few montlis after birth the child has undergone an enormous mental development; it has become a mind, in some inchonte way recognizing itself as the subject of states, and perceiving a little surrounding world of objects of sense. It has also begun to attend to the objects presented in consciousness, and to direct its attention by voluntary choice. The mind's relating activity las been aroused; and acts of memory, discrimination, and judgment, as the basis for those concepts which require articulate language to express them, are repeatedly taking place. The assumptions of reason, as involved in all human experience of things, and of their action and reaction upon each other, are found to be shaping the growth of the mental powers.

As accompanying and forming the ground for this sudden blossoming of the mind in the use of its conscious powers, there is a continuous and yet diminishing monthly increase of the substance of the brain. No new organs are formed within the cranial cavity; but those which hare been formed previous to birth are further developed under the changed conditions of nutrition. In respect to the quantity and arrangement of its molecules, the nervous mechanism certainly undergoes no development during the first year of the child's life which at all corresponds to, or accounts for, the derelopment of the child's mind.

It may be claimed, however, that the most important development of the nervous mechanism has been overlooked in the foregoing description. This development does not consist so much in the increased quantity of the brain's substance, or in the more intricate arrangement of its elements with relation to each other. It consists rather in the forming of what has alrealy been alluded to (Part II., chap. X., $\$ \S 18$ f.) as "dynamical associations" among the existing elements. The statement that such is the nature of the developing activities of the nervous mechanism, and the assumption that such activities are an indispensable physical condi- 
tion for the growth of the mind, may both be taken for granted. But even then the argument is far from complete upon which the development of mind as a real being, with a nature of its own, and with a history controlled by its own laws, is denied. The formation of the so-called "dynamical associations" among the molecules of the nervous mass furnishes no adequate account of the development of mind. This development is not in the direction simply of associating together states of feeling, each one of which has an exact physical correlate in a physical association among the molecules of nervous substance. It is rather a development which for its very existence requires something different from such associations. The child might go on forever merely associating together affections of its own mind in correspondence to dynamical associations among the nervous molecules, and yet have no growth of experience such as it actually attains. The fact is that within a single year, or within two years, the child has learned to know "Things," to attend to some in preference to others, to refer its states in some crude way to itself, to form concepts and judgments by the nind's relating activity, and to underlay the world of its sensuous experience with another world of assumption respecting certain non-sensuous realities. To account for this boundless expansion of the activities of consciousness, with its surprising new factors and mysterious grounds of synthesis and assumption, by proposing an hypothesis of "clynamical associations" among the particles of nervous substance in the brain, is a deification of impotency. So far as we really know anything about the development of both brain and mind, we are compelled to say that the latter, when once started by the sensations furnished through excitation of the former, proceeds to unfold its activities with a rapidity and in an order for which no adequate physical canses can be assigned.

During the period of young manhood, or young womanhood, the dependence of the development of the mind on that of the body is most strikingly seen in the influence over the emotions and imagination from the sudden unfolding of certain bodily organs and powers. The indirect influence of these acts of feeling and imagination upon the more purely intellectual progress of the mind is, of course, correspondingly great. But the dependence of mind on body is by no means such as to favor the view that there is no ground in a real being, other than the brain, for the order and rate of the mental development.

This same statement is emphatically true of the long period of maturity which constitutes what we call the "middle life" of man. During this time the nervous matter undergoes scarcely any dis- 
cernible development. Nothing that microscope or electrometer can detect distinguishes the brain of the man of twenty-five from that of the man of fifty. A few grammes of weight have perhaps been added to it during this long period of years. Anyone is at liberty to speculate as to the immense development of so-called "dynamical associations" which has taken place during the same period. We are far from denying the possibility of such development. But the fact that a large development of mind may have taken place during the same period cannot be denied. If it be true that large numbers of mankind remain mentally stationary for most of their adult life, this truth in no way favors a materialistic view of the development of mind. Most observing persons will rightly find the chief account of the failure of mental growth in precisely those kinds of mental activity which least admit of being explained by physical analogies. It is from want of mental curiosity, attention, careful and comprehensive judgment, sound moral purpose, etc., that most men fail to develop during adult life in their mental powers. And these are mental activities for explaining which no one as yet has been able to conjecture any analogous or corresponding class of cerebral changes.

Many minds, however, not only make vast acquisitions, but also experience a large unfolding of mental capacities during the period of middle life. How mature and wide-reaching do the judgments of some men then become! How profound the insight into the most abstract and difficult speculations comes to be! What cerebral evolution shall be conceived of as being the only true cause, and the exact physical correlate, of the mental development of Kant during the years preceding the appearance of the "Critique of Pure Reason," or of Newton while he was unfolding the calculations and conjectures of the "Principia?" To hold that the changing molecules of the brain-substance of these thinkers were the sole subjects, really being and acting in the unrolling of these great dramas of buman speculations, involves an astonishing credulity. On the contrary, we seem compelled to affirm that no important activity, or law, or fact, in the order of such mental development, fails to demand the assumption of a real and non-material unit-being, unfolding its powers according to its own nature, although in dependence upon certain elements and conditions furnished through the brain.

§. Advancing old age is doubtless, as a rule, characterized by a simultaneous decline both of certain mental and of certain bodily powers. In this period of life, however, the correspondence between the changes in the character of the phenomena of conscious- 
ness and the altered vigor and quality of the nervous mechanism is not such as to suggest that the two have an altogether common basis. In healthy normal old age the course of the mental life is distinguished chiefly by the dropping out or diminished action of certain factors that are relatively prominent in youth. The circulation is slower; the vital energy is declining; the muscles are less promptly and completely under the control of the volitions; the end-organs of sense are less sensitive under impressions; and certain emotions and passions whose physical basis is of the most obvious sort become greatly modified or disappear. As to the marked effect of these bodily changes upon the mental development there can be no doubt; and if the previous mental development has been chiefly along liues indicated by organic activities the apparent decay of mental vigor when the physical basis begins to fail is, of course, also most plainly marked.

On the other liand, there are many other cases, where no notable difference can be detected, or even fairly assumed, in the course of the psychical evolution down to the "feebleness "of old age; where the course of mental development continues substantially undisturbed in all its most important features. The mind of the cultivated old man, with calm and broad judgment, with refined kindliness and fixed moral principles, is not to be spoken of as suffering a decline which keeps pace with the failing of his physical powers. It may justly be claimed that the final period of human life, on the whole, favors that theory which regards the mind as by no means wholly conditioned upon the brain for the character, order, and laws of its development.

$\S 9$. The same general view of the development of mind, which is most consistent with the facts of the different stages of life, is also farored by considering those sudden checks or changes in the course of this development that are caused by disturbing or destroying considerable portions of the nervous matter. The phenomena which follow experimental extirpation of the substance of the brain in the lower animals, and loss of it by serious lesions in the case of man, do not favor a materialistic theory of mental development (see Part II., chaps. I. and II.). Extensive losses in certain areas of the cerebral hemispheres are often followed by no appreciable disturbance even of any sensory or motor activity. When lesions are followed by such disturbance, their effects may in time wholly or partially disappear. When such disturbance is permanent, it is not necessarily connected with loss in the power of judgment, in the higher intellectual, æesthetic, and ethical activities of feeling, intellect, or will. Even where aphasia is so severe 
as to include the loss of all power to utter or understand articulate language, the patient may still show a good degree of mental acuteness by ability to make calculations or play games of skill.

On the other hand, the much more serious interruption or complete loss of mental developrent may occur when no adequate explanation can be detected in the disturbance or arrest of cerebral development. It is, of course, natural to conjecture that, in all this latter class of cases, more accurate information would show us some discased condition of the brain as the physical antecedent of the mental defects. We know that subtle changes in the character of the blood-supply, such as we have no plysical means whatever for detecting, are often the causes of most profound changeseither temporary or more permanent-in the train of ideas. None the less, however, do both classes of cases above mentioned favor the theory we are advocating, rather than the so-called materialistic theory of mind.

$\S 10$. All the foregoing considerations suggest the conclusion that the mind is primarily and chiefly dependent in its development upon the nervous mechanism for furnishing and directing the combination and order in recurrence of those sensations which enter into all presentations of sense. Let any person of normal and sane brain and mind consider how intimate and extensive is the connection between his sensations and his whole mental development. Fickle and confused experience of sensation involves fickleness and confusion of judgment on all matters of sense. Loss of any class of sensations, as a whole or in part, involves a distinct impairment of mental powers. Such loss necessarily changes to a considerable extent the character of the subsequent mental life. Such loss, however, is regularly compensated for, to some extent, by an increase of mental activity along the lines which remain open to the mind. The man blind from birth can never have the same course of mental unfolding, with respect to his perceptions of things, his idea of space, his feelings before the beautiful, etc., as that open to his more fortunate fellow. But he is not necessarily inferior in mental capacity and activity; because the development of his mind, as conditioned upon the other senses, proceeds with the ordinary pace, although along a different path.

The mind is absolutely dependent upon the nervous organism for its awakening and furnishing in the life of conscious sensation. The case of Laura Bridgman, and others similar, show how large mental development is possible with even a greatly diminished outfit of the senses. But, of course, if touch and muscular sensation, as well as smell, taste, hearing, and sight, were lacking, no con- 
scious mental life would be possible in any form known to human experience. The form in which the sensations shall combine, and the time-order of their recurrence, are also depenclent upon the character, number, and succession of the cerebral excitations caused by external or internal stimuli. But when once the mind is started upon its career of unfolding its powers, it maintains a relative independence of its physical basis. Not that sensations and resulting presentations of sense, together with the reproduced images of such mental products, do not always continue to furnish indispensable factors and conditions of all mental development. In the most abstract thought, and in the highest flights of the imagination, the mind never wholly gets away from the world of sensation and perception, with its immediate dependence upon the activities of the physical and nervous basis. On the other hand, the course and extent of its unfolding are such as to show that its stages and laws do not all correspond to those which characterize the evolution of this basis. Its general dependence upon such basis, in all its development, is through the sensations and their reproduced images.

$\S 11$. Several references to the second argument (comp. \& 3) for our view of the development of mind have already been made. This argument is based upon the fact that certain indispensable elements enter into the development of mind which have nothing similar to them, or strictly correlated with them, in the evolution of the material mechanism. The mind can, indeed, undergo no development except as conditioned upon these elements. But the elements themselves cannot be regarded as the expression in consciousness of merely physical causes, or as flowing necessarily from more primitive activities of the mind which may possibly be regarded as the expression of such causes.

All three of those fundamental forms of activity which are recognized in the ordinary threefold division of the soul into facultiesnamely, acts of feeling, acts of knowledge, and acts of will-necessarily enter into the development of the mind. Its development consists in the unfolding of these three classes of acts, in their mutual dependence and according to the laws which belong to each. Among each of these three great classes of acts there are certain subordinate kinds that defy all attempts whatever to correlate them with the changes in the nervous mechanism, or to explain them as necessarily or actually arising out of such physical changes. Such are the feeling of moral obligation, the sentiment of justice, the love of truth, and certain of the higher asthetic feelings. Among the acts of knowledge, such are the mind's relating activity, its use 
of the principle of reason and consequent in drawing degductions, its confident assumption that similar phenomena are signs of like realities, and that the world of sensuous individual experience is but the manifestation of an invisible world of real beings, with permanent properties and forces, acting and reacting under law. Such, also, are the acts of deliberate choice among courses of conduct, under the influence of moral considerations-the acts of "free will " in the highest sense of the term.

Not one of the higher acts of feeling, knowing, or willing, so far as its sui generis character is concerned, admits of being correlated with, or represented under, any of the conceivable modes of the motion and relation of molecules of nervous substance. Certain sensations and perceptions connected with the rise and growth of the higher forms of feeling have, undoubtedly, a pliysical basis; but such basis is not assignable to the feelings themselves. Sensations and perceptions, which are resultants (in some meaning of the word) of physical processes, are discriminated by judgment and made the basis of deductions and inductions. But admitting this does not one whit the better enable us to conceive of a physical process which can account for the sui generis character of the relating activity itself. Acts of "free will," so called, always take place under certain conditions of sensation and perception, as well as of desire; but the physical correlates of these conditions can in no respect be conceived of as being also correlates of the conviction that the choice is responsible and free.

Now, if such activities as the foregoing do actually constitute indispensable elements of mental development-and it is obvious that they do-this development cannot properly be accounted for by assigning it to a mass of nervous matter undergoing a physical process of evolution, after the manner of the growing human brain. Such development rather implies a real being of another than the plysical order. This being must be thought of as stimulated by the rise and recurrence of sensations and images of past sensations, to unfold its own activities as conditioned by its own inherent powers. Like every other real being, the history of its unfolding is dependent upon the relations in which it is placed to other real beings; but it is nevertheless a history determined also by what the being $i s$.

$\S 12$. The trial will doubtless be made to escape from the conclusions hitherto reached, by means of help derived from a certain psychological theory of the development of mind. It may be admitted that the attempt to find, directly, an adequate physical basis for all these so-called higher faculties, or modes of the behavior of 
the mind in its development, must be abandoned. But the higher faculties themselves-it is said-are to be regarded as developments of the simplest activities. These highest faculties of all may then be directly connected with the evolution of the body, or of the cranial mass, through the simplest mental activities. In this way a kind of necessitated psychical mechanism is set up, which is itself entirely explicable as a development from one kind of element (the sensation); and then, by regarding this one kind of element as connected with the motion of nervous molecules in a purely mechanical way, the need is obviated of supposing any real being called Mind as undergoing a process of mental development.

For example, it may be claimed that the one simple and undifferentiated element of all psychical experience is the "nervous shock." This nervous shock is merely the simplest expression or result in consciousness of a nerve commotion set up by the action on the nerrous mechanism of external or internal stimulus. By differentiation and combination of the nervous shocks, the so-called simple seusations arise. By reproduction of similar combinations of fainter shocks, the images of memory are produced. By " agglutination" and "agglomeration" of the sensations and ideas, judgments take place-only, since some new kind of idea does certainly seem to be inrolved in the essence of judgment, it must be held that a "feeling of relation" is somehow slipped in between the agglutinated and agglomerated sensations and ideas. By still more elaborate groupings of the simple ideas, systems of thought and socalled ideas of the highest order of abstraction-like the ideas of space, time, etc.-come into mental being.

In the foregoing way, all the so-called mental processes that constitute the development of the mind are strictly correlated, under laws analogous to those which control the relations of physical elements, with the processes that go on in the nervous system; thus so-called "psychology" results in bringing the mind of man into the same strict subjection to the energy of outside nature, under the law of the conservation and correlation of energy, that characterizes all the phenomena with which modern physical science is accustomed to deal.

The above-mentioned theory is doubtless admirably simple and thorough-going. But its somewhat extreme simplicity and thoroughness constitute very important objections to it. In so crude a form it scarcely deserves detailed consideration. It is enough in this connection to eall attention to the fact that the theory is built throughout upon unverified assumptions; and that, even granting its assumptions, it affords no adequate description whatever of the 
real process of human mental development. No theory of nervecommotion has yet been devised to connect it with the external stimuli under the law of the conservation of energy. If by "nervous shock" be meant a psychical event, the break between such shock and the nerve-commotion which is its antecedent is absolutely impassable; no physical energy, under the general law of its conservation and correlation, can pass this break.

Moreover, there is no actual or conceivable psychical event corresponding to the undifferentiated nervous shock. Sensations are always, as such, and from their very nature, of this or that definite quality. An undifferentiated psychical element is a pure abstraction. Nor do sensations and their remembered images constitute such existences that they can be spoken of as "agglutinated" or "agglomerated." The so-called "feelings of relation," slipped in between the single ideas and sensations, if by this be meant anything less than relating activities of the mind, are absurdities in no way fitted to explain or represent the act of judgment. And, finally, this entire account of the course of mental evolution is an utterly inadequate description of what actually takes place in the history of even the poorest and weakest human minds.

$\S 13$. All theories of the mental development which account for the different so-called faculties and stages of the growth of mind as though they flowed necessarily from some one fundamental activity are inadequate and misleading. The mind is indeed a unit-being, but its unity is not of the kind alleged by these theories. Its different constitutional modes of behavior are not to be resolved into each other, or into any one most primitive activity ; nor do they all necessarily flow forth from such a primitive activity. They manifest the rich variety of the mind's nature. They do, indeed, preserve a certain order in time with regard to the relative amount of their unfolding. The different periods of life are characterized by different stages of mental development; these different stages of mental development are characterized by a relative prominence of particular faculties, or modes of the behavior of the mind. But because such a time-order is followed in the development of the mind, we can by no means conclude that the faculties latest developed are any the less native and essential to the character of the mind. Nor is it true that these latest and highest faculties can be explained as mere developments from, or modifications of, the earlier and simpler.

Strictly speaking, none of the faculties, or constitutional modes of the behavior of the mind, admit of being explained as mere developments of other faculties. This is true even of those minor 
forms of activity which it is customary to class under the same faculty. Thrat I have the sensation "red" is no reason why I should have the sensation "green;" and that I have the sensations "red" and "green" is no reason why I should also have the sensation "blue." Neither does the existence of all these so-called fundamental color-tones, of itself, form any reason why the mind should be affected with any of all the thousands of sensations supposed to be compounded from them. None of these color-tones, psychologically considered, can be regarded as a development from the fundamental color-tones. That I am affected with a certain sensation of color, lying at the bottom of the spectrum's scale, when several billion ribrations of ether strike the retina, and with a qualitatively different sensation when the number of vibrations is increased by several billions more, cannot be explained as an evolution. The same remark holds within the limits of each of the other senses. Their scales of quality are not such that experiences at one place of the scale can be erolved from those at other places of the scale. Some of them, such as smell and taste, do not admit of being referred to any form of a scale or diagram representing relations of quality. The feeling of heat is not another phase of the feeling of cold; neither of the feelings of temperature is to be explained as arising out of feelings of pressure or motion.

When the sensations of the different senses are compared with each other, the impossibility of considering any of the classes as developing from any other becomes yet more apparent. That a sentient being has an experience of hearing musical tones which rise in pitch as the nunber of acoustic vibrations varies from thirty to thirty thousaud is no reason at all why the same being should have an experience of seeing colors that change their "color-tone" as the number of light-vibrations varies from about four hundred billions to about seven hundred billions. In the development of the mind, the senses may actually awaken in a certain order more or less definitely fixed. But this is very different from holding that they derelop out of each other, or that they are all developments of some undifferentiated sense-element, the psychical correlate of the nervous shock. Moreover, our percepts, or knowledges of "Things," cannot be regarded as mere developments of sensations. That a sentient being should have sensations of sound and smell and taste, and even of light, color, temperature, and pressure, is not of itself a sufficient reason for its having perceptions such as belong to human experience. The only way in which such perceptions can be regarded as the necessary resultants of the sensations which enter into them as component parts is by taking the nature of the mind into the ac- 
count. But this implies that perceptions are not developed forms of sensations; that they are rather advanced forms of the activity of that real being which is developing under the experience of sensation-elaborate products of the synthetic activity of mind.

$\$ 14$. The knowledge of things by pereeption involves the activity of the mind as memory and judgment. But acts of memory and judgment are not derelopments from pereeption; they are not merely modified forms of sensations as recurring or combined under the action of physical antecedents. All talk about the "image " of memory as though it were merely a faint or faded-out impression of sense is quite unavailing; it does not hit the real point of inquiry, and consequently does nothing to explain the mystery (comp. Part II., chap. X., $\$ 18$ ff.). The vital element in memory, that which makes it to be memory, is neitler a sensation, nor a modified form of sensation, nor a development of sensation. The same statement is true of judgment.

The relating activity of mind, the power to bring two objects together in the unity of consciousness, and, while keeping their ideas distinctly separate, to bind them into one under the mental affirmation of their likeness or unlikeness-this is a new and startling mode of the activity of mind as contrasted with merely being affected in sensation. Minimize it as we may, we cannot look upon this aetivity as a mere "resultant" of two sensations or images of sensations arising simultaneously in the mind. We cannot consider judgment under the principle of the conservation and correlation of energy. To treat it as such involves the grossest misapplication of the laws which control the coincidence or conflict of physical forces. Nor are the different forms of the relating activity of the mindconcept, judgment, deduction, induction-to be regarded, strictly speaking, as developments from each other or from any one mental activity simpler than any of them. They may all, indeed, be considered as modes of the relating activity, beeause they involve discrimination, the discernment of likenesses and unlikenesses. But each one of them involres somewhat more than simple diserimination; each one involres other elements peeuliar to itself. That a sentient being should simply judge, or affirm this of that, is not of itself a sufficient reason why it should also make inferences by syllogistic processes or arrive at general laws by induction. Indeed, the former may belong to many animals which are incapable of the Iatter.

$\S 15$. We may properly continue the foregoing line of remarks into the consideration of the mind's most general activities. Modern psychology, we have seen, is accustomed to distinguish faculties of 
knowing, feeling, and willing as belonging to the mind. But it is emphatically true that no one of these three faculties can be regarded as dereloped from any other one, or from any two combined. That a being feels-that is, is affected with a state of consciousness more or less pleasurable or painful, and having a characteristic quality-is in itself no ground for explanation of its knowing "Things" through sense-perception, and inference. Conversely, a being is conceivable with the knowledge of an archangel, but without experience of desire, emotion, or sentiment of attraction or repulsion. Sucl a being would indeed have to attain its knowledge in other ways than those open to us, and we find it difficult or impossible to imagine precisely what such knowledge could be like. But growth in knowledge is a different thing from the unfolding of mere feeling; and the former cannot be explained as arising out of the latter. Acts of will are, indeed, always actually dependent upon knowledge and feeling, and cannot even be conceived of as taking place without this dependence. But acts of will are not mere derelopments of those acts of knowledge and feeling on which they undoubtedly depend. The act of choice involves a new element, an element not to be necessarily evolved from the other activities of mind.

$\S 16$. We are so accustomed to the action in common, in the unity of consciousness, of all the so-called faculties, that any attempt to account for them as different modifications of one form of energy meets with a farorable reception. Nothing thus far said is, of course, to be construed to the prejudice of the unity of the mind. But, on the other hand, the incomparable wealth in variety of its natural achievements should not escape our notice. From the beginning to the end of conscious life, the forth-puttings of the mind continue. They are all actual concrete events, happenings in consciousness which hare no permanent existence and are never twice precisely alike. That they are, however, alike in certain particulars and unlike in others, we can observe in consciousness itself. Indeed, it is upon this fact that the possibility of any orderly progress, any true development of mind, depends. But the different classes of mental activities are not to be regarded as though they could themselves be explained each from the other, as the different stages of the embryo of an animal or of the germinating and growing seed of a plant are successively evolved.

The development of mind, therefore, cannot be explained after the analogy of the accretion of molecules within a germ, and the resulting division, multiplication, and advancing arrangement of the living cells into separate organs of the entire system. No real 
elements of the mind exist which can aggregate to themselves other elements by absorbing them as pabulum, or can grow by arranging the new material thus gained according to the energies inherent in the material already organized. The life of consciousuess is a neverceasing change of states. Yet the result of this change of states is an orderly history, a true development. Such development is not merely the expression of the evolution of the material basis of some of these mental states. For it does not follow the same order or the same laws as govern the material evolution; and some of its most important factors cannot be regarded as having any physical correlate, or as evolved from other factors which have such a correlate. The development of Mind can only be regarded as the progressive manifestation in consciousness of the life of a real being which, although taking its start and direction from the action of the physical elements of the body, proceeds to unfold powers that are sui generis, according to laws of its own. 


\section{CHAPTER III.}

\section{REAL CONNECTION OF BRAIN AND MIND.}

§1. That certain uniform relations exist between the mental phenomena and the action of stimuli upon the nervous system, is a most general conclusion of Physiological Psychology. These relations are chiefly concerned with variations which take place in the quality, intensity, combination, and time-order of the states of consciousness, as dependent upon the varying amounts and order of different modes of physical energy as applied to the end-organs of sense. But evidence enough exists to show that the more ultimate psycho-physical relations are those which exist between states of the brain and states of the mind. The dependence of mental states on physical events outside of the body, or at its periphery, is gained by means of the central organs of the nerrous system. In the case of man, at least, what happens beyond the cerebral hemispheres is significant for the states of consciousness only as the hemispheres themselves are affected by it. What happens beyond the cerebral hemispheres becomes the cause or antecedent of what happens in consciousness, through this portion of the brain. If our information were sufficient, then, the empirical science of the connection of body and mind would comprise a statement of all the relations which exist between the mental phenomena and the changes with respect to chemical constitution, structural form, and plysiological function, which take place in the molecules of the cerebral areas.

But even if the conditions were already fulfilled for a complete science of Physiological Psychology, we should scarcely find our speculative inquiries satisfied by this science. The desire would doubtless still be strong to discover some more general statement for the real connection between physical and psychical phenomena. The question would still be raised: What, then, is the one inclusive proposition, or word, or term, which gives the essence of all the relations between the brain and the mind? It would seem tedious and disappointing to reply to this question by again enumerating all the particulars which psycho-physical science has eliscovered. Let it be taken for granted that, when lesions happen in certain areas of the 
cerebral cortex, such or such disturbances of the phenomena of consciousness take place ; that when so many molecular vibrations of a given ware-form and intensity occur within the cerebral elements, sensations of a certain fixed quality and quantity arise in the rnind; that when certain fainter vibrations of like wave-form return in the same elements, reminiscences of the aforesaid sensations are experienced, etc., etc. We are still inclined to ask: What is the meaning of all this? or, How are brain and mind, actually and in principle, related to each other?

It is in deference to the raising of inquiries of the foregoing rational sort-inquiries which are perpetually repeated all the way along the path of psychological research-that we speak further of a real connection between brain and mind. Of course every such form of speech inrolves the assumption that the mind is a real being which can stand in relation to other real beings, and not merely the formal or grammatical subject of mental phenomena. This assumption has already been made and partially verified. In continuing to make it for the purposes of the present chapter, we shall find it still further verified.

§2. Various attempts have been made, from one or another point of view, to sum up in some single word the relations that maintain themselves between the body and the soul. Thus, the body has frequently been spoken of as the "seat" or "organ" of the soul. Looking at these relations from the more materialistic point of view, we have already seen how mental phenomena may be regarded as the "products" or "resultants" or " manifestations" of the functional activity of the brain. More highly figurative terms even have often enough been employed. The body has been called the "prison " or "tenement" or "tabernacle" of the soul. Not seldom, also, has the mind been represented as mastering and controlling, and even "moulding" the body-somewhat as the rider subdues and guides his horse, or the worker in clay and metal shapes the product of his toil. One form of the doctrine of "Animism" has held that the mind is identical with the vital principle, which is busy from the very impregnation of the ovum in shaping its increasing molecules according to an unconscious or dimly conscious plan. Much debate has also been held as to whether the conception of "cause" is applicable to any of the relations in which body and soul stand to each other-whether, indeed, it must not rather be held that what happens in one is only the "occasion" on which some underlying cause, common to both, operates to produce a change in the other.

$\S 3$. The inquiry in what sense, if at all, the brain can be said to 
be the "seat" of the mind is more easily answered in a negative than a positive way. Nothing but the crudest notions, both of the nerrous mechanism and of the mind, would be consistent with any of the more literal and direct interpretations of this word. Few would seriously regard the mind as a special entity, whether constructed of ordinary material atoms or constituted in ethereal form, that maintains a sitting or other posture amidst the cerebral masses. Nor is it any more correctly conceived of as thinly diffused orer the entire mechanism of nerve-cells and nerve-fibres, or as wandering about among the nerre-molecules to find its temporary "seat" where occasion seems to require its presence. And, although some of the phenomena of mind and brain perhaps admit very well of being brought under the conception of the atom, acting and acted upon in varying relations to other atoms of kinds different from itself, no essential gain is made by the attempt to regard the mind as in reality an atom. In brief, there is no literal meaning of the words in which we can speak of the mind as seated in the brain. The phrase, the brain is the "seat" of the mind, is, however, very well adapted to raise the whole question of the spatial qualities of the mind, and of its alleged spatial relations to the molecules of the central nervous system. We shall, then, briefly consider the question in this form.

$\$ 4$. There can be no doubt that ordinary language justifies us in speaking of the soul as in the body, in some sense in which this term does not apply to any other collection of material atoms. The human soul is in the liuman body as it is not in the bird, the tree, the house, the star. Even that way of regarding the mind's nature which does not hesitate to speak as though it were a thinly diffused and half-spiritualized form of matter, assents to the necessity of asserting a special relation in space between it and the body. Hence some old-time philosophies represented the soul in perception as streaming out through the arenues of sense in order to get the sensuous object into its embrace : or else pictured some etherealized copy of this object as streaming into the soul by the same arenues. But eren such a view of the nature and activities of the mind is based upon the claim that the body is, in some sort, the peculiar dwelling-place, or "seat," of the mind. A correct account of the process by which the world of things becomes known shows that all our experience is connected with the establishing and justifying of this claim. There are no "things" known to experience except as our sensations, or modes of being affected, are both localized and projected extra-mentally. Inducements and considerations, such as have already been treated in great detail (Part II., 
chaps. VI. and VII.) irresistibly urge the mind to arrange all its phenomena into two great classes-phenomena which are qualities of outside things, and phenomena which are mere states of internal experience. But the same inducements and considerations compel us to look upon certain phenomena of the first class as related to our mere states of consciousness in a peculiar way. The world of things outside always (at least in ordinary experience) affects usis perceived by us or modifies our consciousness-through the body. The mind is, therefore, said to be in the body.

The conclusion from the foregoing general experience is confirmed by certain experiences of a special order. The feelings of pleasure and pain, which have so immediate and incontestable a value for the life of the mind, are all connected with sensations more or less definitely localized in the body. Hence men say, "My nose is offended by this smell," "My tooth is aching," or " $\mathrm{My}$ limb is suffering." So close is the connection between the localized sensations and the painful or pleasurable states of the mind, that the mind actually seems to be suffering in that part of the body where the sensations are localized. When the localizing of sensations connected with feelings of strong "tone" is very indefinite, as it is in cases where the feelings arise from the condition of large areas of the internal organs, the soul seems to be suffering in, and throughout, almost the entire body.

Furthermore, both ordinary experience and scientific observation require us to regard the mind as standing under certain special relations to parts of the body. The ancients located the soul in the heart or lower viscera, because of certain marked connections between the states of the soul and the condition of these organs. But to speak of the soul as seated in the heart or other viscera plainly applies most pertinently only to the soul as an emotional being; the obrious connection of the head with most of the more obtrusive sensations tends to confirm us in the belief that the mind, as perceptive, has its "seat" in that region of the body. For reasons already given in detail (see Book II., chaps I. and II. and elsewhere), modern scientific researches justify us in narrowing more precisely the local domain within which we can affirm the mind to have its seat. The mind is certainly in the nerrous system, in a sense in which it is not in any other of the systems of the animal body. More precisely yet, it is pre-eminently in the brain; and, among all the complex groups of encephalic organs, the final and special claim of the cerebral cortex to be the "seat" of the mind is most easily maintained. Here, in this convoluted rind which forms the interlaced "projection-systems" of sensory and 
voluntary motor-impulses, here-if anywhere-must it be held that the subject of the states of consciousness has its dwellingplace and home.

§. At this point, however, the results of modern scientific inquiry become unfarorable to the effort yet more particularly to designate a material "seat" for the mind. The eager imagination having, as it were, hunted the soul down as it retreats inward and upward to the higher regions of the supreme central organs, requires some more precise information as to just where in these regions its existence may be pointed out. Is there any one mathematical point, or minute area in the cerebral cortex that is most especially of all the dwelling-place of mind? If so, might it not be properly conceived of as ordinarily remaining at this point to receive the messages despatched to it from the various parts of the periphery; and as executing its will over those peripheral parts by sending back to them corresponding messages despatched from the same central point? The pineal gland has undoubtedly lost the significance which Descartes gave to it as the special seat of the soul. But can no substitute be found to take and hold so important a place? The answer of cerebral histology and physiology to the foregoing questions is, on the whole, a decided negative (comp. Book I., chap. II., and Book II., chaps. I. and II.).

Certain areas of the cerebral cortex do, indeed, appear to have a particular connection with the execution of certain functions of the mind ; the exact nature of this connection, however, cannot as yet be clearly indicated. But the very phenomena on which reliance is placed for establishing the foregoing connection, forbid us to regard the mind, in its special relations to the brain, as limited to any point or small area of the cerebral cortex. Considerable parts of all the cerebral areas can be destroyed without impairment of any of the essential functions or faculties of mind. Moreover, both gross and microscopic anatomy show us that the cortical part of the brain, like all its other parts, is not constructed on the plan of having its uses for the mind concentrated in any one minute circumscribed spot. In any sense in which the mind can be saicl to have its "seat" in the brain at all, in that same sense, and with equal propriety, may the entire cerebral cortex, with its vast complexity of nerve-fibres and nerve-cells, be said to be the "seat" of the mind.

\$6. And now the puzzling question recurs: What that is intelligible can be meant by designating the supreme central organs of man's nerrous mechanism as the "seat" of his conscious mind? No one is directly conseious of these organs. The subject of con- 
sciousness is not a being which can be conceived of as "posturing" within or amongst a certain larger or smaller group of material molecules. And yet, plainly, in some sense the mind is to be thought of as in the brain, as it is not in any object outside of the body, or in any of the non-nervous organs of the body (bones, hair, nails, fat, muscular tissue as such, etc.), or even in the remainder of the nervous system.

The only solution for such a puzzle as the foregoing-if solution it can be called-must always consist in calling attention anew to the essential facts of the case. Certain particles of very highly organized chemical constitution, when grouped into nerve-fibres and nerve-cells, and when further associated into organs, may be acted upon by appropriate stimuli. These material particles are locally in the cranial cavity, and, more precisely, in this or that area or organ of the cranial contents. Moreover, a large and important part of the phenomena of consciousness consists in localized bodily sensations of a painful or pleasurable character. To these facts investigation adds the inference as based upon experiment and observation in the case of others, that the localized sensations are themselves ultimately dependent upon the behavior of the aforesaid material molecules in the brain. That is to say, we directly localize many of our mental affections in this or that part of the body; by remote processes of observation and argument we infer that the last material antecedent of them all is the beharior of certain invisible parts of the body within the brain. Therefore we say : The mind is in the brain ; or the seat of the mind is the brain. By this, nothing further can be meant of an assured or intelligible character than the emphatic repetition of the same principal facts; the sensations which we localize at the periphery of the body, or project from the body in space, all have a sui generis connection with the condition and action of that portion of the same body which is contained in the cranial cavity. Our modes of being affected are directly localized in space outside of the body, or in the various peripheral parts of the body. The part of the body on which the activity of having these percepts is immediately dependent is localized by science in the brain. Other activities of mind are probably also thus dependent on the brain. In no other sense can the brain be said to be the seat of the mind.

As to the possibility of such a sui generis relation between material elements which exist in space, and the localizing and other activities of a being not to be conceived of as, strictly speaking, in space, only experience is entitled to pronounce. Such a relation is an accomplished fact. The fact is not to be disputed on any so- 
called a priori grounds whatever. Both the dicta which have sometimes been made to bear on the case are alike inapplicable. On the one hand, it has sometimes been claimed that a being cannot act where it is ; on the other hand, that a being cannot act where it is not. Nothing, however, can be known as to how and where beings can or cannot act, except through experience of how they actually do act. Building our conceptions upon the basis of facts, we should be inclined to say that beings act upon, and are acted upon by each other, according to their differences in constitution and relations in space. Gravitation keeps constantly before us the example of all bodies acting unceasingly upon each other, in many cases over distances that are immense. The amount of this action depends, indeed, upon the distance orer which it takes place; but the action at all is an instance of beings acting where they are not.

When material molecules are approached nearer to each other than a given small distance we at once discover new modes of behavior set up, which depend upon what the molecules are, and what their condition, etc. New laws, such as those of cohesion and chemical affinity, have now to be taken into the account. But gravitation, cohesion, and chemical affinity are all alike to be unclerstood as expressive simply of the regular modes of the behavior of material elements, with reference to each other, under varying conditions. All these modes of behavior modern physical science reduces to motions of various kinds, directions, durations, and velocities. What is true of all material elements is true of those of the brain; they can do nothing but move.

If, then, we are to speak of the mind as having its "seat" in the brain, in a literal way, we must regard it as one among the many molecules or atoms of which the brain is composed-wandering (that is, moving in a peculiar fashion) among the others, and so variously acting on them, and being acted upon by them. But if the mind were such a molecule or atom, the only affection it could receive from the rest of the brain-molecules would be to change the kind and direction of its own motion; the only effect the mind-atom could produce in the material atoms of the brain would be to modify their motion with respect to kind and direction. But it would still be just as difficult as before to understand how the phenomena of consciousness should result from the movement of one atom among other atoms-no matter how peculiar in constitution each of these two kinds of atoms (the mind-atom and the brain-atoms) might be.

It does not follow, however, that the relation of the mind to the brain is any more ultimately mysterious than that of the molecules 
of the brain to one another. Nor does it form an insuperable objection to the former relation that it is not, like the latter, a relation of changes of position in space. For who shall undertake to affirm that beings which are not extended and movable in space, because their very nature is of another order, cannot exist in relations of any kind to beings which are thus extended and movable? If the existence of the former kind of beings consists essentially in states of consciousness, this fact does not prevent their dependence upon the changing relations in space of extended and movable beings. It is, in reality, in this way that the mind is related to the brain. To speak of the mind as having its "seat" in the brain is to reaftirm the reality of such relations.

§ 7. The term "organ" (or instrument) of the mind, as applied to the body, is particularly calculated to emphasize the relation of the ideas and volitions which arise in consciousness to the control of the muscular apparatus. But the same term may also be used, though with less of propriety, to describe the relation of the brain to the mind in sensation and thought. Thus we may be said to feel or think with the brain, in some manner supposed to be analogous to that in which the workman accomplishes his task by availing himself of a particular tool or instrument. It is obvious, however, that the figure of speech suggested by these terms also will not admit of a literal interpretation. We cannot conceive of the mind as a peculiar kind of material entity which, when it desires or wills to move the bodily members in a certain way, lays a clutch-as it were-upon the nervous substance of the central organs, and so makes the body serve as an "organ" of the desire or volition. Even less are we to conceive of the brain as a complex tool or mechanism which the mind uses in thought and feeling, somewhat as senses and fingers avail themselves of a calculating machine or of a musical instrument.

In producing changes of shape and position in masses of matter outside of our own bodies, we ordinarily find it convenient to use some material medium between those masses and the various movable parts of our own bodies. We throw up the ground with spade or shovel, cut down the tree with an axe, feed ourselves witl knife and fork, etc. We can, by means of much more complicated mechanisms, accomplish a great variety of changes which it would be quite impossible to accomplish without such aid. On the other hand, we sharpen, define, and multiply our sensations and percepts of things in similar manner. The deaf man hears with a trumpet or other acoustic contrivance; and the scientific observer contrives an instrument for observing the absolutely simple tones as analyzed 
out of the composite clang. With the lenses of his spectacles the man of defective vision sees what would otherwise be invisible; and with a prism the optician beholds the colors of the spectrum. Remote objects are brought near with the use of the telescope, and very minute objects near by are revealed by the microscope.

It is characteristic of all the most skilful use of tools and instruments that they come to seem to the observer like a part of his own bodily mechanism. By feelings of "double contact" (see Part II., chap. VI., \& 31) the workman comes to know, with the chisel, the wood or metal which he is carving-just as the blind man seems to extend his conscious life to the very end of the stick he is accustomed to carry. In these cases the mental picture before the practised mind is not that of the hand and the way it must be moved, but of the graving tool and the motion to be imparted to it-as though the instrument itself were immediately subject to volition.

\$ 8. The conception of an "organ" or instrument may with propriety be extended so as to cover the relation which exists between the nervous system and the muscular, and between the central and the peripheral parts of the nervous system. Thus it may be said that the spinal cord and brain move the limbs with the use of the afferent nerves, or that the cerebral hemispheres employ the lower ganglia of the brain in effecting certain co-ordinations of sensation and motion; it may even be said that the end-organs of sense communicate with the supreme central organs by means of the afferent nerve-tracts and the lower ganglia. All such language expresses, correctly enough for popular usage, the undoubted fact that, in the complicated relations of position and motion which are maintained among the different members of the nervous system, a certain order of action is constantly preserved. Clanges originate in one part, and are propagated to other contiguous or more distant parts. In such propagation of the changes a regular tract of the advancing motions is assumed always to exist; and thus the parts that lie between the extremes may be looked upon as means or. media-as instrumental to the completion of the process. For example, in quick succession upon a certain idea of motion, and a volition to a definite motion, my arm is raised or my whole body changes its position in space. How can this come about? To the inexperienced person the result seems to be an "immediate" effect of the will-that is, no apparent media or instruments stand between the volition and the subsequent changes in the relations of the masses of the body to other objects.

The vulgar persuasion undoubtedly is, that a man immediately 41 
knows himself to be the cause of the morement of his own arms or legs; that he knows that he can move them if he will. Little investigation, however, is needed to discover that such is in no respect the state of the case. A thousand hidden livks, any one of which might drop out without our being immediately aware of it, intervene between the volition and the actual motion. No onc directly knows that one can move as one will; one knows that one can will, and infers that, if one will, the movement will follow. In tracing the line of physical sequences backward from the motion of the limb toward the arising of the volition in consciousness, we bring it to a termination in a hypothetical nerve-commotion in some (socalled "motor") area of the brain. At this point the line of sequences, considered as a succession of modes of motion, drawing constantly nearer to the instant of the volition, comes to an end. The connection beyond and into that state of consciousness which is called a "volition" cannot be conceived of as the progressire propagation of a peculiar molecular motion called nervecommotion.

\$9. It is obvious from the foregoing remarks that one part of the nervous mechanism can be said to be the "organ" or instrument of another part, in a meaning of the word which cannot properly apply to the relation of the brain and the mind. In a certain justifiable meaning of the word, all the rest of the body may be said to be the organ of the brain. That is to say, those changes in the molecules of the brain's substance which arise there-whether because of certain ideas and rolitions of the mind, or because of changes in the character of the blood-supply, or of sensory impulses thrown in from the periphery or other lower nervous centres-get themselves expressed through the other members of the body. All this system of instrumentalities or interdependent organs is of one nature. It consists of material particles having a definite chemical constitution, structure, and arrangement in space; its functions are all modes of motion of such particles. One part serves as an instrument or "organ" for another, because the changes in the former. effect changes elsewhere, not directly, but through contiguous and connected parts. If the necessary contiguous parts are wanting or their relations disarranged, if the connection is interrupted or destroyed, then the work cannot be done; the "organ," "instrument," or " means," is lacking.

What is true of the relations described by the word "organ," as these relations exist between any two parts of the nervous mechanism, is only partially true of the relations which exist between any particular portion of this mechanism and the phenom- 
ena of consciousness. In other words, only a part of the real relations existing between mind and brain can properly be described under such terms as "organ," "instrument," etc. The brain, with its appropriate functions, is an indispensable medium between certain changes in the peripheral parts of the body and corresponding changes in the states of consciousness. If ideas of motion and volitions to move are to be followed by actual motions corresponding to the ideas and rolitions, then the brain must act with its appropriate functions. The motions can be executed, if at all, only through the brain. As much as this is true of all the efferent tracts which lead from the cerebral cortex through the lower portions of the encephalon, along the spinal cord, and out to the particular groups of muscles. Something more and special is, however, true of the brain. It is the first of the indispensable plyysical linlss in the whole chain; it stands nearest, as it were, to the mind. All the other steps in the execution of the ideas and volitions of the mind depend upon what takes place in the brain. If nothing takes place here, nothing at the periphery of the body will come from the volitions; if anything wrong takes place here, all that goes on at the periphery will be wrong, and the mind will not get its volitions executed. In this sense, at least, the brain is the particular organ of the mind; it is the most intimate and indispensable means for the execution of all its ideas or volitions of motion.

It does not appear that the foregoing statement by any means exhausts the description of the experience, reflection upon which induces us to regard the brain as the "organ" of the mind. For the brain seems to serve as the special physical basis of the ideas and rolitions of motion themselves. After experience in moving a particular member of the body has once been gained, that member may be lost; and yet if the proper areas of the brain remain unimpaired, the ideas, feelings, and rolitions connected with the movement of the lost menber will still arise in the mind. The man whose leg or arm has been amputated can still feel it, can form the image of how it should be mored to be in this position or in that, and even will to have it moved. The leg is not, then, the organ of these ideas, feelings, and volitions.

Experiments with animals, by extirpating the cortical areas, and observation of human pathological cases-especially, perhaps, in certain forms of aphasia (comp. Part II., chaps. I. and II.)- seem clearly to show that a much more intimate relation exists between the brain and the mind. With the destruction or derangement of certain of its areas, the power even to form such ideas and volitions, or to have such feelings, seems to be impaired or lost. We cannot 
say, to be sure, that the mind has lost a part of its general faculty to conceive, to feel, and to will. It has, however, suffered in respect to its power to frame a certain set of definite ideas and volitions as respects the motion of the peripheral members. This class of facts is certainly calculated to emphasize strongly our conception of the brain as being, in a special sense, the indispensable means through which the states of consciousness are related to changes in the position of molecules and masses of matter. Thus much, then, we are also entitled to include in our declaration that the brain is the "organ" of the mind.

There is another most important class of facts which may be partially described under the same terms as the foregoing. The brain is the indispensable means for furnishing the mind with its sensations, and so with its presentations of sense or perceptions of things. This statement is not to be understood as though the brain could, of itself, construct the sensations and perceptions and hand them over ready-made, as it were, to the mind. Sensations are states of consciousness, not modes of the brain; and even when they are synthetically united, localized, and projected to the periphery of the body, or into surrounding space, they are brought under no essentially new relations to the nerrous mechanism. Sensations are not nerve-commotions, "etherealized" by the optic thalami and cerebral convolutions, and then handed over to consciousness. Therefore the instrumental relation between brain and mind is not that of transmitting a peculiar kind of motion from one phase into another, or from one being to another: The brain is not the "organ" of the mind in the sense in which a being that starts or receives some mode of motion becomes instrumental for the production of changes in another being. Nevertheless, no sensations will arise in the mind unless the brain be affected in a certain way. Looking at the chain of sequences as it runs from without inward, we might say: The brain is the organ, or instrument, through which the stimuli of the outside world, acting on the end-organs of sense, finally reach the mind.

What is properly meant by speaking of the brain as the mind's organ of sensation is, strictly speaking, to be described as follows : The brain is the last and most important physical antecedent to the mind's being affected with the different sensations. The eye, ear, nose, etc., are popularly called organs of sense. Nothing is more obvious about the whole process of sensation than the part played in it by the peripheral sensory organs. It is apparently through these, by means of these, only on condition of the acting of these, that sensations arise in the mind. Further examination shows, 
however, that the end-organs of sense are more remotely connected with the origin of sensations than might at first be supposed. Excitement of the afferent nerve-tracts between the end-organs and the brain will also produce sensations. If these sensations are not so refined and complex as those which are caused by stimulating the end-organs themselves, the reason is to be found in the fact that we cannot stimulate the afferent nerves in the way to produce such sensations except through the appropriate end-organs. Still further examination shows that the value of the stimulus applied to the afferent nerves for the production of sensation is entirely dependent upon what the conducting organs convey to the brain, according to their specific nature and appropriate connections. Sensations can be equally well produced by stimulating the cerebral substance directly. When drugs, or gases, or diseases, or increased action of the blood-vessels change the character of the blood-supply, we see, and hear, and feel a world of things that has no existence for the end-organs of sense. The brain is then the "organ" of sensation for the mind, in the sense of being the indispensable and most inmediate means for the production of sensation.

Nothing that has just been said interferes in the least with the valid claim for the mind, that it alone is the producer of every sensation; or, in other words, all sensations are modes of the behavior of a being that is non-material and a unit-being, and is called Mind. When the physical conditions are fulfilled in the brain, and according to the way in which they are fulfilled, the mind itself puts forth the phenomena of sensation. For the sensations are not copies of outside material molecules, whether acting on the end-organs of sense or acting as excited nervous substance in the brain; it can scarcely be repeated too often-they are modes of the conscious activity of mind.

$\S 10$. Still another class of attempts to generalize, and embody in a single term, the various essential relations of the brain to the mind leads to the inquiry after some one special "connection" or "bond" between the two. How are mind and brain connected? What real tie binds them, so that they are obliged to have regard to each other in the modes of their behavior? Here, again, any too literal answer to this inquiry leads at once to manifest absurdity. A material bond designed to unite mind and brain might perhaps be conceived of as connected with the latter, and yet as remaining material; but in order to make it connect with the former (the mind) it would have to become non-material, unless we are ready to concede that the material and the non-material can stand connected without some special bond. In case this con- 
cession is once made, however, we cease to feel the need of a special bond between the mind and the brain.

If it be at once admitted that no connection is to be sought, or can be found, between the mind and the brain, beyond the fact that their modes of behavior are mutually dependent, it will not be necessary to appeal to any special mystery. What bond connects together the planets of the solar system so that each one moves invariably with reference to the position of all the others, and yet in a path peculiarly its own? We can only respond by talking of the force and laws of gravitation. These "laws," however, are simply a mathematical statement of the uniform modes of the behavior of certain physical beings; this "force" is no entity or bond connecting the individuals with each other, as the rods of the orrery bind its parts to a common centre. Did such rods exist to bind the planets to the sun, we should still have to inquire for some bond between the particles of the rods ; and for another bond to unite the atoms into these particles. Nor would it be an answer to such inquiry to discourse of cohesion and of chemical affinity, or of the laws which control the action of those forces. For cohesion and chemical affinity are not special bonds; they, too, are but expressions for the facts that the elements of material reality, under certain conditions and according to the kind to which they belong, behave as though bound; these elements behave, that is to say, according to what they are, and according to the relations in which they stand to each other.

No more obscure and unsatisfactory is our knowledge concerning the "bond" which unites body and soul, or, more especially, the mind and the brain. The brain is a vast collection of material molecules, connected together in a great variety of ways, which always act, as it were, with their own chemical constitution, and relations to other similarly constituted bodies, fully in mind. Even the molecules are not bound to each other by any one discoverable or conceivable bond. So far as we can speak of them as "connected" at all, they are connected by a great variety of bonds. Each of these bonds depends upon the nature of the molecules which enter into it, and upon the manner in which each molecule is related to other molecules. Essentially the same thing is true -and perlaps with no more of ultimate mystery in its truthfulness - of the connection between mind and brain. The mind is a conscious being, a being that perceives, feels, remembers, imagines, thinks, and wills. In respect to certain classes of its activities, at least, what it does depends upon what is done by the molecules of the brain with which it is, as we say, specially connected. The con- 
nection is not, however, such as can be explained by assuming one special form of a "bond" between the two. An infinite variety of relations, some of which are in a measure reducible under law, and others of which elude all attempts thus to subject them, exists between the unit-being called mind, and the composite structure and varied functions of the brain. The connection is no less real, however, because invisible; no less valuable and certain, because not one bond, but an infinite variety of relations.

$\S 11$. It will scarcely be supposed that information of scientific value concerning the nature of the real connection between the body and the soul can be obtained from terms which are alnost purely figurative and poetic. The limited and defective nature of our sense-perceptions, the misery of much of life, the unrealized longings for knowledge and happiness, and the work of imagination in framing a picture of some state of existence in which the limitations are removed and the longings realized, have led men in all ages to regard the body as the "prison" of the soul. Because the senses are not more in number than they really are, or more far-reaching and accurate than their construction permits them to be, they are regarded as restraining the soul, rather than as bringing it information which has the character of satisfying reality. The brevity and uncertainty of life, and the speed with which accident and disease impair or dissolve the bodily functions, together with the persuasion that the thinking principle will have a continued existence, suggest the reflection: the body is the "tenement" or "tabernacle" of the soul.

However true and comforting the foregoing hopes and reflections may be, it cannot be claimed that they throw any clear light on the subject of our investigation. Physiological Psychology rests upon such facts as show a most intimate and unceasing correlation between the body and the soul. It can never, therefore, consider the ultimate connection of the two as though it were as unimportant and superficinl as that between the prisoner and the prison which holds him, or between the tenant and the tenement or tent which for the time being is his abode. We are not at present engaged in considering the evidence that the mind is immortal, and can exist apart from this body and in another body, if not apart from all bodies; nor even the proof that its nature is vastly superior to that of all the material structures to which it might become related. We are rather testing the assumption that the mind, as connected with the brain, is a real being which, although dependent upon what occurs in the brain for its character and the order of its activities, has nevertheless that existence which belongs to 
all real beings - a nature and a development of its own. This assumption, indeed, is applied and confirmed in every attempt to characterize the real comnection which exists between the mind and the brain-whether the words "seat," "organ," "instrument," or other corresponding words be used.

$\S 12$. Thus firr little las been explicitly said as to the propriety of applying the terms of " causation" (such as "energy," "action," "force," "impulse," "effective agency," etc.) to the case of mind and brain. Yet everything which has been said has implied that these terms are really applicable. There would be no advantage to the mind in being "seated" in the brain-that is, in being under any special relations to a given extent of nervous matter-unless it were somehow influenced or acted upon by this nervous matter, and could in turn influence and act upon it. No "organ" or instrument is of any use whatever-that is, no thing can become an organ or instrument-unless it can be acted upon by that which employs it as an organ, and can in its turn act upon other things. Action of mind on brain is implied in calling the latter the organ of the mind's volitions; action of brain on mind is implied in calling it the organ of the mind's sensations. To act and to be acted upon is equivalent to standing in the relation of cause and effect.

It is not at present necessary to point out in detail how much of obscurity and contradiction are involved in all the more popular ways of mentally representing the foregoing relation. The transmission of energy (or force) is popularly spoken of as though such energy streamed off from one body and attached itself to another; and as though the quantity of energy thus given off were dependent upon the strength of the blow given by one body to another. Let it be supposed, however, that the application of the law of causation to the case of brain and mind is made in the most approved manner. It is simple matter of fact, as tested by thousands of observations and experiments, that changes in the condition and functional activity of the nervous centres are followed by changes in states of consciousness, in a regular way ; and that, conversely, changes of the latter sort are followed by changes in the relations of the masses of the body, and of the functional activity of nervous centres and end-organs of sense. Now, unless we are ready to be satisfied with simply stating the facts, without making the attempt to find any rational account for them, we are obliged to consider these correlated changes under the terms of cause and effect. That is to say, we regard the mind as a real being with activities called states of consciousness, and the brain as a collection of real beings called moving molecules of nervous matter, and we as- 
sume that the latter acts upon the former and is acted upon by it in turn. In other words, brain and mind are conceived of as really connected under the law of causation.

Were it not for the influence of prejudice derived from speculation upon certain philosophical, ethical, and religious questions, no one would think of hesitating to apply the terms of causation to the case of mind and brain. The stoppage of the arteries leading to the cerebrum, by outside pressure or by embolism, is speedily and regularly followed by a disturbance or cessation of consciousness. Who doubts that a man loses liis senses as truly as he loses a portion of his brain-mass, because he has been struck a blow upon the head? The falling of waves of light or sound upon the eye or ear, the contact of the hand with the hard substance of the metal or wood, the breathing of the air into the nostrils, are universally regarded as the causes of the sensations and perceptions which follow. The general impression undoubtedly is, that the act of will is the cause of the motion of the different limbs or of the entire body. In each of these cases more careful observation results in supplying many links in the chain of causation which the popular account has overlooked. The result is a more minute and careful picture of those molecular changes which take place in the cerebral substance, as induced by the severe shock of the blow or by the gentle stir of the stimulus acting through the end-organs of sense. Science explains the way in which the visible changes in the positiou of the ponderous masses of the body are due to antecedent invisible changes in the molecules of the muscles, of the efferent nerves, and of the lower and the supreme nervous centres. But all this explanation implies the application of terms of causation to the entire chain of physical events ; and if these events are to be considered as in any measure explaining the psychical events with which they are connected in time, the relation of the two classes of events is also assumed to be one capable of statement in the same terms.

How impossible it is to avoid speaking of the connection of mind and brain, in terms of causation, may be illustrated by the relations between the condition of the intercranial blood-supply and the states of consciousness. The character of the cerebral circulation is said to have a great " influence" upon the condition of the mind. A slight increase of this circulation, resulting from a small quantity of alcohol or other drugs, or from the hearing of interesting news, produces an increased speed in the mental train. Reaction-time is found to vary witl changes in the circulation. In the delirium of fever the wild and quickly moving condition of the thoughts, fan- 
cies, and sensations is a direct expression of the kind of work which is going on, because of the accelerated heart-beat and the disordered character of the blood, within the cerebral arteries. Schroeder van der Kolk tells of a patient who, when his pulse was reduced by digitalis to 50 or 60 beats per minute, was mentally quiet and depressed; when it was allowed to rise again to 90 beats, his mind was in maniacal confusion. Cox narrates the case of a sick man who, at 40 pulsations in the minute, was "half-dead ; " at 50 , melancholic; at 70, quite "beside himself ;" at 90, raving mad. The character of dreams is determined, to a considerable extent, by the position of the head and the way in which this position affects the cranial circulation. Hallucinations not infrequently are immediately made to cease, when the person having them assumes the standing posture, or lias leeches applied to the head.

$\S 13$. Objections have arisen from various sources, and have been urged with various degrees of skill and intensity, against applying the conception of causation to the relations of mind and brain. So far as these objections are more purely ethical or religious, it is not consistent with the purpose of the present investigation to consider them. But certain objections are more purely scientific, or perhaps philosophical, upon a basis of observed physical and psychological facts. A brief examination of such objections is not only consistent with the present investigation, but even required by it.

Among the followers of the Cartesian philosophy it was held that body and soul cannot really act upon each other, because of the obvious difference in the essential characteristics of the two. The body is extended and material ; the soul, being non-material, does not possess the characteristic most distinctive of all that comes under the conception of matter. Matter and mind, as being in their very essence opposed, are separated from each other by the whole diameter of being. They cannot be regarded as united directly through any real tie, but stand at the mutually exclusive poles of being. That a certain marked correspondence exists between the phenomena of the extended and material body and the phenomena of the conscious non-material soul plainly cannot be denied; and some account for this correspondence must be given. No one can doubt that his sensations, in their quality and order of succession, are related to certain events in the physical organs of his own body; neither is it easy to persuade one's self that one's movements are not, at least in some indirect way, "ordered" by one's desires and volitions respecting them.

To account for the obvious regular relation between bodily changes and mental phenomena, two or three somewhat different 
theories may be proposed. One of these is the so-called doctrine of "Occasionalism." According to this doctrine body and mind do not stand in the relation of cause and effect toward each other ; neither one ever really acts upon the other. But on occasion of some event of a definite kind happening in the bodily realm, a corresponding event of its own definite kind happens in the domain of consciousness ; and vice versa. To say this, however, is plainly in itself nothing more than to repeat the facts of experience over again, but without offering any explanation of them. Since some real "ground" or reason that shall have causal efficiency seems needed in order to explain why body and mind should take "occasion" to act at all, in view of each other's action, theology readily finds such ground in the Divine Being. God, it is said, on occasion of an event occurring in either of the two diametrically opposed spheres, causes the right corresponding event to occur in the other sphere. Matter and mind are not causally connected immediately with each other; they are causally connected only through a common ground in God. Pure Occasionalism, however, seems to make too large demands upon a pious credulity. To be always observing mere "occasions," in order to cause body and mind to keep the right pace with each other, may well be regarded as unworthy of Divine Being. To meet this difficulty the theory of "Pre-established Harmony" is devised. According to this theory God has eternally predestined the entire succession of events in the world, down to every minutest detail. Body and mind, therefore, may be regarded as like two clocks which have been so constructed that, without either having any effect upon the other, they go on exactly as though one were actually moved by the other.

It would scarcely be worth while to consider seriously these older forms of the denial that any real causal relation exists between body and mind, were it not for the fact of their essential agreement with more modern forms of the same denial. Two remarks upon the foregoing theories, in special, are necessary. The assumption that matter and mind are separated from each other "by the whole diameter of being," if it be held to mean that the two forms of being are so disparate in nature as to be unable to act on each other, is an unverifiable assumption. It eren goes squarely in the face of many of the most important psycho-physical facts. We know nothing about what kind of beings can or cannot act on each other, except through our experience of what beings do actually act upon each other. The mystery involved in any one being acting on any other is equally deep and unfithomable, in whatever direction we attempt to explore it. Before ex- 
perience with the facts, we should be quite at $a$ loss to tell whether atoms of oxygen could act on atoms of hydrogen, under the laws of chemical affinity, or not; whether molecules of iron could act on other molecules of iron, under the laws of cohesion, or not, etc. How it is that material masses or molecules can "influence" each other, or what is the real nature of the force which binds them together, physical science is quite unable to say. So that, even if we were entitled to regard matter as somewliat, the very essence of which it is to be spread out, and mind as somewhat, the very essence of which it is to be conscious and not to be spread out, we should still be quite without justification in asserting ( $a$ priori, as it were) that one cannot act upon the other. But-just the contrary -if we are to accept, unbiased, the obvious witness of the facts, we are compelled to affirm: The phenomena of mind and the conditions of the brain are related so constantly and immediately under law, that we are warranted in believing in the action of each upon the other.

Moreover, the theory of Occasionalism, Pre-established Harmony, and all similar theories, do not in the least assist us to escape the difficulties which attach themselves to every conception of causation. We cannot regard the Divine Being as bringing about a change in either mind or body, on "occasion" of some other change, without assuming that mind (the Infinite Mind) stands in the causal relation to matter. Furthermore, we cannot conceive of a "reason" why this Being should effect one change rather than another, without regarding $\mathrm{Him}$ as subjecting himself to the same relation.

$\S 14$. It is interesting to notice certain relations, both of similarity and of difference, between a prominent modern theory as to the mutual action of mind and brain and the now-abandoned views of Occasionalism and Pre-established Harmony. Modern science raises most of its objections, against regarding the conditions of the central nervous system and the states of consciousness as connected by a real causal tie, out of a profound regard for matter and the laws of physics. The great value and significance of physical phenomena, and the regular modes of their recurrence, if not the independent and eternal existence of material beings, are taken for granted by this theory, whatever difficulties, fears, or hopes to the contrary may arise from the sphere of mind. Elements of material reality (called "atoms") are assumed to exist; the universal form of their relation is held to be the law of the conservation and correlation of energy. By "energy" we are to understand that which moves or tends to move the elementary atoms, or their 
aggregations, into molecules and masses. The energy which is regarded as causing actual motion is kinetic; that which is to be regarded as tending to produce motion is stored or potential. But inasmuch as we have no test or suggestion of the presence of energy except motion, we seem compelled to consider the so-called "tendency" to move (potential energy) as motion that is beyond the splere of the senses, because distributed over so vast a number of minute portions of matter whose amount of motion is too small to be discoverable. All physical elements and masses are, accordingly, always in motion, and the total quantum of this motion is invariable throughout the entire universe. All forms of energy must be classified, as respects quality, by the kind of their motion ; and as respects degree, by the amount of their motion.

On attempting to account for the whole world of phenomena in terms of motion, kinetic or potential, under the law of the conservation and correlation of energy, we are met with insuperable difficulty as soon as we enter the domain of consciousness. States of consciousness are not modes of motion. If they were, the general theory of physics would compel us at once to attempt a strict mathematical correlation between physical and mental phenomena. Just as the momentum of masses can be expressed, with a tolerable approximation to exactness, in terms of heat as a mode of motion, so would some formula be conceivable for indicating what amount of chemical changes, or nerve-commotion, in the matter of the brain, is the mathematical equivalent of the conception of home, of the sense of obligation, or of the idea of God. In other words, it seems impossible to regard any amount of physical energy as abstracted from the brain, so to speak, and expended or stored up in conscionsness. Energy is stored by the process of nutrition in the nerrous elements of the brain ; it becomes kinetic in connection with the phenomena of consciousness. But between the mind, whether regarded as merely the formal subject of consciousness or as a real unit-being whose faculty or power it is to be conscious, and the physical basis of mind in the brain, no correlation, no passing back and forth of energy, can occur.

Representing the same truth in another way, we may declare: The entire circuit of the transmission and distribution of energy is complete within the brain itself. Not a single atom enters its substance that does not come forth unchanged, with all its forces inherent in it. No atom is transferred from brain to mind, as all the atoms are transferred from the blood to the nervous substance of the brain. 'Not the most infinitesimal amount of energy exists, stored in the constitution of the molecules of this substance, which 
is not either used up there or returned to external nature in connection with the constitution of the molecules separated from this substance. The stricter we make our application of the law of the correlation and conservation of energy within the physical realm, the more impossible does it become to apply it at all to the relations of body and mind.

$\S 15$. It is not surprising that, in the estimate of one who is unaccustomed to regard with faror any explanation of phenomena which does not come under the most general law of all physics, the case of the mind and the brain should seem to demand the most extraordinary treatment. In any event, the facts of consciousness, as facts, cannot be denied. Whether we can explain them or not, with or without use of the law of the conservation and correlation of energy, they are equally plain and persistent. Men perceive, and imagine, and remember, and reason, and believe in the invisible, and choose, etc. All this they do, as possessed of a bodyand, particularly, of a nervous mechanism, the activities of whose central portion are related in some special and unique way with the doing of all this. And yet sure, beyond doubt-it is arguedis the existence of the atom, with its host of inherent energies; and supreme is the law of the conservation and correlation of these energies regarded as modifications of one fundamental form.

In view of so grave difficulties it has been of late customary to escape from them in one of several different ways. The general claim may be set up that all hopeful inquiry as to the nature of real beings, which act upon and are acted upon by each other under the law of cansation, must be abandoned. Knowledge, it is said, consists simply in the relating of phenomena under certain constant and regular forms of their recurrence, called "laws." This is substantially the position of Positivism.

It may also be held that all mental phenomena are to be regarded as merely transitory appearances-shadows cast, as it were, by the changing activities of the material molecules; and that the latter are the only realities. In this case the constitution and activities of the molecules are all to be regarded as determined by the interaction of the ultimate atoms which compose them, according to their inherent and inseparable forces, under the law of the conservation and correlation of physical energy. Whenever a certain constitution and consequent modes of activity are brought about in the molecules, under this general law, then it is of their own incomprehensible nature to exhibit, in addition to the various forms of motion known as nerve-commotion, another peculiar class of coexisting phenomena, called mental phenomena. The latter 
phenomena do not require a new subject; their appearance is the necessary resuit simply of the special and unique constitution and relations of the physical molecules of the brain. The mental phenomena are one form of expressing the fact of the real existence of these molecules, with such a constitution and in such relations. And just as we do not require a new subject for the mysterious and unique phenomena of magnetism, or of crystallization, but believe them to be only the expression of the new relations into which the same subjects of all phenomena-the imperishable atomshave been brought under the one law of the conservation and correlation of energy; just so do we find no particular need of a new kind of subject, other than the aggregated atoms, for the mysterious and unique phenomena of consciousness. This position is Materialism.

Still further, the impossibility of binding together by a causal tie, under the law of the conservation and correlation of energy, phenomena so utterly incomparable as are those of mind and brain, and the difficulty of assigning the mental phenomena to the same subject as that which, otherwise, manifests itself only as modes of motion rather than modes of thought, have led to more recondite speculation. Hence a return to the "mediæval" view has been made. The real connection of mind and brain has been found in a third somewhat, which is neither mind nor brain, as we know them, but is the ground of both. There is, then-it is claimedonly one substance as the real subject for the two sets of properties. "The one substance, with two sets of properties, two sides, the physical and the mental-a double-faced unity-would appear to comply with all the exigencies of the case." This position may, in general, be designated as that of Monism.

But immediately the inquiry arises and presses for an answer, whether we may not know something as to the real nature of this "double-faced unity," besides the mere fact that, phenomenally considered, it has two faces, or sides-the physical and the mental. Why does it manifest itself both as physical motion and as mental states-one Being, in two utterly incomparable modes of manifestation? Is it itself extended and movable, a material reality? or is it unextended and conscious, a psychical reality? To refuse to attempt the answer to this question is to take refuge in Agnosticism-and that at a critical point, to which we have brought ourselves unnecessarily through having been already overwise. For no one who claims already to know enough about the nature of socalled matter and of so-called mind to affirm confidently that they cannot be two forms of real being, acting on each other and being 
acted upon by each other, is entitled, just beyond this advanced line of knowledge, to make a run sideways into the refuge of confessed ignorance. Furthermore, if the "double-faced unity" is lield to be, in reality, either matter or mind, we raise again all the difficulties as to a real connection between two sets of phenomena so incomparable. Both Materialistic Monism and Idealistic Monism have, then, to undertake the task of showing how the one reality can appear under these two plienomenal forms of being-matter and mind - with its two sides causally connected.

$\S 16$. So far as the theories, which are proposed in order to escape the difficulty of admitting a direct causal connection between mind and brain, involve the assumption that the phenomena of consciousness can be regarded as modes of motion, or can be attributed to the molecules of the brain as their sole subject, they have already been refuted. So far as these theories resolve themselves into the speculations proposed by different schools of philosophy concerning the supreme philosophical inquiry, What is the nature of the Ultimate Reality (the Absolute)? psycho-physical researches have no direct answer to offer to them. But our present inquiry is a more modest one, namely: What is the nature of the real connection between human mind and human brain, so far as psycho-physical science throws any light on such connection? Our general reply is: This connection is, in reality, such as we find between all socalled real beings, to whichever of the two supreme classes (material or spiritual) such beings may belong. The molecules of the brain (so far, at least, as psycho-physical science knows anything of them) are composed of elements of material reality, called "atoms;" these atoms act by way of motion, according to their constitution and relations to each other and to their environment. The mind, on the other hand, is a real unit-being of another order than that of the atoms. Its acts are the various modes or states of consciousness. This being called mind is causally related to the beings called atoms; the relation is mutual. The mind behaves as it does behave bccause of the constitution and behavior of the molecules of the brain. The molecules of the brain behave as they do behave because of the nature and activities of the mind. Each acts in view of the other. The action of each accounts for the action of the other. But the action of neither is to be explained as solely due to the action of the other; neither mind nor brain can be regarded as the subject for the phenomena ordinarily ascribed to the other.

The position just taken is, of course, the most unmistakable Dualism. It assumes two kinds of real beings for the two incomparable classes of phenomena. Whether this position is the ulti- 
mate one attainable by human reason or not, the facts of Physiological Psychology afford no basis for speculation. It is possible that some higher point of view might enable us to resolve the Dualism, and to discover a common ground for the body and soul of man, and even for all physical and spiritual phenomena. But psycho-physical science, simply observing the facts and building upon them, and upon such assumptions as it, in common with all the sciences, is compelled to make, establishes this Dualism of brain and mind, and then hands the case over to philosophy for further consideration. Moreover, there is nothing in any science, physical or psychological, which offers a single valid reason why both mind and brain should not be regarded as real beings, material and spiritual, mutually interacting. This last statement we shall now justify by considering, briefly, the objections to it, which have induced the resort to the before-mentioned other theories.

$\S 17$. The law of the conservation and correlation of energy-as far as it has been observed, or can reasonably be assumed to hold good-offers no valid objection to the existence of a real causal connection between the mind and the brain. The present position of this law is that of an empirical generalization, found to hold approximately true for a large number of classes of phenomena, and presumably true for yet other classes. To exalt it to the place of a universal and necessary relation among all phenomena of every class - mental as well as physical-would be unwarrantably to extend its application. Even in the sphere of physical erents the law is as yet demonstrably true only to a limited extent. The various forms of physical energy in the inorganic world are as yet by no means all reducible to the terms of this law. Gravitation, on the one hand, and magnetism, or chemical affinity, or cohesion, or the forces that act when every crystal is formed, on the other hand, cannot be as yet related together so as to be expressed in these terms. No mathematical formula, or picture framed by the imagination, has thus far bridged over the gap between the molecular energy of inorganic and that of organic structures. In discussing the phenomena of general nerve-physiology, it was made obvious at every turn that even the behavior of the vital nerve-muscle machine under the influence of electrical or other excitation cannot be accounted for by any conceivable application of the known laws of those forces that move unorganized particles of matter. Nerveforce-what it is and what it will do ; what it is as judged by what it will do-cannot, at present, be correlated with any of the forms of energy which act as nervous stimuli. Yet who would for a moment hesitate to say that the action of the electrical current, or 
of the irritating acid, or of mechanical impulse when applied to the nerve, is a "cause" of the contraction of the muscle?

The effort of certain scientific observers to bring all causal relation, all action of one being on another, under the law of the conservation and correlation of physical energy is mistaken, and must prove unsuccessful. The discovery that all the action of physical beings is to be understood only in terms of motion, and that all relations of such leings are to be expressed as comparable quantities of motion, either obvious or potential, has, of course, greatly stimulated this effort. The effort is to a certain extent laudable. It has unified the physical universe ; it has showed to us this universe all alive, as it were, with unceasing, correlated, wondrous motions, which it is indeed conceivable should be all commensurable one with another. But it should never be forgotten that this picture of an objective world composed of beings called atoms, eternally moving with reference to each other and according to the law of the conservation and correlation of energy, is itself a picture constructed by the imaging and reasoning mind. As such a mental picture, it is, and must always remain, dependent on the imagination. Mind, as reasoning and imagining, follows the moving beings into minutiæ of forms and into places where observation can never reach them. Hence the talk of atoms having "forces inherent" in them, of energy "potential" as well as kinetic, of the "influence" or "action" of molecule on molecule, and mass on mass, under this one great law discovered by modern physics.

For the principle of causation is of far wider application, and of far more secure foundation, than the law of the conservation and correlation of energy. The one is a law which, in the form of the principle of reason and consequent, is worked into the very structure of the mind, and is of universal and necessary application to all phenomena; the other is an empirical generalization, of doubtful import and uncertain extent of application. Indeed, we should not accept the physical law at all, or seek to establish its further application, were it not that the mental principle is already taken for granted. It is in our search for causes, and as a result of our persuasion that real beings exist, which act on and are acted on by each other, that we hit upon the hypothesis of the sum-total of their energies as shown by motion remaining unchanged, and of its different kinds being all measurable one against the other. But no objection exists, either in the nature of the mind or in the nature of things, so far as we know, to the reverse of this being true. For example, a world might be constructed in which a certain number of physical beings, of a certain kind (molecules and masses), remained abso- 
lutely motionless and unchanged, while all other beings were in perpetual motion. Or a world might be constructed in which the activities of different physical beings, as expressed by motion, should be related in a totally different way from that formulated by the present law of the conservation and correlation of energy. In this imaginary world, some linds of beings might put forth an amount of energy which was proportional to that of all the energy of the beings acting on them, as the square to the square-root, or as the cube to the cube-root; and other kinds of beings might act and be acted upon under very different laws of relation with respect to the quantity of energy. In fine, the fact that the law of the conservation and correlation of energy cannot hold true as to the connection between physical and psychical phenomena furnishes no sort of proof against the reality of the mind or of the causal connection between it and the brain.

$\S 18$. Nor is there anything in the nature of the so-called "causal nexus" itself which forms a reason why it should not be assumed to exist between brain and mind. For what do we mean when we speak of one thing or event as the cause of another? What do we mean when we speak of "influence exerted," "force transmitted" or "passing over" from one being to another, etc.? Nothing that can be explained or illustrated after the analogy of any series or collocation of phenomena, of any relation of one object to another as discernible by sense or picturable by imagination. Nothing passes from the match to the gunpowder which explains why the latter explodes; or from the bat to the ball which explains why the latter, when struck by the former, changes the direction of its motion. The proximity of the earth and the unsupported condition of the apple, shaken by the wind from its stem, are indeed spoken of as the cause of the apple's fall; but no invisible hands are reached up from the ground to draw the apple down. Were such feelers put out to clasp the smaller body and draw it to the larger, the energy of the clasp and its effect would still as truly need an explanation as does the action of the so-called force of gravitation. None of the senses is capable of discovering or appreciating the energy that is assumed to act; the causes of an event cannot be seen, handled, heard, smelled, or tasted. The world of experience given to us by the activity of the senses is a world in which a ceaseless change of objects takes place, but any evidence of a tie connecting the physical phenomena with real beings as their attributes, or connecting our minds with these physical real beings, so that they may be said to affect us, is quite beyond the range of the senses. 
In general, it may be said that the world of appearances is found by an analysis of our adult experience to be assumed to rest, as it were, upon an invisible world of reality. The popular and uncritical mode of the assumption is, that the world is made up of a great number of real "Things;" that these things exist extra-mentally, just as they appear to us as objects of experience ; that our knowledge of them is a more or less true copy, obtained through the senses, of what they extra-mentally are; and that these things are constantly doing somewhat to each other-acting on each other and being acted on by each other. Scientific researches greatly modify the character of the popular assumption. They show that it is demonstrably false in almost every particular; and yet they re-establish it in other forms. Physics, by a series of careful observations and subtle and remote inferences, constructs an extra-mental world of moving atoms; it shows us how these atoms always have regard to each other when they move, and are ceaselessly moving with reference to each other; it strives to image the direction and velocity of the most infinitesimal of these motions, and to formulate their laws or constant modes of relation. Psychology shows how the world of mental objects, the only world of immediate experiences, is built up by the synthetic activity of mind ; it calls upon the physicist to remember that he has no other way of reaching these atoms, and of discovering the laws of their relations, except by the path of mental activity; and it reminds him that this activity cannot escape the control of mental law. But both the popular view and the scientific attainment are in substantial agreement as to their fundamental view of the world. Both believe that our experience is explicable only on the general hypothesis of the existence of a vast number of real beings which perpetually act on each other and are acted upon by each other.

$\S 19$. The effort to restrict the working of the above-mentioned assumption, in which common-sense and scientific analysis both agree, just at the point where the relation of mind and brain is subjected to scientific treatment, is as needless as it is unavailing. Because it is both needless and unavailing it is often absurd. If it be granted that the law of the conservation and correlation of physical energy cannot possibly be applicable to the connection of mind and brain, and yet that all which we know of the nature and extent of this law forms no valid objection to regarding both mind and material atoms as real beings standing in certain relations to each other-What good reason can be urged for refusing to affirm a causal connection between the two? The fear that either of the two parties will suffer in dignity or integrity by such connection with the other 
may easily be laid to rest; and if it could not be, it would have no right to interfere with the only reasonable interpretation of psychophysical facts. Beings do not lose their reality, or characteristic nature, or value in the universe of Being, because they are causally connected with other beings. On the contrary, none but real beings can be thus connected with each other; none but real beings can act and be acted upon. The so-called causal connection is no bondage of such nature as to destroy the nature of the beings which act under it. Only beings that have natures of their own can be causally connected. In other words, all that appears to us as a causal relation between the objects of our experience is, ultimately considered, due to no material spur or whip which urges, or band that represses, as though one kind of real being could thus dominate and subdue another. No atom acts without being acted on; what it does depends both upon what it is and also upon how it stands related to other atoms.

$\S 20$. We affirm, then, that we are entitled to say: The changes of the brain are a cause of the states of consciousness; and the mind behaves as it does behave, because of the behavior of the molecules of the brain. Modify the constitution and functional activity of the material atoms, and you make the activities of the mind, its acts and states of consciousness, to be differently put forth by the mind. The nature and extent of this "influence" of the material basis upon the psychical subject can never be determined a priori, or brought under any general formula applicable only to a restricted sphere of physical action, like the law of the conservation and correlation of physical energy. The nature and extent of such influence must be learned by investigation. It has been the special task of this treatise on Physiological Psychology to investigate and, as far as possible, to formulate the causal action of brain on mind. Such action has been seen to consist chiefly (if not wholly) in determining the intensity, quality, mode of combination, and of recurrence in time, of the sensational elements of the mind's activity, and of its other activities so far as dependent upon the sensational elements.

The affirmation of a causal influence of the brain on the mind, however, does not really work any prejudice to the claims of the mind to be considered a real being, or to be spiritual and free. For the sole account or cause of the mind's activities can, in no instance, be found in the molecular condition and changes of the brain. The simplest sensation must be referred also to the nature of the mind as its cause. It must be considered, not simply as caused by a certain form of nerve-commotion in the cerebral cortex, but also 
as a psychical activity put forth by the being called mind. There is no incompatibility in these two ways of regarding each state of sensation. Even in the case of some physical event, the nature of each of the factors combining to form the event must be taken into the account. For example, atoms of oxygen will, under certain circumstances, unite with atoms of hydrogen to form water ; under other circumstances they will unite with atoms of iron to form iron-rust; they may also be mechanically mixed with nitrogen-atoms to form air, etc. In each case the cause of the result is to be found in the presence with the oxygen, under certain definite circumstances, of atoms of hydrogen, iron, nitrogen, etc. But in each case the cause is also equally to be found in the nature of the atom of oxygen. So every sensation, however closely it may be correlated with the condition and functional activity of the molecules of the brain, must be explained by referring it to the nature of the mind which has the sensation. Nothing which Physiological Psychology has ever discovered, or can hope to discover, in the least mitigates the necessity of saying, when the question is asked-Why does the mind behave in this particular way under such circumstances?-It is the nature of the mind so to behave when its circumstances are such. In other words, our explanations of the causes of mental phenomena, as lying in the physical basis of such phenomena, does not at all satisfy the need of a real and spiritual subject of the phenomena.

Moreover, we have seen that there are large and most important classes of mental activities which can scarcely be conceived of as standing in any direct relation to the nerve-commotions of the cerebral cortex. These classes are indeed always allied with phenomena of sensation and feeling for which we can trace a bodily basis. But this fact only makes their connection with the brain presumably more indirect. For the explanation of such classes of mental phenomena we are driven much more imperatively and exclusively to an appeal to the existence of a spiritual subject, with a nature and laws of action very different from those ascribed to its physical basis, the brain.

$\S 21$. We affirm, also, that we are equally entitled to say: The states of consciousness are a cause of the molecular condition and changes of the nervous mass of the brain, and through it of the other tissues and organs of the body. And just as no fear for the reality, integrity, and dignity of the mind prevents us from accepting its dependence for the mode of its activity upon the condition of the brain, so no fear for the reality of physical substance, and for the value and extension of physical law, prevents us from as- 
serting the dependence of the brain, for the mode of its activity, upon the states of the mind. Of course-it need scarcely be said again-no relation exists between these two kinds of beings which can be represented as an interchange of physical energy, under the law of the conservation and correlation of such energy. This fact, however, affords no objection to our recognizing a true causal comnection between the two, unless we are ready to insist upon the monstrous claim that modern physical science is entitled to affirm the impossibility of any interaction (or conditional action) taking place in the universe otherwise than between material atoms under the aforesaid law.

The phenomena which indicate that mind operates as true cause within the structure of the body are innumerable. They are quite as numerous, though perhaps not so obvious and impressive, as those which indicate the reverse relation. The chief reason why these phenomena are relatively little regarded in psycho-physical researches is, that the real causes are in this case not readily made the objects of observation and measurement. External stimuli constitute the causes of mental changes which we can most easily observe and estimate. Ideas, feeling, and acts of will arising in the consciousness, and cousidered as causes of the resulting bodily changes, cannot be treated by the same methods of experimental science as apply to the physical stimuli. But that the mind acts on the body is one of the inost familiar of experiences. Such action penetrates and modifies all the life of the body. Hence the material mechanism of the animal structure can never be considered, with a view to explain what is going on within it, as though it were disconnected from the consciousness of the animal. The most purely vegetative of the processes of the human body are dependent for their character upon the states of the human mind. The nutrition of the tissues, the circulation of the blood, the secretion of different kinds of fluids, the healthy or diseased nature of the vital processes, are dependent upon the states of the mind. If abnormal digestion produces melancholy, it is equally true that melancholy causes bad digestion. In the case of the rise of strong emotions, like anger or grief, the increasing affection of the mind builds itself up upon a physical basis of increasing disturbance of the organs ; but it is equally obvious that the starting of the emotion in consciousness, and the letting of it slip from control, are necessarily followed by gathering momentum to the organic disturbance. Irregular action of the heart, caused by organic defect or weakness, occasions a feeling of indescribable alarm in the soul ; fear is followed, through the action of the mind upon the nervous 
centres, by functional incapacity of the heart. The impure condition of the arterial blood which is characteristic of certain diseases brings about a chronic state of mental lassitude or anxiety; care, chagrin, and ennui poison the arterial blood. The lesion of the cortical substance produced by a growing abscess or broken bloodressel impairs the mind's powers of sensation and thought; excessive thought and over-excited feeling wear away the brain.

The entire class of phenomena which we are entitled to call "voluntary," in the widest sense of the word, might be appealed to in proof of the same principle. Whether they show that the mind is "free," in the highest ethical meaning of the word, or not (and upon this question psycho-physical science cannot pronounce), they certainly do show that the condition of the bodily organs is made dependent, through the nervous elements of the brain, upon the states of the mind. Aud here are, in point, the phenomena of the voluntary innervation of the organ by fixing the attention, of the dependence of reaction-time upon the exercise of the will through attention of the person reacting, of the abstraction of regard from the images of sense when occupied in reflective thought, as well as all the more marvellous instances of self-control in determining the results of disease, etc.

The elevation of the bodily activities to the most astonishing precision, under the influence of high and strong artistic feeling, or sense of duty, is also a noteworthy fact of the same order. The mind has not the power to constitute, in opposition to fixed chemical affinities, a single molecule, or to execute the slightest movement of a single muscle, without involving the nervous system in the expenditure of the requisite energy. Moreover, this energy must be started in the appropriate cortical area and descend along the allotted motor tracts. We cannot explain how it is that molecules of nervous matter can be acted upon in view of states of consciousness. But neither can we explain how one kind of atoms comes to act as it does in view of the presence and action of atoms of another kind. Nevertheless, we can just as little assume to explain away the fact of such obvious causal connection, because we cannot bring the measure of the connection under the same law as that which maintains itself among certain modes of physical motion.

§22. No valid objection, therefore, can be urged against conceiving of the connection between mind and brain in the following way, at once most natural and most philosophical: The brain is a vast collection of material molecules, whose constitution and arrangement are such as to connect them, in a unique way, with cer- 
tain forms of physical energy outside of the body. Whenever these appropriate forms of energy act upon the parts of the nervous system lying below, and the impulses are transmitted to the brain, or whenever the chemical character of its blood-supply is altered, then the molecules of the brain are capable of undergoing very remarkable and intricate changes of constitution and arrangement. That is, the brain can be stimulated to certain of its peculiar combinations of nerve-commotion by external stimuli. Moreover, it is constantly initiating other combinations of nerve-commotion that are apparently not due to such stimuli. Some of its actions, that is, are of a kind constantly arising within the system itself ; they are called automatic. We have as yet no adequate means whatever for making a quantitative statement of the relations which exist between the energy of atoms thus constituted and arranged and the energy of the masses or molecules that serve as stimuli of the system composed of these atoms. Nervous energy is not an entity to be dealt with by a sum in addition and subtraction of momenta. For aught we know, it is of the nature of atoms, when they are brought into relations so extraordinary as those which prevail in the nerrous system, to behave with reference to each other in a way that is wholly irreducible to any simple formula like that of the conservation and correlation of energy. If this should finally appear to be indubitably true, the fact would not be specially mysterious. All action and reaction of the atoms is mysterious; the methods of it are to be learned from experience as ultimate and inexplicable facts.

Still further, the molecules of the brain are so constituted and arranged as to be capable of standing in yet more surprising and unique relations to a being of a different nature from their ownthat is, to the mind. These relations involve a causal connection as truly as any relations of real physical beings in which such beings, as we are compelled to believe, act on each other and are acted on by each other. That molecules thus constituted and arranged are causally connected with the subject of consciousness is an ultimate fact ; it involves the nature of both classes of beings thus connected -of the brain and of the mind; it involves also the action of each upon the other. In speaking, however, of mind and brain as acting on each other, we accomplish notling whatever for the completion of the picture by trying to introduce the conception of energy "transmitted" or "passed over" from one to the other. The simple, ultimate fact remains, that how each behaves depends upon the behavior of the other. It is the business of psycho-physical science to discover, if possible, the general modes of this depend- 
ence-that is, the laws of the relation between the mind and the brain.

\&23. In more particular description of the connection between the mind and the brain, it may be said that all intercourse between material objects and the spiritual subject inrolves three processes -a physical, a physiological, and a psychical. In these processes the perceived object and the perceiving subject mutually condition each other. This fact, however, does not destroy the necessity, under which all scientific investigation finds itself, of assuming that both object and subject exist as real beings. The physical process consists in the action of the appropriate modes of physical energy upon the nervous end-apparatus of sense. The bringing of such modes of energy to bear upon the apparatus is accomplished through mechanical contrivances-such as the means for forming an image on the retina in the eye, and for conveying the modified acoustic impulses to the organ of Corti in the ear.

The second process consists in transmuting the physical energies, in part at least, into a physiological process, a nerve-commotion within the nervous system; and in propagating such nervecommotion along the proper tracts and diffusing it over the various areas of this system. Inasmuch as the physiological process is also a physical process-that is, a mode of the motion of material molecules, accompanied by chemical and electrical and other changes-it must be conceived of as standing in certain relations of quality and quantity to the first, or more distinctively physical, process. But that the law of the conservation and correlation of energy, as formulated for much simpler cases of the relations of forces between inorganic bodies, applies to the relations of the nervous system and its stimuli, or within the different parts of the nervous system itself, we are not yet able to affirm with confidence.

The third process is psychical; it is a process which is a psychical event, a forth-putting of the energy of mind. It is directly correlated with the physiological process only when the latter has been realized in certain cerebral areas. It is not to be explained as a resultant of the cerebral physiological process, but as an action of the mind which is conditioned upon that process. So, also, are we entitled to say that, when certain psychical processes, by way of feeling, ideation, and volition, take place, then, and as conditioned upon these processes, certain corresponding physiological processes occur in the brain; the physiological processes, being propagated from the central nervous system, end in physical processes returning energy to the world outside of the body. 
When the mental process is a perception of some object, called an "external" object, it is no less truly a psychical process. The mind creates its own objects; presents itself with its own presentations of sense; acts to bring forth that which it knows as not itself. But it does all this as dependent upon the processes which take place outside of itself, and with the assumption of extra-mental realities as existing, to which it stands in the relation of cause and effect.

\$24. Finally, then, the assumption that the mind is a real being, which can be acted upon by the brain, and which can act on the body through the brain, is the only one compatible with all the facts of experience. There is nothing which we know about the nature of material beings and the laws of their relation to each other, or about the nature of spiritual beings and their possible relation to material beings, or about the nature of causal efficiency whether in the form of so-called physical energy or in that of activity in consciousness, which forbids the aforesaid assumption. On the contrary, everything which we actually know, as distinguished from what we conjecture to be true, or would like to have true, for the satisfaction of certain of our quasi-scientific or ethical impulses favors this assumption. And no other assumption, substantially different from this, is compatible with the facts of experience. 


\section{CHAPTER IV.}

\section{THE MIND AS REAL BEING.}

\$1. No attempt need be made to conceal the fact that the last three chapters have given to the phenomena and laws of Physiological Psychology a "metaphysical" treatment. In the introduction (see $\$ 5$ ) to the scientific discussion of the subject, the intention finally to raise and answer certain metaphysical questions as to the nature of Mind was frankly avowed. Indeed, since all discussion of those assumptions which underlie our experience of what we call "reality" is metaphysical, it is not easy to see how the science of mind-from whatever point of view approached-can be thorough and conclusive without involving metaphysics. In this, the concluding chapter, certain still more distinctively metaphysical inquiries must be briefly pursued. The mind has been spoken of as "real," "spiritual" (or non-material), a "unit-being," etc. These are terms which require further explication. What is meant by speaking of the mind as a real being? What is it to be, in reality, spiritual rather than material? What is the real nature of that unity which belongs to mind; and on what grounds do we affirm that the mind is a "unit-being?"

Thus far the effort has constantly been made to maintain a close connection between the answer given to semi-metaphysical inquiries and the facts of physiological psychology. In rendering such answer the appeal has constantly been taken to the facts. Should the facts, in any case of appeal, not bear out-or, at least, should they contradict-the conclusions alleged to be based upon them, then the conclusions must be modified, or change the basis on which they assume to rest, or utterly fall. But in answering the more distinctively metaphysical questions now raised as to the nature of mind, the psycho-physical facts are of little direct assistance. Such questions are fitly raised at the conclusion of psychophysical researches only because these researches have led us to a certain view as to the nature of the subject of the researchesnamely, as to the nature of the mind.

§ 2. The mind is a "real" being in the highest sense in which 
any finite being can be real. Indeed, its claim to be considered real is more indisputable than the same claim as put forth for any material thing; it is unique. The reality of mind underlies and makes possible all our knowledge of other real beings, and all our assumptions as to the existence of such beings. It is only on condition of granting its reality, in the highest sense of the word, that we can affirm the reality of other beings.

There can be no doubt that the popular impression attributes a reality to material things which it does not consider to be possessed by the mind. This impression makes the clearly visible and hard, tangible substances, experience of which constitutes so important a part of ordinary working-day life, the test and standard of the most indubitable reality. Substantial as a rock (that is, a presentation of sense which consists of certain qualities made known especially to the tactual and muscular senses); and unsubstantial as a day-dream (that is, a series of representative images largely free from all admixture of presentations of sense)-thus does the popular estimate express itself with respect to the reality of the phenomena referable to things as compared with the pure states of mind. Materialistic objections to the reality of mind, when made to rest upon scientific data, repeat and confirm the popular impression. These objections ordinarily assume that no doubt can be raised as to the reality of material "Things." Such things as are real in the highest sense of the word, however, are not now understood in the same way as the things indicated in the popular impression. Only the atoms, or elemental and permanent factors which enter into the composition of all the objects of sense, are held from the scientific point of view to be real in the highest sense of the word.

The things of experience by the senses are admitted to be constantly changing, and at no time, extra-mentally, to resemble the unchanging material realities with which science deals. Things, as they appear to the eye and to touch, are spread-out, continuous, without empty space between them, and for the most part motionless, except as they are moved in masses by application of external energy. Things as they really are, however, are neither spreadout, nor continuous, nor motionless. On the contrary, they consist of a countless number of invisible and intangible real beings, called atoms, that are ceaselessly moving, with incredible velocity and intricateness of changing directions, in empty space, and according to forces inherent in them. These atoms are real, and have always been-the sole element of all which appears as real; so this form of quasi-scientific metaphysics goes on to declare. 
On the other hand, it is argued by certain advocates of the foregoing view that the so-called mind is wanting in every characteristic which could justly entitle it to be called a reality. Certainly it is not adapted to win the popular respect as a hard and solid substance, which it is difficult to move, and impossible to remove from the sphere of possible sensations. What is the mind, in reality? It cannot be seen or touched, or apprehended by any of the senses. It cannot be imaged as spread out in space, or as space-filling, by virtue of some physical energy streaming minterruptedly forth from the mathematical point at which it is situated. It can do nothing except through the body ; that is to say, all that is done, which could possibly be referred to the mind, is really done by the body. And the body is a material mechanism, which is nothing except as it is constructed out of the same atoms, with their inherent forces, which have composed the star, the crystal, the flower; and which can do nothing except as the ceaseless play of the energies of great Nature (of which it is a point, a part) are liept playing through it. Without the physical mechanism, as a real existence, there is no manifestation of so-called mind, no manifestation actual, possible, or conceivable. And when this mechanism is dissolved, the mental phenomena, so far as appears, wholly cease. What, then, is Mind? What claim to reality can it possibly make valid?

More particularly, certain puzzling questions regarding the nature of the mind's behavior may be raised by the advocates of the same foregoing view. Where is the real mind, it may be asked, when consciousness is gone, as in swooning or deep sleep? What becomes of the mental Being when the mental faculties one by one drop away, as in cases of general paralysis? What worthy kind of reality can belong to the subject of phenomena so evanescent and temporary, so incapable of being measured, and weigherl, and related to the permanent forces and beings of the material world? Moreover, if mind is a real being, what shall be said in answer to the inquiry, why certain of the lower animals can apparently divide up their souls by fission of their physical structure? And cannot even man's proud unity of real being become disturbed by the accident or disease which results in a double consciousness, or in the loss of all previously acquired knowledge- of the mind as previously existing and developed?

§3. That many puzzling, and even unanswerable, questions can be asked concerning the nature of the mind, we have no interest to deny. Doubtless, if difficulties growing out of our inability wholly to clear up our ideas of "real being," "self-identical and permanent existence," etc., are objections to believing that any real beings 
exist, they are also objections to our believing in the reality of mind. But certainly it is one thing to ask unanswerable questions regarding the ultimate nature of any particular real beings, so called, and another thing to prove that our belief in the existence of such real beings is unfounded. Moreorer, the fact that we cannot conceive of or define the real being of the soul, in terms which apply to material things, is cheerfully conceded. Indeed, it is this general fact upon which chief reliance is placed to prove that the being of the soul is unlike that of "Things"-is non-material, or spiritual. But how can this fact prejudice the claim of the soul to be real, unless it has previously been established that to be hard, and round, etc., or to be a minute material bit (an atom) ceaselessly in movement, is necessary in order really to be at all? The truth is, however, that both the popular impression and the more scientific theory, just so far as they can cogently be urged against the reality of spiritual being, themselves rest on the most unverifiable and absurd assumptions.

The popular conviction of the indubitable, and, as it were, superior reality of certain classes of things is easily explained as the necessary result of the development of experience. All things which are pre-eminently real, in this meaning of the word, are cognizable by means of tactual and muscular sensations marked by a strong color-tone of feeling. Things merely smellable have no "reality," in this sense of the word, because they are not appreciable by touch and offer no muscular resistance. We cannot put our hands on the effluvia which excite the olfactory nerve; the air when laden with sweet and sickening odors is not tangible or impenetrable. Real things - that is, things which can be seen and handledare, however, regarded as the sources of our sensations of smell. What is true of sensations of smell is also true, in less degree, of sensations of sound. But in the case of sound we are generally able at once to refer the origin of the acoustic sensation to some so-called real "Thing." Objects tasted are popularly regarded as real, because they are objects which are handled before tasted, and constantly touched as they are being tasted in the mouth. A "bad taste in the moutl " is not regarded as giving evidence of the presence of any real thing; it may simply be regarded as a sensation located in that region. Accordingly, one does not consider one's self to be tasting one's own mouth as a real thing, although one may say that the mouth has a bad taste.

Even when the presentation of sense is a clearly visible object, it does not necessarily seem to have the characteristics of a real thing. For the object of vision readily and quickly changes its 
color, apparent magnitude, characteristics of superficies, and visible outlines as a solid. Moreover, everybody knows that his eyes have often deceived him; even when they have been closed visual images have appeared before the mind, such as could not possibly represent any so-called reality. But the nature of tactual and muscular sensations is different from the visual in several important particulars. Such sensations, on bestowing the requisite attention, may ordinarily be brought strongly into consciousness. They do not so readily change their quality regarded as coming to us from an apprehension of the properties of things. The feeling of eftort color's them highly; and the pain from being struck, pressed, pinched, or impeded, is a frequent accompaniment. Therefore, children are educated in their knowledge of, and belief in, a world of reality by being constantly resisted by material things; and adults naturally suppose that when they can lay hands on an object they know that it really is, and what it really is, with a certainty impossible in any other way.

It wholly escapes the ordinary observation that the same assumptions - whether they be deemed verifiable or unverifiable-underlie the conviction of the reality of things tangible which belong to the operation of all the senses. It is true, as experience shows, that tactual and muscular sensations are, from their very nature, and from the manner and frequency of their recurrence, peculiarly adapted to serve the mind well in those acts of synthesis by which it constructs the real things of its experience. But this fact does not in the least diminish the force of the other factnamely, a certain assumption or postulate as to an extra-mental reality (an $X$, which is not any one of the attributes- $a, b$, $c$, etc.of the "Thing," but which is the subject or ground of them all) underlies and conditions all the apprehensions of sense. Without granting and using this assumption we cannot affirm that even by tactual and muscular sensations we know any reality whatever, beyond the real fact that so our own minds stand affected with the presentation of an object of sense. If the popular impression concerning the reality of "things" does not extend beyond this simple act of self-knowledge, as it were, it certainly forms no ground for affirming the superior and undoubted nature of such reality.

$\S 4$. The cause of the scientific objector to the reality of mind, as standing on an equality, with respect to the cogency of its evidence, with those material atoms about whose reality he tolerates no doubt, is not a whit better off than is that of the popular impression. Indeed, it is by no means so good. That ready-made "things" really exist in independence of mind (meaning by such "things" the ob- 
jects of everyone's immediate experience) is a proposition which it involves fewer doubtful elements to maintain than the proposition that so-called "atoms" have such existence. It is time to raise the question: How can one know, so confidently, that those cerebral molecules exist extra-mentally, with all their incalculable and almost inconceivable motions, on whose real being the phenomena of mind are sometimes made to depend? The brain-it is claimed by $\mathrm{Ma}$ terialism-may be made responsible for mental phenomena, for the latter are mere manifestations in consciousness of the changes which are going on in the material constituents of which this organ is really composed; there is no need of a real non-material being as the subject of the mental phenomena; the physical phenomena, however, must have some real being as their subjects; such reality is to be found in the molecules of the brain. But what are the grounds and the nature of our linowledge of this wonderful conjurer styled the brain?

The so-called scientific argument against the reality of mind, as often applied, may be stated in terms somewhat like the foregoing. Little examination is needed, however, to show that its conclusiveness involves certain assumptions which cannot themselves be validated without weakening or destroying the very ground on which the argument is itself based. Let the case be tried by making a beginning with that sort of testimony with which everyone is most familiar. I know that I think, feel, will; that is to say, phenomena take place in consciousness which there is no conceivable way of describing except by attributing them to the subject of all consciousness-to the self-conscious "me" called mind. But because I cannot perceive this subject of all consciousness as an extended and external somewhat-a "Thing" so lar'ge, and shaped and colored in just such a manner, with a definitely hard or soft feel-that is to say, because I do not appear to myself in consciousness to be just such a lind of being as are some of the objects of my perception, I begin to raise the question whether this subject (the " $\mathrm{I}$ " that thinks, etc.) has any real being at all. May it not in fact be, I ask myself, that some "thing," or collection of things, like those which I have often seen and felt, is the subject to which the thoughts and feelings and acts of will that $I$ have called "mine" should be attributed? Of course, if this question is to be answered in the light of modern physiology with even a provisional affirmative, the particular " thing," to which such activities as those I am conscious of are to be attributed, is my brain. Nothing, surely, but my brain can think, and feel, and will-so to speak-for me. For if physical science has established anything whatever with regard to a particu- 
lar organ or substratum of the mental phenomena, it is that such organ or substratum is the brain.

But the inquiry must next be raised: How does one know that one has a brain, which may serve as the real substratum of the phenomena of one's consciousness? It scarcely need be said that no one has ever had any evidence presented directly to the senses that such organ exists within his own cranial cavity. To be conscious, and at the same time to observe the substratum of one's consciousness, is an unattainable opportunity. It may even be that the ego (the "I" of consciousness) which is engaged in the search for its own real being in a material substratum has never seen so much as a single human brain. It is certain that no ego has directly observed the molecular changes of any central nervous mass, whether belouging to another or to itself, when such mass was engaged in the activities whose resultant the phenomena of consciousness are claimed to be. Since there is such scarcity of direct ocular and tangible demonstration of a special relation between the brain and mental phenomena, it is plain that the testimony of experts must be summoned. Resort must be had to the great anatomists and experimental physiologists who have had most experience as to the structure and functions of the brain-mass.

It must, of course, be confessed that no expert has any more direct evidence than every self-conscious ego has of the existence of a real material structure called brain, which may account, by its presence and activities, for his own mental phenomena. Nor can he offer any evidence peculiar to himself for his belief that the particular ego which each one calls "myself" is connected with a brain. How many soever other brains he may have seen, he only knows by a series of very indirect and complicated inferences that any individual whose brain he has not seen really possesses one. But whence these inferences? and, What are the grounds on which the confidence attached to them is based? To these questions only one answer is possible. The inferences themselves are acts of knowledge, modes of consciousness, phenomena of mind. The only possible grounds of confidence in them, as valid inferences, must be referred back to our inherent faith in the power of the mind rightly to infer, from its own phenomena, the real existence of beings the phenomena of which it has never perceived. Moreover, if the mind had perceived the phenomena of its own brain, there could be nothing in the phenomena themselves to account for the power to make inferences which belong to it as mind. On the ground, then, of an inferred reality called the brain, I am asked to dispense with my confidence in the reality of the being which 
makes the inference, and which, at the same time, makes a much more irresistible inference as to its own reality as an active inferring force.

$\S 5$. The case is, however, by no means so favorable, as the statement just made would imply, for that phase of scientific materialism which refers the phenomena of consciousness to the brain as their sole cause. For it is not in the brain, as a mere mass of matter whose structure and mechanical functions can be made obvious to any intelligent observer, that the real substratum of mental phenomena must be sought. Considered as such a mass, this organ is no better than any other similar soft and pulp-like bulk. It is the wonderful molecular constitution, atomic play, and changing dynamic relations of the invisible particles of this mass, which are responsible for its unique functions. In all the first Part of our investigation we saw how necessary physiology finds it to regard the nervous centres as molecular mechanisms. Nothing that is in itself of first importance appears to the eve of the observer who looks upon the freshly extracted mass of the human brain. And when this mass has been skilfully prepared for investigation under the microscope, the investigation itself cloes not reveal directly, to the highest magnifying powers of the glass, the ultimate agents in the wonderful drama it has been playing. These agents are the atoms, to whose real being and so-called "inherent" forces all that is done by the complicated mechanism must be referred. But the existence of the atoms as real beings, capable of acting on each other and of being acted on-how shall this remote and obscure fact be ascertained? And how shall we learn what is the nature of these beings, so as to determine whether or not they are capable of performing the stupendous task of bringing forth the various mental phenomena?

In attempting to answer the last two questions we are in great danger of losing completely all that we have taken most pains to gain. It is to the all-powerful "atoms," with their potent forces, that we are now looking as the real subjects at once of the molecular changes in the brain-mass and of the phenomena of consciousness. From these real beings and their relations there must be derived, not only the activities which all ascribe to nervous matter, but also those which some are constrained to ascribe to conscious mind. And yet, how do we know that any real beings whatever called atoms exist? Certainly not by direct evidence of any of the senses. Not even the most pronounced materialist would venture to affirm that he has seen or touched an atom, or can demonstrate its existence and nature to ordinary observation through the human 
senses. Atoms are supersensible beings. Moreover, they are hypothetical existences, or beings whose existence is inferred in an extremely roundabout way in order that we may be able to give to ourselves a rational account of the grounds on which certain classes of phenomena rest.

The phenomena whose rational explanation seems most peremptorily to demand some hypothesis of atoms are the phenomena of chemistry. When, however, the further inquiry is raised as to the real nature of the atoms, it is found that modern plysical science is by no means satisfied with its own answer. Dynamical theories, tending to resolve the atoms into mathematical points acting as mere centres of force, contend with other more realistic theories which regard the atoms as simply the smallest bits of matter into which we can by any known means break up the larger collections. What is meant by the forces being "inherent" in the atoms is a still more difficult question to answer. Indeed, to this question no answer can be given which gets much beyond the simple declaration of the facts of experience ; that is to say, these hypothetical and yet sole real material beings are always supposed to behave, with respect to their motions, in the same way under the same relations, and something can be done by science toward measuring their various motions in terms one of the other.

Moreover, the best efforts of modern investigation to describe the nature of the atoms appear, not ouly incomplete, but also, in certain particulars, self-contradictory. It is certain that the atom cannot be regarded as an independent reality. What it is can only be described by telling what it does; but in telling what it does we always find ourselves implying certain relations to other atoms. That is to say, we know nothing about the nature of any of the atoms which does not involve also complicated hypotheses concerning its mode of behavior as caused by the presence and mode of behavior of other hypothetical beings. In this way the reality of the atoms is made ultimately to depend on the reality of some form of being that binds them together, as it were, and makes them work to a unity of plan. But here, again, we are reminded that we can form no conception of a "plan" which is not a phenomenon of mind, and no conception of a "unity" that does not depend upon the unifying actus of the mind. Moreover, all ideas of "relation" are dependent upon mental activities that are quite without physical analogy. All "Things" are made into the units which they appear to be by the unifying action of the mind. Such action is implied in perceiving the things; for the study of perception, from the physiological point of view even, has enabled us to show that 
no so-called "thing" is a ready-made material product, apprehended by mind in a form which is a copy of some extra-mental being. In trying, therefore, to comprehend what is the nature of those real beings (the atoms), on whose existence, activity, and relations all mental phenomena are assumed by Materialism to depend, we find that the picture we frame of them is the work of the mind.

$\S 6$. Accordingly, the whole course of argument and the whole weight of conviction appear to be the reverse of what is assumed by the objector to the reality of mind. The material molecules of the brain are not beings about the reality and exact nature of which we have the most indubitable evidence-evidence so indubitable that we may venture to press it into the contradiction of the more immediate data of consciousness. If these elements of all physical being are real, they come to us as inferences and hypotheses; they involve a vast amount of conjecture, indirect inference, and unsolved difficulties, or even contradictions. And if we ask, On what authority are these inferences made? Whence comes the demand for any rational explanation whatever? Where do the conjecture, hypothesis, and sense of difficulty and seeming contradiction exist? then the only answer to be given to all these questions refers them to the Mind. What atoms and forces and laws can be, or mean, without the being and activity of self-conscious mind, is even harder to conjecture than what a color can be which is not seen, a sound which is not heard, an odor that is not smelled.

And now let the attempt of materialistic theory be made anew; let it be assumed that the phenomena of consciousness have no real subject in the mind. Such phenomena must, accordingly, be attributed to the peculiarly constituted and mutually interacting molecules of the brain. But these supreme physical beings are themselves, as far as they are the object of knowledge, pre-eminently mental creations; and the sole warrant for carrying them over into the realm of extra-mental reality consists in certain irresistible convictions or assumptions of mind. To make their real being the account of the mental phenomena, and thus to deny the real being of the subject of mental phenomena, is not only to explain what is most direct and certain by what is most indirect and uncertain ; it even involves the wonderful paradox, that the one being in whose active energizing all conceptions of all real being arise, feels justified in denying its own reality in the supposed favor of certain of its most remote and doubtful conceptions.

§. What is meant by affirming the reality of mind may be made obvious by pursuing the following train of reflections: In the development of the mental life its phenomena come inevitably 
to divide themselves into two great classes. As it appears to adult experience, not only the unfolding, but even the very existence of self-consciousness seems to involve the distinction between the ego and the non-ego-between the "I" with its states, and the "Things" which it knows with their manifold properties or attributes. Each of these two classes of phenomena-the so-called subjective and the so-called objective-is inevitably attributed in consciousness to a different subject ; the one to the "I" as its own states, the other to somewhat left undefined, except that it is not the "I," and is called "matter," "material substance," etc. (the unknown $X$ which is not I). It is only as involving all this mental process that any real being is known or believed to exist; but the mind in the development of experience inevitably completes the process, which involves the assumption that real beings do exist, and that all these real beings are either "things," such as I know, or myself and other conscious beings, such as I am. What any real being is can only be told by an enumeration of its so-called attributes; and this is as true of myself as of the things which I know. It is also as true of them as it is of myself. If the foregoing statements covered the entire case, it would simply be true that $I$ have no better reason for attributing a real Being to any material thing than to the subject of consciousness. But we have already seen that the process by which we reach the real being of the molecules of the brain is much more indirect and doubtful than that by which we reach the affirmation of a real being for the things of daily experience and for the subject of all that experience.

§8. Peculiar and cogent reasons may be given, however, which further enforce and verify the assumption of a real existence for the Mind. We have seen (comp. Part II., chap. X., and the preceding chaps. of Part III.) that there is a class of so-called mental faculties, most important and distinctive, for the distinguishing: characteristic of which no plyysical analogies or correspondences whatever can be discovered or imagined. This is true of memory as active reminiscence, of the unity of consciousness, of voluntary attention, and of the relating activity. The existence of these modes of mental behavior requires the assumption of a characteristic real being, other than the molecules of the brain, to which they may be referred. Some of these modes of behavior are conspicuously unintelligible and meaningless without granting such an assumption. For example, an act of recollection involves the presence in consciousness of a state the very essence of which is that it claims to represent (or stand for) an absent past state of consciousness. No way of verifying this claim which does not 
involve its acceptance can possibly be devised. But the present state of memory is a state of my consciousness, and the state which it claims to represent was also a state of my consciousness. To recollect the past state of another consciousness than my own involves an absurdity; to recollect a past state otherwise than as represented in a present state of my own consciousness also involves an absurdity. Of course, such reflection upon the nature of the act of memory affords no demonstration of the claim that the subject of the present state is one and the same real being with the subject of the past state. On the contrary, all demonstration itself rests on this assumption ; for without accepting it as valid we could not reach the conclusion of any demonstration. The premises of every syllogism are connected with one another and with their conclusion in a living unity of thought, only on the assumption that one real being is the subject of each of the thoughts which constitute the syllogism.

To "be veally," and to be the one permanent subject of changing states, are, in our conception, but different ways of expressing the same truth. That really is which is such a subject of its own states. It is for this reason that modern plyysical science regards the atoms as having a permanent reality which does not belong to the composite structures-the things of our experience-into which the atoms enter. Every "Thing" may perish-that is to say, as such thing, it may cease to be the object of observation, the subject of states. But the atoms are supposed to remain with unchanged natures through all the changes of relation which they nay undergo toward other beings with somewhat similar natures. Even if we were obliged to adopt the hypothesis of a constant change of states in the interior of the atoms, since every atom shows a variety of possible activities according to the relations in which it stands for the time to other atoms, it is not considered to have lost its real being or distinctive nature by changing its states. For it-the atom-can be brought into the same relations again, and then it will again display the same modes of behavior. Its reality does not depend upon its interior rigidity, the unchanging nature of its being; it rather depends upon its eapacity for being the subject of so-called states, and for following a law or an idea which recalls it, as it were, to the same states when the same circumstances recur.

How can it be denied that all our conceptions of the atoms as enduring subjects of various states are derived from our experience with ourselves? The "I" which is the subject of all consciousness is accustomed to attribute to itself every state of that great variety 
into which consciousness may be shaped. The states are changing; they have a transitory and phenomenal being. But they are all states attributable to one subject. On what ground, then, shall one undertake to deny the confidence which the soul comes to have in itself as the real and permanent subject of its own states? For we can form no conception of real being at all which is not modelled after this pattern. To have a variety of changing states attributed to it as the subject of them all-this is to demonstrate in consciousness a claim to real Being. Unchanging rigidity, the permanence of the mathematical point or of the material atom, on the supposition that the latter undergoes no interior changes whatever, if such rigidity and permanence anywhere exist, constitutes no claim to the title of real being.

The soul exists in reality, above all other kinds of being, because it alone, so far as we know on good evidence, knows itself as the subject of its own states; or, indeed, knows the states of which it is the subject as states belonging to itself. But its law is that of development; and, unlike all "things" which are subjects of various kinds of evolution, so called, the soul can recognize the law of its own being. When, therefore, we are asked what the Mind really is, we can respond by telling what it comes to be as the result of its unfolding under the fixed conditions of its native powers. But these "powers" cannot be called native, as though they were actual achievements of the mind's inborn faculties, or separate forms of energy inherent in it, after the analogy of the forces said (somewhat unintelligibly, it must be admitted) to be "inherent" in the atom.

But we do not define the nature of any real being simply by stating how it appears and behaves in its most germinal and undereloped form. The tree explains the seed; the adult bird, the egg; the character of the highly differentiated product must be studied in order to know the full description of the energies that are potential in the simpler stages. It is an undoubted fact that the mind has a history in each individual case ; and in each case such history is a development. The great service which Physiological Psychology has rendered to the general science of mind consists in its description of the nature and stages of this development, so far as concerns the phenomena of sensation and perception by the senses. This self-recognizing unity of development which belongs to the mind is a striking proof of the validity of its claim to be considered a real being. As the being which acts and knows itself as acting, which is acted upon and lnows itself as affected, which is the subject of states and itself attributes these states to 
itself, which derelops according to a plan and so remembers and comprehends the significance of its past states that it can recognize the fact of its own development-as such a being the Mind is more entitled to consider itself "real " than to consider real any of the various objects that, immediately or indirectly, appear before it in the course of its history.

\&9. The question whether the mind is to be spoken of as nonmaterial or "spiritual" scarcely merits the grave and lengthy discussion to which it has often been carried. Materiality, as predicated of any real being, is only a complex term including a number of so-called attributes, which are all the subjects of experience only as belonging to individual things. All real things are to be called material which have these attributes, so called. Primarily, as has been frequently shown already, the attributes are simply modes of the affection of the mind which we have learned to localize and objectify as belonging to extra-mental reality. But if we raise the question whether the Mind, too, is known to itself as haring those attributes which make up our complex, general notion of "materiality," no one would find it easy to think of giving this question an affirmative answer. The mind attributes to "things" the qualities of extension, impenetrability, and all the various subordinate modifications of these qualities. It perceives these things as colored, cold, hot, rough, smooth, etc. But it does not attribute such qualities to itself; it can find nothing in the modes in which it manifests itself to itself which would warrant the application of similar terms to these modes of its own behavior.

Indeed, all the terms which do apply to the recognized qualities of mind have to be understood as figurative when, having been borrowed from physical relations, they are made to apply to psychical states. Even in those cases where the analogy seems almost to amount to an identity, closer inspection shows that this seeming does not correspond to the actual fact. For example, we do attribute quantity to sensations and feeling. But when the suffering from pressure becomes more intense, we do not regard the mind as actually passing, like some material thing, under a heavier load (sub-fero), against which it must either bear up or break, through the physical strain. Just so, movements of the mind are not to be defined as changes of its position with relation to other things in space. We are, then, surely warranted in affirming that, so far as the mind has any immediate information as to what qualities should be assigned to itself and what to "things"-which it always looks upon as not-itself-it is compelled to regard itself as non-material. 
We have no way, however, of telling what is the nature of any so-called real being except by enumerating its qualities, or those modes of behavior which we attribute to it on account of its affecting our consciousness in certain definite ways. To attempt to regard the mind as material, when it manifests itself to itself as non-material, compels us either to use the word "material" in an unwonted and unauthorized way, or else to attribute to matter in general certain occult powers which it never manifests itself to the mind as possessing, and which make it really to be quite different from what its manifestation of itself would indicate.

The only way of maintaining the materiality of mind would then appear to be that of denying its real existence at all, and of attributing its phenomena to the material molecules of the brain as their real and material substratum or basis. But the untenable nature of this view has already been sufficiently indicated. Or perhaps a strong temptation may be again felt, at this point, to recur to the hypothesis of a third somewhat, a "two-faced unity," which is the ground of the phenomena of both body and mind. But such hypothesis can throw no light whatever on the inquiry whether the mind is material or non-material. The phenomena we call "mental," and attribute to the subject of consciousness, would remain just as radically unlike those which we call "physical," and attribute to matter, after making the hypothesis as before. And to the hypothesis itself the same objections would remain opposed.

The negative conclusion that mind is non-material is quite inevitable for everyone who admits that mind is a real being with any nature whatever.

$\S 10$. It is not difficult, also, to show that we must make the corresponding positive statement, and affirm the spirituality of mind. This we can do with confidence, however, only so long as we menn by the term "spirituality" simply to sum up and express in one word the list of attributes which describes the known activities of mind. To perceive, feel, think, will-in brief, to be conscious in some one of the various forms of conscious life-this is to be positively spiritual, in the only sense in which we are entitled to affirm spirituality of mind as such. As soon as we conceive of spirituality as some ethereal extension of thinking substance, we enter upon the vain effort to conceive of mind under terms of matter, and at the same time escape the consequences of so conceiving of it. Nor can we hope to vindicate for the mind such spirituality as wonld be implied in its being freed from all relations to material things, or from dependence for the modes of its being upon the material substratum of the brain. How spirit, in the sense of disembodied or unem- 
bodied mind, would perceive, and feel, and think, and will, is a question toward the answer to which we can make no beginning. To attempt its answer at all involres us in the vain effort to use the very relations which are most inseparately connected with the conscious activity of the mind in such way as to escape from the control of the relations themselves.

It is true, nevertheless, that a marked difference exists in the directness and intimacy which belong to different classes of mental states, as regards their comparability to the classes of physical stimuli which rouse the mind to its fundamental activities. Those which appear to be most indirectly, and, as it were, loosely related to these fundamental activities are fitly most relied upon to show the spiritual nature of mind. To control the mental train as distinguished from being a passive member of a mental mechanism, to reason so as to deduce conclusions and make inductions to general laws, to recognize the call of duty, and to call up and classify in the consciousness the lofty and complex ideas which answer to words like "beauty," "truth," and "God "-these and other similar operations of the mind pre-eminently emphasize its spirituality.

$\S 11$. In somewhat the same way must it be admitted that the question of the unity of mind has given rise to much fruitless and by no means altogether pertinent debate. The attempt to conceive of the mind as a unit-being, constituted after the analogy of those physical structures which we are accustomed to regard as unities, ineritably leads to confusion and error. The important psychological fact is, that there is no one of these physical unities which does not derive its unity from the unifying actus of the mind. This statement is true of each such so-called unity, whether it is perceived as one or is conceived of as one. The unity which belongs to the percept finds its source in the synthetic activity of the perceiving mind; the unity of the conception, in the unifying activity of the mind's relating faculty. It is sometimes supposed, howerer, that an atom which should have no parts, be perfectly homogeneous throughout, and so incapable of changes of its interior states, would be the highest possible type of a unity of real being. Nothing could ever happen to disturb or destroy such a unity. Wherever in all space it might be moved, or whatever in all time might happen to it, it could ex hypothesi never be made two. If, now, such a unit-atom were to be endowed with consciousness and spiritual being, how secure would its unity continue to be! Unlike the mind of man, it could not fear that some rude concurrence of other atoms, not of the right affinities, or setting themselves in untoward relations, would dissolve its complex material substratum and so 
destroy its spiritual oneness. The molecules of the human brain are in number beyond computation; they are highly complex and unstable compounds; they are not so protected by their inclosure in the cranial carity as to make them invulnerable against all manner of assaults. In how dangerous a position, then, is this so highly valued unity of our present spiritual organization!

Now, it must be admitted that such a thinking atom would be in far less danger of suffering from the death of the physical basis of its thought than is the thinking man. But two considerations of great importance are likely to be orerlooked in the mere making of the lyypothesis of such an atom. Surely such an atom could hardly have any experience corresponding to what we call the unity of our consciousness ; and if it had any unity of consciousness whatever, such unity could no more be explained as arising out of, or conditioned upon, the simplicity of the physical being of the atom than the unity of our consciousness can be explained as arising out of, or conditioned upon, the complexity of our physical being.

It is impossible to see how a unity of consciousness at all resembling what we understand by the term could find an adequate material substratum in a single rigid atom. In other words, if a spiritual being having a unity of consciousness were brought into special psycho-physical relations with a material being incapable of any interior changes, because possessed of no parts to undergo change, these relations would have to be totally different from any which we can conceive of as holding between the body and mind of man. For the very nature of the mind's unity is dependent upon that variety of experiences which is occasioned in the mind through the changing states of the brain. The physical basis of the human mind is undoubtedly an extremely complex system of interacting molecules. Certain relations can be traced between the character of these physical interactions and the character of the states arising in the mind. These states depend for their character, and even for their very existence, upon the occurrence of the corresponding material changes. A brain that is not in a ceaseless change of activities of the peculiar sort called "neural" is a dead brain, so far as its influence on the mind is concerned; such a brain could not serve as the substratum or physical cause of mental phenomena. Comparative anatomy shows us that the greater the number of molecules, and the larger the variety and the size of the organs specially related to the mental processes, the richer in variety and nobler in quality the mental processes themselves become. Moreover, so far as we can ascertain, the highest unity of consciousness belongs in connection with the greatest complexity of the material 
substratum. The animals which have the largest cerebral development appear to have, too, not only the most manifold and extensive mental life, but also, in the highest legree, the capacity for attributing the phenomena of that life to one subject. Those psychical activities which are connected with the physical interaction of the greatest number of material elements are the most numerous and significant; and they are, also, actually most perfectly harmonized into a higher unity of spiritual self-conscious being.

\$12. No information derived from the study of Physiological Psychology warrants us in affirming that a highly developed selfconscious existence must, from the universal necessities of the case, be united with a vastly complex material structure like the human brain. Such study does, however, compel us to affirm that such a unity in variety as is the human mind cannot be conceived of in dependence upon the movements in space of a single perfectly rigid and unchanging atom. The development of human experience is conditioned upon the arising in consciousness of many sensations of varied quantities, qualities, and orders in time; upon the synthesis of these sensations into presentations of sense; and upon the recall of the presentations in the form of representative ideas. What experience would be, if its basis were not laid in such rise and combination and recurrence of sensations, we cannot even conjecture. In the highest flights of imagination, in the profoundest explorations of reflection, we never escape out of the influences arising from this basis. The nature of this psychical basis of sensation and perception depends upon the nature of the physical basis of the living and acting brain. In other words, what sensations and perceptions constitute, at least in part, the "stuff" of all consciousness depends upon what the molecules of the central nervous system are doing. We cannot even conceive of any other relations as possible between the mind, on the one hand, and the brain, on the other, than relations between a system of moving molecules and a corresponding change of conscious states.

$\$ 13$. Furthermore, the unity of a single indestructible and eternally unchanging atom would afford no explanation of a mental unity. In the case of man's mind and brain, the variety of the nervous changes in part explains the variety of the mental states; but nothing in the changing relations of the innumerable moving molecules throws any clear light on the origin of the unity of mind in consciousness. A material being absolutely without distinction of parts would be, for that fact, no better fitted to become conscious of itself as one. A series of states of consciousness can indeed be attributed by our imagination to such a being. From the purely 
psychological point of view we can conceive of the unit-atom as having an experience resembling our own. We, in our conscionsness, can imagine such a being as the subject of states, and as attributing each of these states to one and the same subjectnamely, the "I" of the unit-atom-after the fashion of our customary mental behavior. But this is quite a different thing from explaining the consciousness of such an atom as arising, with respect to its unity, out of the material nature of the atom. By the very hypothesis, the material nature of this particular kind of atom can have no states; it never changes; it is always the same. But consciousness is always some particular definite state; and self-consciousness is always the being aware of some particular definite state. There is no consciousness in general ; there is no consciousness which does not involve change of state. Indeed, change is a reality in human consciousness, if nowhere else in the universe of being. No particular state of consciousness, whether considered as involving an attribution of that state to a subject or not, could be explained by reference to the material nature or condition of such a unit-atom.

$\$ 14$. The foregoing remarks have their value chiefly as a warning against supposing that the unity of the soul's real being suffers any prejudice because it is not to be regarded or explained from a point of view furnished by physical analogies. To be one, as a rigid material atom may possibly be regarded as one, would be no advantage to the soul. Or if it be admitted that, in case it lad such unity, it could never lose its real being, it must also be admitted that we are unable to see how it could ever gain any real being as a soul. If the unit-atom could never die, it could also nerer live-as a conscious psychical existence. And it is the unity which the mind plainly has in self-consciousness that is alone worth contending for. If the mind were really - that is, regarded as out of its own consciousness-one, and yet two or more in consciousness, it would be no better, but rather the worse off. If it were really one, but were obliged not to know itself as one, and could never be aware of its own states, or attribute them to the one "I" which is the subject of them all, it would surely be the worse off. To be one, in the only meaning of the word that is of real value, is to have and to keep the unity of consciousness. If this unity were really a mere seeming-a trick of nature to cheat the mind-the seeming would forever seem real, would, indeed, be the ground of all reality; the trick would be the kindest of all illusions, and one from which we should crave never to be set free. When, then, we have recognized the fact that all ordering and development of human consciousness 
implies this kind of unit-being as belonging to the mind, we have gone as far in vindication of the mind's rights as we have any psychological interest in going.

$\S 15$. That the mind attributes its own conscious states to a subject of such states (the "I" in all sentences such as "I think," "I feel," etc.), we have seen it necessary to admit from the very beginning of our psycho-physical researches. As one result of the study of perception by the senses, it was also found necessury to recognize a certain unifying or synthetic activity in order to account for the way in which sensations combine to form the "presentations of sense ;" such unifying activity seems plainly to imply the existence of a unit-being, the so-called Mind. Further arguments in the same direction came to light as the phenomena of the mind in memory, voluntary attention, and judgment were brought under examination. More recently still, an examination of the factors of self-consciousness, from the more purely introspective point of view, has confirmed the same opinion. In this comnection we may add, finally, the argument for the existence of the mind as a real unit-being, which has been so forcefully urged by one of the greatest of modern psychologists (Lotze). The mind is a real unitbeing, not simply because it appears to itself to be such, but chiefly because it appears to itself at all. Granted that all that which only appears to another may be mere seeming, it still remains indisputable that somewhat appears. The somewhat which merely appears may be really many when it appears as one; this happens, in some sort, in the case of all "Things" which appear many or one according to the way we consider them. But how can that to which all else appears, whether as one or as many, and that which also appears to itself-whether it appear to itself as one or as many -really be other than one, in the highest sense of the word unity? No twisting of imagination, or subtlety of argument, can show how a mind not really one could appear to itself at all; or break the strength of the conviction inwrought into the very structure of human self-consciousness, that the real and spiritual being, which we call Mind, is not a fortunate confluence or phenomenal centre of changing modes, but a unit-being, and a reason of ail unity in whatever becomes the object of its thought.

$\$ 16$. As to the first and last things of the Mind-its origin and destiny, its mortality or corruptibility-Physiological Psychology finds itself unable to pronounce. It cannot, indeed, explain the entire being of the mind as arising out of the development of the plyysical germ from which the bodily members unfold themselves. It knows no decisive reason against the belief that such a non- 
material and real unit-being, as the mind is, should exist in other relations than those which it sustains at present to the structure of the brain. On the contrary, it discloses certain phenomena which at least suggest, and perhaps confirm, the possibility of such existence for the Mind. But, in general, if it remain faithful to its own mission, within its own limits, it entrusts the full consideration of these questions, after it has cleared the way from barriers of ignorance and prejudice, to Rational Psychology, to Ethics, to Metaphysics, and to Theology. 


\section{INDEX.}

Agraphis, nature of, 294

Allen, Grant, on nature of feeling, $501 \mathrm{f}$., 521

Aphasia, phenomena of, $292 \mathrm{f}$; kinds of, $294 \mathrm{f}$.

Aqueduct of Sylvius, 88 ; gray matter of, 91

Aqueous Humor, the, 173

Arachnoid, the, structure of, $63 \mathrm{f}$.

Attention, effect of, on reaction-time, 480 f., 495 f.; physical basis of, 538 f., $542 \mathrm{f}$; effect of, on perception and memory, $539 \mathrm{f}$.

Aubert, on measurement of light, $3 \pi 4 \mathrm{f}$.

Automatic Action, nature of, $49 \mathrm{f}$., $130 \mathrm{f}$.; in spinal cord, $138 \mathrm{f}$; ; and brain, $144 \mathrm{f}$; physical basis of volition, $535 \mathrm{f}$.

B.Is, on local signs, 397 (note); theory of feeling, 501 ; feeling of effort, 524

Baxt, on reaction-time, 481

Bechterew, on the olivary bodiez, 150, $161 \mathrm{f}$; ; and central gray matter, $161 \mathrm{f}$.

Bell, Sir Charles, discovery of, $123 \mathrm{f}$.

Beueke, on nature of feeling, 503

Berger, on reaction-time, $4 \% 8$

Bernstein, on exhaustion of nerves, 109.

Betz, "giant-cells" of, 97, 283

Birge, E. A., on number of nervous elements, 46, 70 ; excitability of cord, 143

Blastoderm, the, $200 \mathrm{f}$; layers of, $202 \mathrm{f}$; areas of, $202 \mathrm{f}$.

Blind-spot ( papilla optica), 183

Body, general relations of, to mental phenomena, 560 f.; early development of, 562 f., 567 ; phases of, 565 f.; sexual differences of, 570 ; relative proportions in, $5 \pi 1 \mathrm{f}$; race-characteristics of, $5 \pi 3 \mathrm{f}$.

Brain, chemistry of, $25 \mathrm{f}$, $2 \% \mathrm{f}$; membranes of, $61 \mathrm{f}$.; structure of, $73 \mathrm{f}$., 85 f.; ventricles of, 85, $149 \mathrm{f}$; ganglia of, $85 \mathrm{f}$.; hemispheres of, $91 \mathrm{f}$.; lobes of, $92 \mathrm{f}$; cortex of, $95 \mathrm{f}$.; inhibitory influence of, $143 \mathrm{f}$; ; as central organ, $143 \mathrm{f}$.; development of, $204 \mathrm{f}$; ; general functions of, $239 \mathrm{f}$.; temperature of, 242 ; comparative weight of, $243 \mathrm{f}$.; weight of human, $244 \mathrm{f}$; relation of, to mind, 247 f., 605 f., 633 f., 640 f.

Broca, convolution of, $292 \mathrm{f}$.

Brücke, on neutralization of taste, 403 ; perception of depth, 442

Byasson, on brain-waste, 242

Camerei, on measurement of taste, $376 \mathrm{f}$.

Capsule, the internal, 90,91

Carville and Duret, on stimulation of motor areas, 257

Cattell, on reaction-time, 485, $493 \mathrm{f}$.

Cells, the olfactory, $164 \mathrm{f}$; t the gustatory, $16 \tau \mathrm{f}$. : the auditory, 192

Central Canal, $66 \mathrm{f}$.

Cerebellar tract, $\pi 1$ f., $\pi 5, \pi 7$

Cerebellum, $\pi 4$, structure of, $78 \mathrm{f}$.; peduncles of, 79 ; arborvile of, 79 ; functions of, $152 \mathrm{f}$; ; lesions of, $153 \mathrm{f}$.

Cerebrin, 24, 25

Cerebro-spinal system, axis of, 62, $204 \mathrm{f}$.; development of, $204 \mathrm{f}$., 212

Cerebrum, 74, 82 ; shape of, 82 ; gyri of, 84, $91 \mathrm{f} ., 95$; sulci of, $84,91 \mathrm{f}$; nervous elements in, $91,95 \mathrm{f}$; ; layers in its cortex, $95 \mathrm{f}$; fibres of, $97 \mathrm{f}$; nervous paths in, $127 \mathrm{f}$, $269 \mathrm{f}$.; functions of, $150 \mathrm{f}$., 156 f., 239 f.; development of. 204 f.; localization in, 239 f., 250 f., $255 \mathrm{f}$, $269 \mathrm{f}$; significance of, $249 \mathrm{f}$.; effects of injury to, $25 \mathrm{~s}$., $269 \mathrm{f}$.

Charcot, scheme of decussation, 290 
Charcot and Pitres, on localization of Dobrowolsky, on measurement of colorcerebral function, 283

Cha ussier, ou growth of fætus, 566

Chemistry, of nervous system, $21 \mathrm{f}$., 217 f.; of cells and tibres, 28 ; of pliysiological function, $28 \mathrm{f}, 111 \mathrm{f}$., 2:2: ; of vision, $184 \mathrm{f}$.

Chodin, measuring power of the eye, 451

Cholesterin, $23 \mathrm{f}$.

Choroid, the, $171 \mathrm{f}$.

Clarke, columns of, 70

Cochlea, the, 191

Color, stimulus of, 323 f., 335 f. ; saturated, 323 ; tones of, 329 ; brightness of, 330 , $3 \% 6$; shades of, $331 \mathrm{f}$; complementary, 333 f., 343 ; dependence of, on time, $334 \mathrm{f} . ;$ and place of the retina, 335 , 338; blindness to, $335 \mathrm{f}$; contrast of, 337,460 ; Young-Helmboltz theory of, $33 \mathrm{f}$; symbolism of, $342 \mathrm{f}$; sensitiveness to, $3 \pi 5 \mathrm{f}$.

Consciousness, the circuit of, $494 \mathrm{f}$; physical basis of, $544 \mathrm{f}$.; possibility of a prenatal, 565 f.; psycho-physical explanations of. 596 ; phenomena of, 597 f.; unity of, $60 \tau \mathrm{f}$, $631 \mathrm{f}$.

Cornea, structure of, 171, 173, 175 f.; index of refraction of, 176 ; function of, $176 \mathrm{f}$.

Corona Radiata, 91

Corpus albicans, 83,87

Corpus callosum, 82, 85; function of, 98

Corpus dentatum, of the medulla, 78 ; of the cerebellum, 79

Corpus geniculatum, $87, \$ 9$

Corpus quadrigeminum, position of, 87 ; structure of, $90 \mathrm{f}$; functions of, $156 \mathrm{f}$; ; development of, $208 \mathrm{f}$.

Corpus striatum, 86 ; nuclei of, $86 \mathrm{f}$; paths in, 129; functions of, 158 f., 160 f.; development of, $208 \mathrm{f}$.

Corpus subthalamicon, 89

Cortex of Cerebrum, structure of, $95 \mathrm{f}$.

Crura Cerebri, 83, 8\%; crusta and tegmentum of, $87 \mathrm{f}$; fibres in, $8 \%$ f.; functions of, 156

Crusta, see Crura Cerebri

Crystalline Lens, the structure of, 173

Deiters, processes of, 43, 70 ; conical hair-cells of, 195,197

Dietze, on the circuit of consciousness, $494 \mathrm{f}$. sensations, $375 \mathrm{f}$.

Donders, on localization of depth, 465 ; time of mental processes, $468 \mathrm{f}$., $4 \mathrm{rg} \mathrm{f}$.

Dove, the experiment of, 442

Drbal, on nature of feeling, 503; kinds of feeling, 505.

Du Bois-Reymond, discoveries of, 104, $112,115,117$; theory of nervous action, $227 \mathrm{f}$.

Dura Mater, structure of, $61 \mathrm{f}$; processes of, 63.

EA1r, 185f,; the external, 185; the middle, $186 \mathrm{f}$; bones of, $187 \mathrm{f}$; ; tympanum of, 188 ; vibrations in, 188 ; the internal, $189 \mathrm{f}$; vestibule of, 189 ; canals of, $189 \mathrm{f}$. ; cachlea of, 191 ; nerve of, $191 \mathrm{f}$. ; terminal apparatus of, 192 f., 324; problen of, 195; development of, :211 f.; sensitiveness of, 317,319

Ecker, view of cerebral cortex, 263; charts of, $2 \pi 6 \mathrm{f}$.

Eckhard, law of central mechanisms, 161 Electricity, "current of rest" in nerves, $104,106 \mathrm{f}, 11 \%, 227 \mathrm{f}$; ; as stimulus of nerves, $111,112,114 \mathrm{f}, 228 \mathrm{f}$; ; "negative variation" in nerves, $118,22 \mathrm{f}$.

Electrotonus, Pfluger's law of, 113, $115 \mathrm{f}$; theory of, 222 f., $226 \mathrm{f}$.

Embryo, knowledge of, $198 \mathrm{f}$., $212 \mathrm{f}$. ; of the fowl, $199 \mathrm{f}$; ; development of, $200 \mathrm{f}$., $204 \mathrm{f}$, $212 \mathrm{f}$, 618

Encephalon, see Brain

End-organs of Motion, place in nervous system, 60, 164; structure of, 197

End-organs of Sense, place in nerrous system, 60, 164; significance of, $163 \mathbf{f}$; end-organs of smell, $164 \mathrm{f}$.; of taste, $166 \mathrm{f}$; of touch, $168 \mathrm{f}$; of sight, $171 \mathrm{f}$. ; of hearing, $185 \mathrm{f}$.

Engelmann, on continuity of axis-cylinders, $40 \mathrm{f}$.

Estel, on reaction-time, 490

Eustachian Tube, 186 f., 189

Exner, on speed of reflex action, 135; regio olfactoria, 165 ; nature of nervecommotion, $224 \mathrm{f}$. ; general function of the brain, 240 ; cerebral physiology, $254,26 \tau$; views of, on localization, 267 , 259 f., 284 f., $289 \mathrm{f}$. ; methods of, $2 \pi 6 \mathrm{f}$. ; on aphasia, 294 ; reaction-time, $470 \mathrm{f}$, 480, $496 \mathrm{f}$; attention, 538

Eye, structure of, $171 \mathrm{f}$; tunics of, $171 \mathrm{f}$; 
refracting media of, $173 \mathrm{f}$, $175 \mathrm{f}$. ; ap- Gamgee, on chemistry of brain, $25 \mathrm{f}$.

pendages of, $173 \mathrm{f}, 17 \tau$; muscles of, Ganglia, the "basal," $8 \mathrm{~s} \mathrm{f}$.

174 f., 428 f.; problem of, $1 \tilde{4} \mathrm{f}$; ; ad- Ganglion-cell, see nerve-cells

justment of, $1 \% \tau \mathrm{f}$., 433 ; pigments of, 184 ; development of, $210 \mathrm{f}$.; motion of, $428 \mathrm{f}$., $439 \mathrm{f}$; meridians of, $431 \mathrm{f}$.; torsions of, $432 \mathrm{f}$; innervation of, $439 \mathrm{f}$. ; stereoscopy of, $440 \mathrm{f}$.

\section{Fascicules Gracilis, 68, 72}

Fechner, conception of psycho-phyzics, 12 ; on measurement of sensation, $361 \mathrm{f}$., $369 \mathrm{f}$. ; law of, 365 f., 374 f., 594

Feeling, mixture of, in local signs, $398 \mathrm{f}$; of innervation or effort, 415 f., $523 \mathrm{f}$; of "double contact," $41 \mathrm{f}$ f. ; nature of, $499 \mathrm{f}$., 504 ; classes of, $505 \mathrm{f}$; inteusity of, $508 \mathrm{f}$. ; tone of, $509 \mathrm{f}$. ; physical apparatus of, $510 \mathrm{f}$. ; common, 512; of sensation, $514 \mathrm{f}$. ; the emotions, $516 \mathrm{f}$., $519 \mathrm{f} . ;$ sthenic and asthenic, $518 \mathrm{f}$. ; the higher æsthetic and intellectual, $520 \mathrm{f}$, 523

Ferrier, on corpora quadrigemina, 15\%; and striate bodies, 159 ; experiments of, 254,264 ; centres of, 268 f., 285 f., 291 ; on feeling of effort, 524

Fick, on muscle-contractions, 119 ; minute color-sensations, $334 \mathrm{f}$; curre of intensity, 475

Filum terminale, 64

Fissures, of Sylvins, 92, 94, 210, 26 $\mathrm{f}$.; of Rolando, 92, 94, 267

Flechsig, on tracts in spinal eord, $71 \mathrm{f}$.

Flourens, on respiratory centre, $147 f_{\text {. }}$; optic lobes, 156 ; localization of cerebral functions, 253

Foramen magnum, 64

Formatio reticularis, in the medulla, it f.; in the tegmentum, 88

Foster, on the respiratory centre, 148

Fovea centralis, 183

Franck and Pitres, on stimulation of brain, 257

Friedrich, on reaction-time, $48 \% \mathrm{f}$.

Fritsch, expcriments of, $253 \mathrm{f}$,, 264

Fröhlich, classification of sensations of smell, 310

Funke, on Weber's "sensation-circles," 407

GaLL, on cerebellum, 156; phrenological theory of, 252

Gerlach, on intimate structure of the cord, 70 ; and cerebral cortex, 96

Gliky, on nerve-tracts, 261

Goldscheider, on "pressure spots," 346 f., 369,410 ; temperature-spots, $348 \mathrm{f}$., $3 \pi 0,413$

Goll, column of (see fasciculus gracilis)

Goltz, experiments of, on spinal cord, 140 ; on optic lobes, $15 \pi$; view of localization, $264 \mathrm{f}$., $273 \mathrm{f}$; experiments of, $297 \mathrm{f}$.

Grützner, on nature of nerve-commotion, $225 \mathrm{f}$

Gyri (or convolutions) of the cerebrum, $84,92,93 \mathrm{f}$; d development of, 210

Hail, G. Stasiet, on perception of motion, $411 \mathrm{f}$., 416 ; studies of rhythm, 490

Hamilton, on the circuit of conscionsness, 494 ; tone of feeling, 509

Hearing, end-organ of, 185 f.; sensations of, $195,315 \mathrm{f}$; perceptions of, $403 \mathrm{f}$.

Helmholtz, on speed of nervons processes, $120 \mathrm{f}$.; index of refraction of cornea, $1 \pi 6$; accommodation of eye, $1 \pi \tau \mathrm{f}$, 433 ; size of blind-spot, $183 \mathrm{f}$; ; analysis of sound, 196 ; nature of noises, 316 ; consonances of tone, $323 \mathrm{f}$; theory of color-sensations, $339 \mathrm{f}$.; and of perception, 389 f., 452 ; on Listing's law, 431 ; movements of the head, 454 ; localization of depth, 405

Hensen, on function of labrrinth, $194 \mathrm{f}$., $196 ;$ nature of noises, 316

Hering, theory of color-sensations, $340 \mathrm{f}$; of temperature-sensations, $350 \mathrm{f}$.; innervation of the eye, $439 \mathrm{f}, 451,525$; movements of the head, 454

Hermann, on electrical phenomena, in nerves, $118 \mathrm{f}$., 120 ; theory of nervous action, $226 \mathrm{f}$.

Herschel, on brightness of stars, $373 \mathrm{f}$.

Herzen, on sensations of temperature, $352 \mathrm{f}$.

Hill, A., view of "basal ganglia," 89

Hirsch, on reaction-time, 470

Hitzig, experiments of, $253 \mathrm{f}$. 264: on localization of cerebral function, $253 \mathrm{f}$., $\Sigma 6 \tau$; centres of, $26 \pi \mathrm{f}$. 
Horopter, calculation of, 437

Horwicz, theory of feeling

INHibition, nature of, 51, 144; from brain on cord, $143 \mathrm{f}$.

Iris, the, $1 \% 2$

Island of Reil, 92 ; layers in, 97 ; function of, $295 \mathrm{f}$.

JAMEs, Professor, theory of the emotions, $519 \mathrm{f}$.; on the feeling of effort, $524 \mathrm{f}$.

Jastrow, on comparative judgments of eye and hand, 466 ; studies of rhythm, 490

KANT, on sthenio and asthenic feeling, $518 \mathrm{f}$.

Keppler, on measurement of taste, 377

Klug, on localization by temperature, 414

Kölliker, on end-organs of touch, 170

Kollert, on reaction-time, $489 \mathrm{f}$.

Kraepelin, on measurement of visual sensations, $3 \pi 5$

Kranse, end-bulbs of, 169 f; on index of refraction, $1 \pi 6$

Kühne, on chemistry of retina, 28, $184 \mathrm{f}$.; function of nerve-fibres, 54 ; structure of end-plates, 197

Kunkel, on inertia of the retina, 474

Kussmaul, on aphasia, 293

LAMANSKY, on measurement of colorsensations, 375

Lecithin, $26 \mathrm{f}$.

Le Conte, on Listing's law, 431, 439; torsions of the eye, 431 ; nature of the horopter, $437 \mathrm{f}$; ; theory of double images, 44 ?

Listing, the law of, 430 f., 439

Lobcs, of the cerebrum, $92 \mathrm{f}$.

Local Signs, theory of, $387 \mathrm{f}, 396 \mathrm{f}, 398$ f., 409

Löb, on visual areas, $288 \mathrm{f}$.

Lohmeyer, on cases of aphasia, 296

Lombard, on temperature of brain, 242

Longet, on columns of the cord, $125 \mathrm{f}$. ; localization of cerebral function, $252 \mathrm{f}$.

Lotze, theory of local signs, $387,396 \mathrm{f}$., 451 ; on distinctions by the skin, 409 ; perception of magnitude by the eye, 451 ; errors of sense, 455 ; thcory of feeling, $499 \mathrm{f}, 510$; image of memory, $547 \mathrm{f}$; ; differences of the sexes, 573 ; kinds of temperament, $577 \mathrm{f}$.
Luchsinger, on reflexes of the cord, 138 , 141

Luciani, on localization of cerebral function, $269 \mathrm{f}, 285,288,301$

Luys, on basal ganglia, 129; attention and will, $5+4$; memory, 552.

MACH, on fusion of nervons shocks, 473

Magendie, discovery of, $123 \mathrm{f}$.

Materialism, views of, $607 \mathrm{f}$.

Mattencei, on electrotonus, 228

Mechanism, nervous system as, $4 \mathrm{f}$, $19 \mathrm{f}$., $198,214 \mathrm{f}$; the nerve as, 104 ; development of, 198; thcory of the nervous, $214 \mathrm{f}, 222 \mathrm{f}, 2: 26 \mathrm{f}$.

Medulla Oblongata, structure of, $74 \mathrm{f}$; tracts of white matter in, $76 \mathrm{f}$; gray matter in, 77 f.; nuclei of, 78 ; reflexmotor functions of, $146 \mathrm{f}$; as antomatic, $147 \mathrm{f}$; centres of, $147 \mathrm{f}$., 150 ; vaso-motor function of, 148

Meissner, calculation of the horopter, $43 \pi \mathrm{f}$.

Membranes, of the brain, $63 \mathrm{f}$.; the basilar, of Reissner, 191 ; Kïlliker, 194

Memory, reproduction of images of, 491 f., 54l; f.; physiological study of, $535 \mathrm{f}$; physical basis of, $545 \mathrm{f}$., $550 \mathrm{f}$; as retentive, $548 \mathrm{f}$; the organic, $550 \mathrm{f}$; as reproductive, $552 \mathrm{f}$; psychological nature of, $554 \mathrm{f}$.

Merkel, on reaction-time, 483 f., 486 , $495 \mathrm{f}$.

Mesencephalon, development of, $207 \mathrm{f}$.

Meynert, description of brain, $73,98 \mathrm{f}$., 246 ; on layers of cerebral cortex, 95 ; relation of brain to intelligence, 248 ; nerve-tracts in cerebrum, 283

Nind, subject of phenomena, $3 \mathrm{f}$, $585 \mathrm{f}$, $596 \mathrm{f}$.; relation to nervous mechanism, $235 \mathrm{f}$., $560 \mathrm{f}$., $579 \mathrm{f}$.; and to the brain, $247 \mathrm{f}$., 588 f., 59 : f., 605 f., 633 f.; synthetic act of, in perception, $388 \mathrm{f}$., 416 f., $462 \mathrm{f}$., $467,594 \mathrm{f}$; faculties of, 557 f., $600 \mathrm{f}$; physical explanations of, 593 f., $602 \mathrm{f}$., $62.5 \mathrm{f}$; as a unit-being, $596 \mathrm{f}$., $668 \mathrm{f}, 683 \mathrm{f}$; phenomena of. $597 \mathrm{f}$.; as a real being, $606 \mathrm{f}, 611$, 633 f., 656 , $668 \mathrm{f}$; d development of, $614 \mathrm{f}, 623 \mathrm{f}$; seat of, $634 \mathrm{f}$.; physical organs of, 640 $f . ;$ as a causc, $648 \mathrm{f}$.; spirituality of, $\cos 1 \mathrm{f}$.

Moldenhauer, on reaction-time of taste, 479 
Moos, on duration of the image of memory, 549

Motions, the Bodily, classes of, 526 f.; the impulsive, 527 ; the voluntary, $527 \mathrm{f}$, 530 ; the expressive, 5.31

Müller, G. E., on measurement of sensations, 368

Müller, J., on brain as measure of intelligence, 248

Munk, experiments of, $270 \mathrm{f}$; on localization of cerebral function, $2 \% 2$; motor areas of, $2 \% 2 \mathrm{f}$.; visual areas of, $286 \mathrm{f}$; auditory area of, 291

NAHLOWsKY, on nature of feeling, 502 ; kinds of feeling, $505 \mathrm{f}$.

Nerve-cells, chemistry of, 28 ; elements of nervons system, $30 \mathrm{f}$. ; kinds of, $31 \mathrm{f}$.; intimate structure of, $42 \mathrm{f}$; shapes of, $44 \mathrm{f}$; processes of, 44,70 ; size of, 45 ; as a typical element, $45 \mathrm{f}$.; number of, 46 ; functions of, $49 \mathrm{f}, 134,234 \mathrm{f}$; of the embryo, $200 \mathrm{f}$, 212

Nerve-commotion, causes of, 45 ; conditions of, $106 \mathrm{f}$., 12: ; phenomena of, 111 f., 230 ; nature of, 116 f., 122, 222 ; laws of, $118 \mathrm{f} ., 122,230$; speed of, $120 \mathrm{f}, 1: 2$ f. ; paths of, 122 f., $127 \mathrm{f}$., 261, 283 ; summation of, 223 f., $233 \mathrm{f}$. ; facilitation of, $224 \mathrm{f}$.

Nerve-fibres, chemistry of, 28; elements of nervous system, $30 \mathrm{f}$; kinds of, $34 \mathrm{f}$. ; size of, 34, $41 \mathrm{f}$. ; structure of the medullated, $35 \mathrm{f}$; fibrillated axiscylinder of, $38 \mathrm{f}$. ; origin of, $45 \mathrm{f}$; number of, 46 ; in the cord, $68 \mathrm{f}$., $123 \mathrm{f}$., 134 ; of the embryo, 212

Nerve-muscle machine, $104 \mathrm{f}$. ; behavior under electricity, 111 f. ; as a mechanism, $215 \mathrm{f}$.

Nerves, structure of, $33 \mathrm{f}$. ; general function of, 47 f., $54,59 \mathrm{f}$, $106 \mathrm{f}$. ; excitability of, $47,106 \mathrm{f}$., $353 \mathrm{f}$.; conductivity of, $47,60,102-122,118 \mathrm{f}$., 120 ; kinds of, $51 \mathrm{f}$., $60,353 \mathrm{f}$; afferent, $52 \mathrm{f}, 120$; efferent, 52 f., 120 ; the cranial, $100 \mathrm{f}$.; the encephalic, $100 \mathrm{f}$; exhaustion of, $108 \mathrm{f}$.; mechanical properties of, 109 ; thermic influences upon, $110 \mathrm{f}$; chemical influences on, 111 ; processes in, $117 \mathrm{f}$; specific energy of, $300 \mathrm{f}$., $307 \mathrm{f}$. $353 \mathrm{f}$.

Nervous Matter, kinds of, 22 f.; specific gravity of, $22 \mathrm{f}$.
Nervous System, a mechanism, $4 \mathrm{f}, 19 \mathrm{f}$, 198 f., 214 f., 222 f., 226 f. ; general function of, $18 \mathrm{f}$., $57,219 \mathrm{f}$; elements of, 21 f., $30 \mathrm{f}$., $216 \mathrm{f}$.; chemistry of, $21 \mathrm{f}$., $217 \mathrm{f}$.; cells and fibres in, $30 \mathrm{f}$; structure of, 56-101; plan of, $5 \% \mathrm{f}, 219 \mathrm{f}$; sets of organs in, $59 \mathrm{f}$.; the sympathetic, $60 \mathrm{f}$.; the cerebro-spinal, $60 \mathrm{f}$; development of the, $195 \mathrm{f}$; inertia of, $472 \mathrm{f}$.

Neuclein, $24 \mathrm{f}$.

Neuroglia, nature of, $31 \mathrm{f}$.

Neurokeratin, 24

Nothnagel, on striate bodies, 159; fineness of temperature-sense, $369 \mathrm{f}$.

Nuclei of nerve-cells, 43 ; of the medulla, 78 ; of the corpus striatum, $86,88,90$, 159 ; of the tegmentum (red nucleus), 88,89

Nystagmus, $153 \mathrm{f}$.

OLrves, the, 75,78 ; functions of, 150

Optic Thalami, position of, $86 \mathrm{f}$; structure of, $89 \mathrm{f}$.; connections of, $90,127 \mathrm{f}$.; cells and fibres of, 90 ; paths in, 129 ; functions of, $158 \mathrm{f}$. ; development of, $208 \mathrm{f}$

Organ of Corti, $193 \mathrm{f}$.

Organs, kinds in nervous system, 59 f. ; the central, $60,73 \mathrm{f}$; functions of, $130 \mathrm{f} ., 224 \mathrm{f}$.

Ott, on centre of temperature, 161

Pacini, corpuscles of, 169

Paneth, on excitation of cerebral cortex, $269 \mathrm{f}$.

Papillæ, circnmvallatæ, 166 f.; fungiformes, $166 \mathrm{f}$.

Peduncles, of the cerebellum, 79 ; of the cerebrum, 82, $87 \mathrm{f}, 97 \mathrm{f}$

Perception, nature of, 382 f., 462 f., 467 , $53 \mathrm{f}$.; nativistic and empiristic theories of, $399 \mathrm{f}$.; by smell, $402 \mathrm{f}$.; taste, 403; hearing, $403 \mathrm{f}$; t touch, $405 \mathrm{f}$; of motion, $411 \mathrm{f}, 452 \mathrm{f}$; by temperature, $413 \mathrm{f}$; of sight, $421 \mathrm{f}, 440 \mathrm{f}$., $448 \mathrm{f}$.; of depth, 441 f., 459, 464; of spatial relations, 448 f., 464 f.; development of, $462 \mathrm{f}$; ; physical basis of, $538 \mathrm{f}$.

Ptuger, table from, 113; law of, $113 \mathrm{f}$, 115 ; on reflexes of the cord, $137 \mathrm{f}$.

Physiological Psychology, definition of, $1 \mathrm{f}$., $4 \mathrm{f}$.; combines two sciences, $6 \mathrm{f}$; divisions of, $8 \mathrm{f}$; method of, $9 \mathrm{f}$., 12, 53: ; claims of, 13 ; successes of, $304 \mathrm{f}$., 
532 f., 532 f.; theory of perception of, $382 \mathrm{f}$; limits of, $532 \mathrm{f}$.

Physiology, relation to psychology, $1 \mathrm{f}$.; of nerves in general, $103 \mathrm{f}$.

Pia Mlater, structure of, 64

Pons Varolii, $\pi 4$; structure of, $81 \mathrm{f}$.

Presentations of Sense, elements of, 304 f., $383 \mathrm{f}$, $46 \mathrm{f}$.; process of construction of, $382 \mathrm{f}$., 387 f., $416 \mathrm{f}$., $448 \mathrm{f}$; space-form belonging to, $385 \mathrm{f}$., $391 \mathrm{f}$., 448 f.; synthesis of, 386 f., 416 f., 467 ; analysis of, $388 \mathrm{f}$., 595 ; nativistic theory of, $389 \mathrm{f}$; empiristic theory of, 389 $\mathrm{f}$; stages of, $400 \mathrm{f}$; by smell, $402 \mathrm{f}$; taste, 403 ; hearing, $403 \mathrm{f}$; by touch, 405 f.; by sight, 421 f., 433 f., 443 f.; time-relations of, $468 \mathrm{f}$; assumptions entering into, 594

Pressure, sensations of, 345 f., 367 f.; spots of, 346

Preyer, on sensitiveness to pitch, 319 f.; fusion of nervons shocks, $4 \% 2$; sensations of new-born child, 560

Protagon, 25 f.

Psychology, conception of, $2 \mathrm{f}$.; method of, $9 \mathrm{f}$., $587 \mathrm{f}$., $605 \mathrm{f}$.; classifications of, $587 \mathrm{f}, 605 \mathrm{f}$.

Psychometry, method of, $469 \mathrm{f}$.; elements of time in, $470 \mathrm{f}$; ; results of, 497

Psycho-physics, Fechner's conception of, $12,380 \mathrm{f}$; method of, $359,361 \mathrm{f}$., 365 $\mathrm{f}$; t the laws of, $359 \mathrm{f}$; $365 \mathrm{f}$; $379 \mathrm{f}$; of sensations of touch, $36 \mathrm{f}$; ; of sound, 370 ; of light, $373 \mathrm{f}$; of smell and taste, $376 \mathrm{f}$.

Purkinje, cells of, 80

Pyramidal tract, 71 f., 77,97

QUETELET, proportions of human body, 566 f., 574

Ranvier, nodes of, $36 \mathrm{f}$., $40 \mathrm{f}$; ; on structure of ganglion-cell, 43

Reaction-time, natnre of, $455 \mathrm{f}$.; influences upon, $476 \mathrm{f}$., $495 \mathrm{f}$.; methods of determining, $479 \mathrm{f}$.; complex processes of, 491

Reflex action, 50 f., 130 f.; kinds of, 131 ; in spinal cord, $131 \mathrm{f} ., 136 \mathrm{f}$; conditions of, $134 \mathrm{f}$., $136 \mathrm{f}$.; speed of, $135 \mathrm{f}$; in the brain, $143 \mathrm{f}$., $224 \mathrm{f}$.

Regio olfactoria, 164 f., 308 f.

Reissner, membrane of, 191

Remak, fibres of, 34,41
Retina, the, $172 \mathrm{f.}$; problem solved by, $174 \mathrm{f} ., 178 \mathrm{f}$., $183 \mathrm{f}$; layers of, $179 \mathrm{f}$, $183 \mathrm{f}$.; nervous elements of, $180 \mathrm{f}$, 326 f; rods and cones in, 181 f., 327 ; own light of, 326 ; relation of, to sight, 335 , $423 \mathrm{f}$; f field of, $423 \mathrm{f}$.; identical and corresponding points of, $43 \pm f$.

Ribot, on physiological study of concepts, 5:2; and of memory, $552 \mathrm{f}$.

Ritter, on sensations of smell, 309

Rolando, funiculus of, $\pi \tilde{\tau}$; tubercle of, 7\% ; fissure of, 92, 267, 282

Romieu, on stimulus of smell, 311

Rosenthal, on speed of reflex action, 135 f.; electrical taste, $\mathbf{3 1 3}$

SCHAFHÃTtL, on limits of sound, $372 \mathrm{f}$.

Schiff, on posterior columns of cord, 125 $f$; on excitability of cord, $141 \mathrm{f}$.; on cerebellum, 153, $155 \mathrm{f}$; t temperature of brain, 242; localization of cerebral function, $273 \mathrm{f}$., $283 \mathrm{f}$.

Schultze, Hans, on structure of axiscylinder, $39 \mathrm{f}$.

Schnltze, Max, on varieties of nervefibres, $34 \mathrm{f}$.; and structure of nervecell, $42 \mathrm{f}$.; on olfactory cells, $164 \mathrm{f}$;; auditory cells, 192

Schwann, sheath of, 36 ; substance of, 36 Sclerotic, the, 171

Seguin, on cases of aphasia, 295

Semicircular canals, the, $189 \mathrm{f}$.

Sensations, end-organs of, $164 \mathrm{f}$.; analysis of auditory, 195, 324 ; quality of, $303 \mathrm{f}$., $325 \mathrm{f}$.; simple, $305 \mathrm{f}$; conditions of, $307 \mathrm{f}$;; of smell, $308 \mathrm{f}$., $3 \pi \mathrm{f}$; ; of taste, $311 \mathrm{f}$., $376 \mathrm{f}$.; of sound, $315 \mathrm{f}$, $370 \mathrm{f}$; of sight, $325 \mathrm{f}$., $373 \mathrm{f}$.; theory of the visual, $335 \mathrm{f}$; 保 temperature, $344,369 \mathrm{f}$; of pressure, $344,345 \mathrm{f}$., $36 \% \mathrm{f}$; the muscular, $344 \mathrm{f}$; quantity of, $356 \mathrm{f}$; measurement of, $359 \mathrm{f}$., $364 \mathrm{f}$, $369 \mathrm{f}$; ; least observable difference in, $361 \mathrm{f}$., $364 \mathrm{f}$; range of, $362 \mathrm{f}$.; spatial series of, $356 \mathrm{f}$, $393 \mathrm{f}$.; localization of, $387 \mathrm{f}$., $405 \mathrm{f}$.

Senses, organs of the, 164 f.; classification of the, $303 \mathrm{f}$.; the geometrical, 386 $f$; errors of the, $455 \mathrm{f}$.

Setschenow, on inhibitory centres, 144.

Sight, end-organs of, $1 \pi 1 \mathrm{f}, 1 ; 4 \mathrm{f}$, $338 \mathrm{f}$; photo-chemistry of, $178 \mathrm{f}$., $184 \mathrm{f}, 326$; sensations of, $325 \mathrm{f}$.; stimulus of, $325 \mathrm{f}$; after-imsges of, $336 \mathrm{f}$.; elements in perception of, $420 \mathrm{f}$., $447 \mathrm{f}$; motion of eye 
in, 428, $431 \mathrm{f}$.; single and double images in, $434 \mathrm{f}$, $438 \mathrm{f}$; stereoscopic and perspective, $440 \mathrm{f}$; ; secondary helps of, 443 f., $455 \mathrm{f}$.

Smell, organs of, 164 f., 308 f.; nerve of, 165,310 ; stimulus of, $165 \mathrm{f} ., 308 \mathrm{f}$., 310 ; sensations of, $308 \mathrm{f}$; kinds of, 310 ; measurement of, $378 \mathrm{f}$; perceptions of, $402 \mathrm{f}$.

Soul, see Mind

Sound, analysis of, 195 f., 324; sensations of, $315 \mathrm{f}$., $370 \mathrm{f}$; kinds of, 316 ; nature of the musical, $316 \mathrm{f}$; limits of, $31 \%, 371 \mathrm{f}$; " entotic," $403 \mathrm{f}$; d direction of, 404

Spinal cord, membranes of, $61 \mathrm{f}$; structure of, $64 \mathrm{f}, 143,207$; fissures of, $64 \mathrm{f}$.; columns of, $66,67,125 \mathrm{f}$; commissurcs of, 66 ; horns of, 67 ; white substance of, 68 ; nerve-fibres in, 68 f., 123 f; gray substance of, $69 \mathrm{f}$; nervous tracts in, $71 \mathrm{f}$., $123 \mathrm{f}$; as mechanism, 72,122 , 133 f., 144; nerves from, 100 f. ; nervous processes in, 122, $134 \mathrm{f}$; roots of, $123 \mathrm{f}$. 207 ; as a central organ, 132 f., $138 \mathrm{f}$; antomatism, $138 \mathrm{f}$; " centres" of, 140 f.; excitability as a whole, $141 \mathrm{f}$.; " "xthesodic" and "kinesodic," 142; influence of brain on, $143 \mathrm{f}$; development of, 207

Stimulus, kinds of, 48 ; heat as, $110 \mathrm{f}$; electricity as, 111 f., 312 f.; of smell, $165 \mathrm{f}$., $308 \mathrm{f}, 376 \mathrm{f}$.; of taste, $311 \mathrm{f}$., $376 \mathrm{f}$; of hearing, $315,3 \% 0 \mathrm{f}$; of sight, $325 \mathrm{f}$., $328 \mathrm{f}$, $373 \mathrm{f}$; measurement of, 359 f., 367 f.; limits of, 362 f., 367 f.

Strabismus, $153 \mathrm{f}$.

Stricker, on common feeling, 513

Stumpf, on judgment of tone, 320

Substantia gelatinosa, 69

Substantia nigra, 87

Snlci, of the cerebrum, $84,91,92 \mathrm{f}$.; development of, 210

Sully, on tone of feeling, 511

Suspensory ligament, 173 ; function of, $17 \% \mathrm{f}$.

Sympathetic System, structure of, $60 \mathrm{f}$.

Talbot, the principle of, $4 \pi 3$

Taste, end-organs of, $166 \mathrm{f}, 313$; nerve of, 168, 314; sensations of, $311 \mathrm{f}$., $376 \mathrm{f}$. ; stimulus of, $312 \mathrm{f}$; kinds of, 314 ; measurement of, $376 \mathrm{f}$. ; perceptions of, $403 \mathrm{f}$.
Tegmentum, see Crura Cerebri

Temperament, theory of, 575 f., 579; kinds of, $575 \mathrm{f}$; physical basis of, 579

Temperature, sensations of, $344,348 \mathrm{f}$, $369 \mathrm{f}$; after-images of, $351 \mathrm{f}$.; measurement of, $369 \mathrm{f}$; sense of locality by, $413 \mathrm{f}$.

Thalamen-cephalon, 208

Things, distinguished from sensations, $3.59 \mathrm{f}, 38 \%$, 594 ; results of mental synthesis, $594 \mathrm{f}$., 609 ; unity of, $609 \mathrm{f}$.

Thudichum, on chemistry of brain, 27

Tischer, on Weber's law, 372

Tones, the mnsical, 316 ; pitch of, $317 \mathrm{f}$; table of, 318; sensitiveness to, 319 , $3 \% 0 \mathrm{f}$; purity of, $319 \mathrm{f}$. ; judgments of, 320 ; relations of, $322 \mathrm{f}$.

Touch, kinds of, $168,345 \mathrm{f}$; end-organs of, $165 \mathrm{f}$. ; sensations of, $345 \mathrm{f}$., $367 \mathrm{f}$; perceptions of, $405 \mathrm{f}$; the field of, 406 $\mathrm{f} ., 416 \mathrm{f}$.

Trautscholdt, reaction-time of complex processes, $491 \mathrm{f}$; on effect of practice, 496

Türck, method of, 71 ; on columns of cord, 126

Tympanum, the, 186 f. ; membranes of, $156,155 \mathrm{f}$. ; windows of, 186 ; muscles of, 187 ; office of, $15 \% \mathrm{f}$.

Tyndall, on stimulus of smell, $311 \mathrm{f}$.

Valextis, on nervous excitation, $2: 3 \mathrm{f}$; sensations of smell, $30 \mathrm{f} \mathrm{f}$; of taste, $314,37 \% \mathrm{f}, 403$; on sense of locality, $406 \mathrm{f}$; fusion of nervous shocks, $47 \%$

Valli, principle of, 107

Vestibule, of the ear, 189

Vierordt, on measurement of sensation, $3 \pi 1 \mathrm{f}$. ; localization by touch, $409 \mathrm{f}$. ; subjectire estimate of time, $4 \mathrm{~S} 8 \mathrm{f}$.

Vitreous Humor, the, 173

Volkmann. A. W., on measurement of sound, $3 \% 1$; of light, $3 \% 4$; of length of lines, 376 ; on sense of locality, 407

Volkmann von Volkınar, on motifs of monocular vision, 420 ; nature of fecling, 503

Volta, on electrical taste, $312 \mathrm{f}$.

Von Gudden, on optic chiasm, 290

Von Kries, on the number of colors, 332 ; on theories of color-sensations, $341 \mathrm{f}$; sense of locality, $39 \%$

Von Kries and Auerbach, on sense of 
locality, 397 ; reaction-time, 476, $481 \mathrm{f}$., Wertheim, on ductility of nerves, 109 48\%, 496

Von Vintschgau, on conduction in nerves, 121 ; reaction-time for multiplying, 493

Von Wittich, on fusion of nervous shocks, 472 ; reaction-time of taste, 478

Vulpian, on nervous function, 54 ; on excitability of cord, 142 ; centres of the medulla, 149 ; and cerebellum, 154 f.

WAGNER (H, and R.), corpuscles of, 170 ; on measurement of brain-mass, 247; effect of fear, 518

Waller, method of, $107 \mathrm{f}$.

Weber, E. H., on temperature-sensations, $110,347,351$; smell, 308 ; law of, 365 f., $368 \mathrm{f}$., $374 \mathrm{f}$., $378 \mathrm{f}$; on dircetion of sound, 404 ; perceptions of touch, 405 f.; "sensation-circles" of, 406 f.; measuring power of the eye, 452

Will, effect of, on bodily motions, $528 \mathrm{f}$;; physiological study of, $535 \mathrm{f}$; physical basis of, $536 \mathrm{f}$.; in attention, $539 \mathrm{f}$.

Wundt, on columns of the cord, 126 ; on cerebellum, 152 ; optic thalami, $158 \mathrm{f}$; and striate bodies, 160 ; mechanical theory of, $231 \mathrm{f}$.; kinds of taste, 314 ; theory of color-sensations, 341 ; on complementary colors, 343 ; theory of apperception, 380, $539 \mathrm{f}$; ; on Weber's law, 381 ; on theories of perception, $389 \mathrm{f}$.; "sensation-circles," 408; visual perception, 422, 425 f., 451; judgment of distance, 433; feelings of innervation, $439,451,524$; psycho-physical time, $471,477,483 \mathrm{f}$., 488,496 ; curve of feeling, 514 ; theory of temperament, $576 \mathrm{f}$.

ZoNuLE of Zinn, 173 





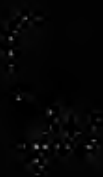

$\therefore \cdot 3$ 